\title{
Scalable Approach to Fluorinated Heterocycles with Sulfur Tetrafluoride $\left(\mathrm{SF}_{4}\right)$
}

Serhii Trofymchuk, ${ }^{\dagger, 1,2}$ Maksym Bugera, ${ }^{\dagger, 1,3}$ Anton A. Klipkov, ${ }^{1,3}$ Volodymyr Ahunovych, ${ }^{1,3}$ Bohdan Razhyk, ${ }^{1}$ Sergey Semenov, ${ }^{1}$ Andrii Boretskyi,${ }^{4}$ Karen Tarasenko, ${ }^{3,5}$ Pavel K. Mykhailiuk ${ }^{1 *}$

${ }^{1}$ Enamine Ltd., Chervonotkatska 78, 02094, Kyiv, Ukraine, www.enamine.net, www.mykhailiukchem.org

${ }^{2}$ Institute of Organic Chemistry NAS of Ukraine, Murmanska 5, 02094 Kyiv, Ukraine

${ }^{3}$ V.P. Kukhar Institute of Bioorganic Chemistry and Petrochemistry NAS of Ukraine, Murmanska 1, 02094 Kyiv, Ukraine

${ }^{4}$ UORSY, Ukrorgsyntez Ltd, PO Box 59, 02002 Kyiv, Ukraine

${ }^{5}$ Chemspace LLC, 85 Chervonotkatska Street, Suite 1, 02094 Kyiv, Ukraine

${ }^{\dagger}$ Equal contribution

Supporting Information

Contents

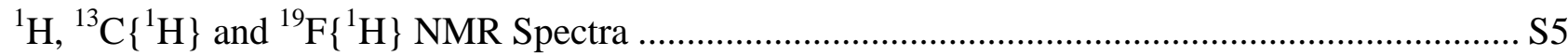

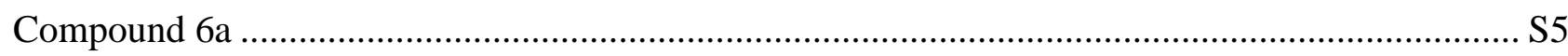

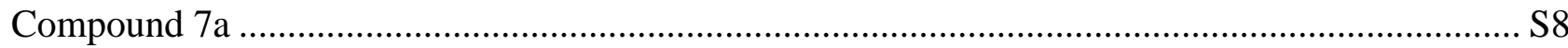

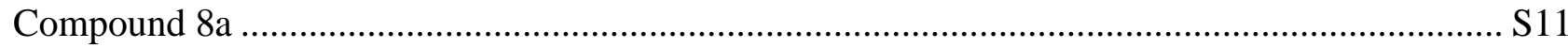

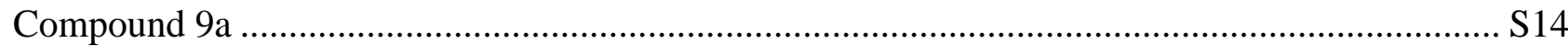

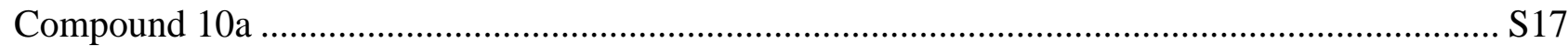

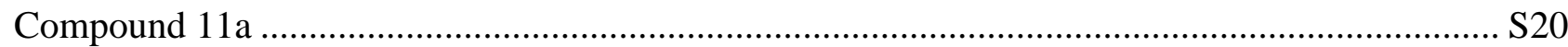

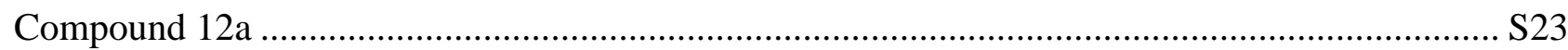

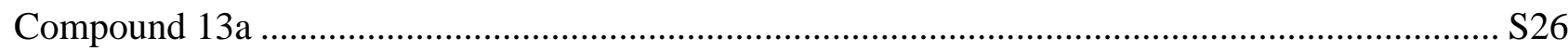

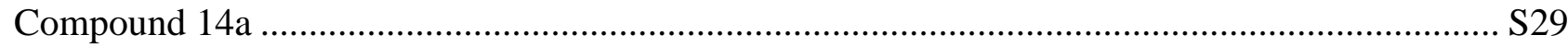

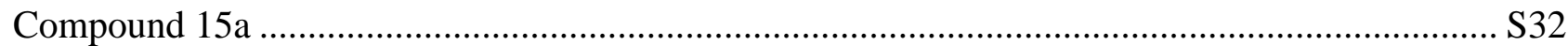

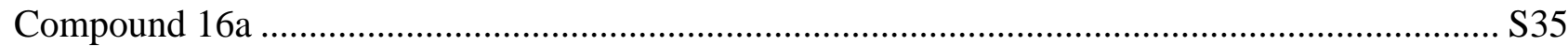

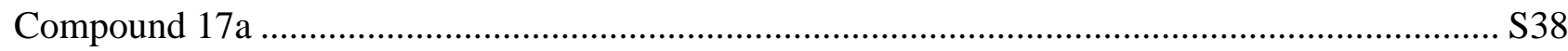


Compound 19a

Compound 20a

Compound 21a

Compound 22a S53

Compound 23a S56

Compound 24a S59

Compound 25a S62

Compound 26a S65

Compound 27a S68

Compound 28a S71

Compound 29a S74

Compound 30a S77

Compound 31a S80

Compound 32a S83

Compound 33a S86

Compound 34a S89

Compound 35a S92

Compound 40a S95

Compound 41a S98

Compound 42a S101

Compound 43a S104

Compound 44a S107

Compound 45a S110

Compound 46a S113

Compound 47a S116

Compound 48a S119

Compound 49a S122

Compound 50a S125

Compound 51a S128

Compound 52a S131

Compound 53a S134

Compound 54a S137

Compound 55a S140

Compound 56a S143 
Compound 57a S146

Compound 58a S149

Compound 59a S152

Compound 60a S155

Compound 61a S158

Compound 62a S161

Compound 63a S164

Compound 64a S167

Compound 65a S170

Compound 66a S173

Compound 67a S176

Compound 69 S179

Compound 70 S182

Compound 71 S185

Compound 72 S188

Compound 73 S191

Compound 74 S194

Compound 75 S197

Compound 76 S200

Compound $40 \mathrm{~b}$ S203

Compound $44 \mathrm{~b}$ S206

Compound $49 \mathrm{~b}$ S209

Compound 58b S212

Compound 61b S215

Compound $63 \mathrm{~b}$ S218

Compound 7c S221

Compound 11c S224

Compound 15c S227

Compound 19c S230

Compound 31c S233

Compound 42c S236

Compound $45 \mathrm{c}$ S239

Compound $48 \mathrm{c}$ S242

Compound 50c S245

Compound 62c S248 


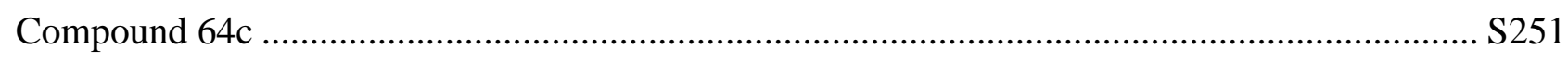

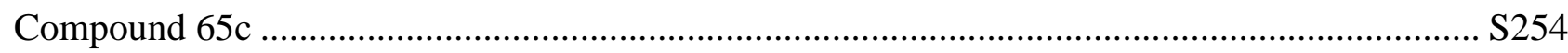

Compound 9d* $\mathrm{d}$

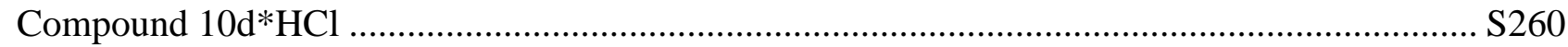

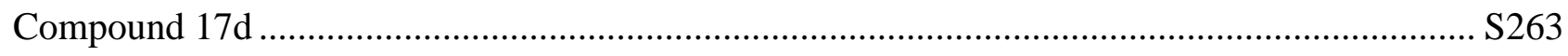

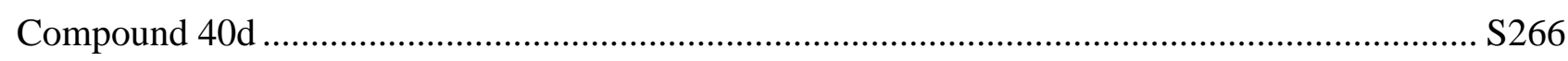

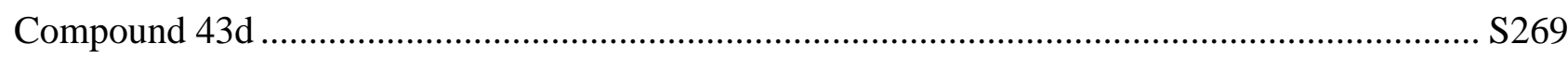

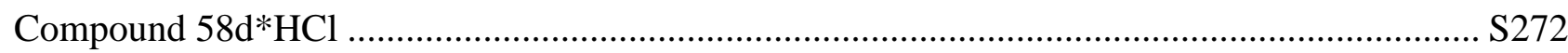

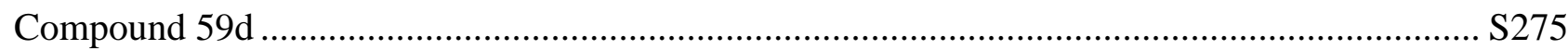

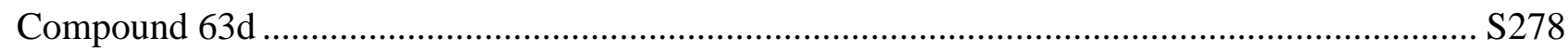

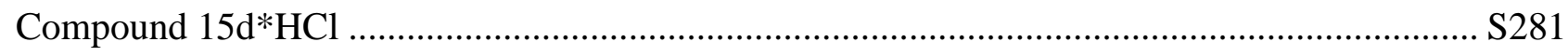

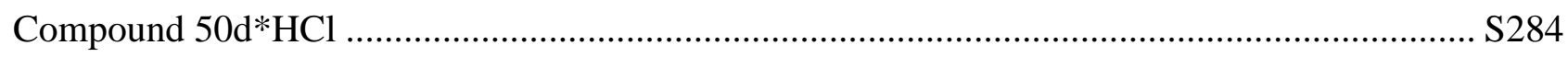

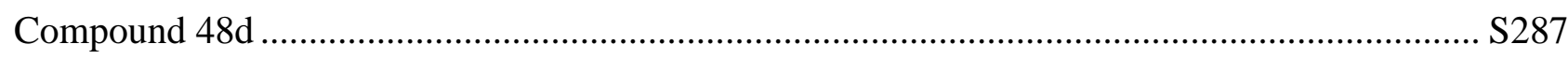

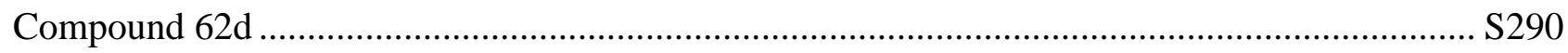

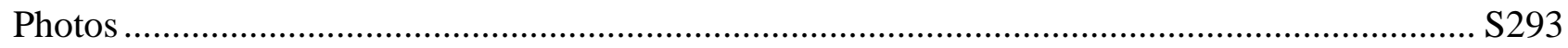


${ }^{1} \mathrm{H},{ }^{13} \mathrm{C}\left\{{ }^{1} \mathrm{H}\right\}$ and ${ }^{19} \mathrm{~F}\left\{{ }^{1} \mathrm{H}\right\}$ NMR Spectra

\section{Compound 6a}

${ }^{1} \mathrm{H}$ NMR (400 MHz, $\mathrm{CDCl}_{3}$ )

IGMA5559

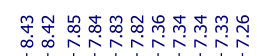

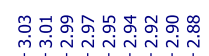

혹ㅇำ

$\overbrace{\mathrm{Br}}^{\mathrm{Fe}} \mathrm{Me}$

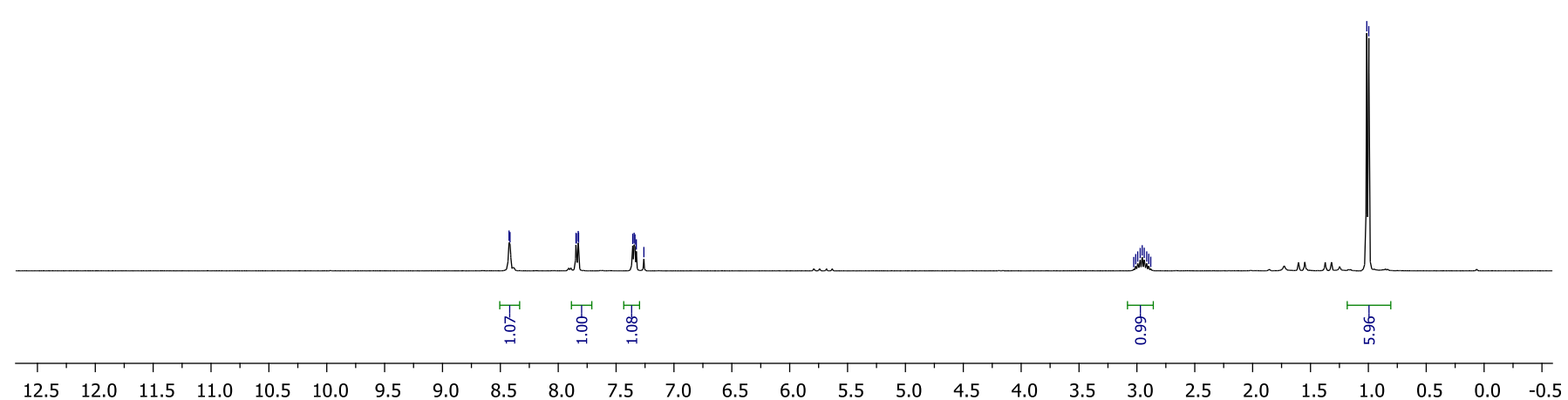


${ }^{13} \mathrm{C}\left\{{ }^{1} \mathrm{H}\right\} \mathrm{NMR}\left(126 \mathrm{MHz}, \mathrm{CDCl}_{3}\right)$

igma5569_C13

13C (1H-decoupled)

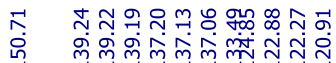

苟

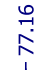

ฉซ్

总是疋

年
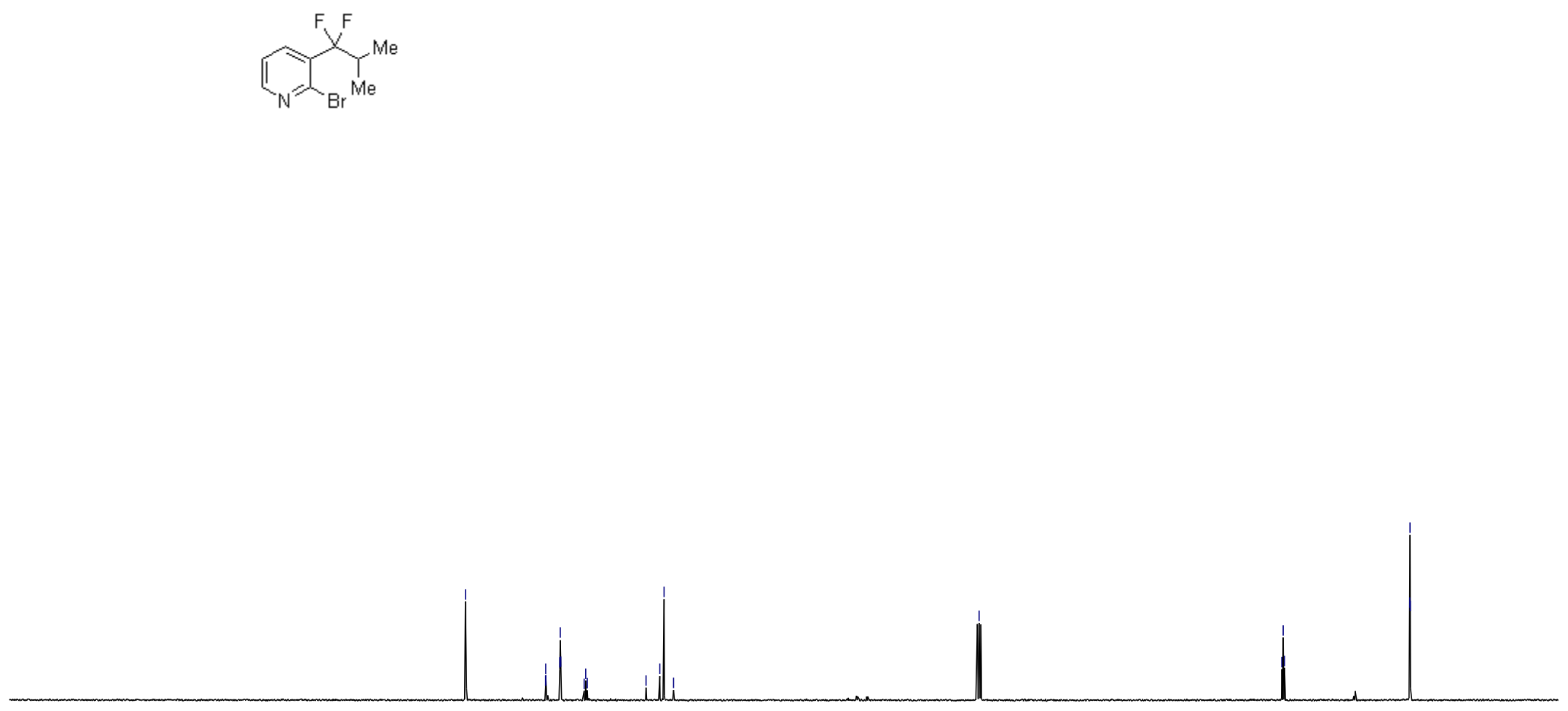

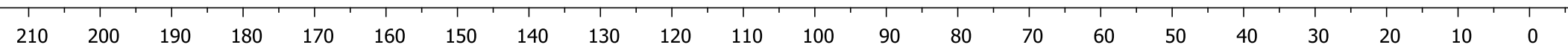


${ }^{19} \mathrm{~F}\left\{{ }^{1} \mathrm{H}\right\}$ NMR (470 MHz, $\left.\mathrm{CDCl}_{3}\right)$

igma5559_F19

$\underset{0}{0}$
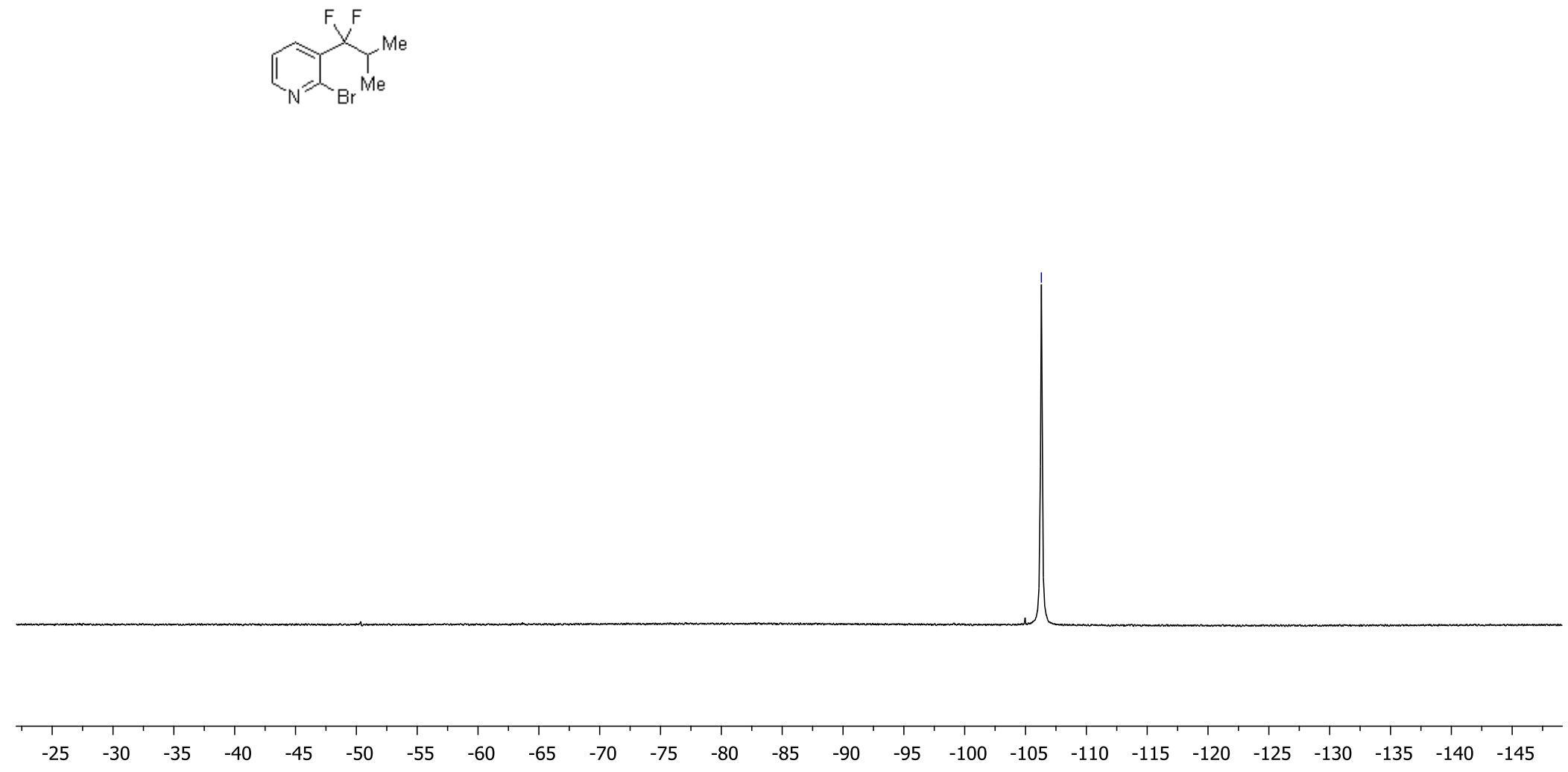

S7 


\section{Compound 7a}

${ }^{1} \mathrm{H}$ NMR $\left(400 \mathrm{MHz}, \mathrm{CDCl}_{3}\right)$

R1214738

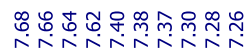

ㄱำ

$\overbrace{\mathrm{Me}}^{\mathrm{Fr}}$

11

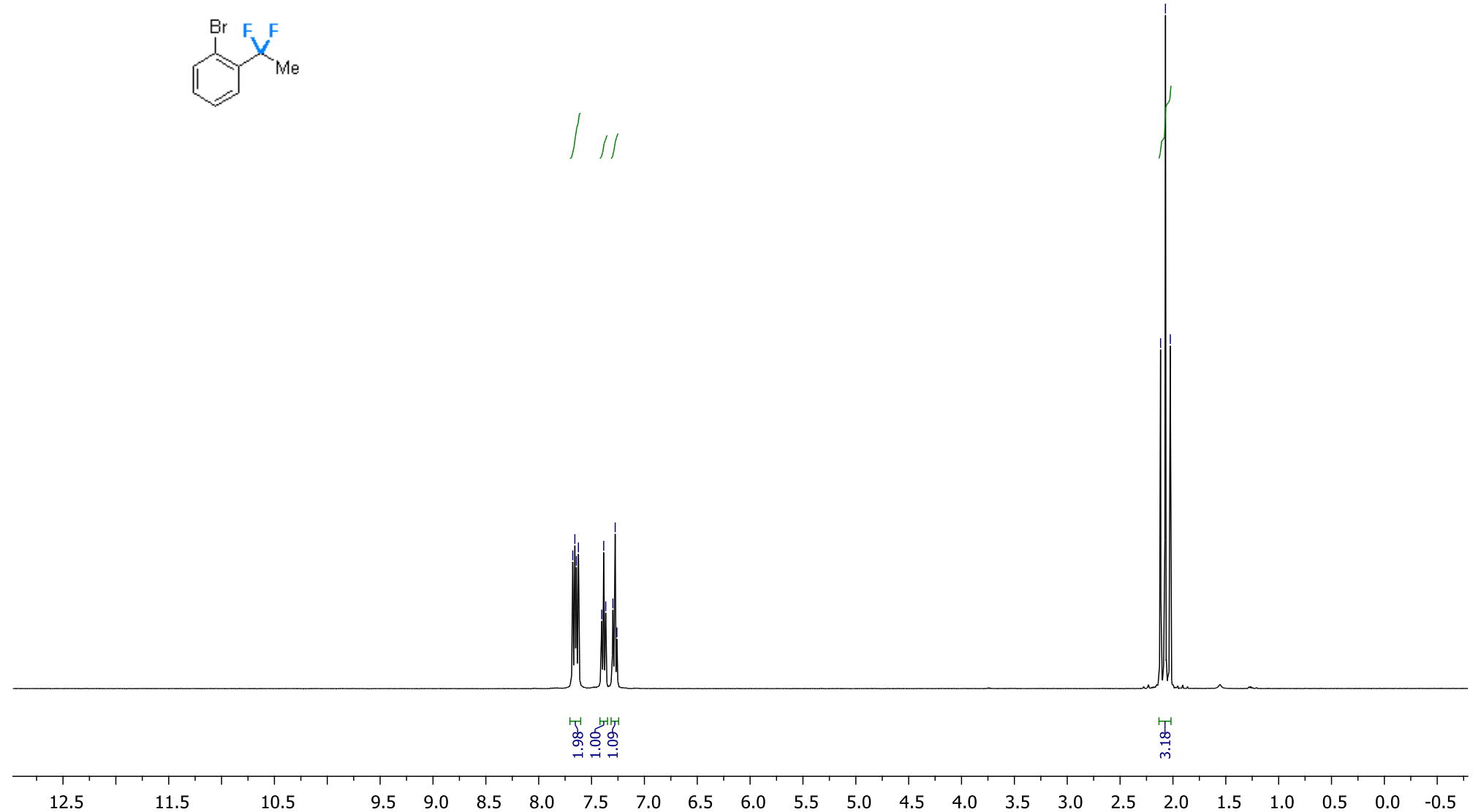


${ }^{13} \mathrm{C}\left\{{ }^{1} \mathrm{H}\right\}$ NMR $\left(101 \mathrm{MHz}, \mathrm{CDCl}_{3}\right)$

R1214738_C13

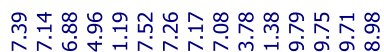

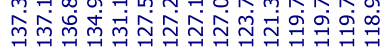

$\stackrel{0}{\stackrel{2}{*}}$

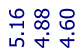

난

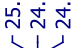

$\left.{ }^{B r}\right)_{M e}^{F}$

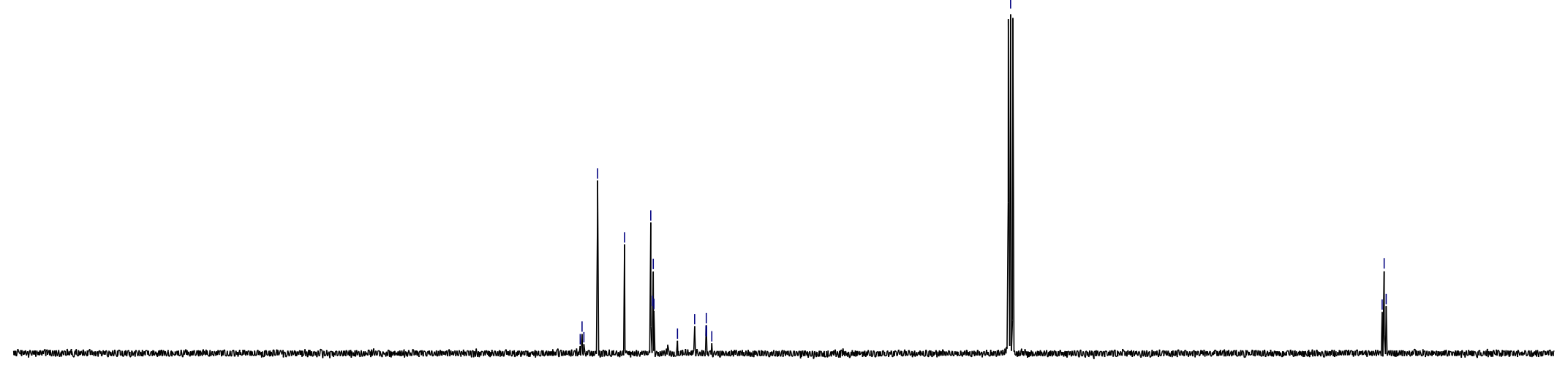

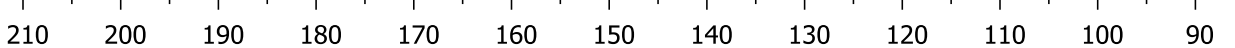

80

70

$60 \quad 50$ 


\section{${ }^{19} \mathrm{~F}\left\{{ }^{1} \mathrm{H}\right\} \mathrm{NMR}\left(376 \mathrm{MHz}, \mathrm{CDCl}_{3}\right)$}

R1214738_F19 $\{\mathrm{H}\}$ $19 \mathrm{~F}\{1 \mathrm{H}\}$
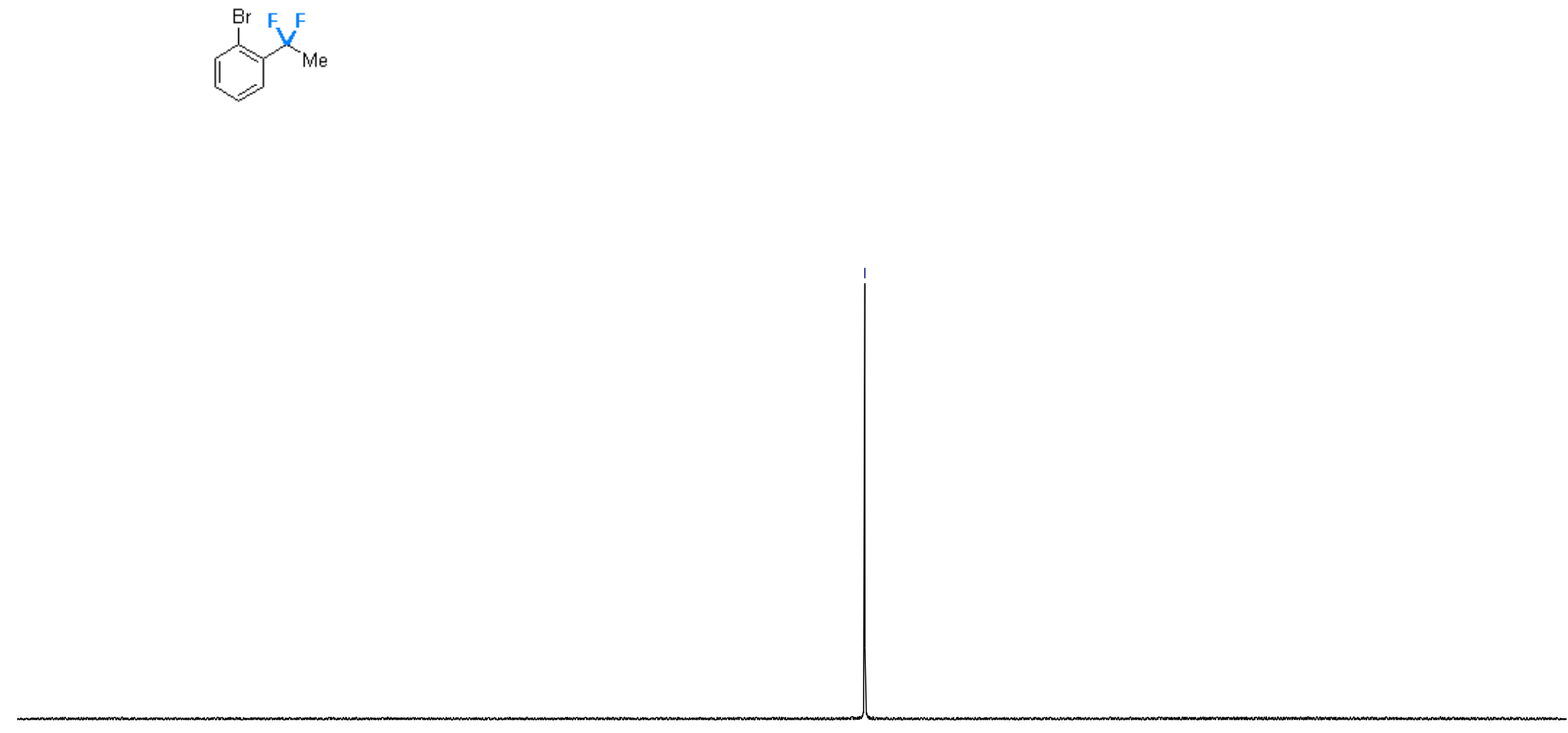


\section{Compound 8a}

${ }^{1} \mathrm{H}$ NMR (400 MHz, $\mathrm{CDCl}_{3}$ )

R2485569

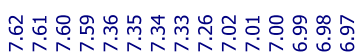

ํํำ

1

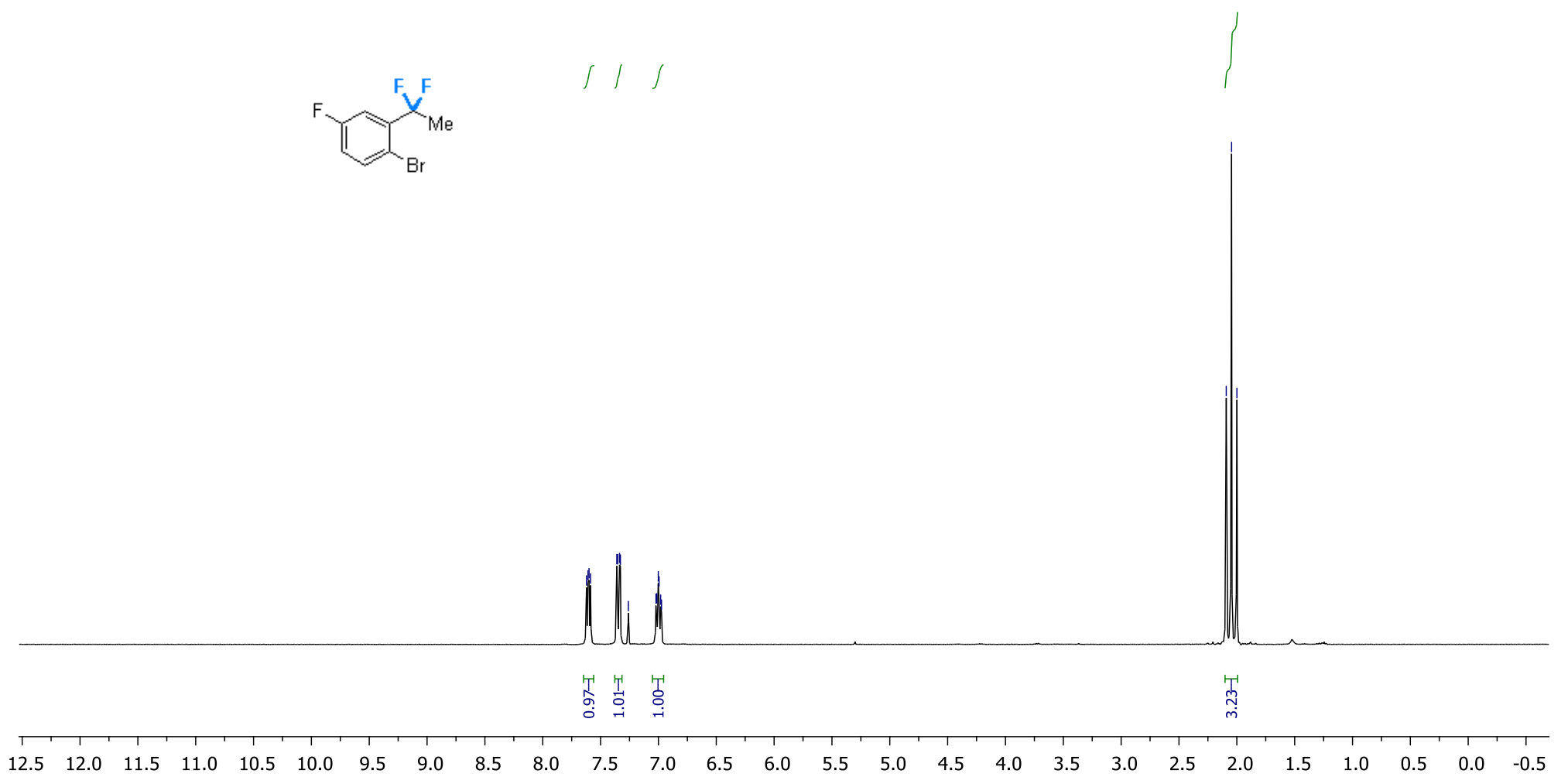


${ }^{13} \mathrm{C}\left\{{ }^{1} \mathrm{H}\right\} \mathrm{NMR}\left(126 \mathrm{MHz}, \mathrm{CDCl}_{3}\right)$

R2485569_C13

$13 \mathrm{C}$ (1H-decoupled)

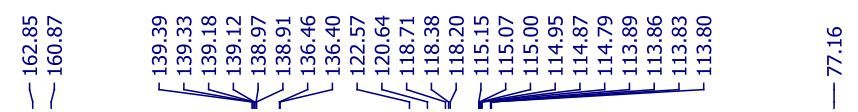

每

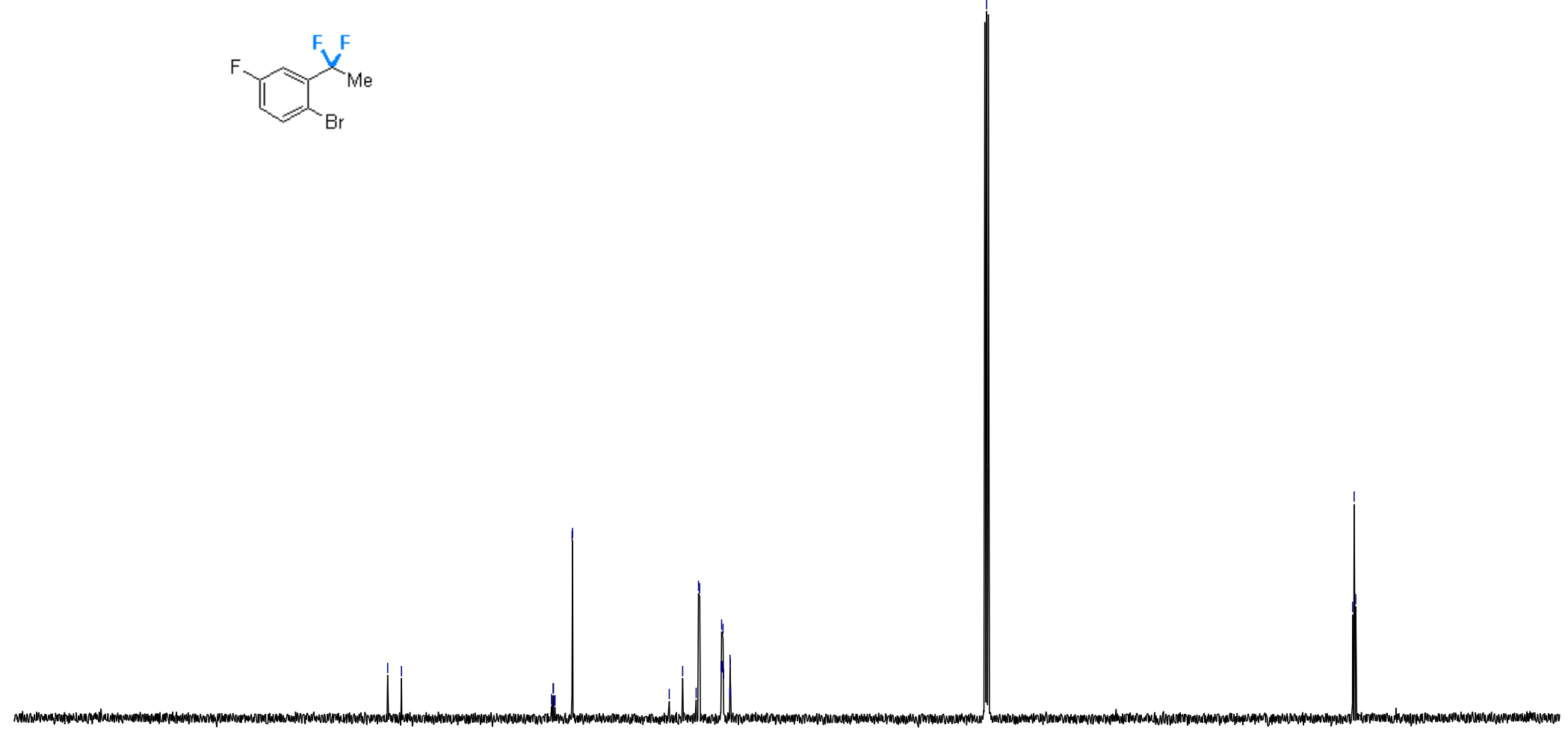

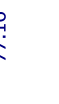

$\sqrt[n]{\operatorname{din}}$

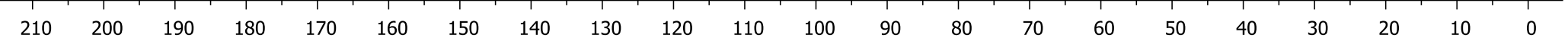




\section{${ }^{19} \mathrm{~F}\left\{{ }^{1} \mathrm{H}\right\}$ NMR (376 MHz, $\left.\mathrm{CDCl}_{3}\right)$}

R2485569_F19 $\{\mathrm{H}\}$

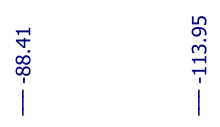
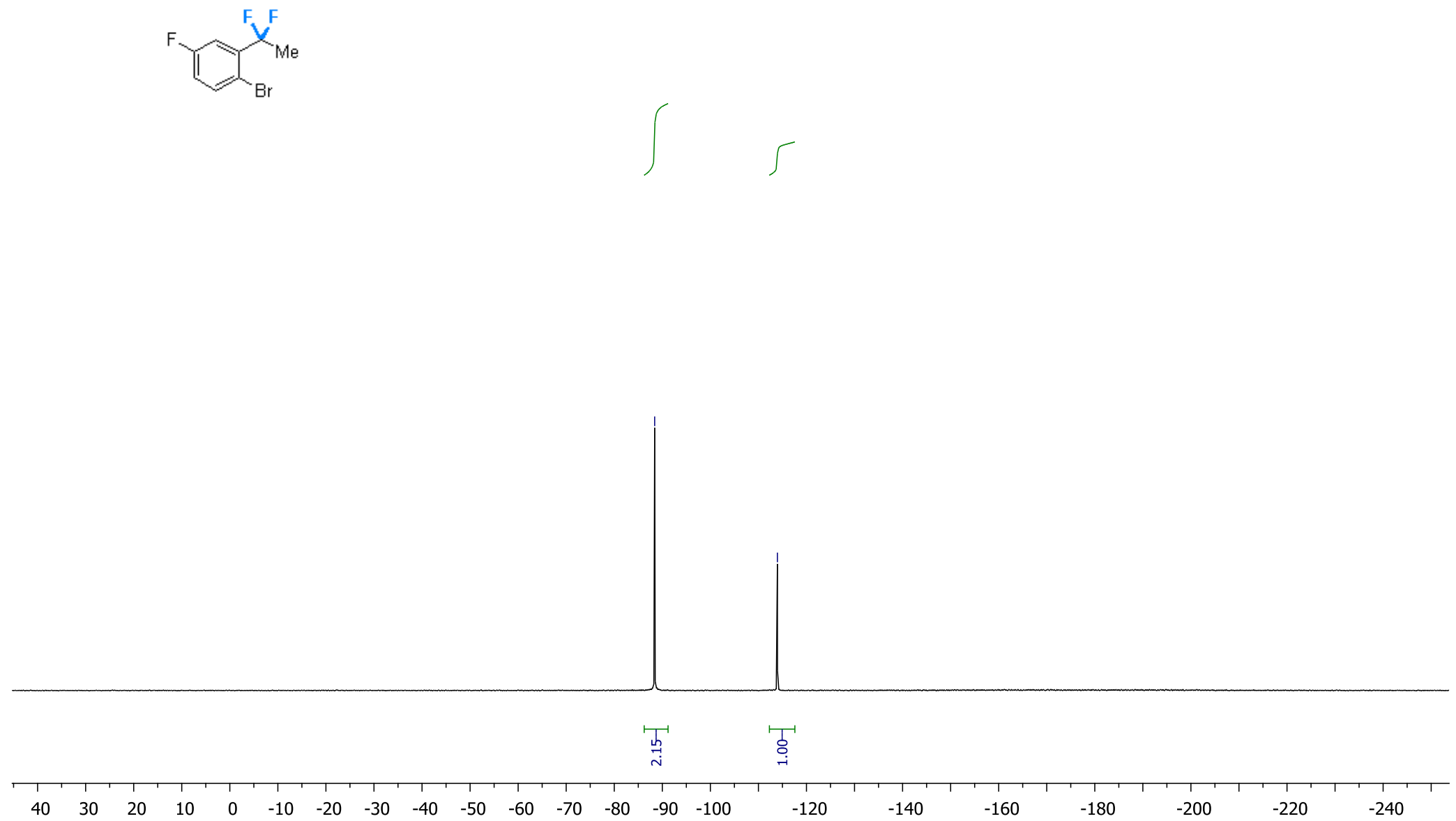


\section{Compound 9a}

${ }^{1} \mathrm{H}$ NMR (400 MHz, DMSO- $d_{6}$ )

R711986

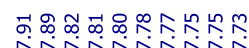

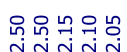

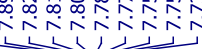

Y \ا।

$\overbrace{\mathrm{Me}}^{\mathrm{NO}_{2} \mathrm{~F}^{\mathrm{F}}}$

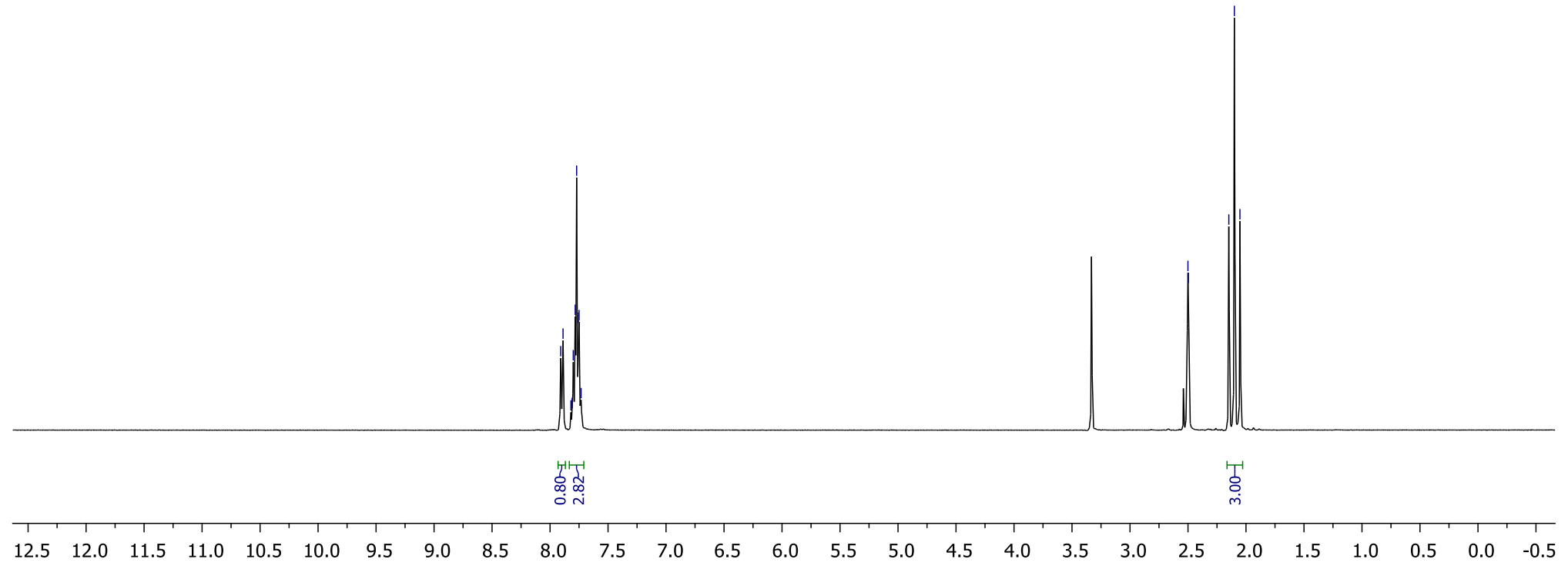


${ }^{13} \mathrm{C}\left\{{ }^{1} \mathrm{H}\right\}$ NMR (151 MHz, DMSO- $\left.d_{6}\right)$

R711986_C13

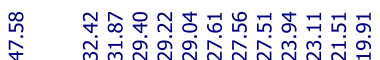

过

11

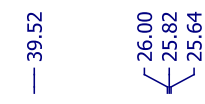

$\overbrace{M e}^{\mathrm{NO}_{2} \mathrm{~F}}$
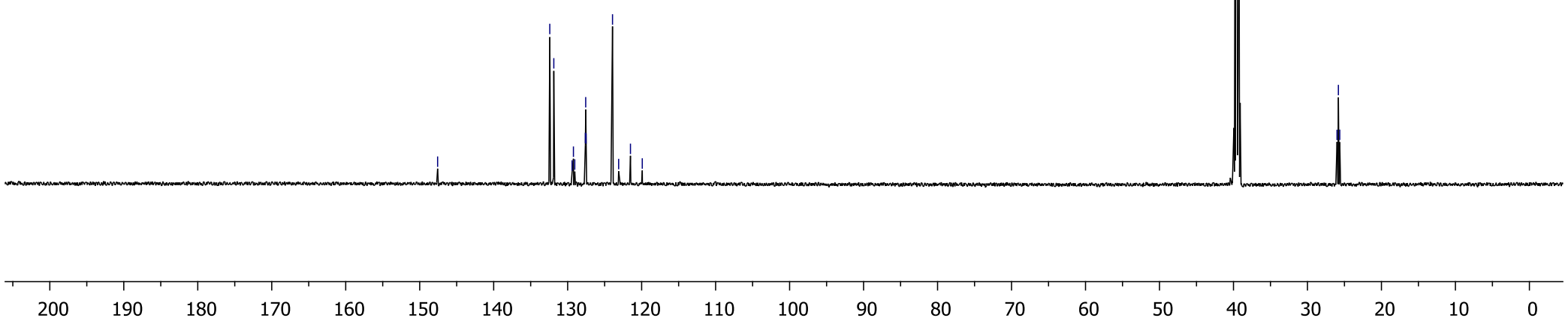


\section{${ }^{19} \mathrm{~F}\left\{{ }^{1} \mathrm{H}\right\}$ NMR (376 MHz, DMSO- $\left.d_{6}\right)$}

R711986_F19 $\{\mathrm{H}\}$
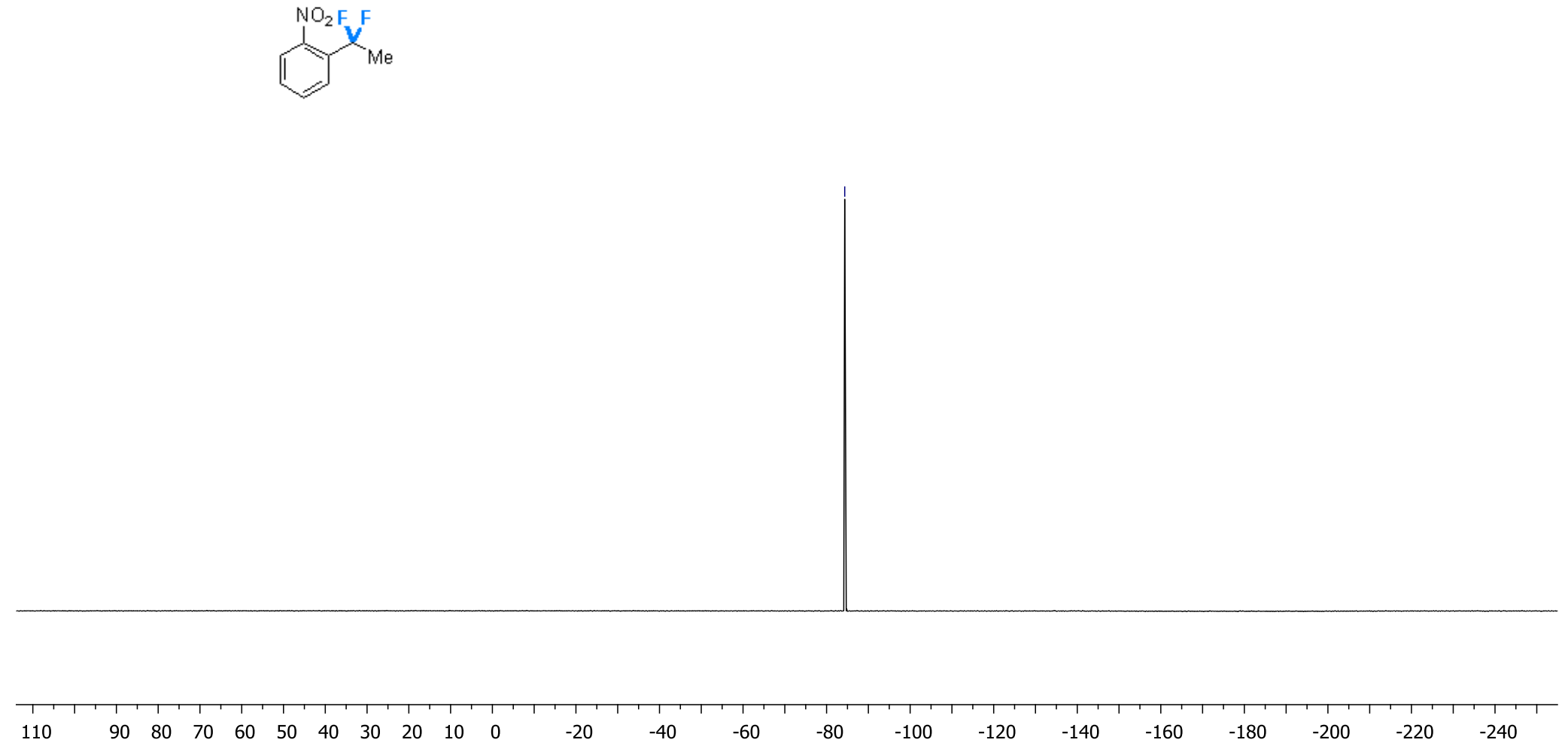


\section{Compound 10a}

${ }^{1} \mathrm{H}$ NMR (400 MHz, DMSO- $d_{6}$ )

R2541459

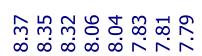

นึำ

Un

।

$\mathrm{C}^{\mathrm{NO}}$

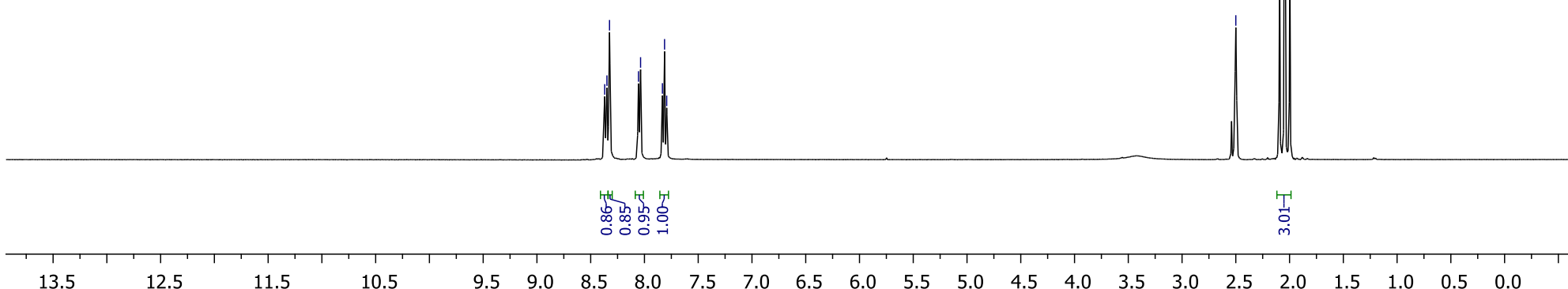




\section{${ }^{13} \mathrm{C}\left\{{ }^{1} \mathrm{H}\right\}$ NMR (126 MHz, DMSO- $\left.d_{6}\right)$}

R2541459_C13

$13 \mathrm{C}(1 \mathrm{H}$-decoupled $)$

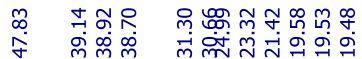

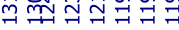

${ }^{\mathrm{F}} \mathrm{X}^{\mathrm{F}} \mathrm{NO}_{2}$

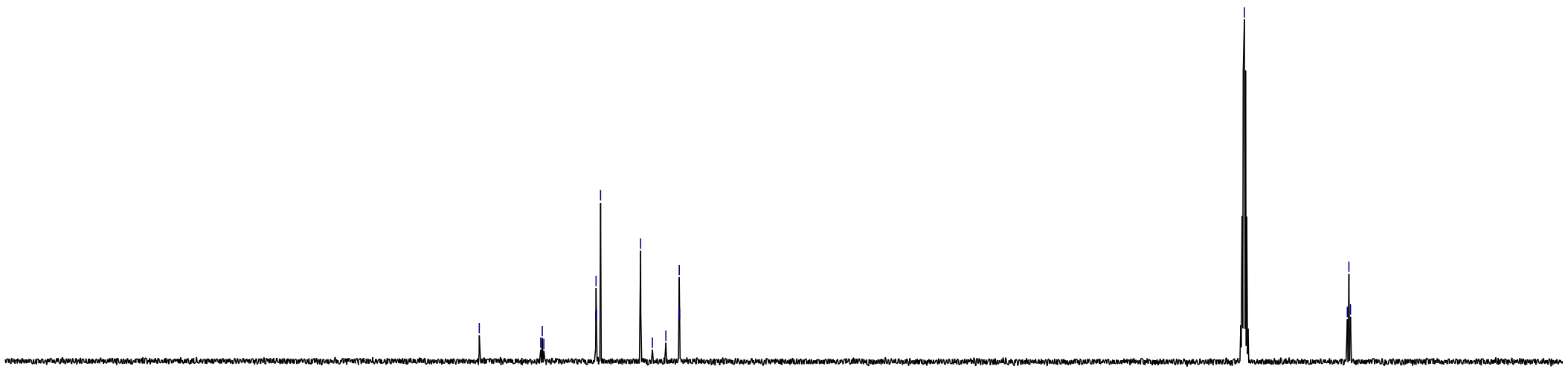

$\begin{array}{llllllllllllllllllllll}210 & 200 & 190 & 180 & 170 & 160 & 150 & 140 & 130 & 120 & 110 & 100 & 90 & 80 & 70 & 60 & 50 & 40 & 30 & 20 & 10\end{array}$ 
${ }^{19} \mathrm{~F}\left\{{ }^{1} \mathrm{H}\right\}$ NMR (376 MHz, DMSO DMSO- $d_{6}$ )

R2541459_F19 $\{\mathrm{H}\}$
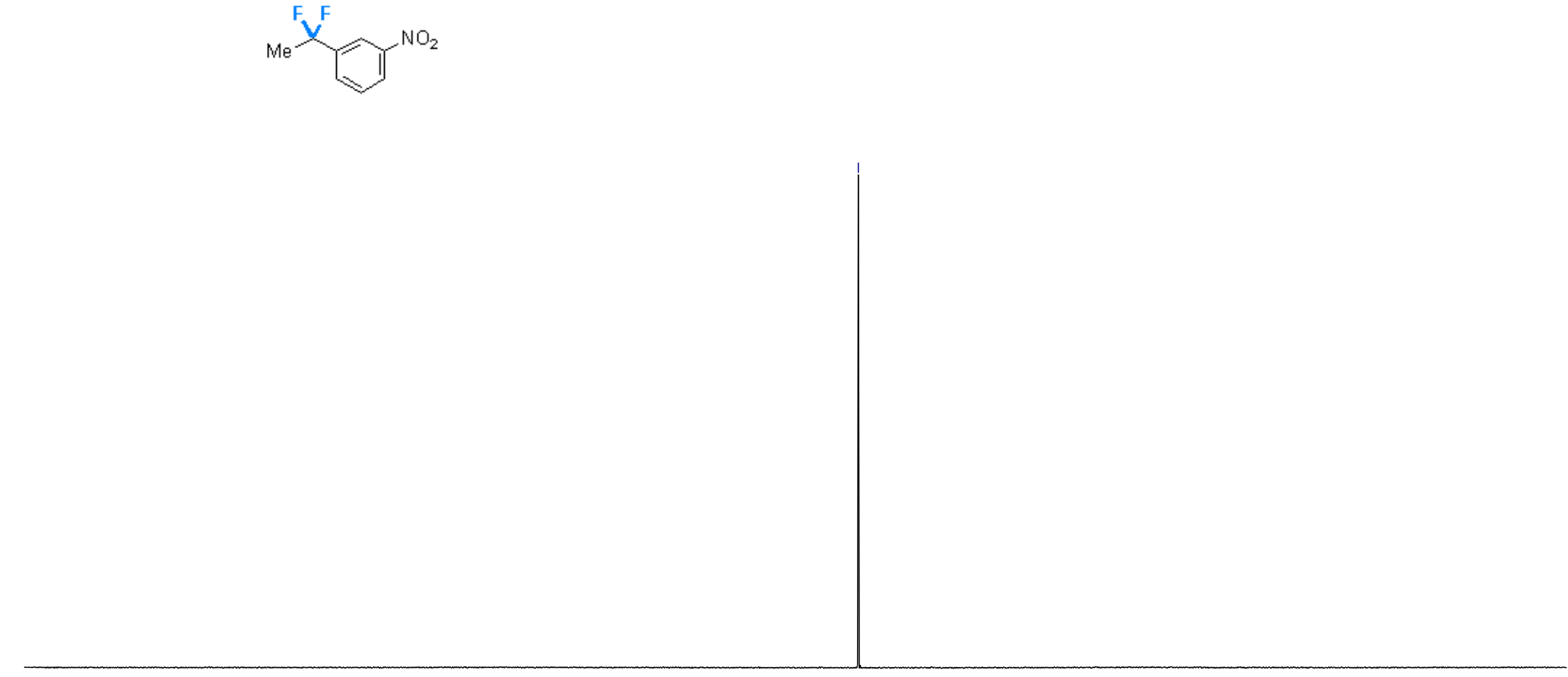


\section{Compound 11a}

${ }^{1} \mathrm{H}$ NMR (400 MHz, $\mathrm{CDCl}_{3}$ )

R1138153

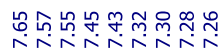

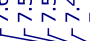

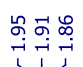

${ }^{\mathrm{F}} \mathrm{F}^{\mathrm{Br}}$

$1 / 11$

广

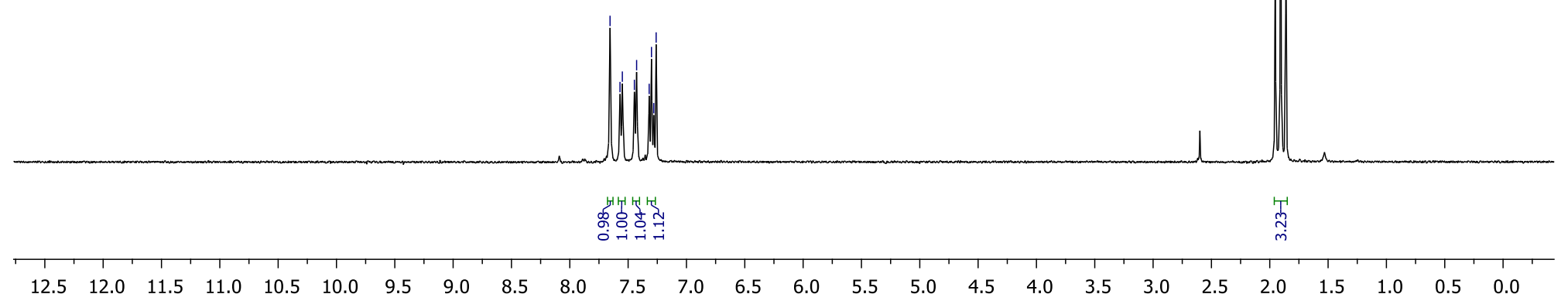


${ }^{13} \mathrm{C}\left\{{ }^{1} \mathrm{H}\right\}$ NMR (101 MHz, $\left.\mathrm{CDCl}_{3}\right)$

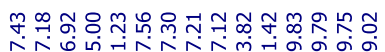

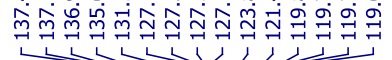

现岽

$\mathrm{C}^{\mathrm{F}} \mathrm{X}^{\mathrm{Br}}$

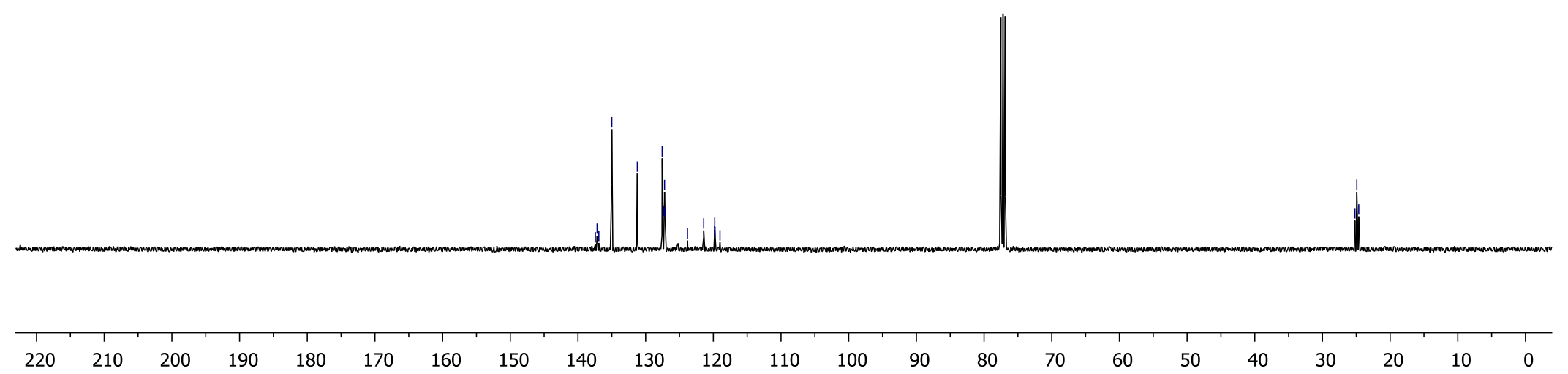




\section{${ }^{19} \mathrm{~F}\left\{{ }^{1} \mathrm{H}\right\}$ NMR (376 MHz, $\left.\mathrm{CDCl}_{3}\right)$}

R1133035_F19 $\{\mathrm{H}\}$
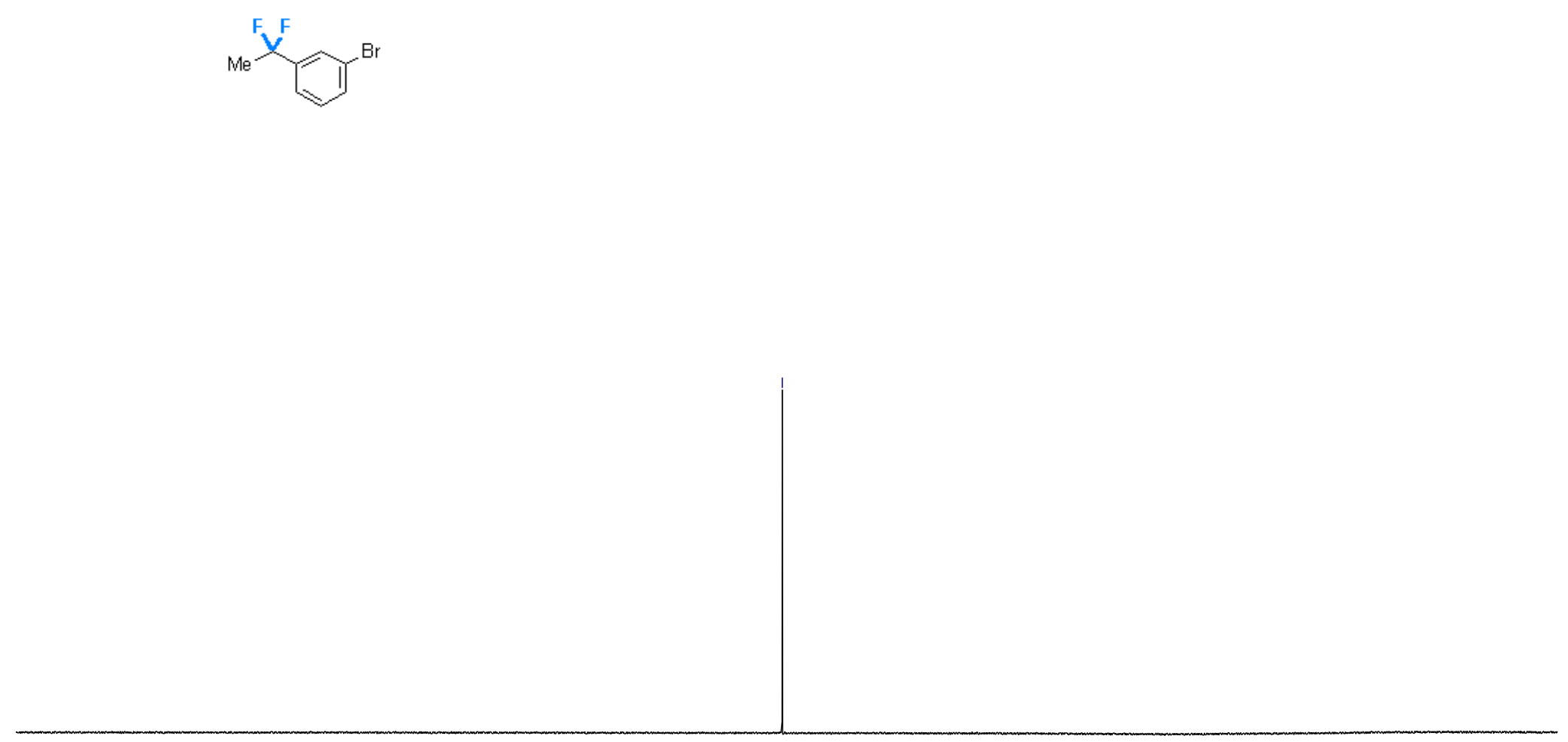


\section{Compound 12a}

${ }^{1} \mathrm{H}$ NMR (400 MHz, $\mathrm{CDCl}_{3}$ )

R1406335

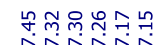

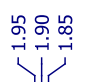

${ }_{F}^{F}{ }_{F}^{F r}$

$\iiint$
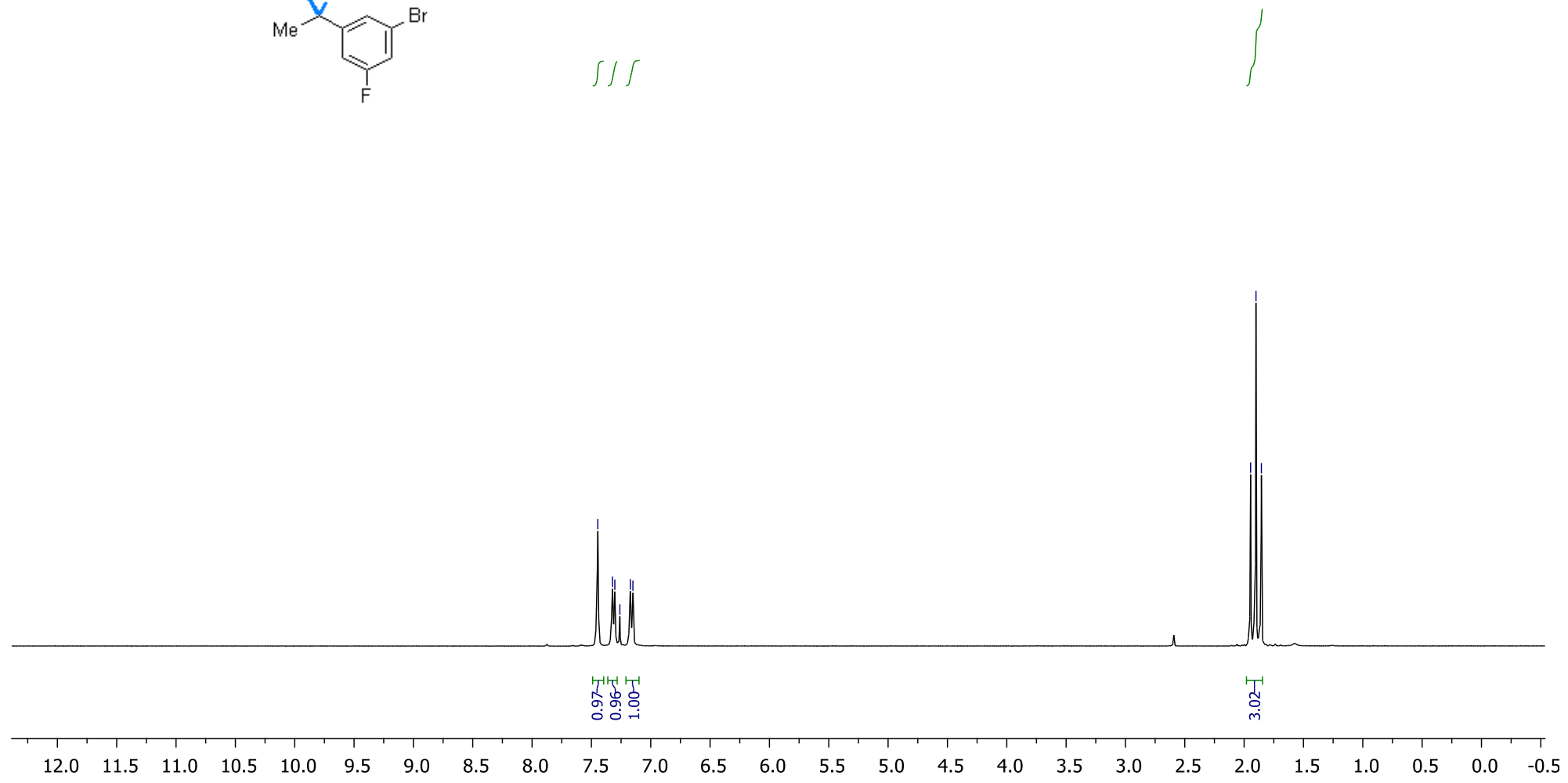
${ }^{13} \mathrm{C}\left\{{ }^{1} \mathrm{H}\right\}$ NMR $\left(151 \mathrm{MHz}, \mathrm{CDCl}_{3}\right)$

R1406335_C13

$P_{F}^{F r}$
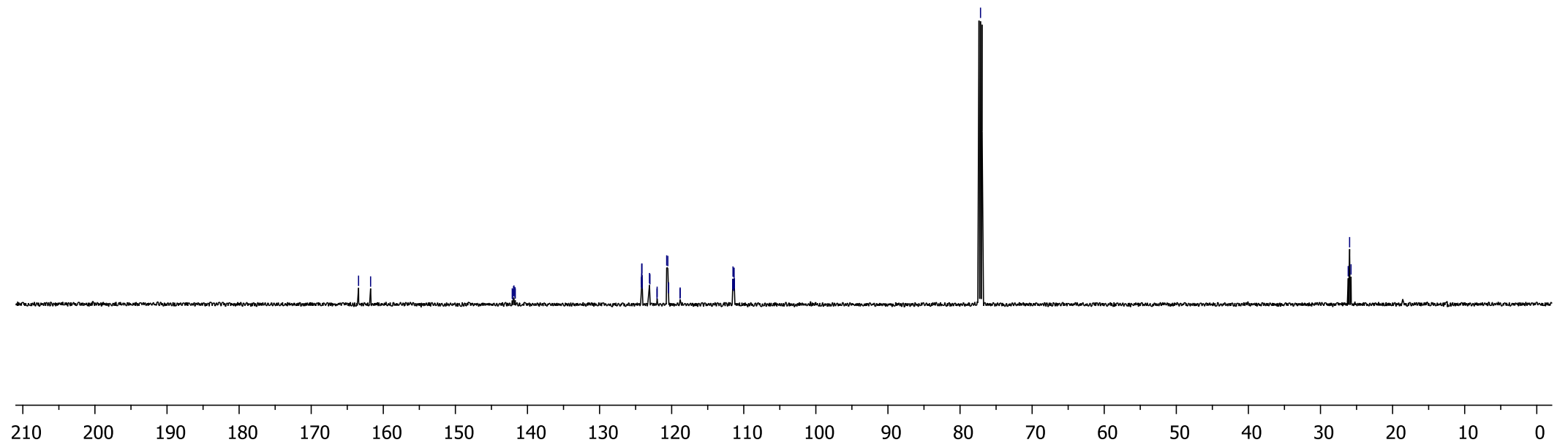


\section{${ }^{19} \mathrm{~F}\left\{{ }^{1} \mathrm{H}\right\}$ NMR (376 MHz, $\left.\mathrm{CDCl}_{3}\right)$}

R1406335_F19 $\{\mathrm{H}\}$

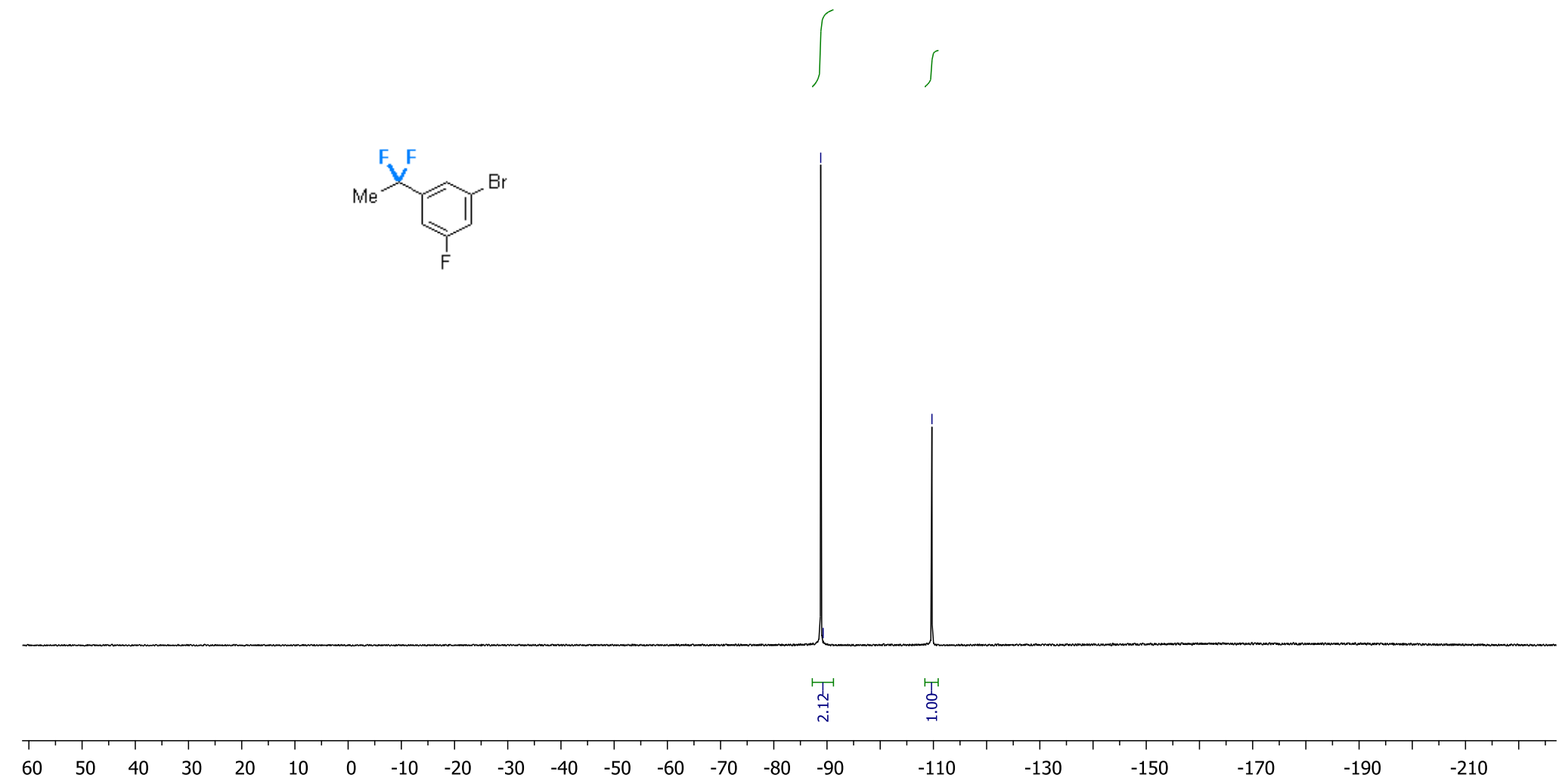




\section{Compound 13a}

${ }^{1} \mathrm{H}$ NMR (400 MHz, $\mathrm{CDCl}_{3}$ )

R540589

$\mathrm{F}_{\mathrm{F}}^{\mathrm{NO}_{2}}$
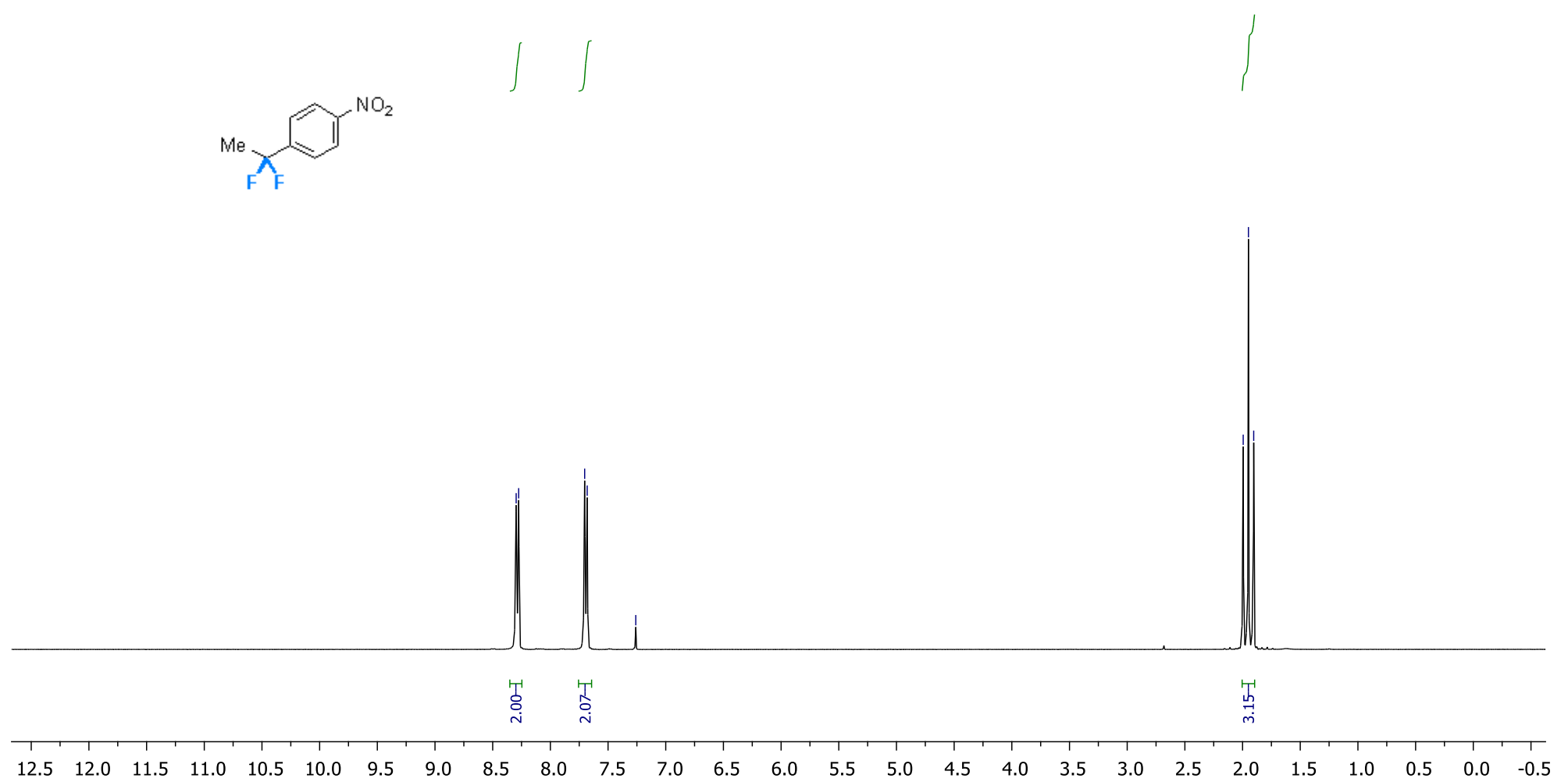
${ }^{13} \mathrm{C}\left\{{ }^{1} \mathrm{H}\right\}$ NMR $\left(126 \mathrm{MHz}, \mathrm{CDCl}_{3}\right)$

R540589_C1:

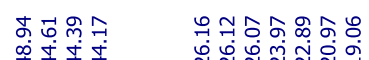

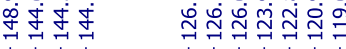

$\stackrel{\substack{i \\ i}}{1}$

急

Vifit

${ }_{F}^{\mathrm{NO}_{2}}$

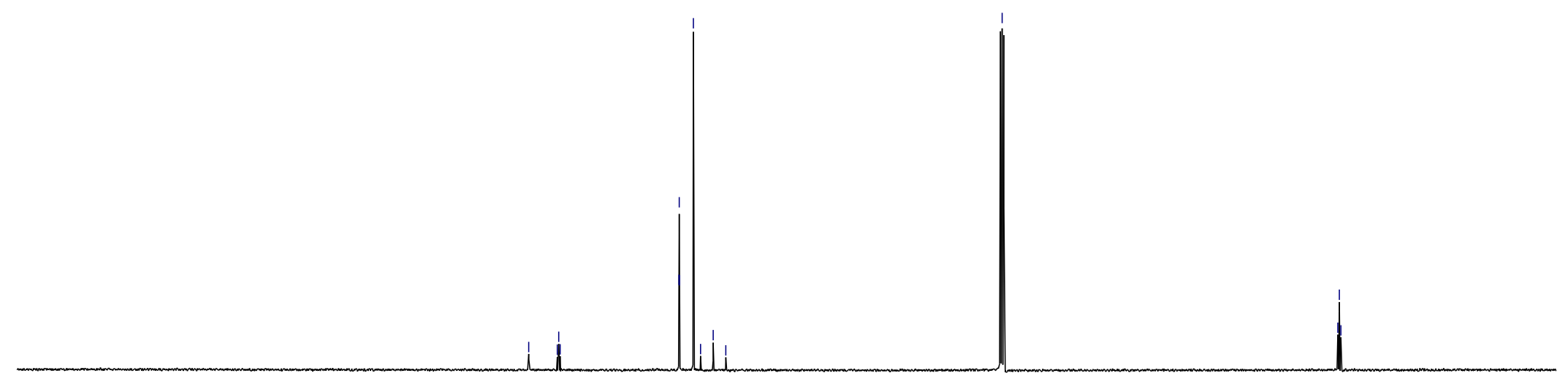




\section{${ }^{19} \mathrm{~F}\left\{{ }^{1} \mathrm{H}\right\} \mathrm{NMR}\left(376 \mathrm{MHz}, \mathrm{CDCl}_{3}\right)$}

R540589_F19 $\{\mathrm{H}\}$

$$
19 F-\{1 \mathrm{H}\}
$$
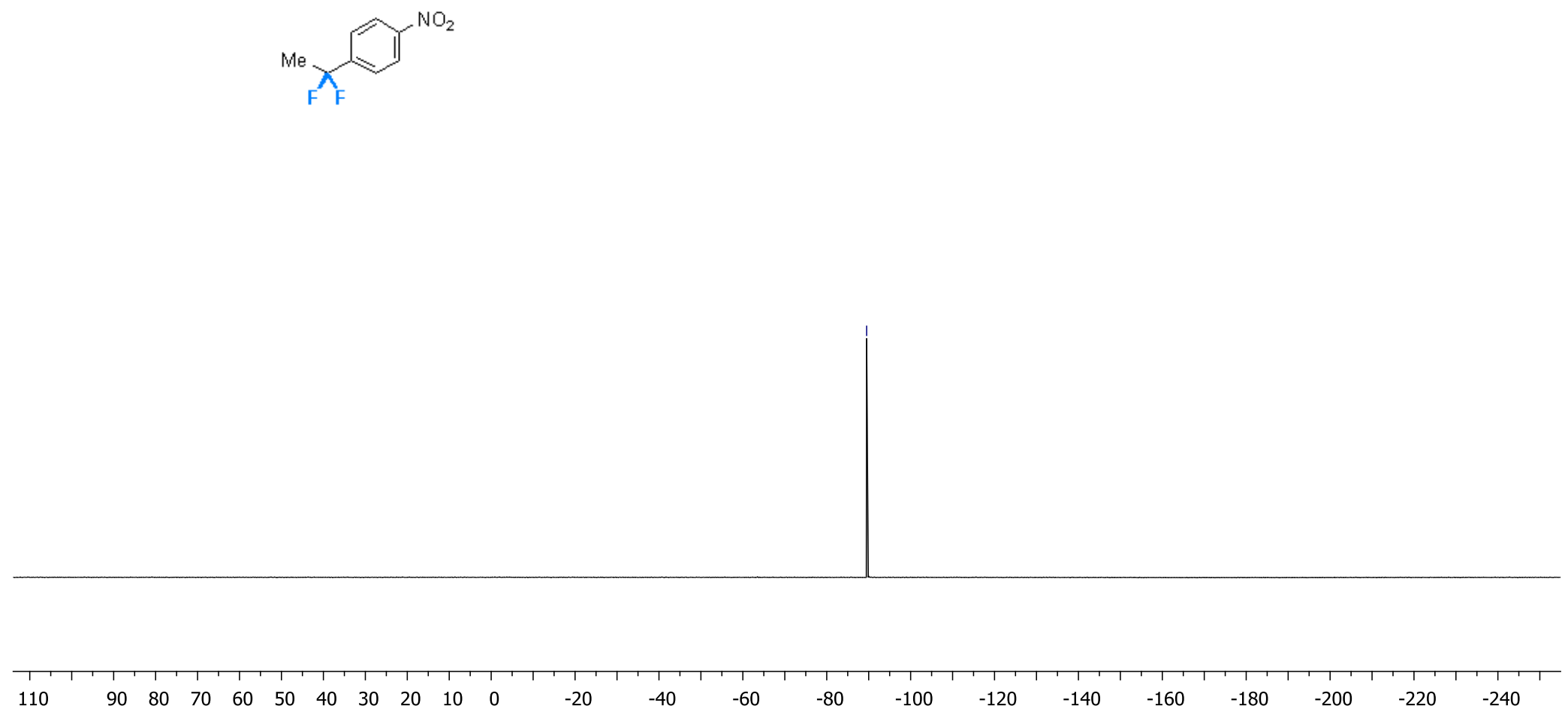


\section{Compound 14a}

${ }^{1} \mathrm{H}$ NMR (400 MHz, DMSO- $d_{6}$ )

R1033382

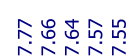

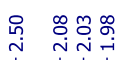

रोif

$\backslash 1$

${ }_{\mathrm{F} F}^{\mathrm{Cl}}$

$1 / 1$

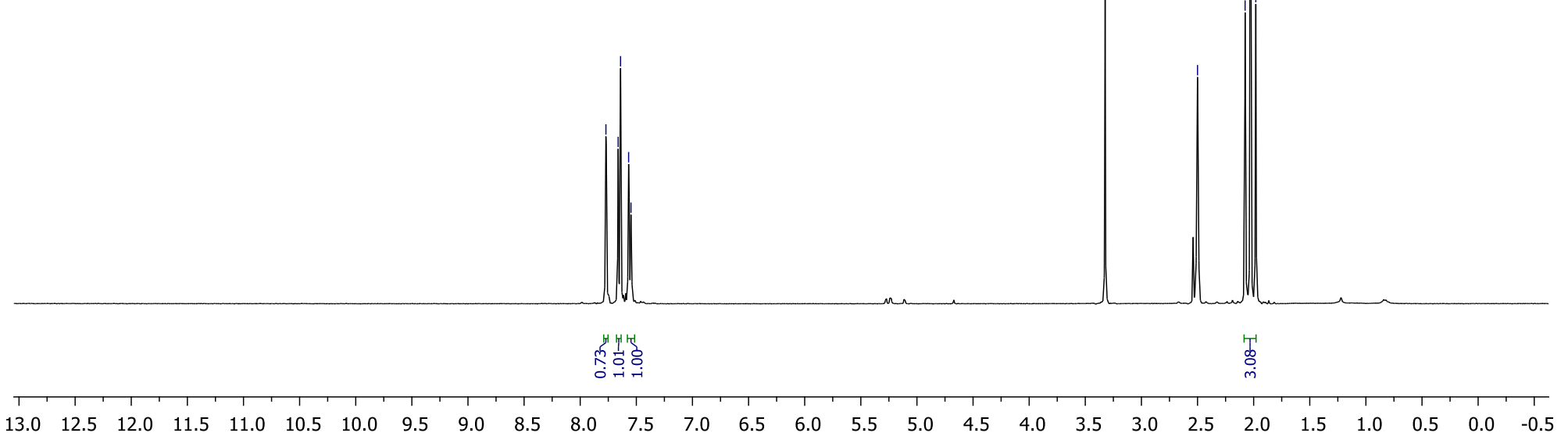


${ }^{13} \mathrm{C}\left\{{ }^{1} \mathrm{H}\right\}$ NMR (151 MHz, DMSO- $\left.d_{6}\right)$

R1033382_C13

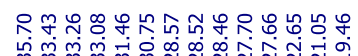

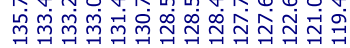

กูบ

$\longrightarrow$

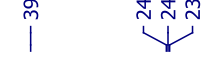

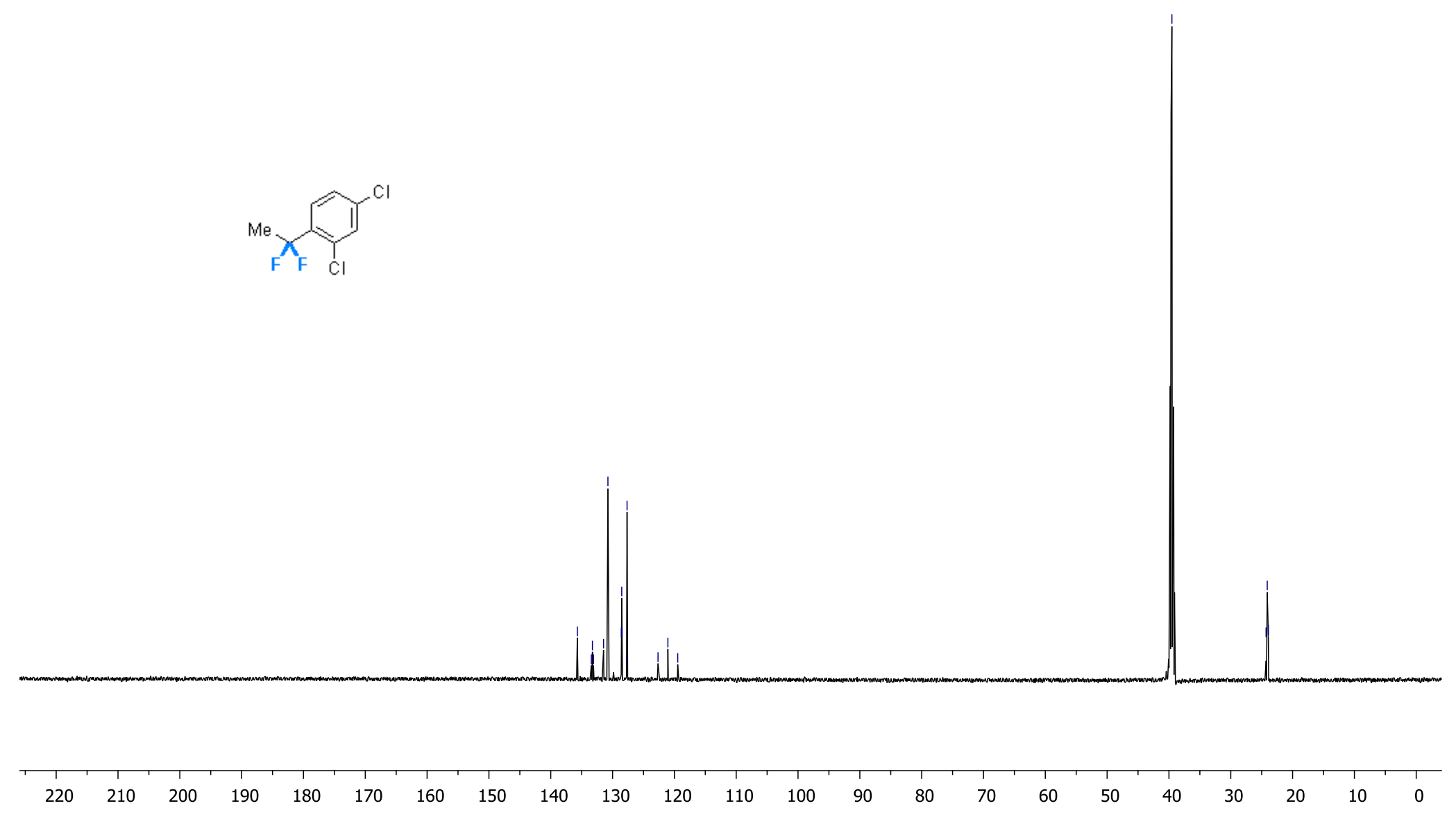




\section{${ }^{19} \mathrm{~F}\left\{{ }^{1} \mathrm{H}\right\}$ NMR (376 MHz, DMSO- $\left.d_{6}\right)$}

R1033382_F19 $\{H\}$
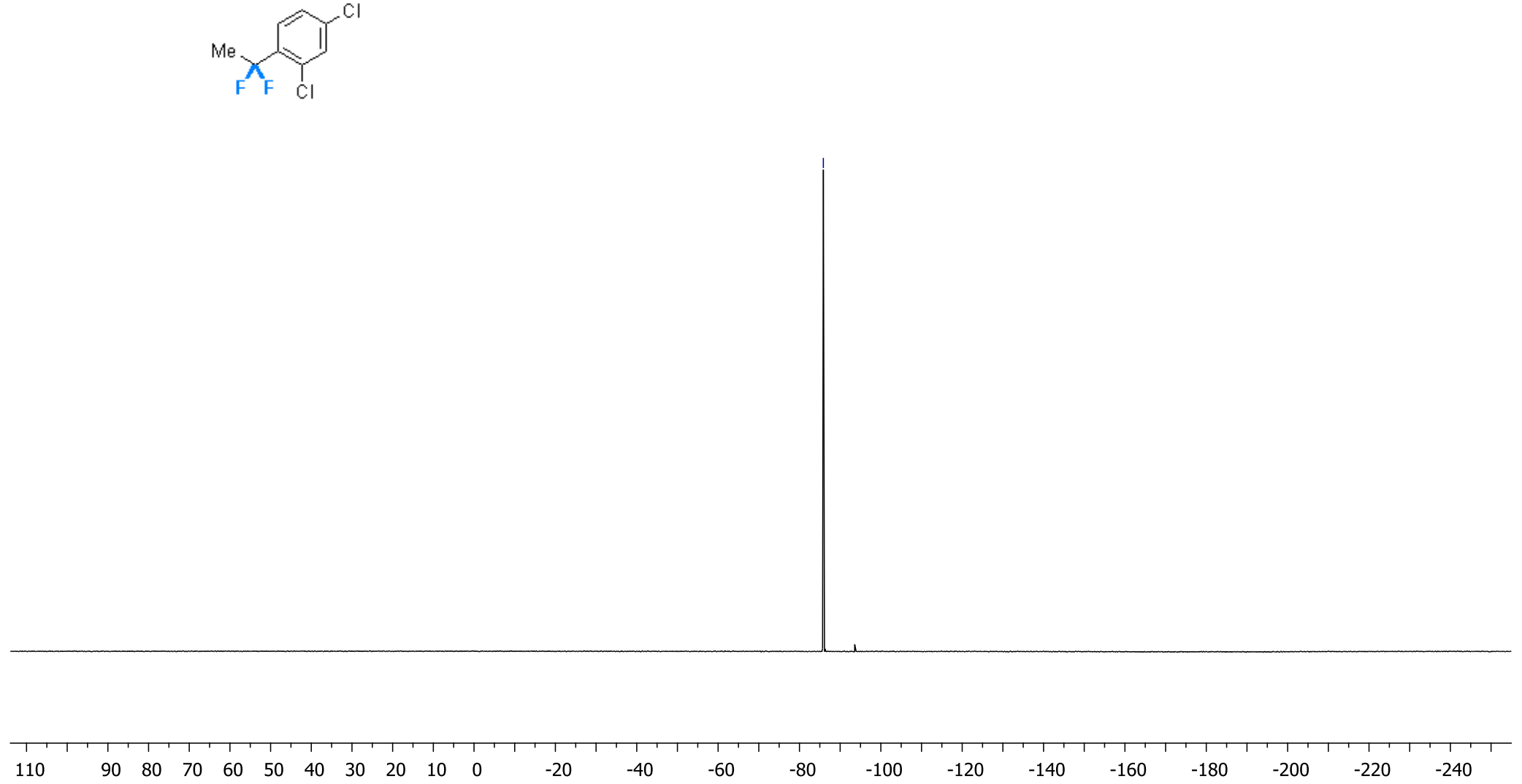


\section{Compound 15a}

${ }^{1} \mathrm{H}$ NMR (400 MHz, $\mathrm{CDCl}_{3}$ )

R1416153

STANDARD PROTON PARAMETERS

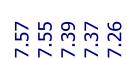

पivi

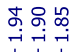
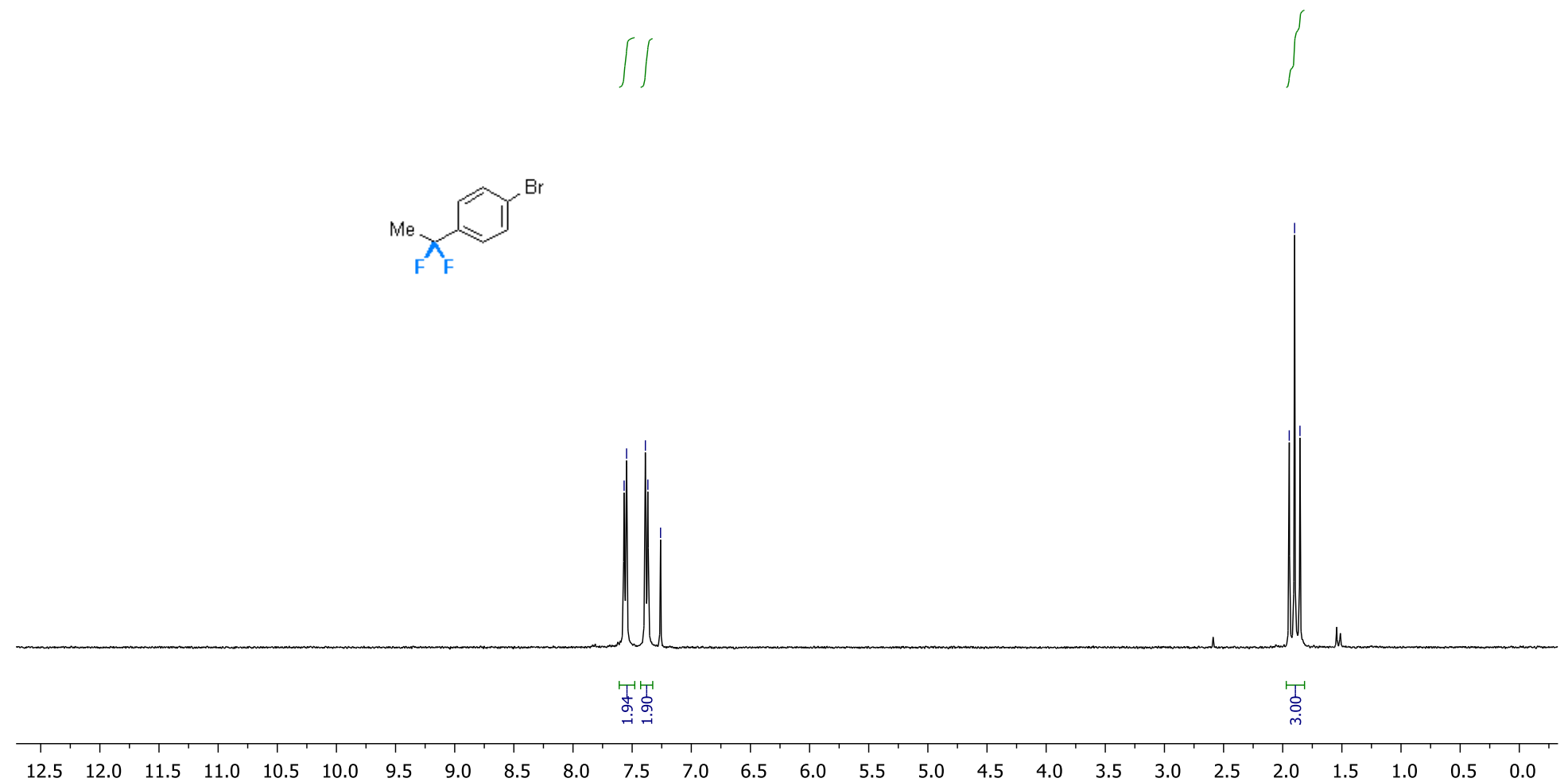


\section{${ }^{19} \mathrm{~F}\left\{{ }^{1} \mathrm{H}\right\} \mathrm{NMR}\left(376 \mathrm{MHz}, \mathrm{CDCl}_{3}\right)$}

R1416153_F19\{H\}

$$
19 \mathrm{~F}-\{1 \mathrm{H}\}
$$
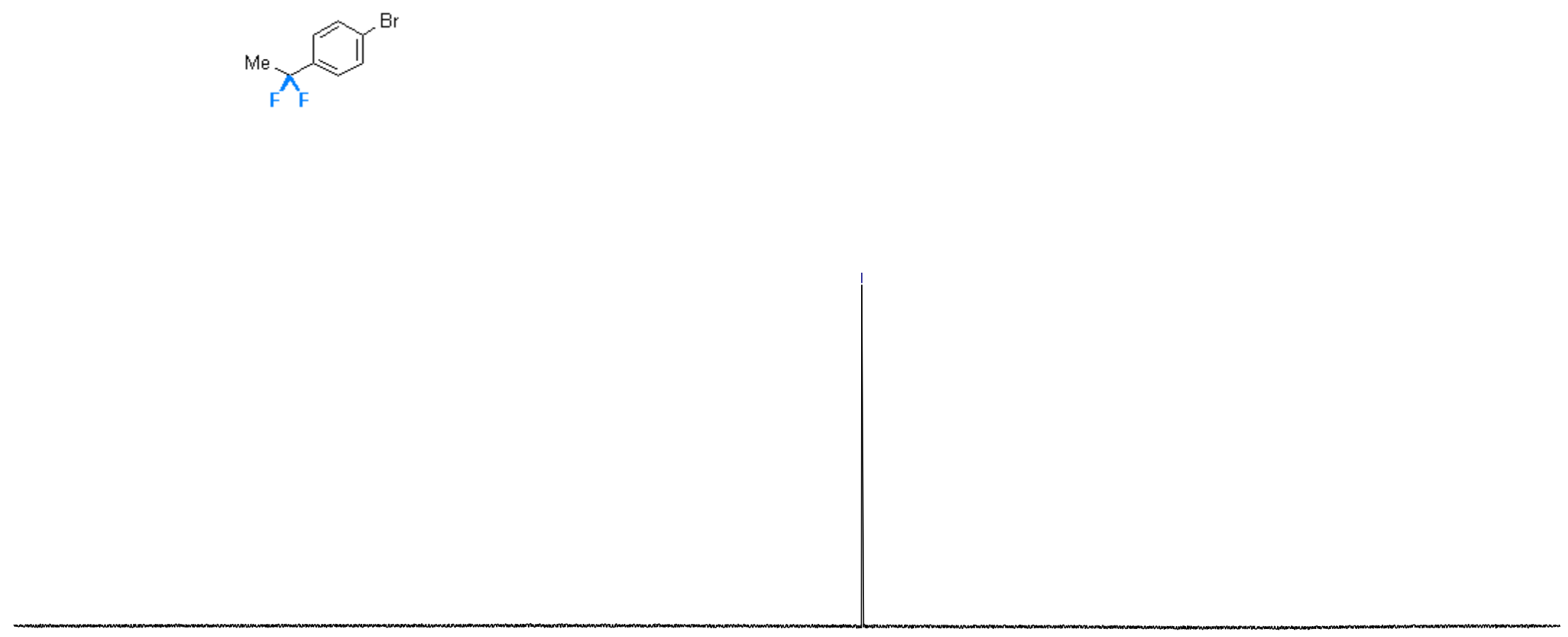
${ }^{13} \mathrm{C}\left\{{ }^{1} \mathrm{H}\right\}$ NMR (126 MHz, $\left.\mathrm{CDCl}_{3}\right)$

R1478978_C13

$13 \mathrm{C}$ (1H-decoupled)
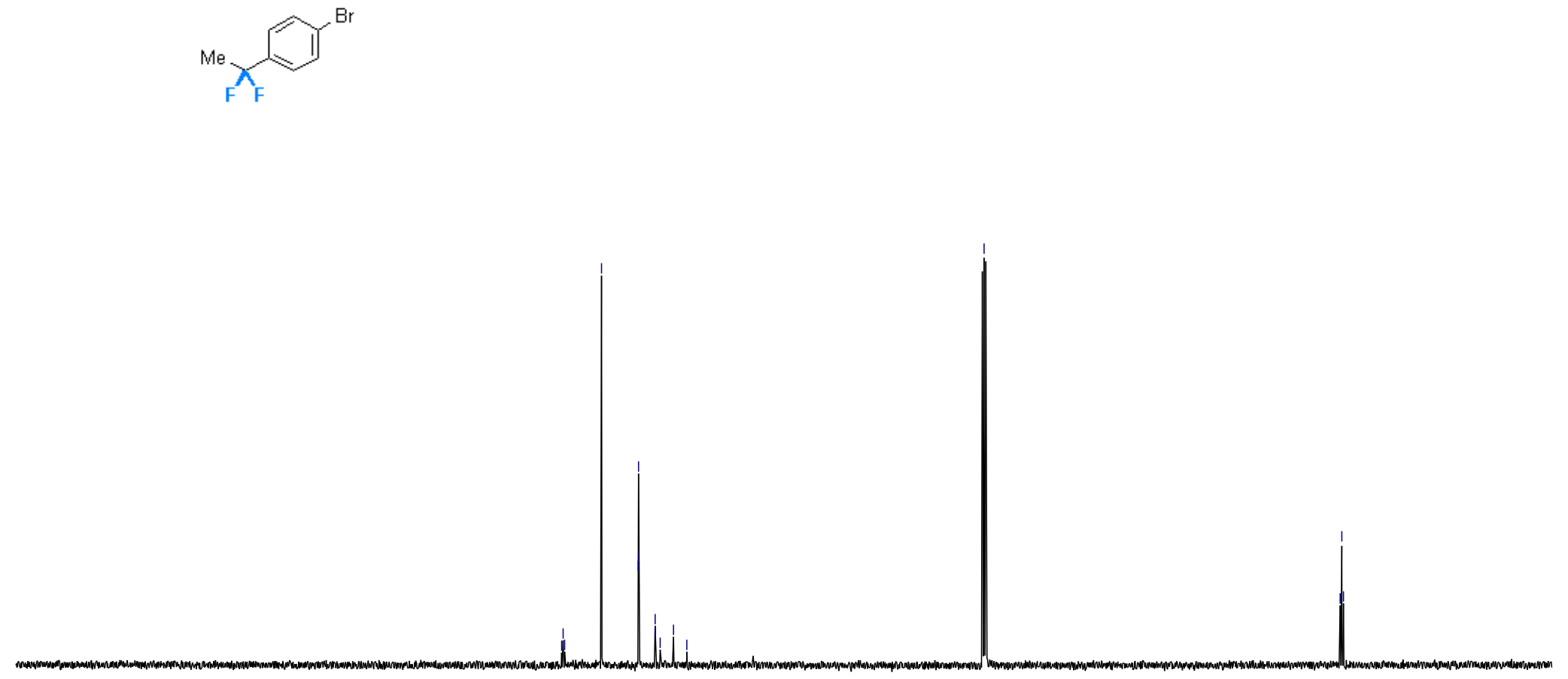


\section{Compound 16a}

${ }^{1} \mathrm{H}$ NMR (400 MHz, $\mathrm{CDCl}_{3}$ )

R1673030

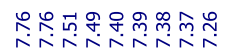

$$
\text { (1) }
$$

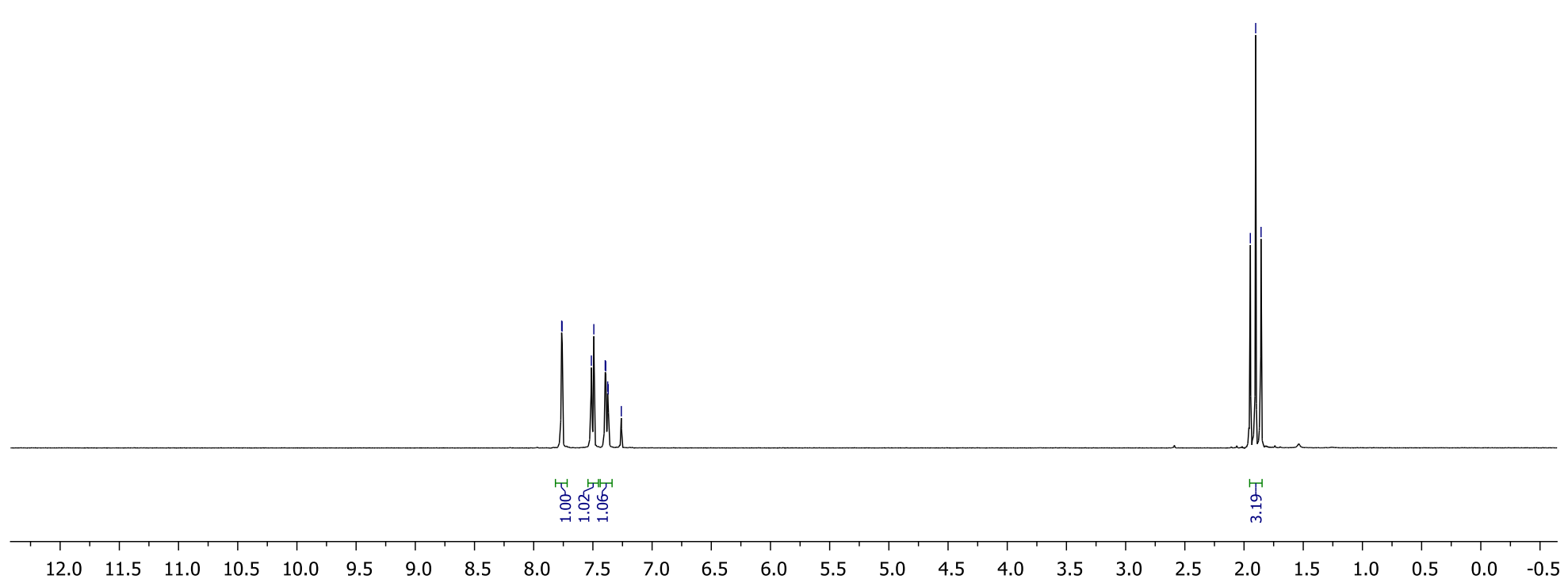


${ }^{13} \mathrm{C}\left\{{ }^{1} \mathrm{H}\right\}$ NMR (151 MHz, $\left.\mathrm{CDCl}_{3}\right)$

R1673030_C13

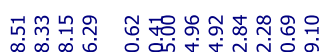

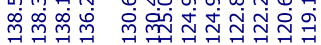

V)

$\stackrel{0}{i}$

ำ

${ }_{\mathrm{F} F}^{\mathrm{Cl}}$

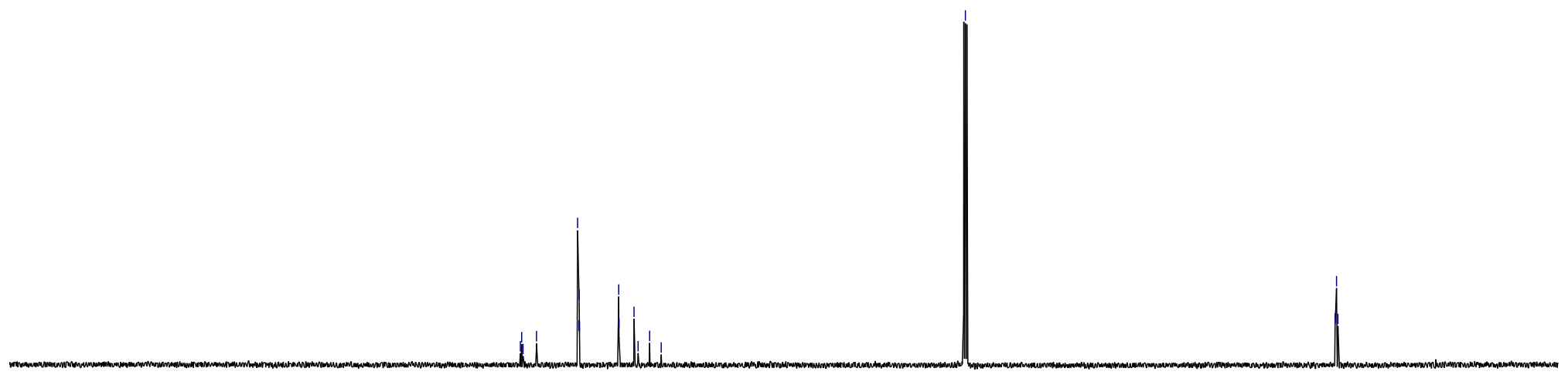




\section{${ }^{19} \mathrm{~F}\left\{{ }^{1} \mathrm{H}\right\}$ NMR (376 MHz, $\left.\mathrm{CDCl}_{3}\right)$}

R1673030_F19

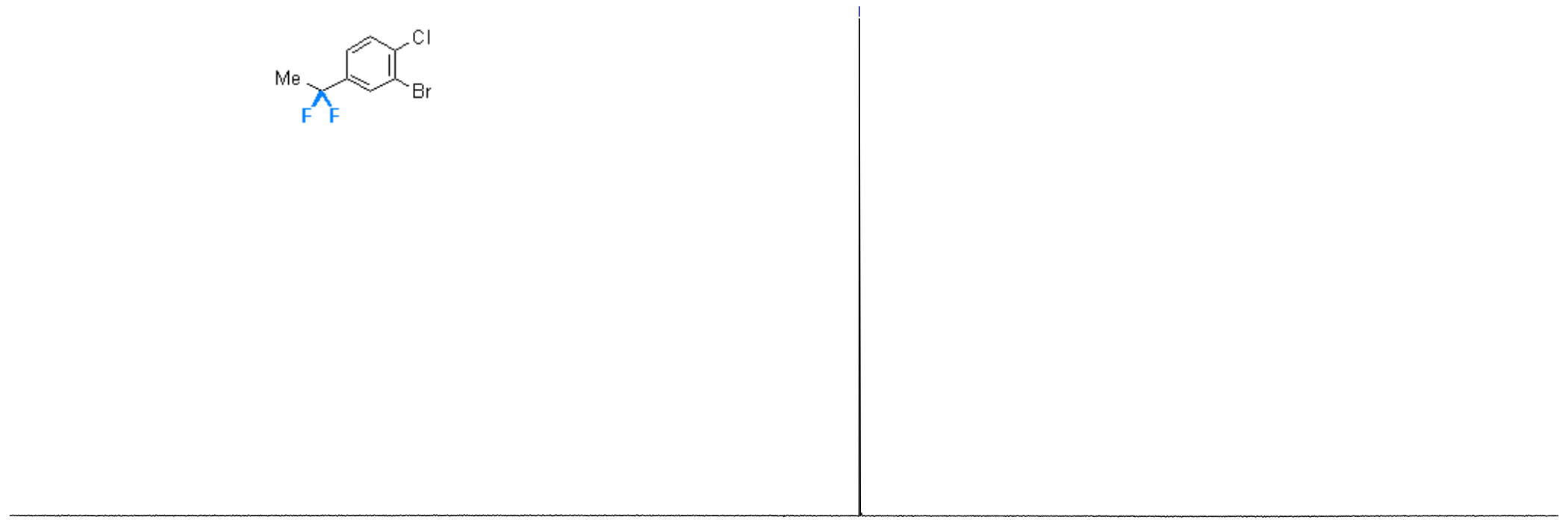

$j$
$\infty$
$\infty$
$\infty$
1

$\begin{array}{lllllllll}90 & 80 & 70 & 60 & 50 & 40 & 30 & 20 & 10\end{array}$

$-20$

$-40$

$-60$

$-80$

$-100$

$-140$

$-160 \quad-180$

$\begin{array}{lll}-200 & -220 & -240\end{array}$ 


\section{Compound 17a}

${ }^{1} \mathrm{H}$ NMR (400 MHz, $\mathrm{CDCl}_{3}$ )

R1985357

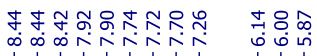
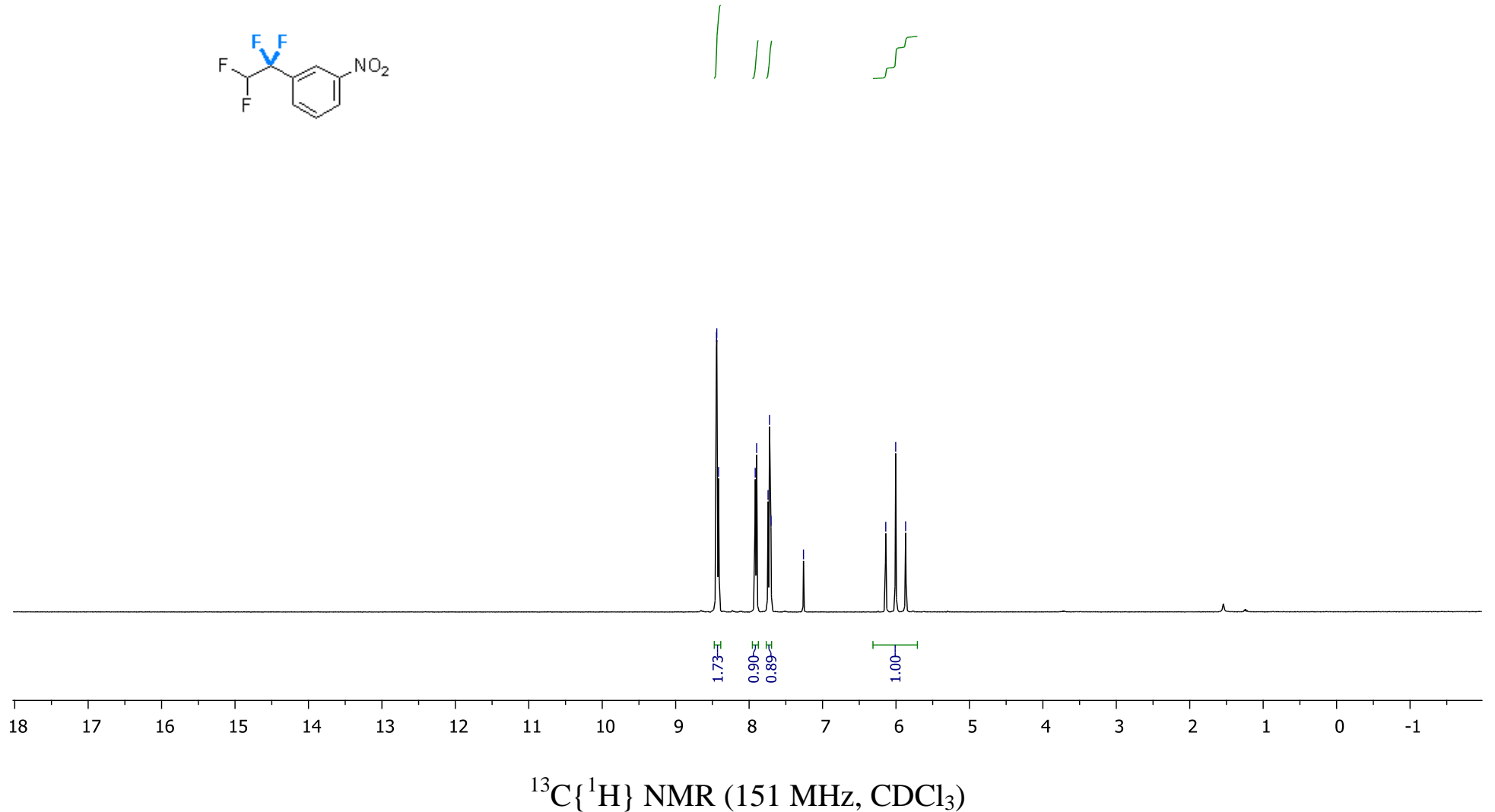

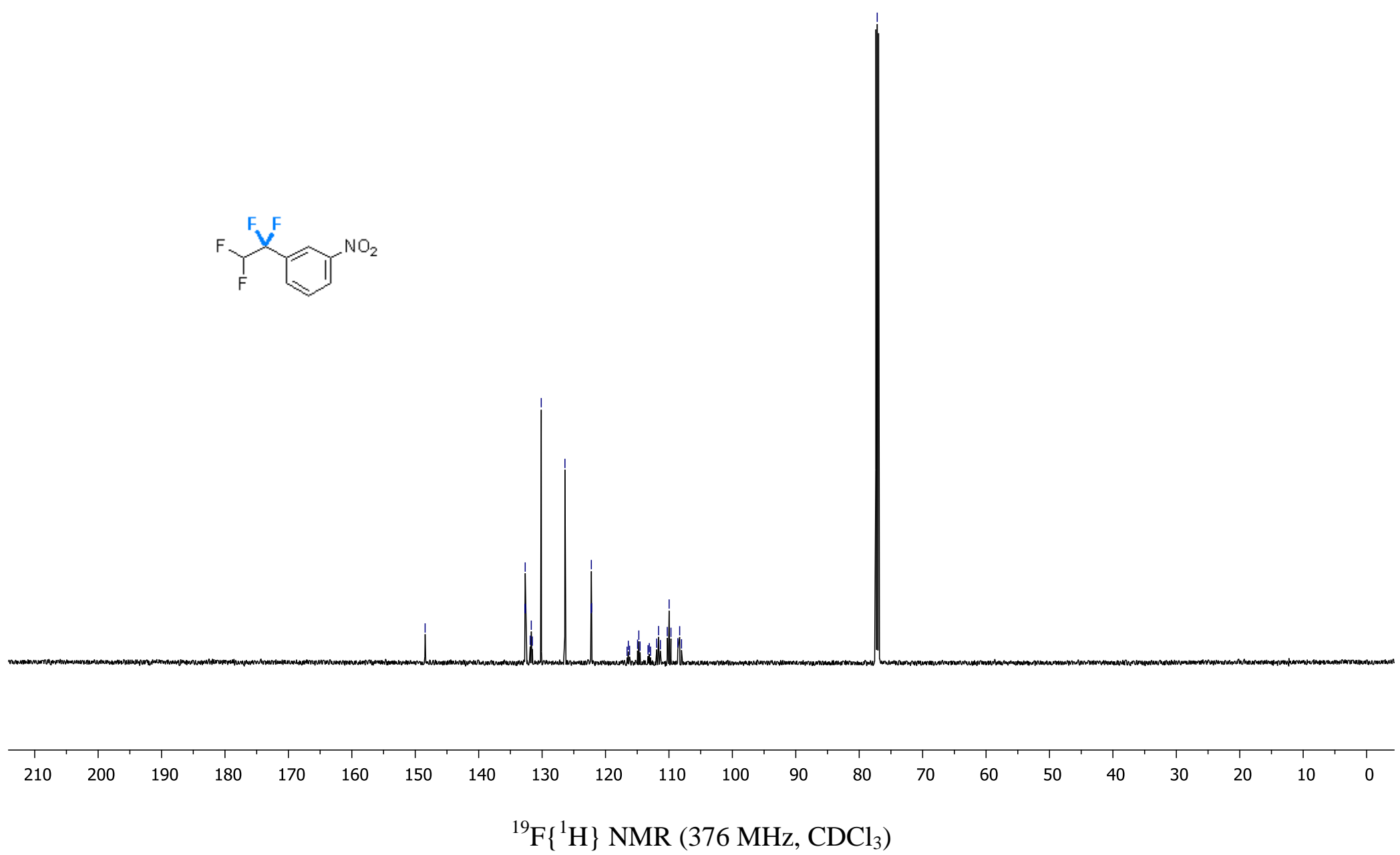
R1985357_F19 $\{\mathrm{H}\}$

$19 \mathrm{~F}-\{1 \mathrm{H}\}$

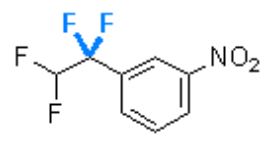

$\iint$

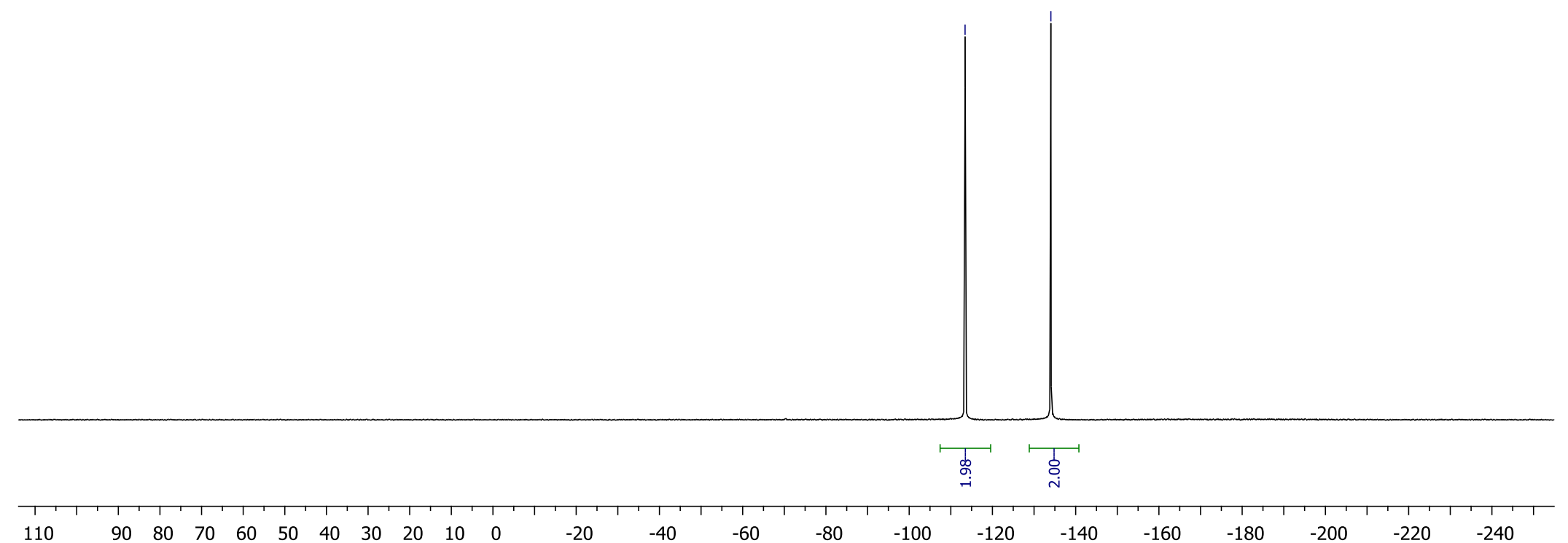




\section{Compound 18a}

${ }^{1} \mathrm{H}$ NMR (400 MHz, $\mathrm{CDCl}_{3}$ )

R1159238

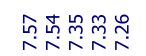

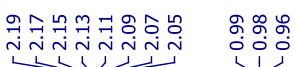

$\checkmark \mathrm{VI}$
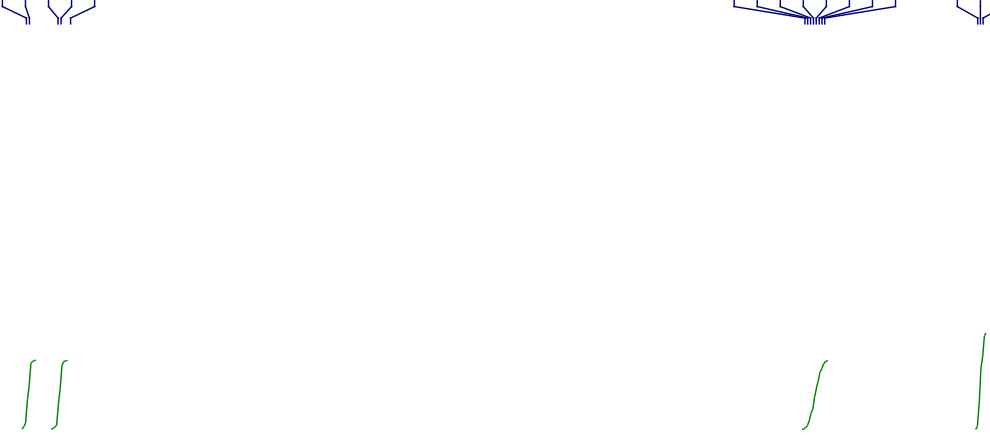

${ }_{F F}^{M e}{ }_{F}^{B r}$

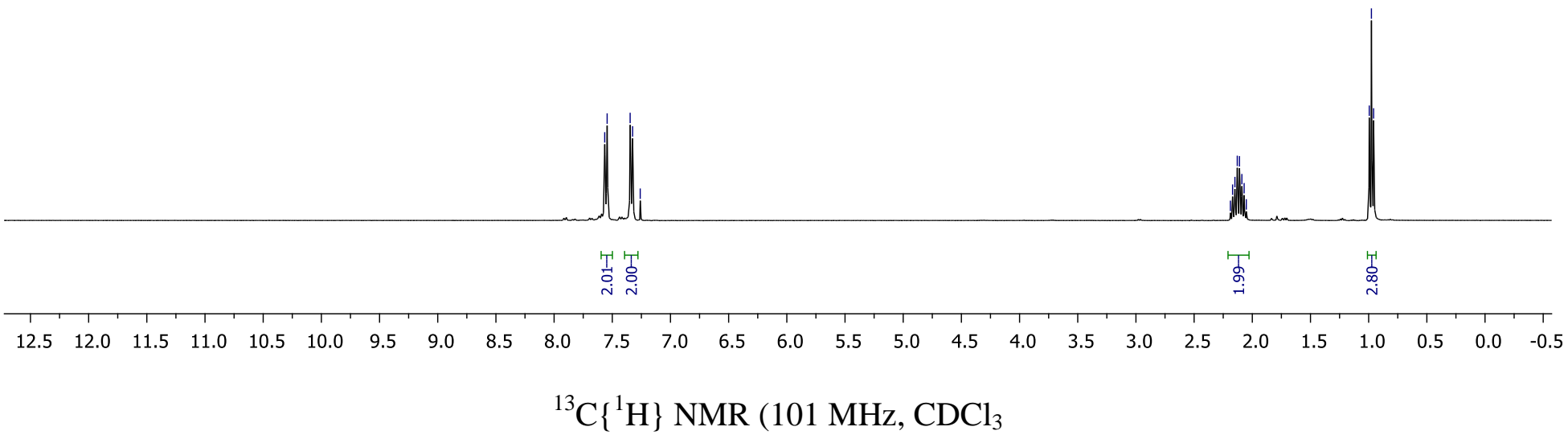



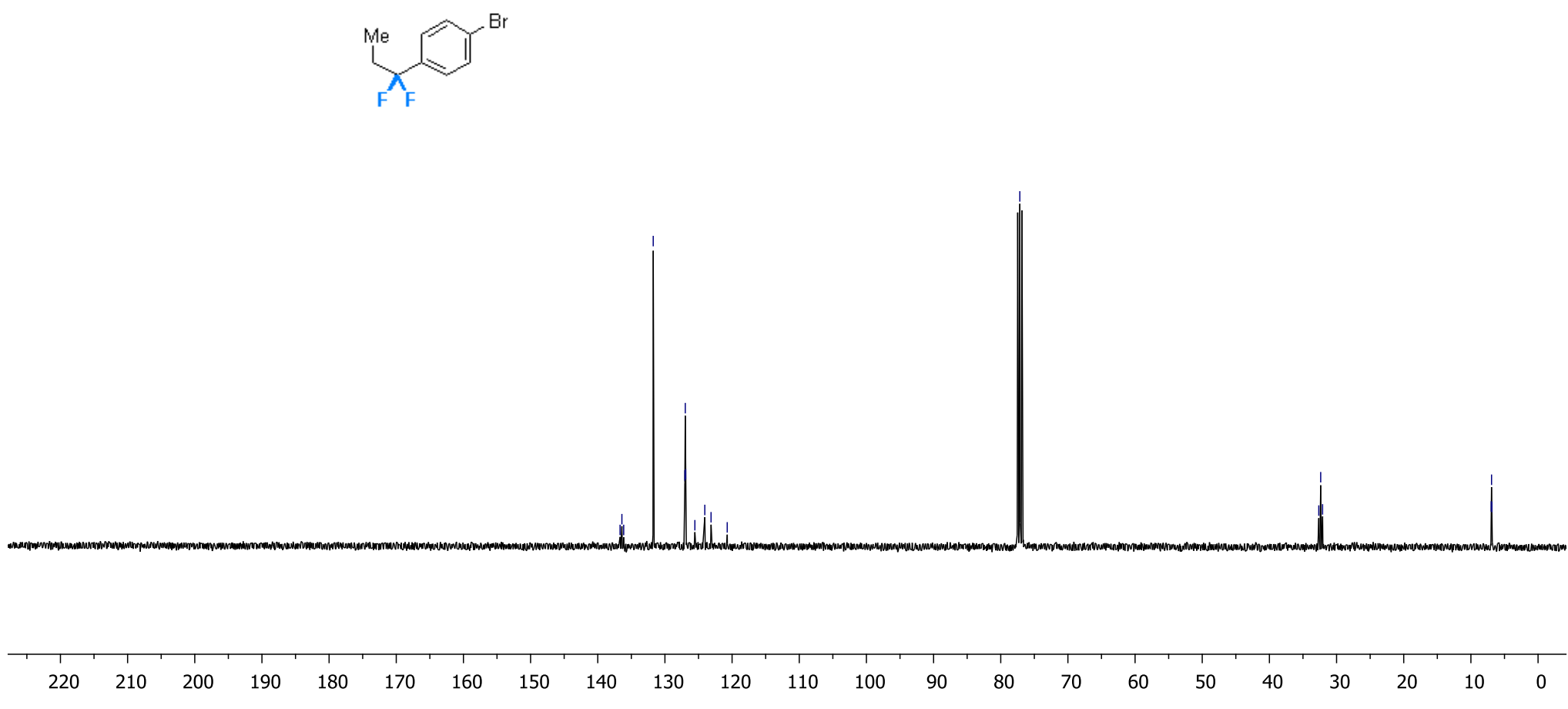

${ }^{19} \mathrm{~F}\left\{{ }^{1} \mathrm{H}\right\}$ NMR $\left(376 \mathrm{MHz}, \mathrm{CDCl}_{3}\right)$ 


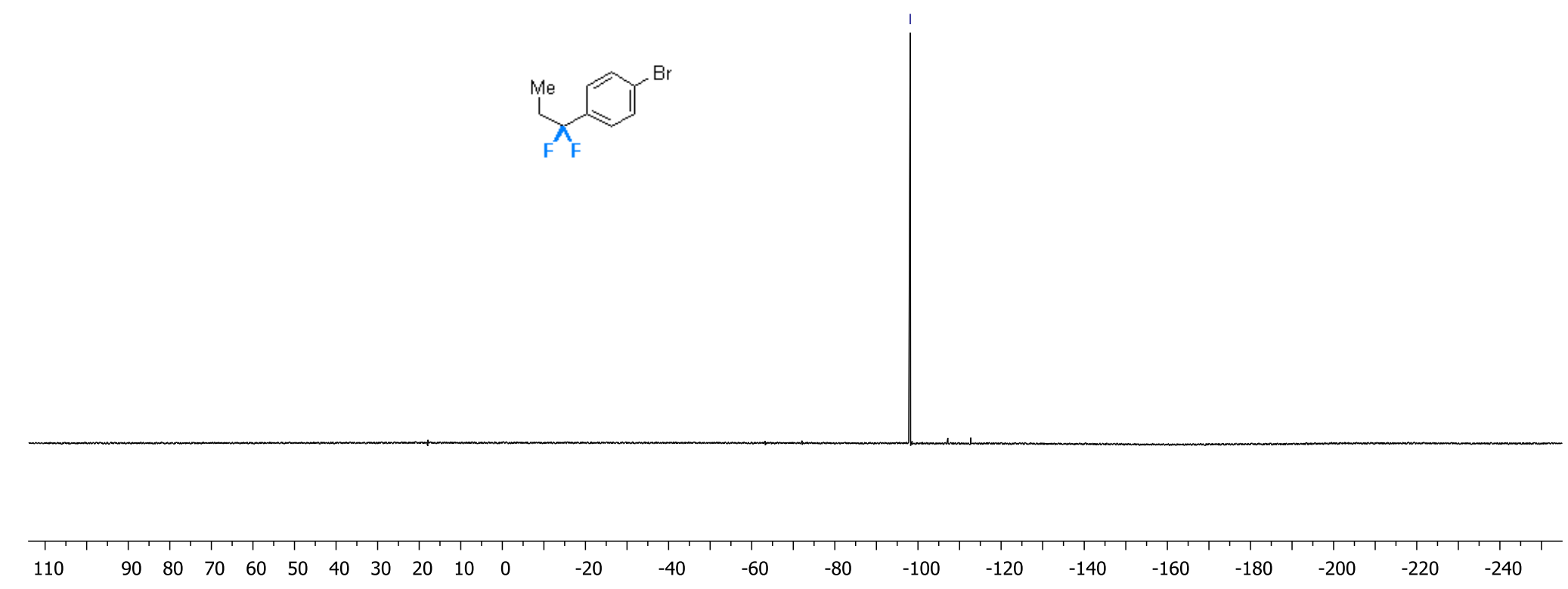




\section{Compound 19a}

${ }^{1} \mathrm{H}$ NMR (400 MHz, $\mathrm{CDCl}_{3}$ )

R2446162

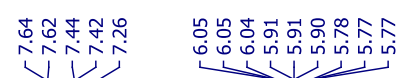

$\sum_{F F}^{F} C_{F}^{B r}$
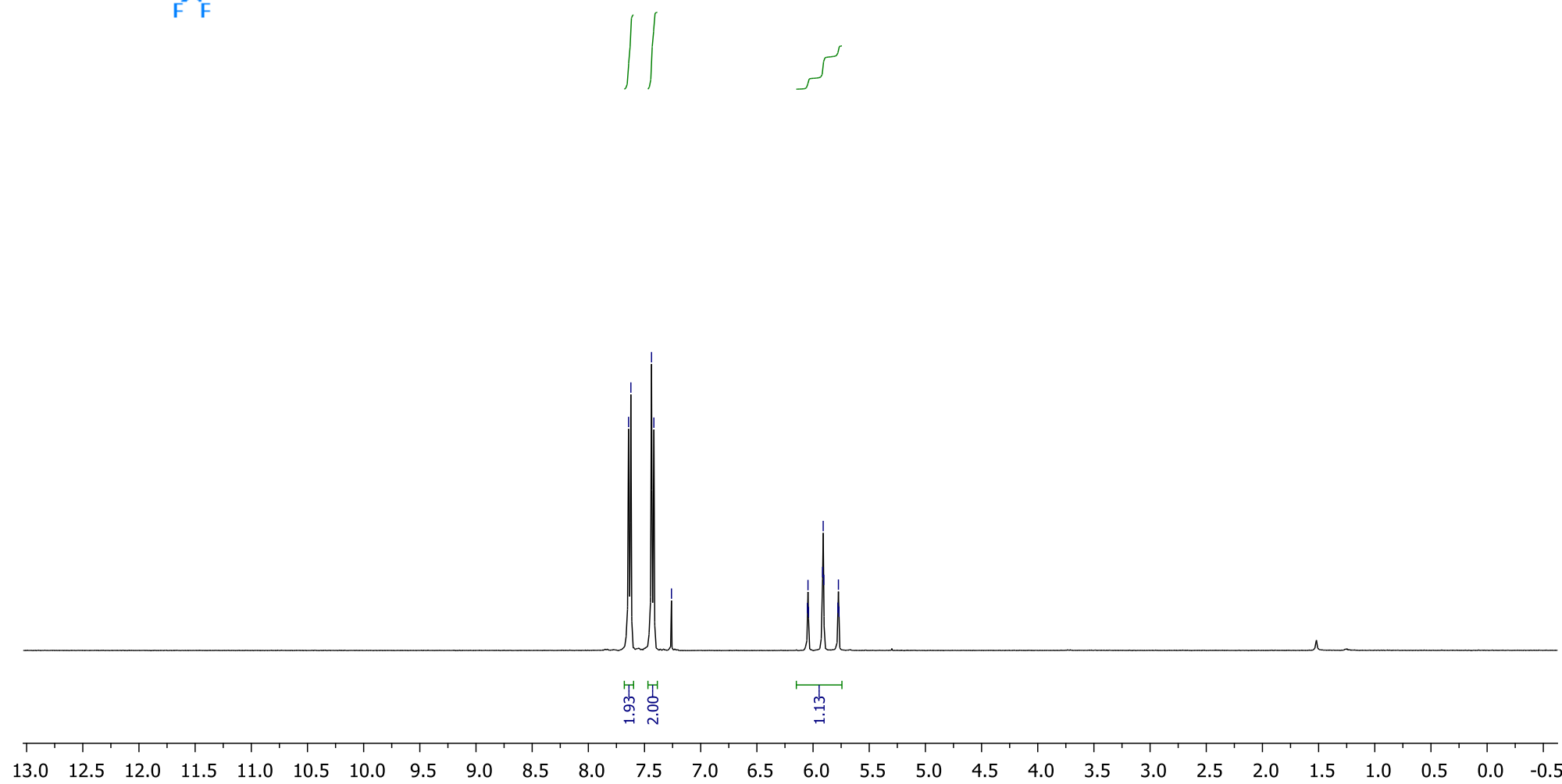
${ }^{13} \mathrm{C}\left\{{ }^{1} \mathrm{H}\right\}$ NMR $\left(151 \mathrm{MHz}, \mathrm{CDCl}_{3}\right)$

R2446162_C13

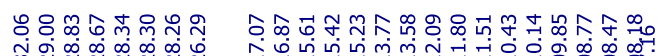

雨

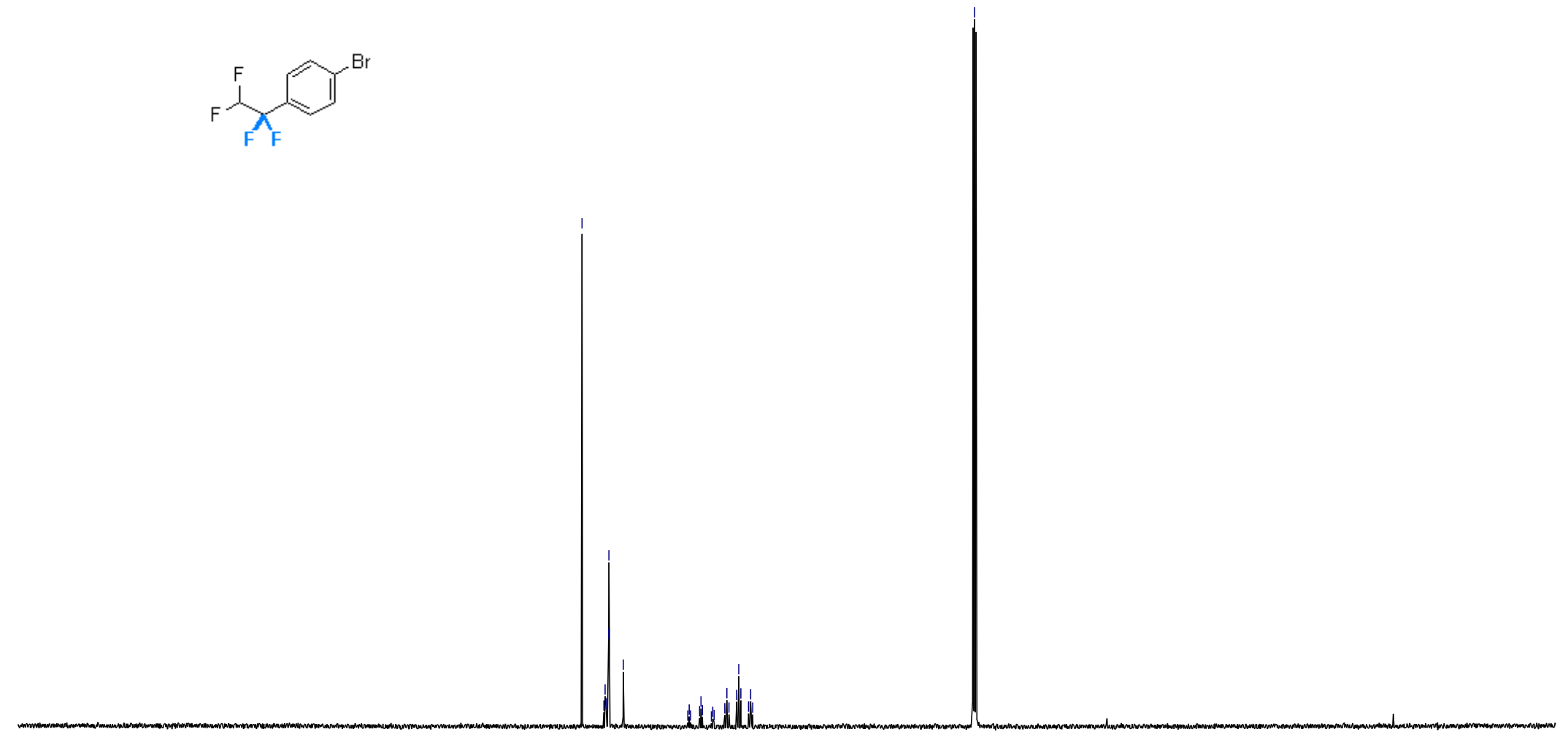

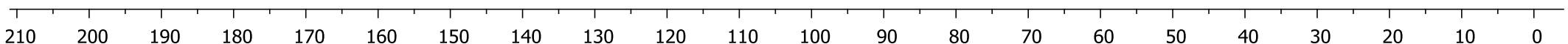


${ }^{19} \mathrm{~F}\left\{{ }^{1} \mathrm{H}\right\} \mathrm{NMR}\left(376 \mathrm{MHz}, \mathrm{CDCl}_{3}\right)$

R2446162_F19 $\{\mathrm{H}\}$

$19 \mathrm{~F}-\{1 \mathrm{H}\}$

형ำ

覃兽

$\psi$

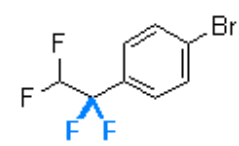

$\iint$

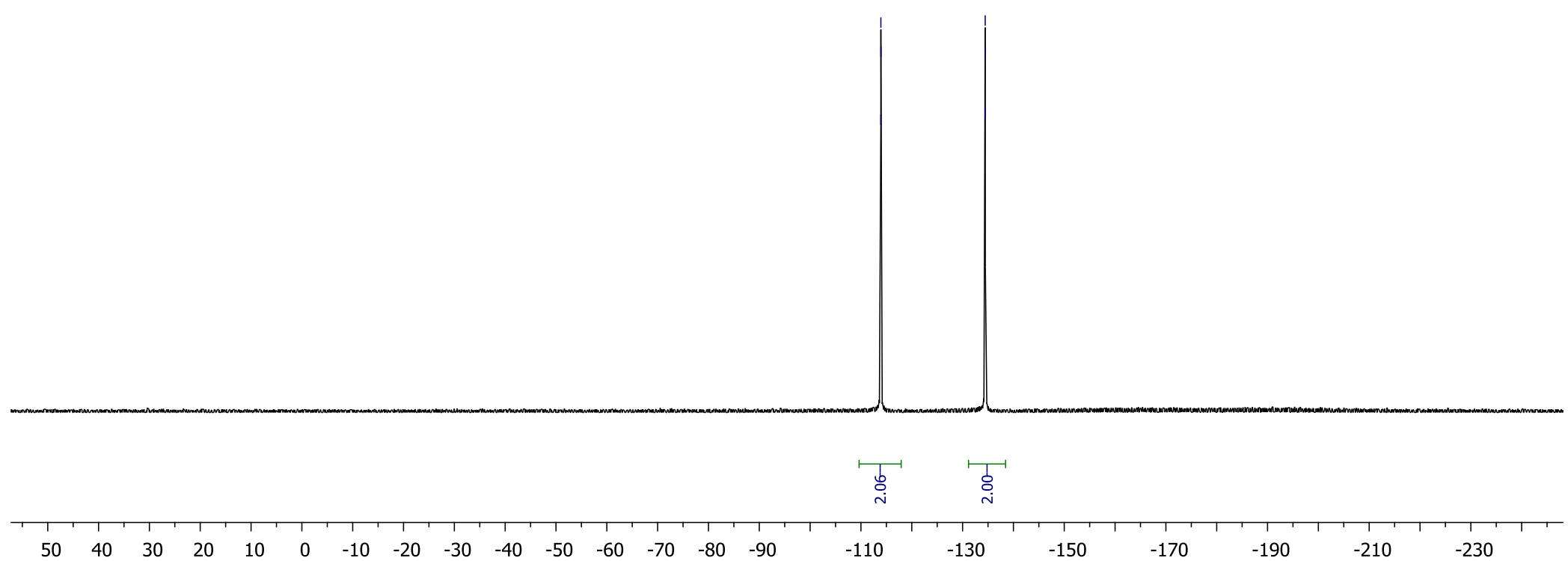




\section{Compound 20a}

${ }^{1} \mathrm{H}$ NMR (400 MHz, $\mathrm{CDCl}_{3}$ )

R1029750

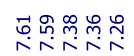

यें⿰

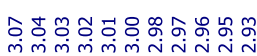

$\iint$
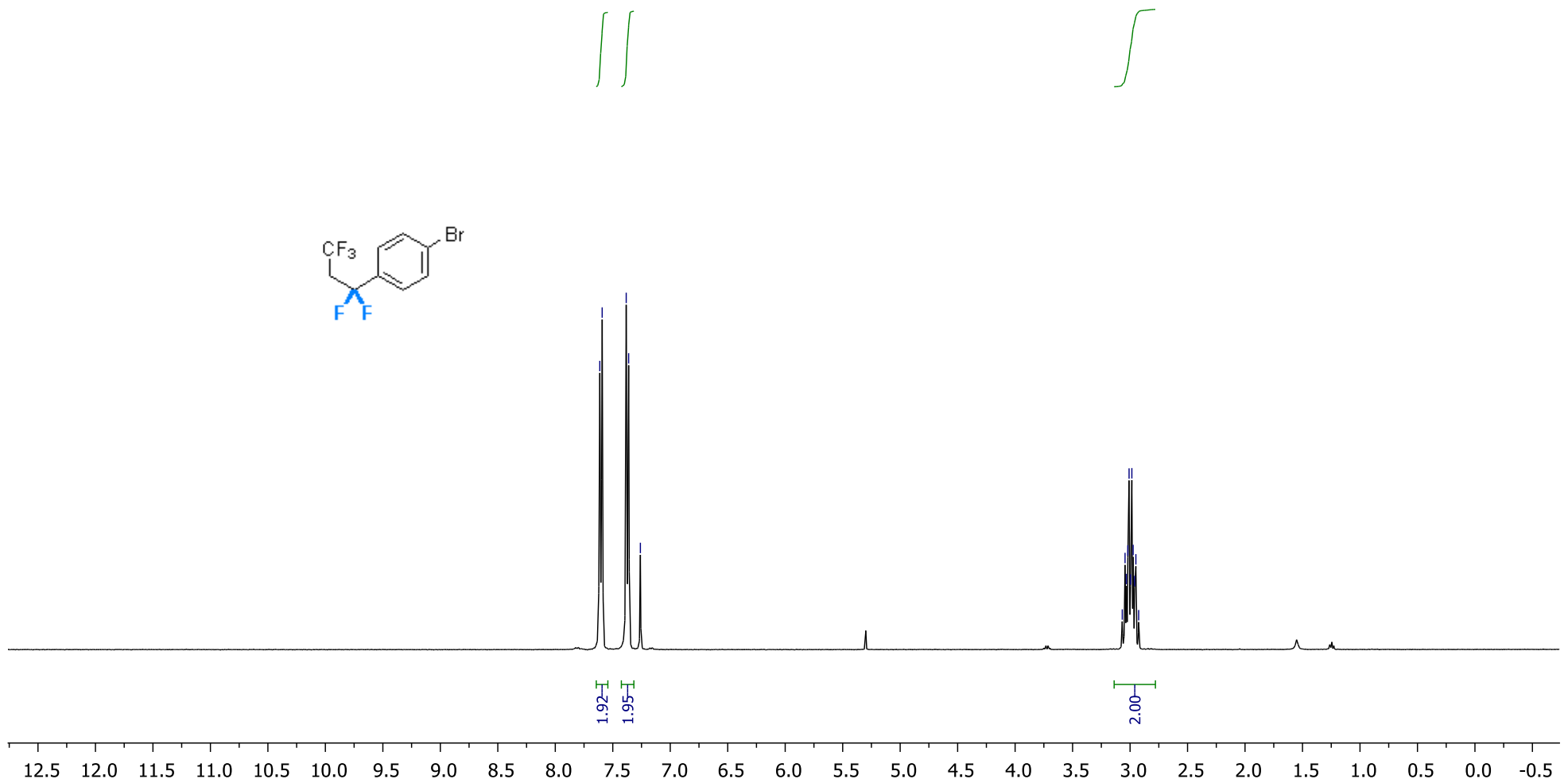
${ }^{13} \mathrm{C}\left\{{ }^{1} \mathrm{H}\right\}$ NMR (151 MHz, $\left.\mathrm{CDCl}_{3}\right)$

R1029750_C13

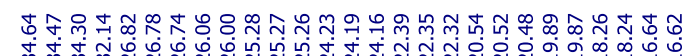

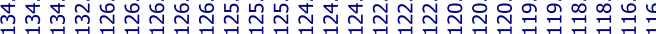

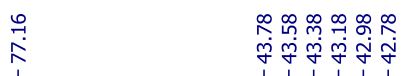

$\overbrace{F F}^{B r}$

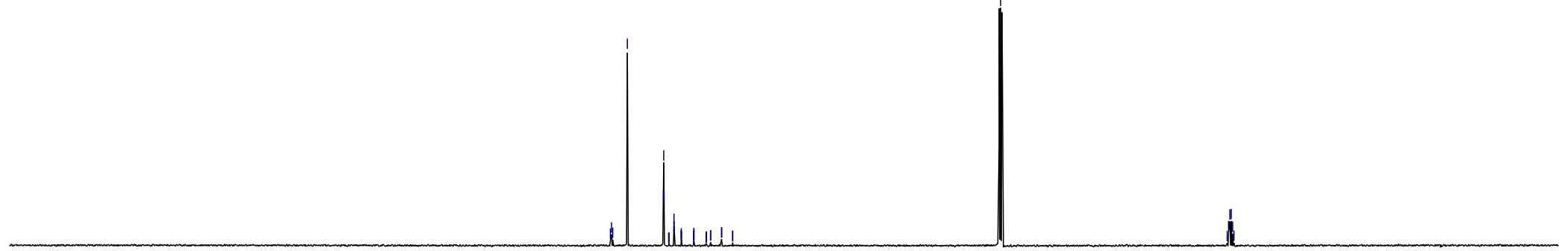

190

$\begin{array}{llll}180 & 170 \quad 160 \quad 150\end{array}$

$140 \quad 130$

120

$\begin{array}{lll}110 & 100 & 90\end{array}$

70

60 
${ }^{19} \mathrm{~F}\left\{{ }^{1} \mathrm{H}\right\} \mathrm{NMR}\left(376 \mathrm{MHz}, \mathrm{CDCl}_{3}\right)$

R1029750_F19 $\{\mathrm{H}\}$

$19 \mathrm{~F}-\{1 \mathrm{H}\}$

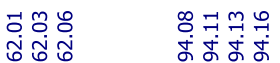

$\psi$ y

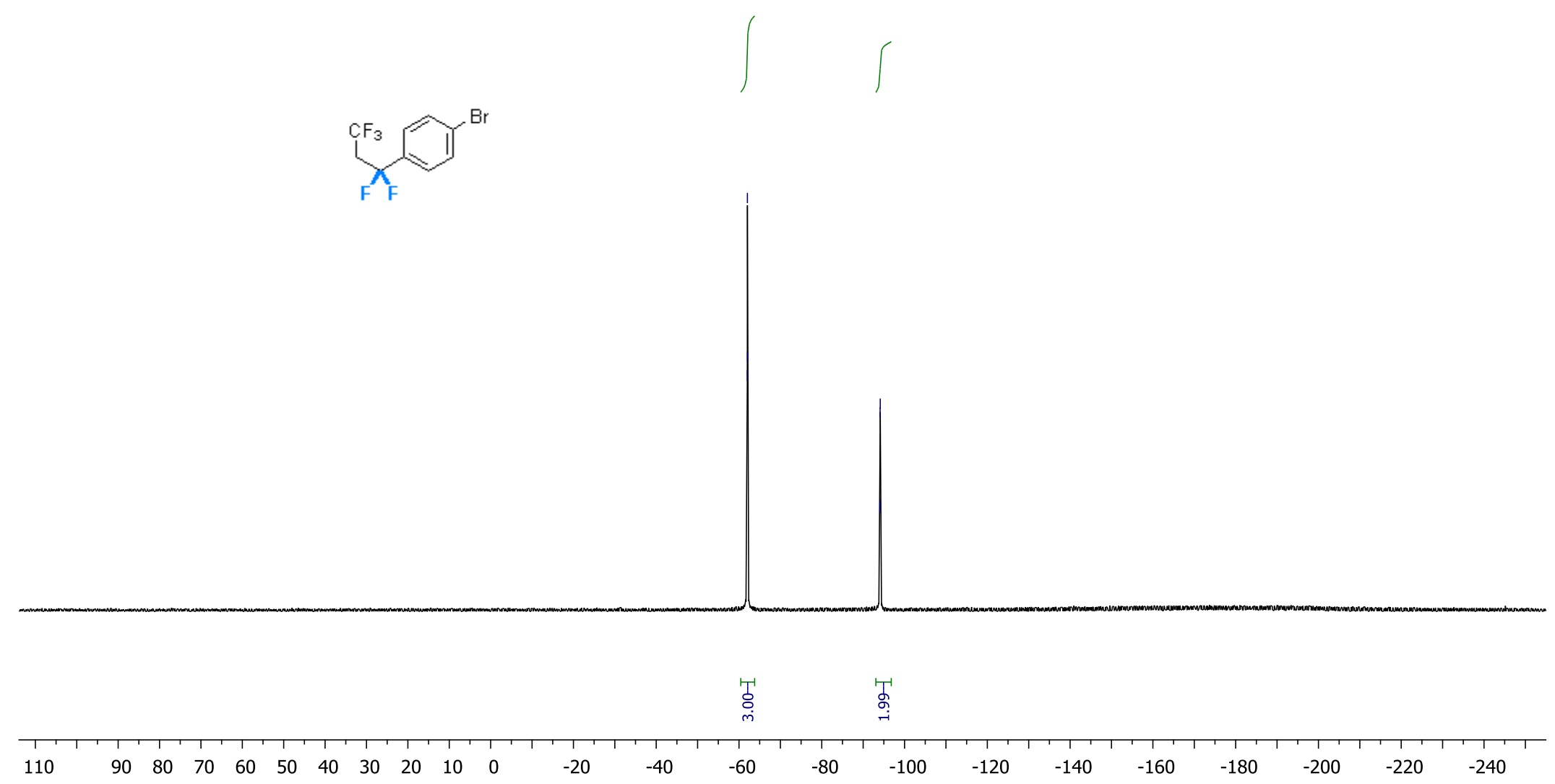




\section{Compound 21a}

${ }^{1} \mathrm{H}$ NMR (400 MHz, $\mathrm{CDCl}_{3}$ )

R688589

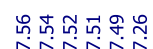

这然

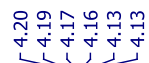

(1)
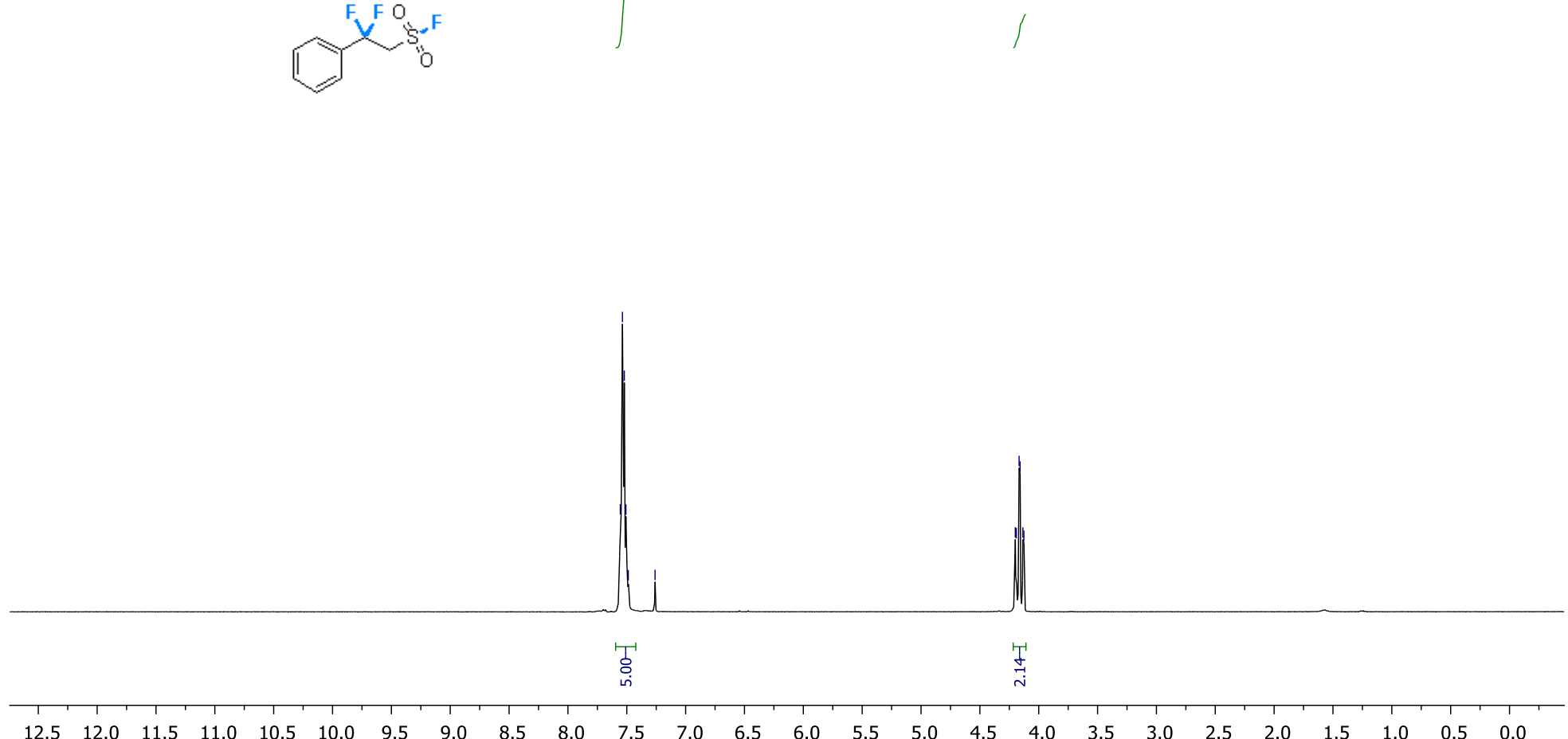
${ }^{13} \mathrm{C}\left\{{ }^{1} \mathrm{H}\right\}$ NMR (151 MHz, $\left.\mathrm{CDCl}_{3}\right)$

R688589_C13

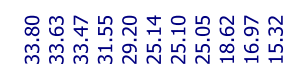

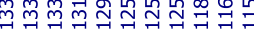

군

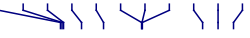

i

$10 x^{F o}$

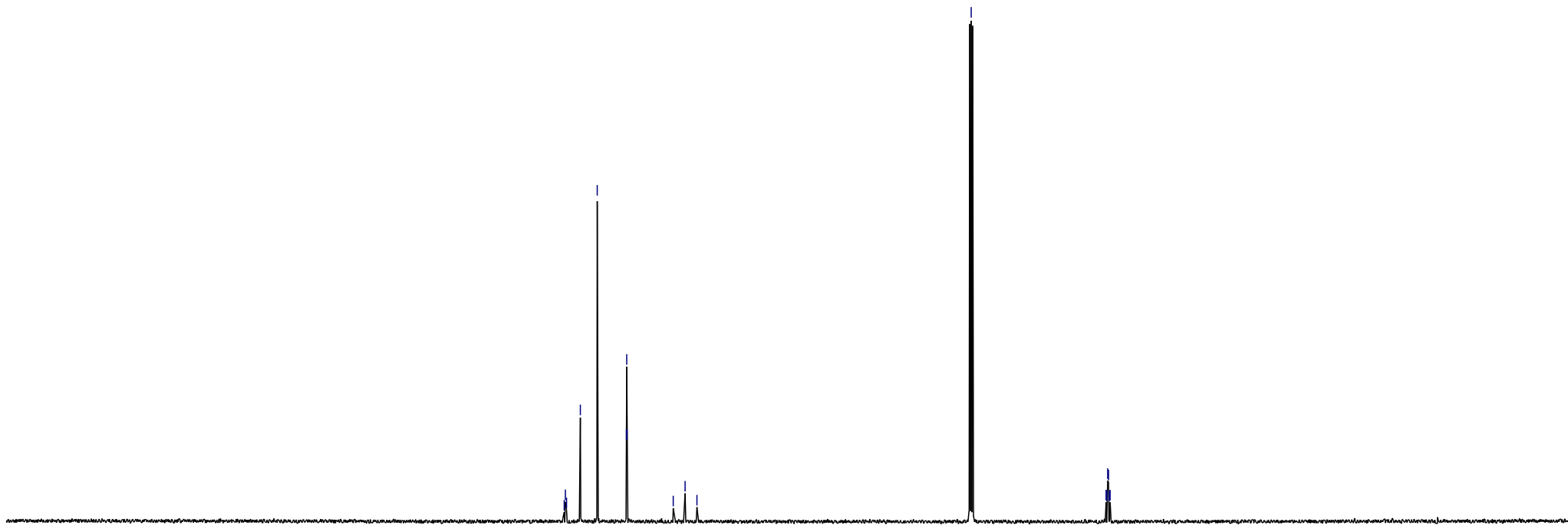

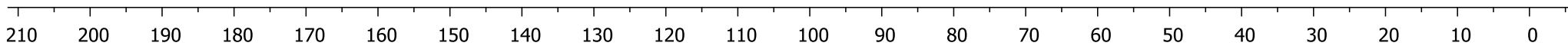


${ }^{19} \mathrm{~F}\left\{{ }^{1} \mathrm{H}\right\}$ NMR $\left(376 \mathrm{MHz}, \mathrm{CDCl}_{3}\right)$

R688589_F19\{H\}

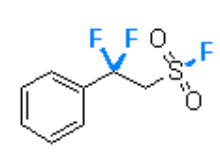

1

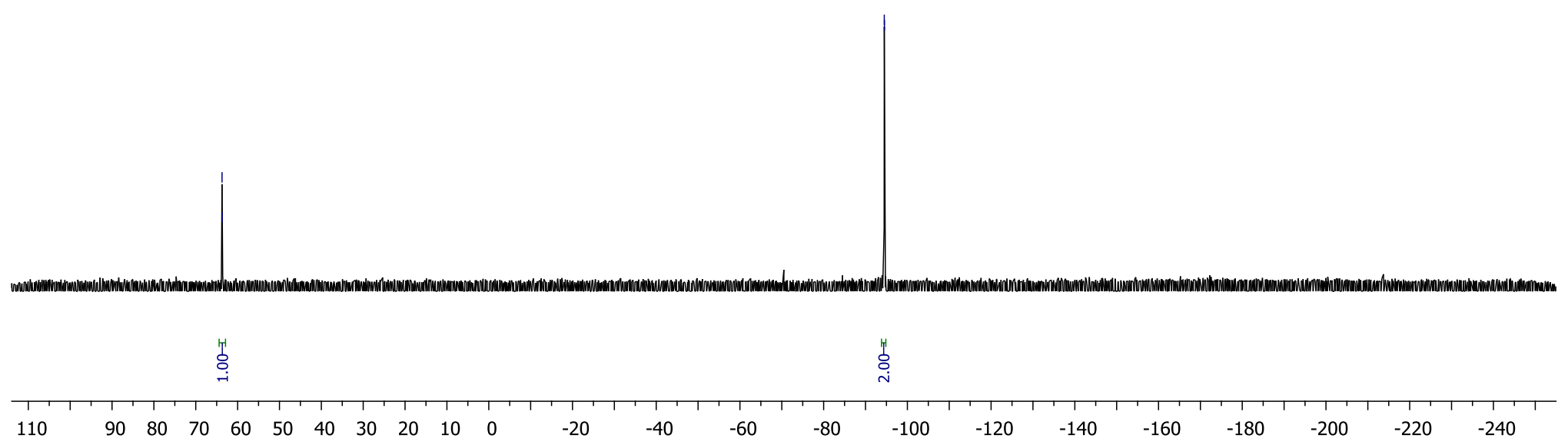




\section{Compound 22a}

${ }^{1} \mathrm{H}$ NMR (400 MHz, $\mathrm{CDCl}_{3}$ )

R1250237
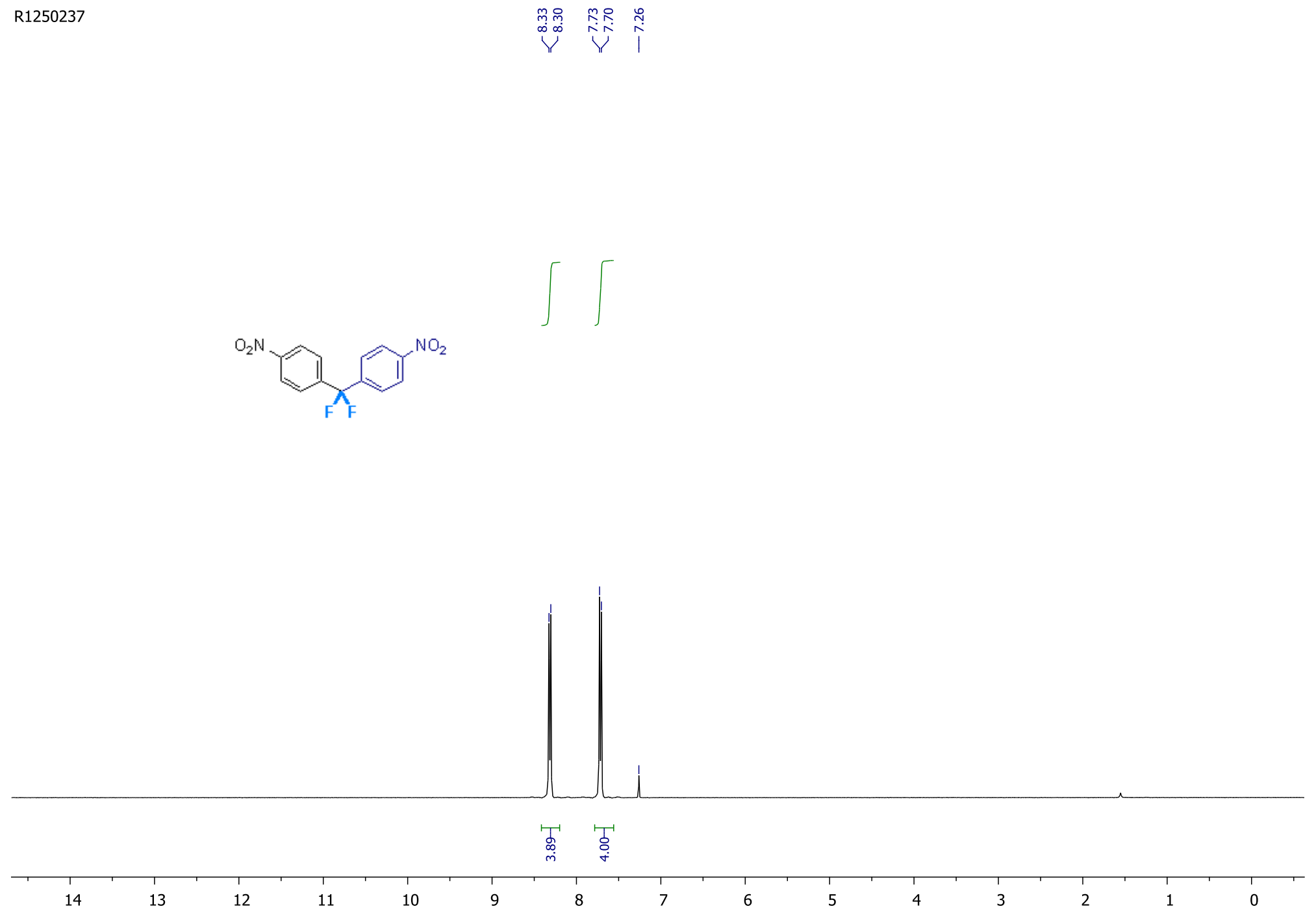
${ }^{13} \mathrm{C}\left\{{ }^{1} \mathrm{H}\right\} \mathrm{NMR}\left(151 \mathrm{MHz}, \mathrm{CDCl}_{3}\right)$

R1250237_C13

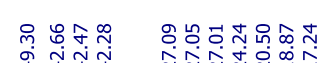

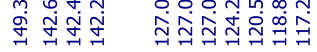

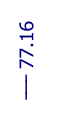

${ }_{F}^{\mathrm{O}_{2} \mathrm{~N}}$

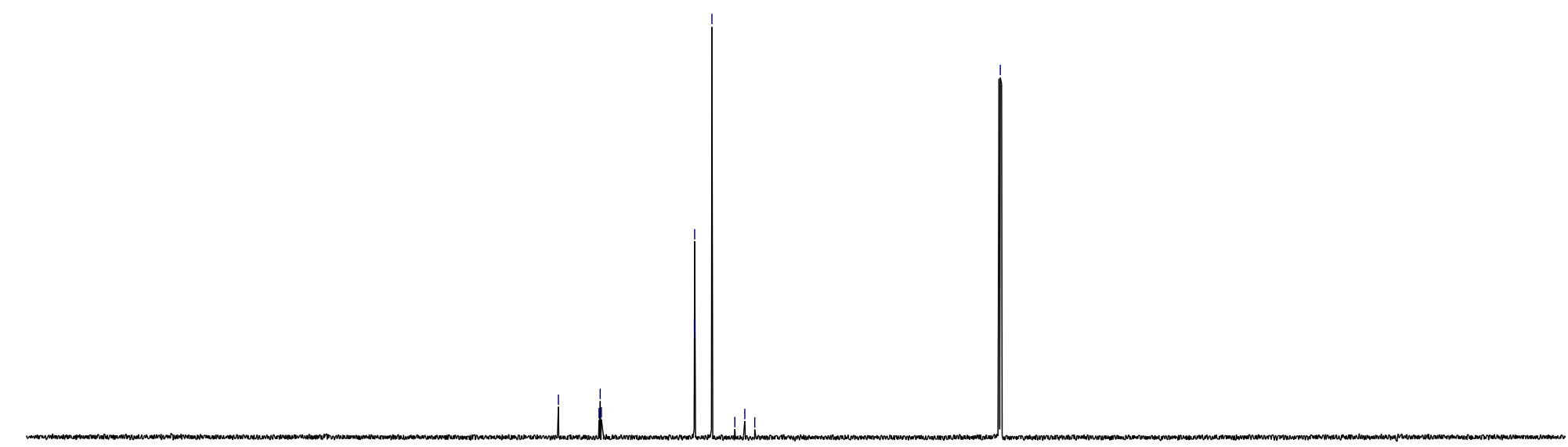

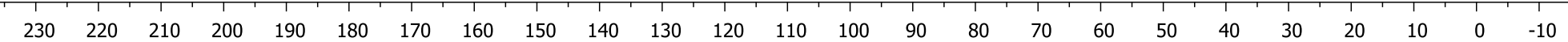


${ }^{19} \mathrm{~F}\left\{{ }^{1} \mathrm{H}\right\}$ NMR $\left(376 \mathrm{MHz}, \mathrm{CDCl}_{3}\right)$

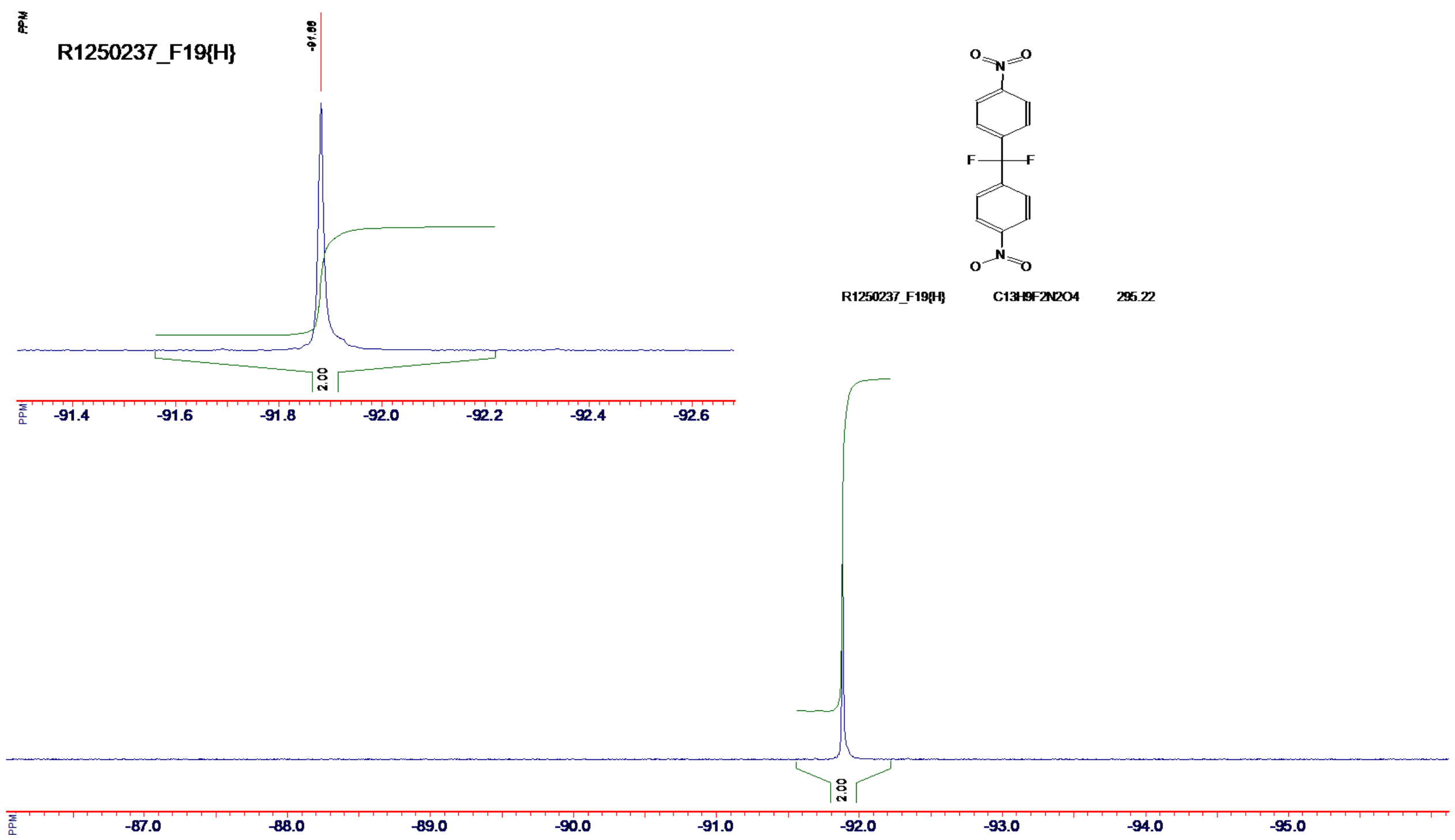




\section{Compound 23a}

${ }^{1} \mathrm{H}$ NMR (400 MHz, $\mathrm{CDCl}_{3}$ )

R1687402

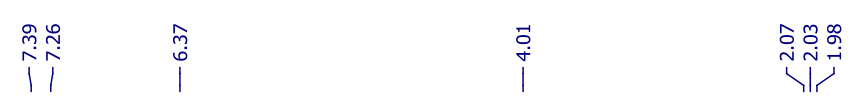

${ }_{\text {Me F }}^{N_{F}^{M}}$

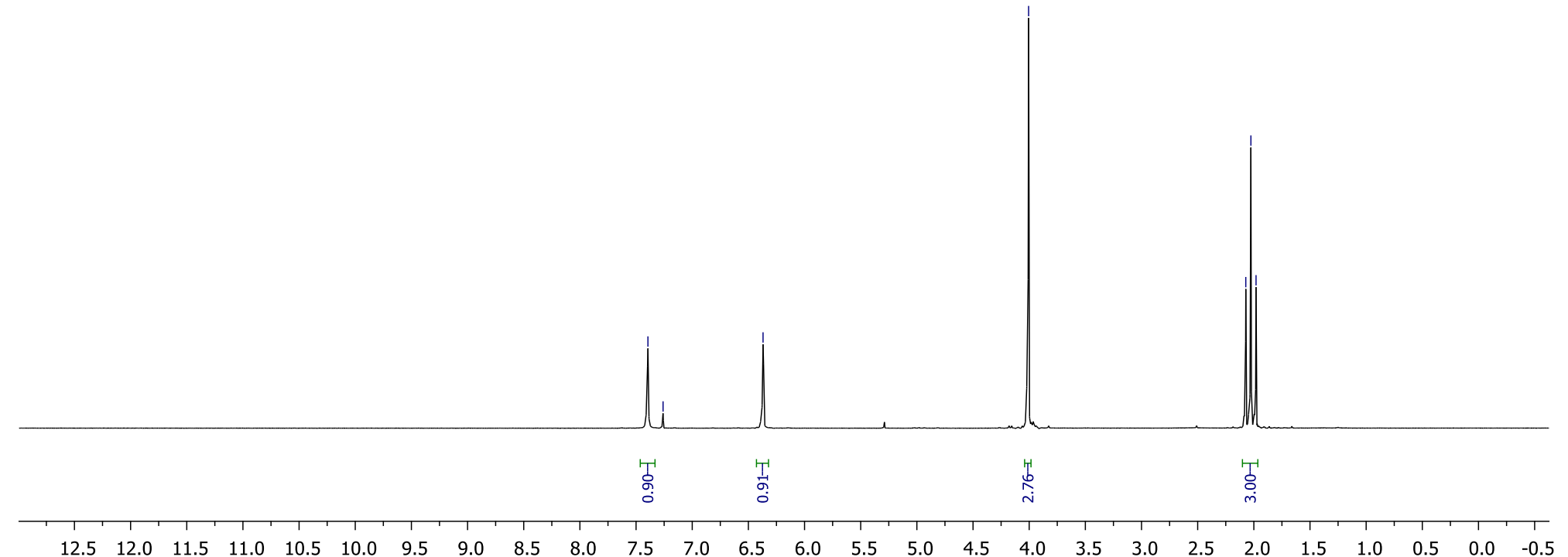


${ }^{1} \mathrm{H}$ NMR $\left(400 \mathrm{MHz}, \mathrm{CDCl}_{3}\right)$

R1687402_C13

${ }_{N K_{F}}^{M e}$
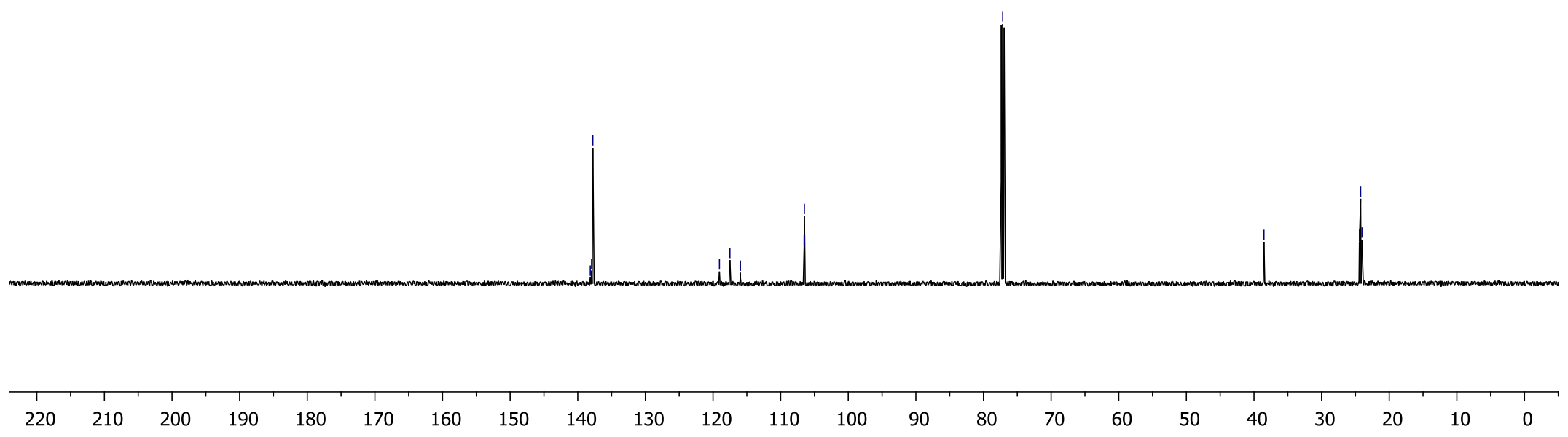


\section{${ }^{19} \mathrm{~F}\left\{{ }^{1} \mathrm{H}\right\}$ NMR (376 MHz, $\left.\mathrm{CDCl}_{3}\right)$}

R1687402_F19 $\{H\}$

$$
{ }_{\mathrm{Me}} \mathrm{F}_{\mathrm{F}}^{\mathrm{Me}}
$$

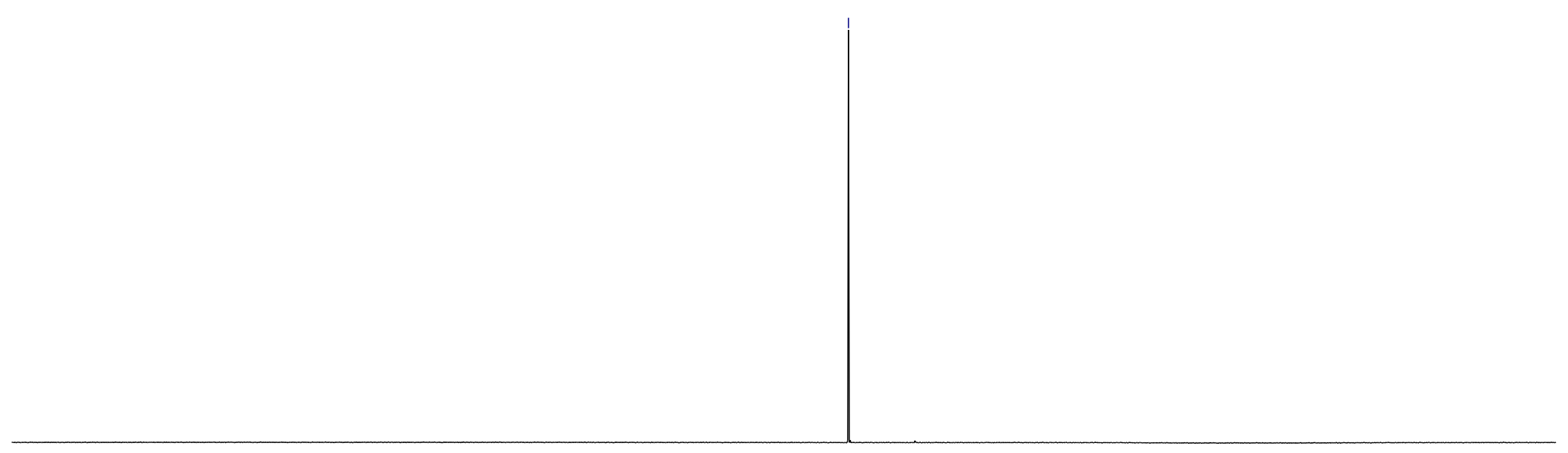




\section{Compound 24a}

${ }^{1} \mathrm{H}$ NMR (400 MHz, DMSO- $d_{6}$ )

R2599631

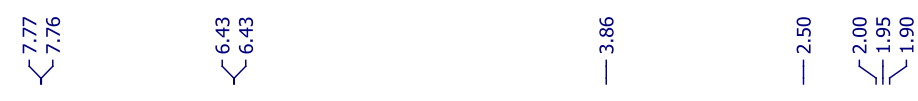

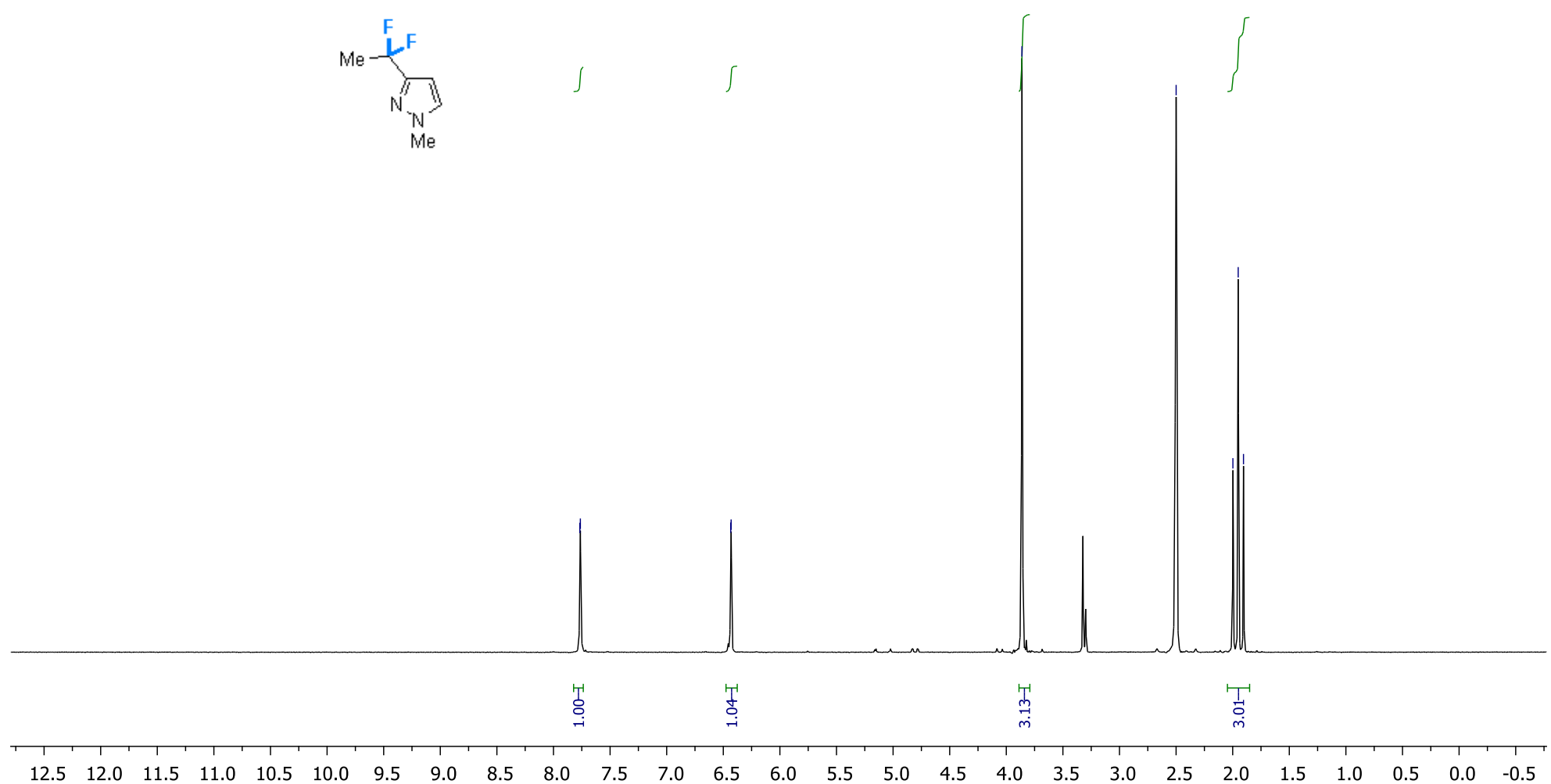




\section{${ }^{13} \mathrm{C}\left\{{ }^{1} \mathrm{H}\right\}$ NMR $\left(126 \mathrm{MHz}, \mathrm{DMSO}-d_{6}\right)$}

R2599631_C1:

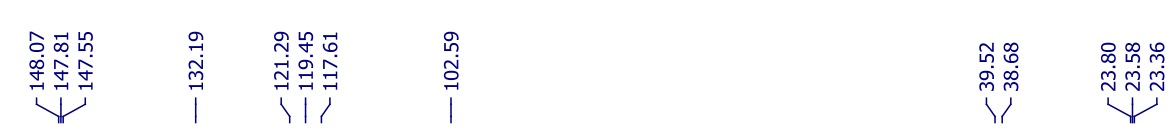

$\sum_{\mathrm{Me}}^{\mathrm{F}}$

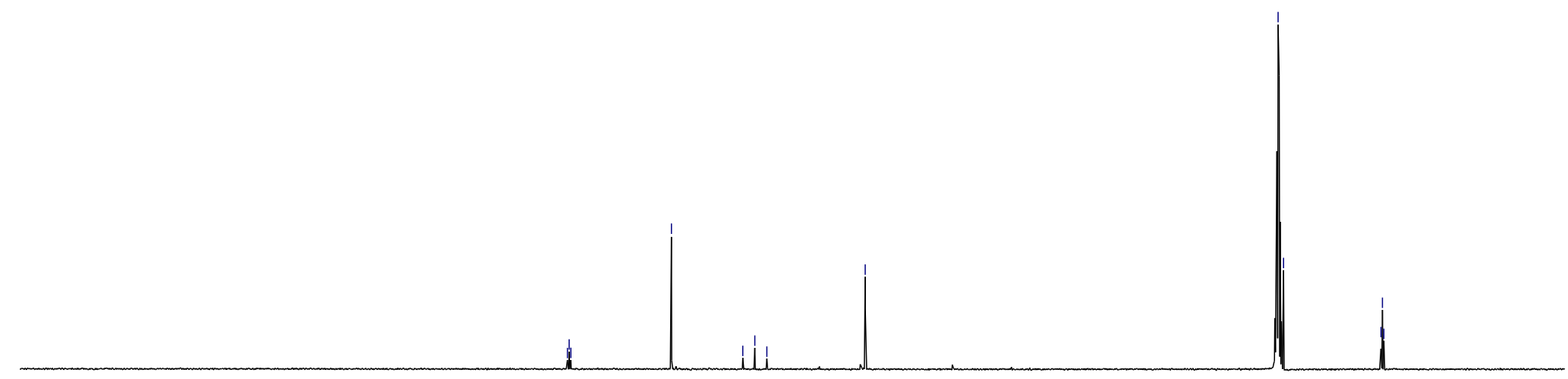

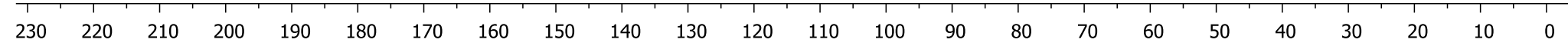




\section{${ }^{19} \mathrm{~F}\left\{{ }^{1} \mathrm{H}\right\}$ NMR (376 MHz, DMSO- $d_{6}$ )}

R2599631_F19 $\{H\}$
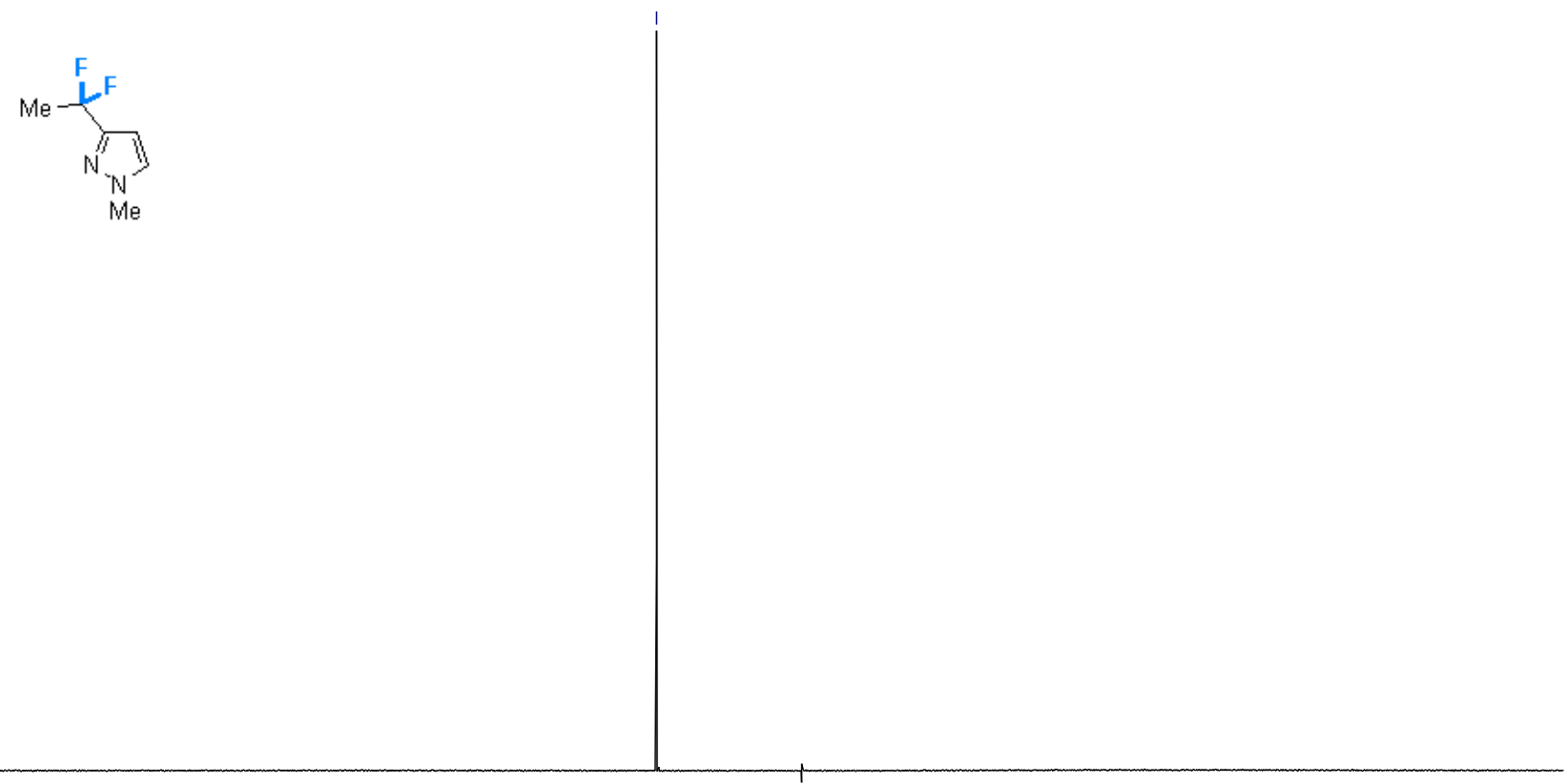

$\begin{array}{lllllllllll}110 & 90 & 80 & 70 & 60 & 50 & 40 & 30 & 20 & 10 & 0\end{array}$ 


\section{Compound 25a}

${ }^{1} \mathrm{H}$ NMR (400 MHz, $\mathrm{CDCl}_{3}$ )

R2595225

$$
\text { Me } \mathrm{FF}_{\mathrm{F}} \mathrm{CO}_{2} \mathrm{Et}
$$
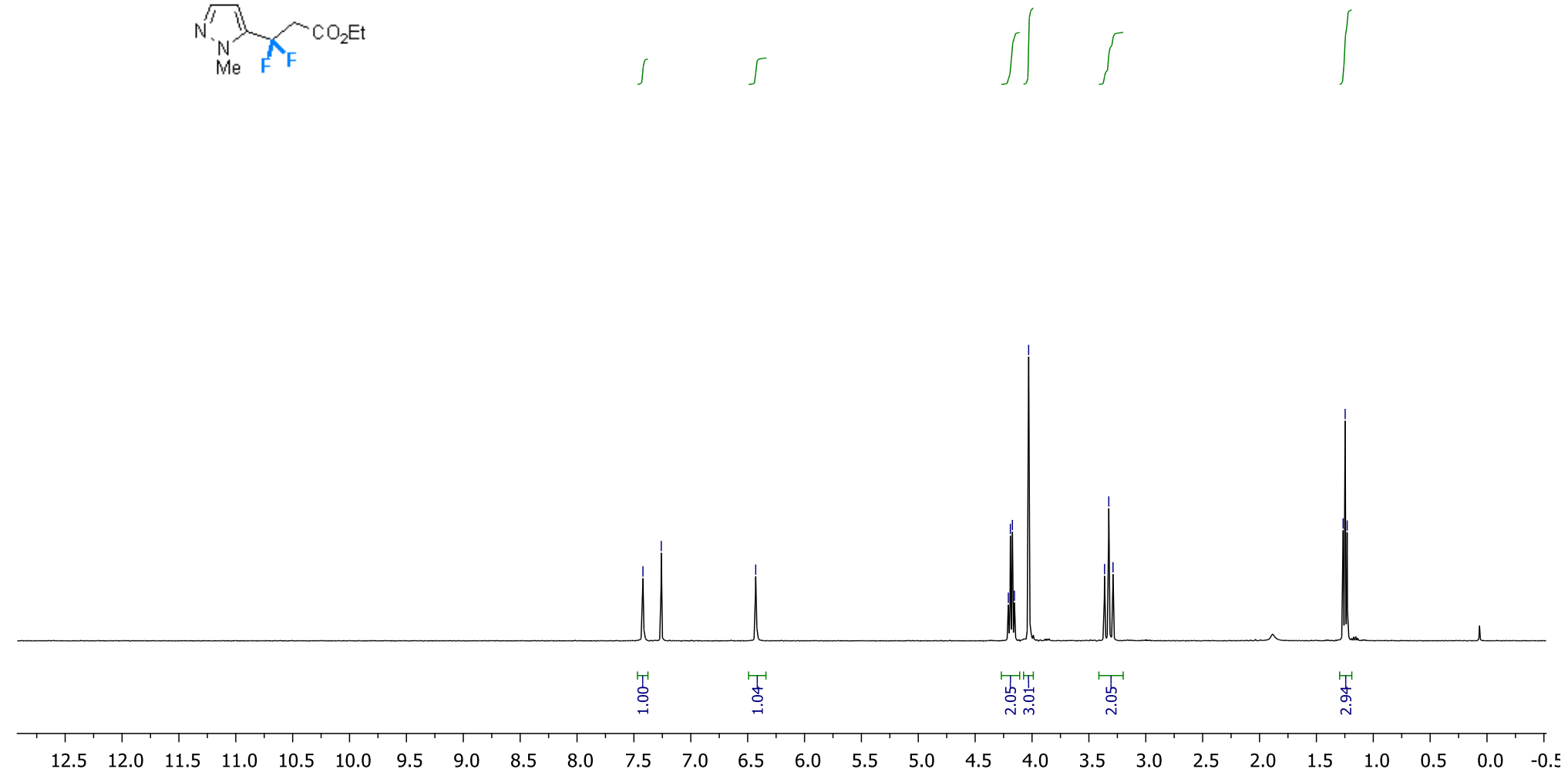
${ }^{13} \mathrm{C}\left\{{ }^{1} \mathrm{H}\right\}$ NMR (126 MHz, $\left.\mathrm{CDCl}_{3}\right)$

R2595225_C1:

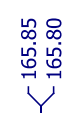

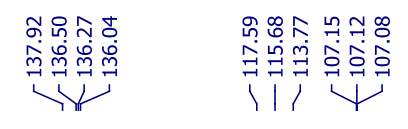

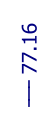

i

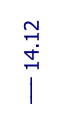
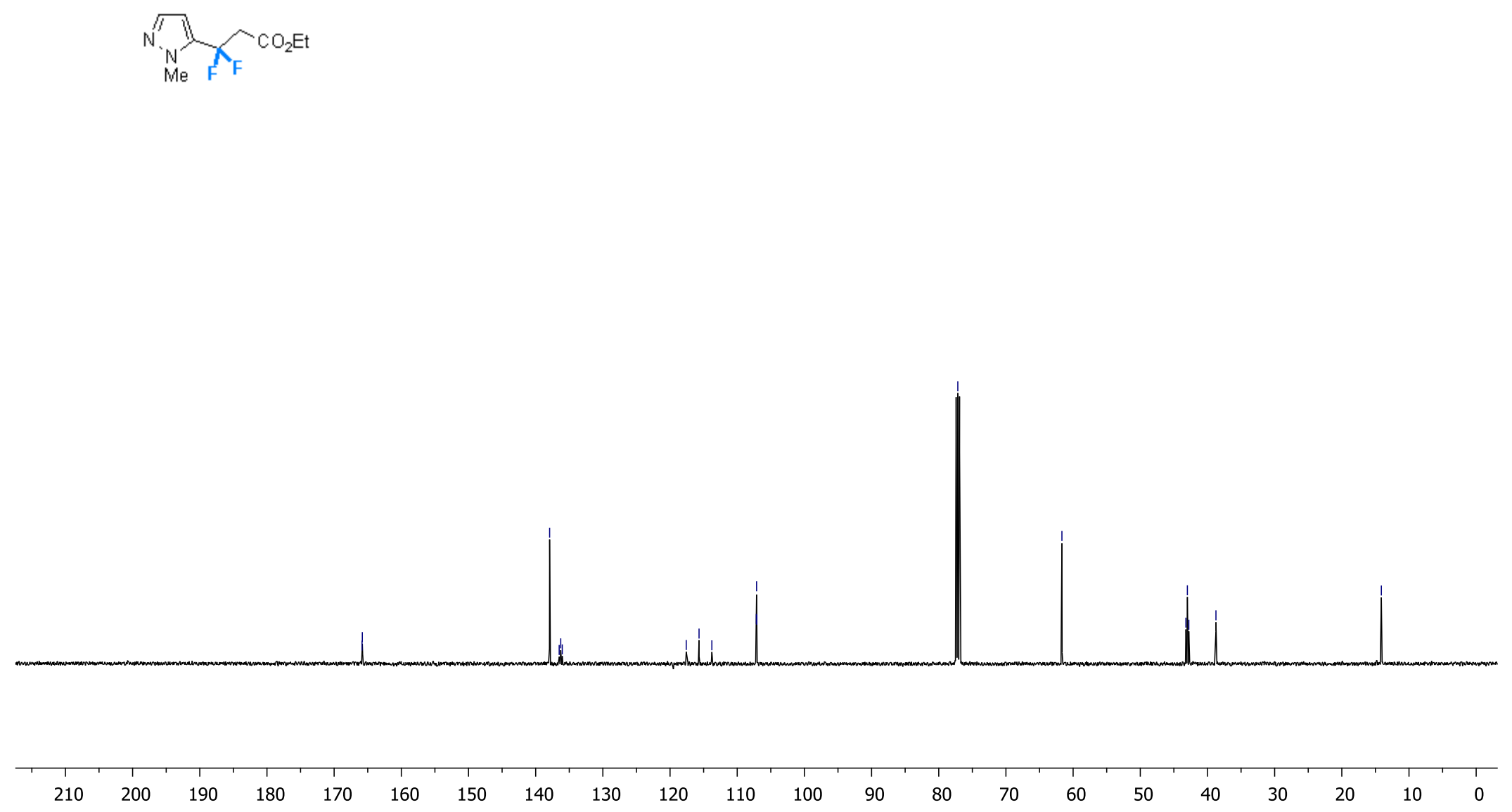

S63 


\section{${ }^{19} \mathrm{~F}\left\{{ }^{1} \mathrm{H}\right\}$ NMR (376 MHz, $\left.\mathrm{CDCl}_{3}\right)$}

R2595225_F19 $\{\mathrm{H}\}$

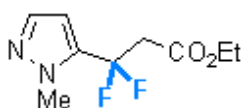

$110 \quad \begin{array}{rlllllllllllllllllllllll} & 90 & 70 & 60 & 50 & 40 & 30 & 20 & 10 & 0 & -20 & -40 & -60 & -80 & -100 & -120 & -140 & -160 & -180 & -200 & -220 & -240 & \end{array}$ 


\section{Compound 26a}

${ }^{1} \mathrm{H}$ NMR (400 MHz, $\mathrm{CDCl}_{3}$ )

R1501113

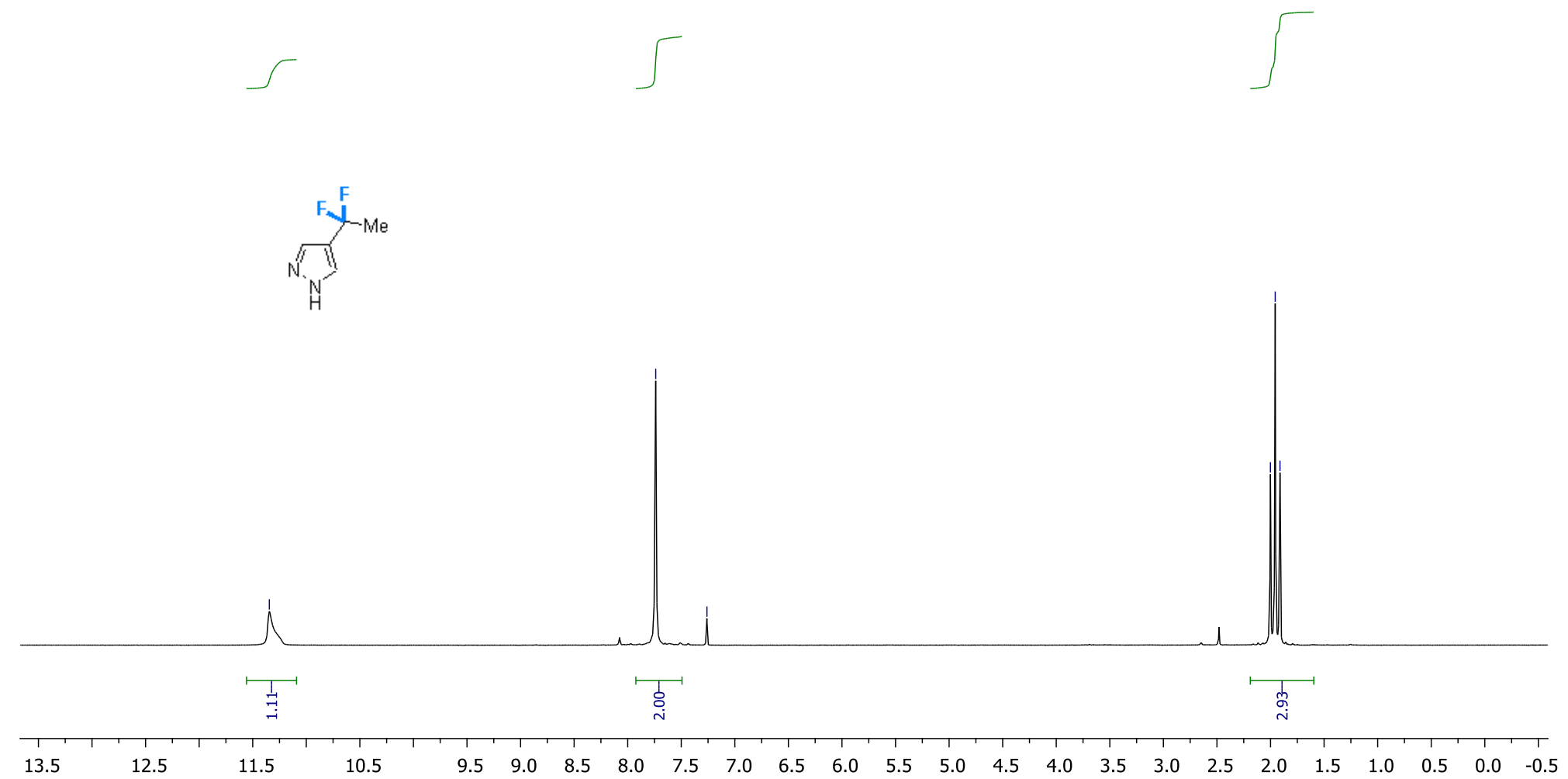


${ }^{3} \mathrm{C}\left\{{ }^{1} \mathrm{H}\right\} \mathrm{NMR}\left(151 \mathrm{MHz}, \mathrm{CDCl}_{3}\right)$

R1501113_C13

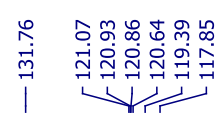

$\stackrel{\substack{i \\ i}}{i}$

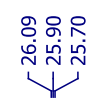

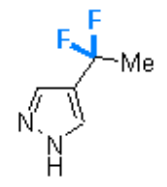

S66 


\section{${ }^{19} \mathrm{~F}\left\{{ }^{1} \mathrm{H}\right\}$ NMR (376 MHz, $\left.\mathrm{CDCl}_{3}\right)$}

R1501113_F19 $\{\mathrm{H}\}$

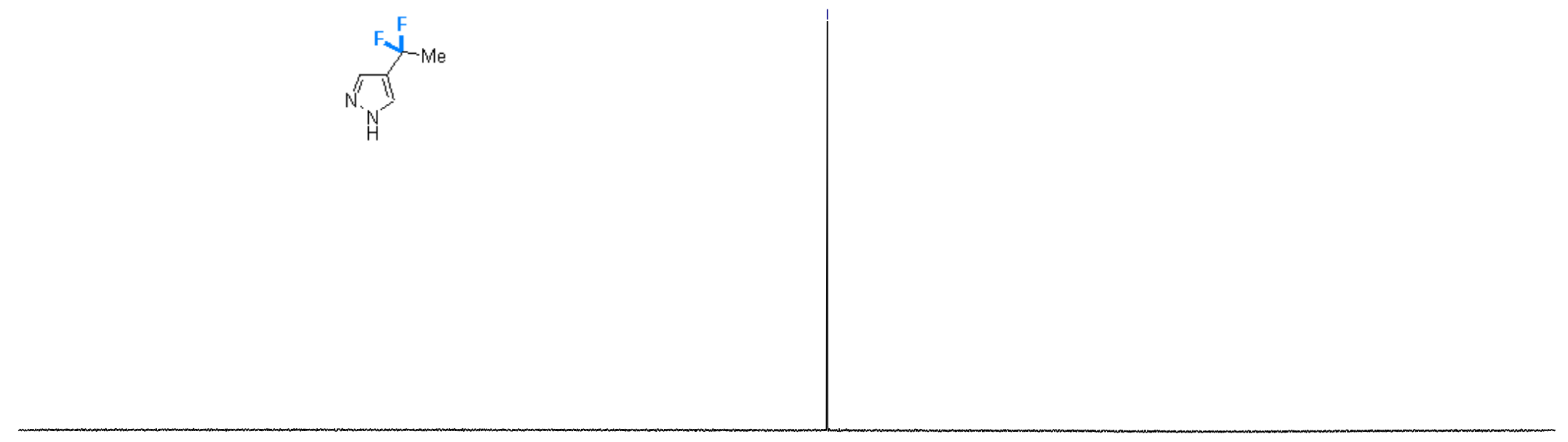




\section{Compound 27a}

${ }^{1} \mathrm{H}$ NMR (400 MHz, $\mathrm{CDCl}_{3}$ )

R1940862

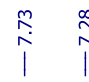

ํㅗำㅇำ

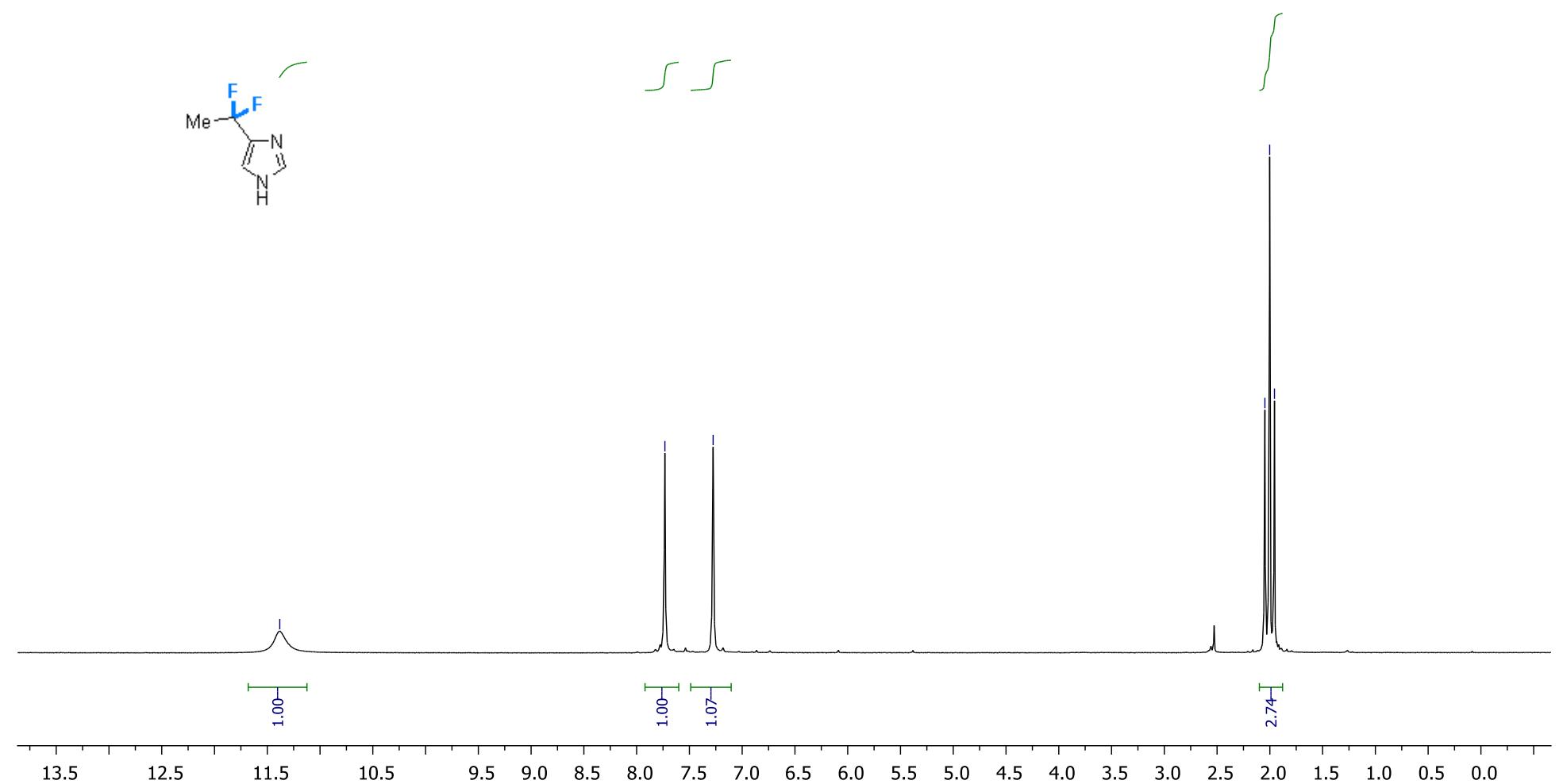


${ }^{13} \mathrm{C}\left\{{ }^{1} \mathrm{H}\right\}$ NMR (101 MHz, $\left.\mathrm{CDCl}_{3}\right)$

R1940862_C13

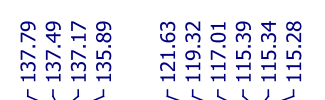

$\stackrel{0}{i}$

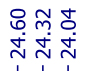

$M=-\sum^{E}$

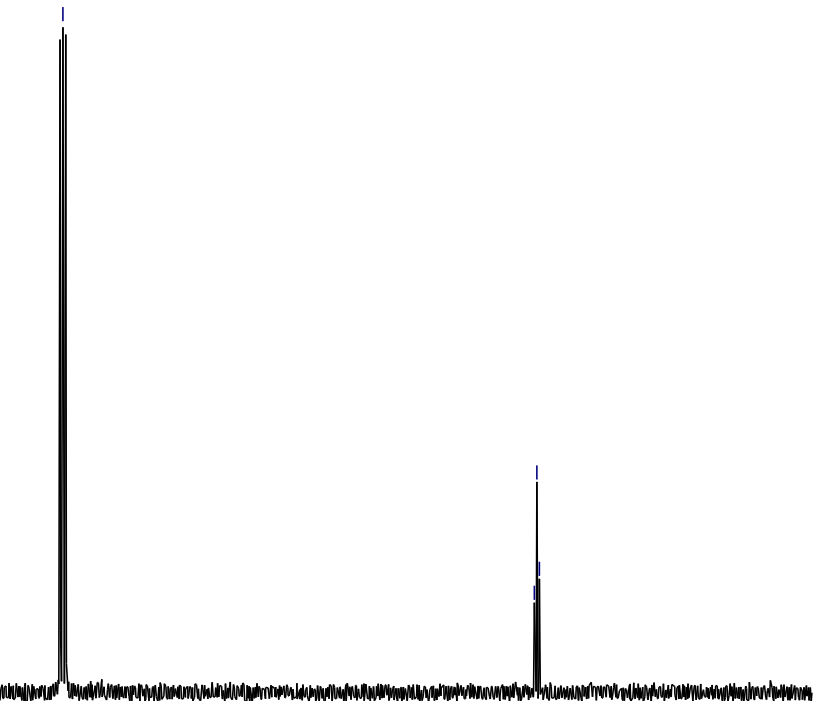




\section{${ }^{19} \mathrm{~F}\left\{{ }^{1} \mathrm{H}\right\}$ NMR (376 MHz, $\left.\mathrm{CDCl}_{3}\right)$}

R1940862_F19\{H $\}$ $19 \mathrm{~F}-\{1 \mathrm{H}\}$
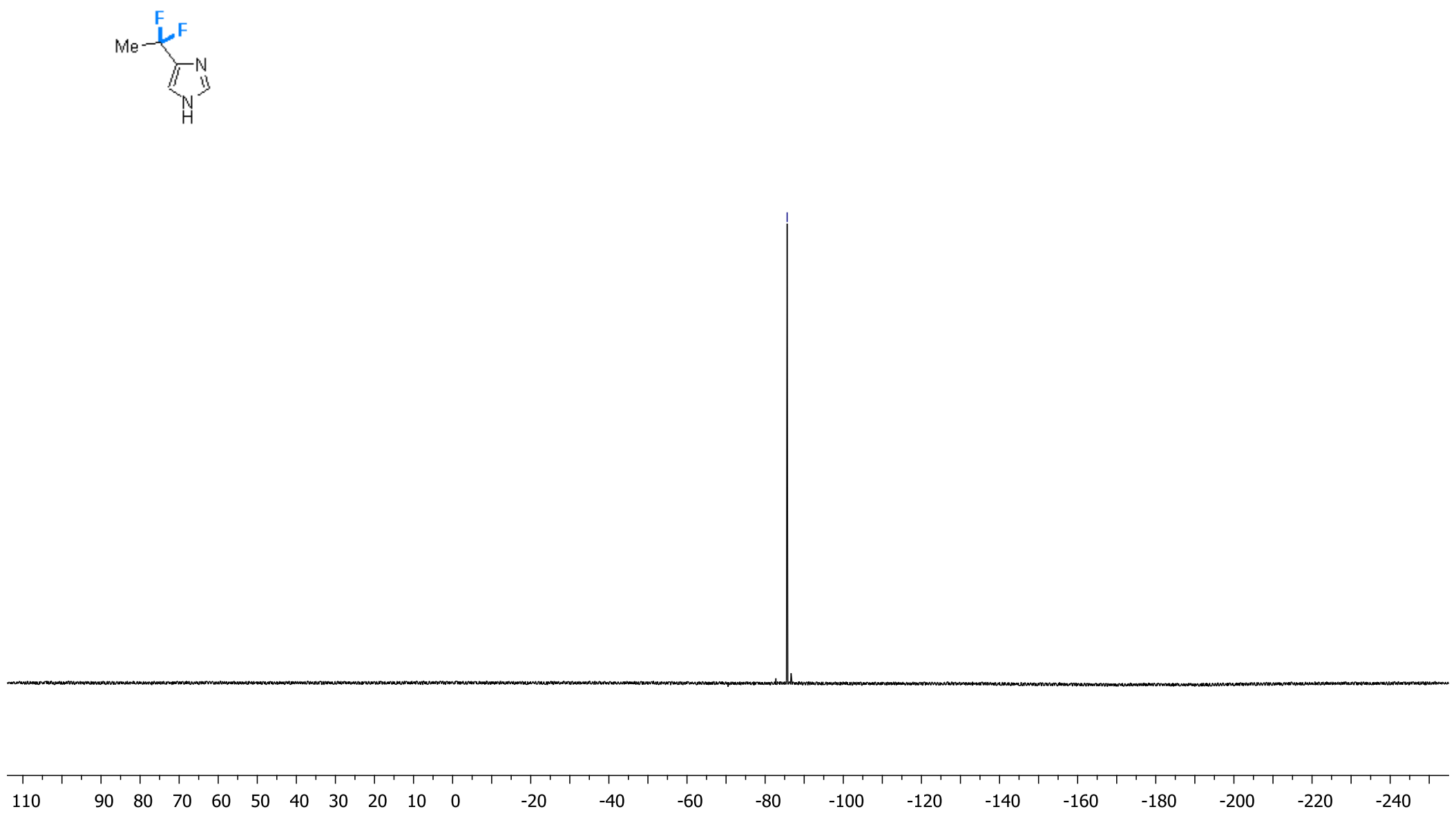


\section{Compound 28a}

${ }^{1} \mathrm{H}$ NMR (400 MHz, $\mathrm{CDCl}_{3}$ )

R1811706

${ }_{B r}^{N} \int_{H}^{M} X_{F}^{M e}$

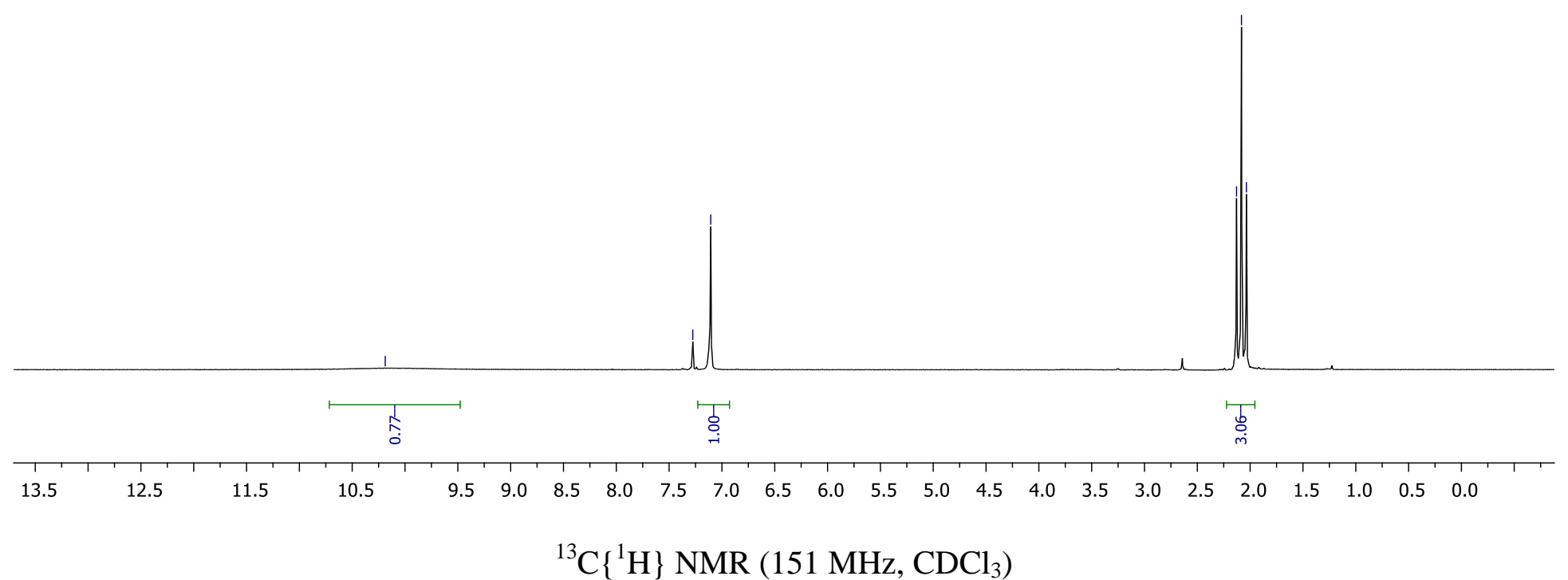


R1811706_C13
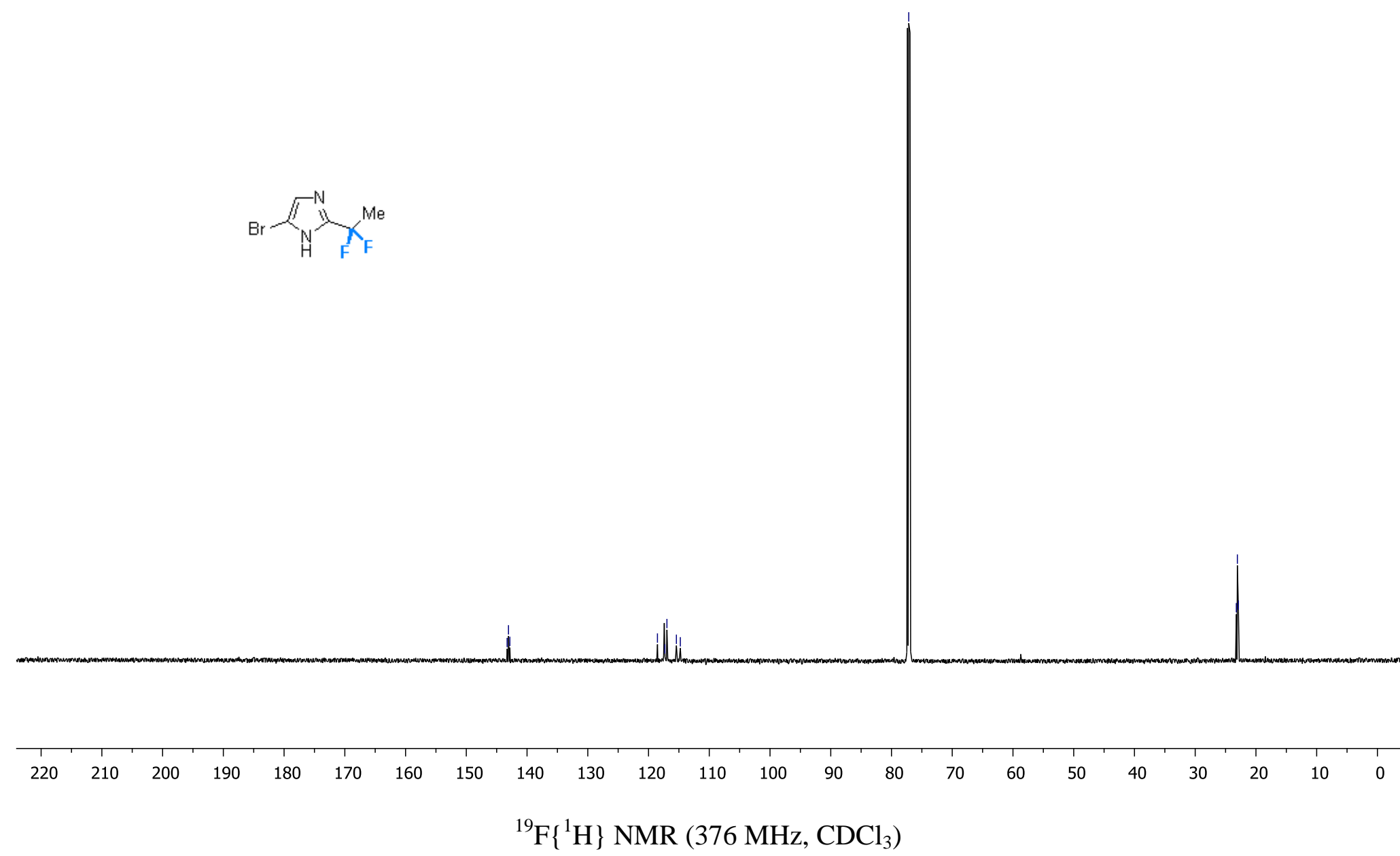
R1811706_F19 $\{\mathrm{H}\}$

${ }_{\int_{H}^{N}}^{N} \int_{F}^{M e}$

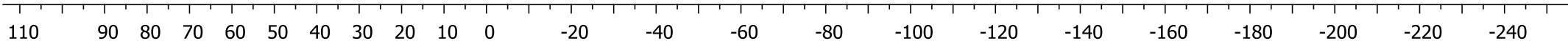




\section{Compound 29a}

${ }^{1} \mathrm{H}$ NMR (400 MHz, DMSO- $d_{6}$ )

R2427533

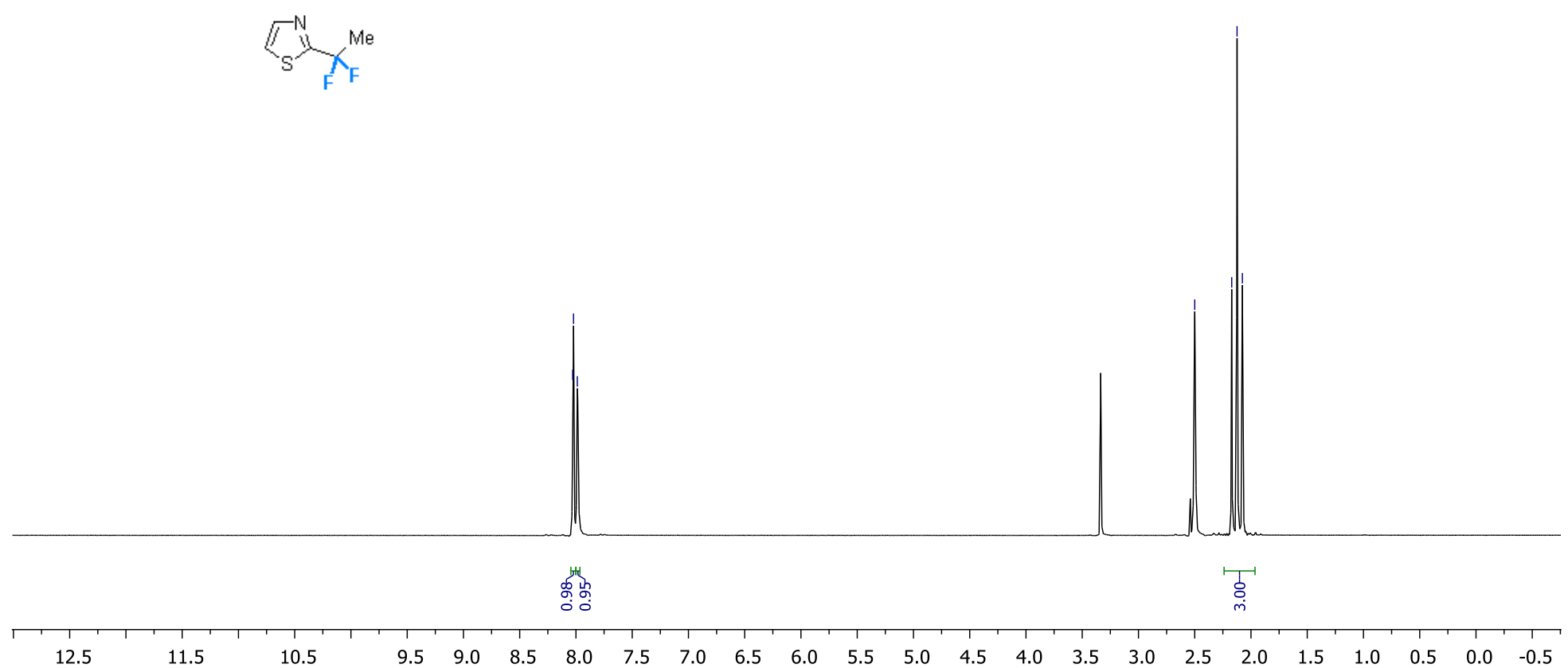




\section{${ }^{13} \mathrm{C}\left\{{ }^{1} \mathrm{H}\right\}$ NMR $\left(126 \mathrm{MHz}\right.$, DMSO- $\left.d_{6}\right)$}

R2427533_C13

$13 \mathrm{C}$ (1H-decoupled)

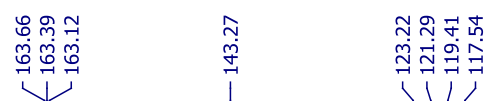

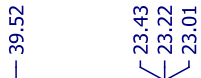

${ }_{S}^{N}{ }_{F}^{N} X_{F}^{M e}$

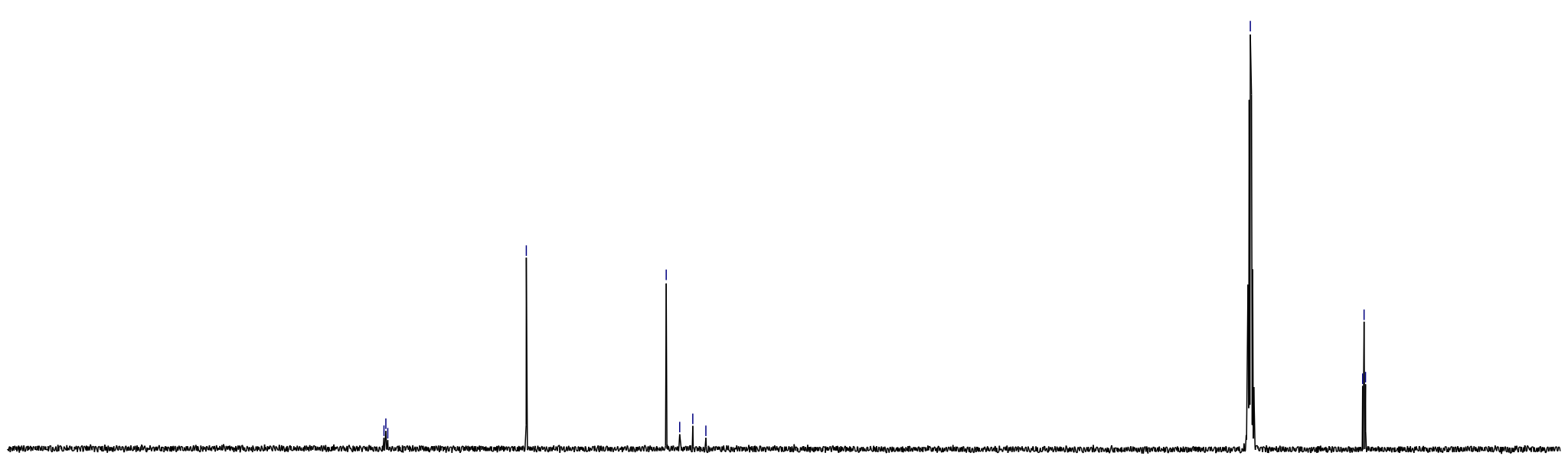

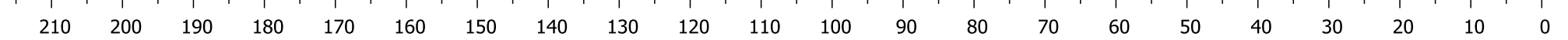




\section{${ }^{19} \mathrm{~F}\left\{{ }^{1} \mathrm{H}\right\}$ NMR (376 MHz, DMSO- $\left.d_{6}\right)$}

R2427533_F19\{H\}

$$
{ }_{S}^{N} \sum_{F}^{N} X_{F}^{M e}
$$

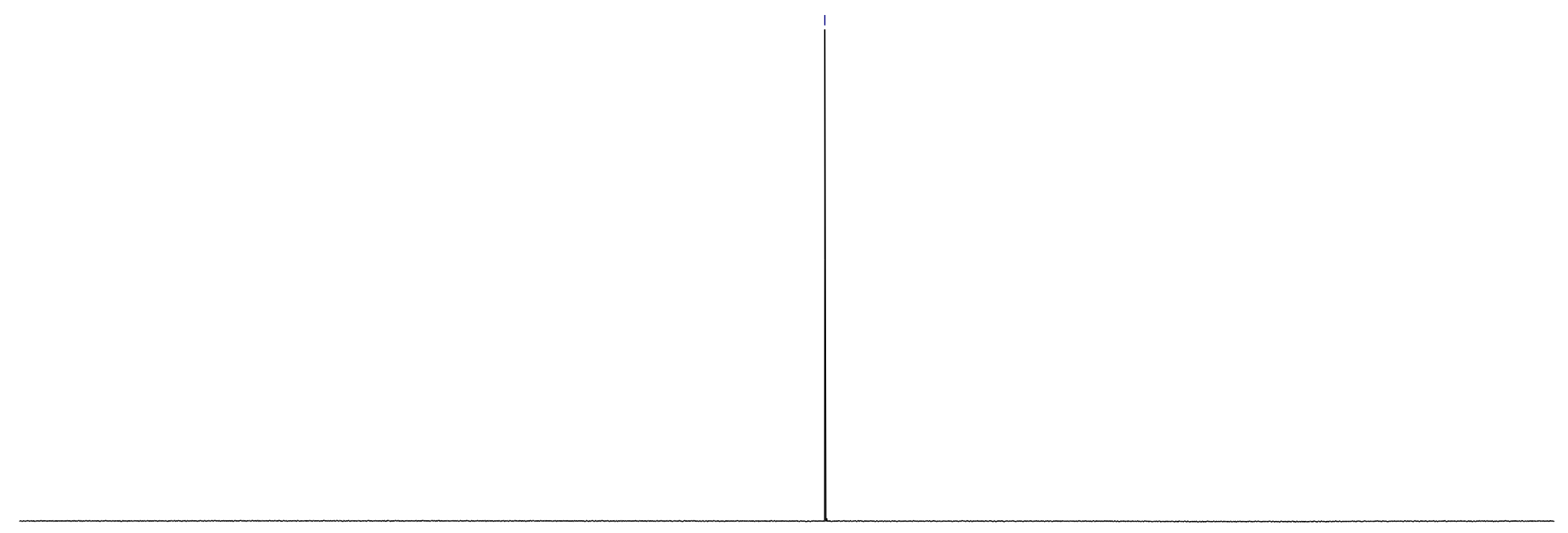

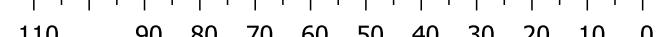




\section{Compound 30a}

${ }^{1} \mathrm{H}$ NMR (400 MHz, $\mathrm{CDCl}_{3}$ )

R1852407

$$
{ }^{\mathrm{Br}} \mathrm{H}_{\mathrm{S}}^{\mathrm{N}} \mathrm{X}_{\mathrm{F}}^{\mathrm{Me}}
$$

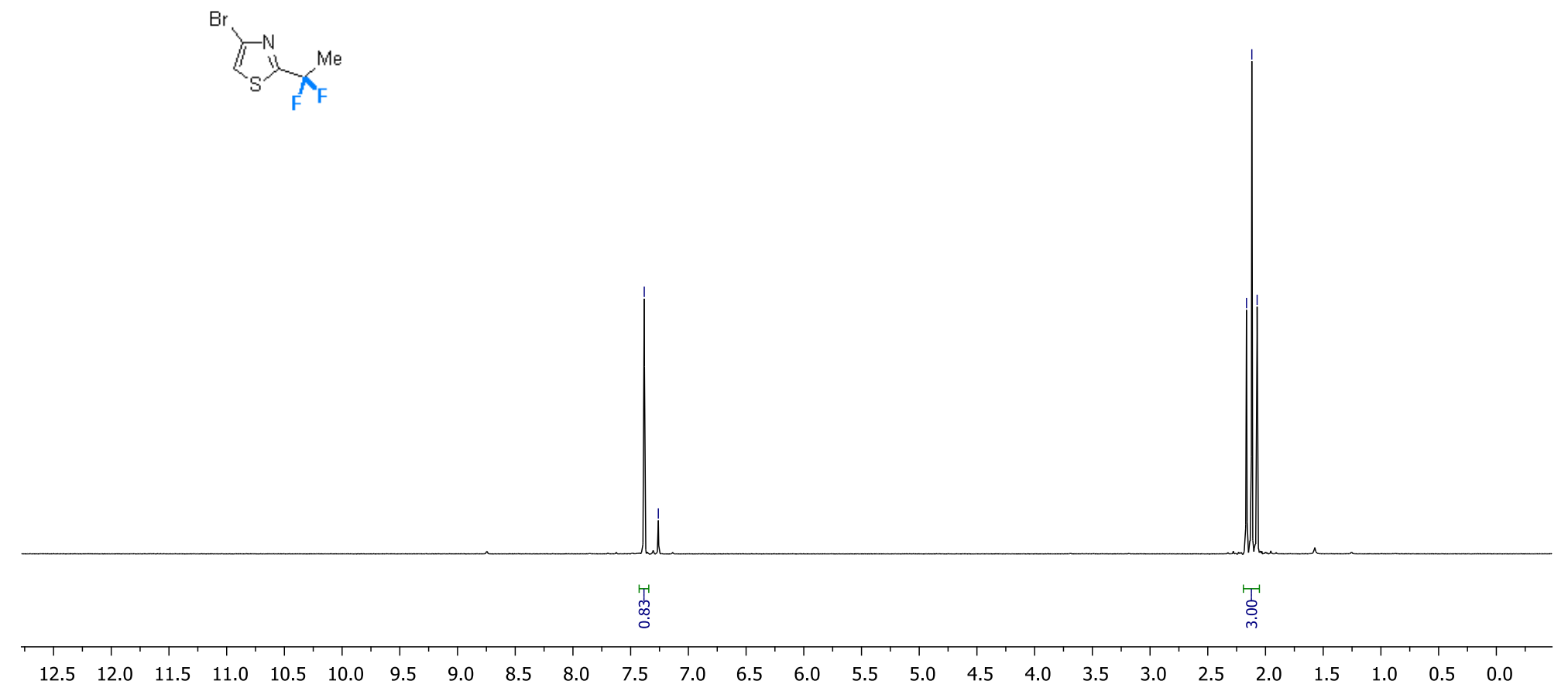


${ }^{13} \mathrm{C}\left\{{ }^{1} \mathrm{H}\right\}$ NMR $\left(151 \mathrm{MHz}, \mathrm{CDCl}_{3}\right)$

R1852407_C13

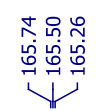

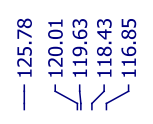

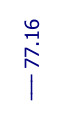

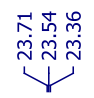
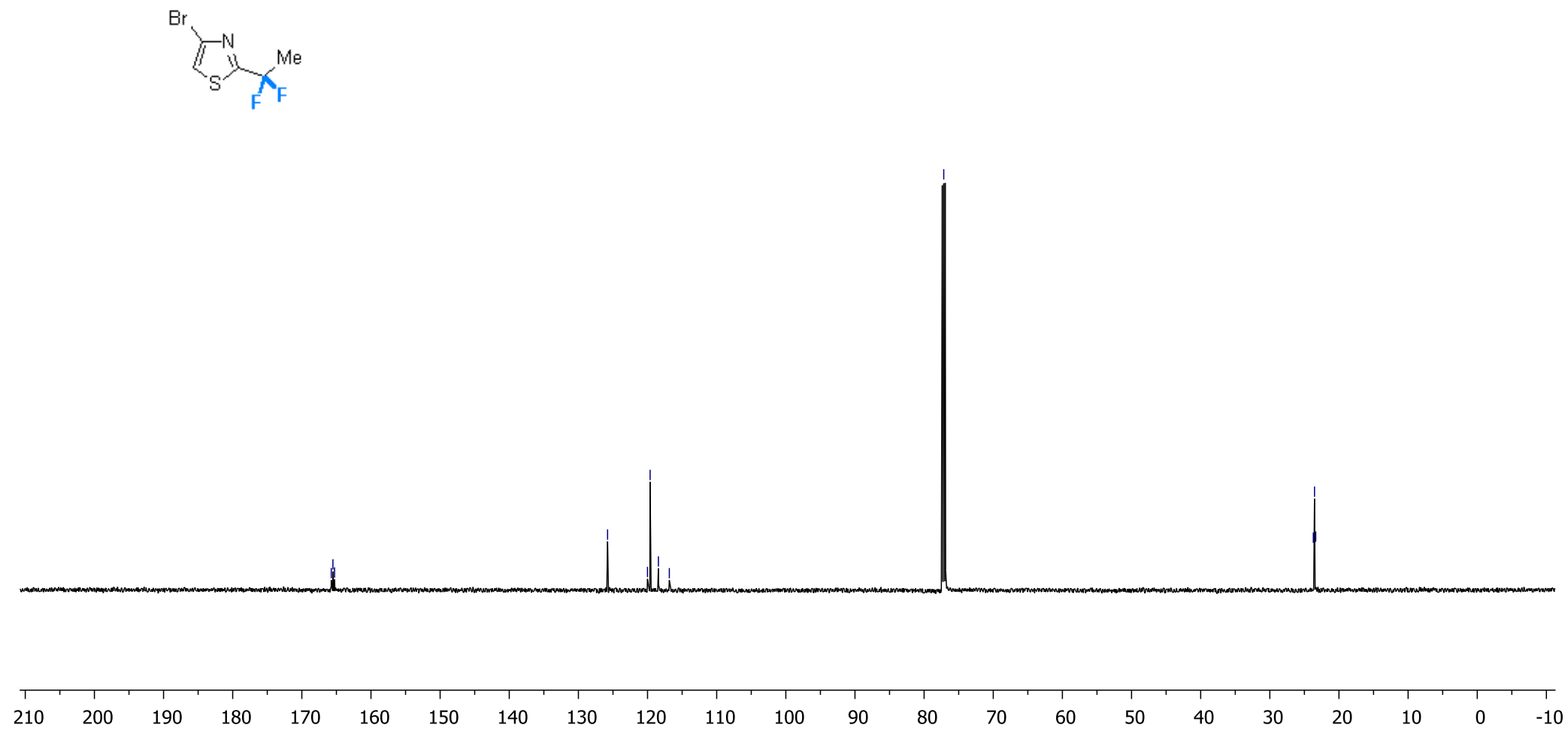

S78 


\section{${ }^{19} \mathrm{~F}\left\{{ }^{1} \mathrm{H}\right\} \mathrm{NMR}\left(376 \mathrm{MHz}, \mathrm{CDCl}_{3}\right)$}

R1852407_F19\{H\} $19 \mathrm{~F}-\{1 \mathrm{H}\}$

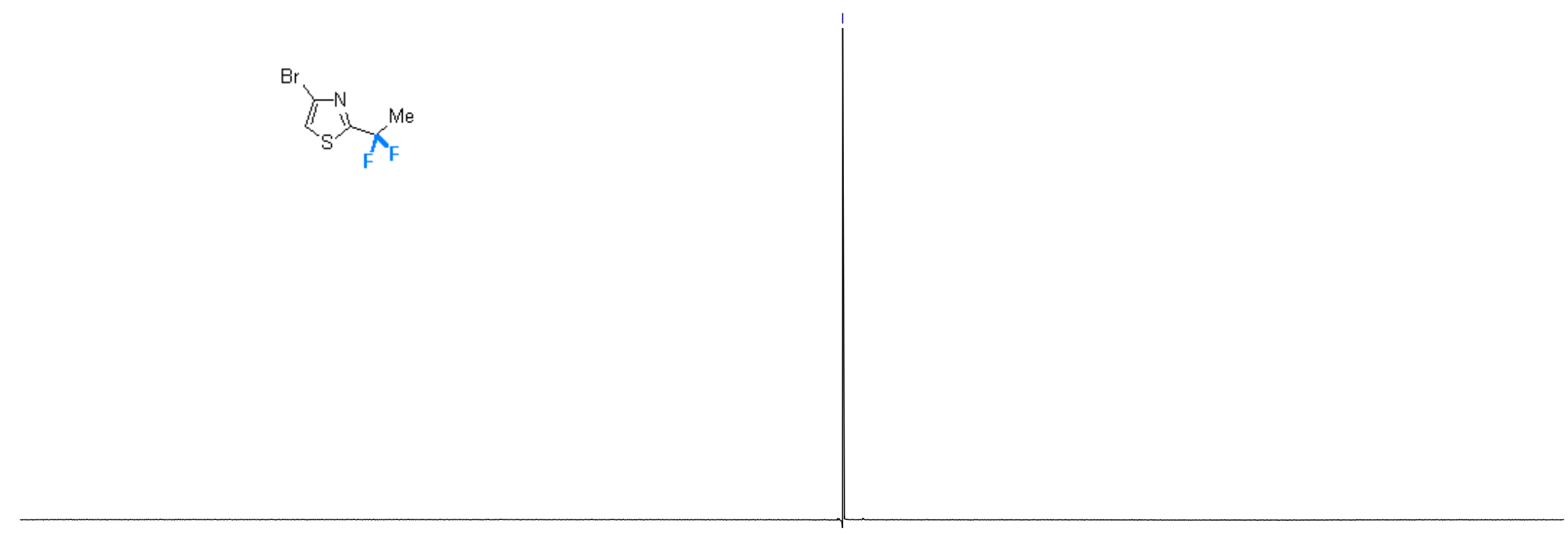




\section{Compound 31a}

${ }^{1} \mathrm{H}$ NMR (400 MHz, $\mathrm{CDCl}_{3}$ )

R1863029

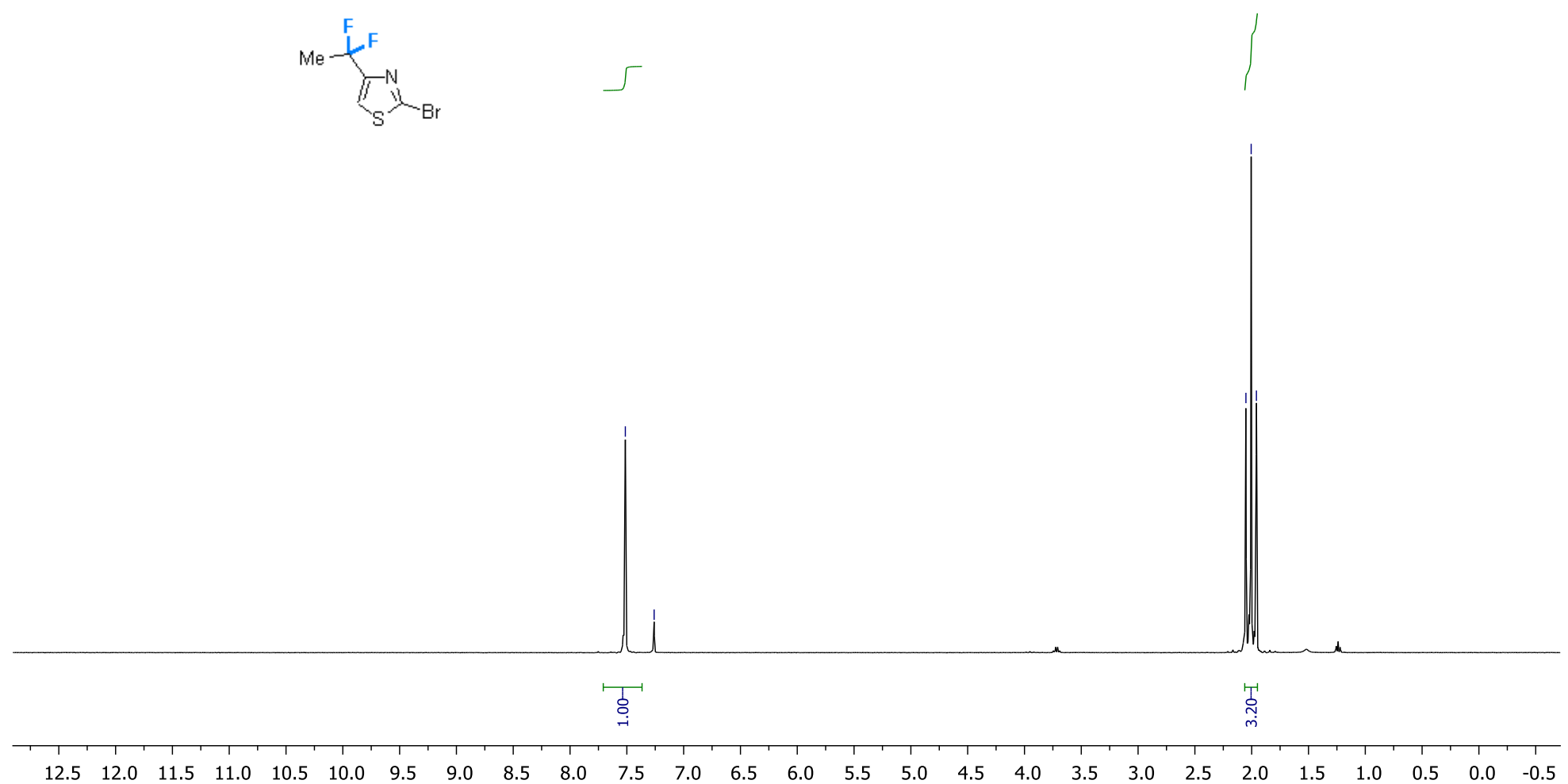


${ }^{13} \mathrm{C}\left\{{ }^{1} \mathrm{H}\right\}$ NMR (151 MHz, $\left.\mathrm{CDCl}_{3}\right)$

R1863029_C13

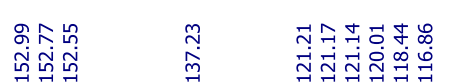

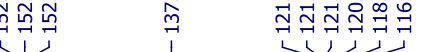

$\stackrel{\stackrel{0}{R}}{i}$

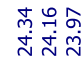

vif

$\sum_{S}^{\mathrm{N}} \mathrm{W}_{\mathrm{Br}}^{\mathrm{F}}$

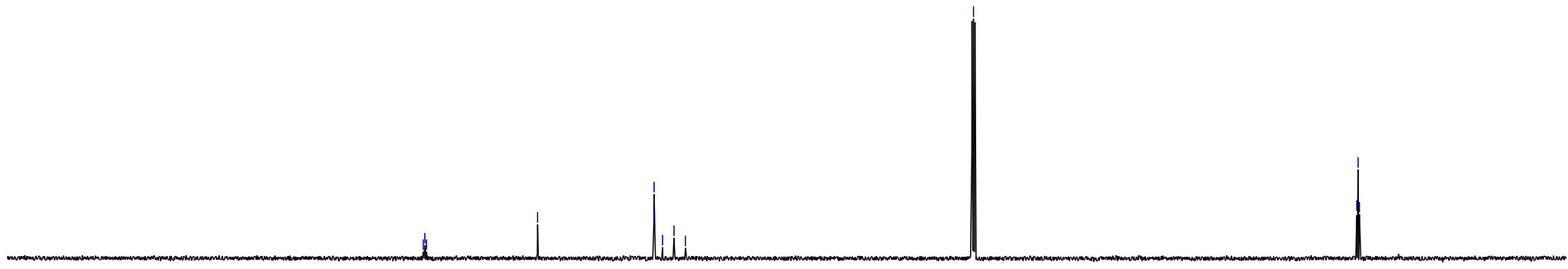

$\begin{array}{rllllllllllllllllllllllllllll}210 & 200 & 190 & 180 & 170 & 160 & 150 & 140 & 130 & 120 & 110 & 100 & 90 & 80 & 70 & 60 & 50 & 40 & 30 & 20 & 10 & 0\end{array}$ 


\section{${ }^{19} \mathrm{~F}\left\{{ }^{1} \mathrm{H}\right\}$ NMR (376 MHz, $\left.\mathrm{CDCl}_{3}\right)$}

R1863029_F19 $\{\mathrm{H}\}$

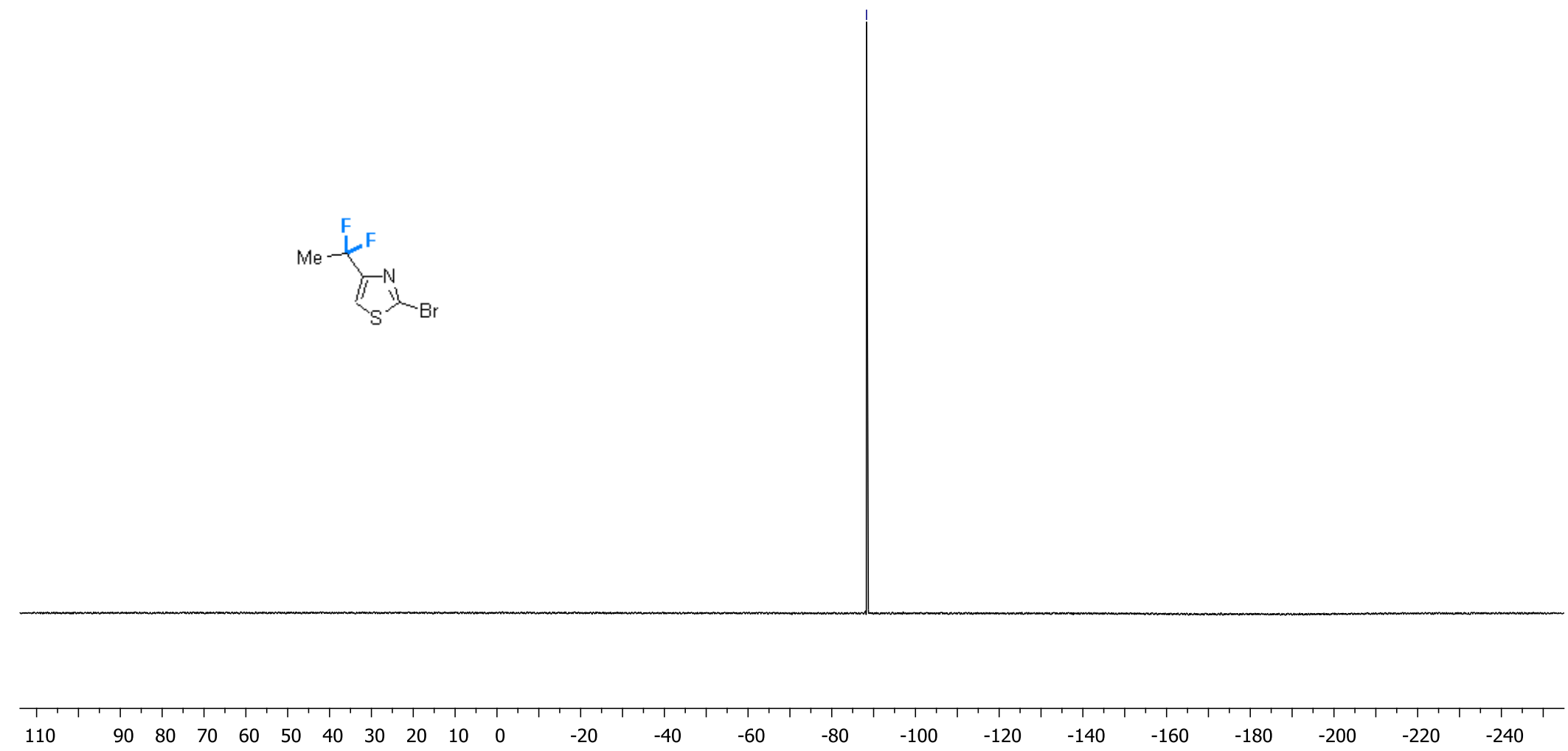




\section{Compound 32a}

\section{${ }^{1} \mathrm{H}$ NMR $\left(400 \mathrm{MHz}, \mathrm{CDCl}_{3}\right.$}

R2430199

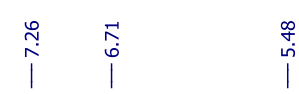

苻
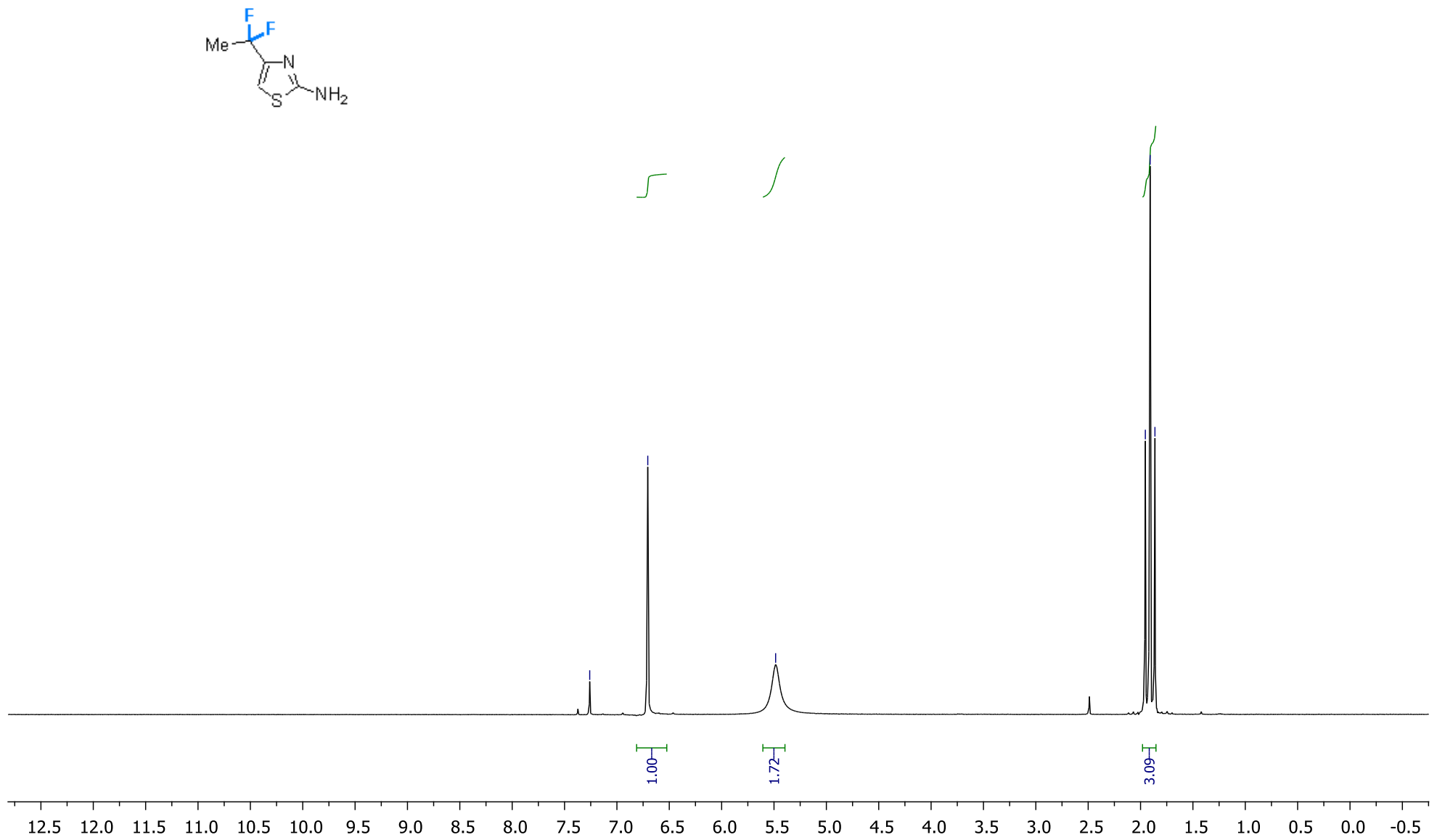
${ }^{13} \mathrm{C}\left\{{ }^{1} \mathrm{H}\right\}$ NMR (151 MHz, $\left.\mathrm{CDCl}_{3}\right)$

R2430199_C13

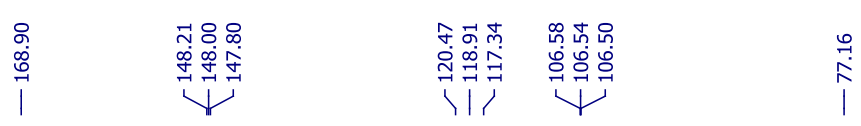

$\stackrel{?}{\stackrel{2}{i}}$

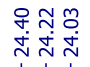
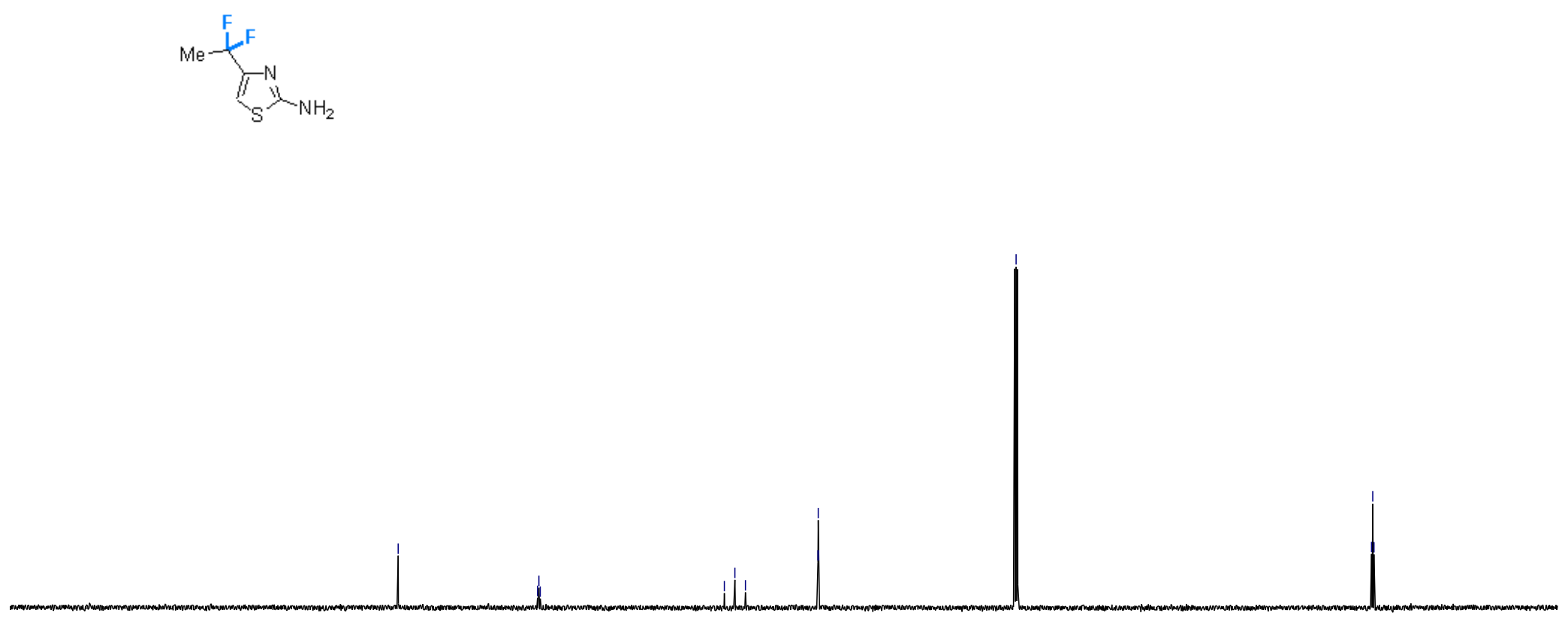


\section{${ }^{19} \mathrm{~F}\left\{{ }^{1} \mathrm{H}\right\}$ NMR $\left(376 \mathrm{MHz}, \mathrm{CDCl}_{3}\right)$}

R2430199_F19 $\{\mathrm{H}\}$

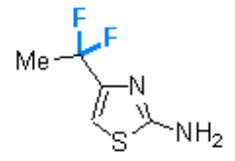

$\begin{array}{llllllllll}110 & 90 & 80 & 70 & 60 & 50 & 40 & 30 & 20 & 10\end{array}$ 


\section{Compound 33a}

${ }^{1} \mathrm{H}$ NMR $\left(400 \mathrm{MHz}, \mathrm{CDCl}_{3}\right.$ )

R1860015

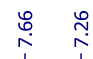

نْ่

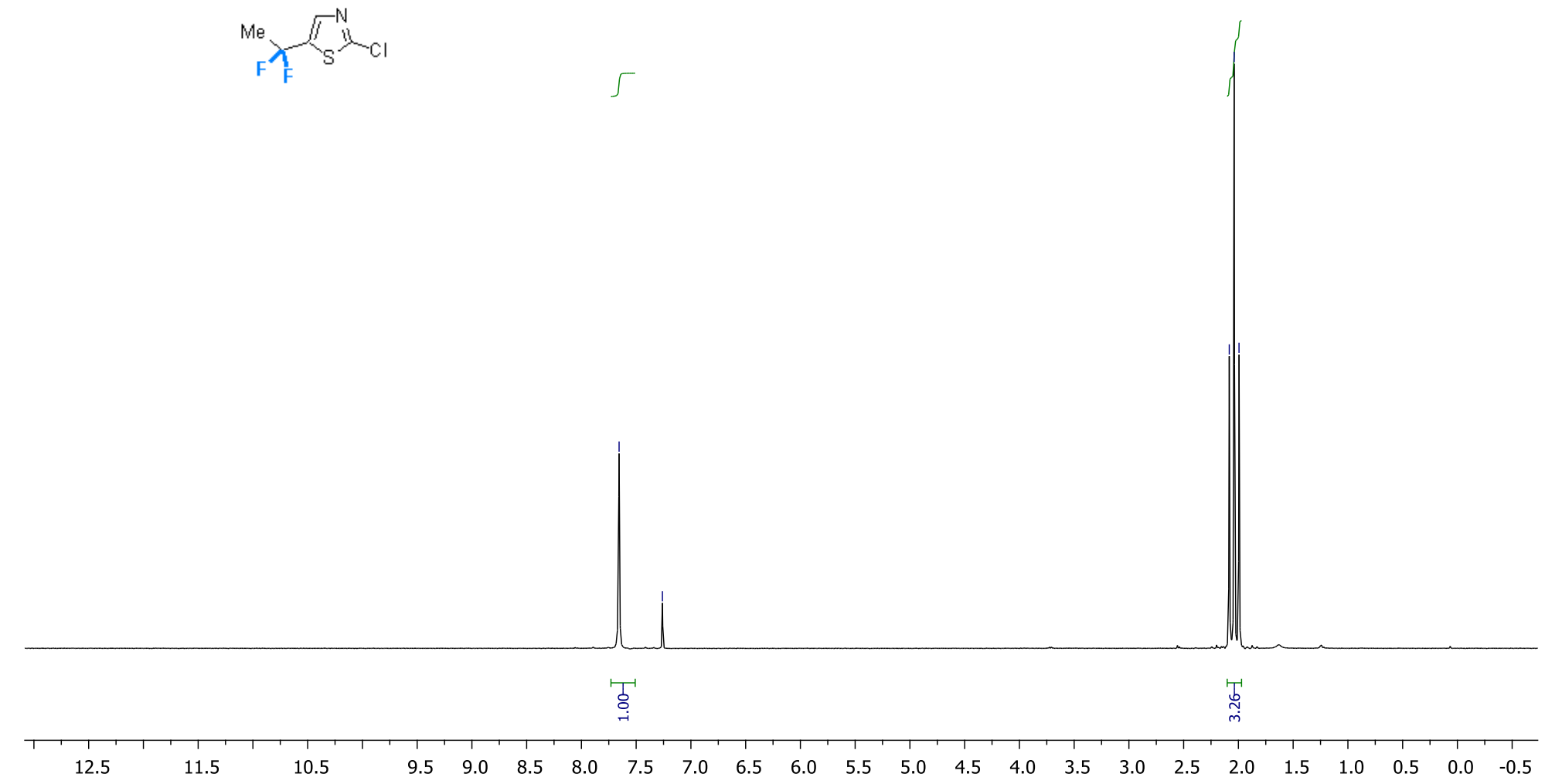


${ }^{13} \mathrm{C}\left\{{ }^{1} \mathrm{H}\right\}$ NMR (151 MHz, $\left.\mathrm{CDCl}_{3}\right)$

R1860015 C13

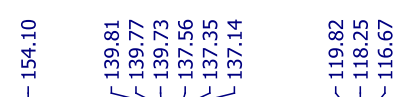

$\stackrel{?}{\stackrel{1}{\gtrless}}$

${ }_{\mathrm{F}}^{\mathrm{M}} \mathrm{I}_{\mathrm{F}}^{\mathrm{N}} \mathrm{Cl}_{\mathrm{Cl}}$

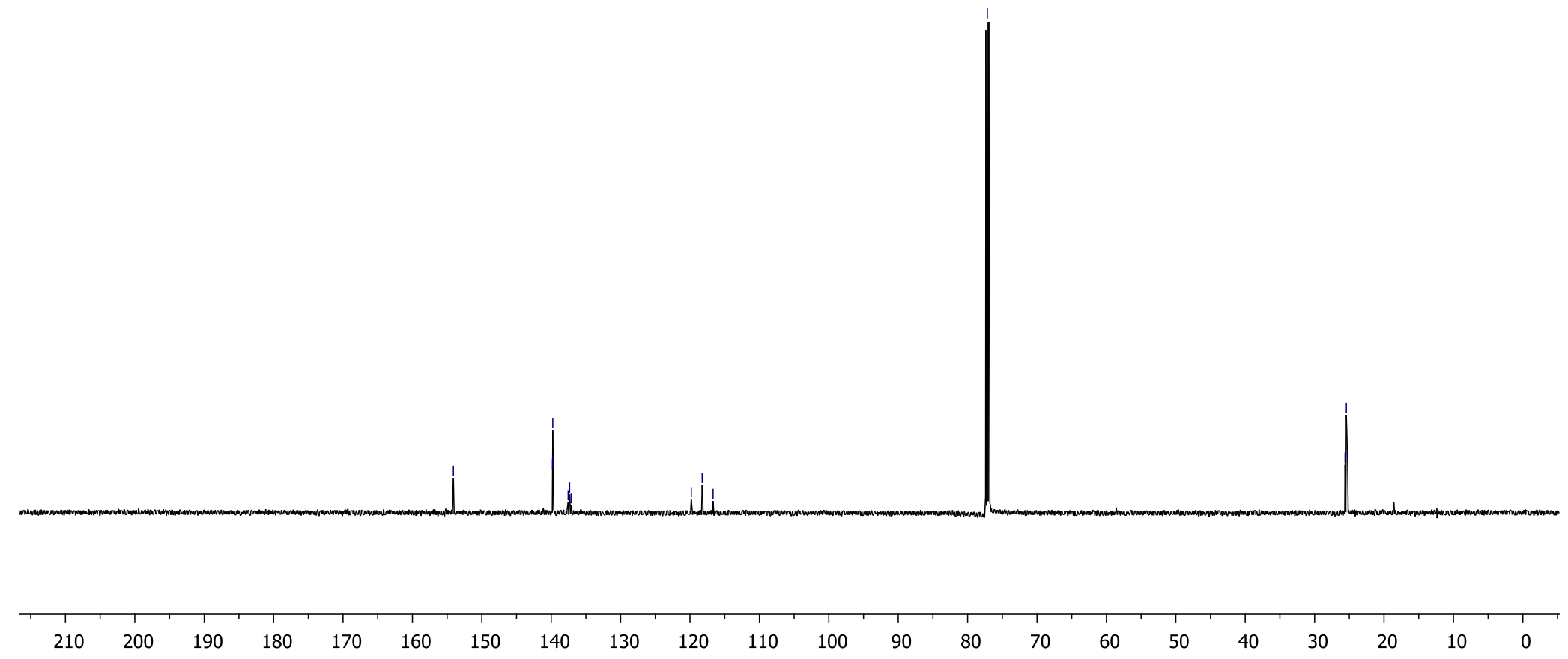




\section{${ }^{19} \mathrm{~F}\left\{{ }^{1} \mathrm{H}\right\}$ NMR $\left(376 \mathrm{MHz}, \mathrm{CDCl}_{3}\right)$}

R1860015_F19

$19 \mathrm{~F}-\{1 \mathrm{H}\}$
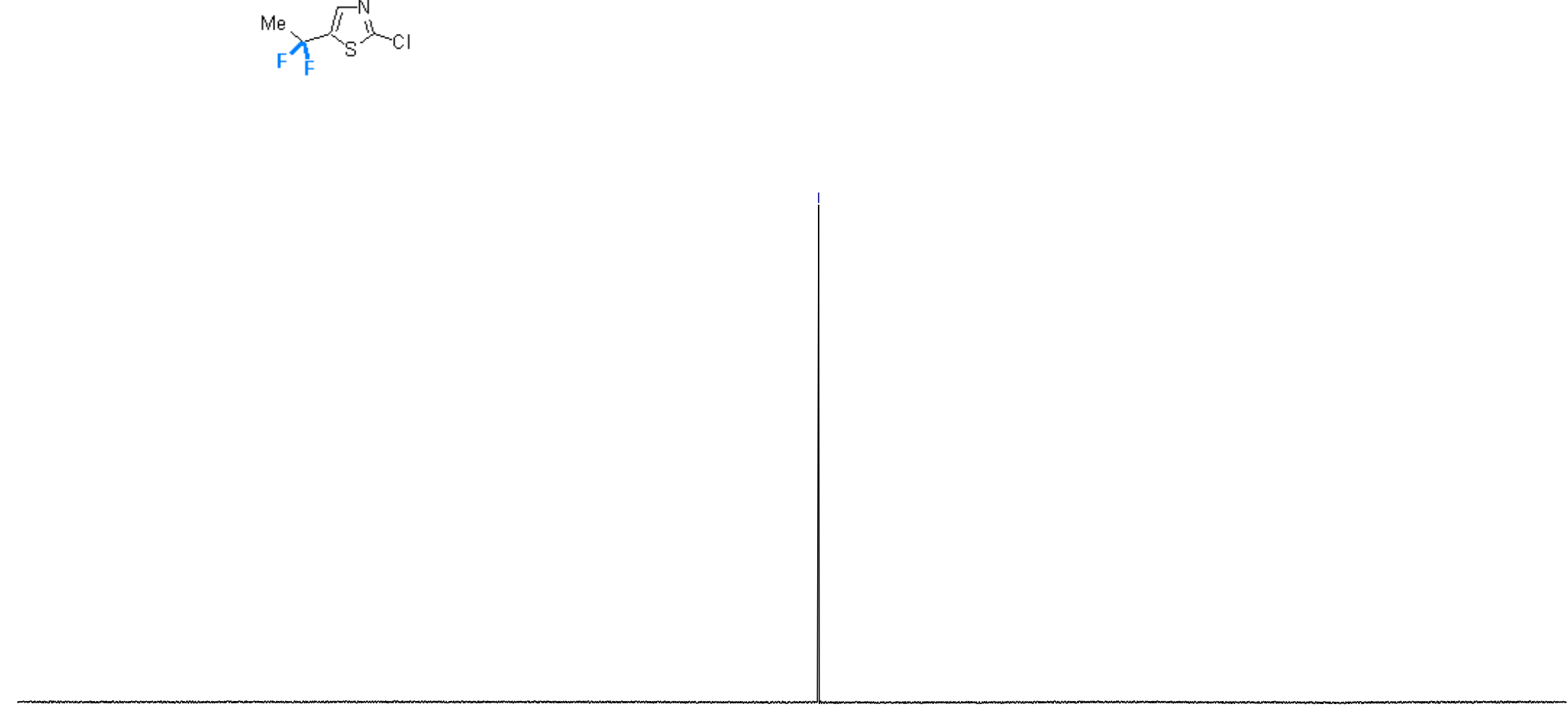


\section{Compound 34a}

${ }^{1} \mathrm{H}$ NMR (400 MHz, $\mathrm{CDCl}_{3}$ )

R1932823

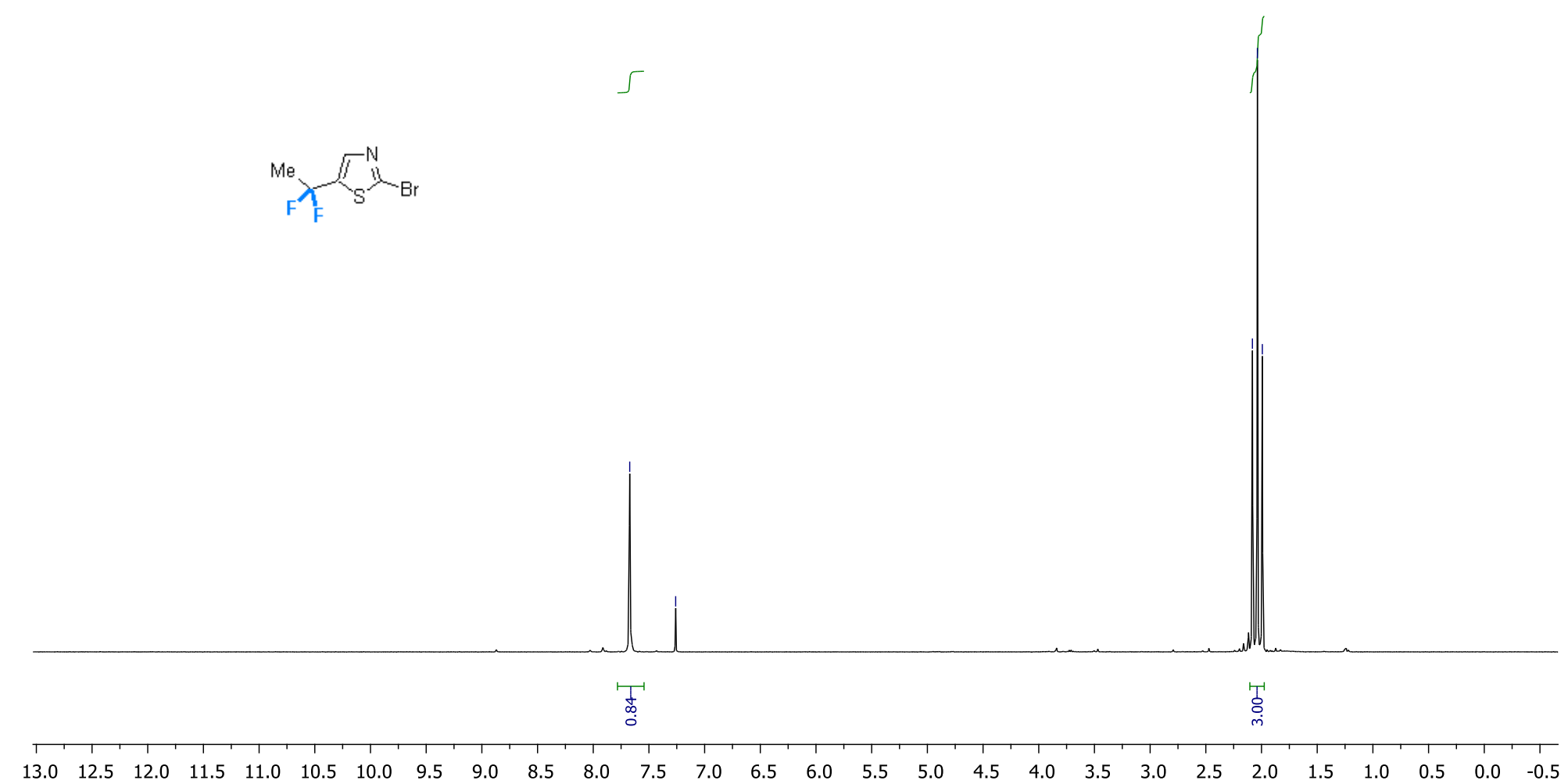


${ }^{13} \mathrm{C}\left\{{ }^{1} \mathrm{H}\right\} \mathrm{NMR}\left(126 \mathrm{MHz}, \mathrm{CDCl}_{3}\right)$

R1932823_C13

$13 \mathrm{C}$ (1H-decoupled)

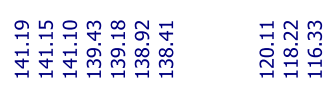

$\stackrel{0}{\stackrel{1}{i}}$

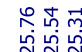

। ।

${ }_{\mathrm{F}}^{\mathrm{M}} \mathrm{F}_{\mathrm{F}}^{\mathrm{N}} \mathrm{S}$

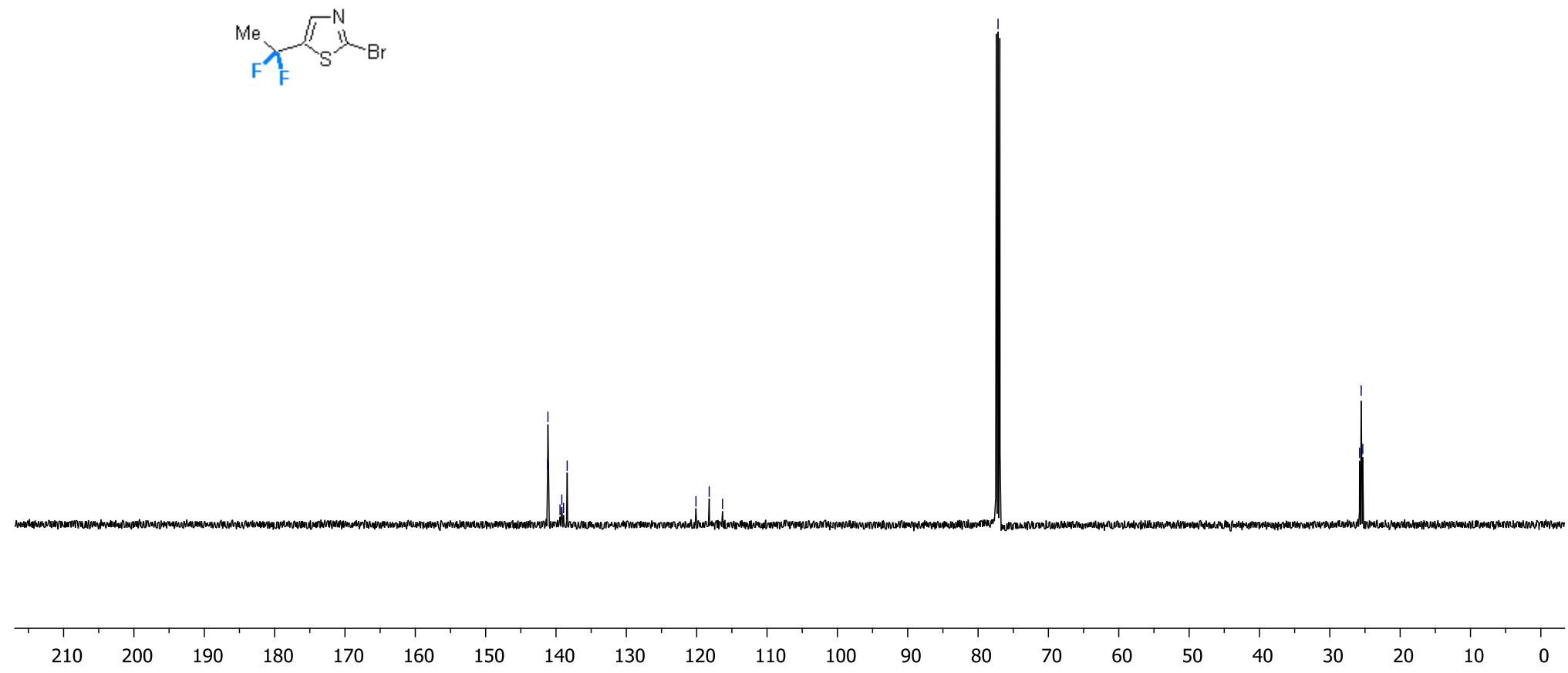




\section{${ }^{19} \mathrm{~F}\left\{{ }^{1} \mathrm{H}\right\}$ NMR (376 MHz, $\left.\mathrm{CDCl}_{3}\right)$}

R1932823_F19 $\{\mathrm{H}\}$
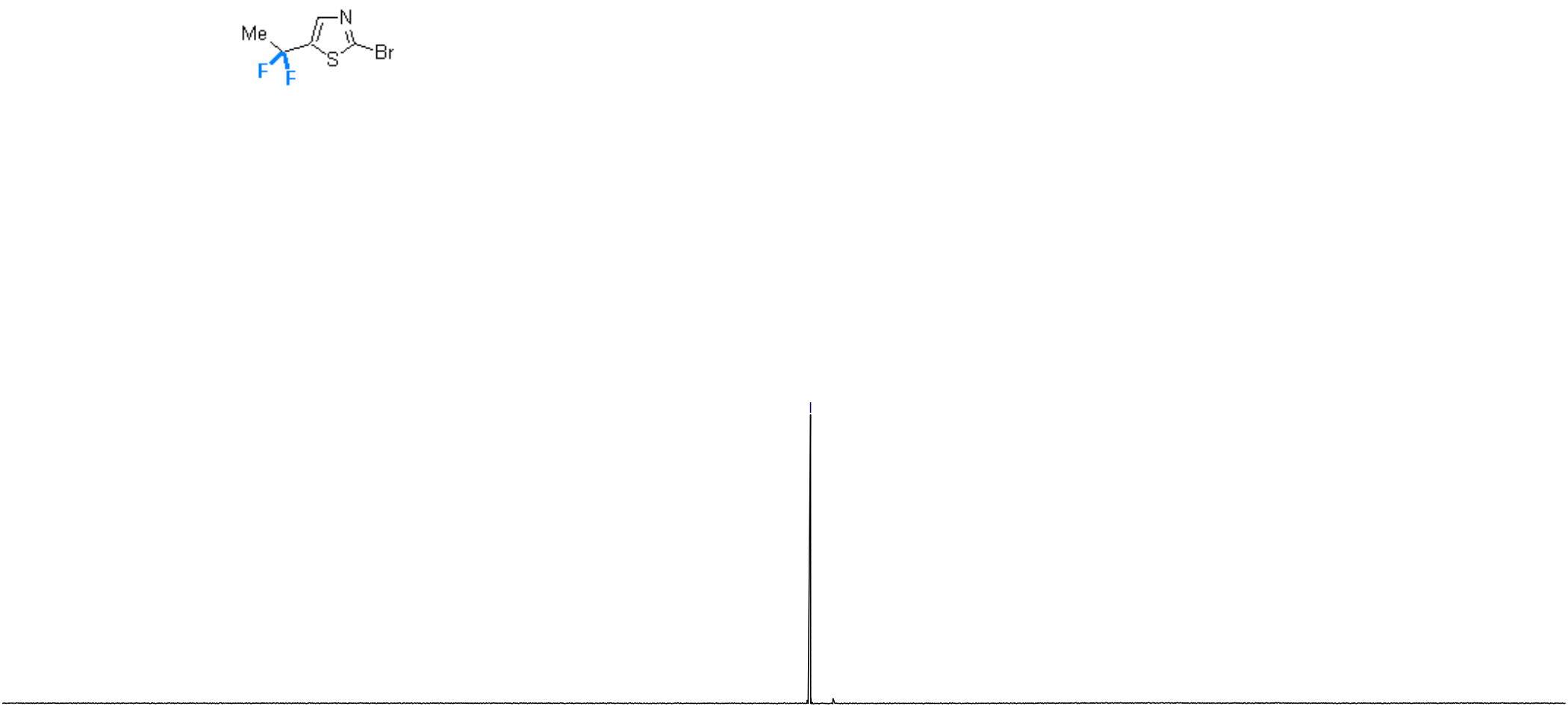

$110 \quad \begin{array}{rlllllllllllllllllllllll} & 80 & 70 & 60 & 50 & 40 & 30 & 20 & 10 & 0 & -20 & -40 & -60 & -80 & -100 & -120 & -140 & -160 & -180 & -200 & -220 & -240\end{array}$ 


\section{Compound 35a}

${ }^{1} \mathrm{H}$ NMR (400 MHz, $\mathrm{CDCl}_{3}$ )

IGMA5482

$\stackrel{\infty}{\stackrel{\infty}{i}} \stackrel{\infty}{\substack{0 \\ i}}$

i

${ }_{\mathrm{Cl}}^{\mathrm{F}}{ }_{\mathrm{Cl}}^{\mathrm{CO}_{2} \mathrm{Me}}$

厂
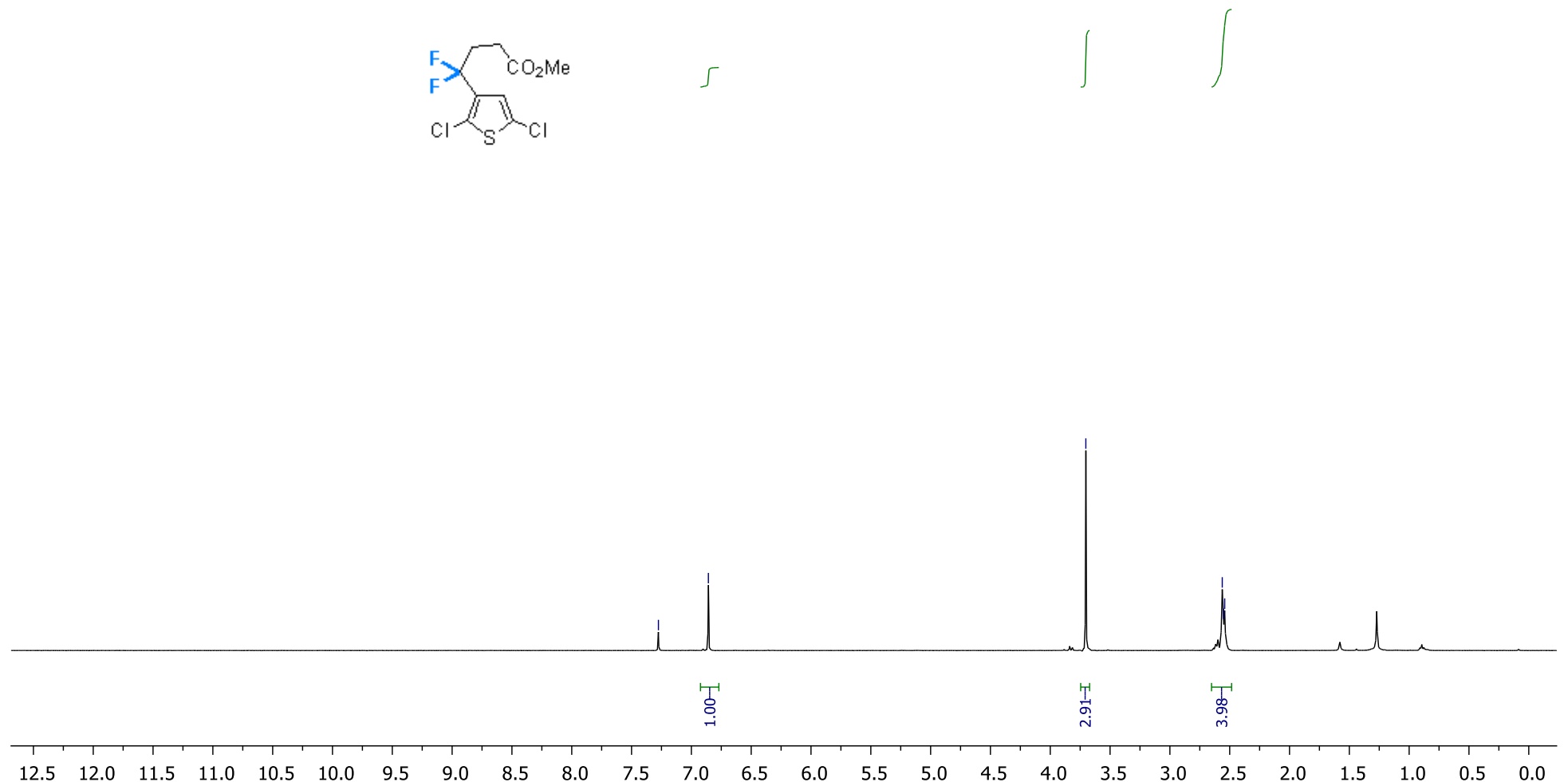


\section{${ }^{13} \mathrm{C}\left\{{ }^{1} \mathrm{H}\right\}$ NMR (101 MHz, $\left.\mathrm{CDCl}_{3}\right)$}

igma5489_C13

$$
\text { (I) }
$$

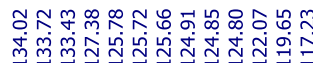


${ }^{19} \mathrm{~F}\left\{{ }^{1} \mathrm{H}\right\}$ NMR $\left(470 \mathrm{MHz}, \mathrm{CDCl}_{3}\right)$

igma5482_F19

${ }_{\mathrm{Cl}}^{\mathrm{F}} \mathrm{CO}_{\mathrm{S}} \mathrm{CO}_{\mathrm{Cl}}$

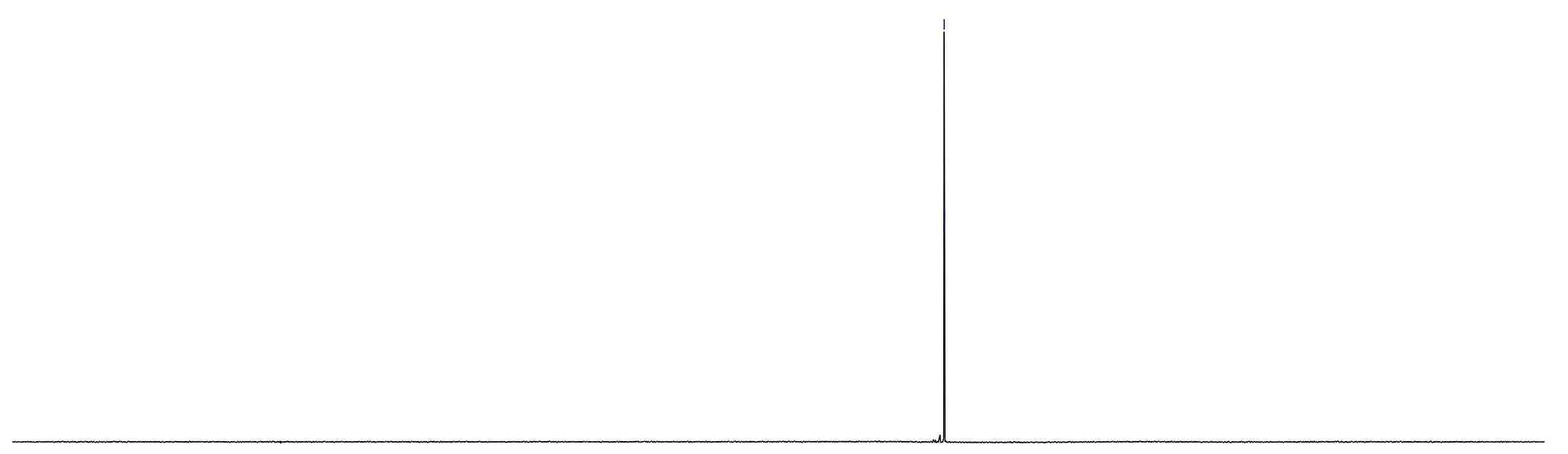




\section{Compound 40a}

${ }^{1} \mathrm{H}$ NMR (400 MHz, $\mathrm{CDCl}_{3}$ )

R1429014

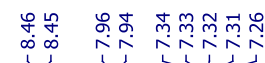

ㄱํำ
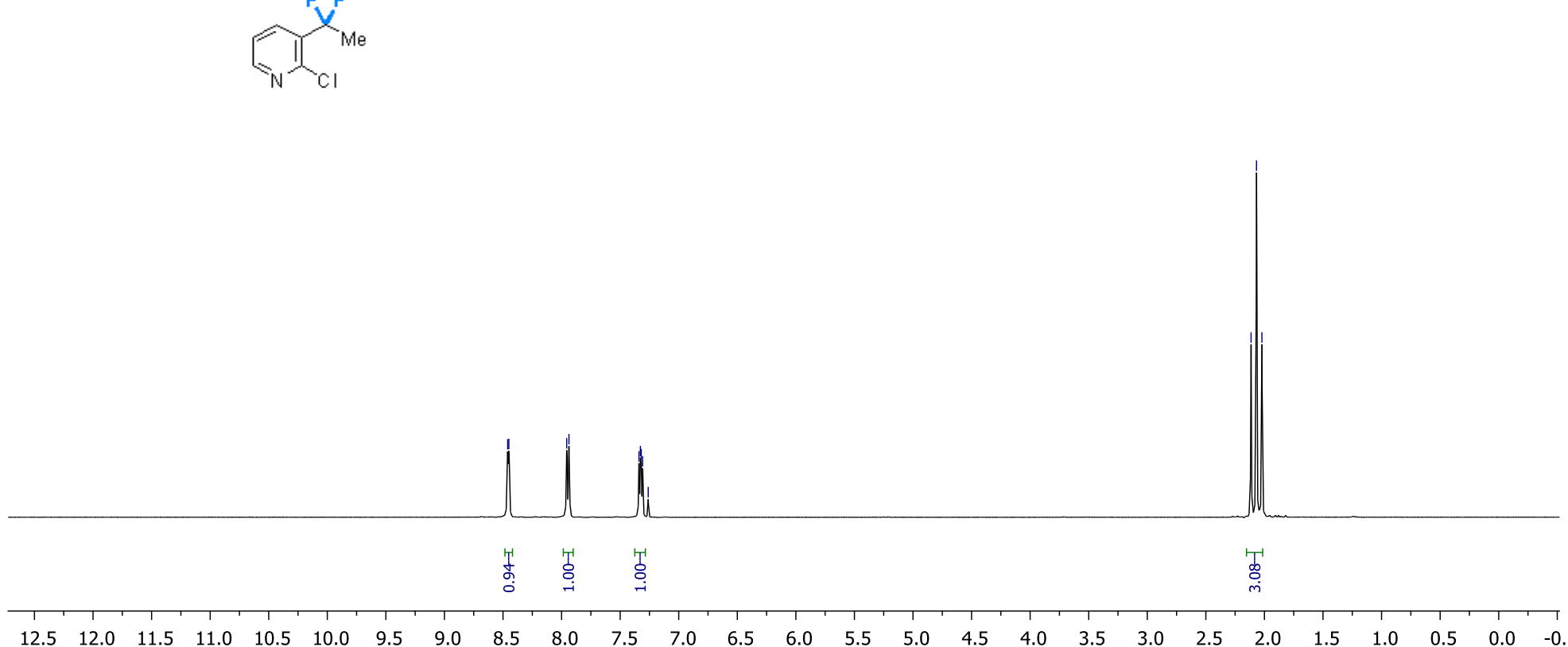
${ }^{13} \mathrm{C}\left\{{ }^{1} \mathrm{H}\right\}$ NMR (151 MHz, $\left.\mathrm{CDCl}_{3}\right)$

R1429014_C13

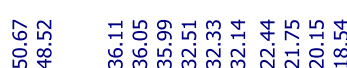

마

$\stackrel{\substack{i \\ i}}{i}$

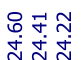

पर पiा

$\overbrace{N}^{F} x_{M e}^{F}$

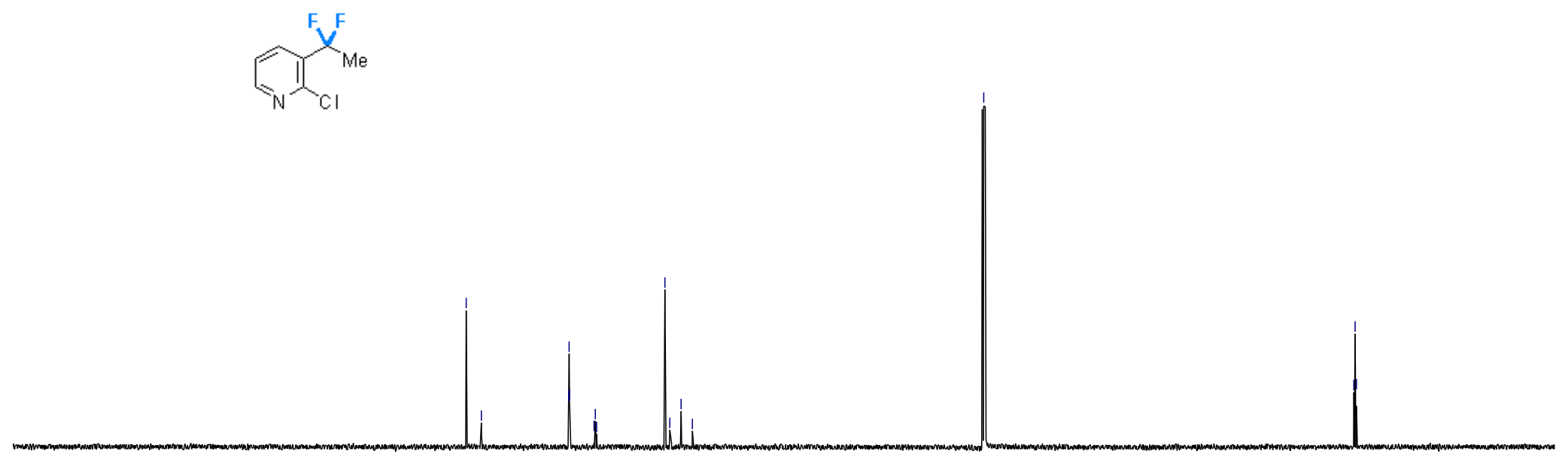




\section{${ }^{19} \mathrm{~F}\left\{{ }^{1} \mathrm{H}\right\} \mathrm{NMR}\left(376 \mathrm{MHz}, \mathrm{CDCl}_{3}\right)$}

R1429014_F19 $19 \mathrm{~F}-\{1 \mathrm{H}\}$
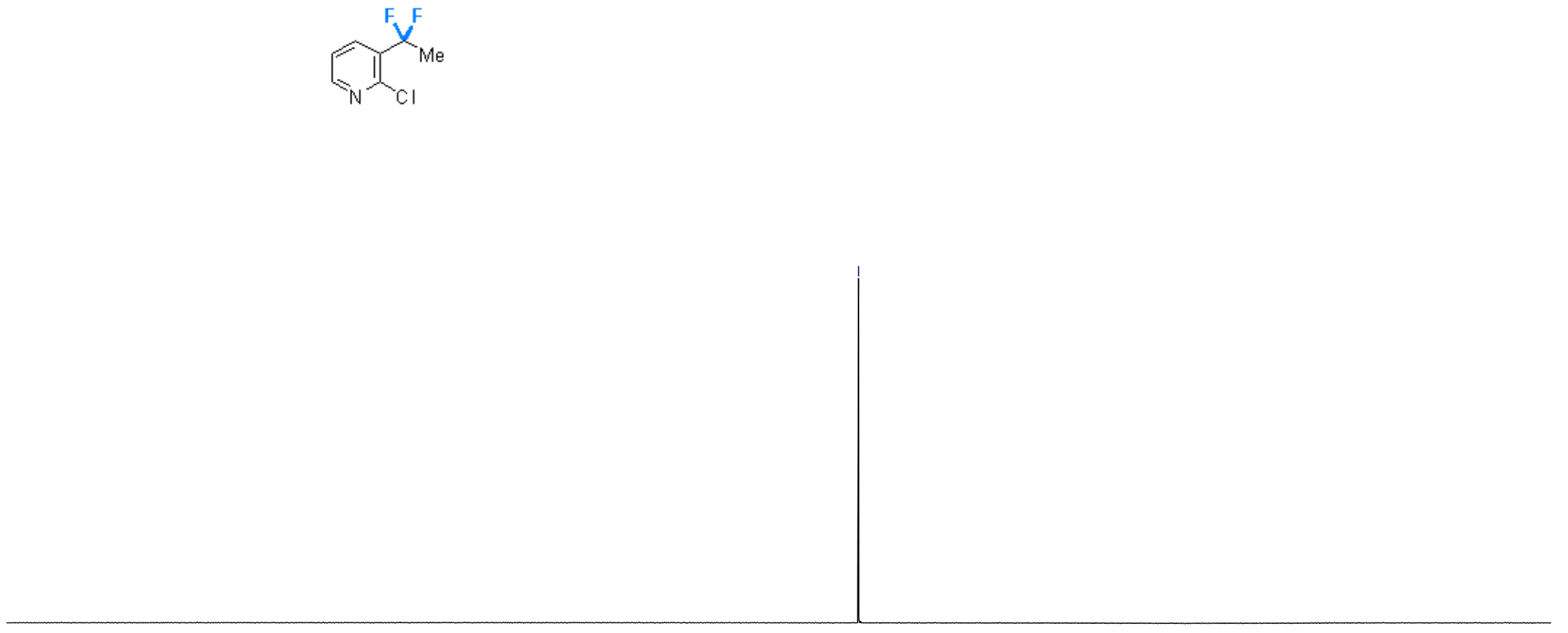

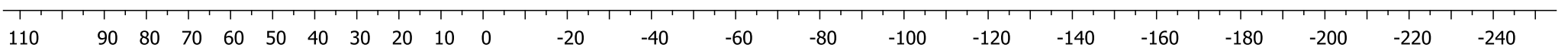




\section{Compound 41a}

${ }^{1} \mathrm{H}$ NMR (400 MHz, $\mathrm{CDCl}_{3}$ )

R1927770

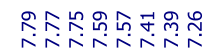

ஸ்ં

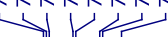

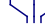

Me

$\iiint$
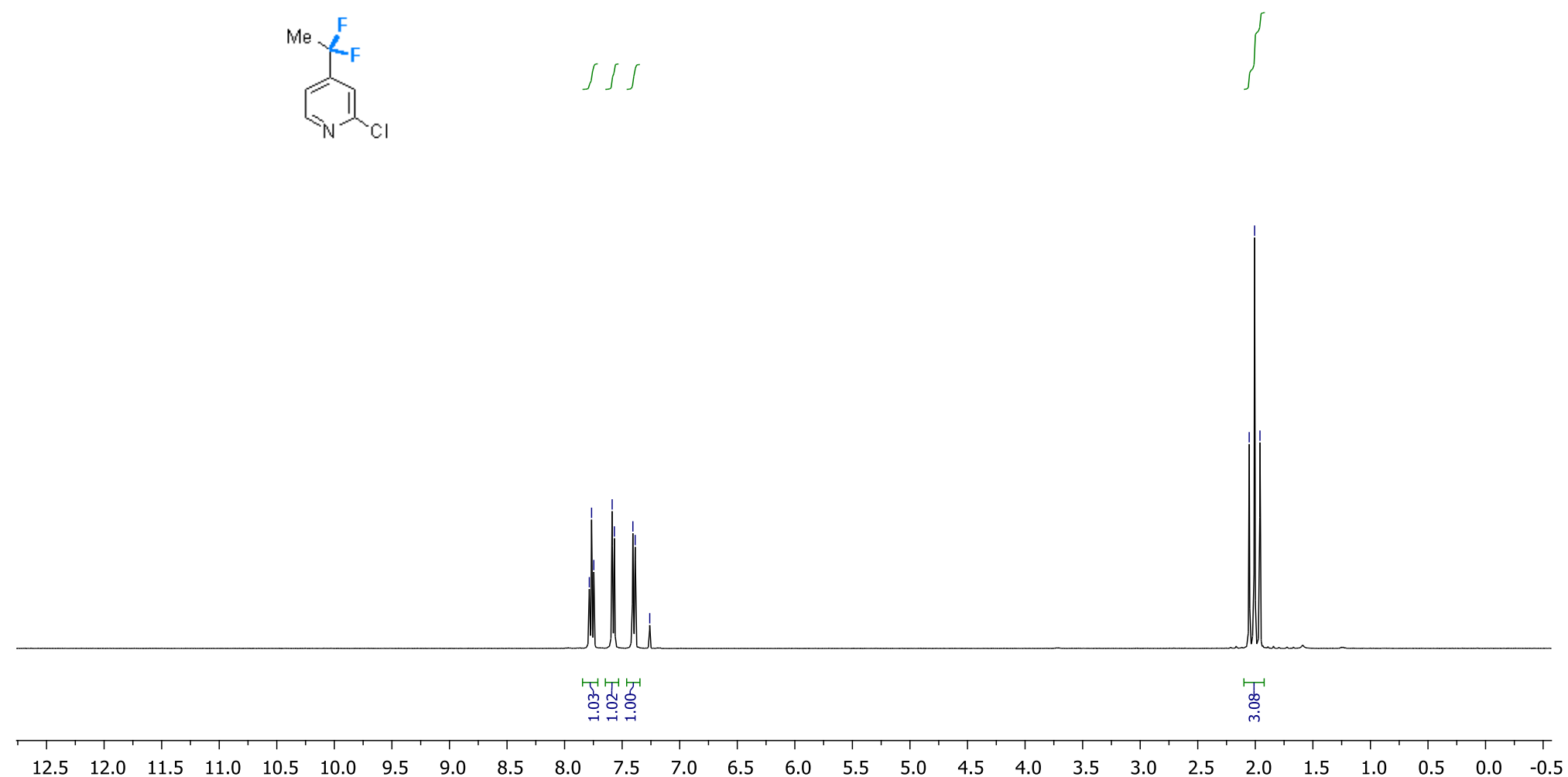
${ }^{13} \mathrm{C}\left\{{ }^{1} \mathrm{H}\right\}$ NMR (151 MHz, $\left.\mathrm{CDCl}_{3}\right)$

R1927770_C13

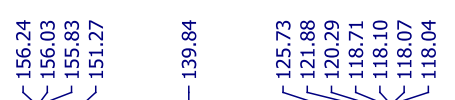

$\stackrel{\substack{i \\ i}}{i}$

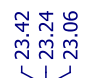

$\overbrace{\mathrm{N}}^{\mathrm{F}}$

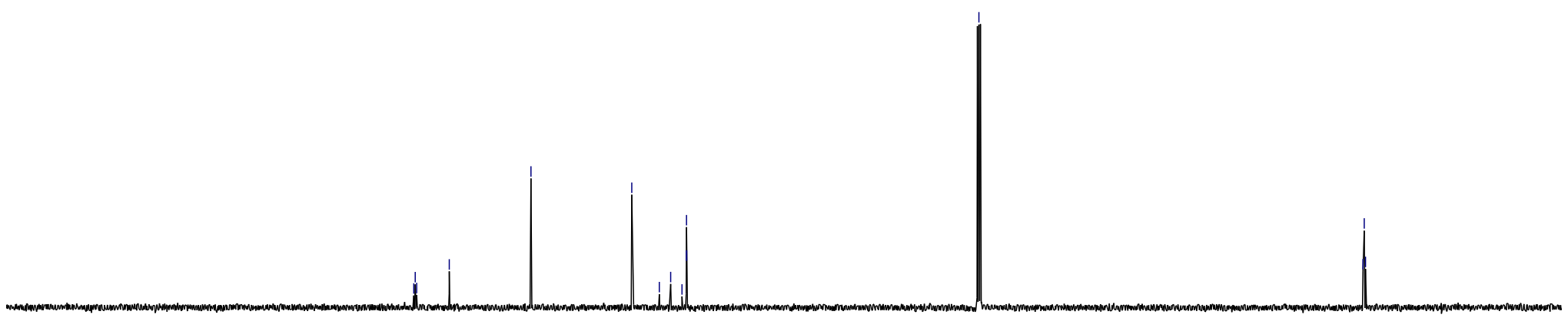




\section{${ }^{19} \mathrm{~F}\left\{{ }^{1} \mathrm{H}\right\} \mathrm{NMR}\left(376 \mathrm{MHz}, \mathrm{CDCl}_{3}\right)$}

R1927770_F19\{H\}

$$
19 F-\{1 \mathrm{H}\}
$$
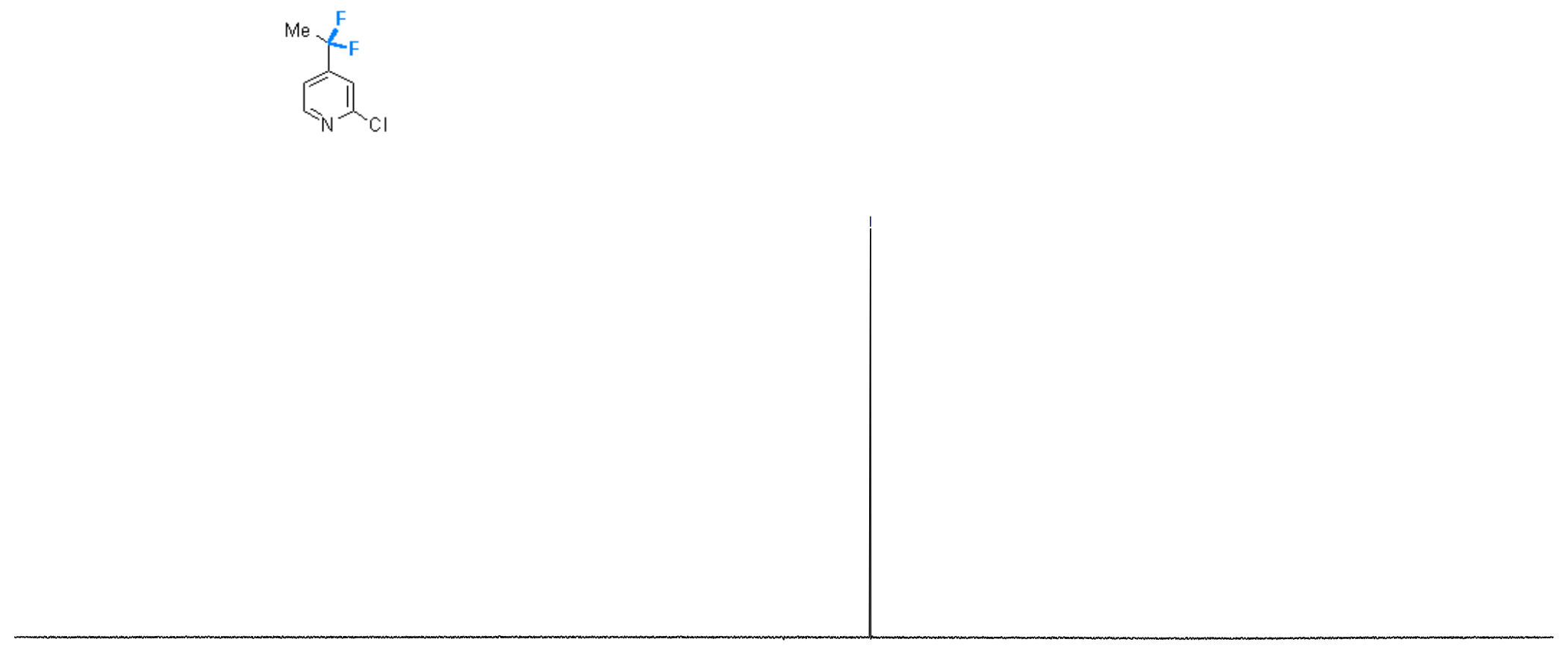


\section{Compound 42a}

${ }^{1} \mathrm{H}$ NMR (400 MHz, $\mathrm{CDCl}_{3}$ )

R1995364
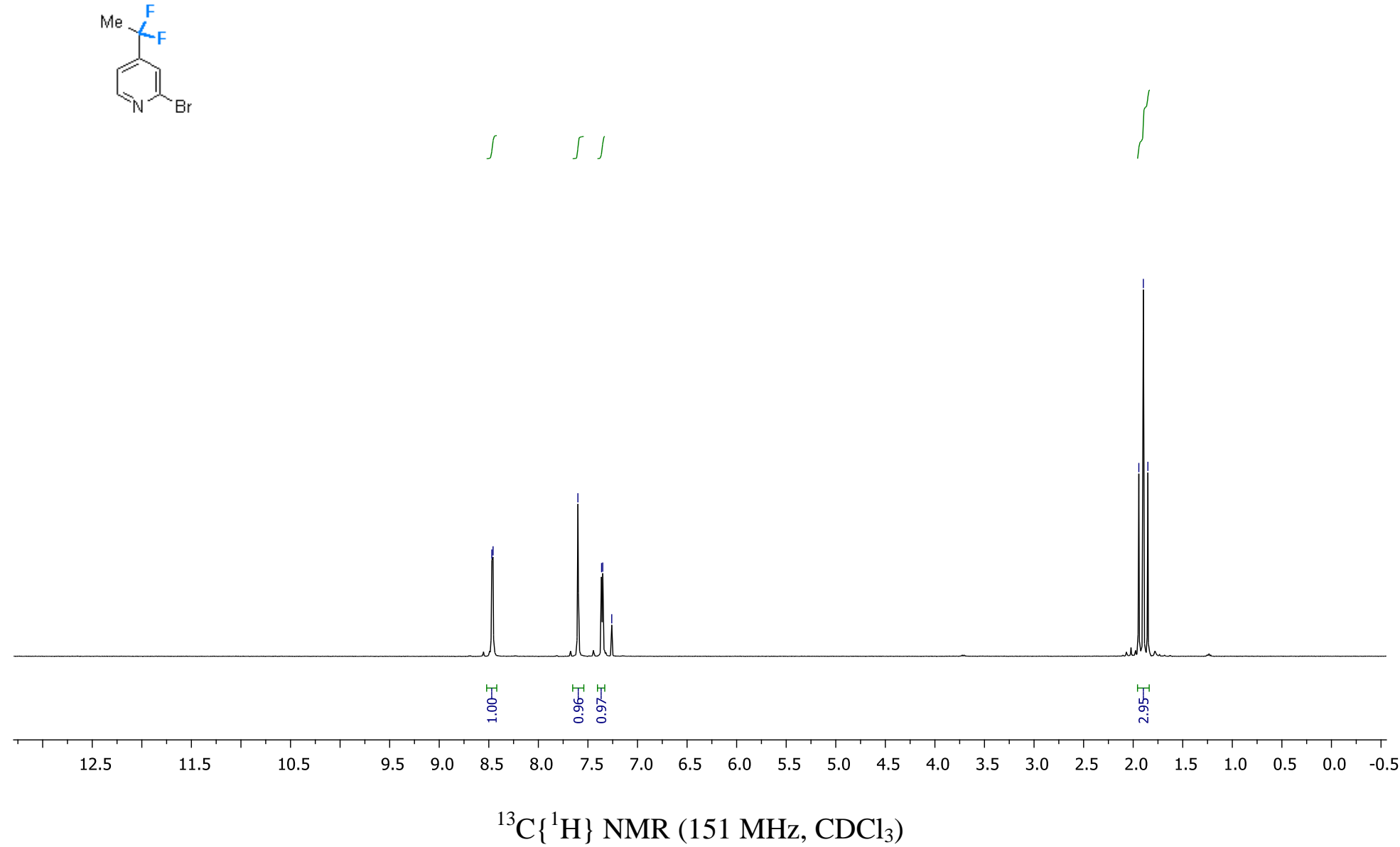


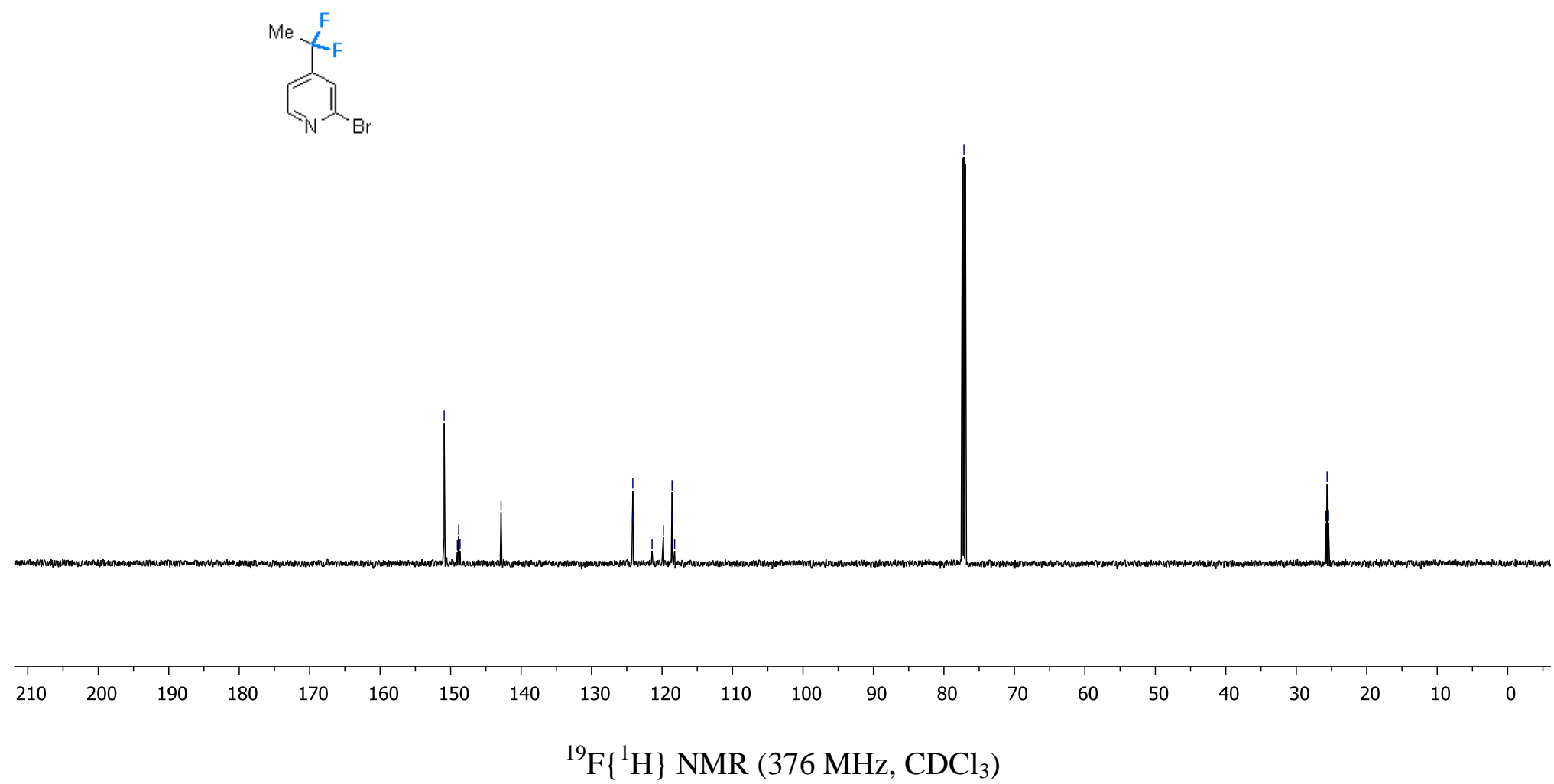



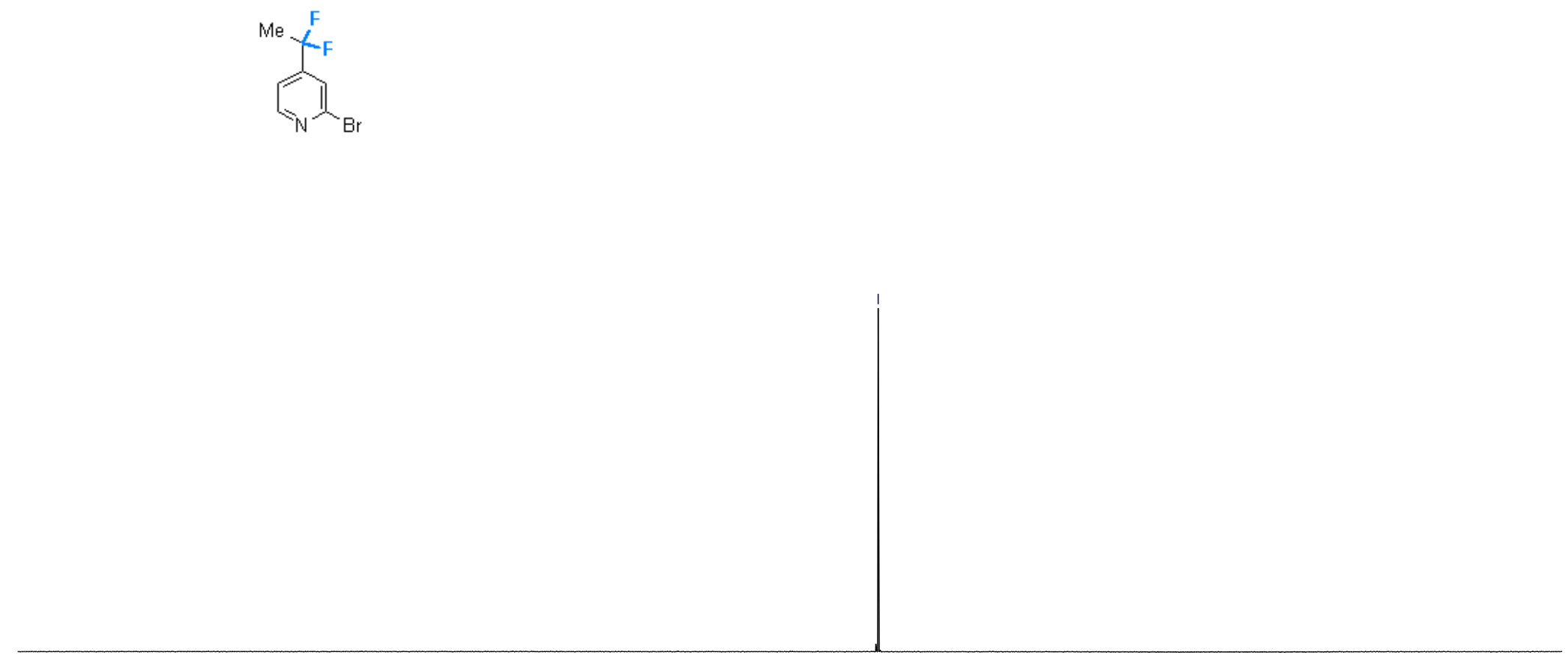

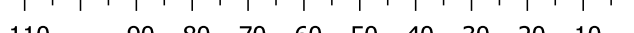

$\begin{array}{llllllllll}90 & 80 & 70 & 60 & 50 & 40 & 30 & 20 & 10 & 0\end{array}$ 


\section{Compound 43a}

${ }^{1} \mathrm{H}$ NMR (400 MHz, $\mathrm{CDCl}_{3}$ )

R1856360
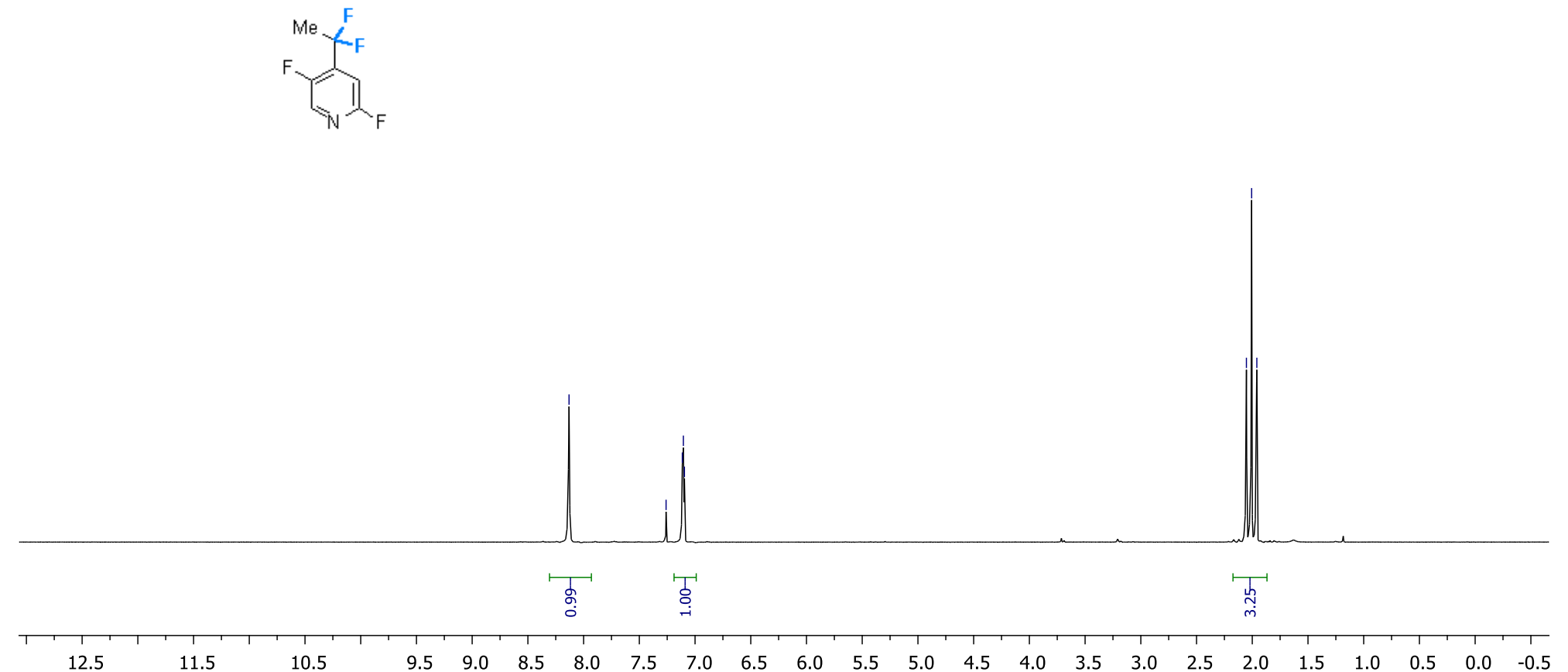
${ }^{13} \mathrm{C}\left\{{ }^{1} \mathrm{H}\right\} \mathrm{NMR}\left(126 \mathrm{MHz}, \mathrm{CDCl}_{3}\right)$

R1856360_C1:

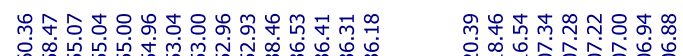

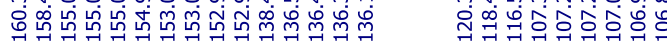

1) 年

$\stackrel{\substack{i \\ i}}{i}$

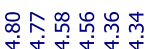

型

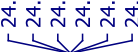
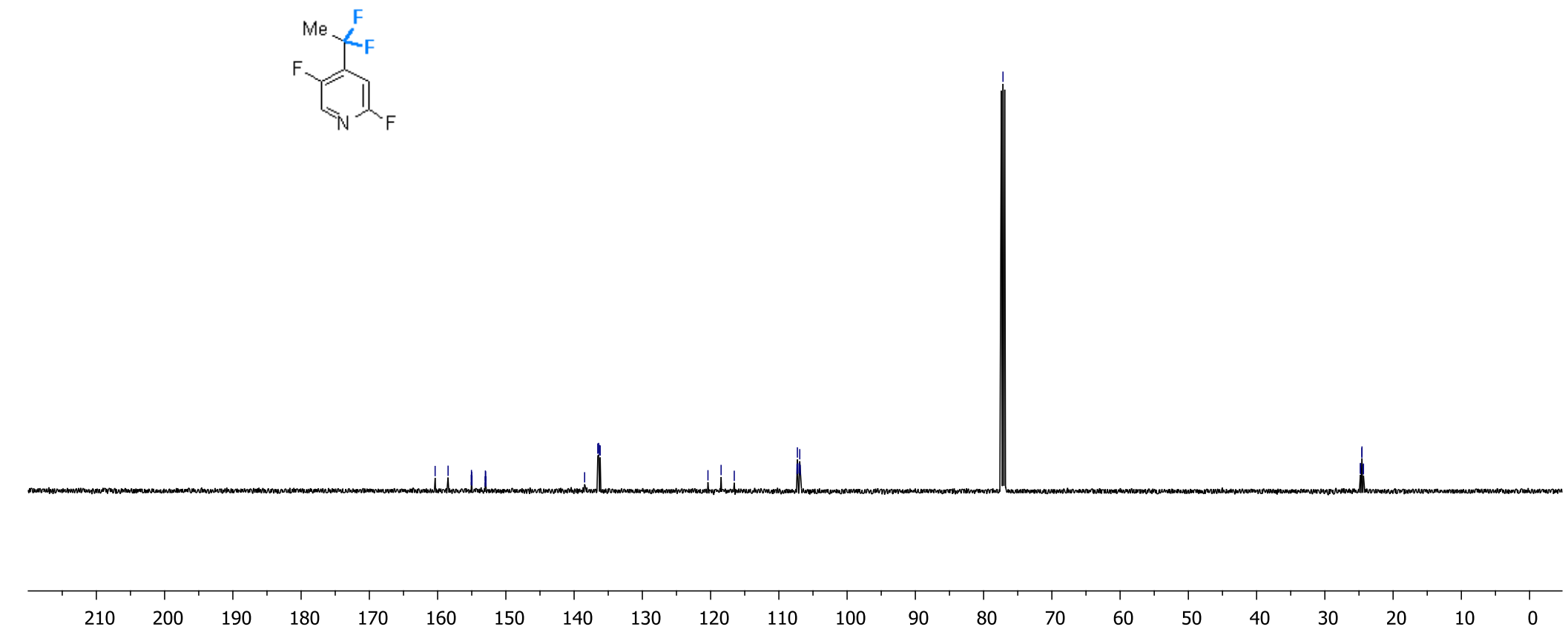
${ }^{19} \mathrm{~F}\left\{{ }^{1} \mathrm{H}\right\} \mathrm{NMR}\left(376 \mathrm{MHz}, \mathrm{CDCl}_{3}\right)$

R1856360_F19 $\{H\}$

vi

$$
\text { Me }
$$

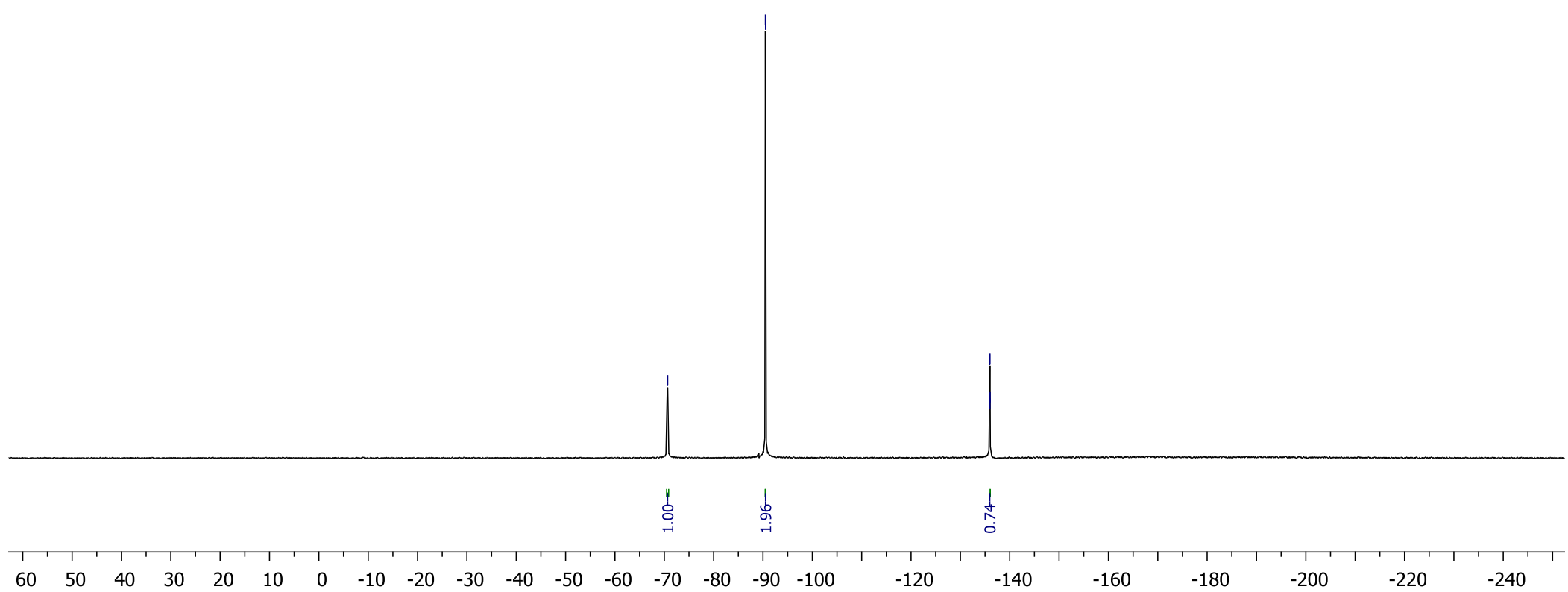




\section{Compound 44a}

${ }^{1} \mathrm{H}$ NMR (400 MHz, $\mathrm{CDCl}_{3}$ )

R1498081

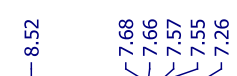

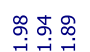

厂 $\int 5$
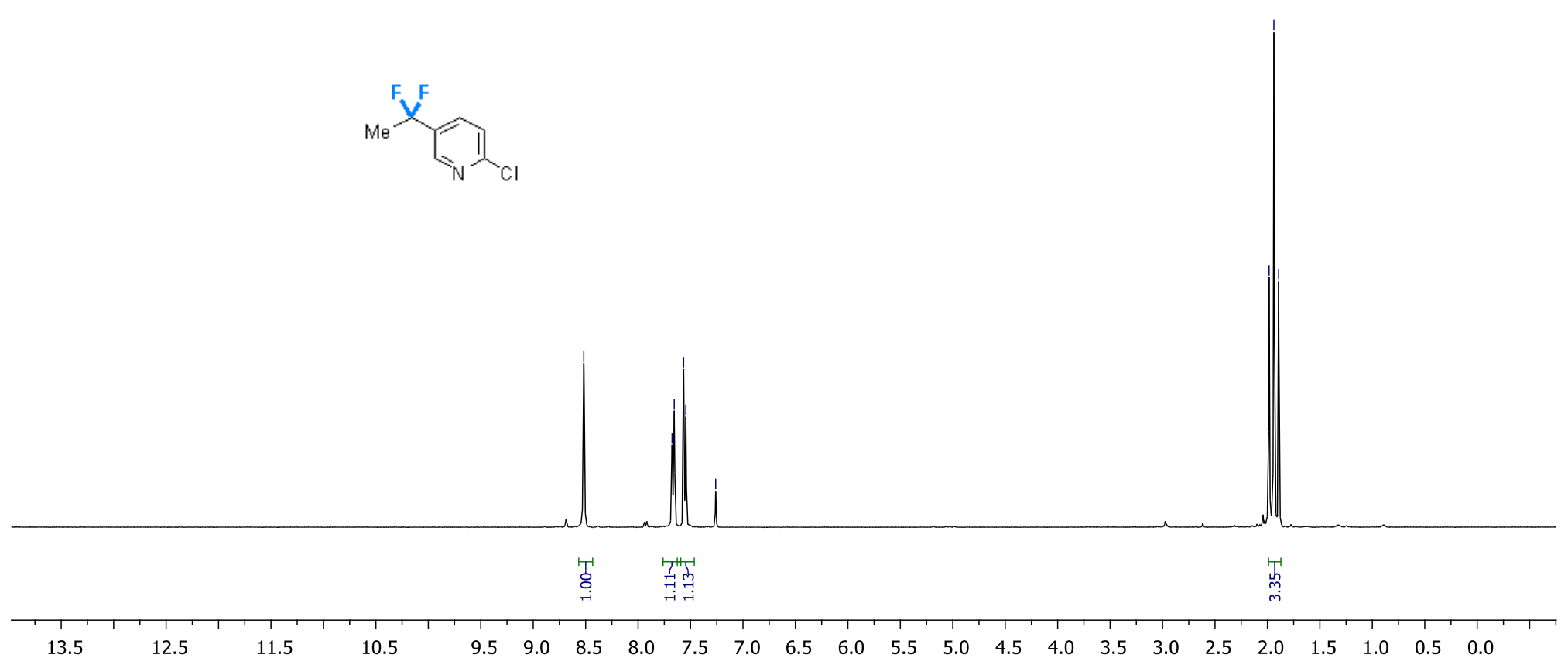
${ }^{13} \mathrm{C}\left\{{ }^{1} \mathrm{H}\right\} \mathrm{NMR}\left(126 \mathrm{MHz}, \mathrm{CDCl}_{3}\right)$

R1498081_C1:

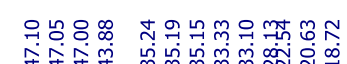

守手年

$\stackrel{\substack{i \\ i}}{i}$

m. 용

स स

ํำ

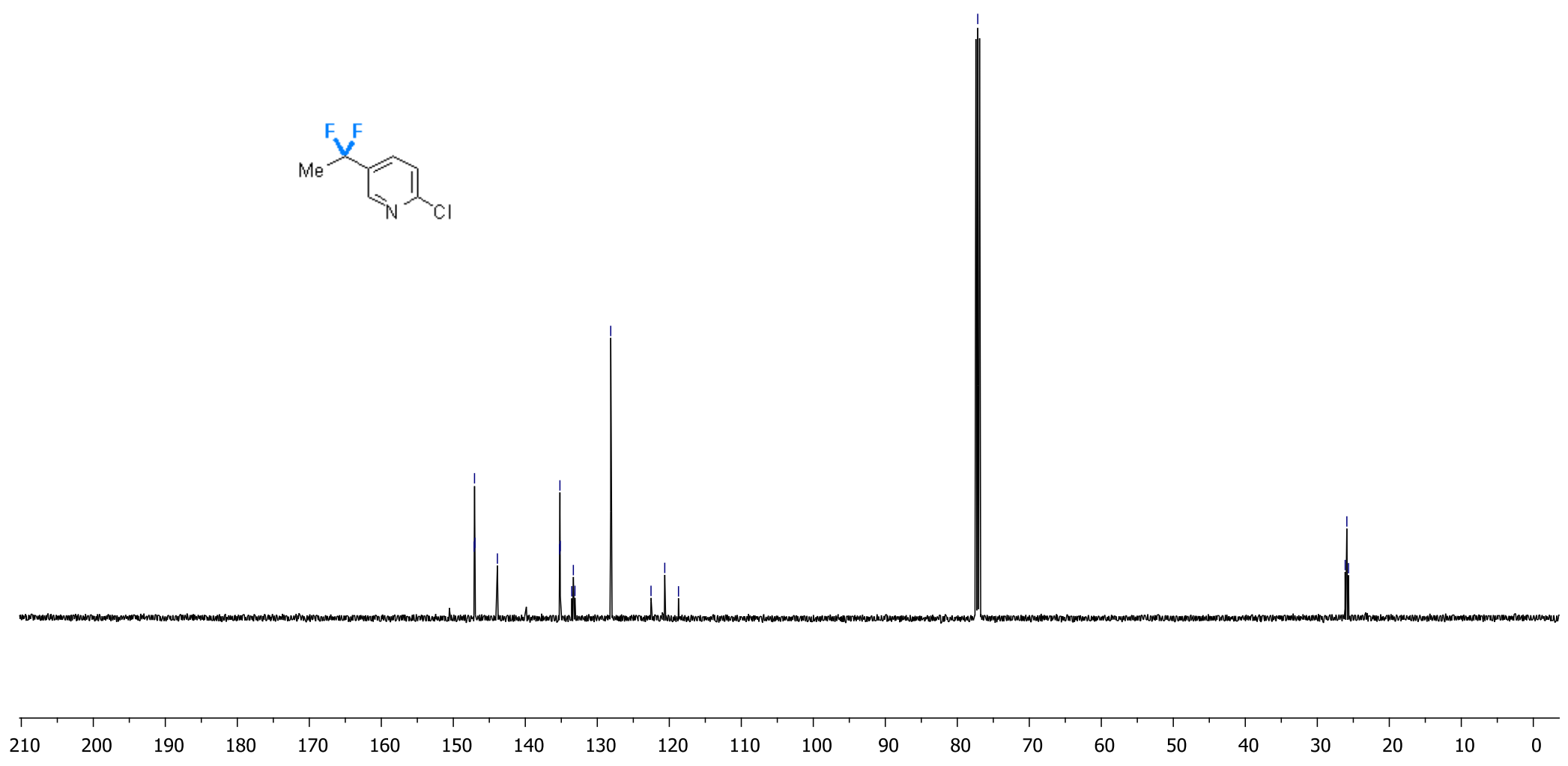




\section{${ }^{19} \mathrm{~F}\left\{{ }^{1} \mathrm{H}\right\} \mathrm{NMR}\left(376 \mathrm{MHz}, \mathrm{CDCl}_{3}\right)$}

R1498081_F19\{H\}

$19 \mathrm{~F}-\{1 \mathrm{H}\}$
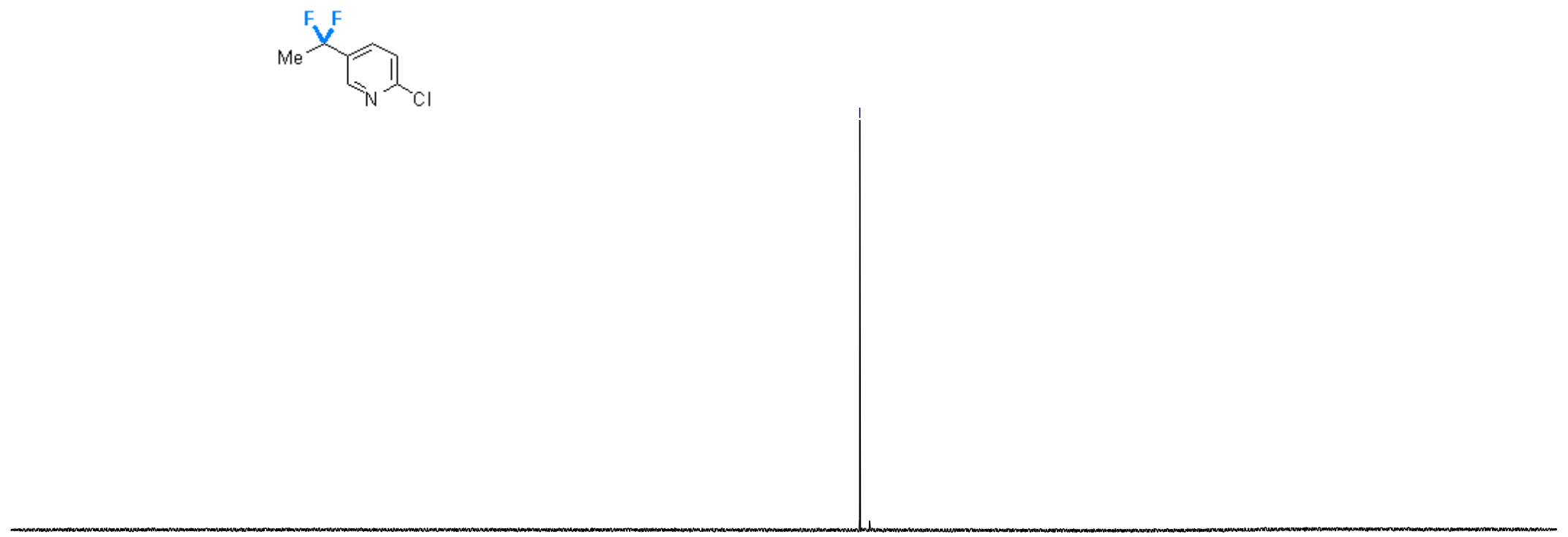


\section{Compound 45a}

${ }^{1} \mathrm{H}$ NMR (400 MHz, $\mathrm{CDCl}_{3}$ )

R1498081

STANDARD PROTON PARAMETERS

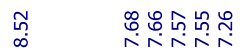

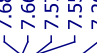

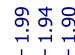
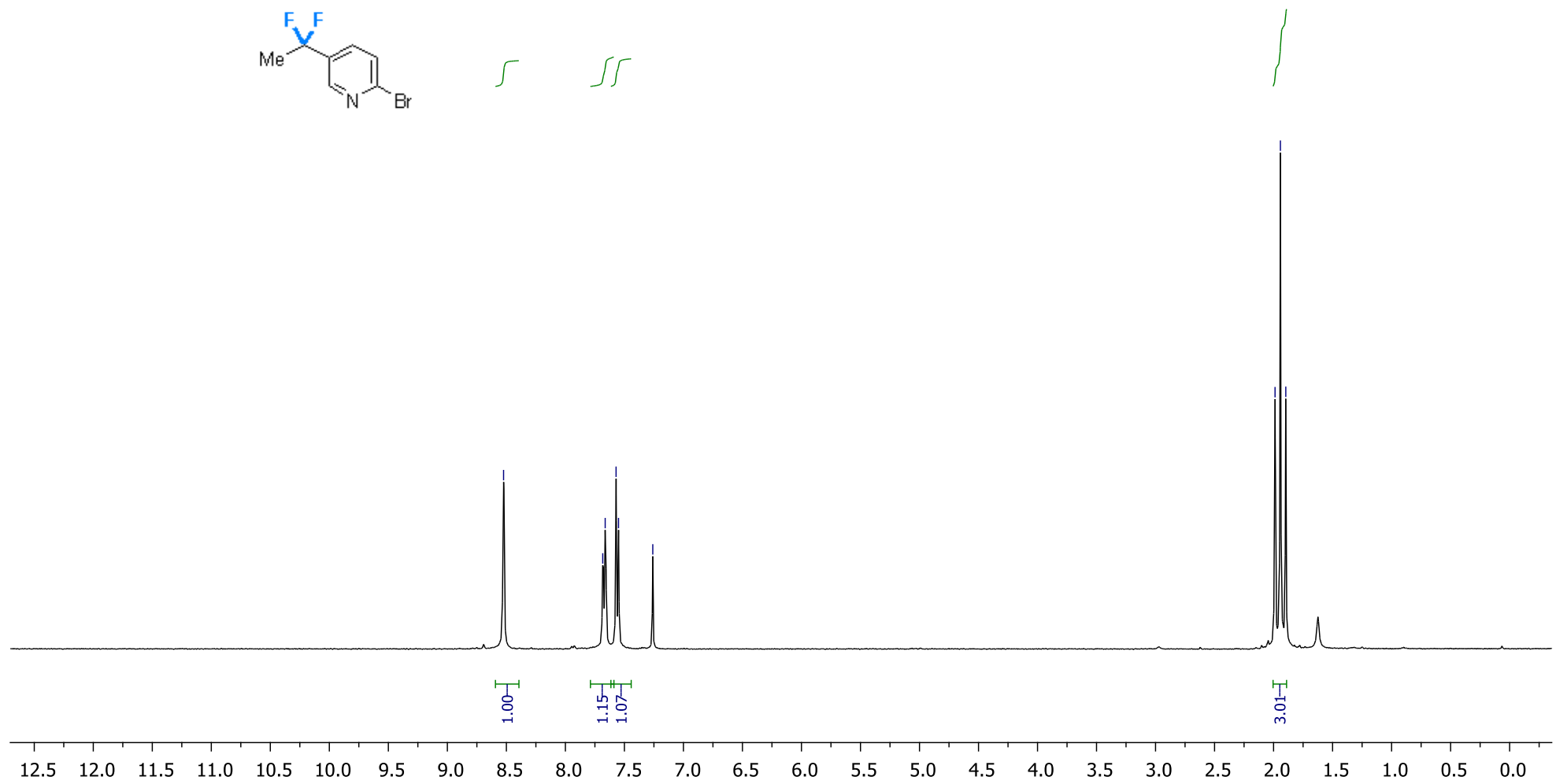
${ }^{13} \mathrm{C}\left\{{ }^{1} \mathrm{H}\right\} \mathrm{NMR}\left(126 \mathrm{MHz}, \mathrm{CDCl}_{3}\right)$

R1498081_C1:

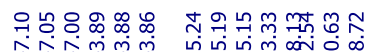

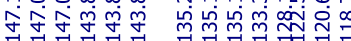

स ।

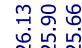

许
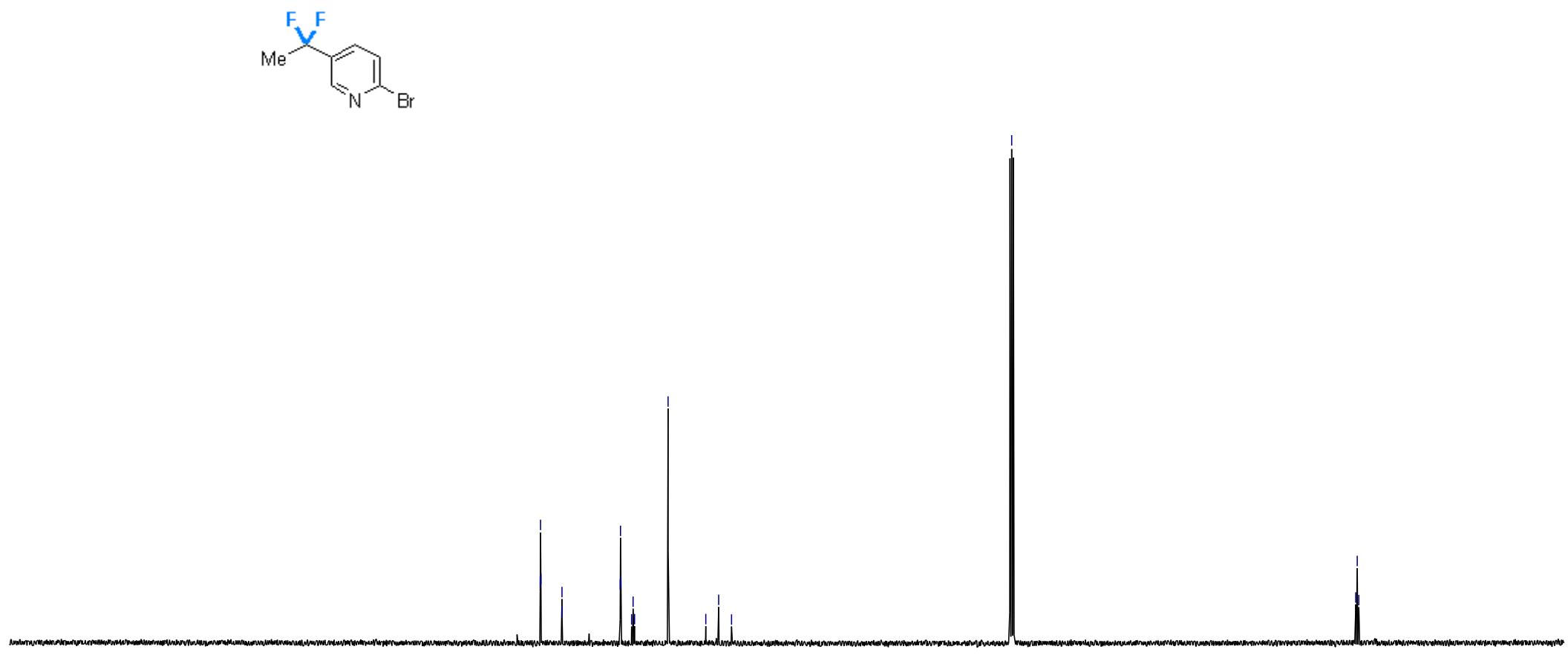

$\begin{array}{lllllllllllllllllllllll}220 & 210 & 200 & 190 & 180 & 170 & 160 & 150 & 140 & 130 & 120 & 110 & 100 & 90 & 80 & 70 & 60 & 50 & 40 & 30 & 20 & 10 & 0\end{array}$ 


\section{${ }^{19} \mathrm{~F}\left\{{ }^{1} \mathrm{H}\right\} \mathrm{NMR}\left(376 \mathrm{MHz}, \mathrm{CDCl}_{3}\right)$}

R1498081_F19\{H\} $19 \mathrm{~F}-\{1 \mathrm{H}\}$
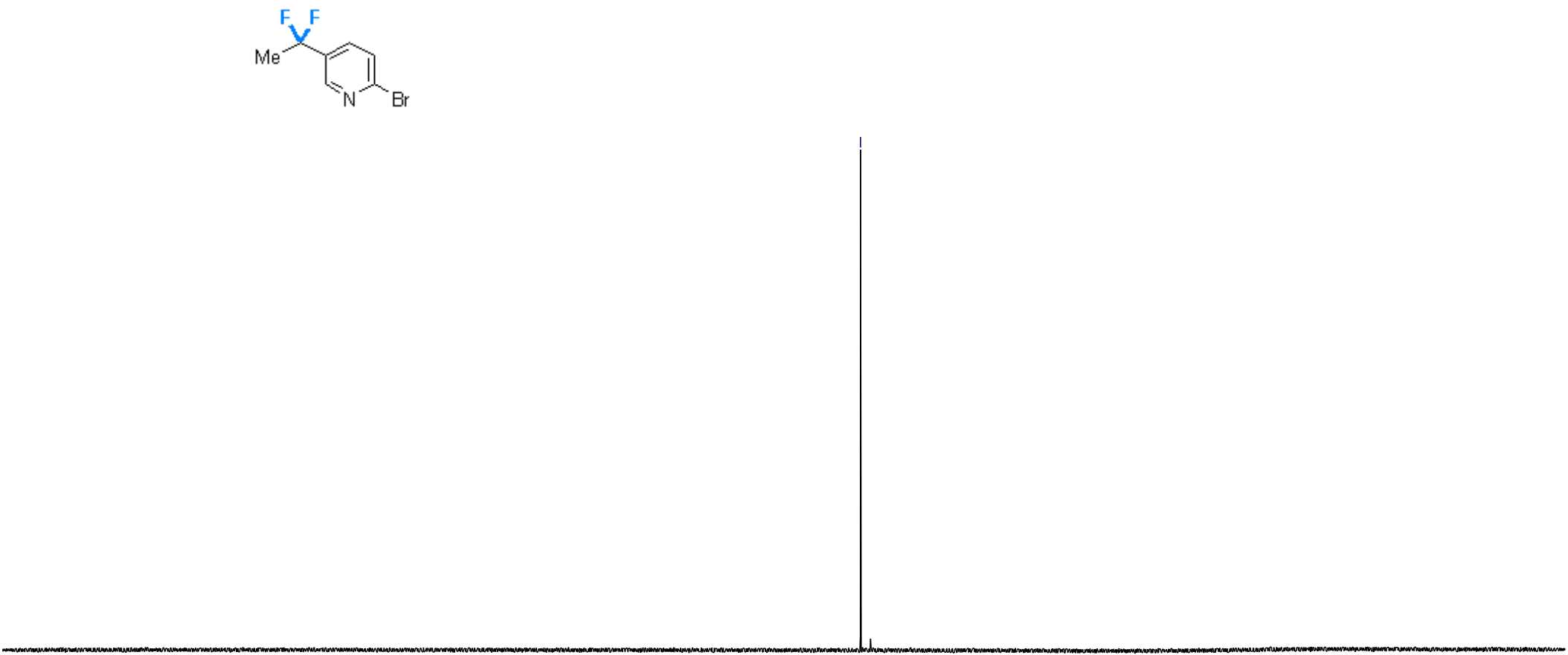


\section{Compound 46a}

${ }^{1} \mathrm{H}$ NMR (400 MHz, $\mathrm{CDCl}_{3}$ )

R1546047

|
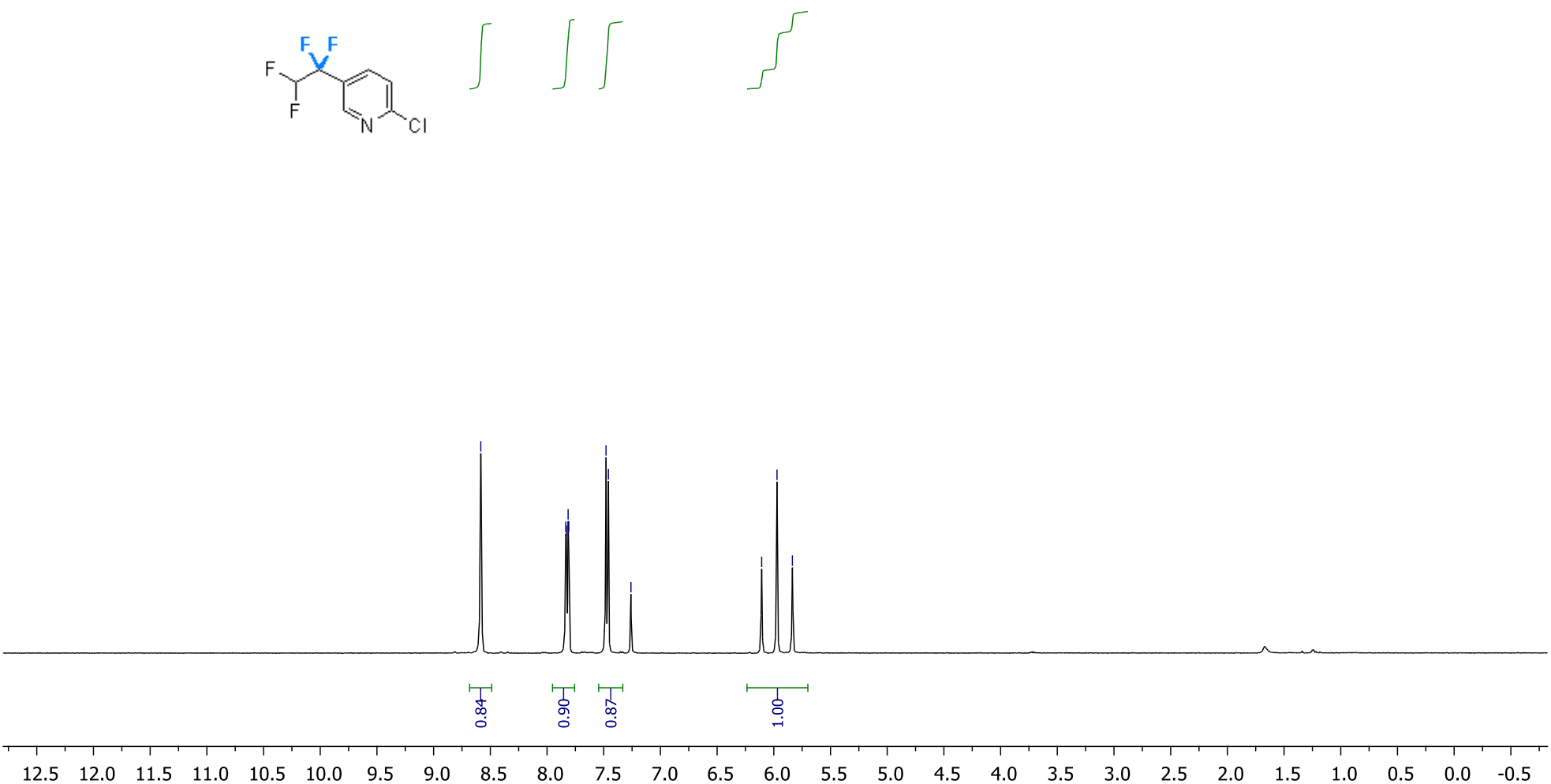
${ }^{13} \mathrm{C}\left\{{ }^{1} \mathrm{H}\right\}$ NMR $\left(151 \mathrm{MHz}, \mathrm{CDCl}_{3}\right)$

R1546047_C13

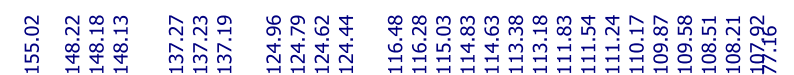

1
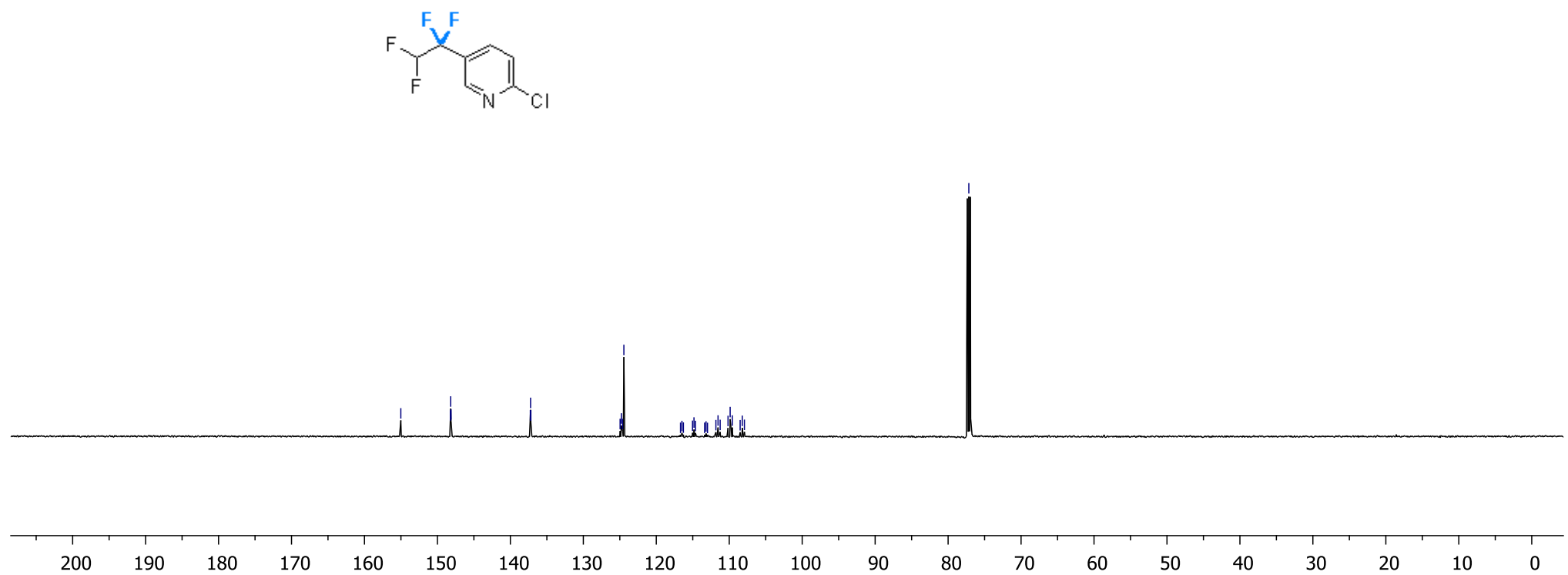
${ }^{19} \mathrm{~F}\left\{{ }^{1} \mathrm{H}\right\} \mathrm{NMR}\left(376 \mathrm{MHz}, \mathrm{CDCl}_{3}\right)$

R1546047_F19\{H\}
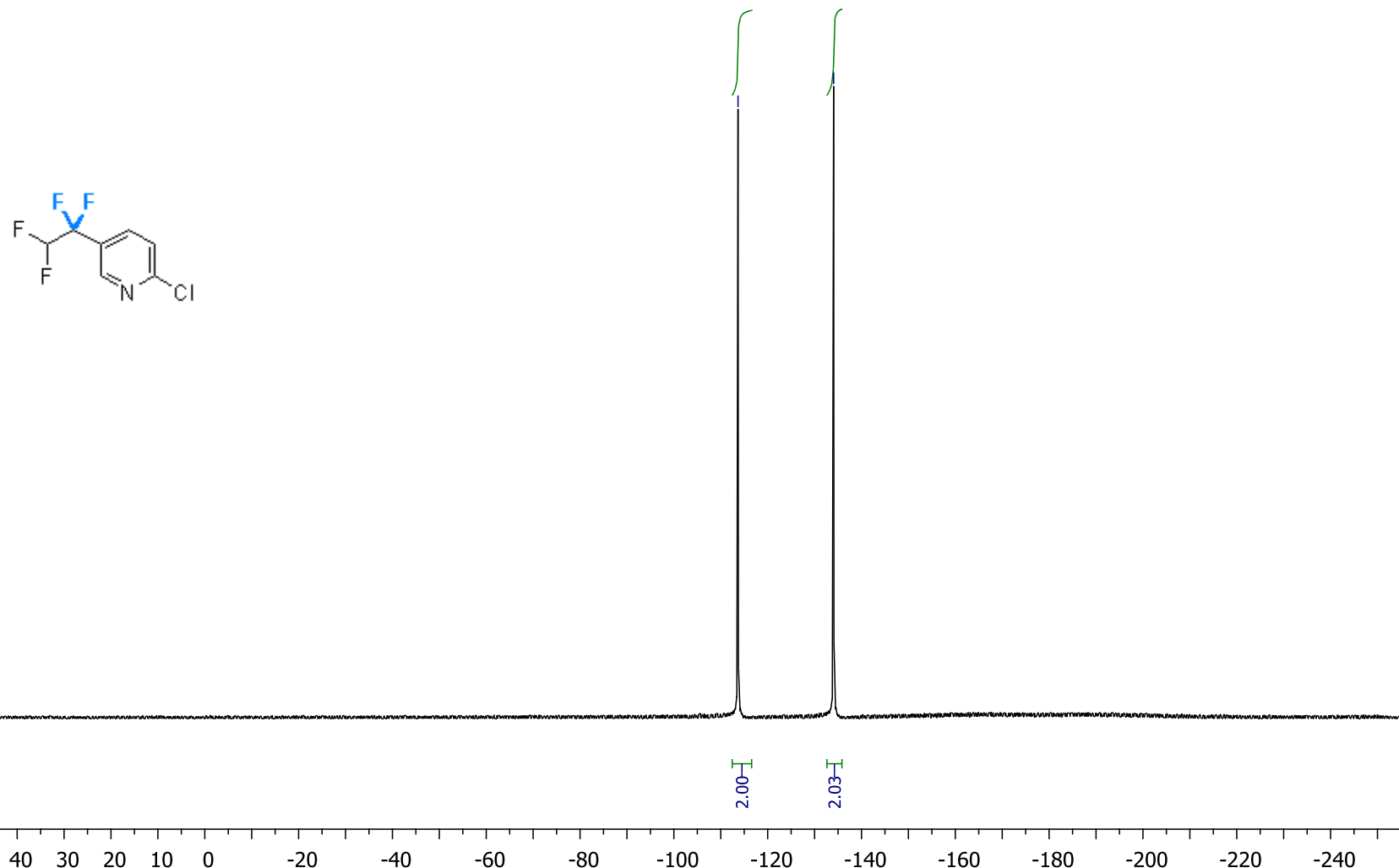


\section{Compound 47a}

${ }^{1} \mathrm{H}$ NMR (400 MHz, $\mathrm{CDCl}_{3}$ )

R1677046

$\mathrm{M}_{\mathrm{N}}^{\mathrm{X}}$
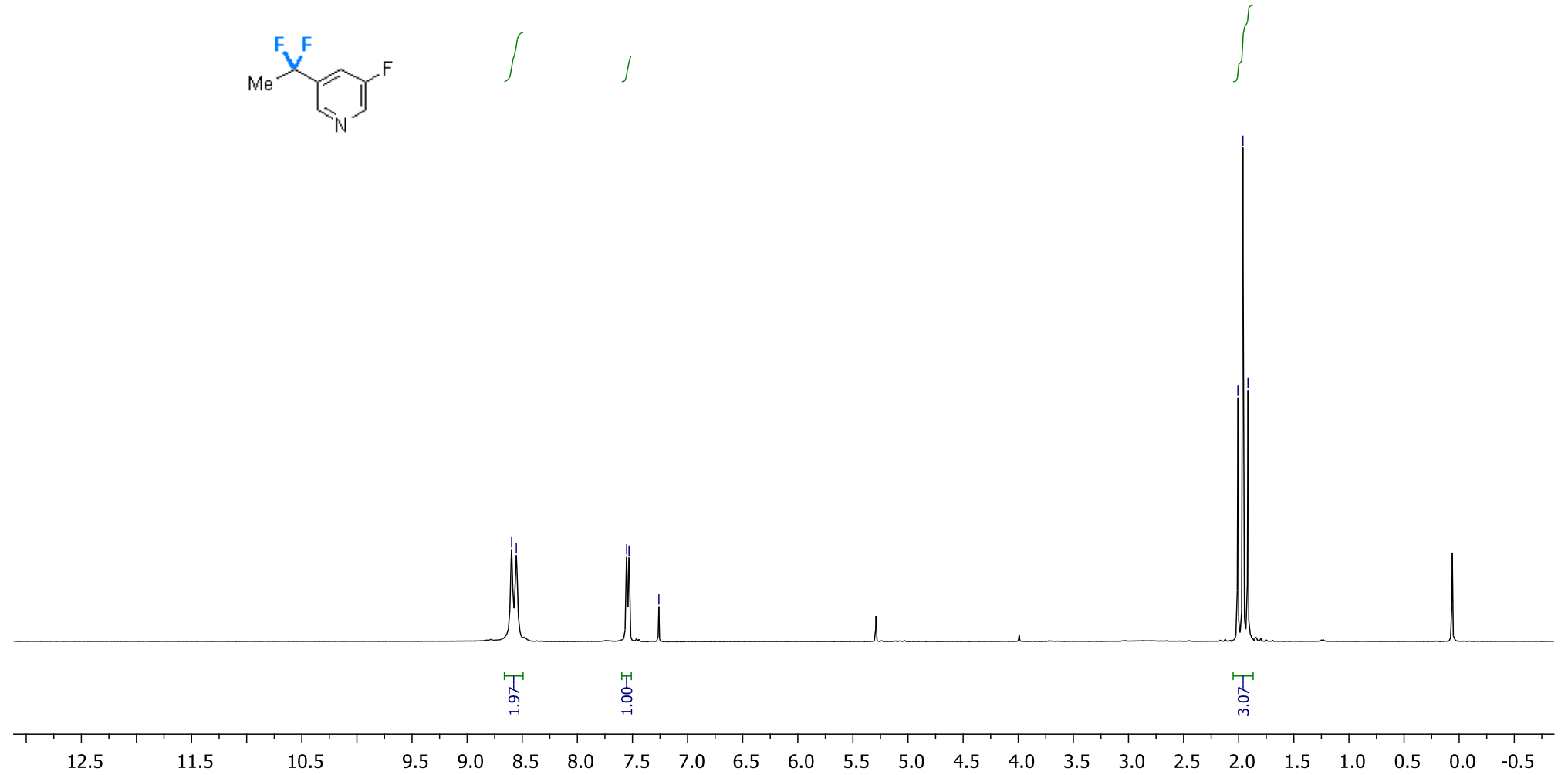
${ }^{13} \mathrm{C}\left\{{ }^{1} \mathrm{H}\right\}$ NMR (126 MHz, $\left.\mathrm{CDCl}_{3}\right)$

R1677046_malo_v-va_C13

13C (1H-decoupled)

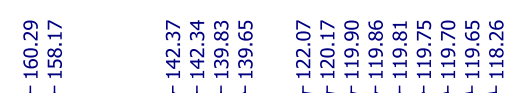

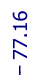

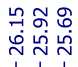

$\mathrm{M}_{N}^{\mathrm{F}}$

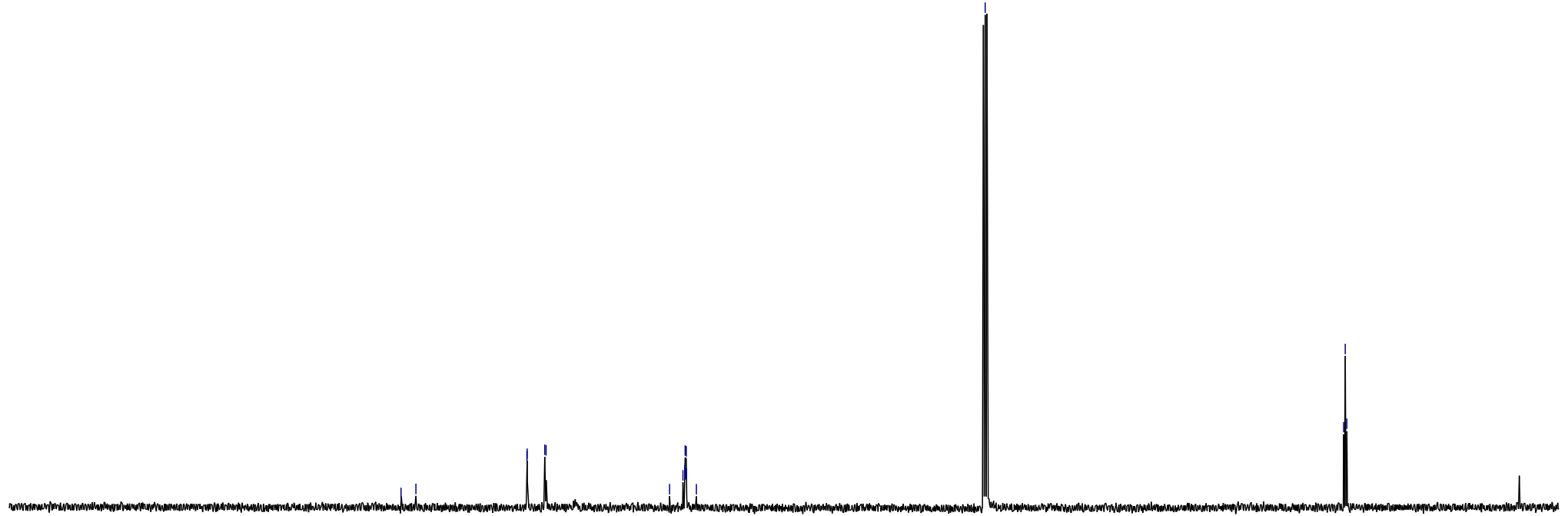

$\begin{array}{lllllllllllllllllllllllllllll}210 & 200 & 190 & 180 & 170 & 160 & 150 & 140 & 130 & 120 & 110 & 100 & 90 & 80 & 70 & 60 & 50 & 40 & 30 & 20 & 10 & 0\end{array}$ 


\section{${ }^{19} \mathrm{~F}\left\{{ }^{1} \mathrm{H}\right\}$ NMR (376 MHz, $\left.\mathrm{CDCl}_{3}\right)$}

R1677046_F19 $\{\mathrm{H}\}$

$19 \mathrm{~F}-\{1 \mathrm{H}\}$

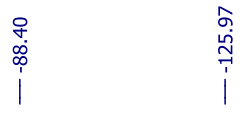

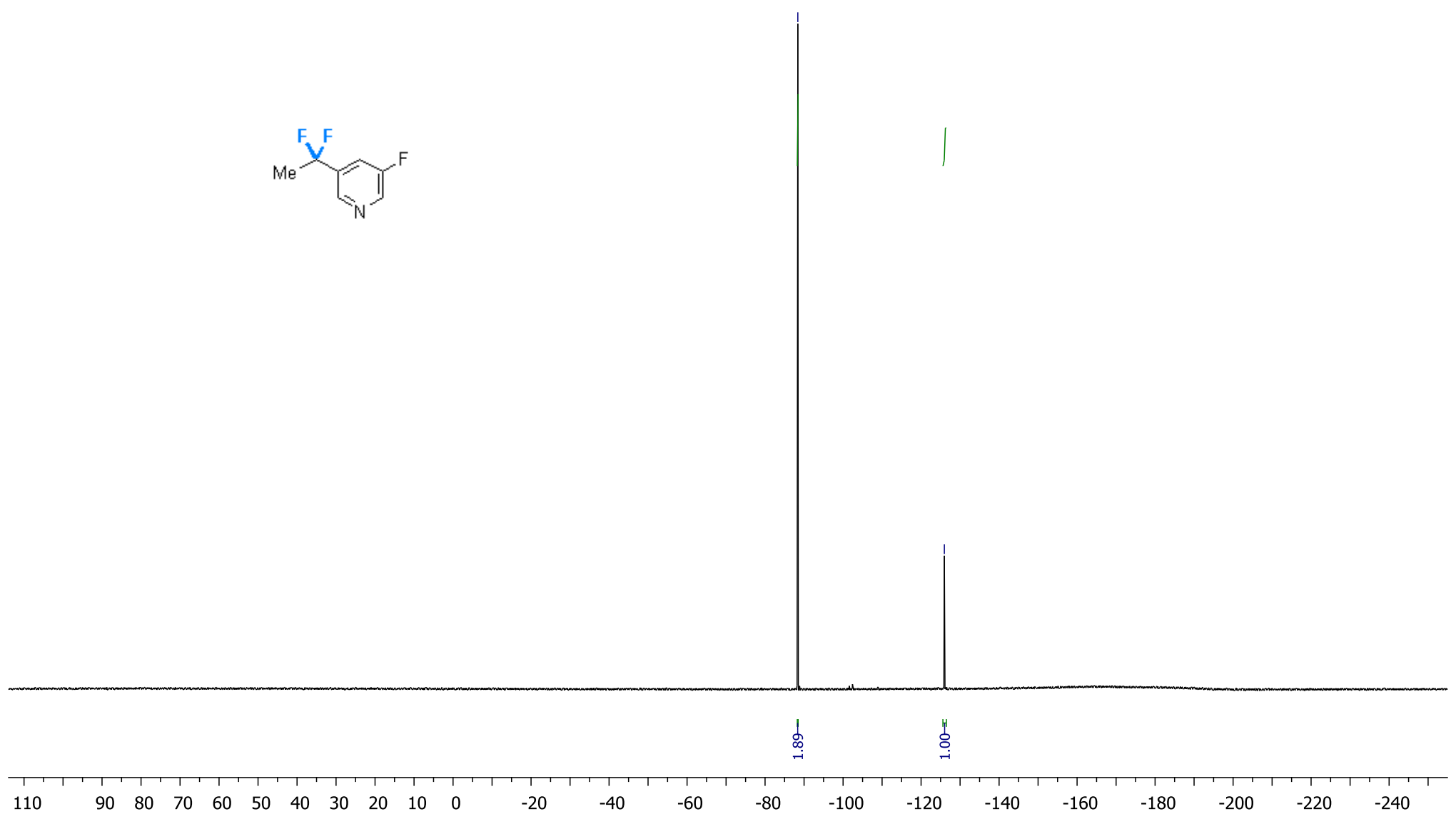




\section{Compound 48a}

${ }^{1} \mathrm{H}$ NMR $\left(400 \mathrm{MHz}, \mathrm{CDCl}_{3}\right.$ )

R1394043

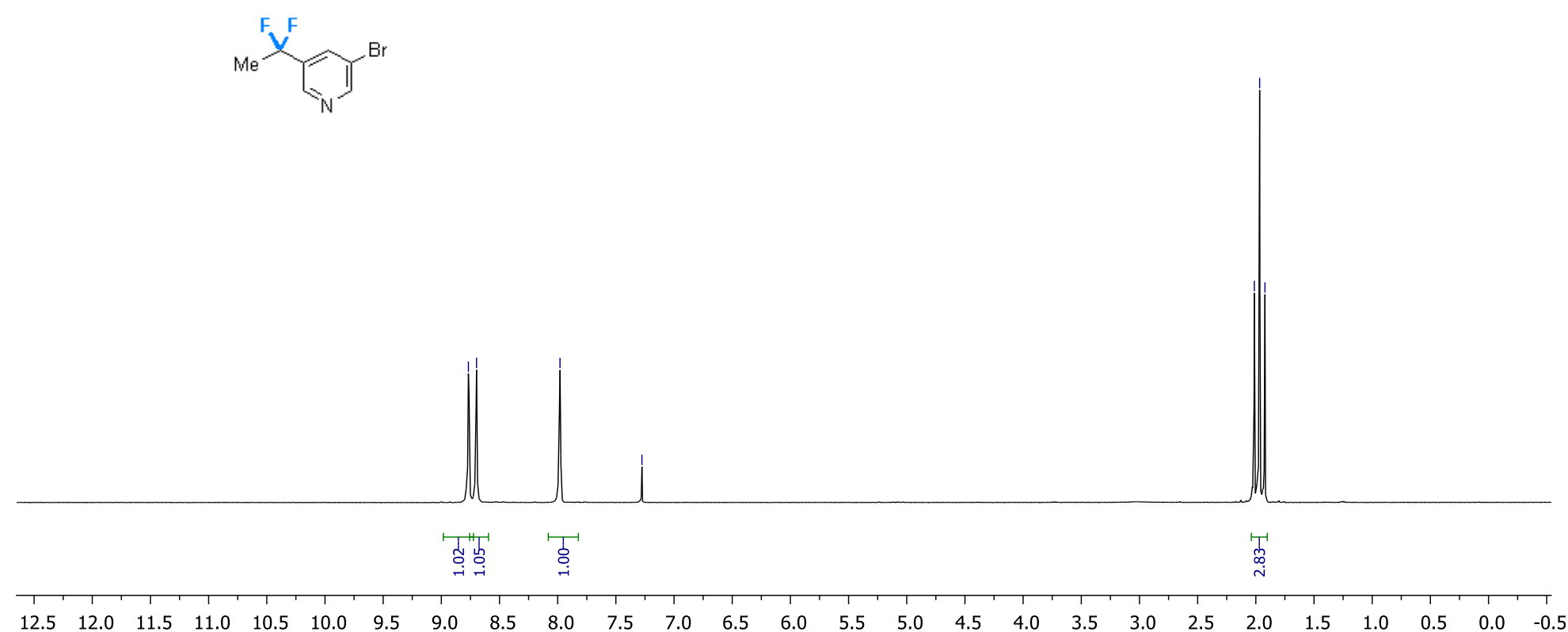


${ }^{13} \mathrm{C}\left\{{ }^{1} \mathrm{H}\right\}$ NMR (126 MHz, $\left.\mathrm{CDCl}_{3}\right)$

R1394043_C1:

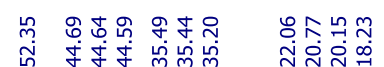

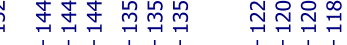

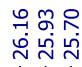

$\mathrm{Me}^{\mathrm{F}} \mathrm{C}^{\mathrm{F}}$

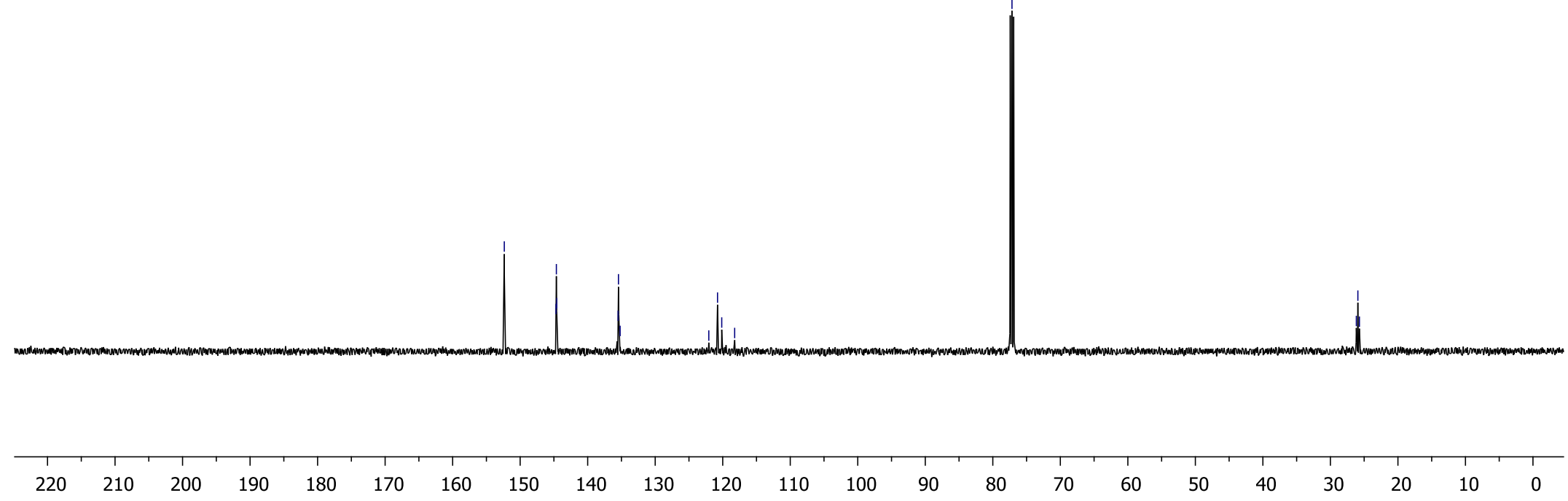




\section{${ }^{19} \mathrm{~F}\left\{{ }^{1} \mathrm{H}\right\}$ NMR (376 MHz, $\left.\mathrm{CDCl}_{3}\right)$}

R1394043_F19\{H\}

$19 \mathrm{~F}-\{1 \mathrm{H}\}$

$\overbrace{N}^{F}{ }^{F r}$

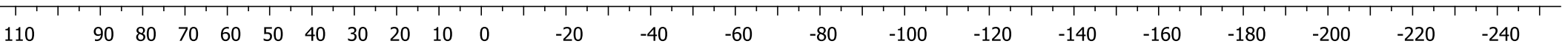




\section{Compound 49a}

${ }^{1} \mathrm{H}$ NMR (400 MHz, $\mathrm{CDCl}_{3}$ )

R1919325

${ }_{\mathrm{F}} \overbrace{\mathrm{F}}$

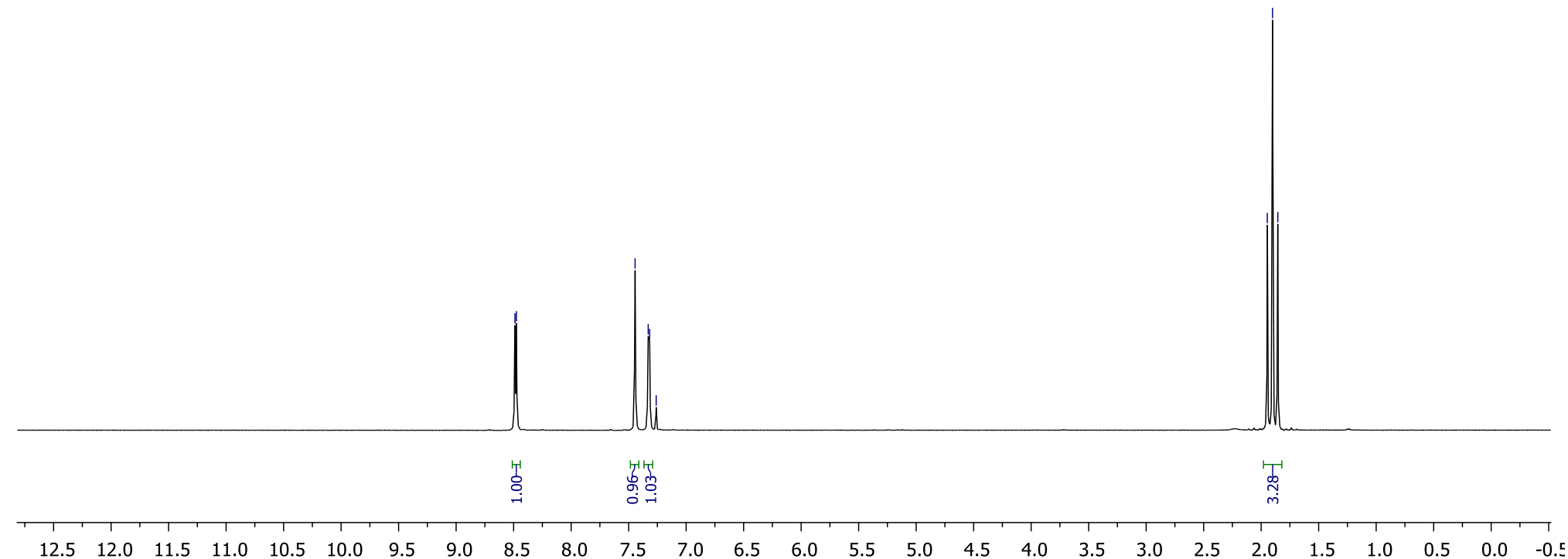


${ }^{13} \mathrm{C}\left\{{ }^{1} \mathrm{H}\right\} \mathrm{NMR}\left(126 \mathrm{MHz}, \mathrm{CDCl}_{3}\right)$

R1919325_C1:

${ }_{F=}$

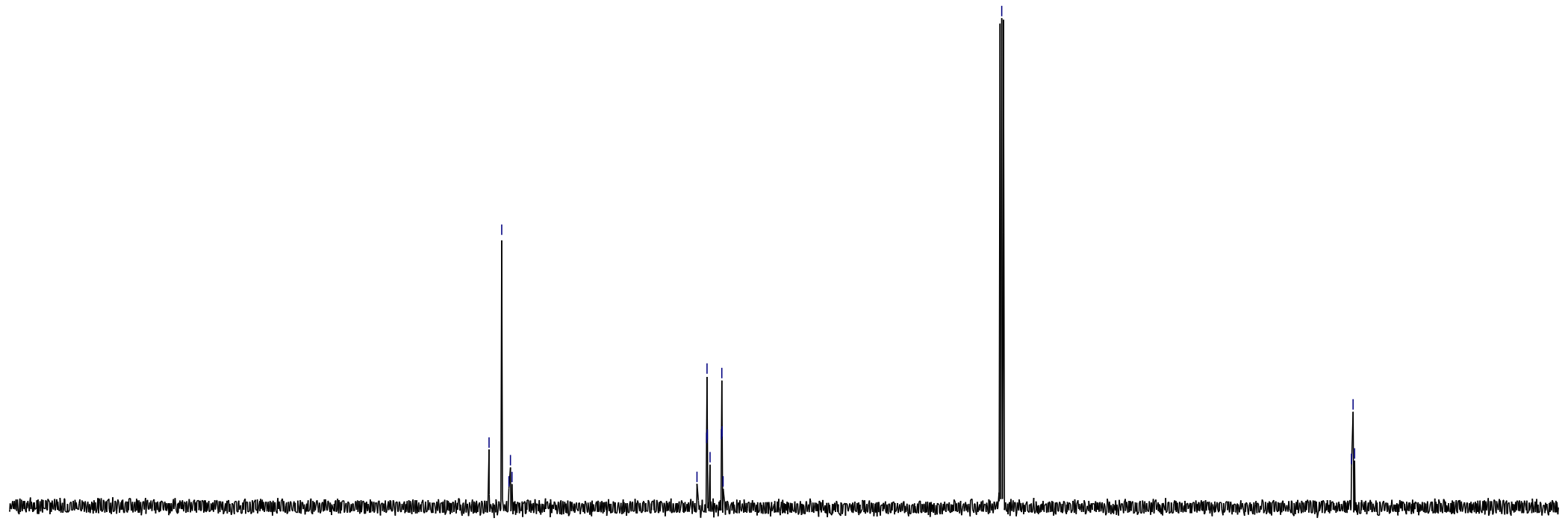

$220 \quad 210 \quad 200 \quad 190$

$150 \quad 140$

130

$110 \quad 100$

80

$70 \quad 60$

50

$40 \quad 30$ 


\section{${ }^{19} \mathrm{~F}\left\{{ }^{1} \mathrm{H}\right\}$ NMR (376 MHz, $\left.\mathrm{CDCl}_{3}\right)$}

R1919325_F19 $\{\mathrm{H}\}$

$\overbrace{F}{ }_{F}$

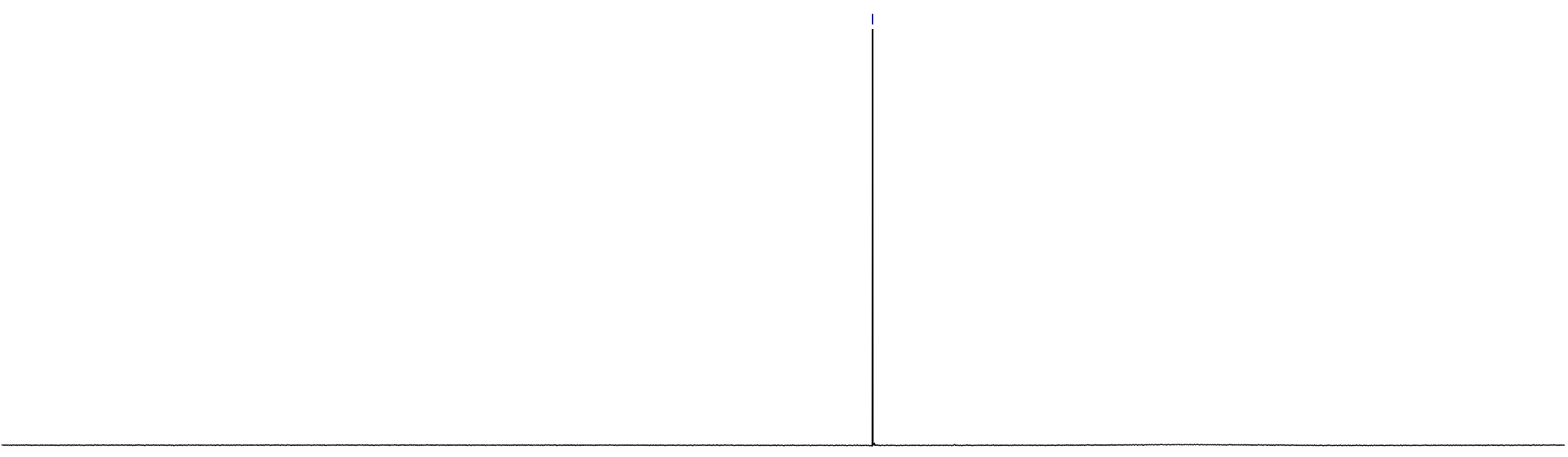

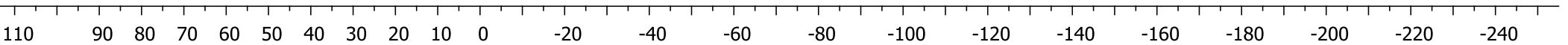




\section{Compound 50a}

${ }^{1} \mathrm{H}$ NMR (400 MHz, $\mathrm{CDCl}_{3}$ )

R1334489

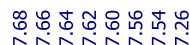

نู่

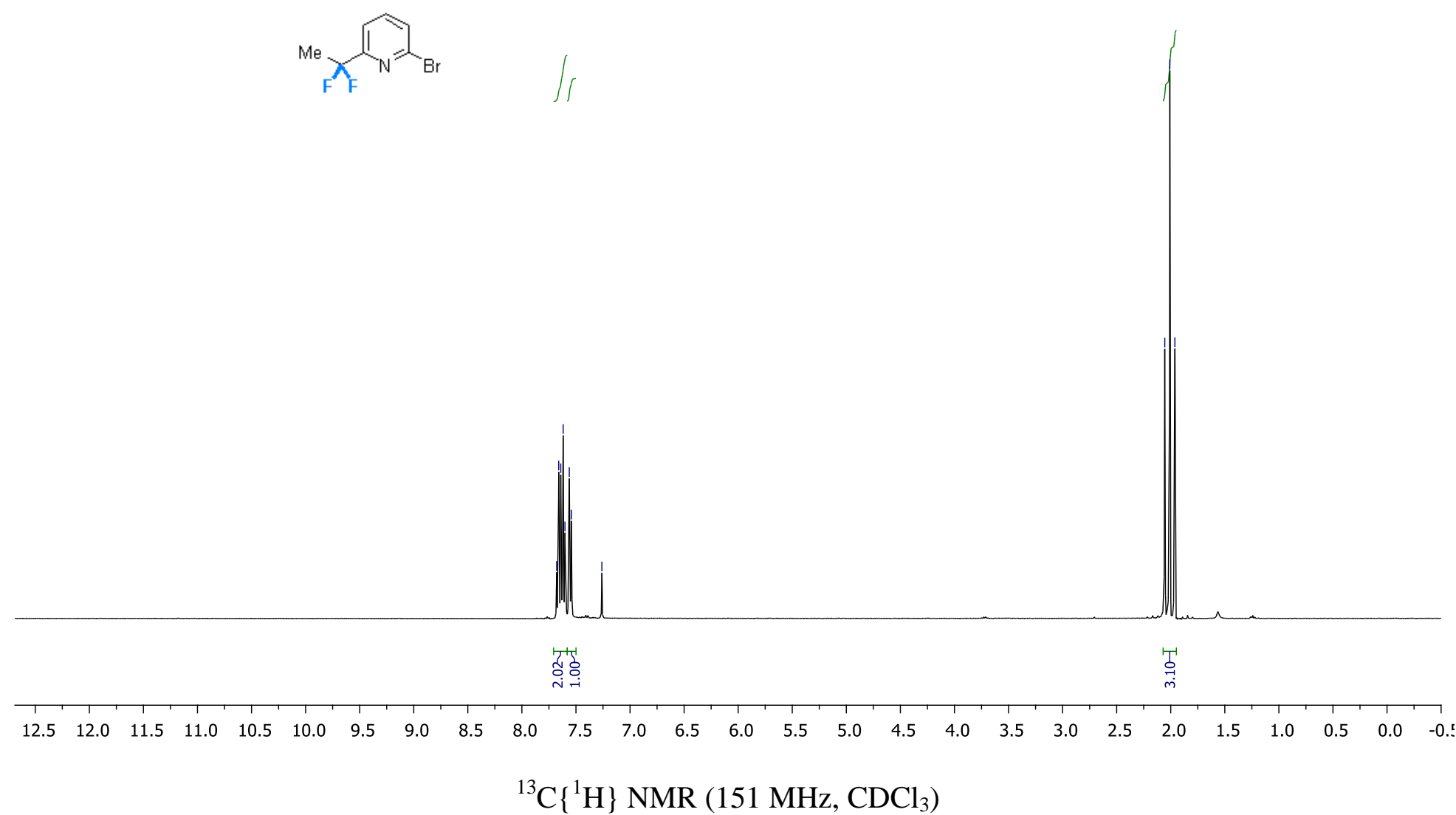


R1334489_C13
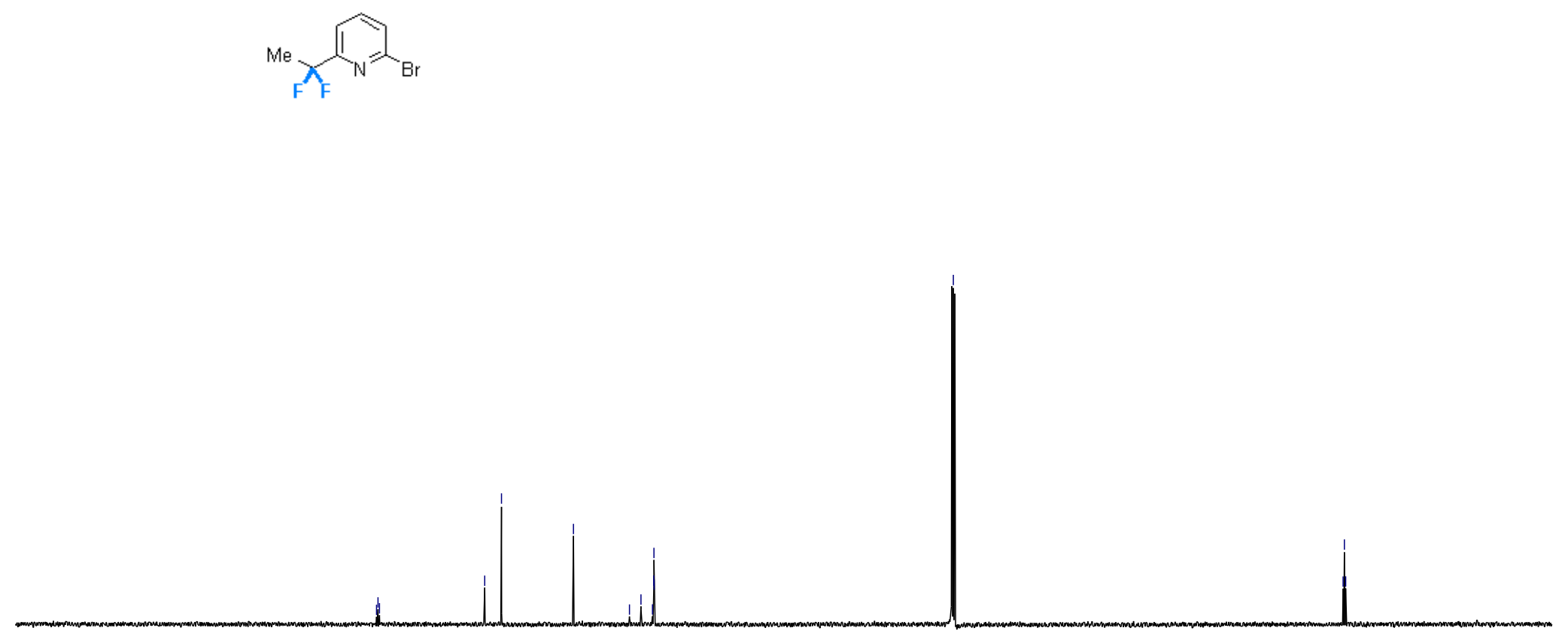

$\begin{array}{lllllll}200 & 190 & 180 & 170 & 160 & 150 & 140\end{array}$

$\begin{array}{lll}120 & 110 & 100\end{array}$

90

$80 \quad 70$

60
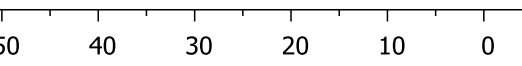

${ }^{19} \mathrm{~F}\left\{{ }^{1} \mathrm{H}\right\}$ NMR $\left(376 \mathrm{MHz}, \mathrm{CDCl}_{3}\right)$ 
R2601618_F19 $\{\mathrm{H}\}$

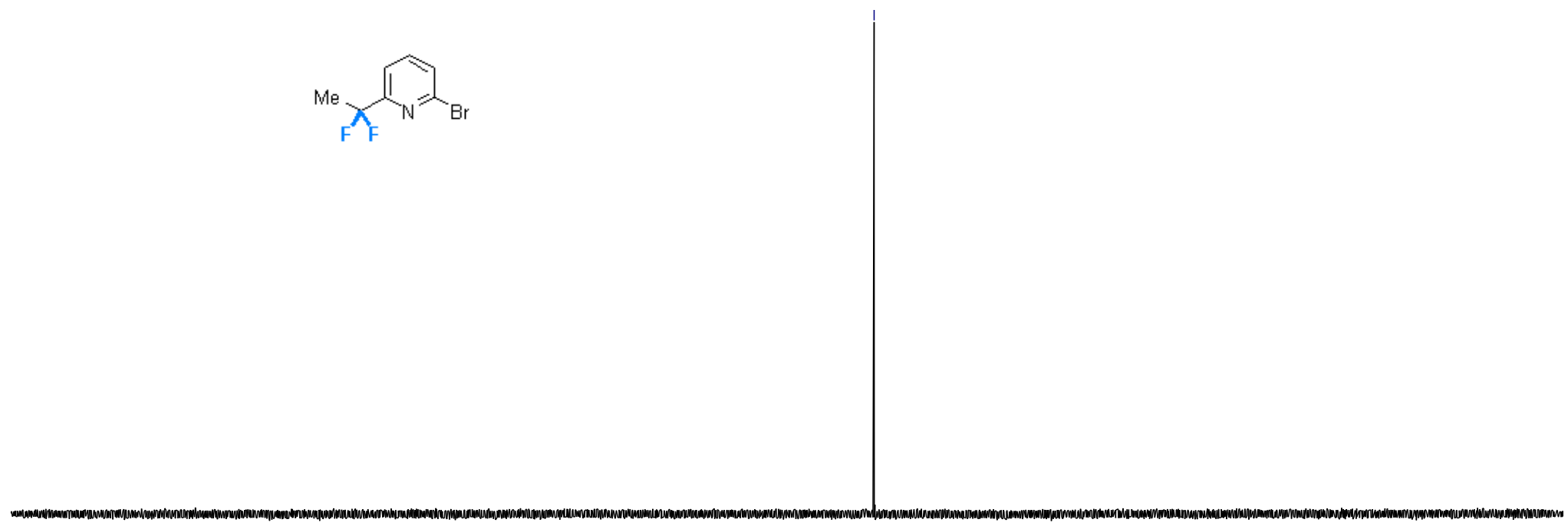




\section{Compound 51a}

${ }^{1} \mathrm{H}$ NMR (400 MHz, $\mathrm{CDCl}_{3}$ )

R1824907

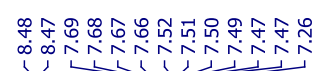

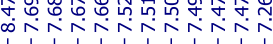

$$
\underset{F{ }_{F}}{M}
$$

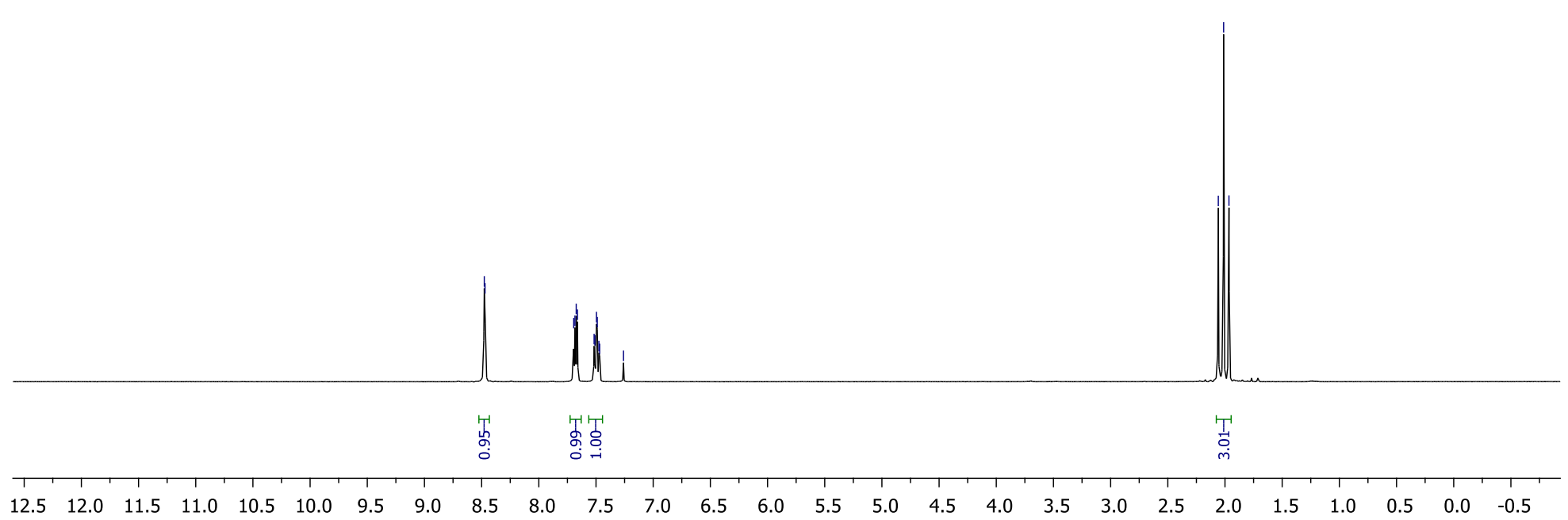


${ }^{13} \mathrm{C}\left\{{ }^{1} \mathrm{H}\right\}$ NMR (126 MHz, $\left.\mathrm{CDCl}_{3}\right)$

R1824907_C1:

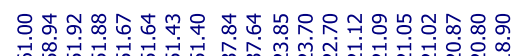

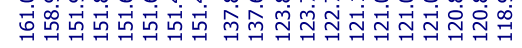

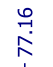

证

।

กับ
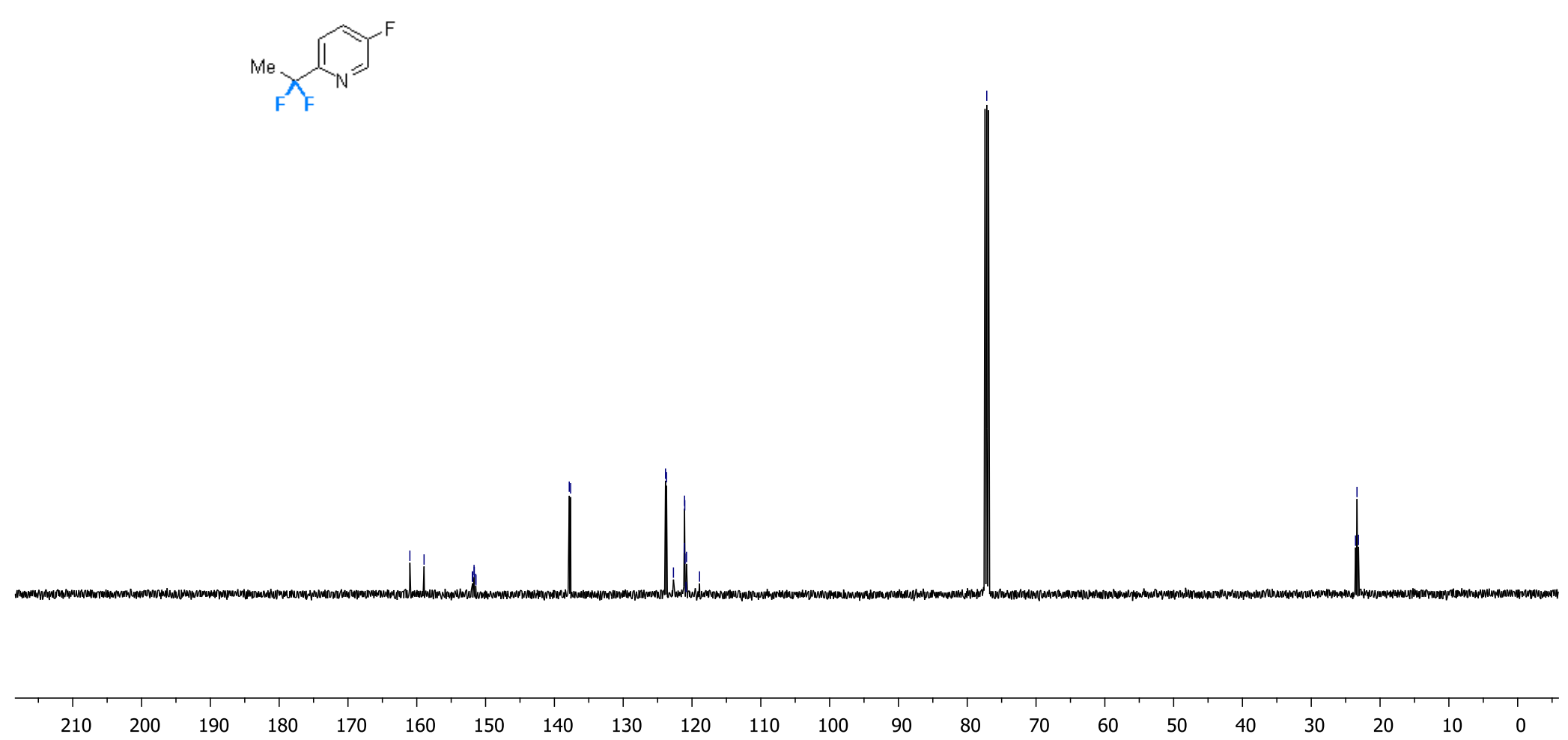


\section{${ }^{19} \mathrm{~F}\left\{{ }^{1} \mathrm{H}\right\} \mathrm{NMR}\left(376 \mathrm{MHz}, \mathrm{CDCl}_{3}\right)$}

R1824907_F19 $\{\mathrm{H}\}$

$$
19 \mathrm{~F}-\{1 \mathrm{H}\}
$$

$$
\mathrm{F}_{\mathrm{F}}
$$
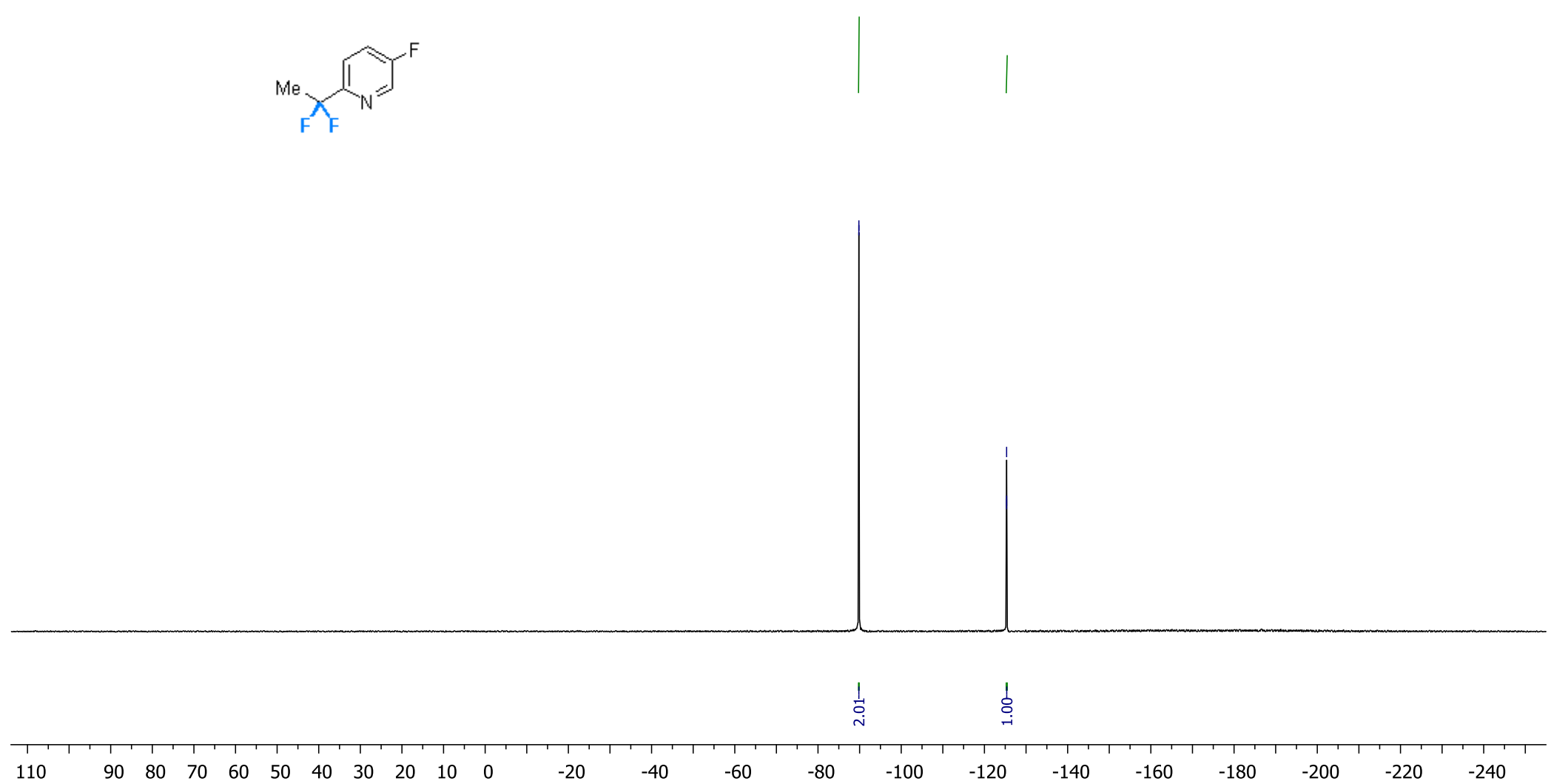


\section{Compound 52a}

${ }^{1} \mathrm{H}$ NMR (400 MHz, $\mathrm{CDCl}_{3}$ )

R1704020

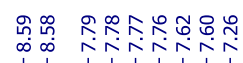

ํㅛㅇㅛㅛ

$Y$ H

$\underbrace{C l}_{F=} \int \quad J j$

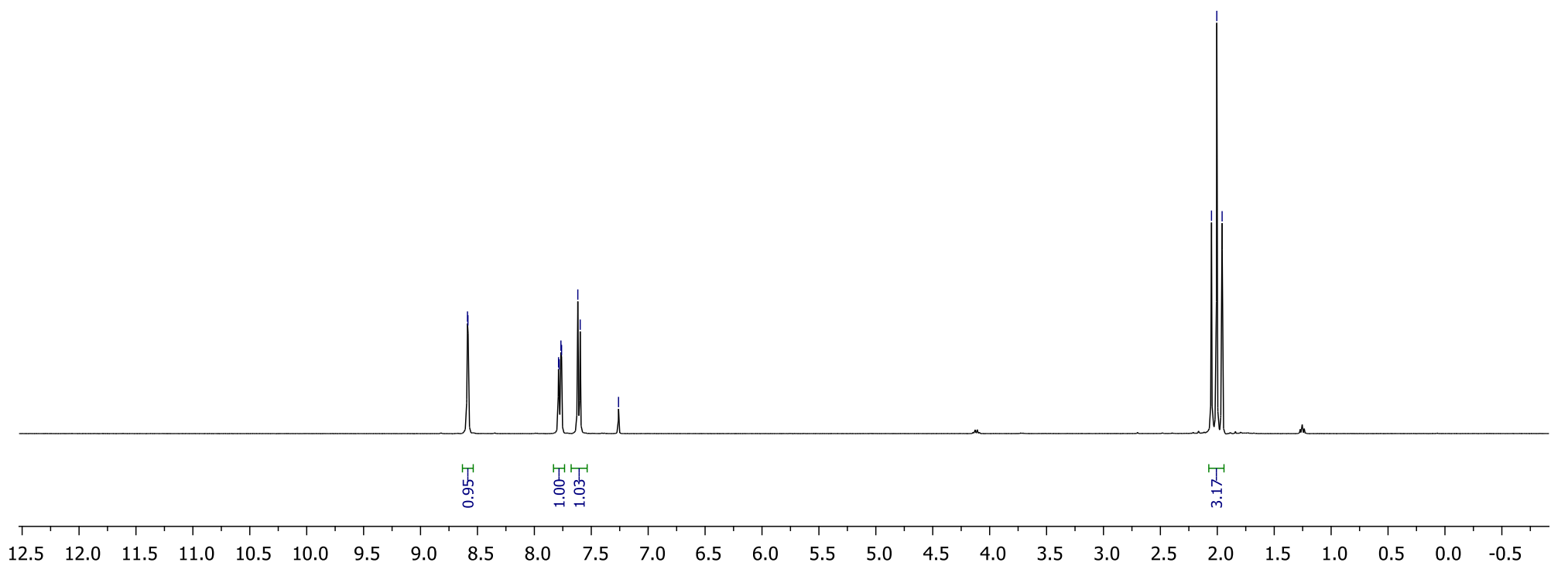


${ }^{13} \mathrm{C}\left\{{ }^{1} \mathrm{H}\right\}$ NMR $\left(126 \mathrm{MHz}, \mathrm{CDCl}_{3}\right)$

R1704020_C1:

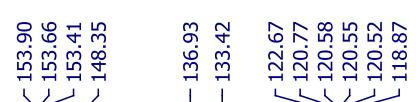

${ }_{F=}^{M e} \overbrace{F}^{C l}$

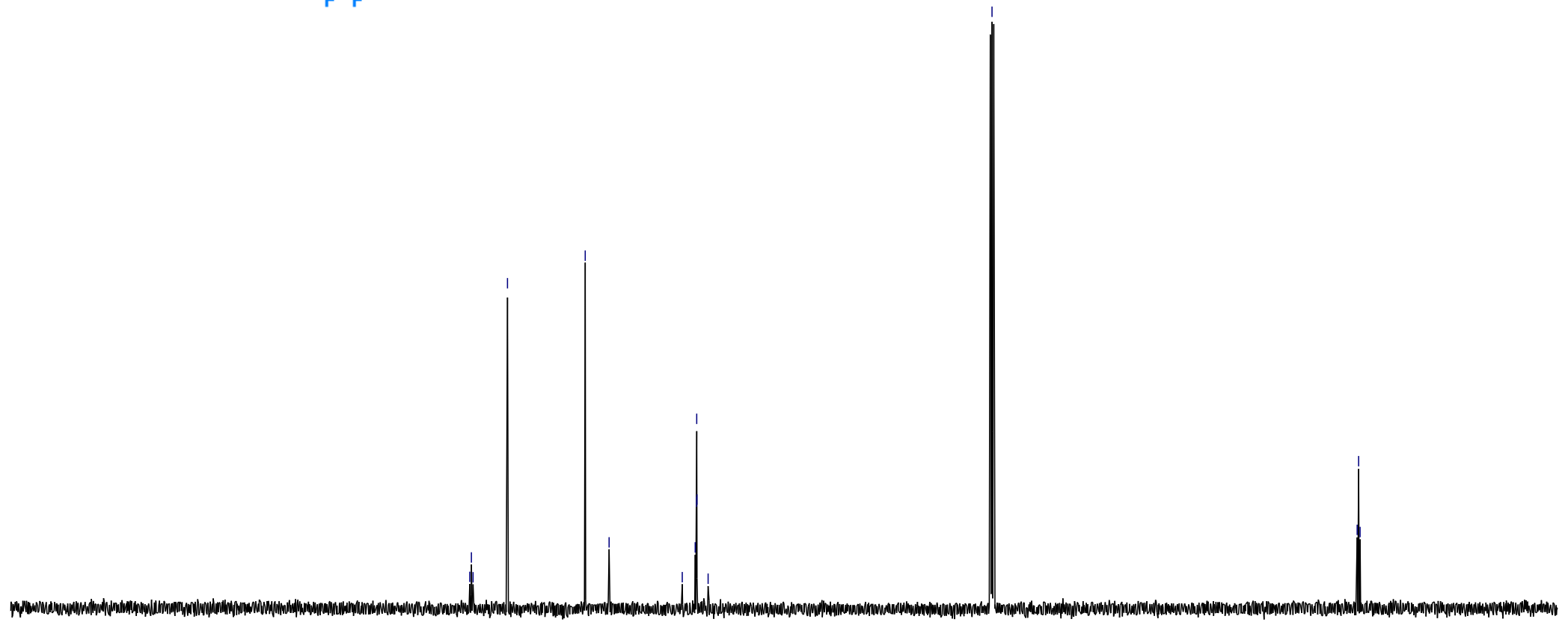

$220 \quad 210 \quad 200$ 


\section{${ }^{19} \mathrm{~F}\left\{{ }^{1} \mathrm{H}\right\}$ NMR (376 MHz, $\left.\mathrm{CDCl}_{3}\right)$}

R1704020_F19 $\{\mathrm{H}\}$

$$
19 \mathrm{~F}-\{1 \mathrm{H}\}
$$
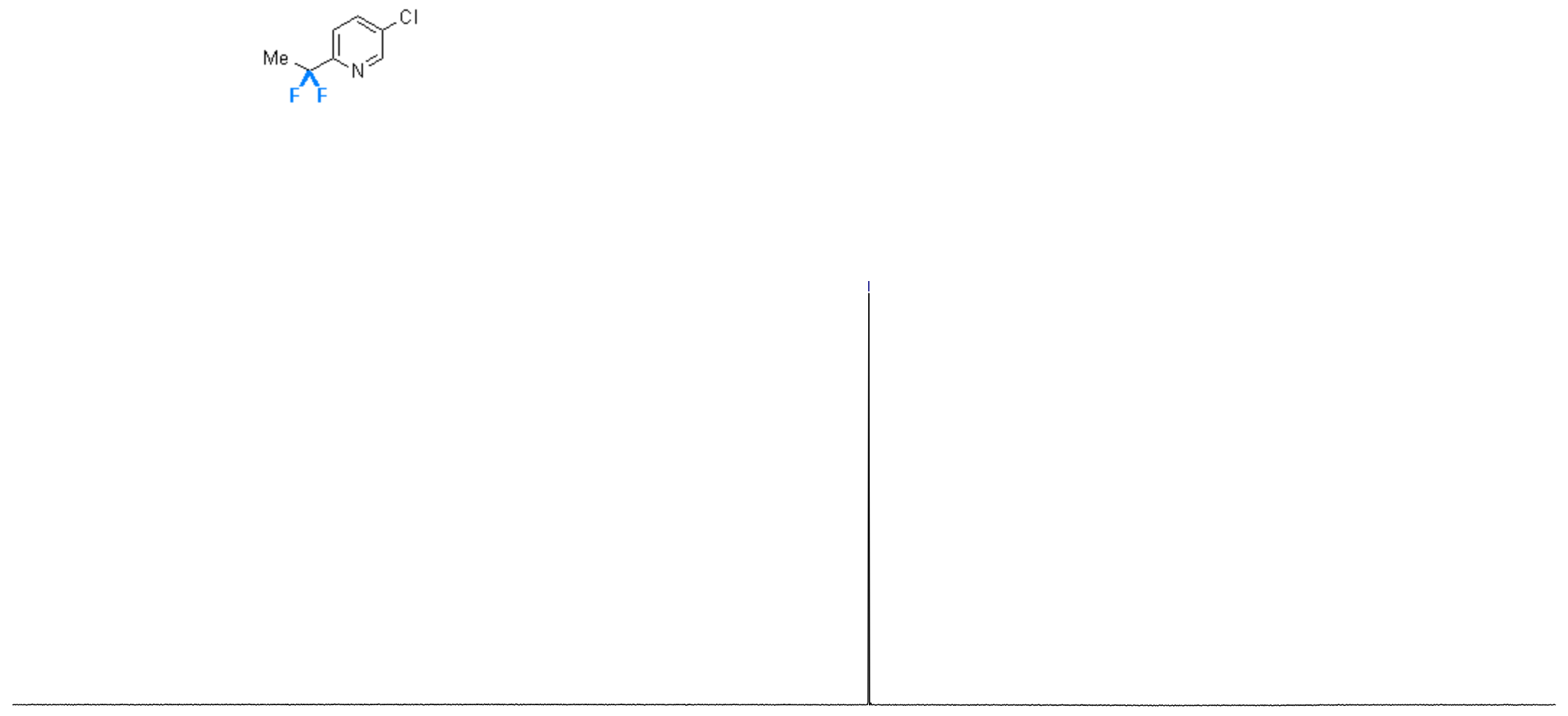

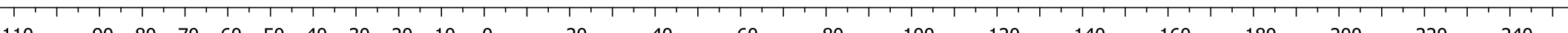




\section{Compound 53a}

${ }^{1} \mathrm{H}$ NMR (400 MHz, $\mathrm{CDCl}_{3}$ )

R1409962

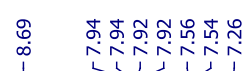

نูi

$\mathrm{F}_{\mathrm{F}}^{\mathrm{Br}}$

$\int \Gamma \Gamma$
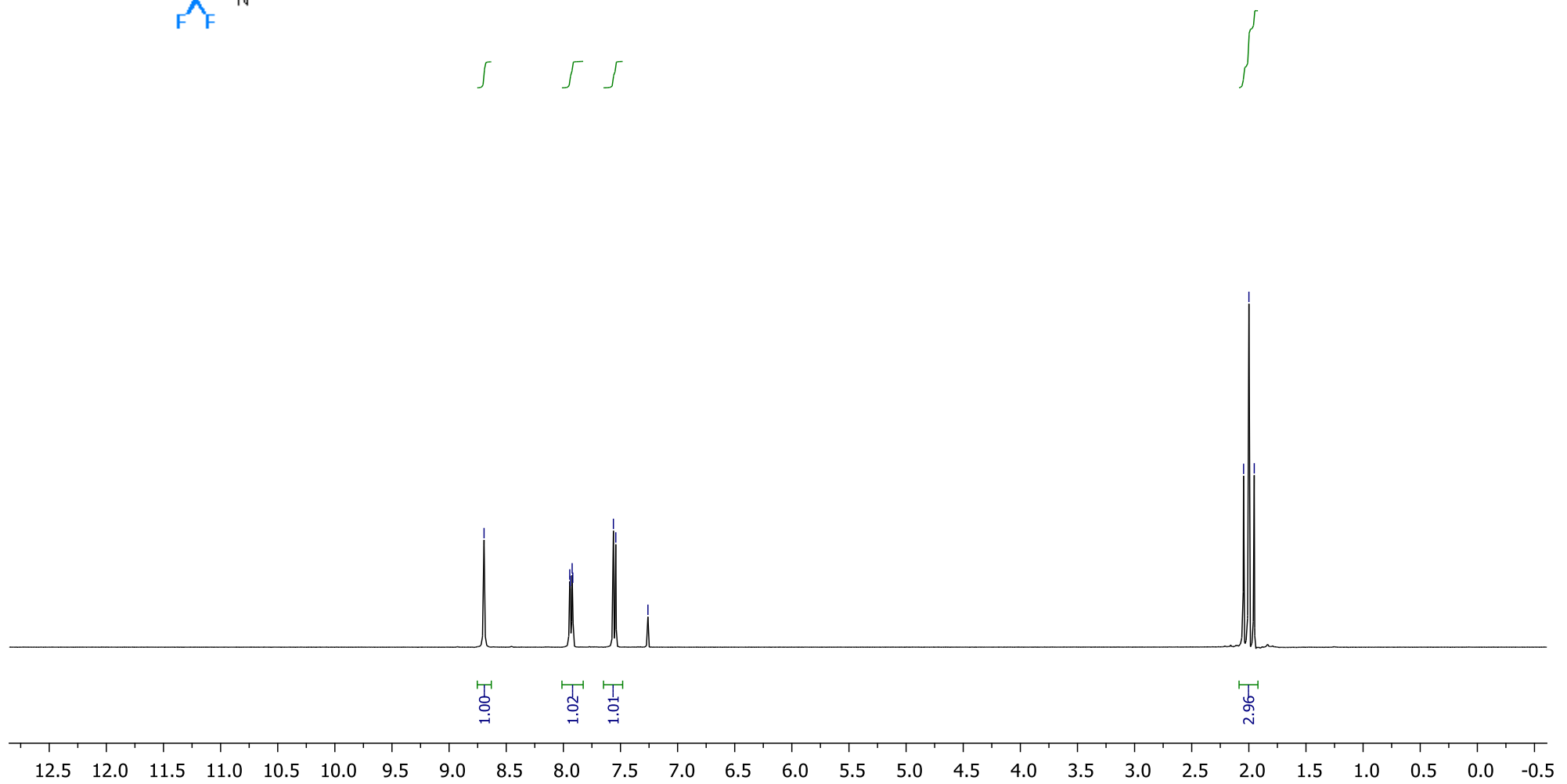
${ }^{13} \mathrm{C}\left\{{ }^{1} \mathrm{H}\right\} \mathrm{NMR}\left(126 \mathrm{MHz}, \mathrm{CDCl}_{3}\right)$

R1409962_C1:

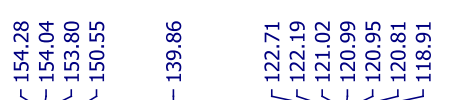

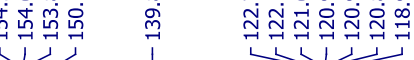

$\stackrel{0}{i}$

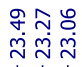

บูก

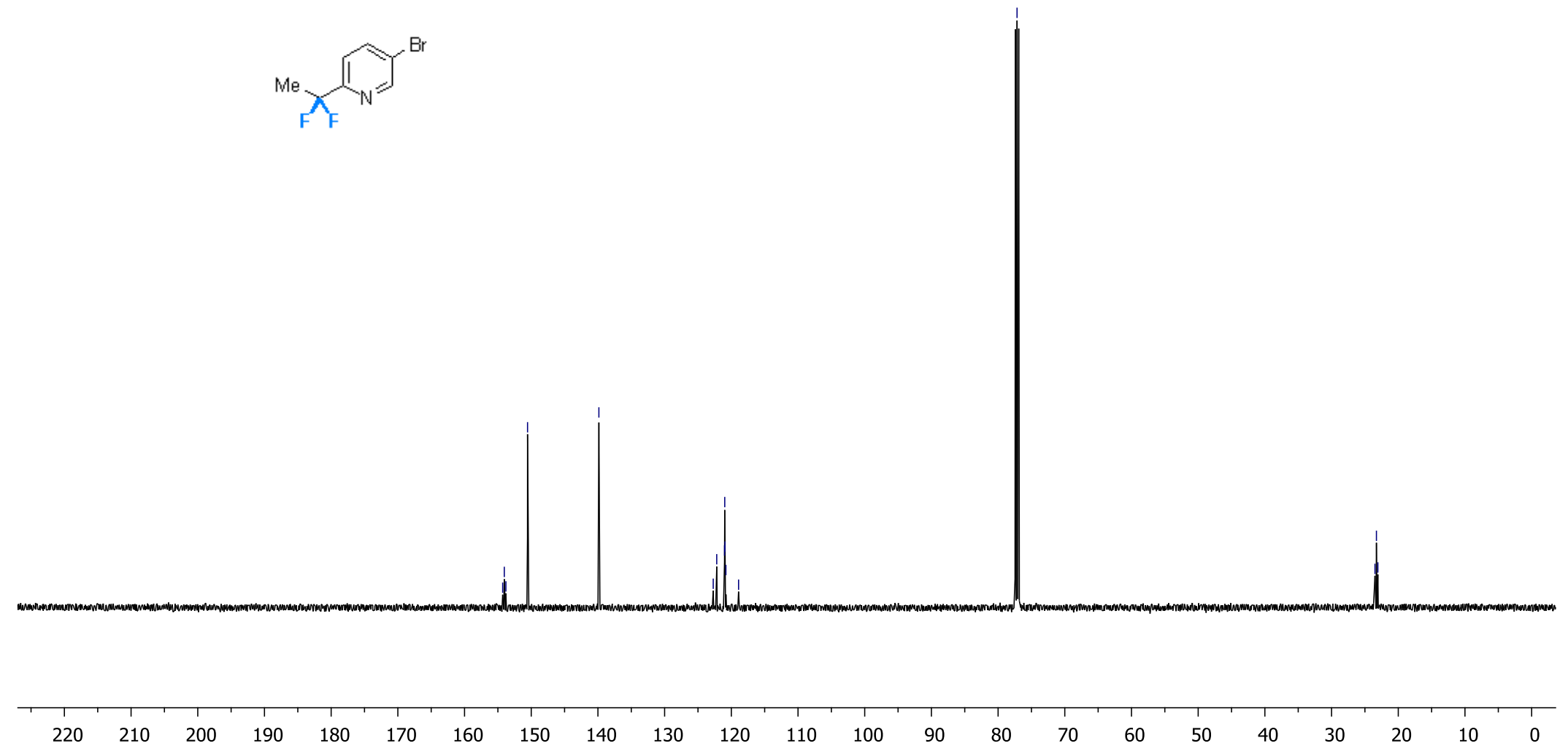




\section{${ }^{19} \mathrm{~F}\left\{{ }^{1} \mathrm{H}\right\}$ NMR (376 MHz, $\left.\mathrm{CDCl}_{3}\right)$}

R1409962_F19\{H\} $19 \mathrm{~F}-\{1 \mathrm{H}\}$
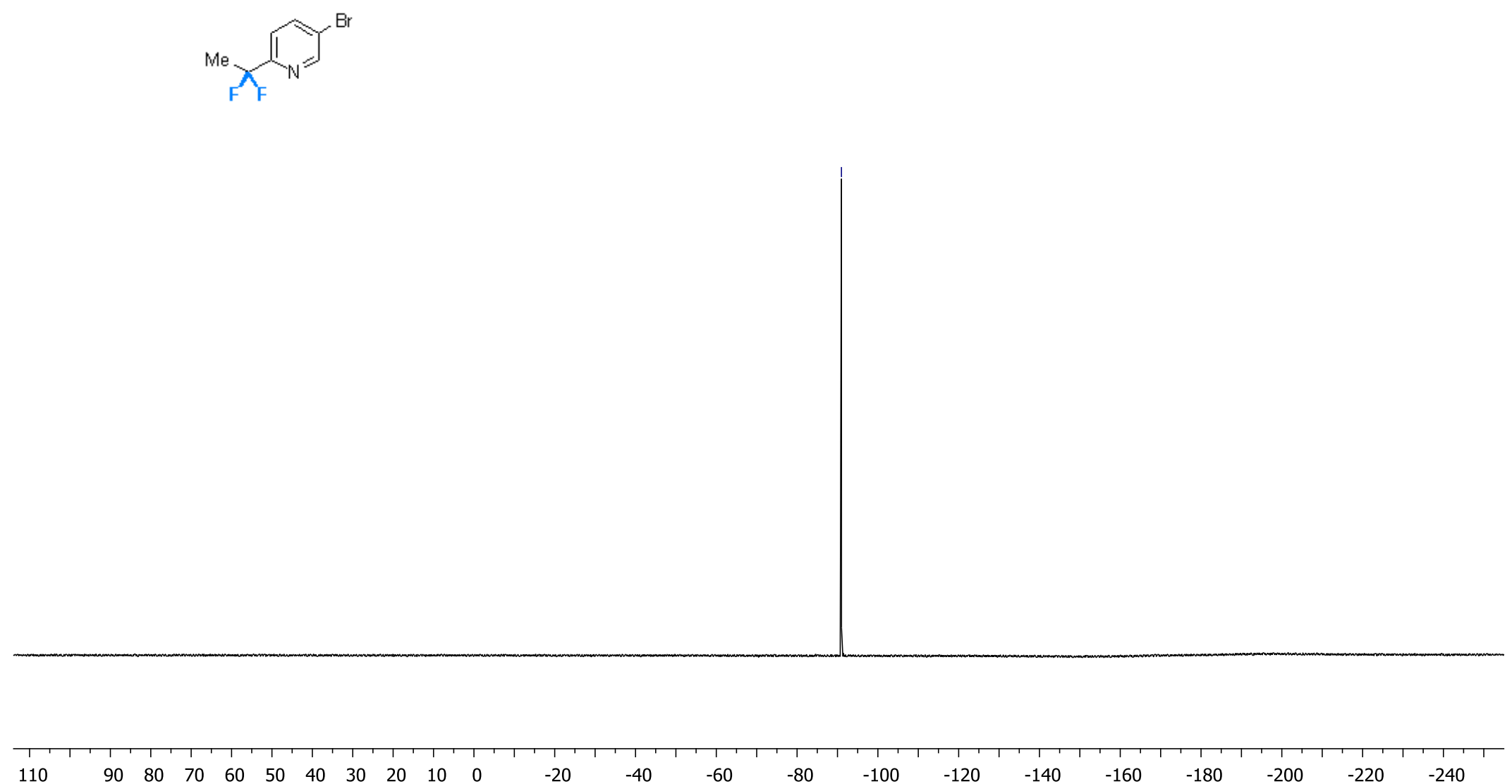


\section{Compound 54a}

${ }^{1} \mathrm{H}$ NMR (400 MHz, $\mathrm{CDCl}_{3}$ )

R2488908

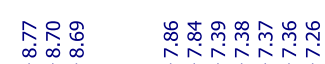

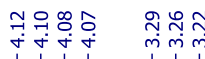

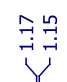

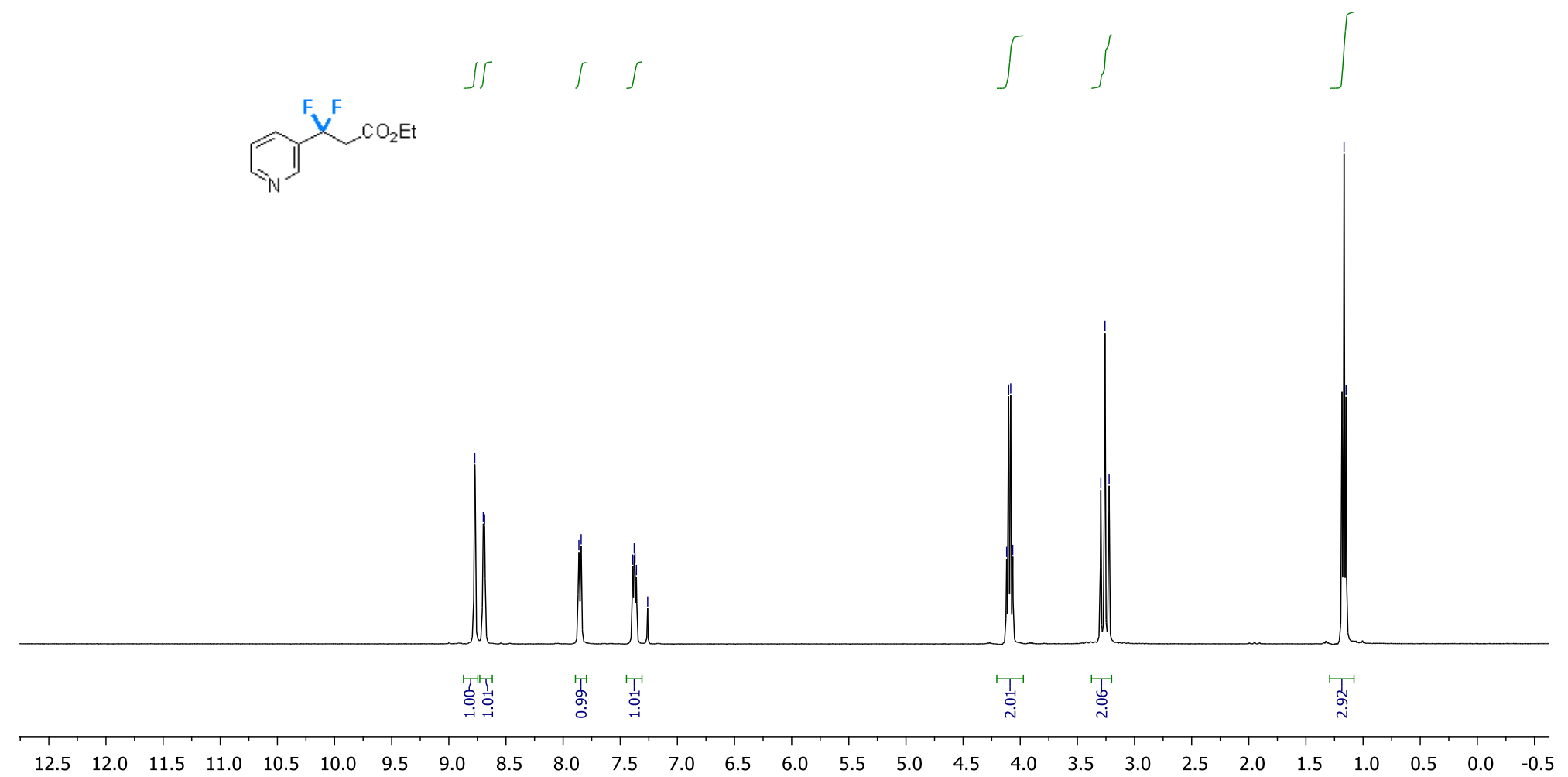


${ }^{13} \mathrm{C}\left\{{ }^{1} \mathrm{H}\right\}$ NMR $\left(126 \mathrm{MHz}, \mathrm{CDCl}_{3}\right)$

R2488908_C1:

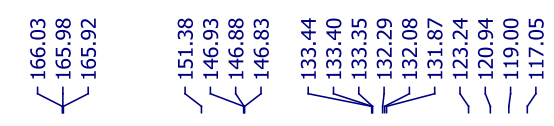

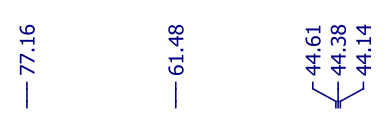

$\stackrel{\substack{0 \\ \dot{+}}}{\mid}$
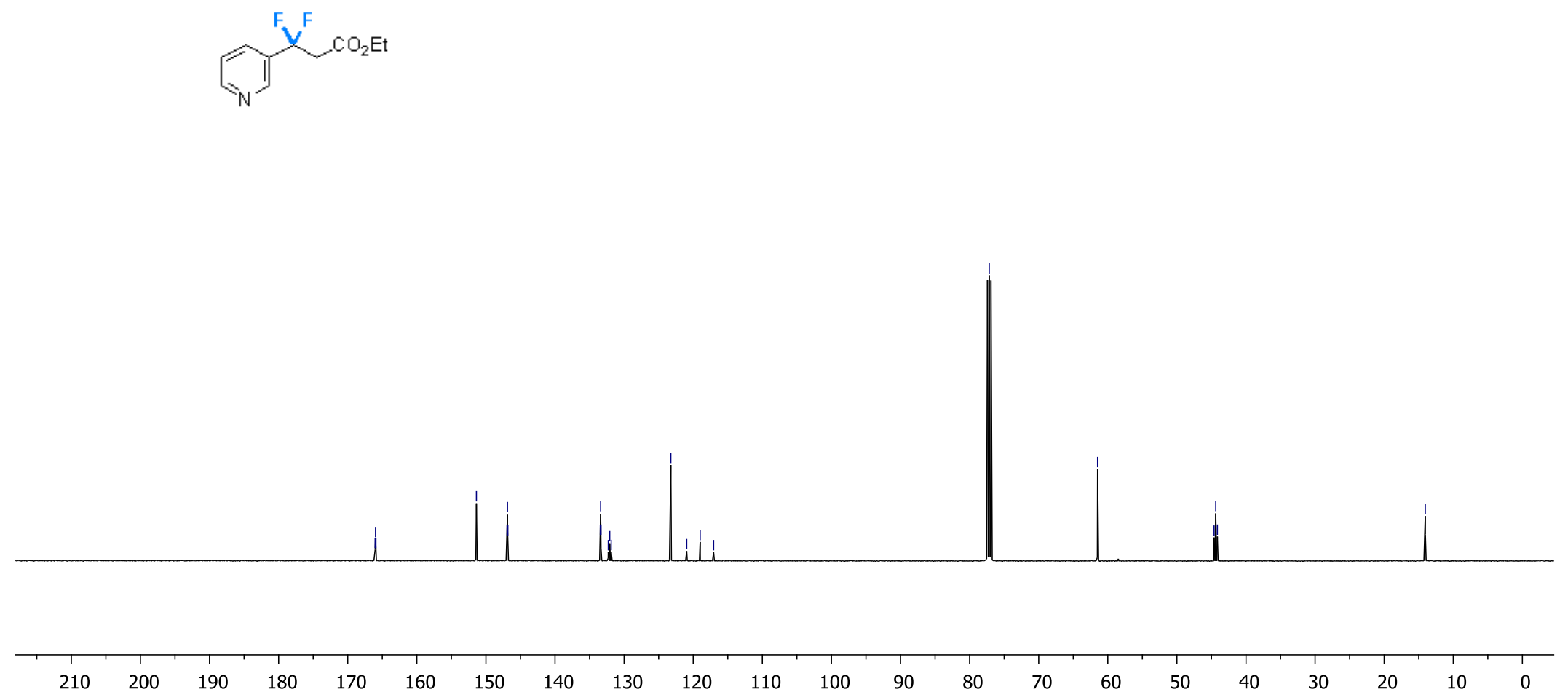

S138 
${ }^{19} \mathrm{~F}\left\{{ }^{1} \mathrm{H}\right\}$ NMR $\left(376 \mathrm{MHz}, \mathrm{CDCl}_{3}\right)$

R2488908_F19\{H\}

$19 \mathrm{~F}-\{1 \mathrm{H}\}$

i্ণ
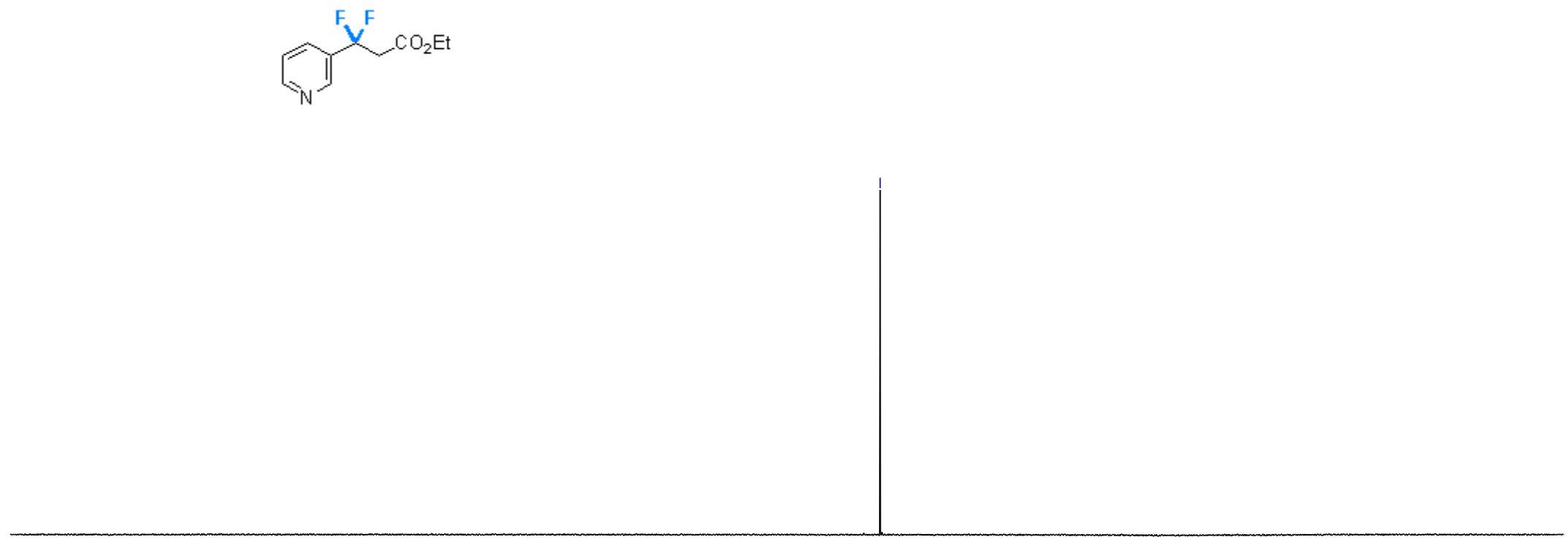

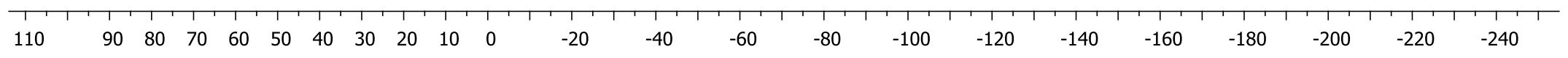

S139 


\section{Compound 55a}

${ }^{1} \mathrm{H}$ NMR (400 MHz, $\mathrm{CDCl}_{3}$ )

R591071

$\mid$

$\int_{F}^{N} x_{F}^{M e}$
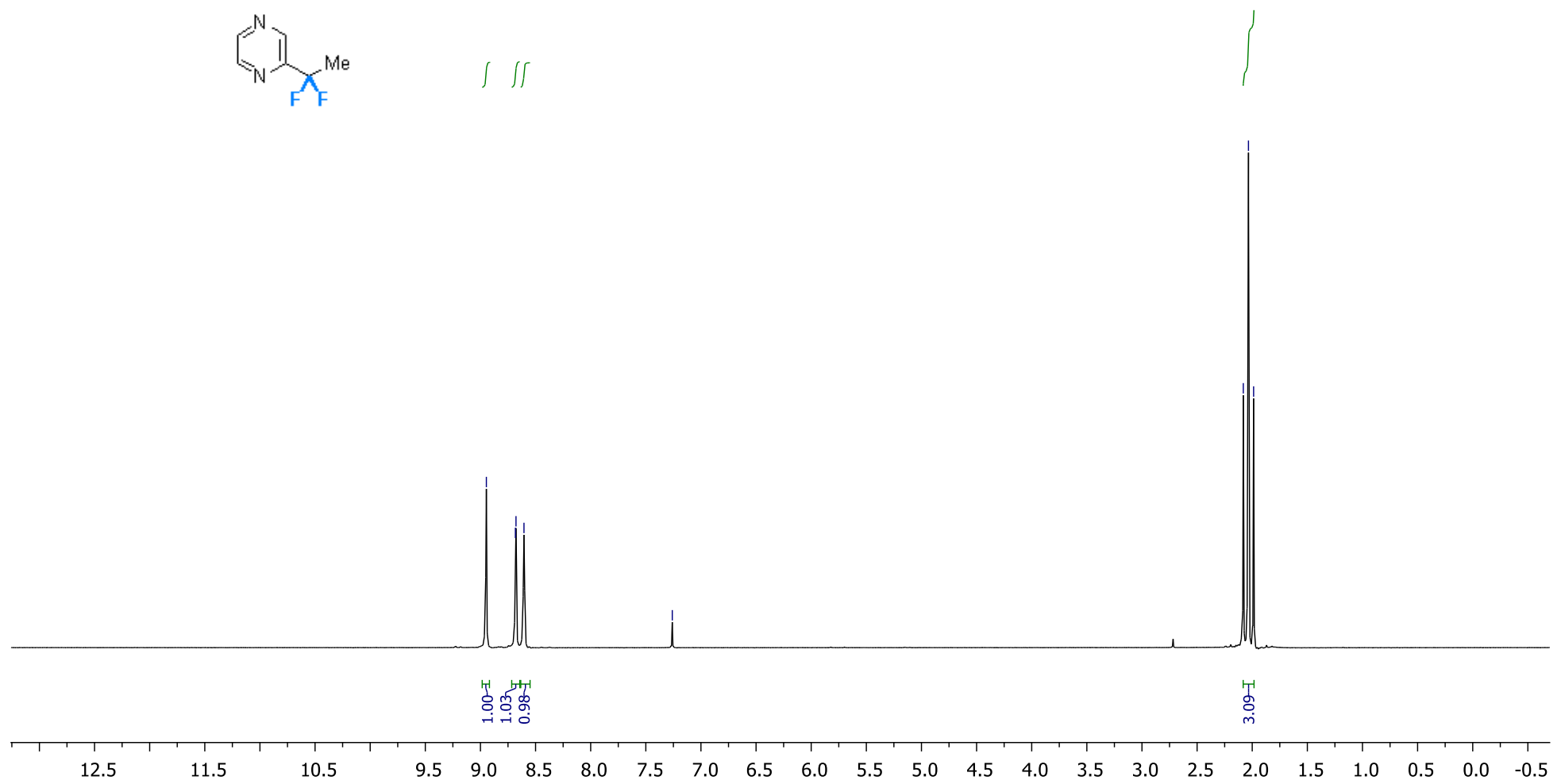
${ }^{13} \mathrm{C}\left\{{ }^{1} \mathrm{H}\right\}$ NMR $\left(126 \mathrm{MHz}, \mathrm{CDCl}_{3}\right)$

R591071_C1:

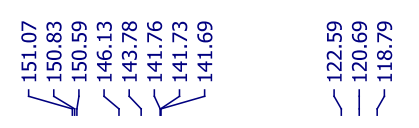

$\stackrel{\substack{i \\ i}}{i}$

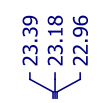
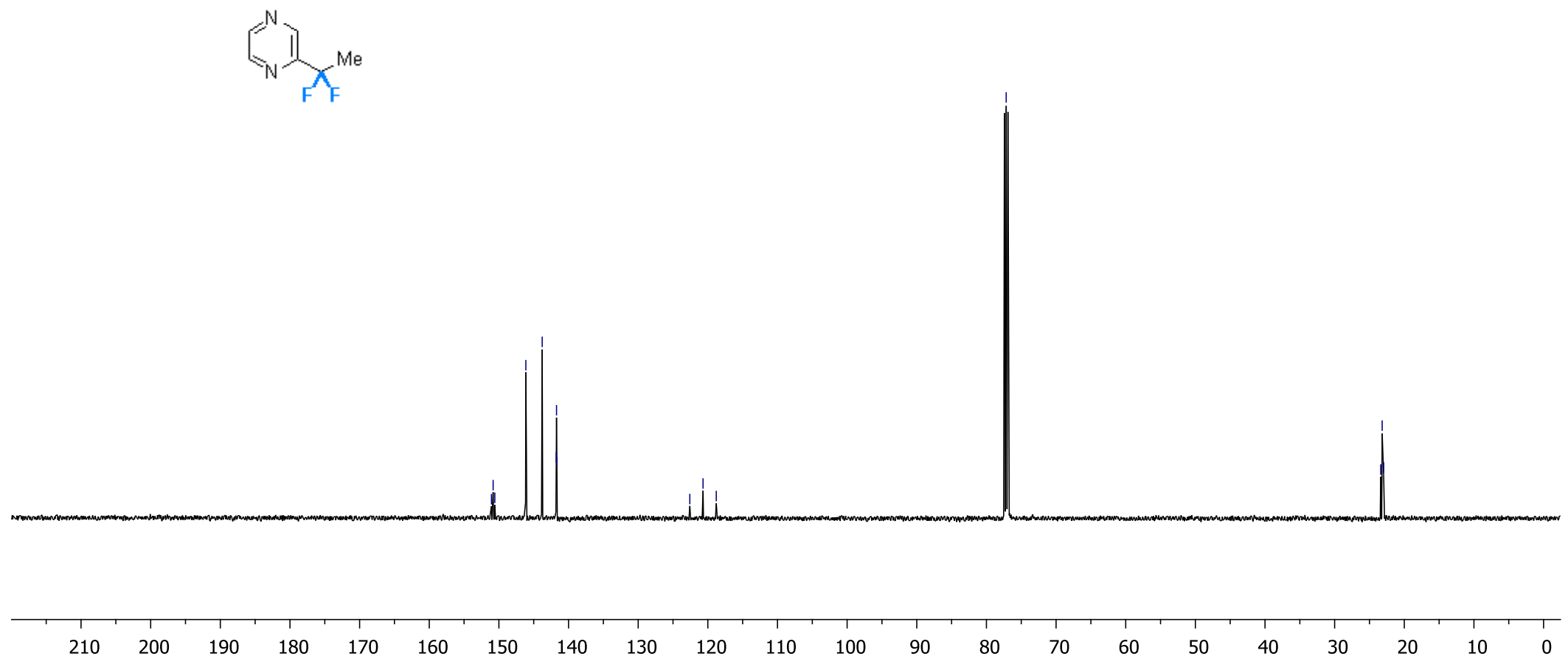

S141 
${ }^{19} \mathrm{~F}\left\{{ }^{1} \mathrm{H}\right\}$ NMR (376 MHz, $\left.\mathrm{CDCl}_{3}\right)$

R591071_F19\{H\}

$19 \mathrm{~F}-\{1 \mathrm{H}\}$

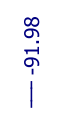
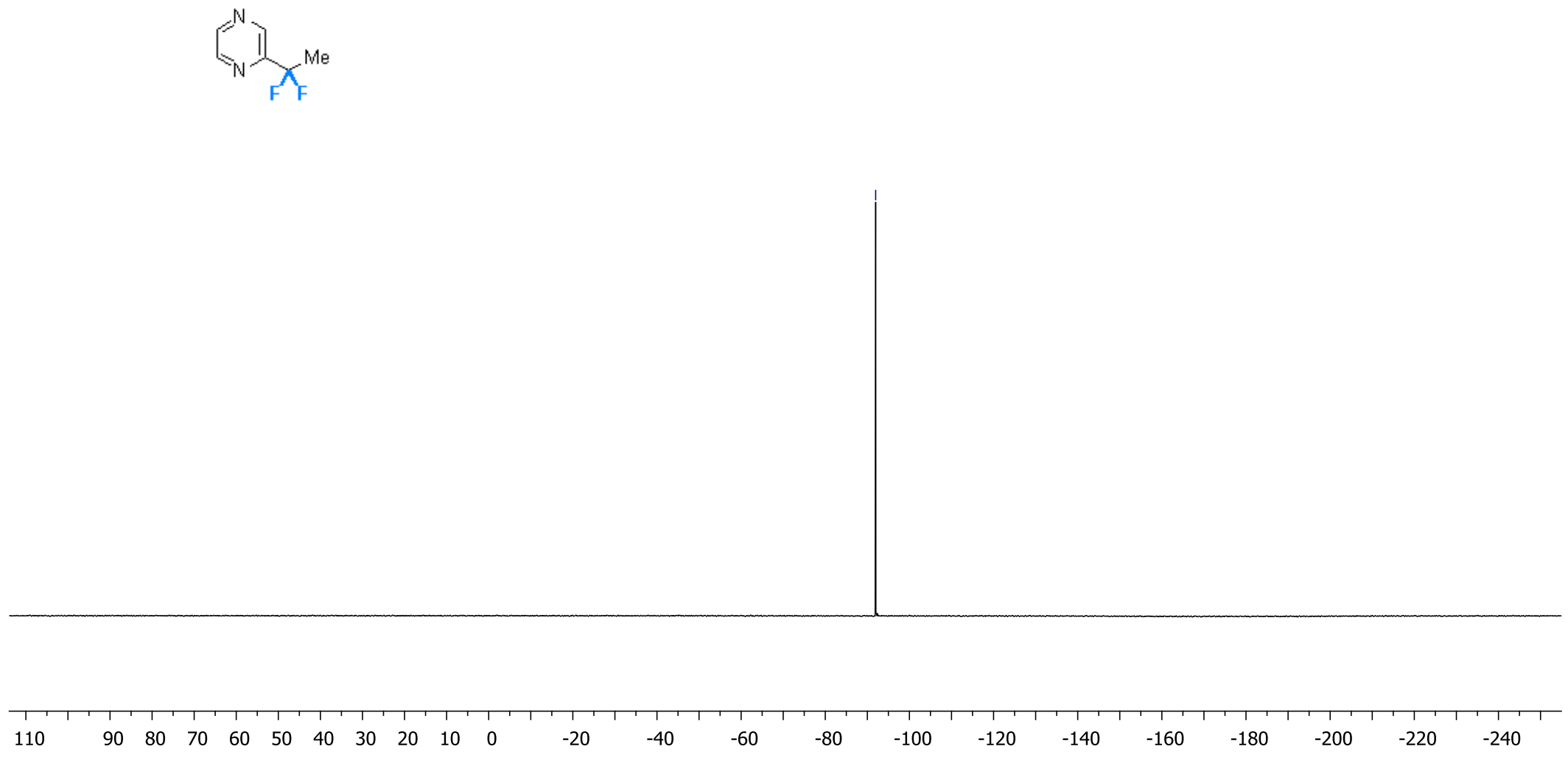

S142 


\section{Compound 56a}

${ }^{1} \mathrm{H}$ NMR (400 MHz, $\mathrm{CDCl}_{3}$ )

R1614690

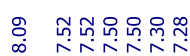
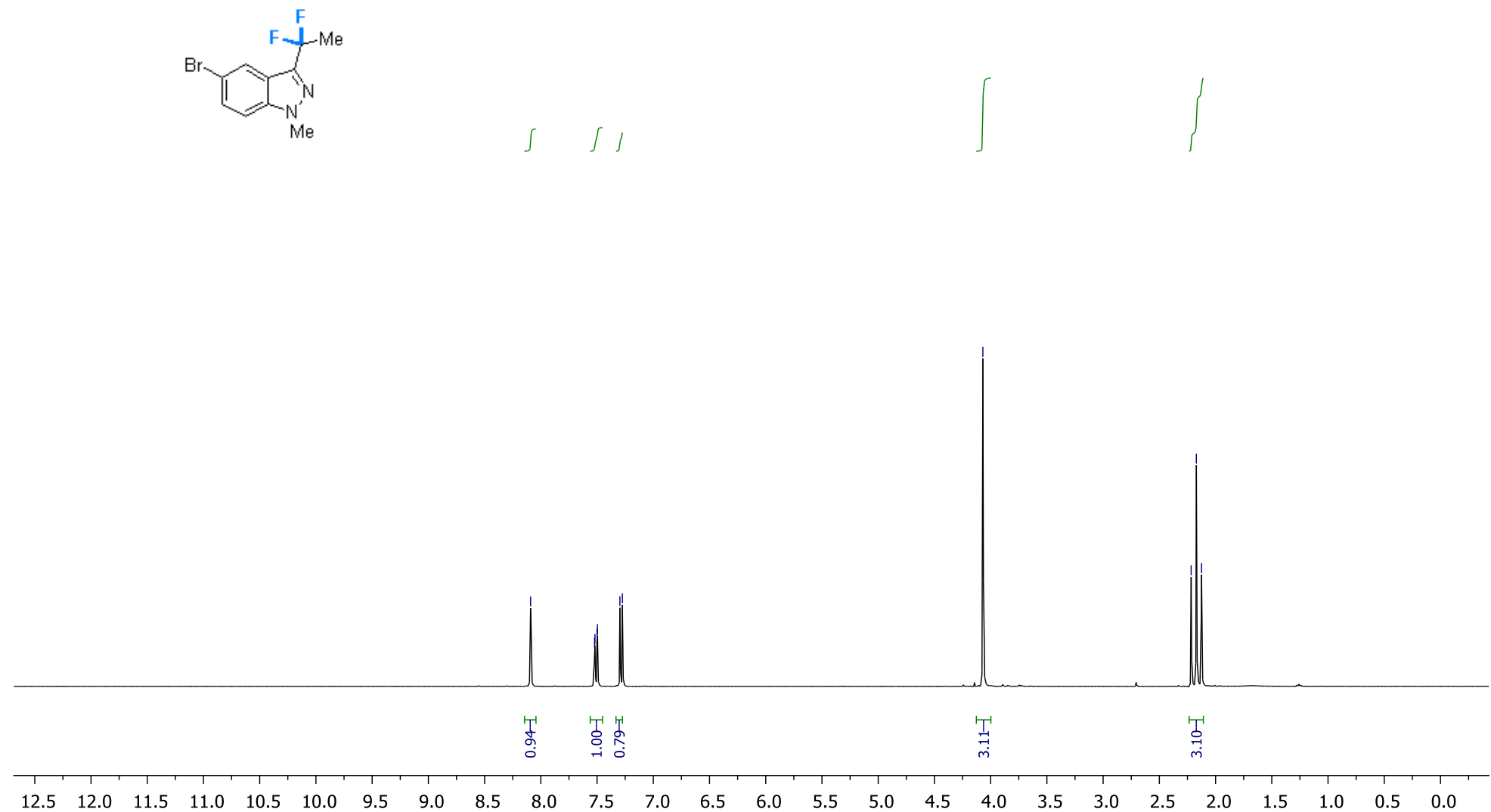
${ }^{13} \mathrm{C}\left\{{ }^{1} \mathrm{H}\right\}$ NMR (151 MHz, $\left.\mathrm{CDCl}_{3}\right)$

R1614690_C13
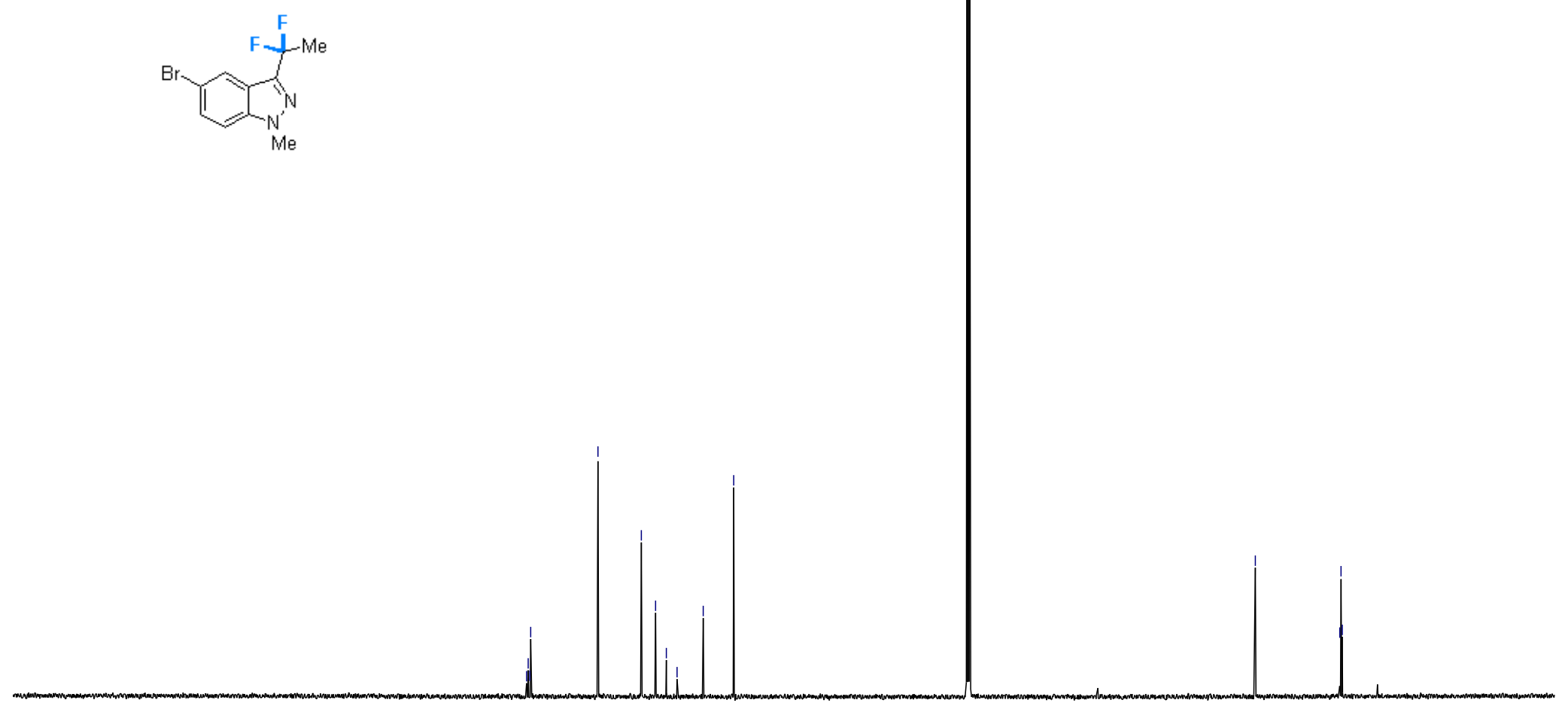

2

$\begin{array}{lllll}210 & 200 & 190 & 180 & 170\end{array}$

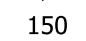

140

$130 \quad 120$

$110 \quad 100$

90
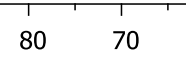

$60 \quad 50$

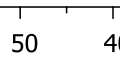

30 


\section{${ }^{19} \mathrm{~F}\left\{{ }^{1} \mathrm{H}\right\}$ NMR (376 MHz, $\left.\mathrm{CDCl}_{3}\right)$}

R1614690_F19 $\{\mathrm{H}\}$

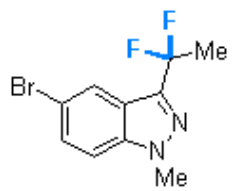




\section{Compound 57a}

${ }^{1} \mathrm{H}$ NMR (400 MHz, $\mathrm{CDCl}_{3}$ )

R2437746

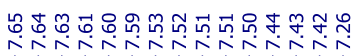

$\overbrace{\mathrm{F} F} \overbrace{\mathrm{F}}$
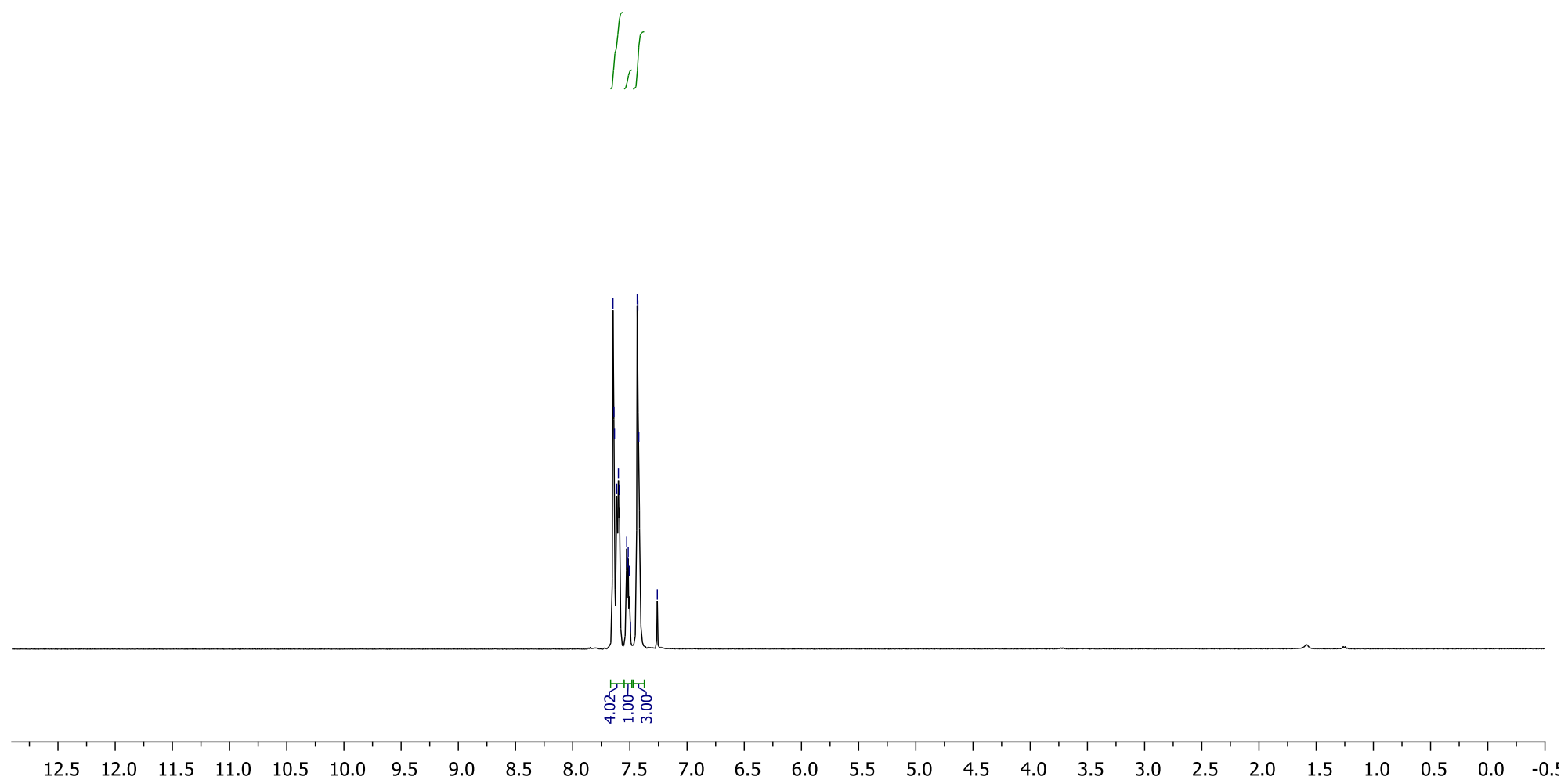
${ }^{13} \mathrm{C}\left\{{ }^{1} \mathrm{H}\right\}$ NMR $\left(126 \mathrm{MHz}, \mathrm{CDCl}_{3}\right)$

R2437746_C1:

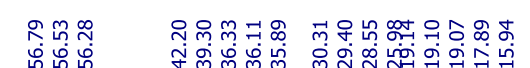

,

$\underset{F}{K_{F}}$

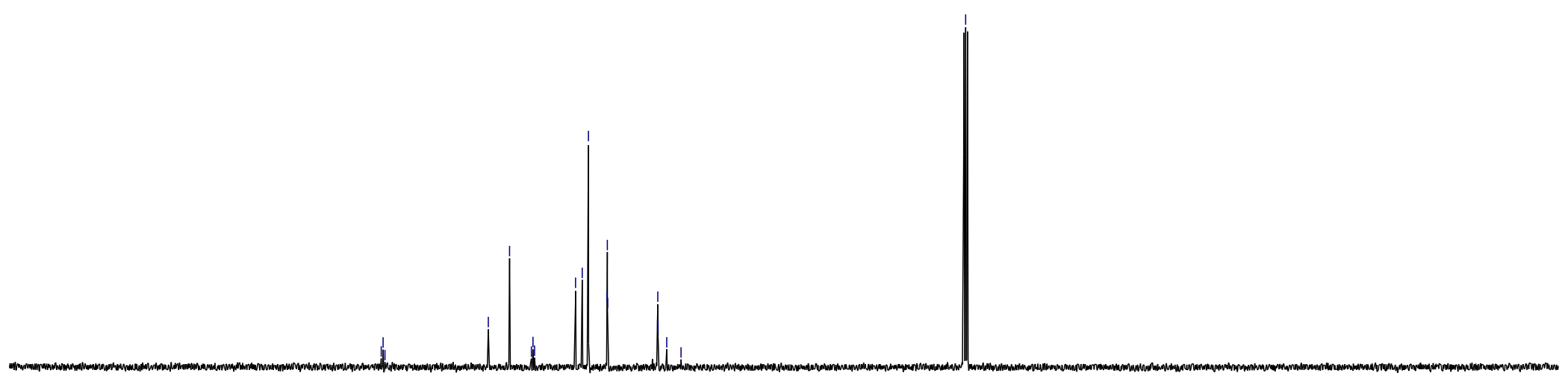


${ }^{19} \mathrm{~F}\left\{{ }^{1} \mathrm{H}\right\}$ NMR (376 MHz, $\left.\mathrm{CDCl}_{3}\right)$

R2437746_F19 $\{\mathrm{H}\}$

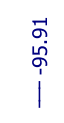
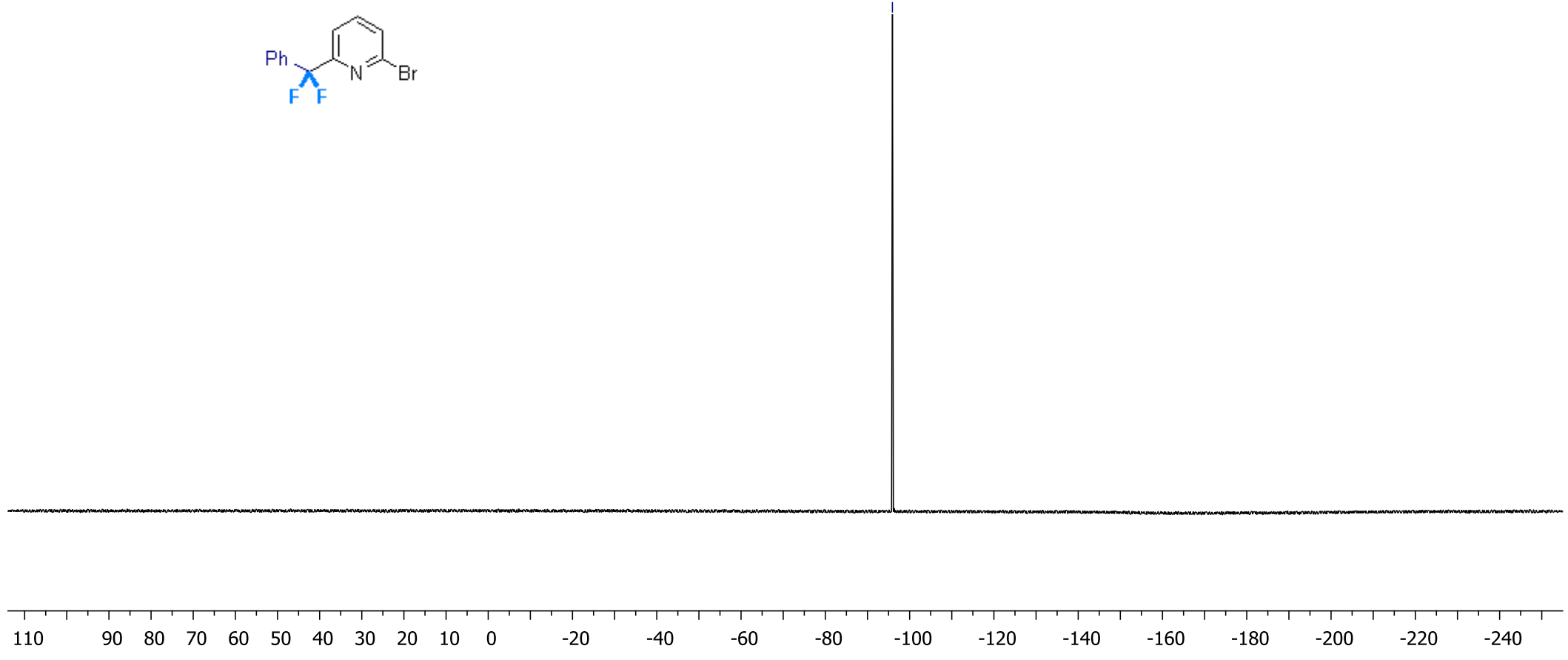

S148 


\section{Compound 58a}

${ }^{1} \mathrm{H}$ NMR (400 MHz, $\mathrm{CDCl}_{3}$ )

R1738922

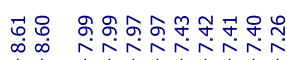

$\sqrt{n}$

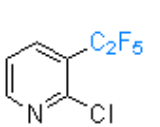

$\iint$

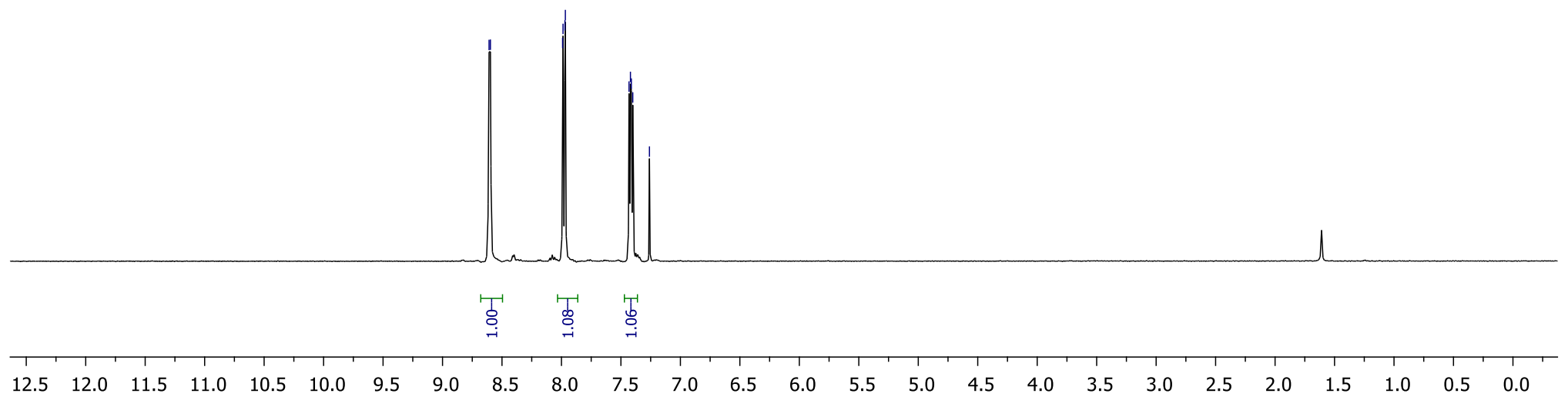


${ }^{13} \mathrm{C}\left\{{ }^{1} \mathrm{H}\right\}$ NMR $\left(151 \mathrm{MHz}, \mathrm{CDCl}_{3}\right)$

R1738922_13C

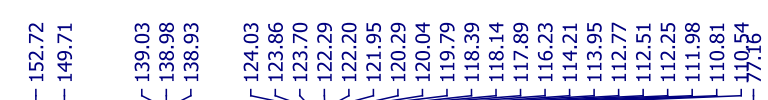

।

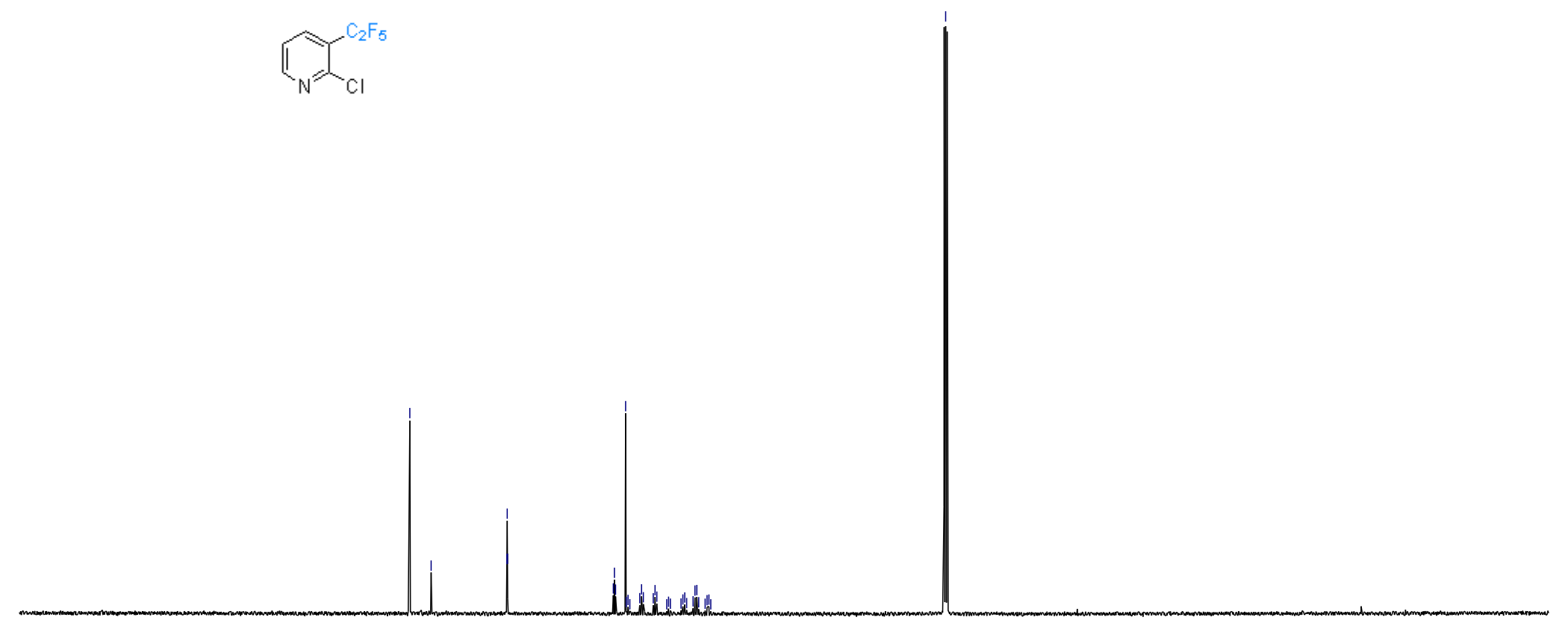




\section{${ }^{19} \mathrm{~F}\left\{{ }^{1} \mathrm{H}\right\}$ NMR (376 MHz, $\left.\mathrm{CDCl}_{3}\right)$}

R1738922_F19 $\{H\}$
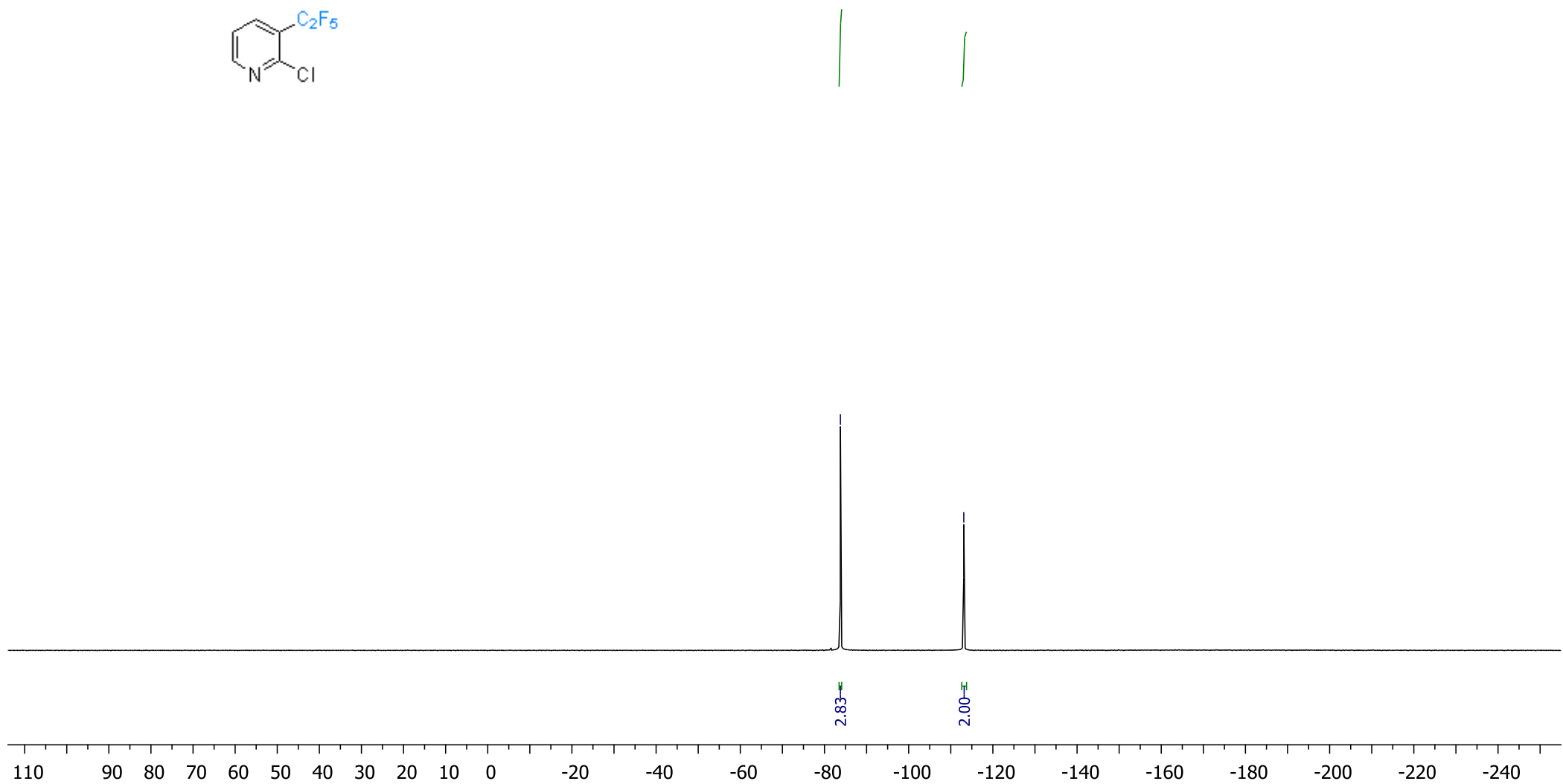


\section{Compound 59a}

${ }^{1} \mathrm{H}$ NMR (400 MHz, $\mathrm{CDCl}_{3}$ )

R1701976

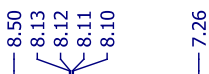
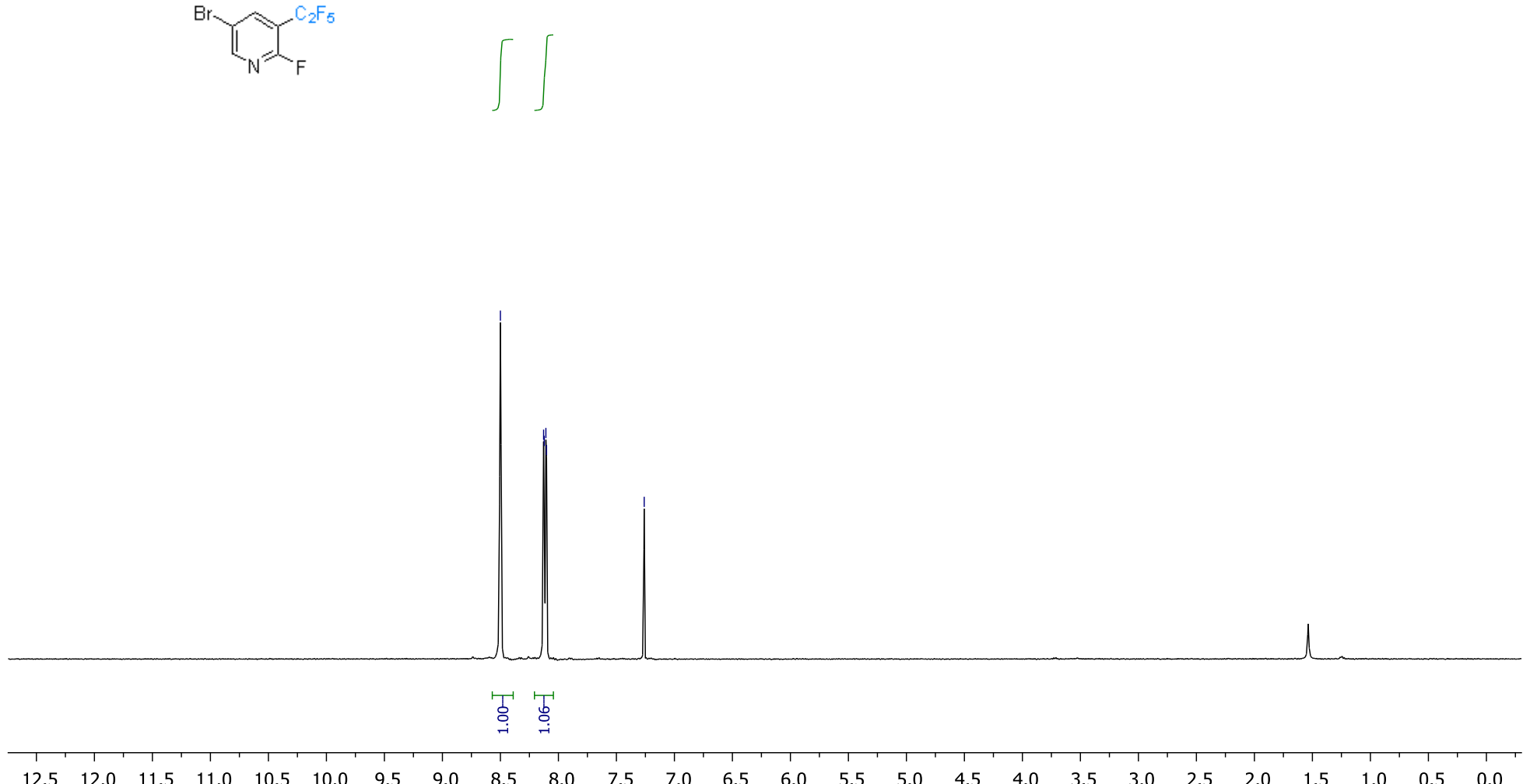
${ }^{13} \mathrm{C}\left\{{ }^{1} \mathrm{H}\right\} \mathrm{NMR}\left(151 \mathrm{MHz}, \mathrm{CDCl}_{3}\right)$

R1701976_C13

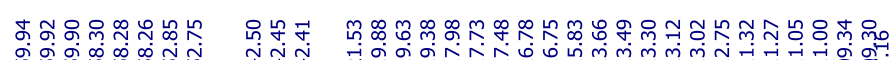

S.

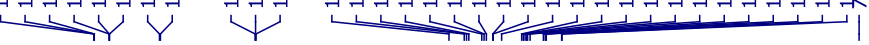

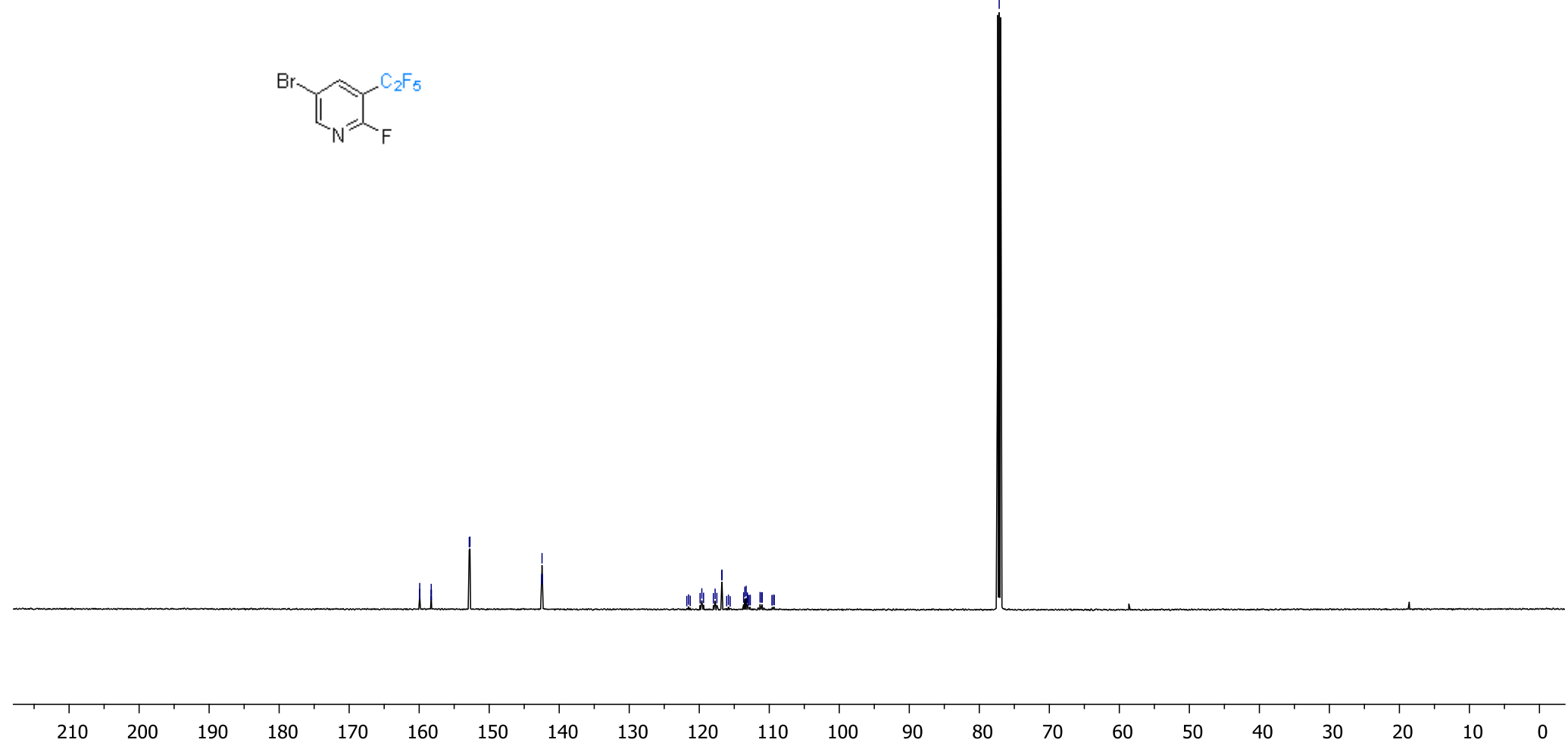




\section{${ }^{19} \mathrm{~F}\left\{{ }^{1} \mathrm{H}\right\} \mathrm{NMR}\left(376 \mathrm{MHz}, \mathrm{CDCl}_{3}\right)$}

R1701976_F19\{H\}
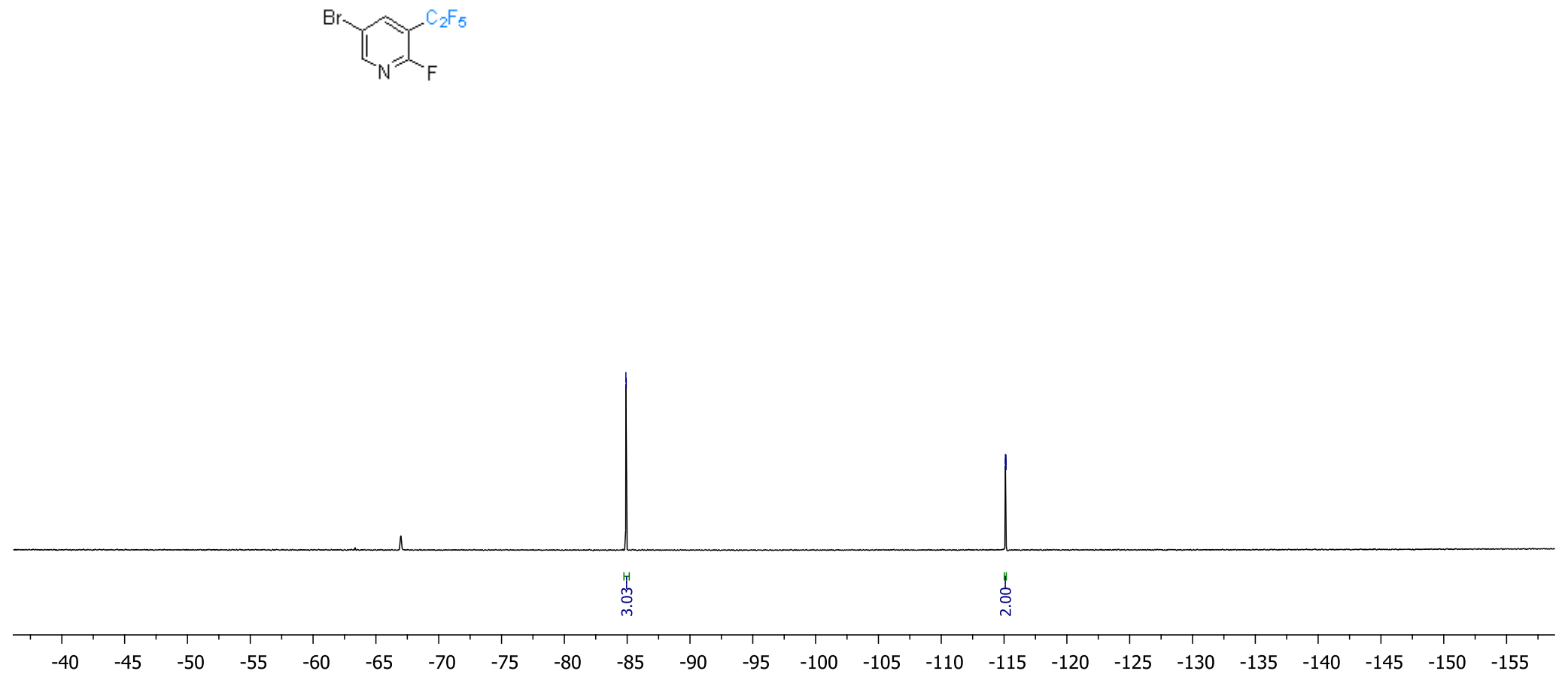


\section{Compound 60a}

${ }^{1} \mathrm{H}$ NMR (400 MHz, $\mathrm{CDCl}_{3}$ )

R1466960

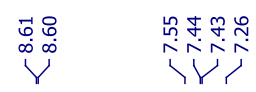

Cl
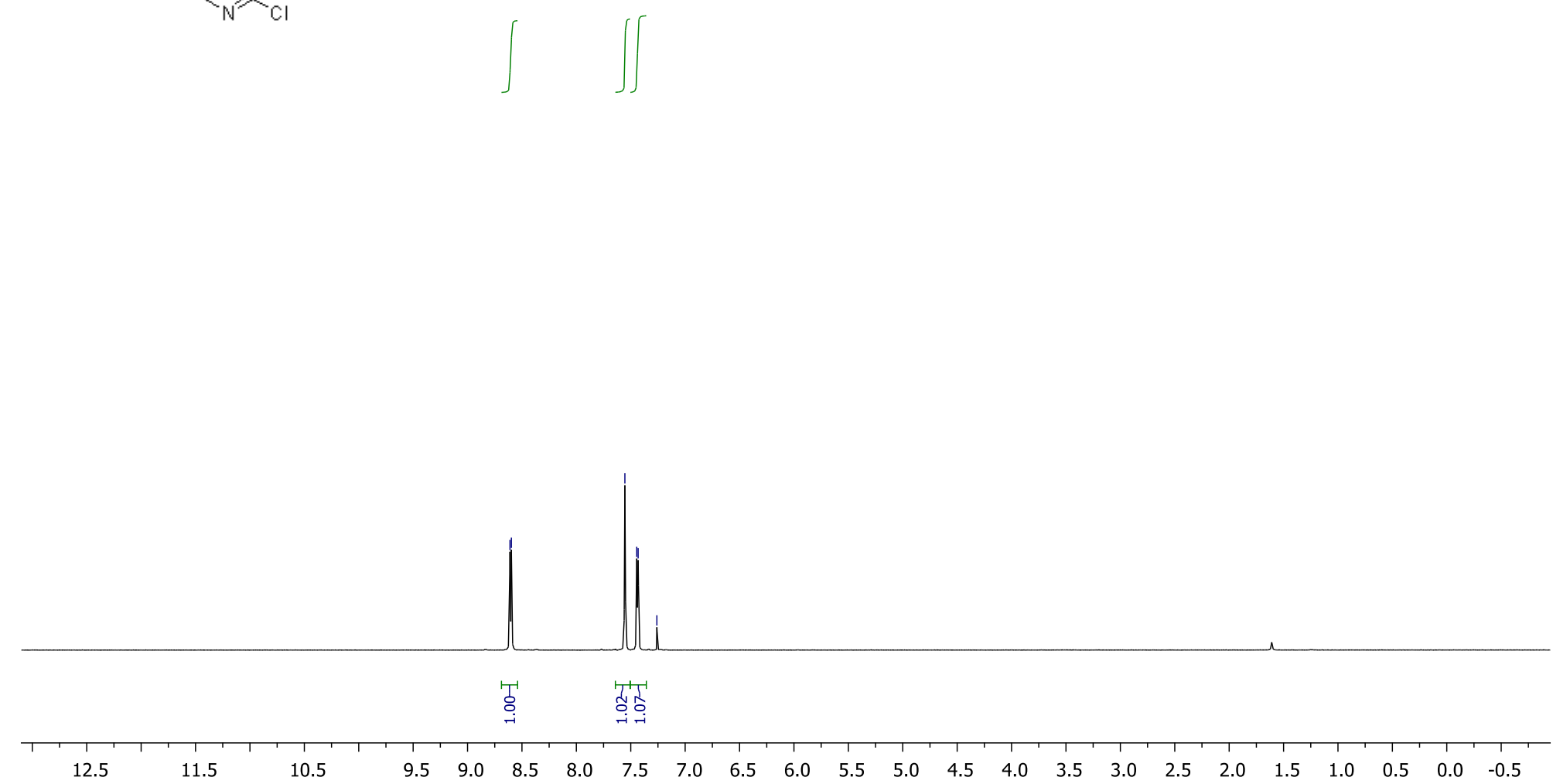
${ }^{13} \mathrm{C}\left\{{ }^{1} \mathrm{H}\right\}$ NMR (151 MHz, $\left.\mathrm{CDCl}_{3}\right)$

R1466960_13C

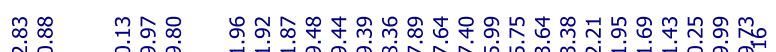

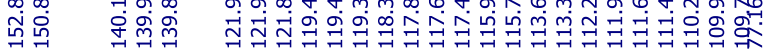

i)

Cl

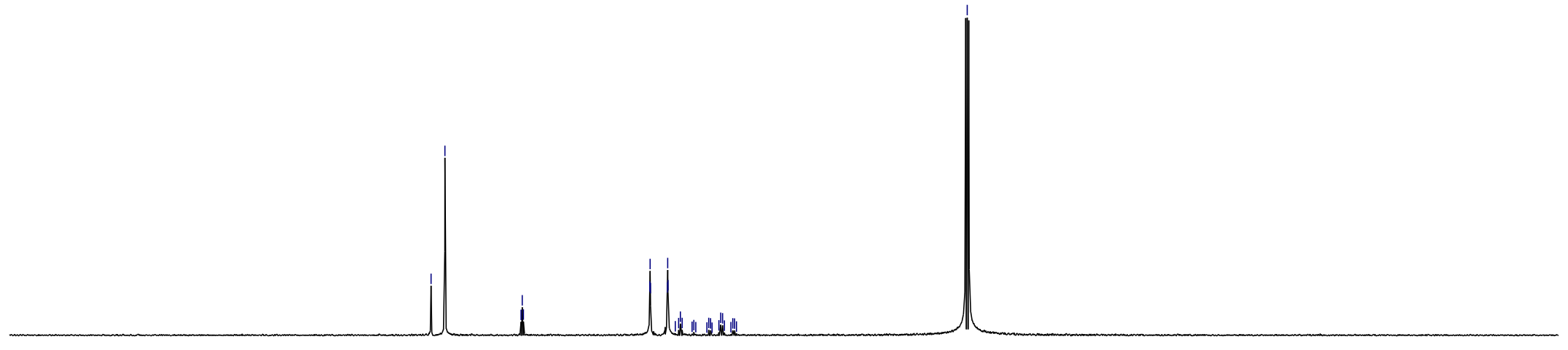

$210 \quad 200 \quad 190 \quad 180$

$180 \quad 170$

$160 \quad 150$

$140 \quad 130$

$120 \quad 110$

100

80

$70 \quad 60$

50

40

$30 \quad 20$

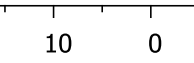


${ }^{19} \mathrm{~F}\left\{{ }^{1} \mathrm{H}\right\}$ NMR (376 MHz, $\left.\mathrm{CDCl}_{3}\right)$

R1466960_F19 $\{\mathrm{H}\}$

$19 \mathrm{~F}-\{1 \mathrm{H}\}$

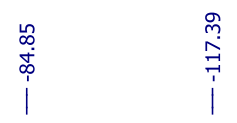

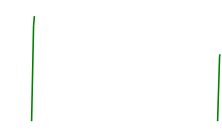

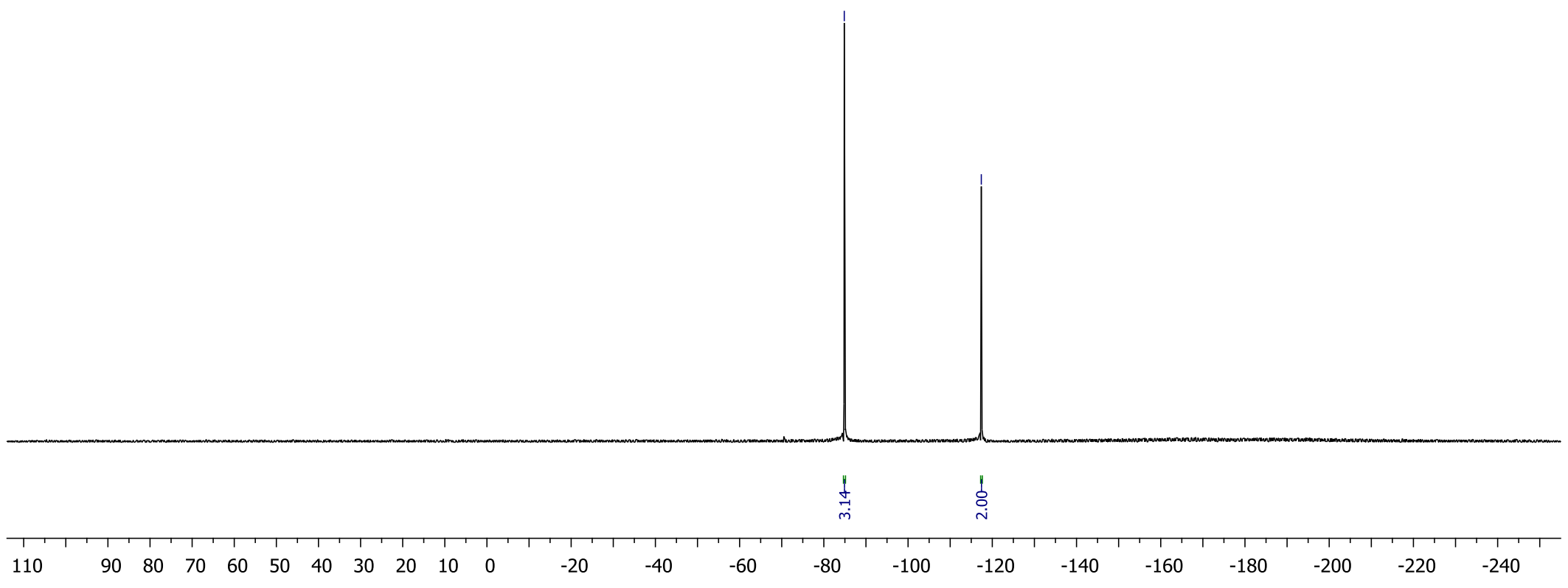

S157 


\section{Compound 61a}

${ }^{1} \mathrm{H}$ NMR (400 MHz, $\mathrm{CDCl}_{3}$ )

R1436539

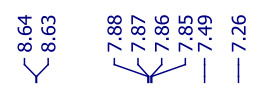

$\overbrace{\mathrm{N}}^{\mathrm{C}_{2} \mathrm{~F}_{5}}$
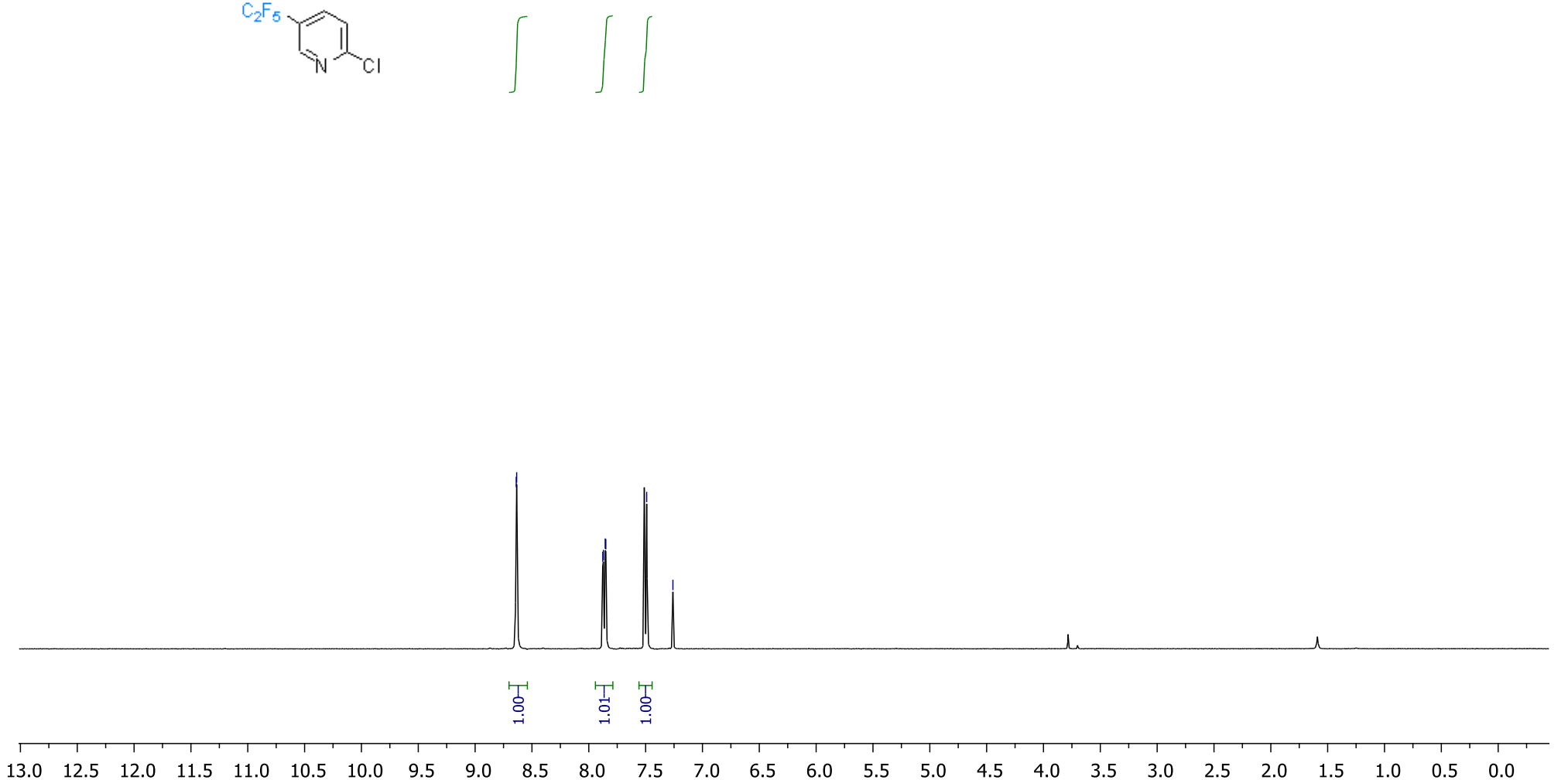
${ }^{13} \mathrm{C}\left\{{ }^{1} \mathrm{H}\right\}$ NMR $\left(151 \mathrm{MHz}, \mathrm{CDCl}_{3}\right)$

R1436539_13C

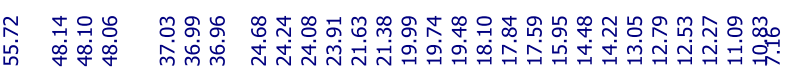

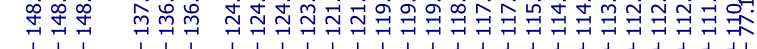

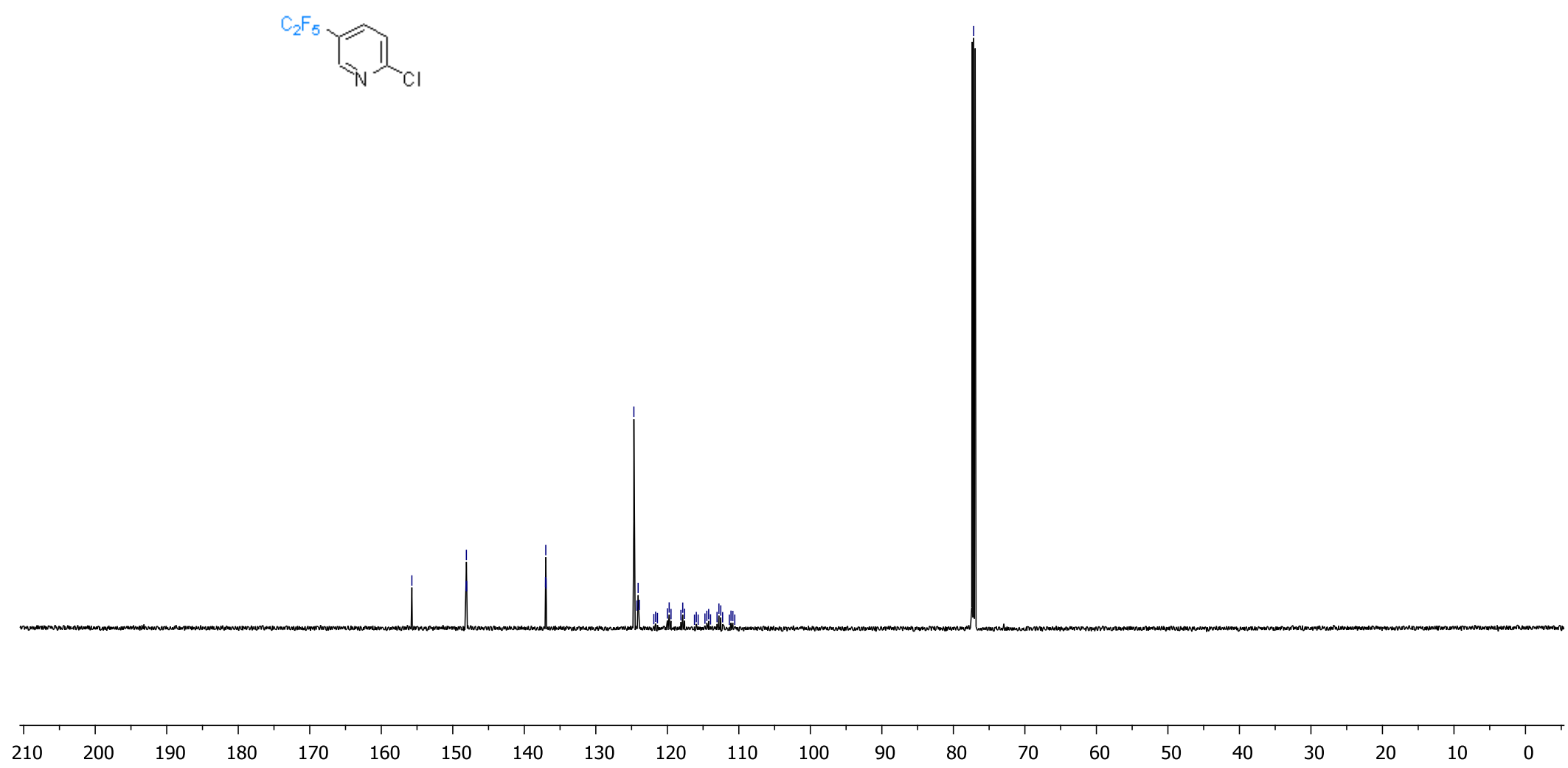


${ }^{19} \mathrm{~F}\left\{{ }^{1} \mathrm{H}\right\} \mathrm{NMR}\left(376 \mathrm{MHz}, \mathrm{CDCl}_{3}\right)$

R1436539_F19 $\{\mathrm{H}\}$

$19 \mathrm{~F}-\{1 \mathrm{H}\}$

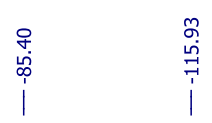

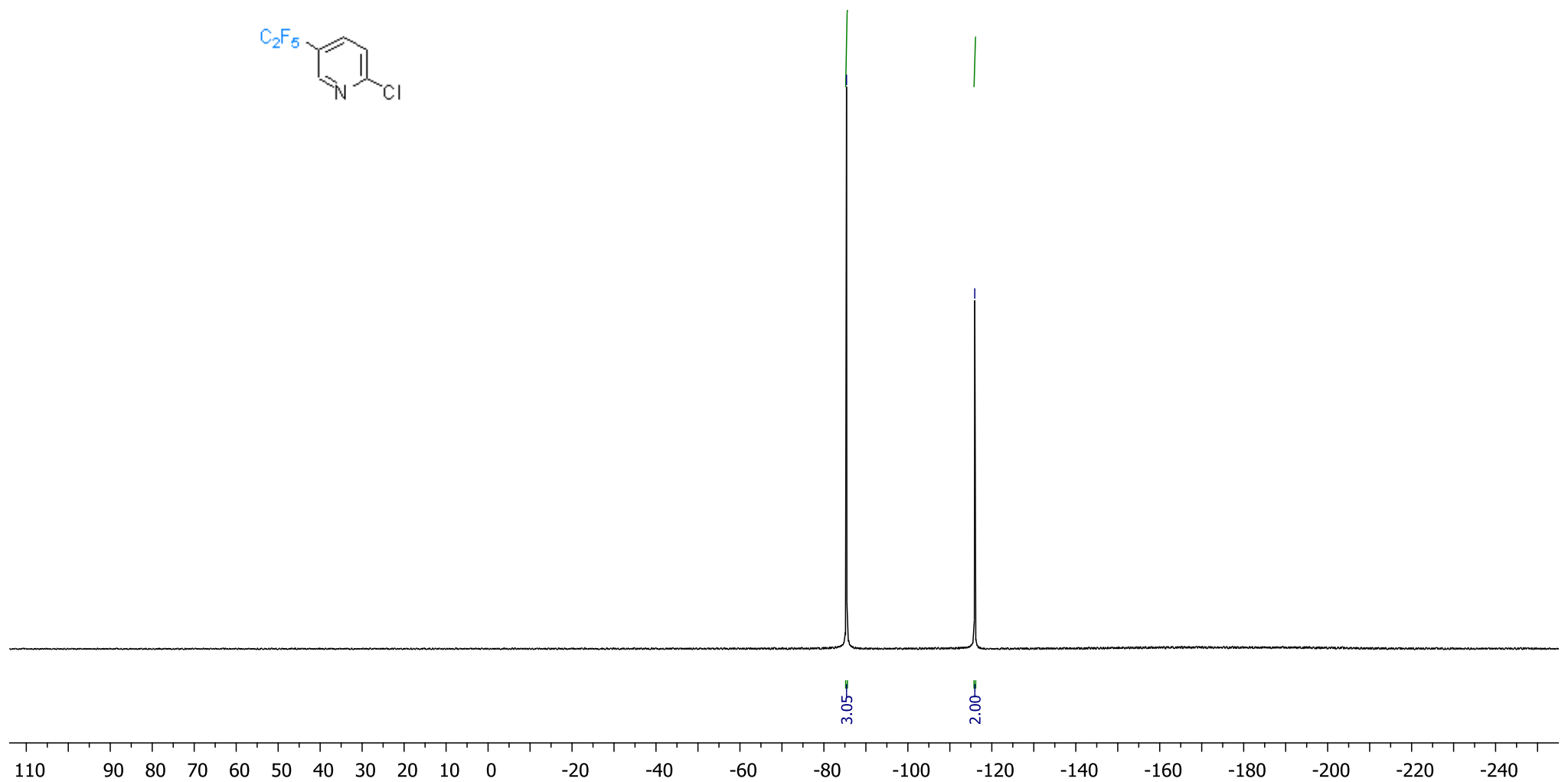




\section{Compound 62a}

${ }^{1} \mathrm{H}$ NMR $\left(400 \mathrm{MHz}, \mathrm{CDCl}_{3}\right.$ )

R1886406

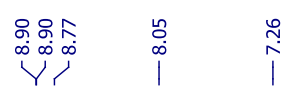

$\mathrm{C}_{\mathrm{N}}^{\mathrm{Br}}$
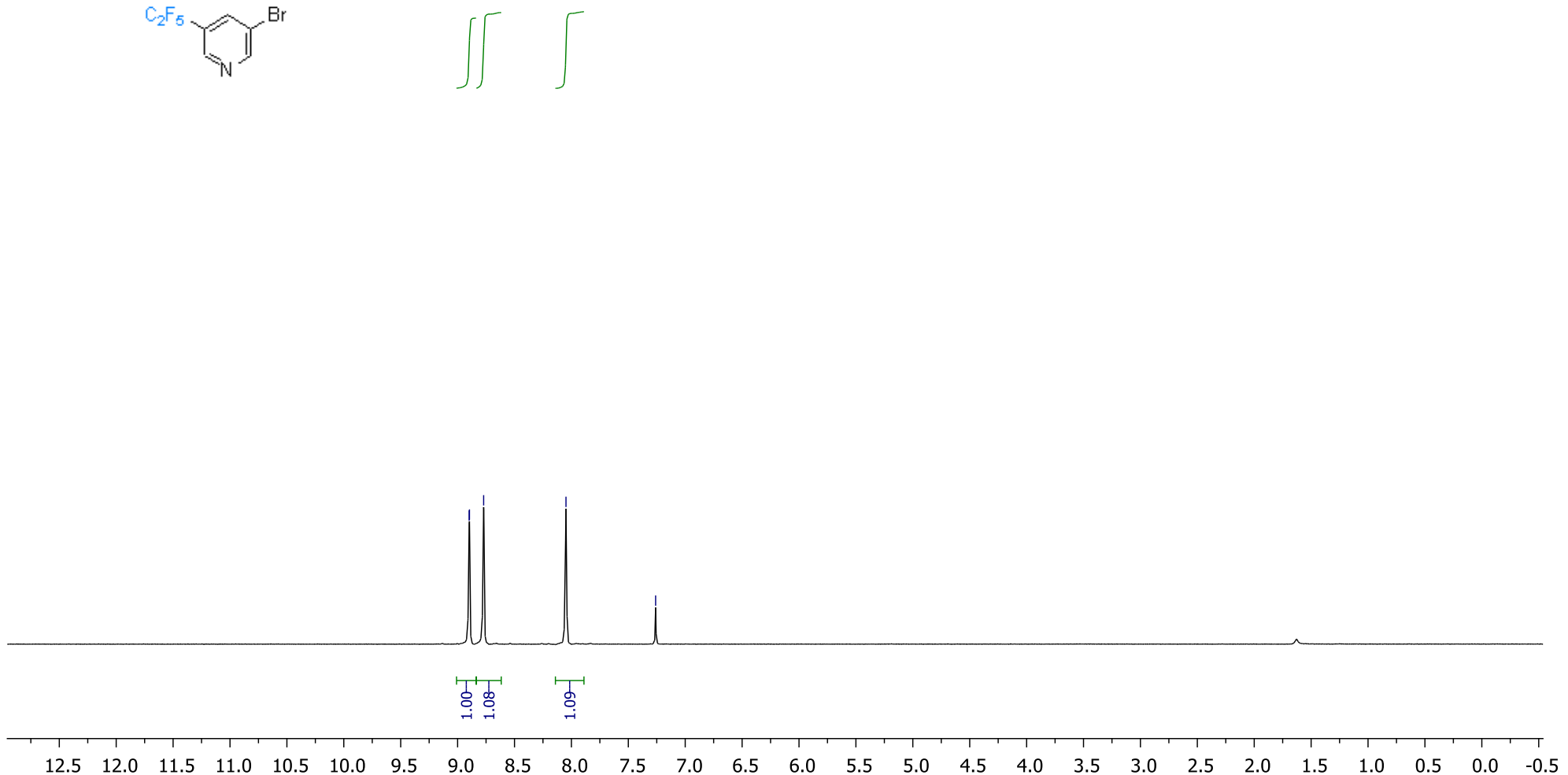
${ }^{13} \mathrm{C}\left\{{ }^{1} \mathrm{H}\right\}$ NMR $\left(126 \mathrm{MHz}, \mathrm{CDCl}_{3}\right)$

R1886406 $13 \mathrm{C}$

$13 \mathrm{C}(1 \mathrm{H}$-decoupled $)$

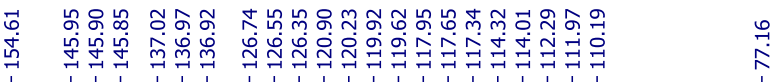<smiles>FC(F)(F)c1cncc(Br)c1</smiles>
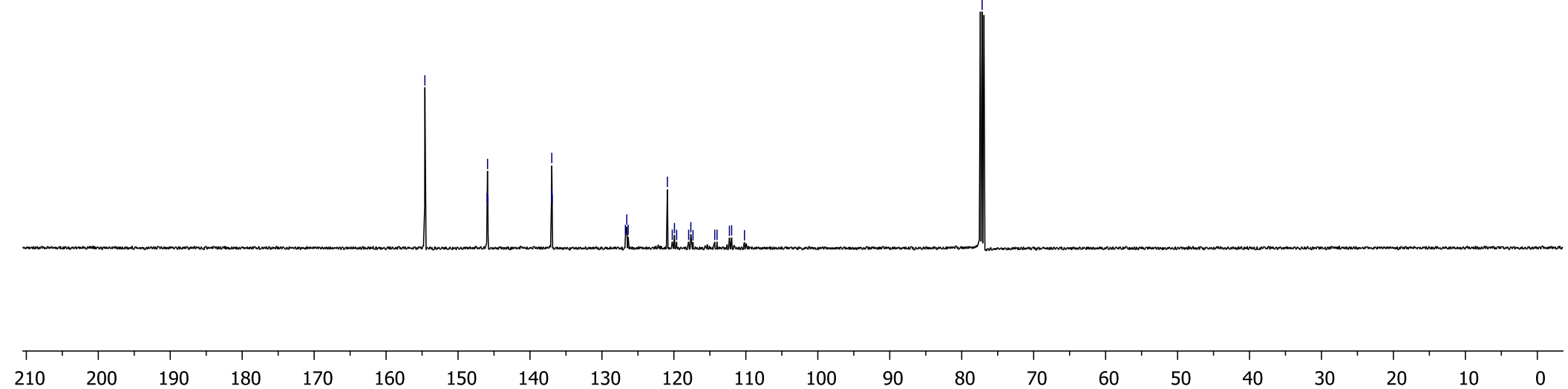

$\begin{array}{lllllllllllllllllllllll}210 & 200 & 190 & 180 & 170 & 160 & 150 & 140 & 130 & 120 & 110 & 100 & 90 & 80 & 70 & 60 & 50 & 40 & 30 & 20 & 10 & 0\end{array}$ 
${ }^{19} \mathrm{~F}\left\{{ }^{1} \mathrm{H}\right\} \mathrm{NMR}\left(376 \mathrm{MHz}, \mathrm{CDCl}_{3}\right)$

R1886406_F19 $\{\mathrm{H}\}$

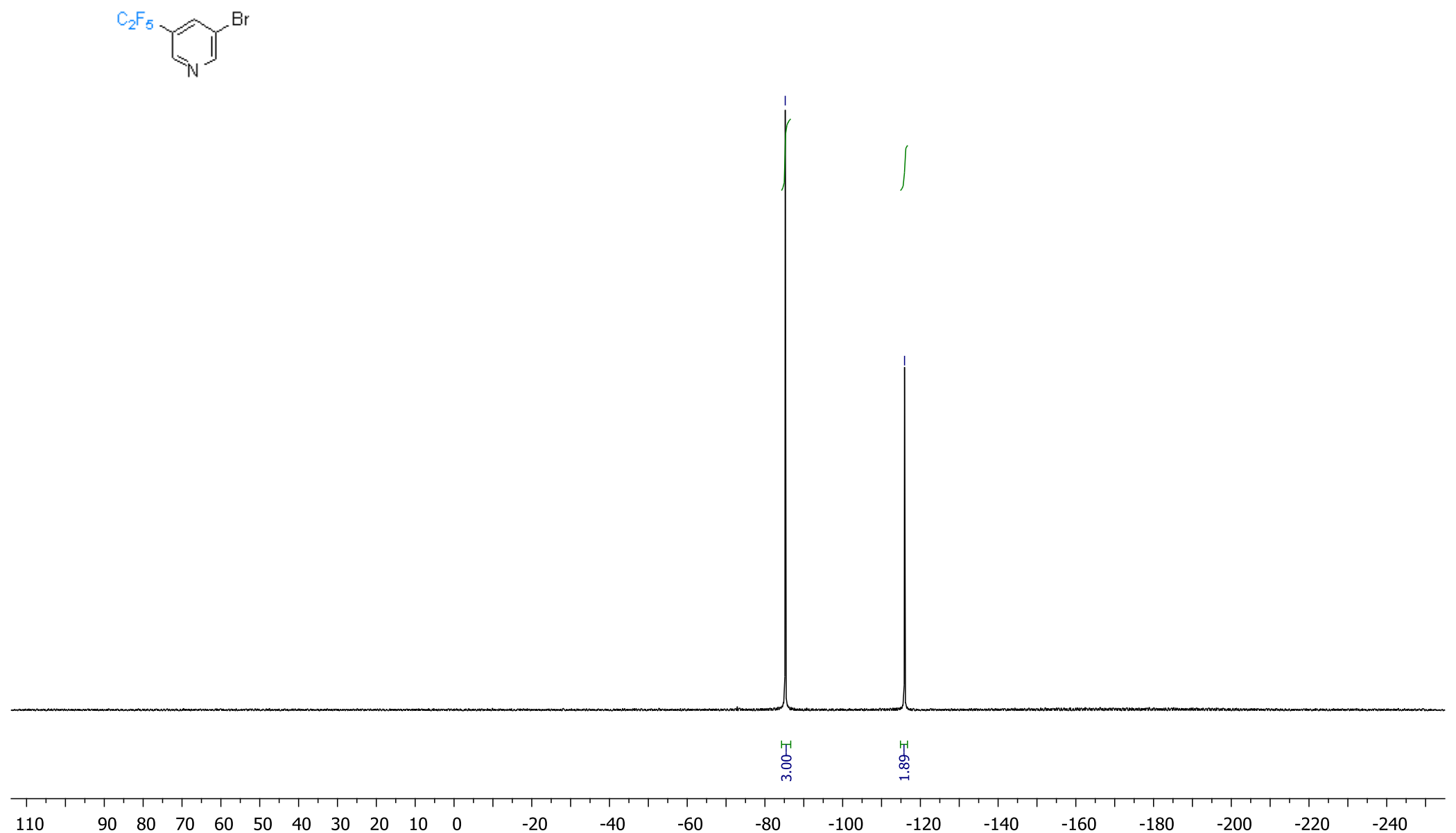




\section{Compound 63a}

${ }^{1} \mathrm{H}$ NMR (400 MHz, $\mathrm{CDCl}_{3}$ )

R2389718

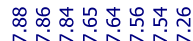

4
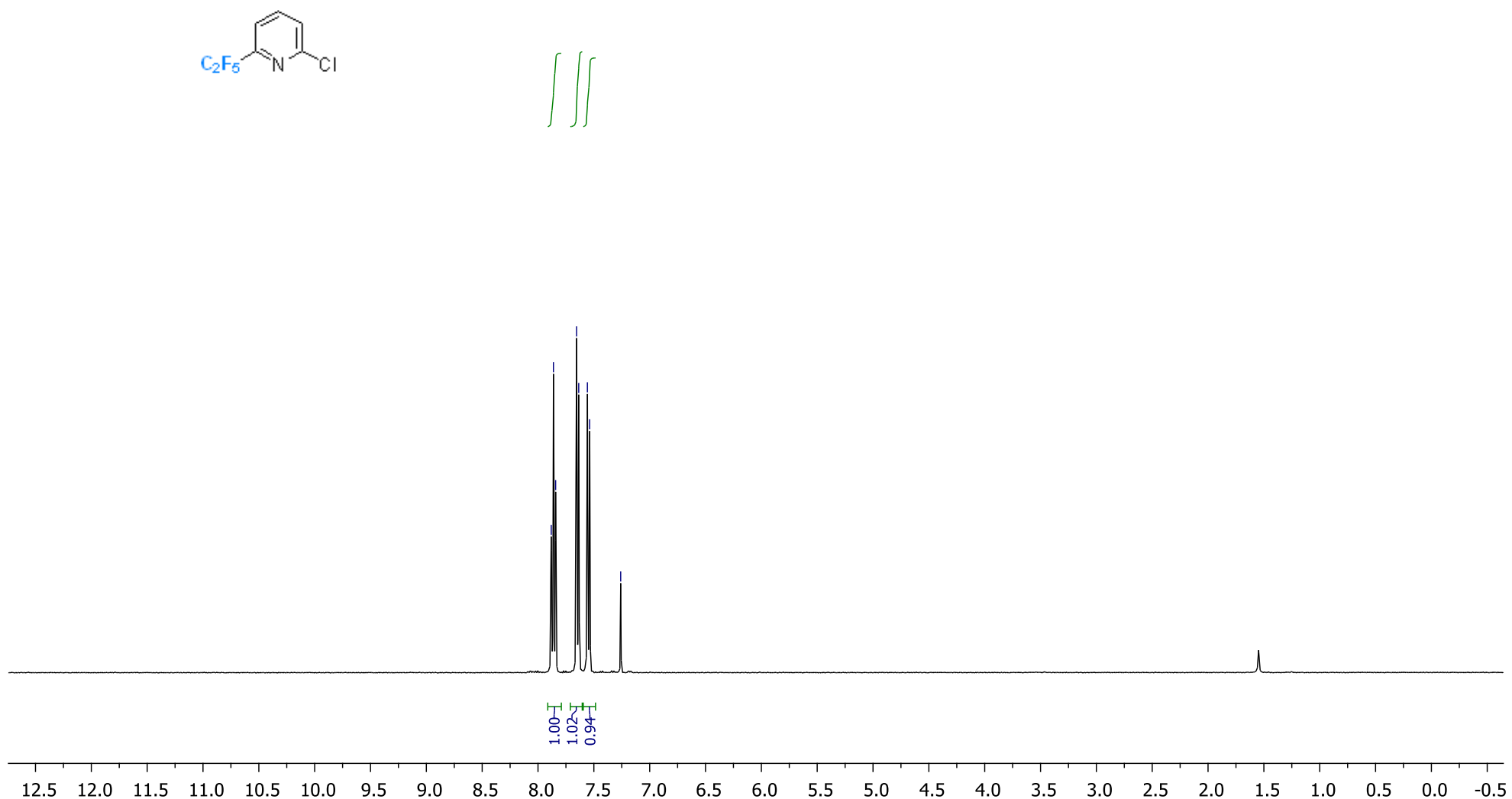
${ }^{13} \mathrm{C}\left\{{ }^{1} \mathrm{H}\right\}$ NMR $\left(151 \mathrm{MHz}, \mathrm{CDCl}_{3}\right)$

R2389718_13C

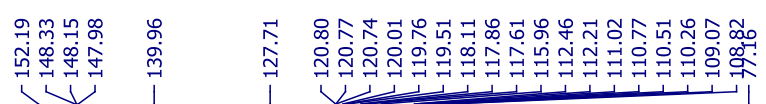

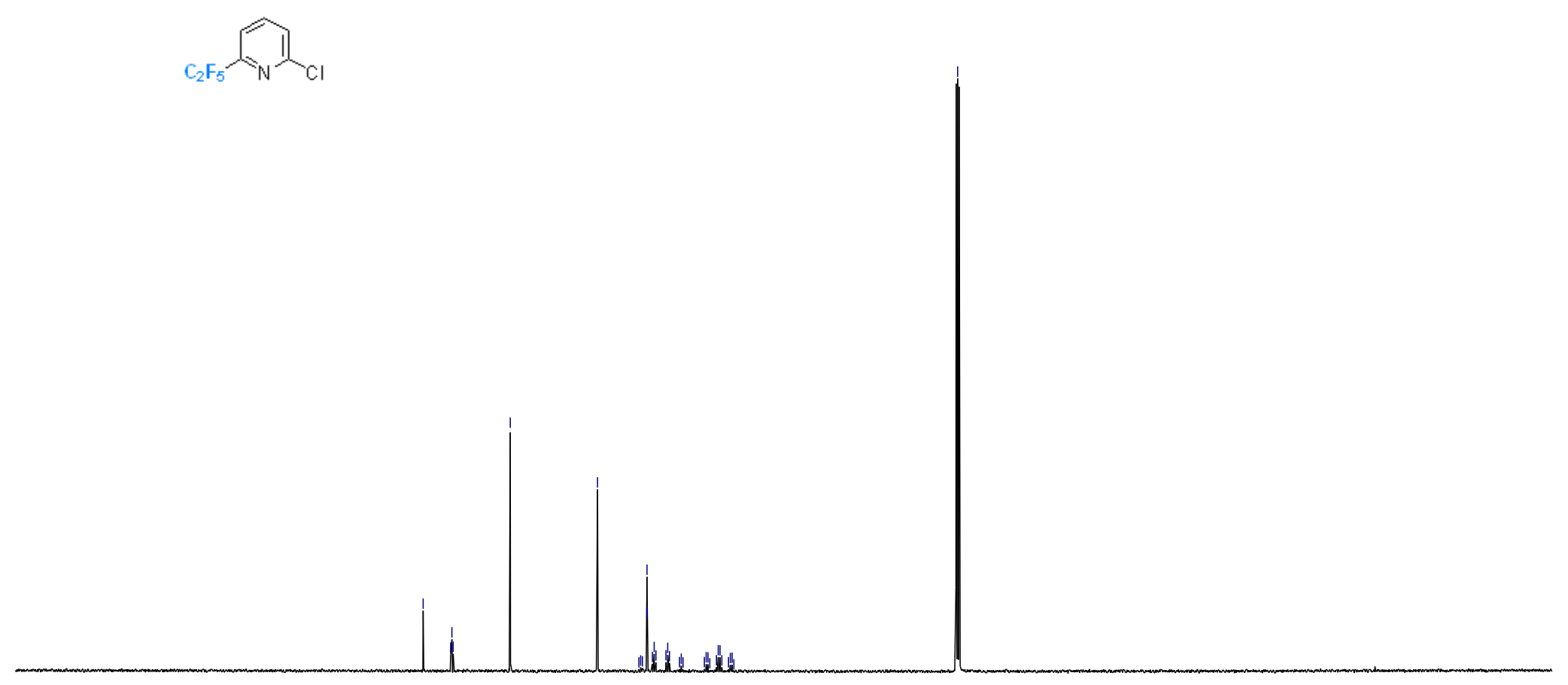

$\begin{array}{lll}140 & 130 & 120\end{array}$

$110 \quad 100$

$90 \quad 80$

$70 \quad 60$

50

40

30

$20 \quad 10 \quad 0$ 
${ }^{19} \mathrm{~F}\left\{{ }^{1} \mathrm{H}\right\} \mathrm{NMR}\left(376 \mathrm{MHz}, \mathrm{CDCl}_{3}\right)$

R2389718_F19 $\{\mathrm{H}\}$

$19 \mathrm{~F}-\{1 \mathrm{H}\}$

$\stackrel{\substack{0 \\ \infty}}{\stackrel{5}{0}}$

$\mathrm{C}_{2} \mathrm{~F}_{5} \overbrace{\mathrm{Cl}}$
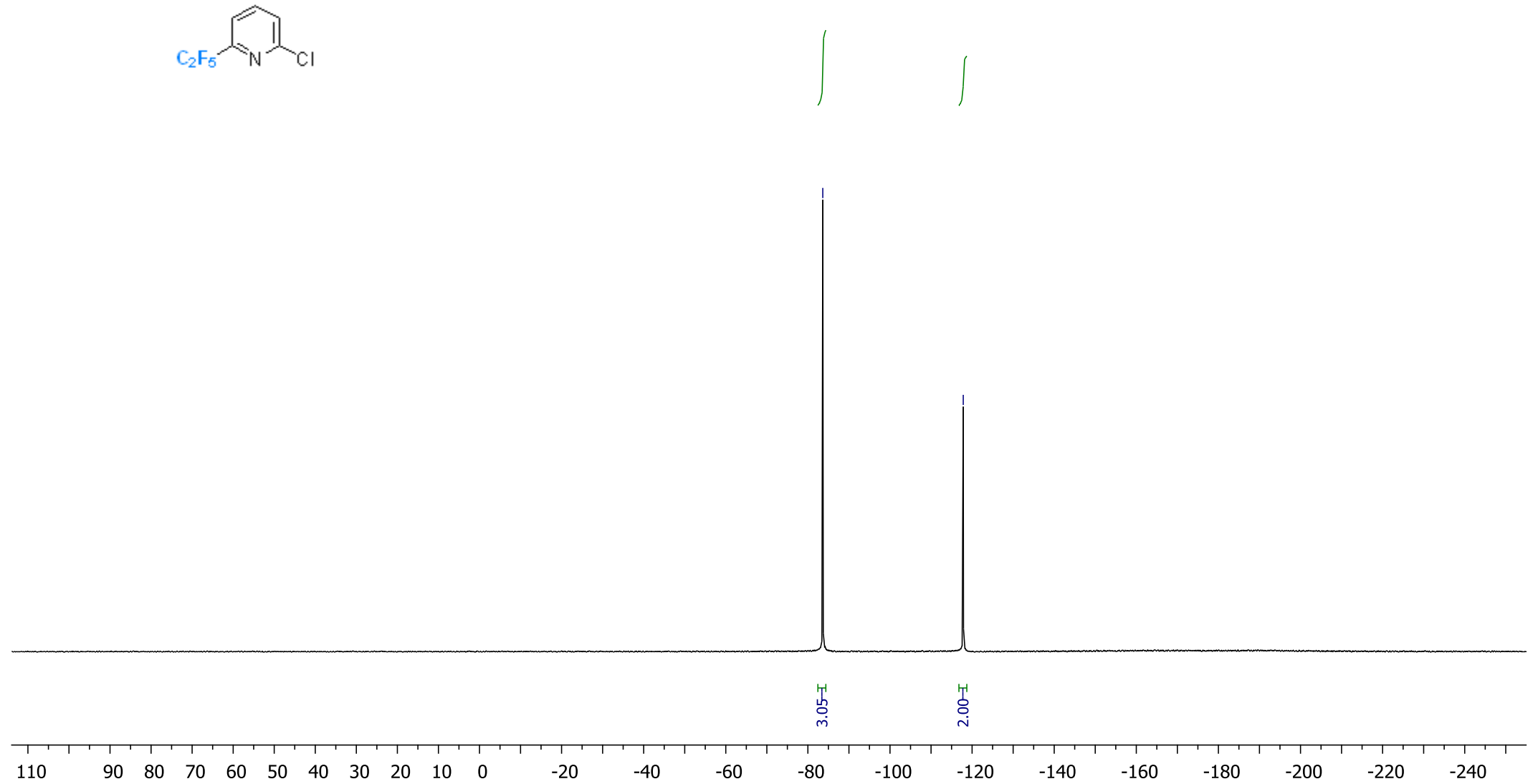


\section{Compound 64a}

${ }^{1} \mathrm{H}$ NMR (400 MHz, $\mathrm{CDCl}_{3}$ )

R2395797

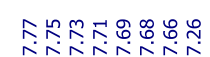

$\mathrm{C}_{2} \mathrm{~F}_{5} \overbrace{\mathrm{Br}}$
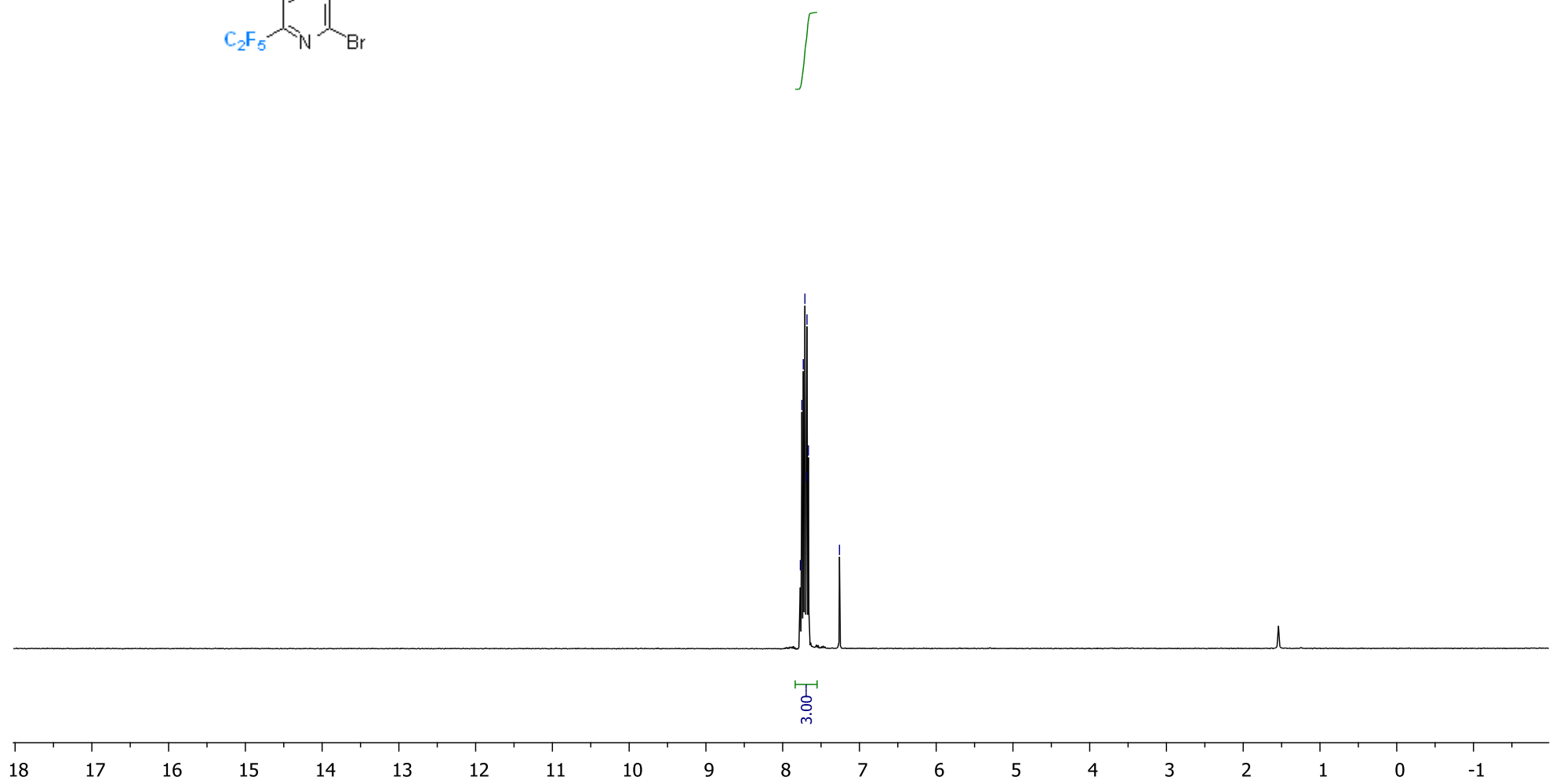
${ }^{13} \mathrm{C}\left\{{ }^{1} \mathrm{H}\right\} \mathrm{NMR}\left(126 \mathrm{MHz}, \mathrm{CDCl}_{3}\right)$

R2395797_13C

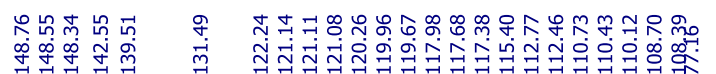

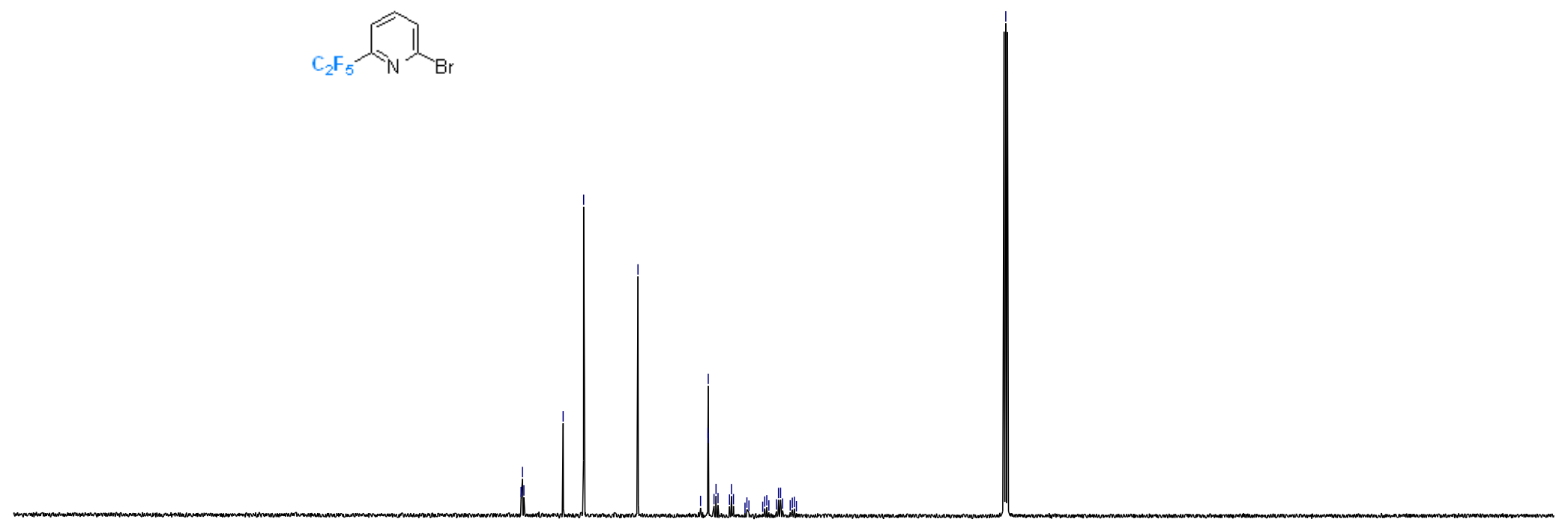

$\begin{array}{lllllllllll}220 & 210 & 200 & 190 & 180 & 170 & 160 & 150 & 140 & 130 & 120\end{array}$

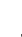


${ }^{19} \mathrm{~F}\left\{{ }^{1} \mathrm{H}\right\}$ NMR (376 MHz, $\left.\mathrm{CDCl}_{3}\right)$

R2395797_F19 $\{H\}$

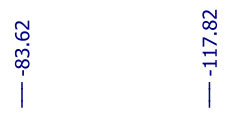

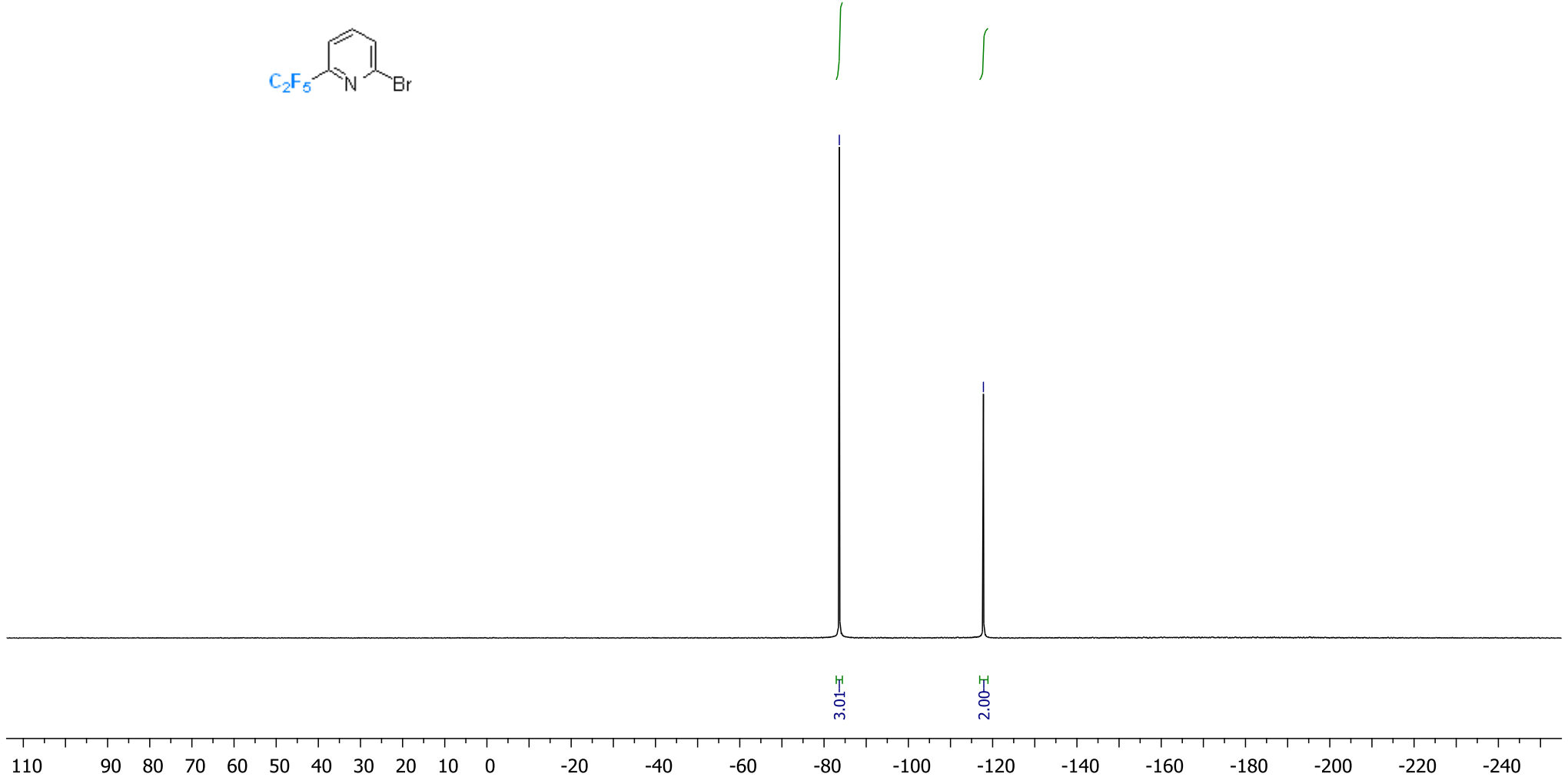




\section{Compound 65a}

${ }^{1} \mathrm{H}$ NMR (400 MHz, $\mathrm{CDCl}_{3}$ )

R1861193

$\sum_{\substack{\infty \\ \infty}}^{\substack{\infty \\ \infty}}$
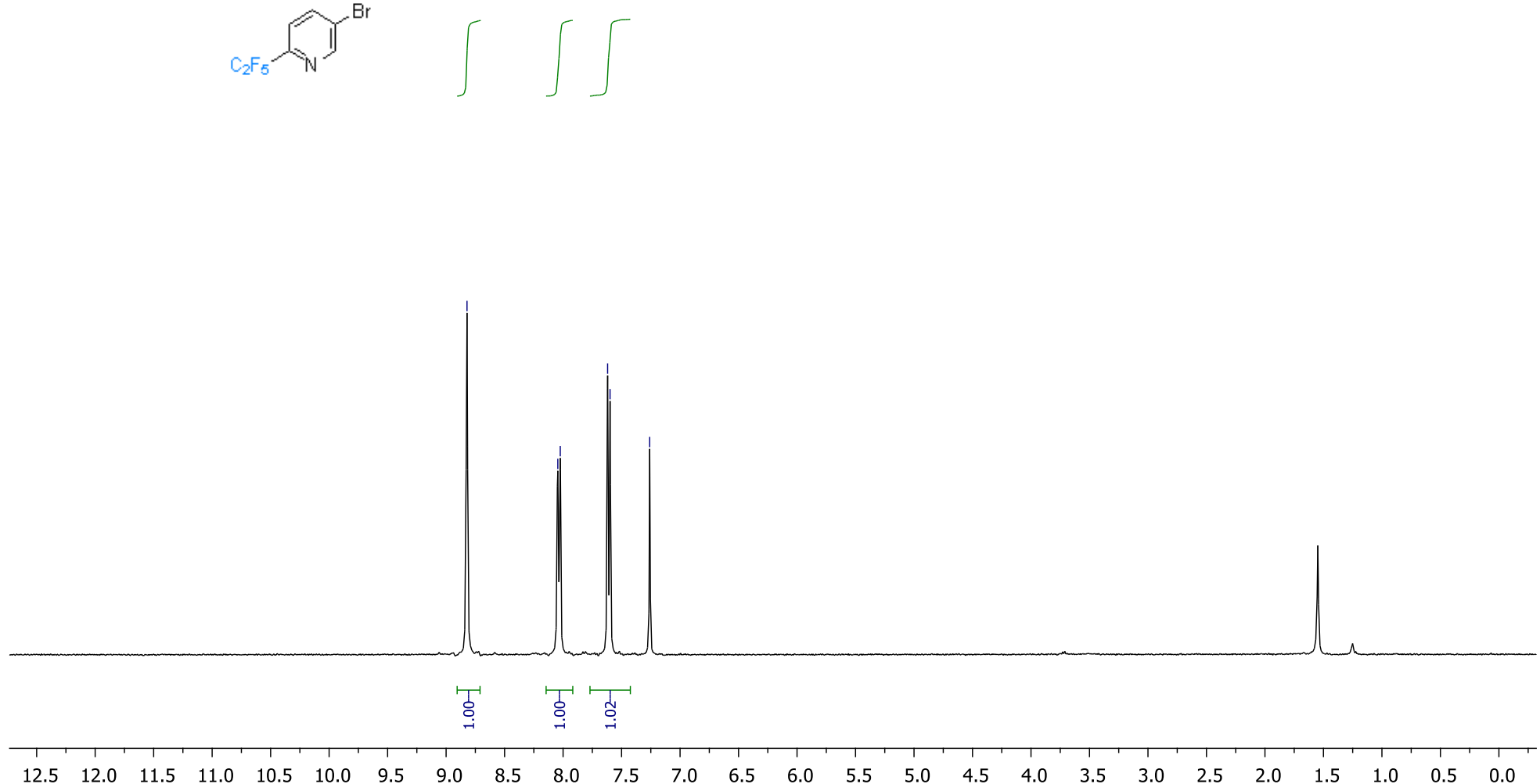


\section{${ }^{13} \mathrm{C}\left\{{ }^{1} \mathrm{H}\right\}$ NMR (126 MHz, $\mathrm{CDCl}$ )}

R1861193_13c

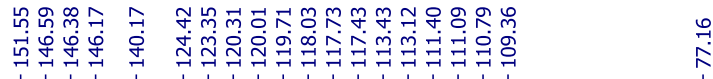

岱

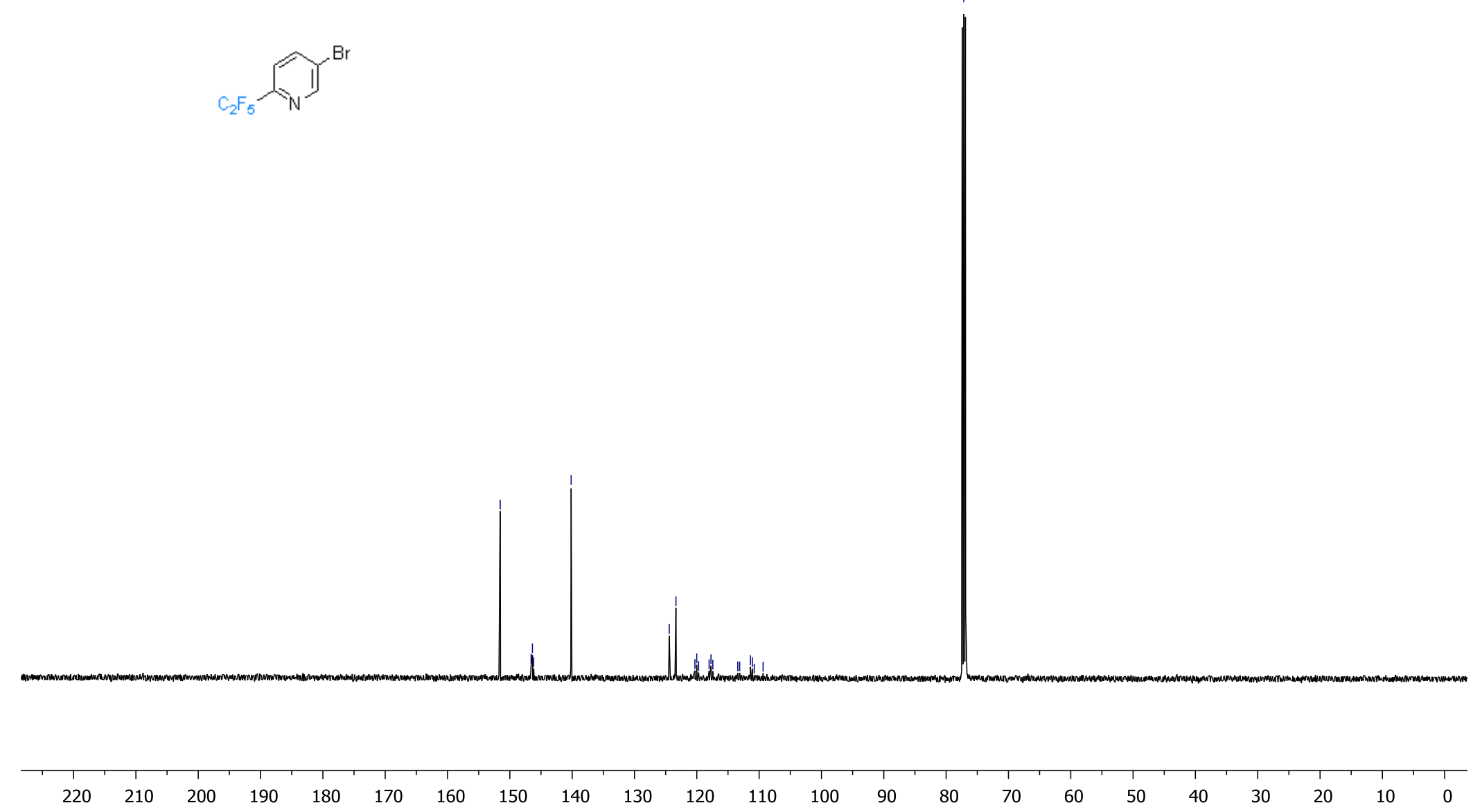


${ }^{19} \mathrm{~F}\left\{{ }^{1} \mathrm{H}\right\} \mathrm{NMR}\left(376 \mathrm{MHz}, \mathrm{CDCl}_{3}\right)$

R1861193_F19 $\{\mathrm{H}\}$

$19 F-\{1 \mathrm{H}\}$

$\stackrel{\substack{i \\ \infty}}{i}$

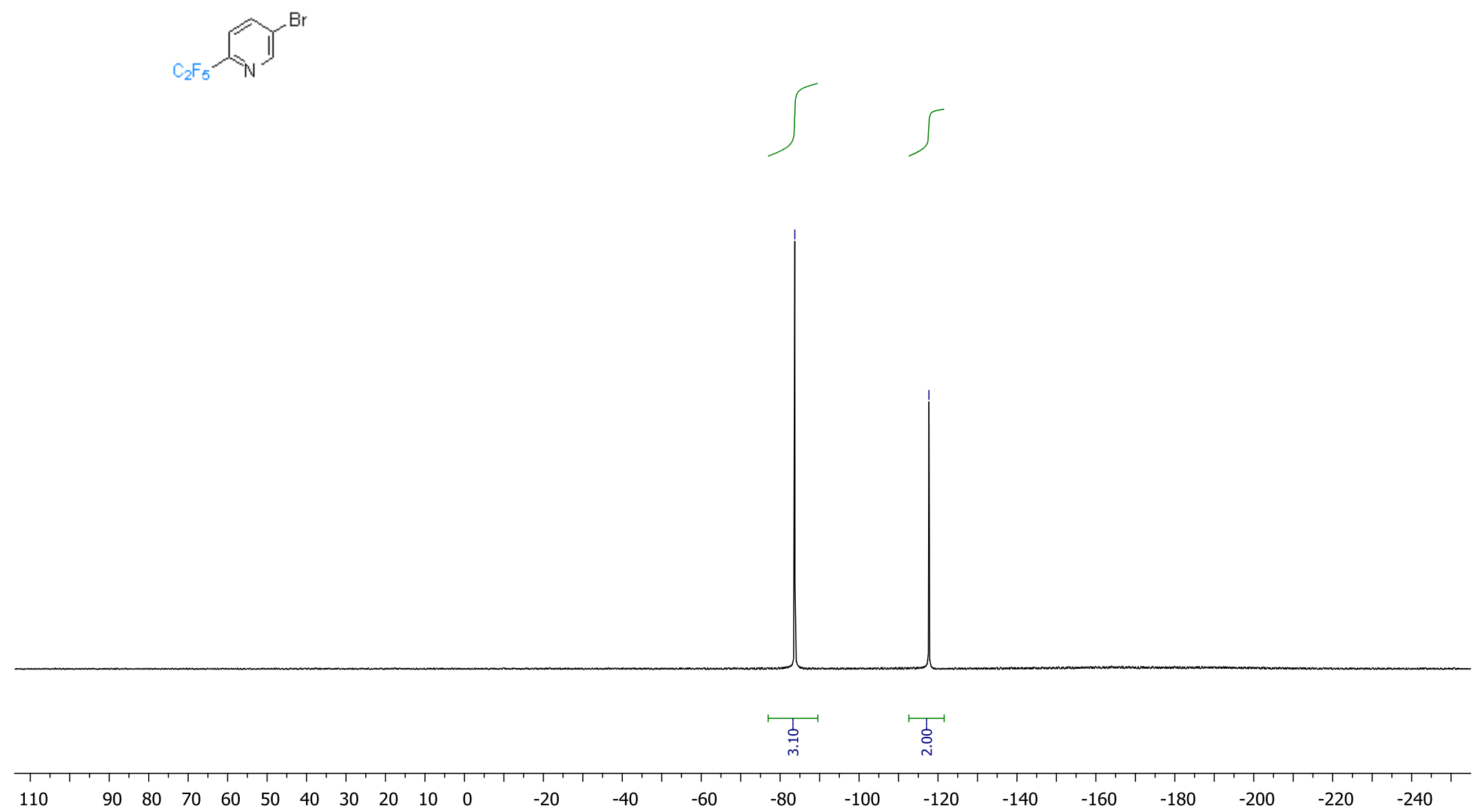




\section{Compound 66a}

${ }^{1} \mathrm{H}$ NMR (400 MHz, $\mathrm{CDCl}_{3}$ )

R1613728

कำำำ

$\mathrm{C}_{2} \mathrm{~F}_{5}-\overbrace{\mathrm{S}}^{\mathrm{Br}}$

$\int$

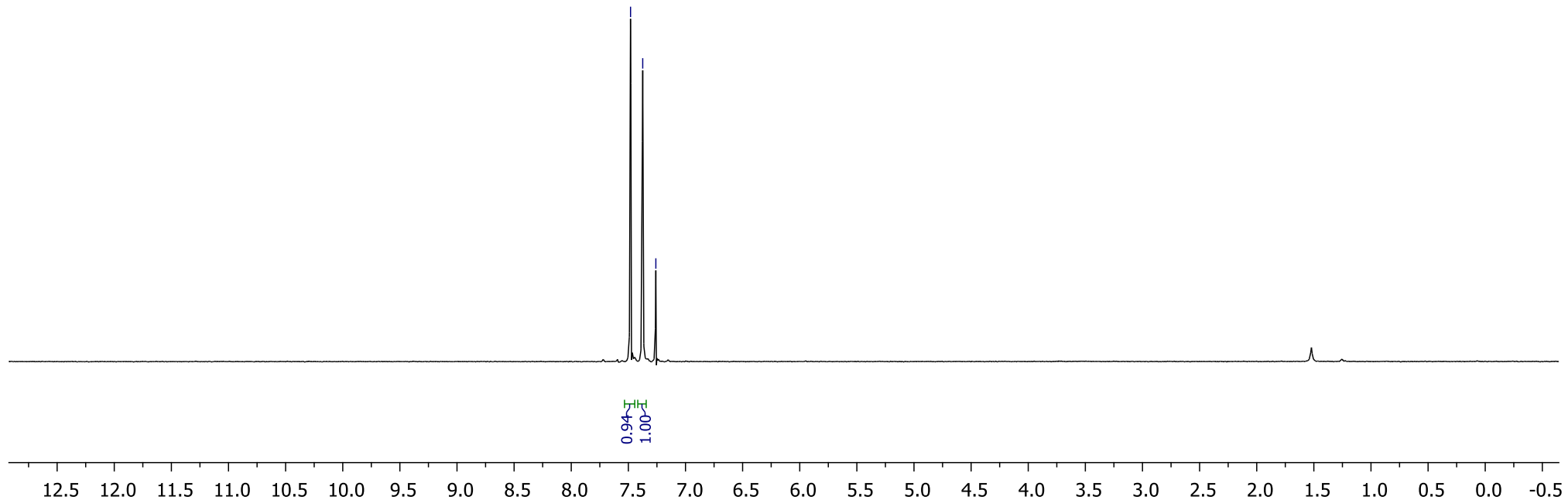


${ }^{13} \mathrm{C}\left\{{ }^{1} \mathrm{H}\right\} \mathrm{NMR}\left(126 \mathrm{MHz}, \mathrm{CDCl}_{3}\right)$

R1613728_136

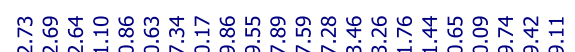

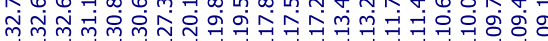

i

$$
\mathrm{C}_{2} \mathrm{~F}_{5} \overbrace{\mathrm{S}}^{\mathrm{Br}}
$$

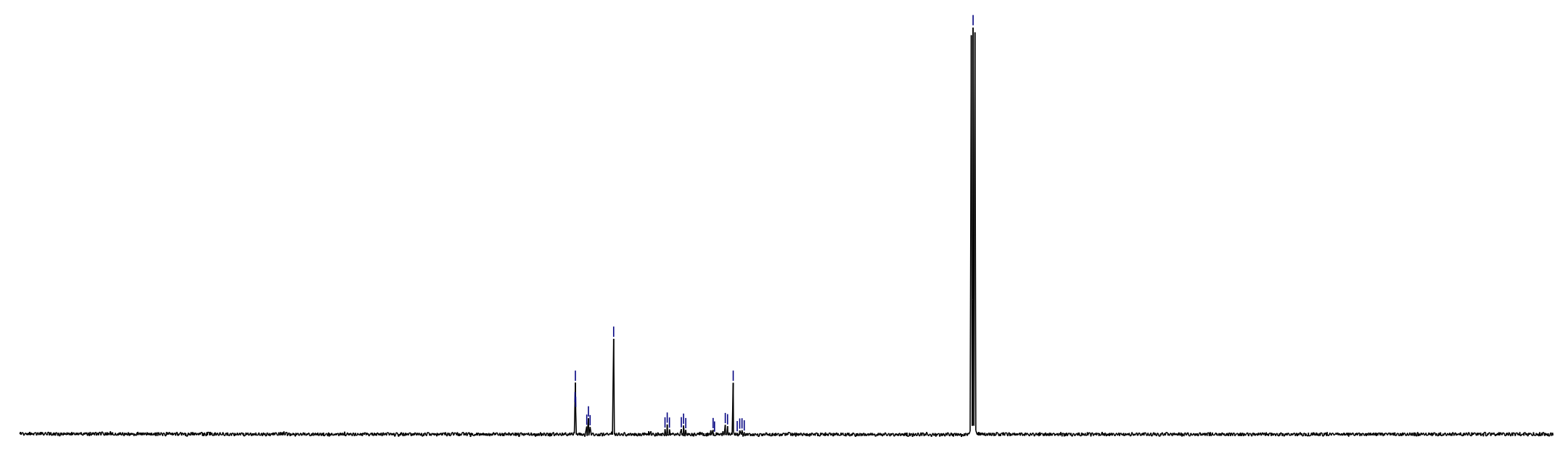

210

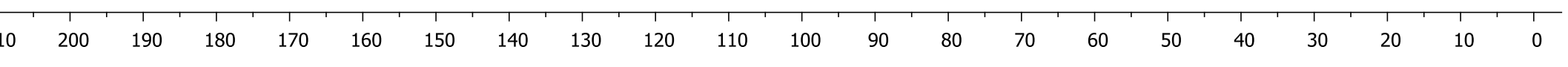


${ }^{19} \mathrm{~F}\left\{{ }^{1} \mathrm{H}\right\}$ NMR (376 MHz, $\left.\mathrm{CDCl}_{3}\right)$

R1613728_F19

$19 \mathrm{~F}-\{1 \mathrm{H}\}$

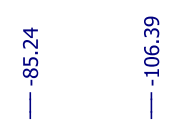
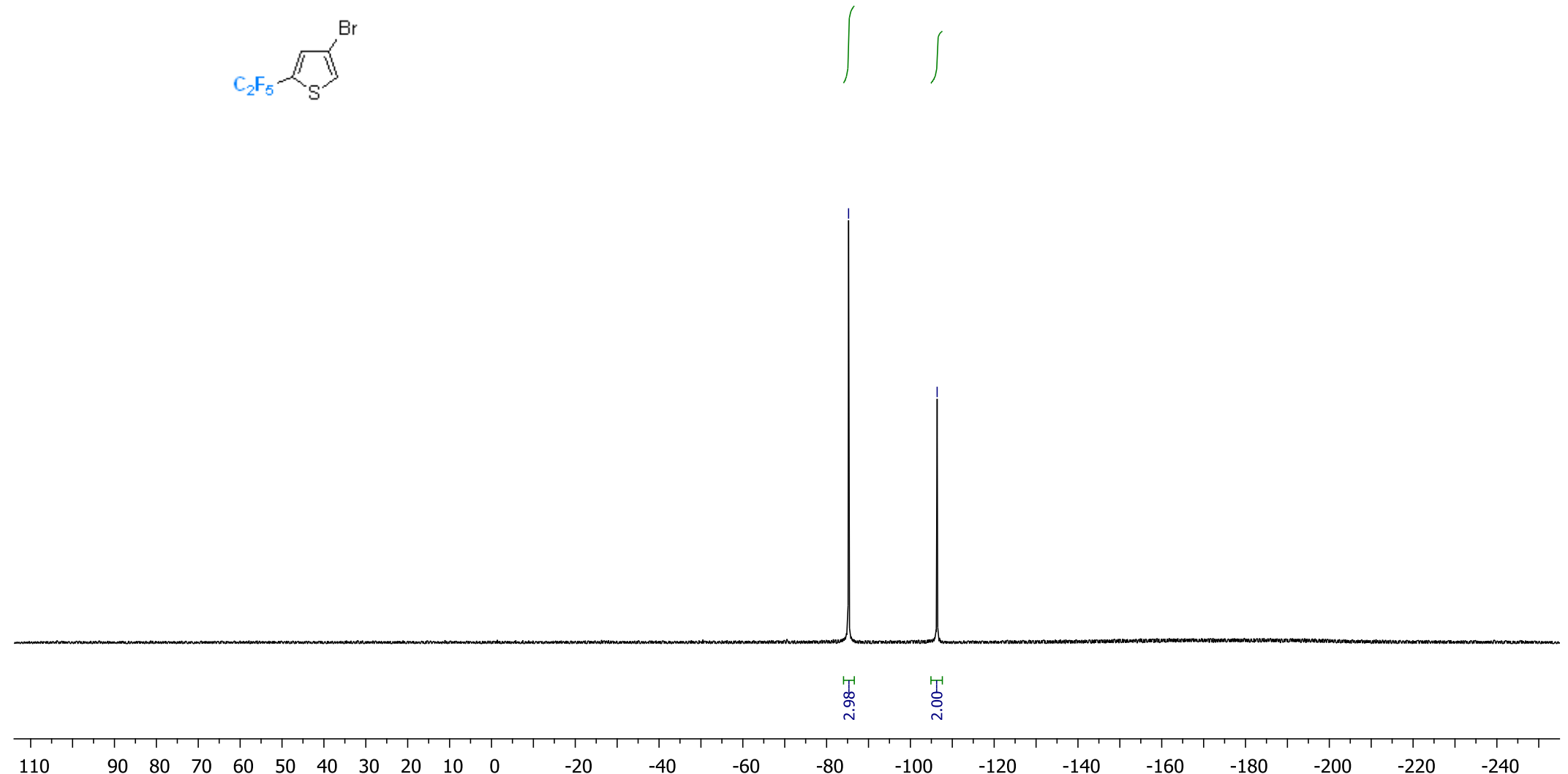


\section{Compound 67a}

${ }^{1} \mathrm{H}$ NMR (400 MHz, $\mathrm{CDCl}_{3}$ )

R1627364

${ }_{\mathrm{S}}^{\mathrm{C}_{2} \mathrm{~F}_{5}}$
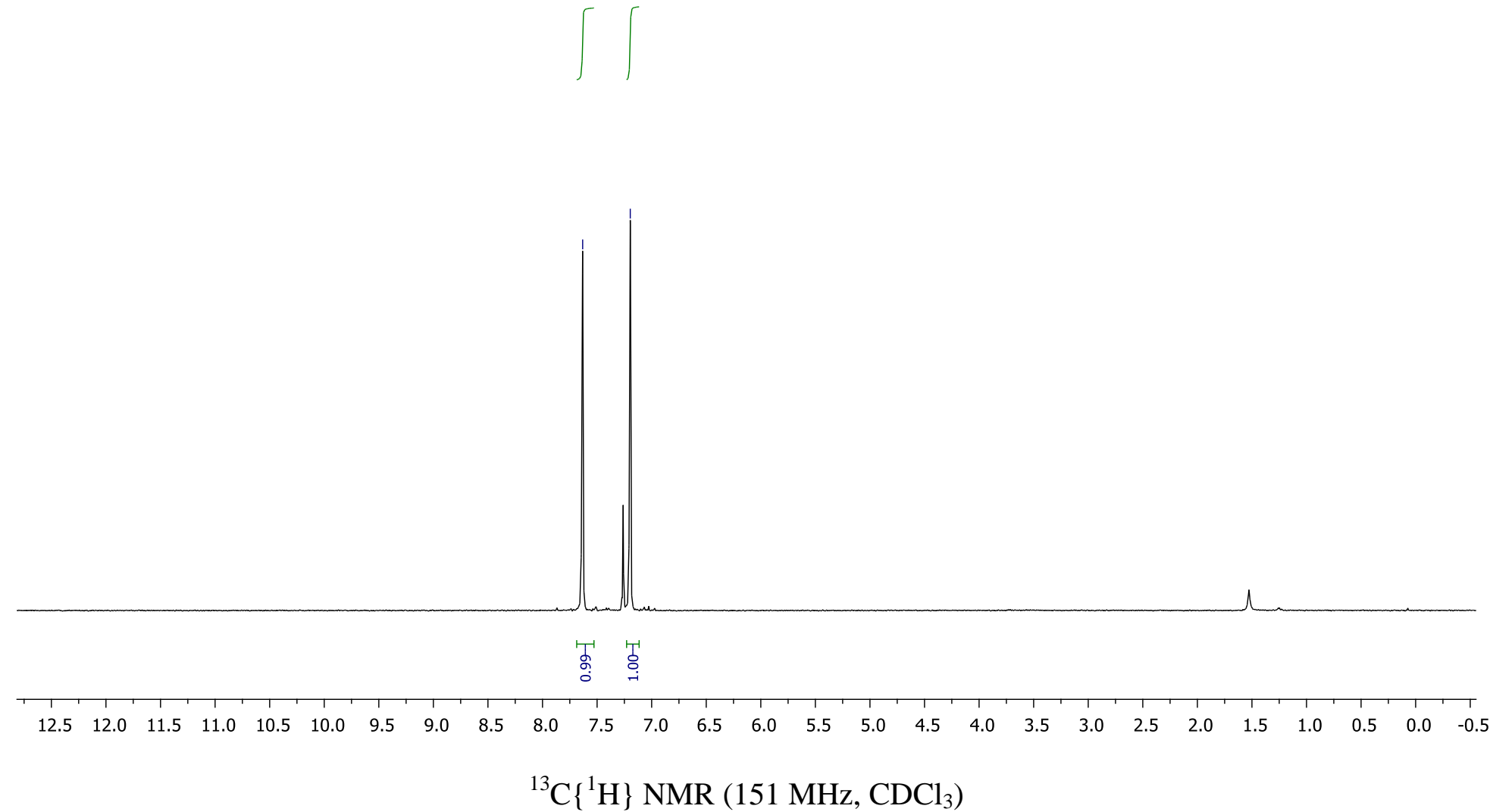


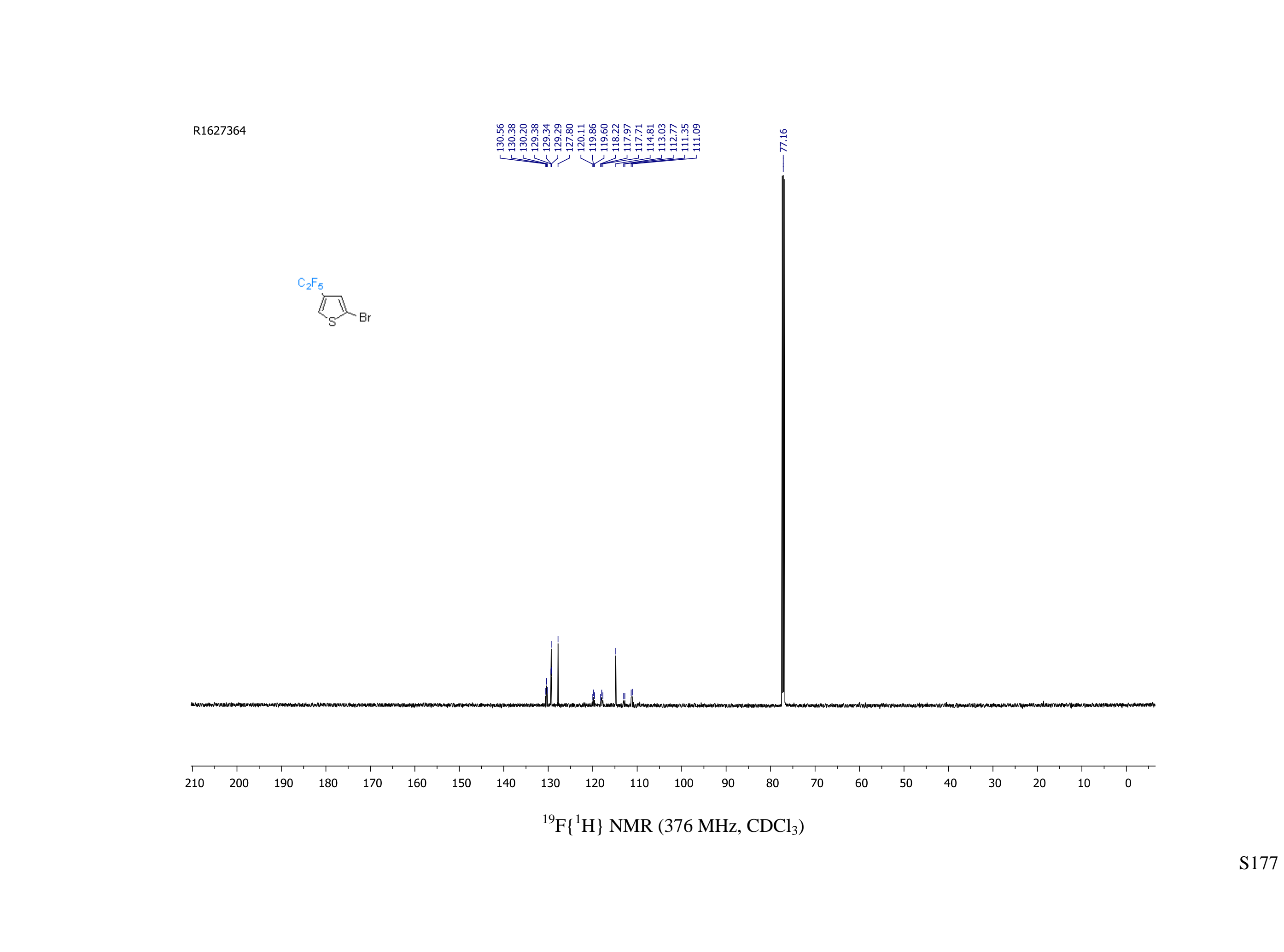


R1627364_F19\{H\}

$$
\prod_{\mathrm{S}}^{\mathrm{C}_{2} \mathrm{~F}_{5}}
$$

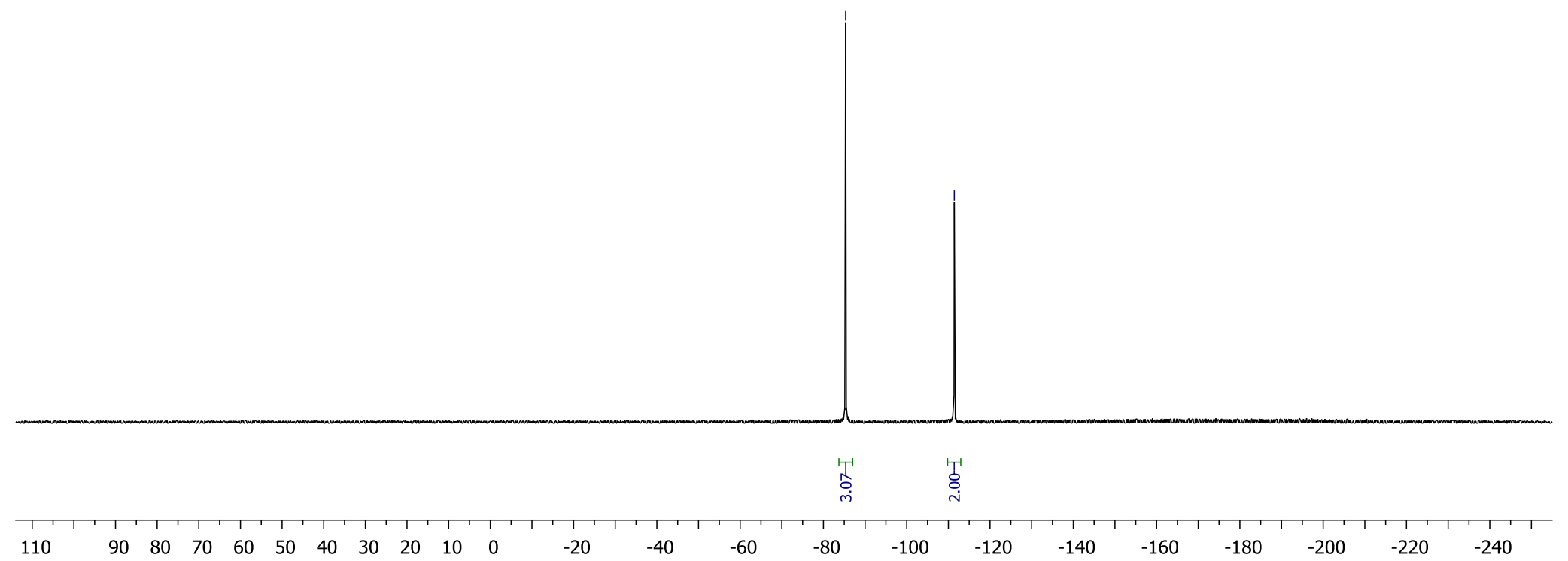




\section{Compound 69}

${ }^{1} \mathrm{H}$ NMR (400 MHz, DMSO- $d_{6}$ )

R2560579

$\stackrel{\vec{N}}{\stackrel{1}{1}}$

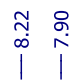

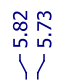

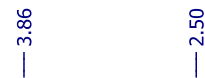
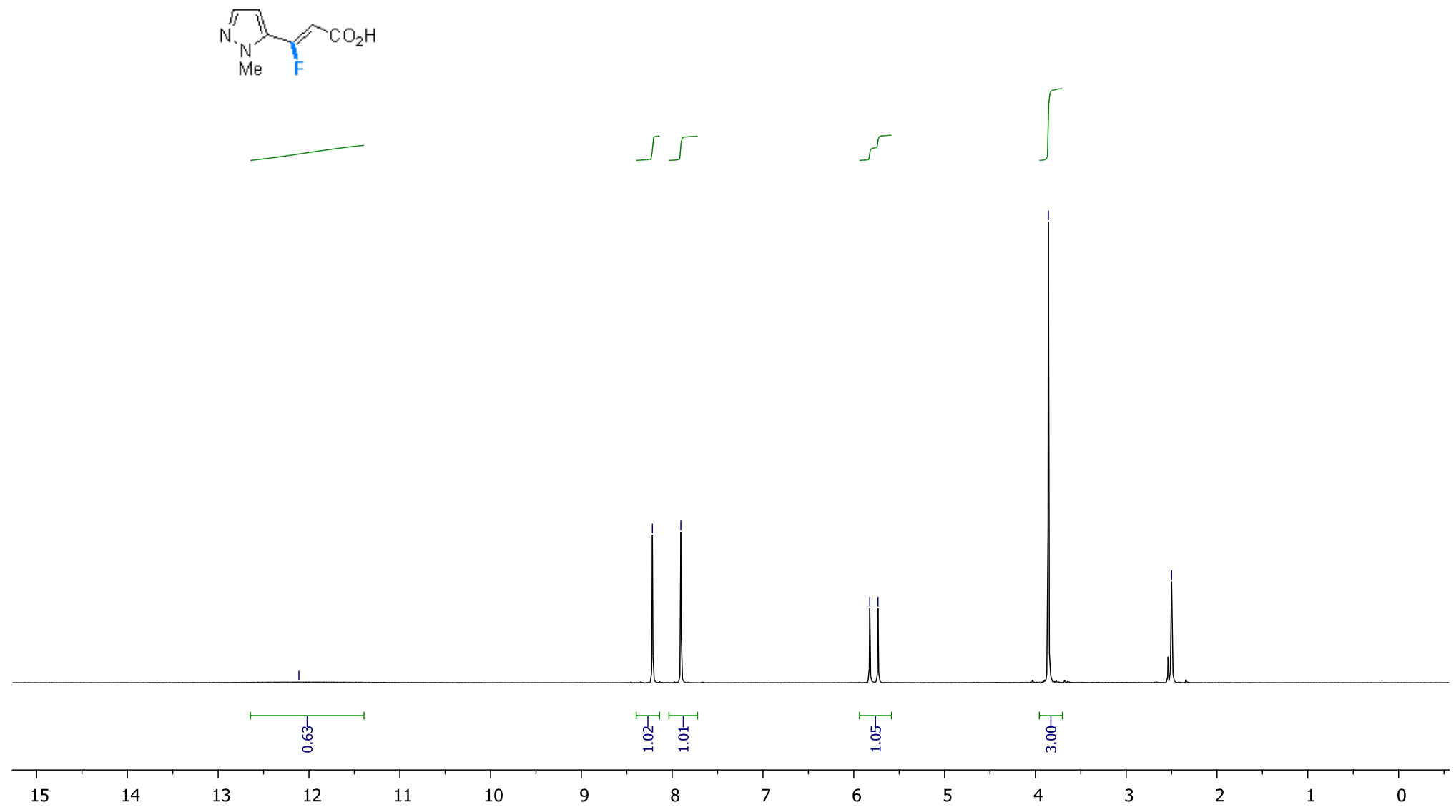


\section{${ }^{13} \mathrm{C}\left\{{ }^{1} \mathrm{H}\right\}$ NMR $\left(126 \mathrm{MHz}\right.$, DMSO- $\left.d_{6}\right)$}

R2560579_13c

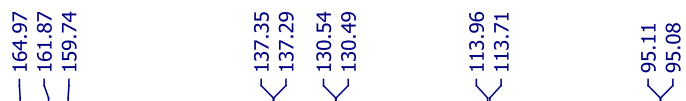

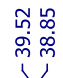
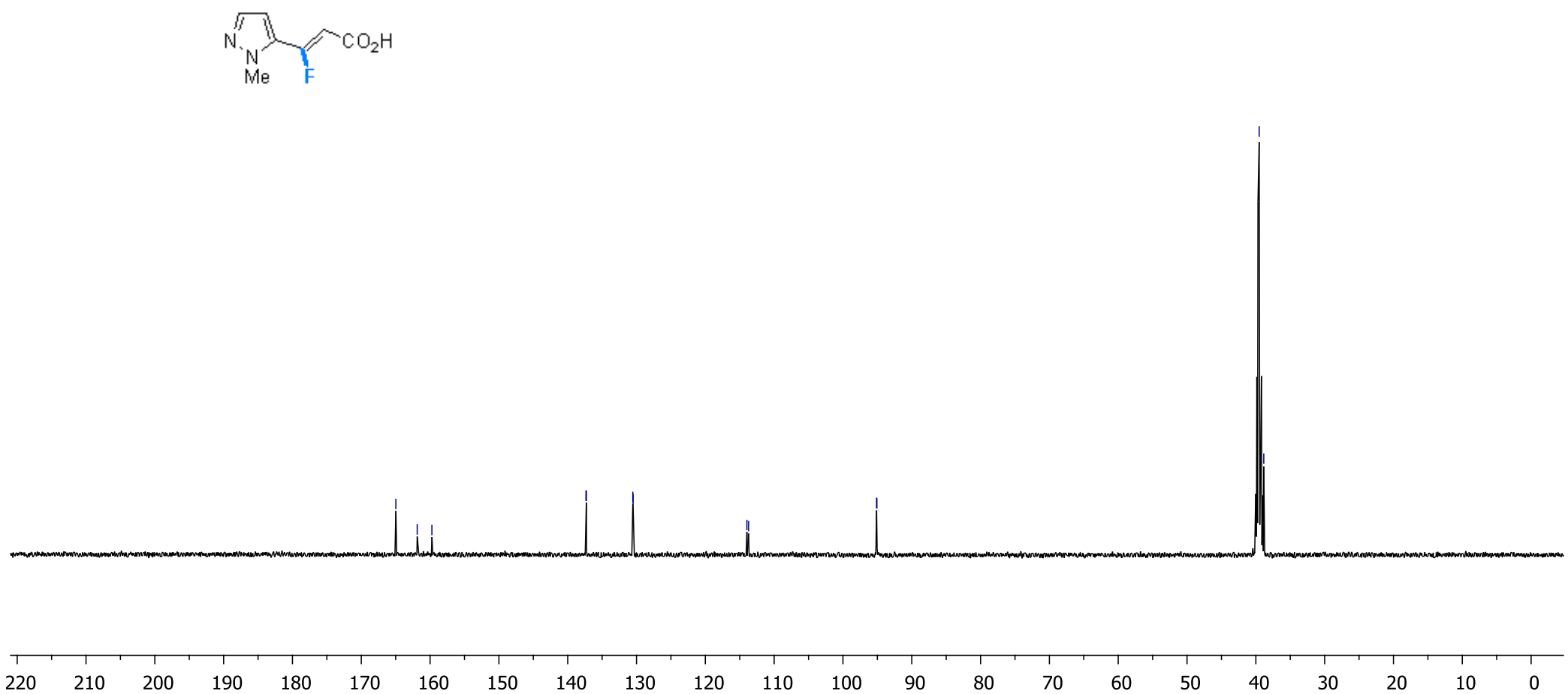


\section{${ }^{19} \mathrm{~F}\left\{{ }^{1} \mathrm{H}\right\}$ NMR (376 MHz, DMSO- $\left.d_{6}\right)$}

R2560579_F19\{H\}

$19 \mathrm{~F}-\{1 \mathrm{H}\}$
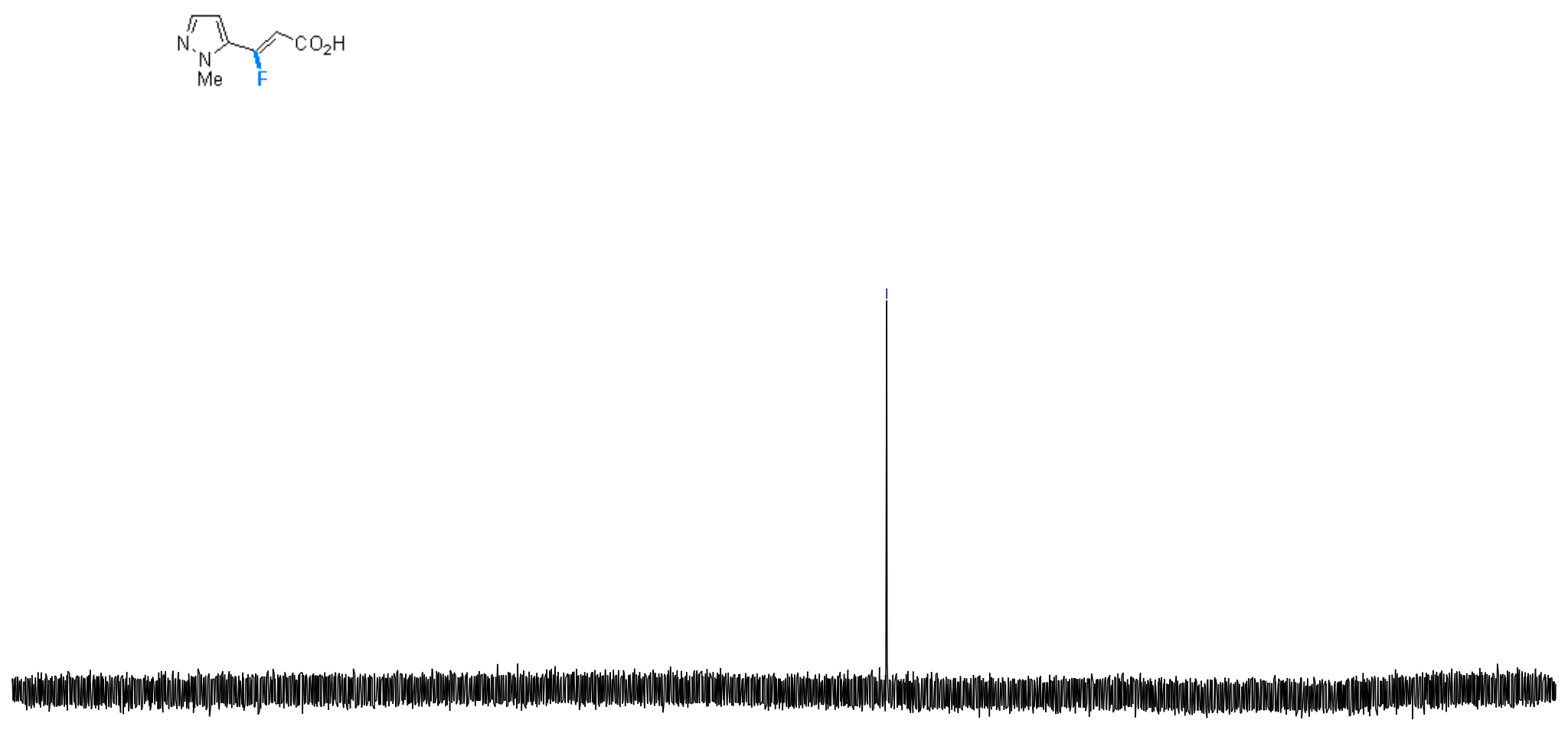


\section{Compound 70}

${ }^{1} \mathrm{H}$ NMR (400 MHz, DMSO- $d_{6}$ )

R2531552

|

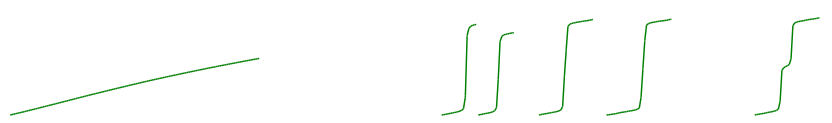

$\overbrace{\mathrm{N}}^{\mathrm{F}} \mathrm{CO}_{2} \mathrm{H}$

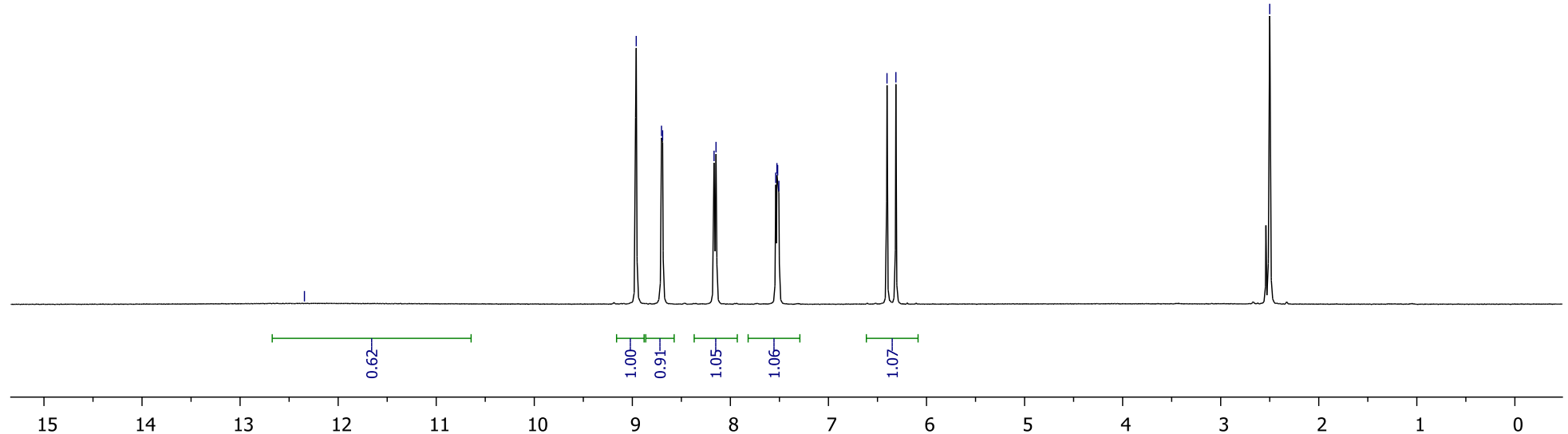


${ }^{13} \mathrm{C}\left\{{ }^{1} \mathrm{H}\right\}$ NMR $\left(126 \mathrm{MHz}\right.$, DMSO- $\left.d_{6}\right)$

R2531552_13c

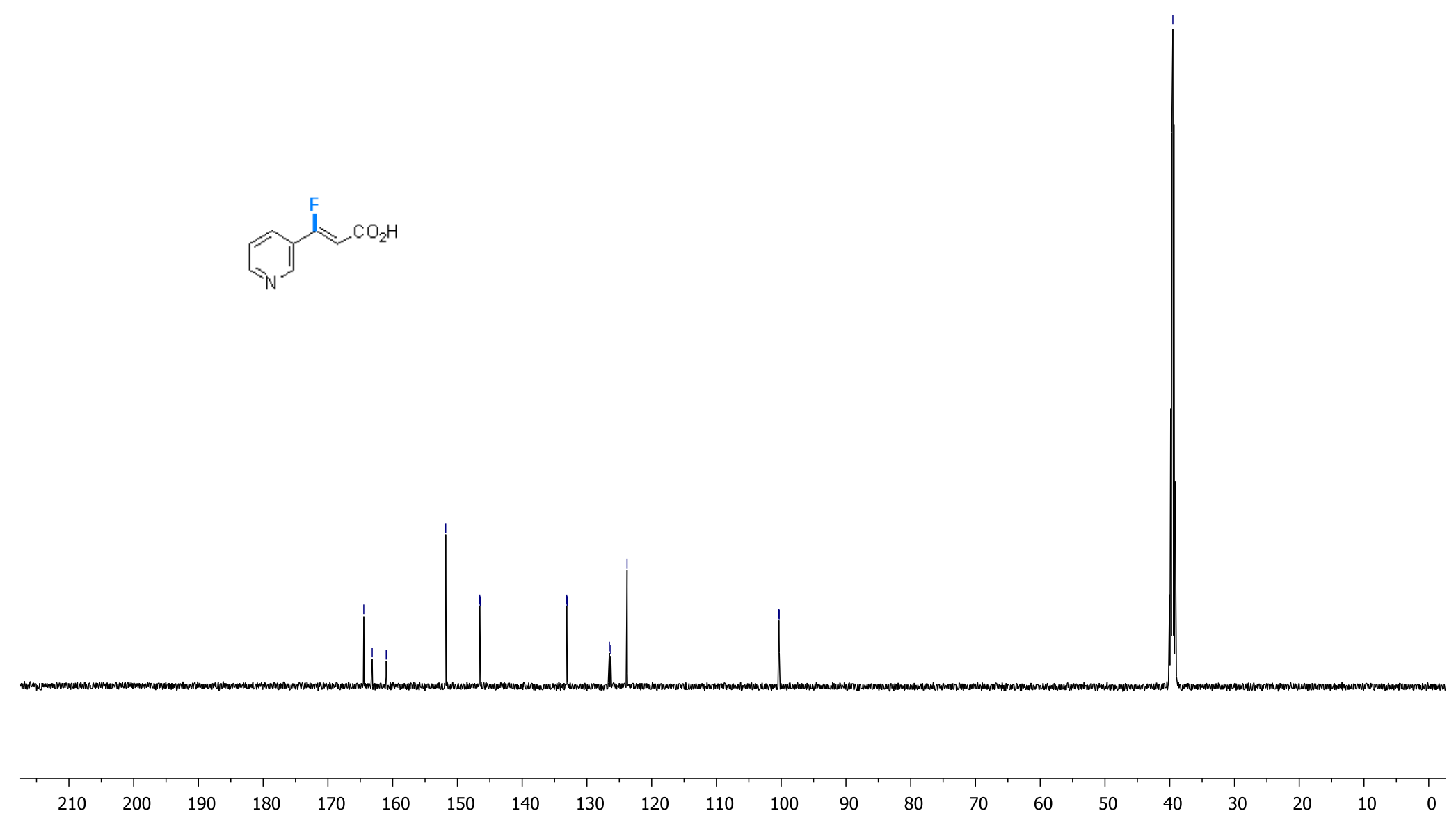


${ }^{19} \mathrm{~F}\left\{{ }^{1} \mathrm{H}\right\}$ NMR (376 MHz, DMSO- $\left.d_{6}\right)$

R2531552_F19

$19 \mathrm{~F}-\{1 \mathrm{H}\}$

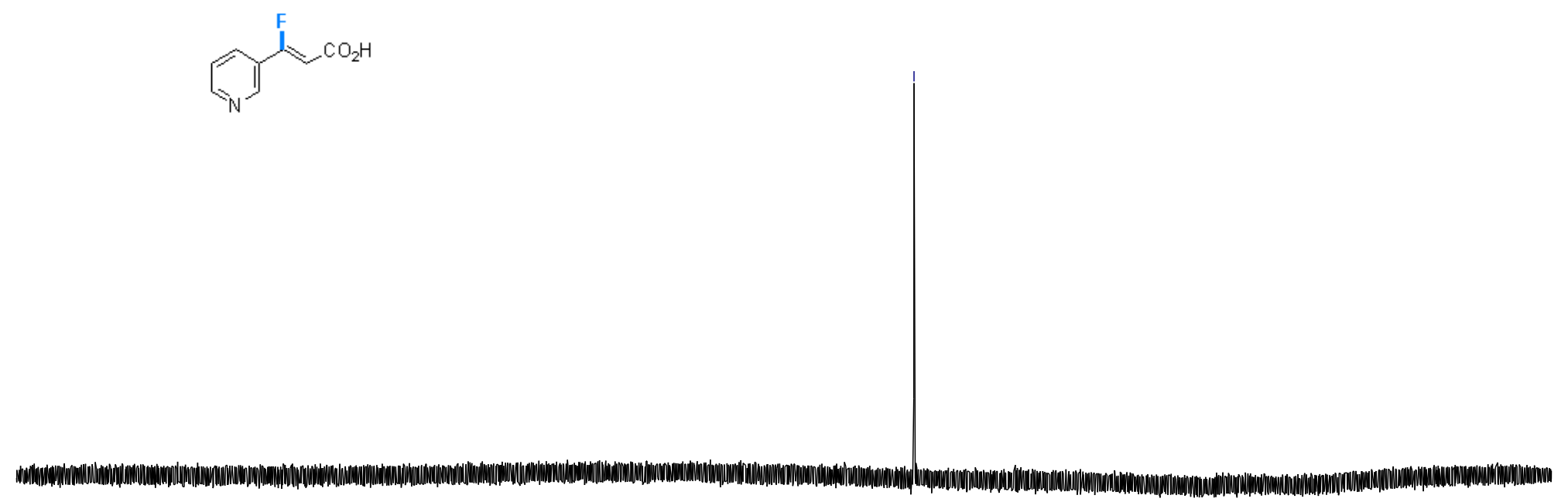




\section{Compound 71}

${ }^{1} \mathrm{H}$ NMR (400 MHz, $\mathrm{CDCl}_{3}$ )

R1634554

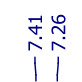

${ }_{\mathrm{Me}}^{\mathrm{I} \mathrm{F}_{\mathrm{F}}^{\mathrm{Me}}}$

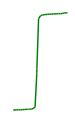

\{

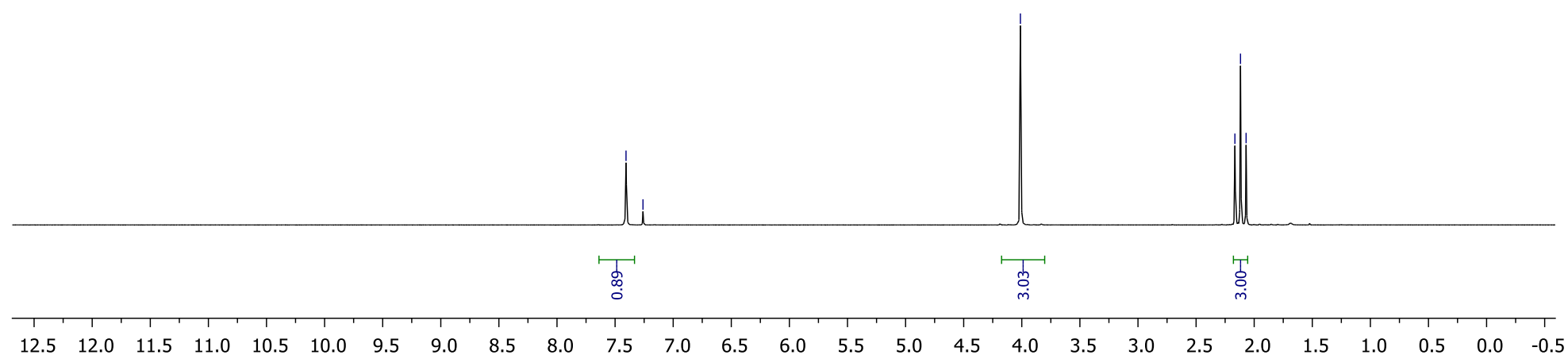


${ }^{13} \mathrm{C}\left\{{ }^{1} \mathrm{H}\right\}$ NMR (126 MHz, $\left.\mathrm{CDCl}_{3}\right)$

R1634554_13c

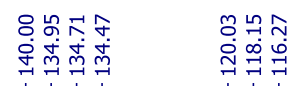

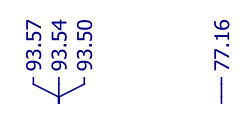

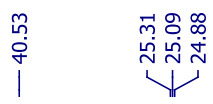

${ }_{\mathrm{Me}}^{\mathrm{Br}} \mathrm{Me}$

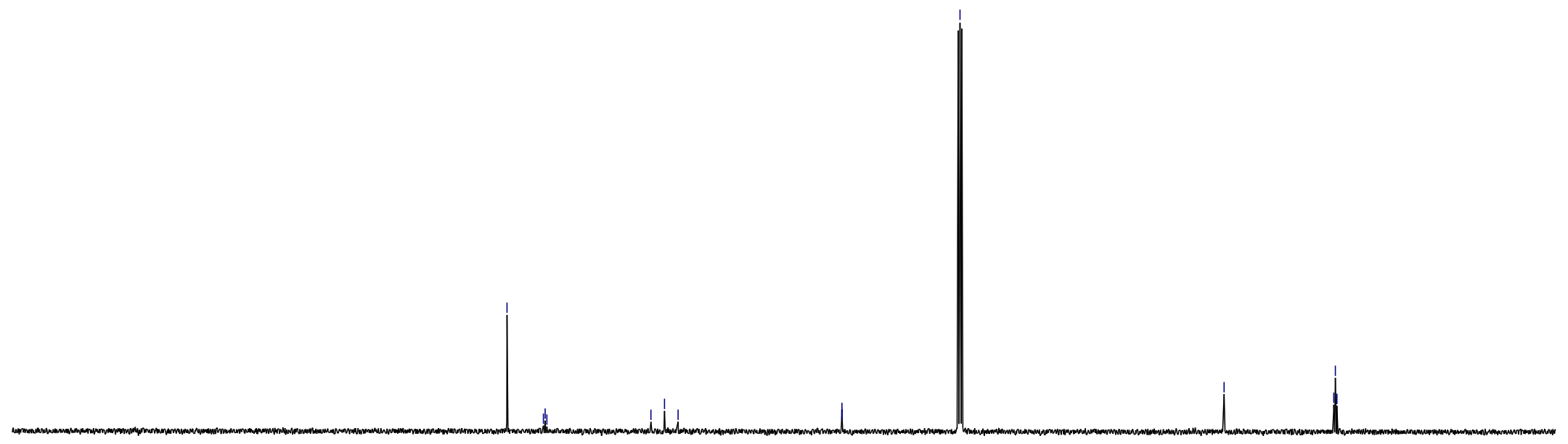

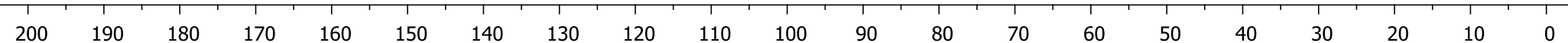




\section{${ }^{19} \mathrm{~F}\left\{{ }^{1} \mathrm{H}\right\}$ NMR (376 MHz, $\left.\mathrm{CDCl}_{3}\right)$}

R1634554_F19 $\{\mathrm{H}\}$
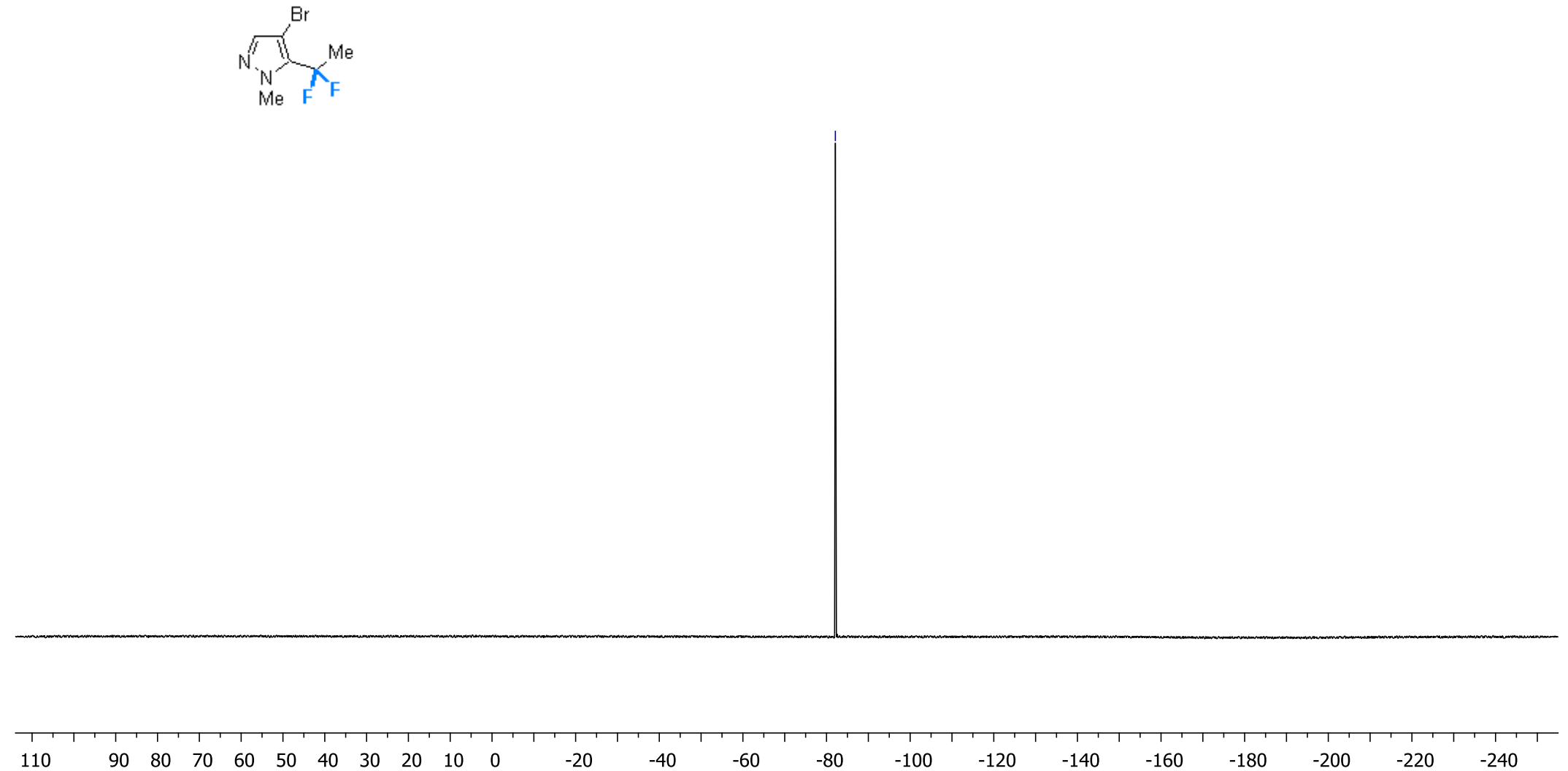


\section{Compound 72}

${ }^{1} \mathrm{H}$ NMR (400 MHz, $\mathrm{CDCl}_{3}$ )

R2513152

$\stackrel{9}{2}$

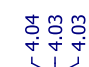

平总总

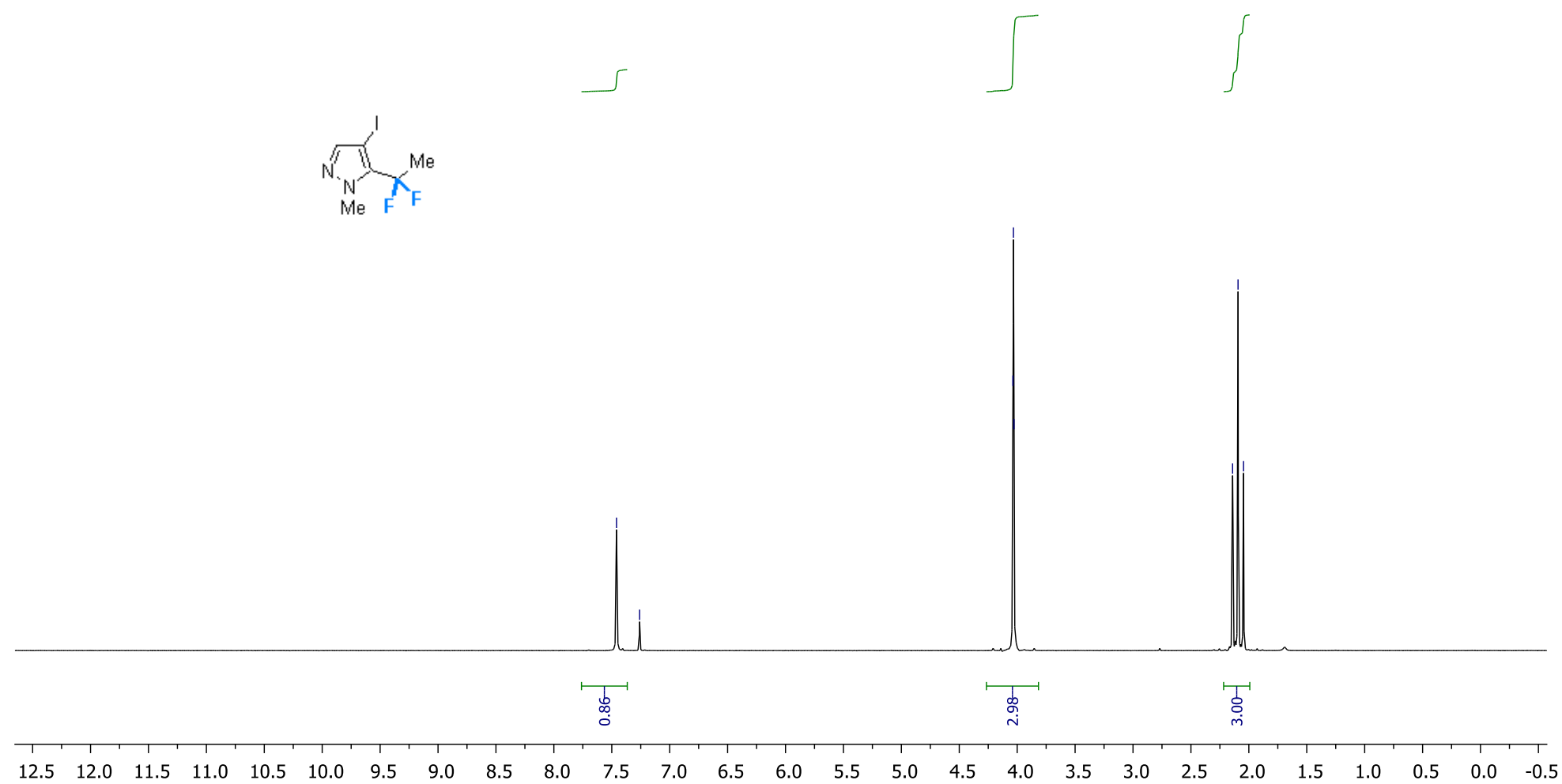


${ }^{13} \mathrm{C}\left\{{ }^{1} \mathrm{H}\right\}$ NMR (151 MHz, $\left.\mathrm{CDCl}_{3}\right)$

R2513152_13C

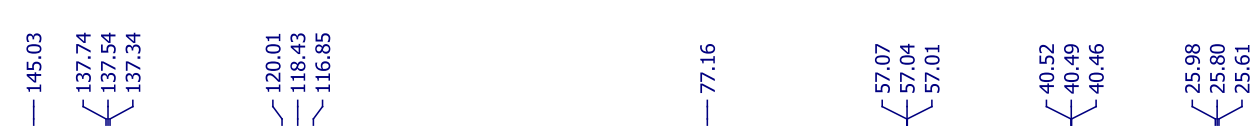

$$
\prod_{M e}^{M}
$$
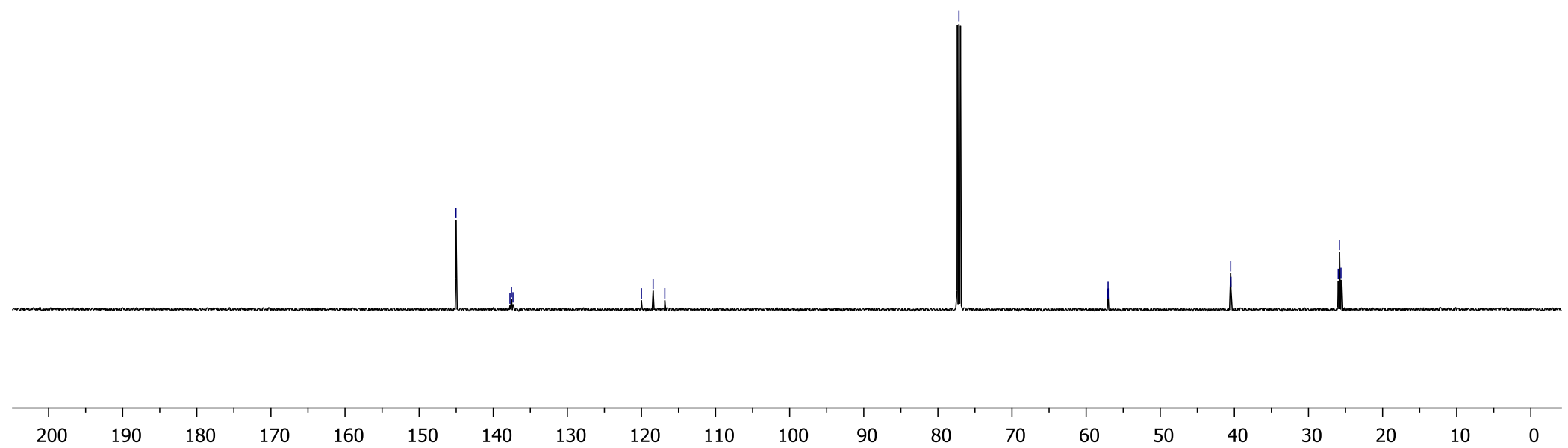

90 


\section{${ }^{19} \mathrm{~F}\left\{{ }^{1} \mathrm{H}\right\} \mathrm{NMR}\left(376 \mathrm{MHz}, \mathrm{CDCl}_{3}\right)$}

R2513152_F19 $\{\mathrm{H}\}$
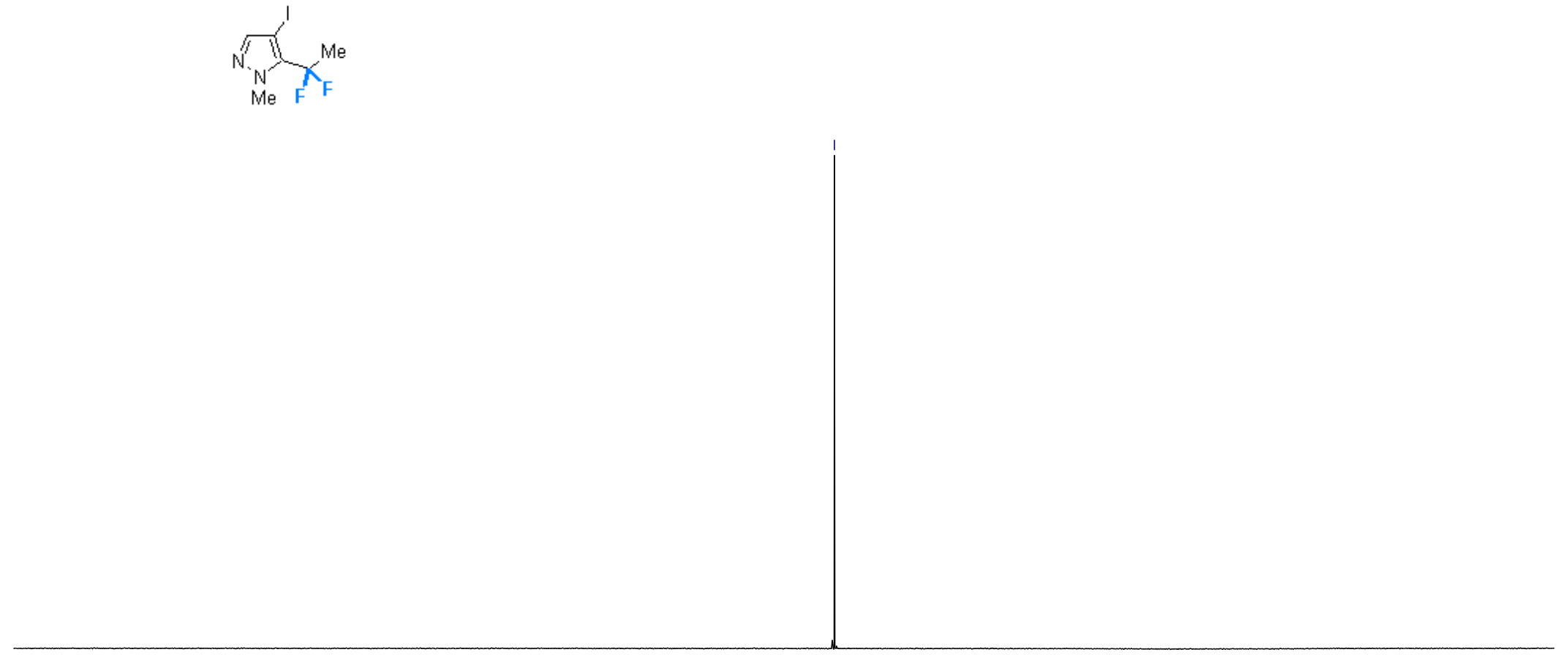


\section{Compound 73}

${ }^{1} \mathrm{H}$ NMR (400 MHz, DMSO- $d_{6}$ )

R2541442 $\stackrel{\text { I }}{\stackrel{n}{\sim}}$

$$
\stackrel{\infty}{i}
$$

ฟ

$\mathrm{Me}_{\mathrm{F}}^{\mathrm{XO}_{\mathrm{F}} \mathrm{H}}$

$\int$

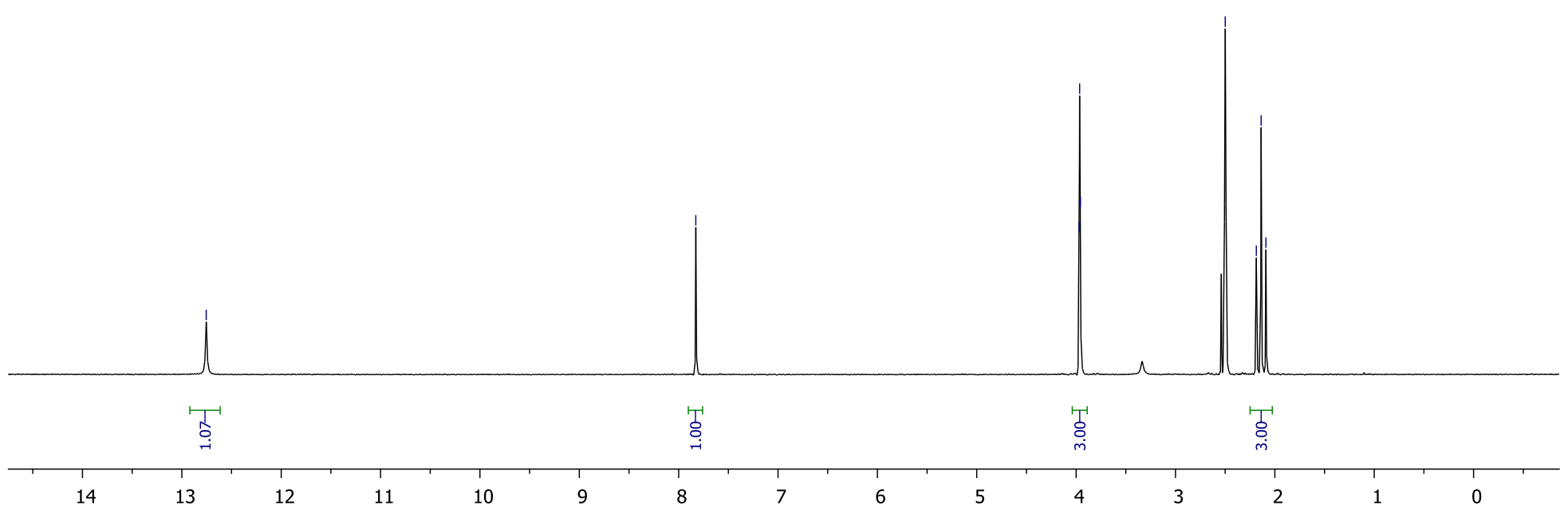


${ }^{13} \mathrm{C}\left\{{ }^{1} \mathrm{H}\right\}$ NMR (126 MHz, DMSO- $\left.d_{6}\right)$

R2541442_13c

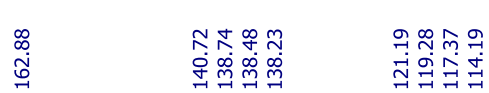

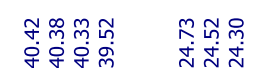

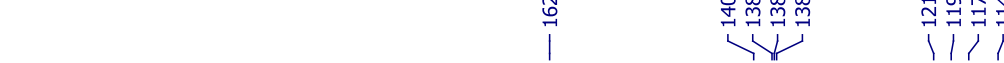

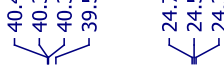

$$
{ }_{\text {Me }}^{N_{N}^{2}} \int_{\text {Me }}^{\mathrm{CO}_{2} \mathrm{H}}
$$
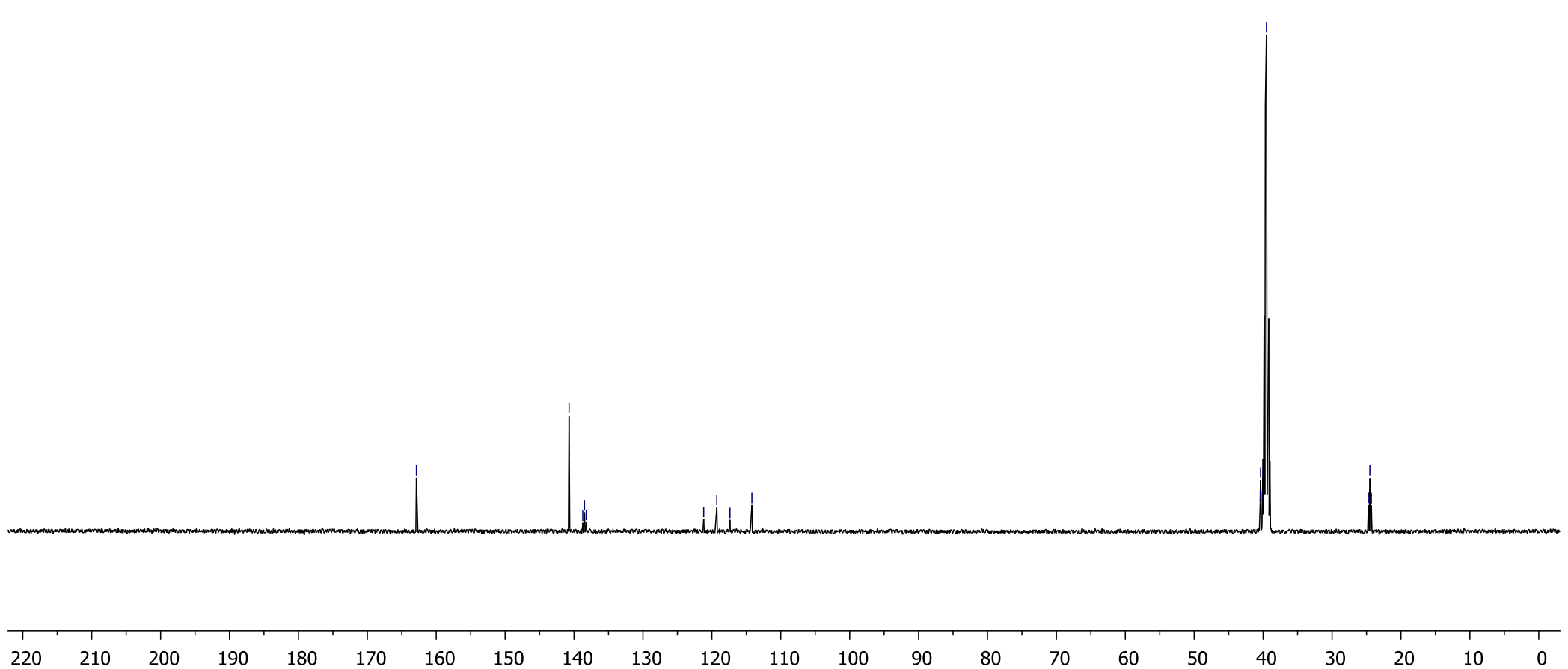


\section{${ }^{19} \mathrm{~F}\left\{{ }^{1} \mathrm{H}\right\}$ NMR (376 MHz, DMSO- $\left.d_{6}\right)$}

R2541442_F19\{H\}

$19 \mathrm{~F}-\{1 \mathrm{H}\}$
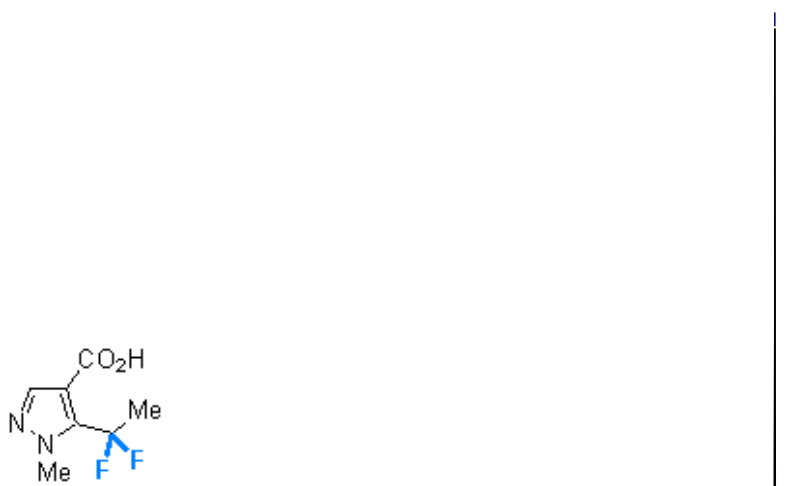

$$
110
$$




\section{Compound 74}

${ }^{1} \mathrm{H}$ NMR $\left(400 \mathrm{MHz}, \mathrm{CDCl}_{3}\right)$

R1405868

|

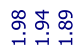

पir

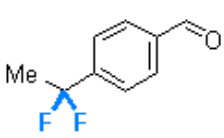

$\iint$

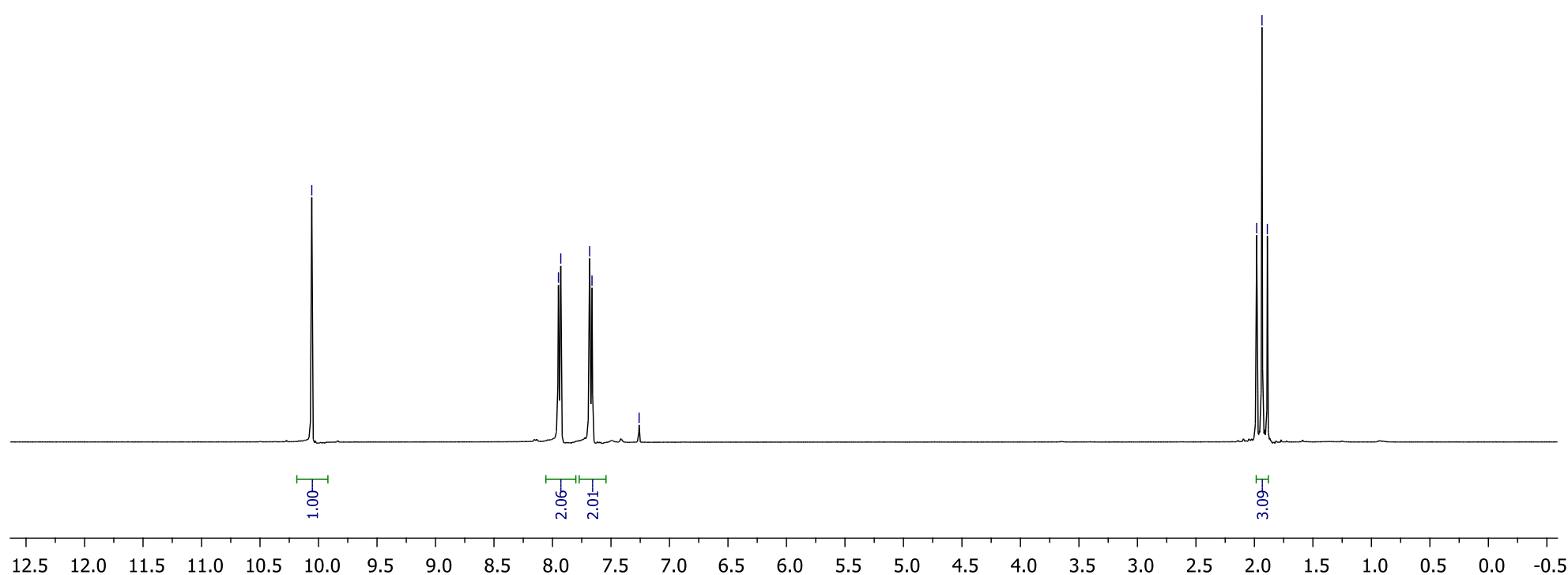


${ }^{13} \mathrm{C}\left\{{ }^{1} \mathrm{H}\right\}$ NMR $\left(151 \mathrm{MHz}, \mathrm{CDCl}_{3}\right)$

R1405868_C13

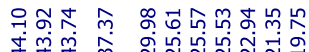

至 V V

$\stackrel{?}{i}$

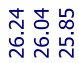

Me

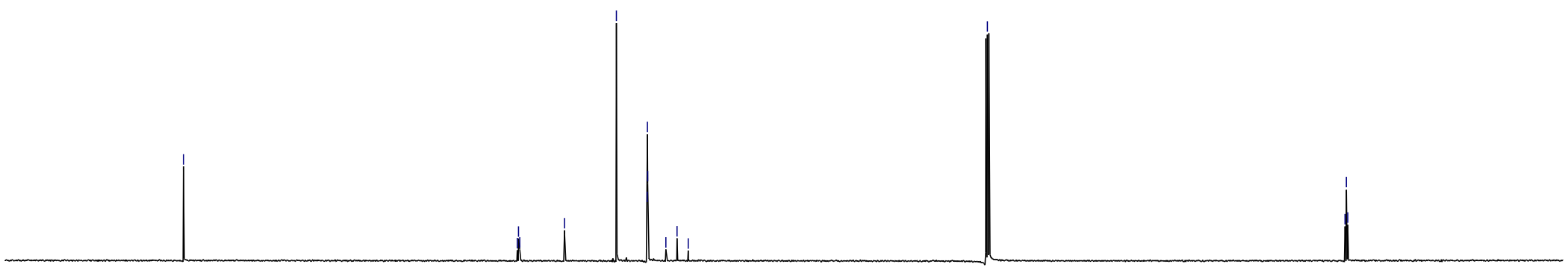

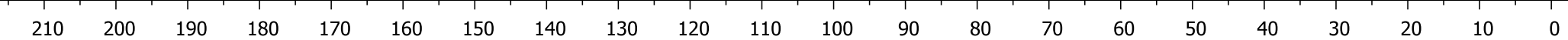




\section{${ }^{19} \mathrm{~F}\left\{{ }^{1} \mathrm{H}\right\} \mathrm{NMR}\left(376 \mathrm{MHz}, \mathrm{CDCl}_{3}\right)$}

R1405868_F19\{H\} $19 \mathrm{~F}-\{1 \mathrm{H}\}$
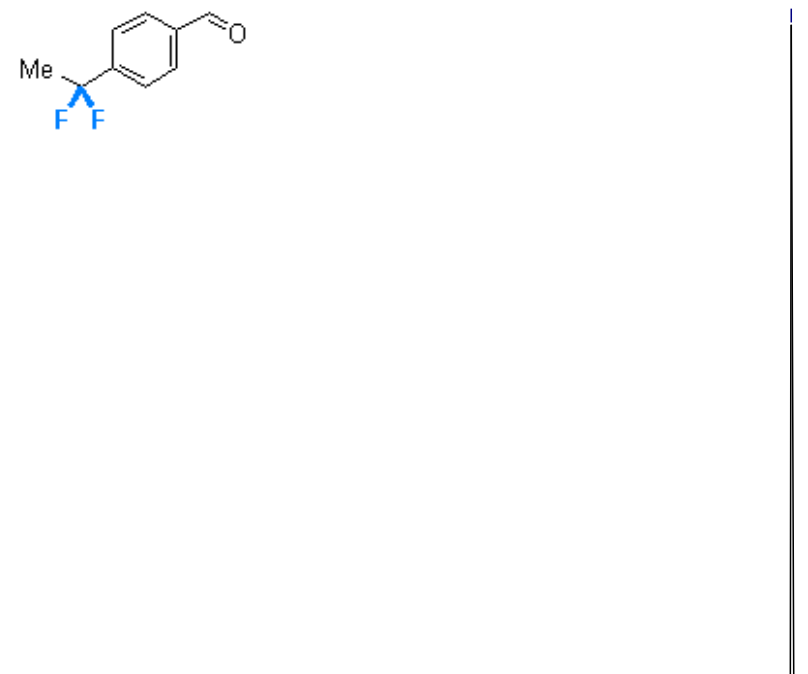


\section{Compound 75}

${ }^{1} \mathrm{H}$ NMR (400 MHz, $\mathrm{CDCl}_{3}$ )

R1418595

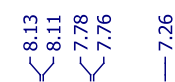

\&

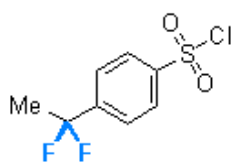

$\iint$
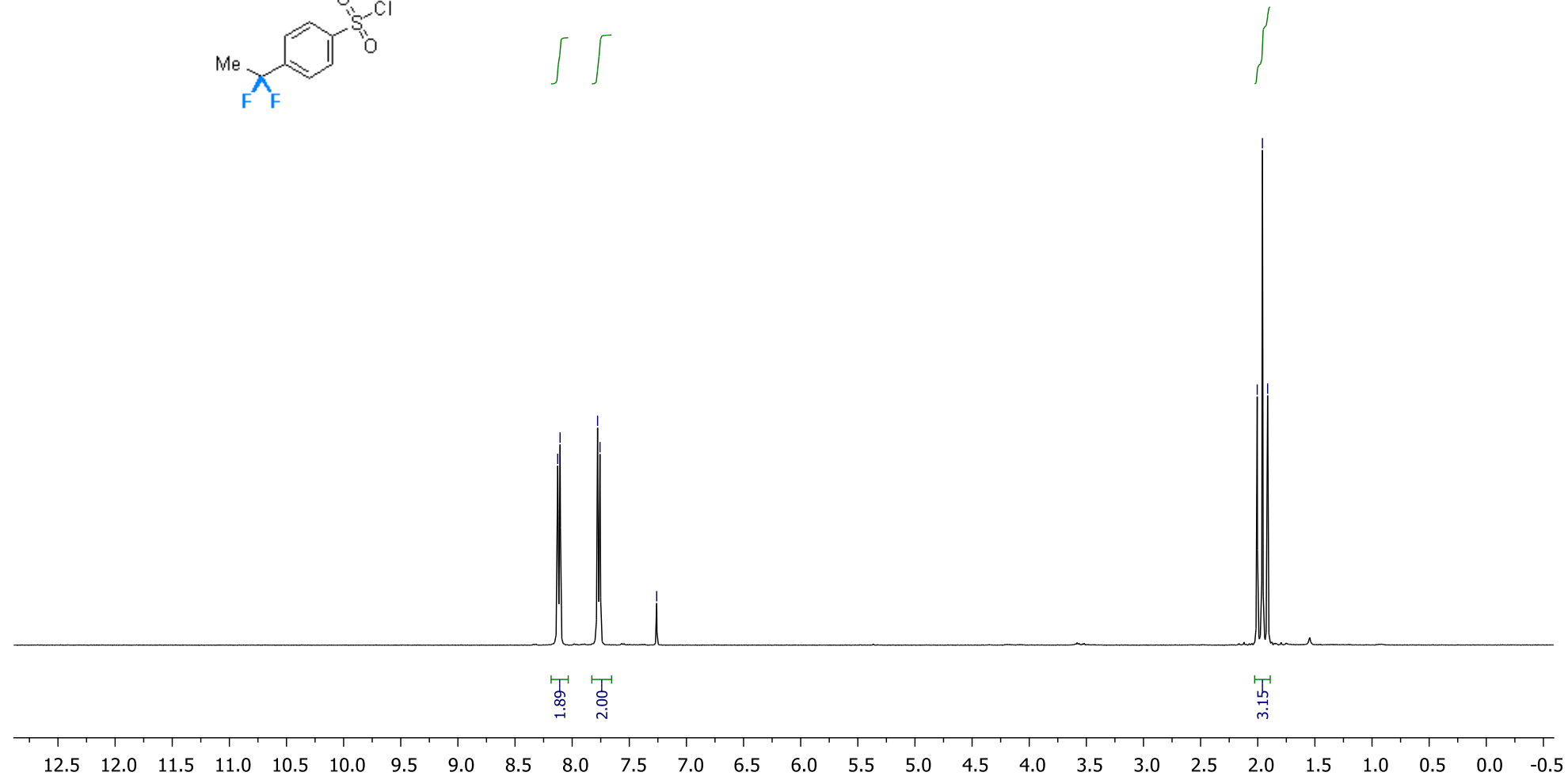
${ }^{13} \mathrm{C}\left\{{ }^{1} \mathrm{H}\right\}$ NMR $\left(151 \mathrm{MHz}, \mathrm{CDCl}_{3}\right)$

R1418595_C13

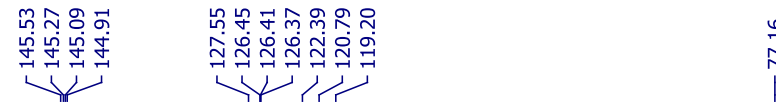

年

年

$\stackrel{\substack{R \\ i}}{i}$

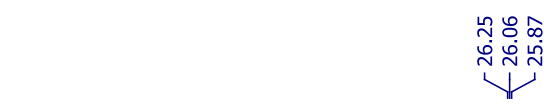

(l)

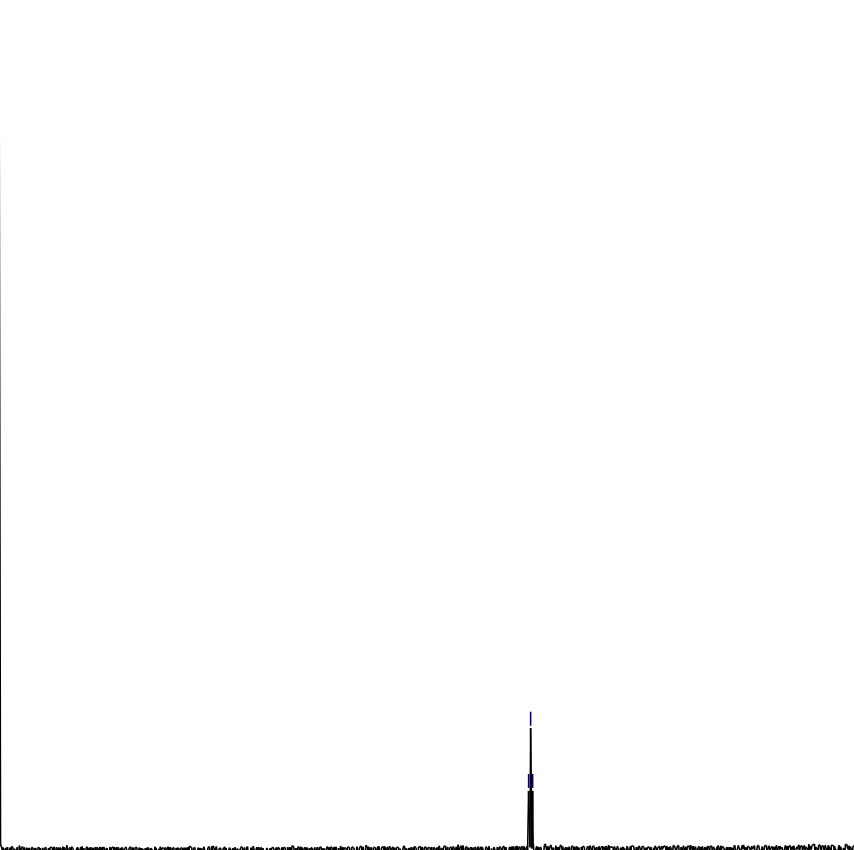

$190 \quad 180$

$170 \quad 160$

$150 \quad 140$

$130 \quad 120$

$110 \quad 100$

$90 \quad 80$

$70 \quad 60$

$50 \quad 40$

$30 \quad 20$

$10 \quad 0$ 


\section{${ }^{19} \mathrm{~F}\left\{{ }^{1} \mathrm{H}\right\} \mathrm{NMR}\left(376 \mathrm{MHz}, \mathrm{CDCl}_{3}\right)$}

R1418595_F19\{H\}

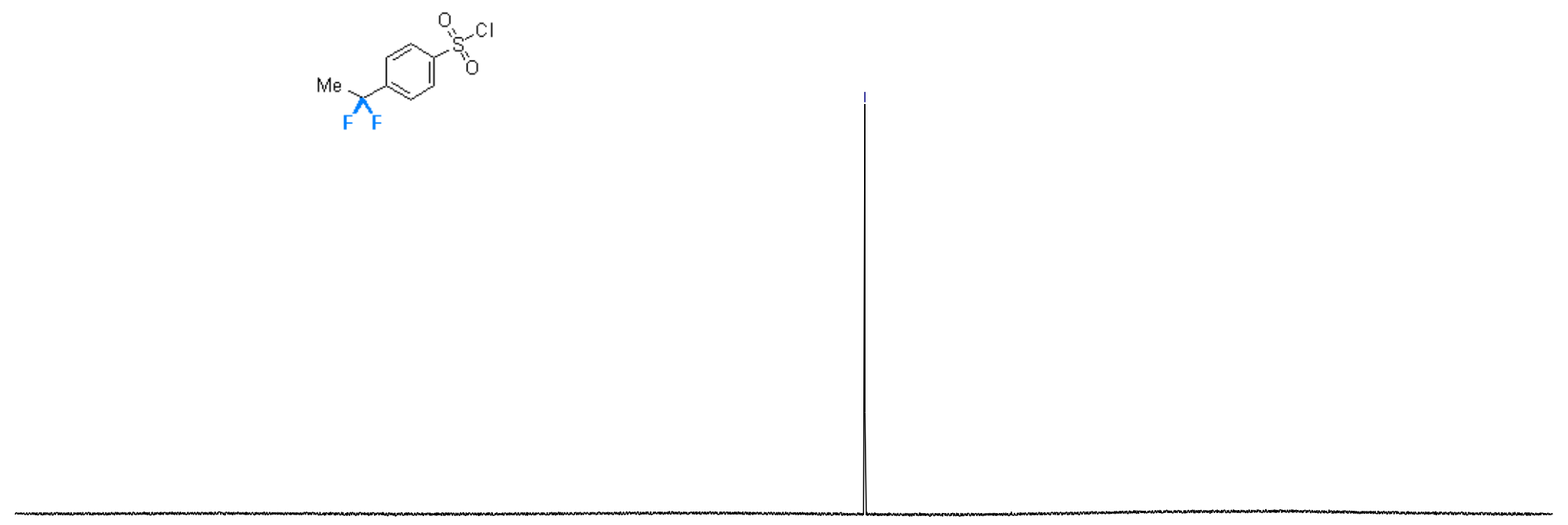




\section{Compound 76}

${ }^{1} \mathrm{H}$ NMR (400 MHz, $\mathrm{CDCl}_{3}$ )

R1406842

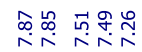

خ้ำ

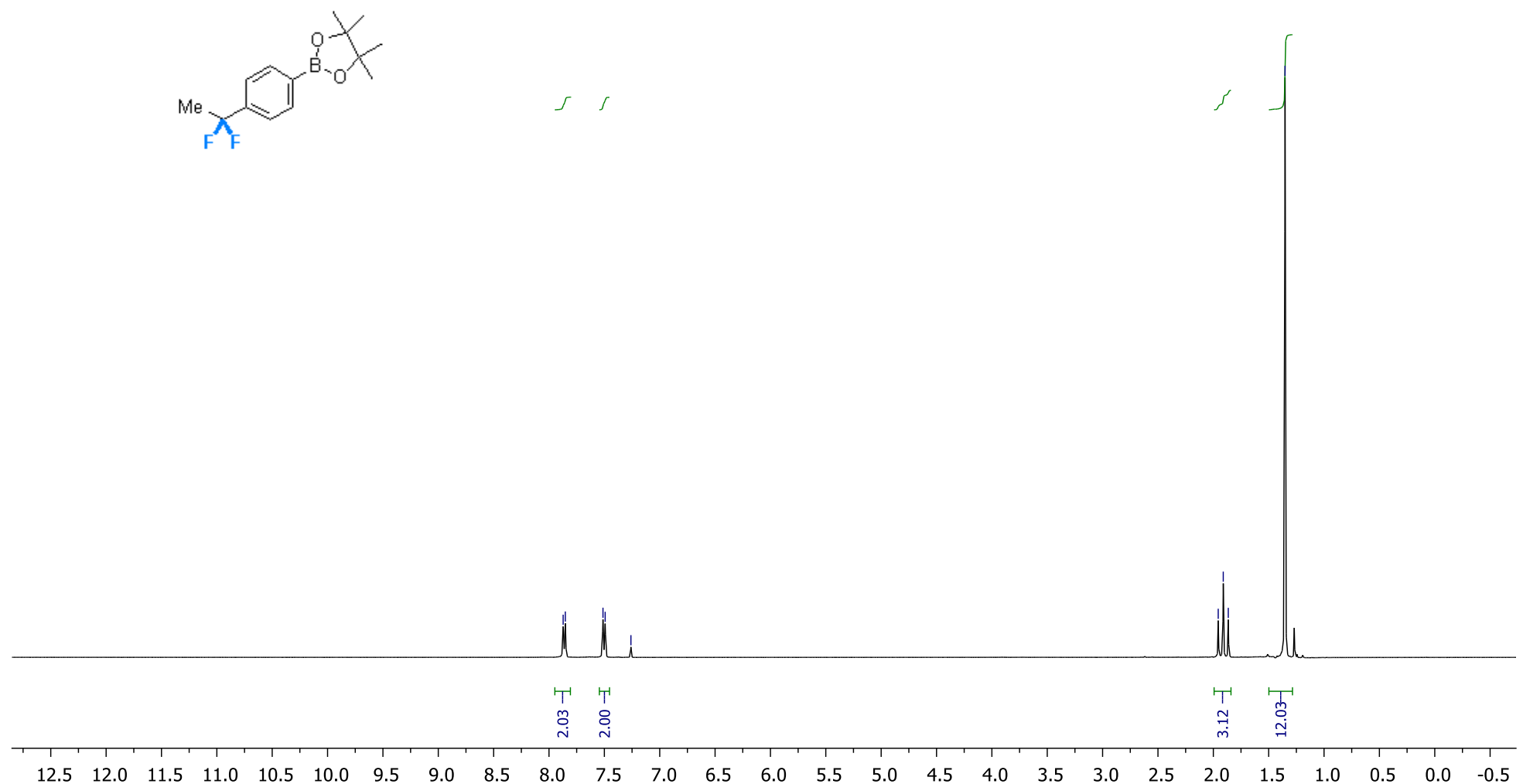


${ }^{13} \mathrm{C}\left\{{ }^{1} \mathrm{H}\right\} \mathrm{NMR}\left(151 \mathrm{MHz}, \mathrm{CDCl}_{3}\right)$

R1406842_13C

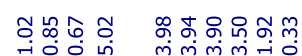

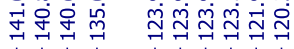

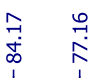

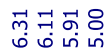

ำำำ
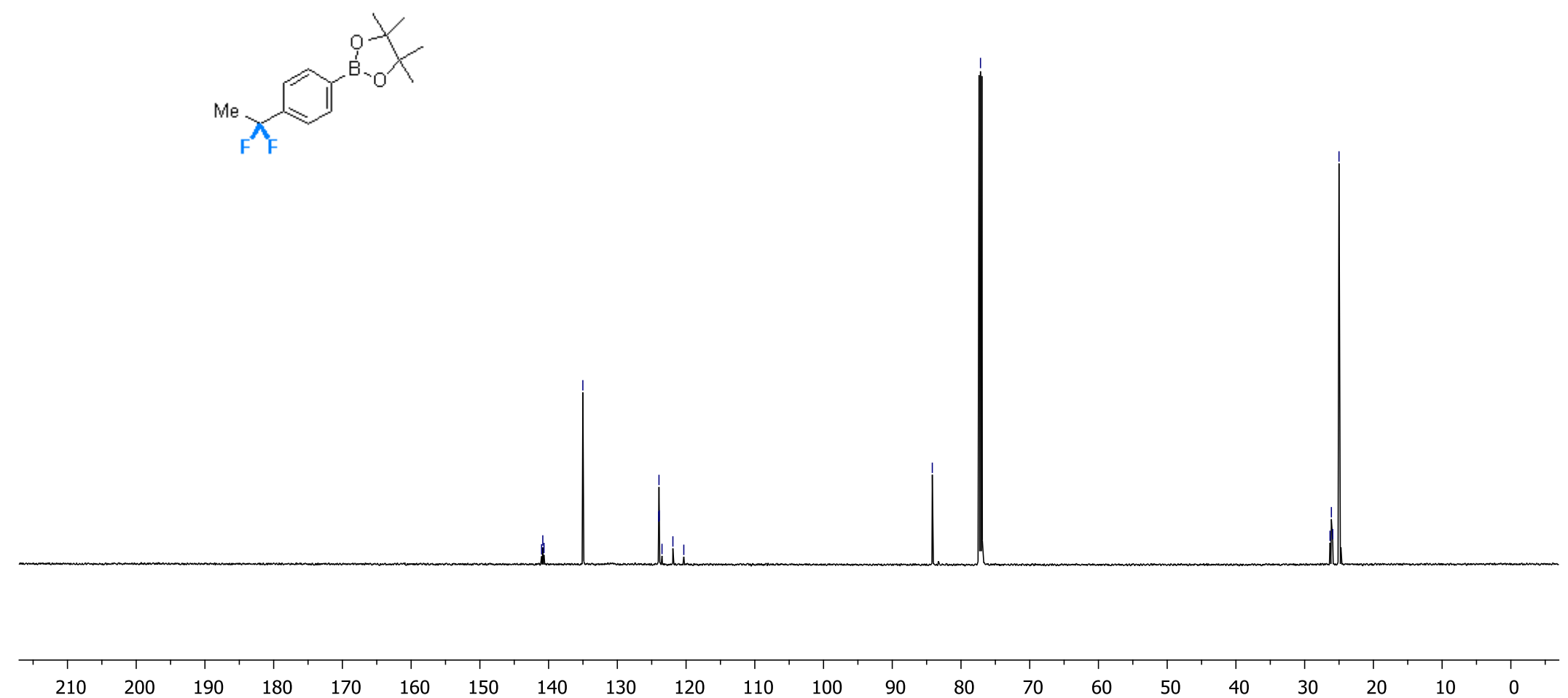


\section{${ }^{19} \mathrm{~F}\left\{{ }^{1} \mathrm{H}\right\}$ NMR (376 MHz, $\left.\mathrm{CDCl}_{3}\right)$}

R1406842_F19\{H\}
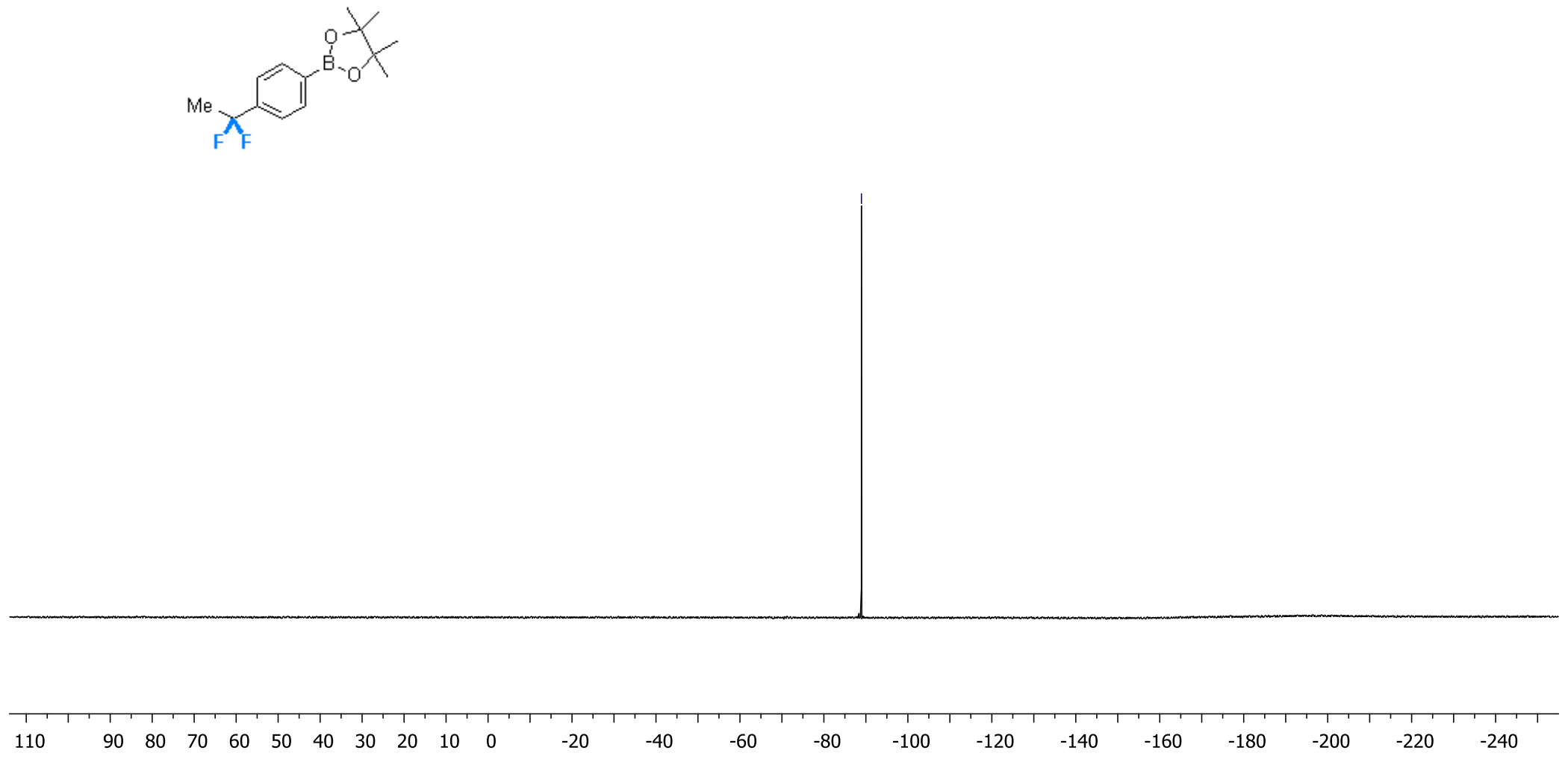


\section{Compound 40b}

${ }^{1} \mathrm{H}$ NMR (400 MHz, $\mathrm{CDCl}_{3}$ )

R1462184

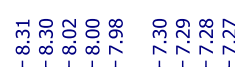

نَّi

${ }_{F}^{F}$
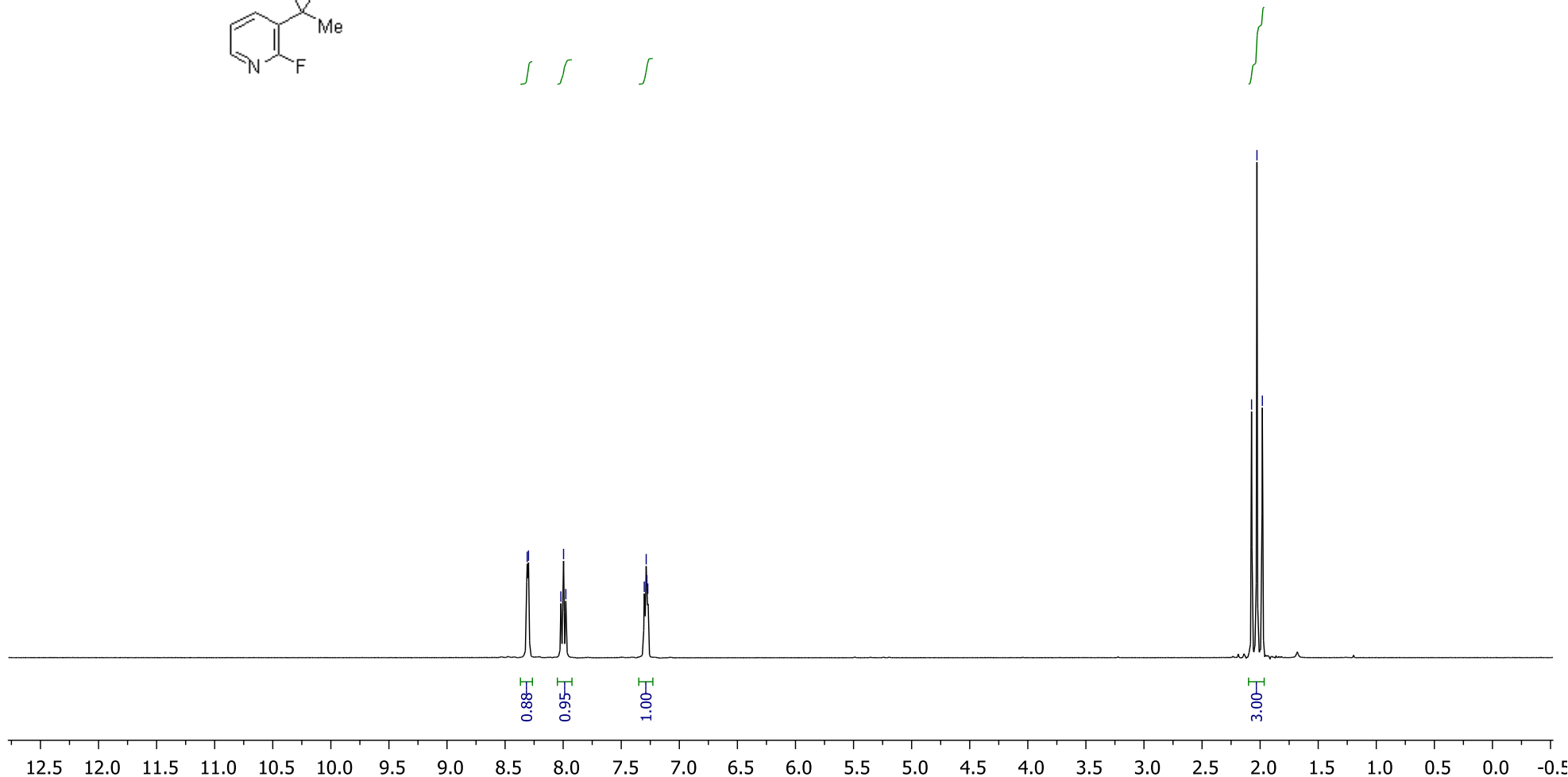
${ }^{13} \mathrm{C}\left\{{ }^{1} \mathrm{H}\right\} \mathrm{NMR}\left(126 \mathrm{MHz}, \mathrm{CDCl}_{3}\right)$

R1462184_C1:

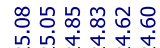
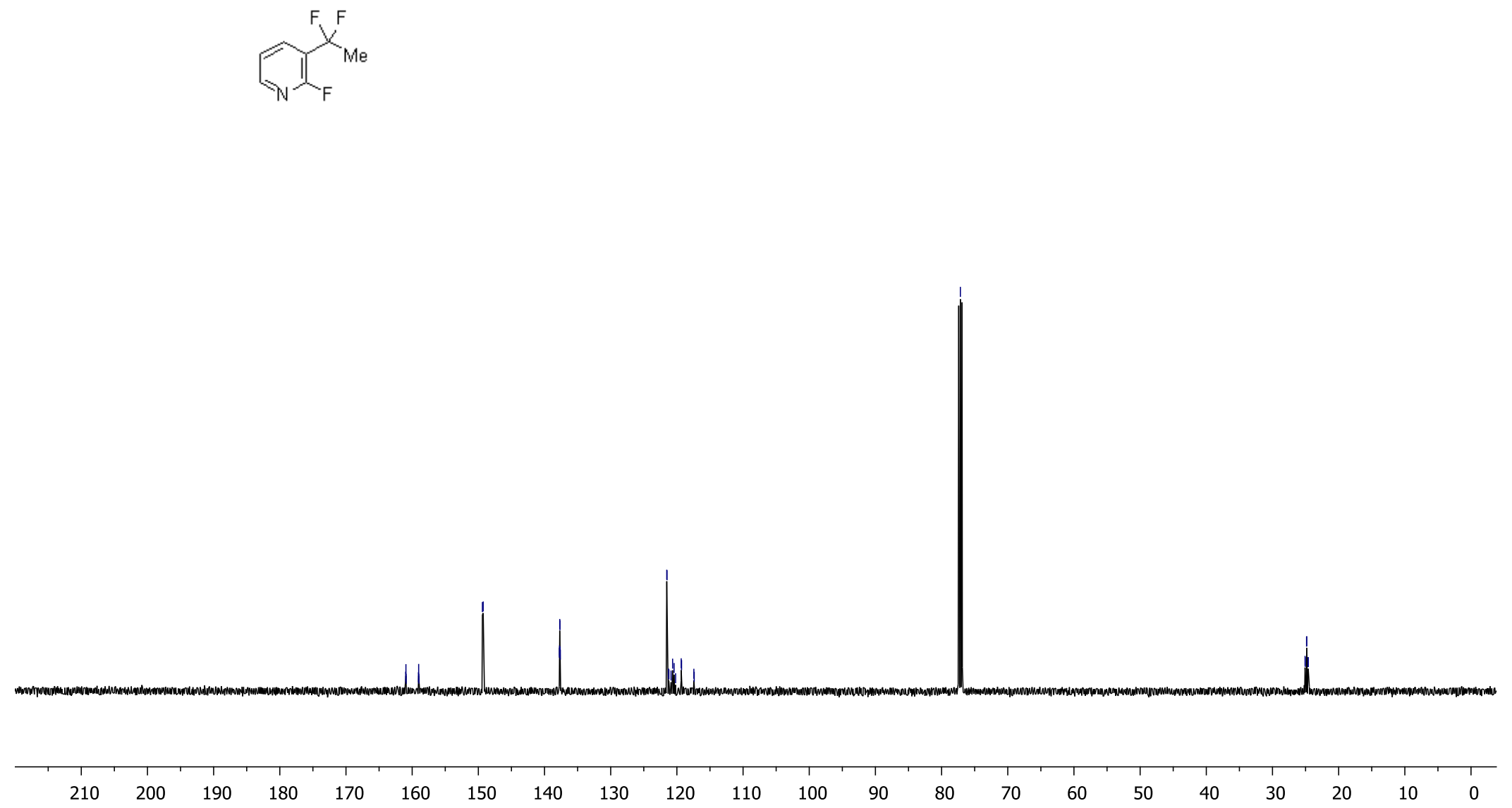
${ }^{19} \mathrm{~F}\left\{{ }^{1} \mathrm{H}\right\}$ NMR (376 MHz, $\left.\mathrm{CDCl}_{3}\right)$

R1462184_F19 $\{\mathrm{H}\}$

$19 \mathrm{~F}-\{1 \mathrm{H}\}$

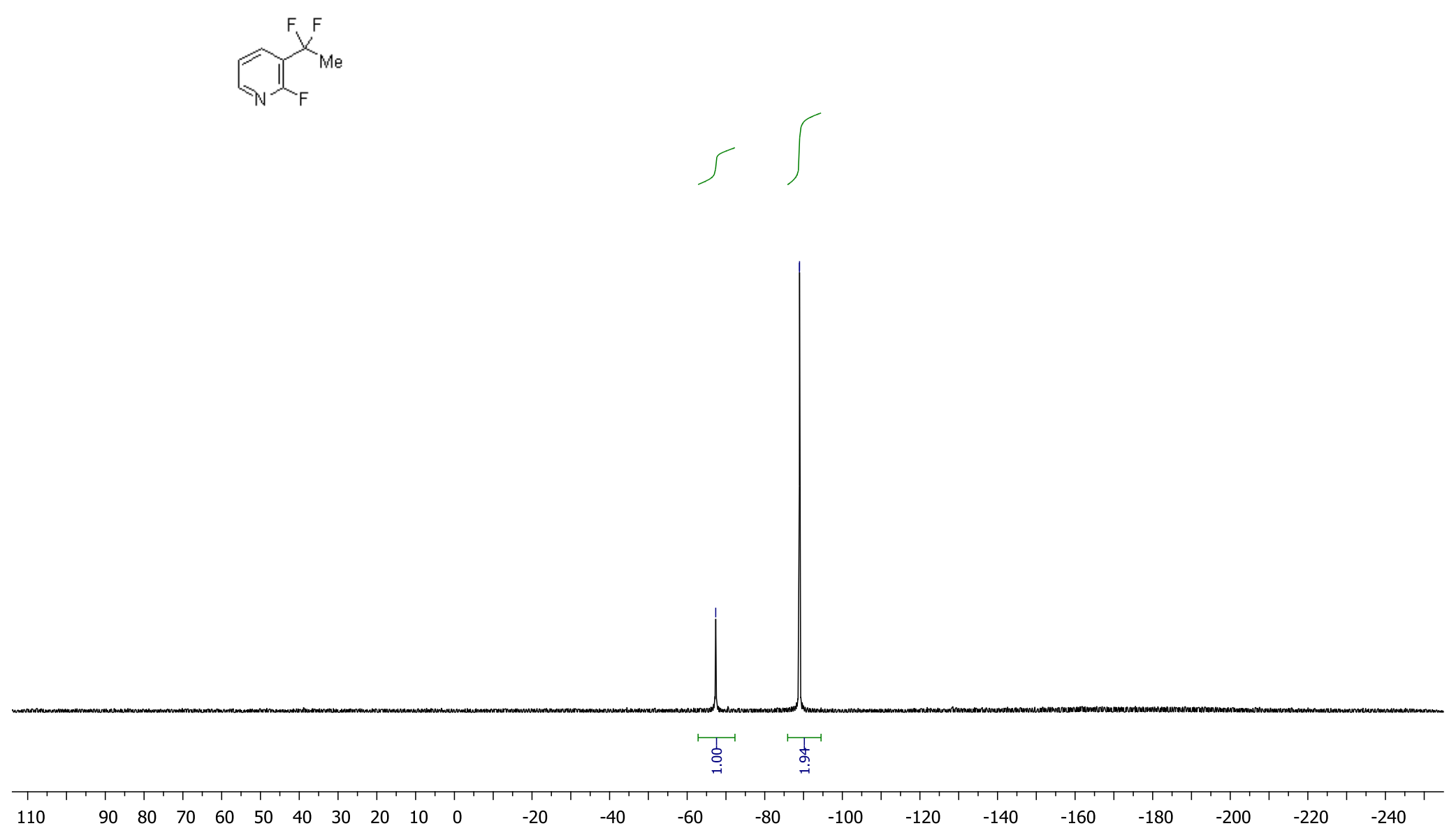

के 


\section{Compound 44b}

${ }^{1} \mathrm{H}$ NMR (400 MHz, $\mathrm{CDCl}_{3}$ )

R1439761

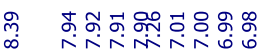

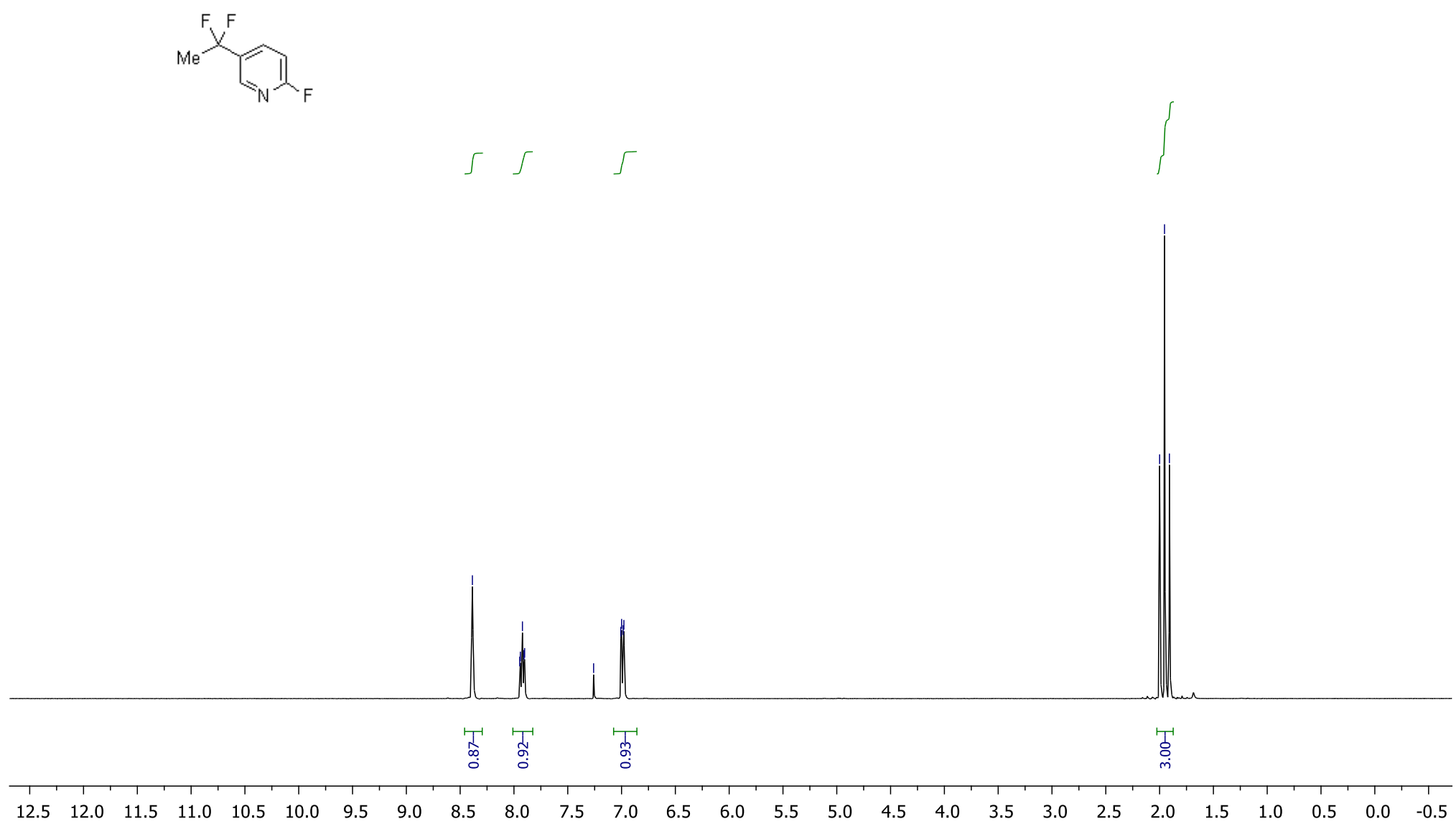<smiles>C[C@H]1[C@@H]2C[C@@H]1C2</smiles> 
${ }^{13} \mathrm{C}\left\{{ }^{1} \mathrm{H}\right\}$ NMR (151 MHz, $\left.\mathrm{CDCl}_{3}\right)$

R1439761_C13
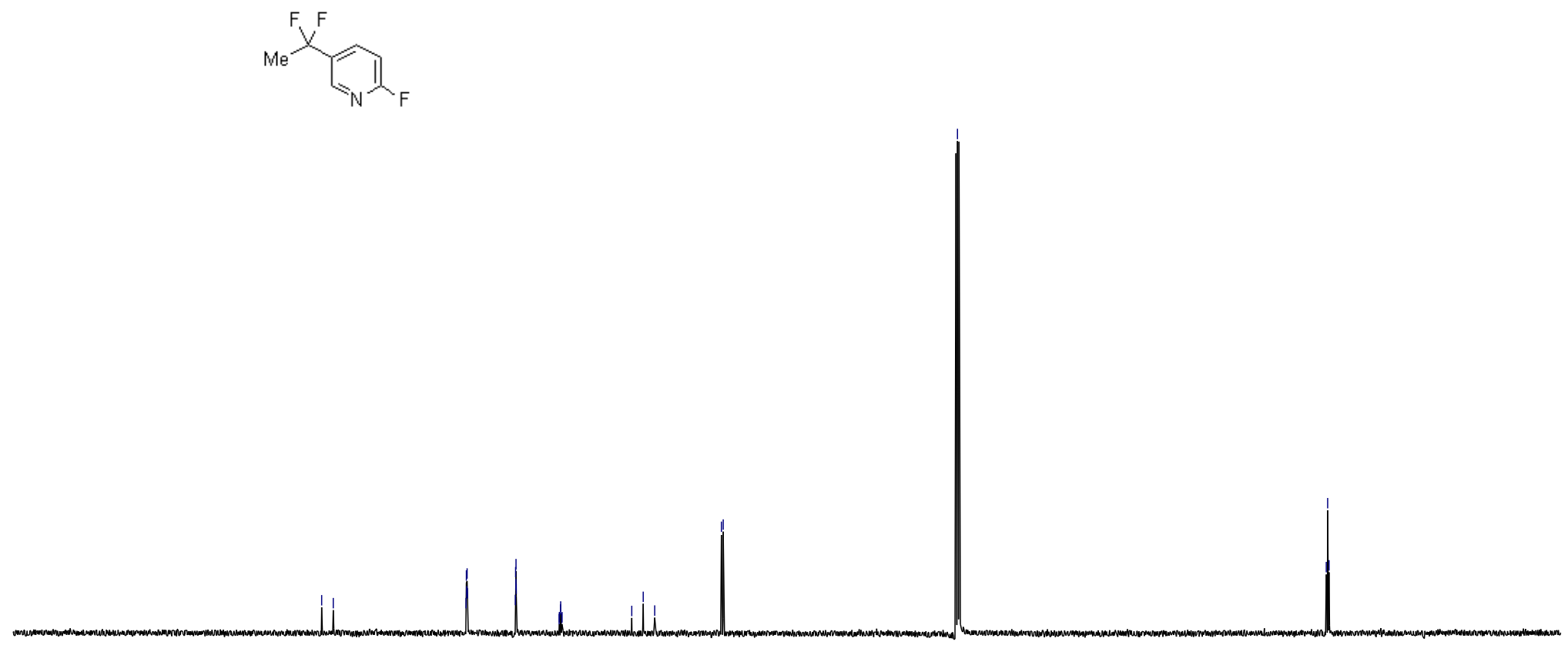
${ }^{19} \mathrm{~F}\left\{{ }^{1} \mathrm{H}\right\} \mathrm{NMR}\left(376 \mathrm{MHz}, \mathrm{CDCl}_{3}\right)$

R1439761_F19

$19 \mathrm{~F}-\{1 \mathrm{H}\}$
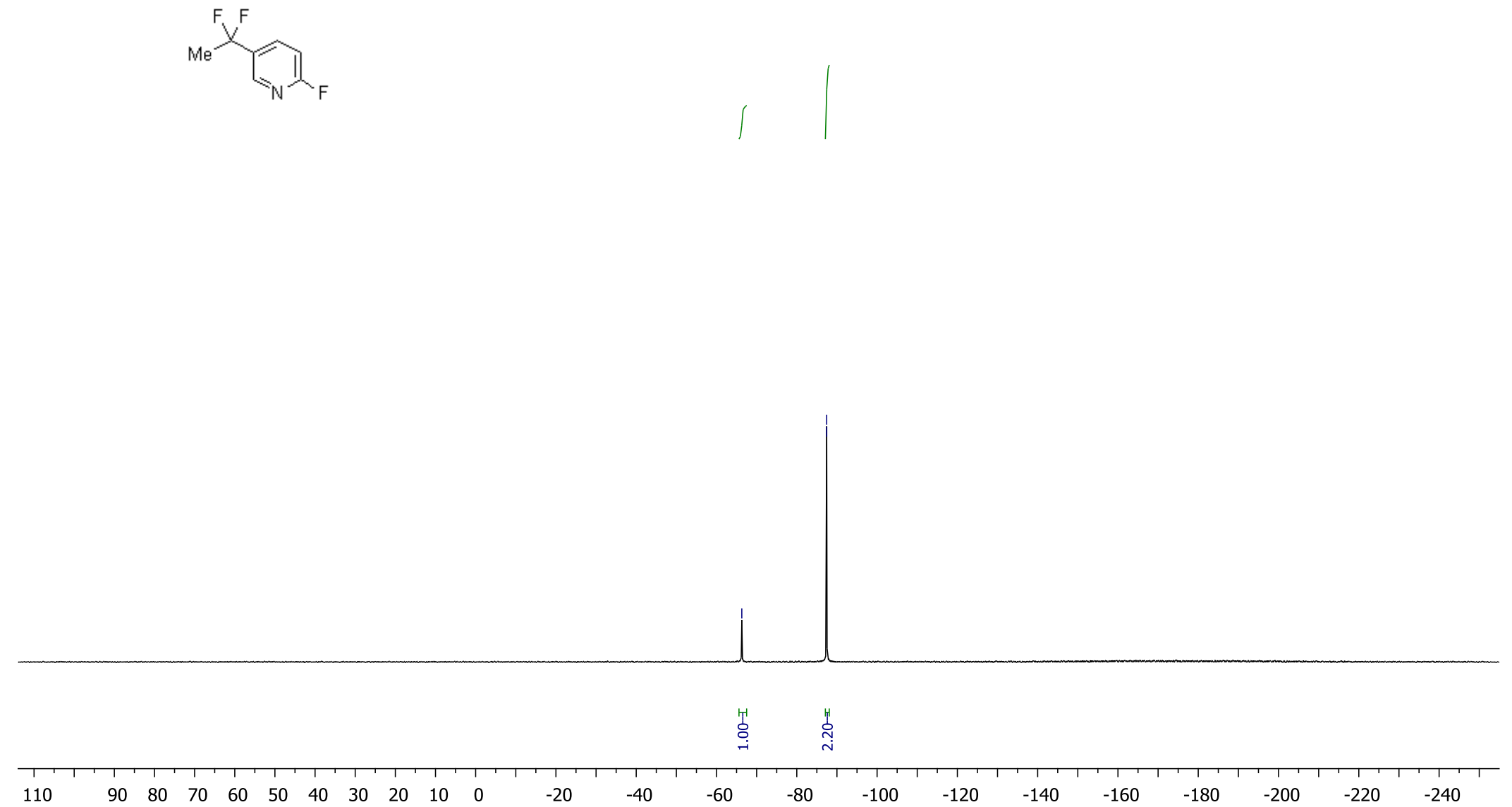


\section{Compound 49b}

${ }^{1} \mathrm{H}$ NMR (400 MHz, $\mathrm{CDCl}_{3}$ )

R2397800

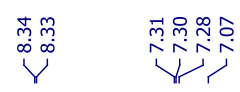

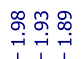

$\underset{F F}{M} x_{F}$

I

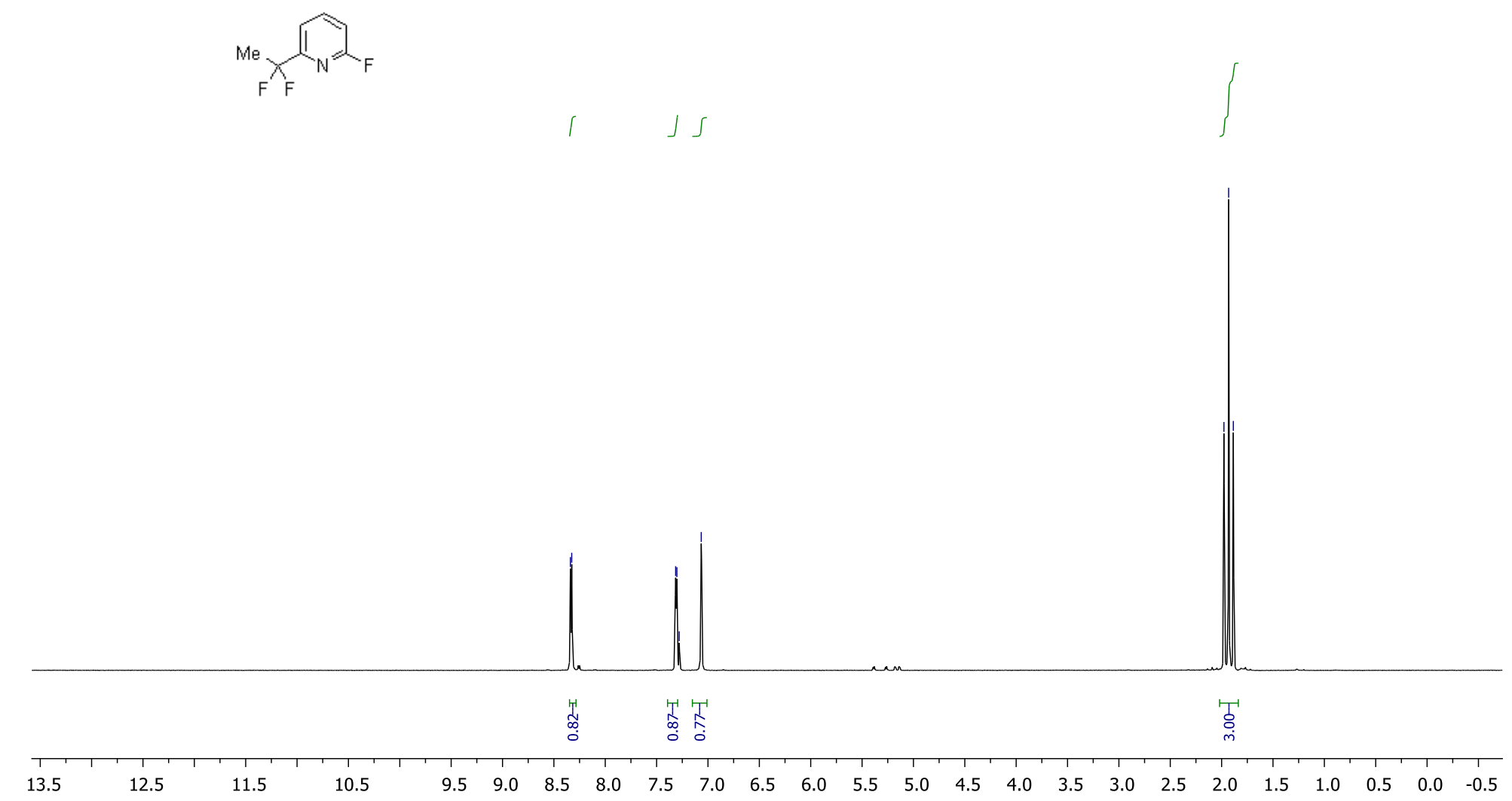


${ }^{13} \mathrm{C}\left\{{ }^{1} \mathrm{H}\right\}$ NMR $\left(151 \mathrm{MHz}, \mathrm{CDCl}_{3}\right)$

R2397800_C13

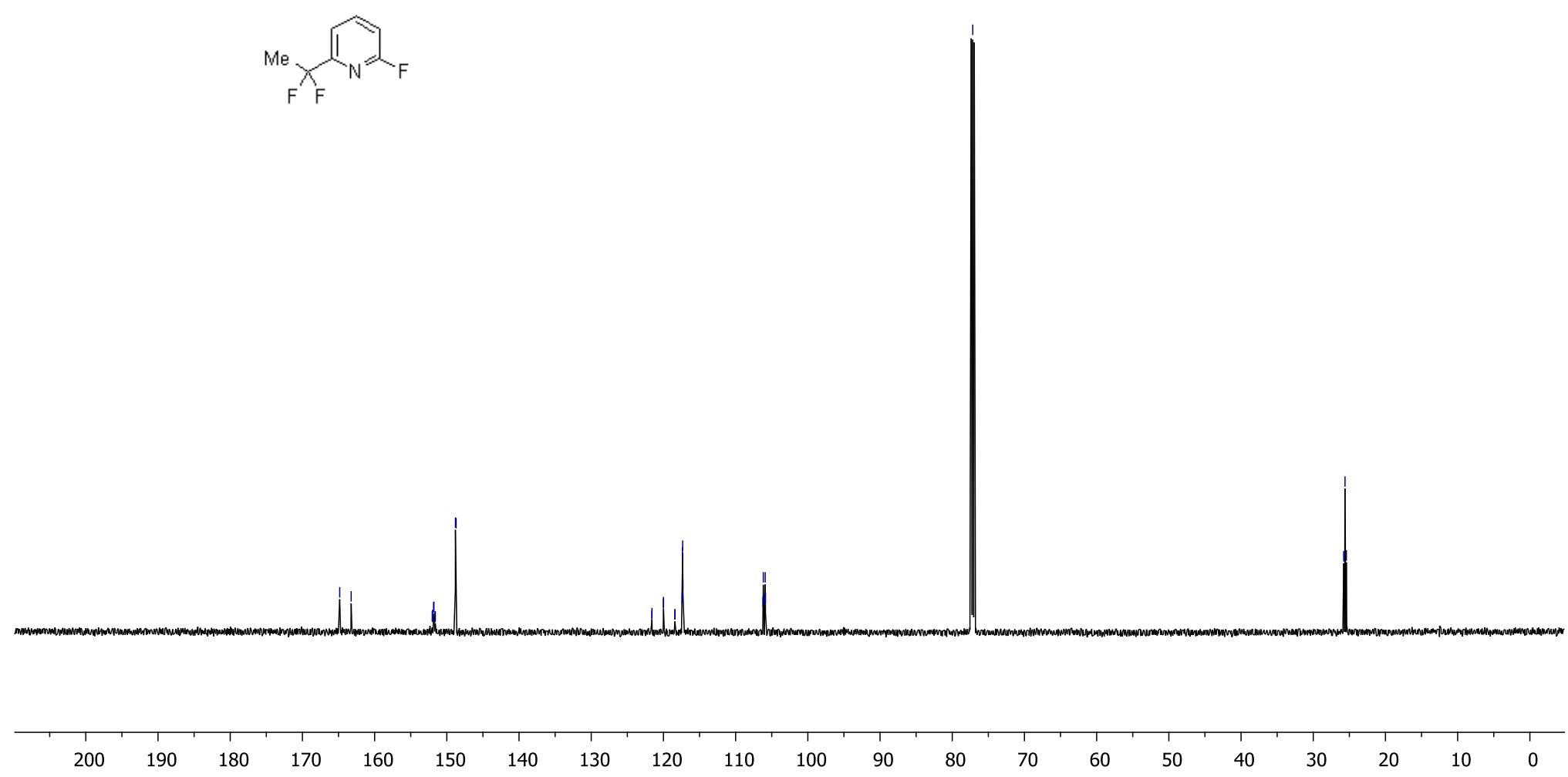




\section{${ }^{19} \mathrm{~F}\left\{{ }^{1} \mathrm{H}\right\} \mathrm{NMR}\left(376 \mathrm{MHz}, \mathrm{CDCl}_{3}\right)$}

R2397800_F19\{H\}

$19 \mathrm{~F}-\{1 \mathrm{H}\}$

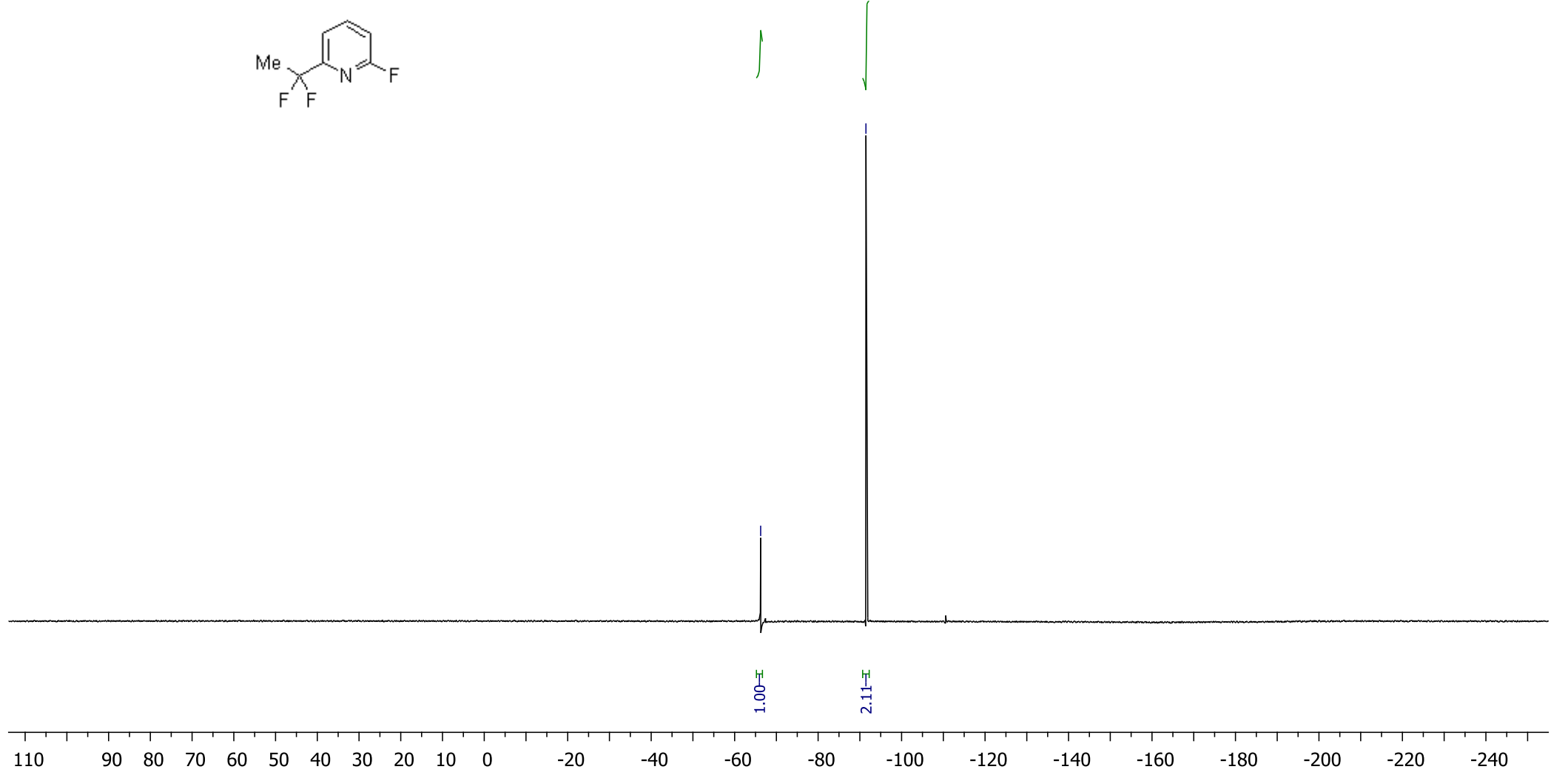




\section{Compound 58b}

${ }^{1} \mathrm{H}$ NMR (400 MHz, $\mathrm{CDCl}_{3}$ )

R1732494

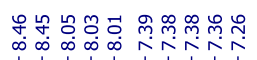

$\overbrace{N}^{C_{2} F_{5}}$
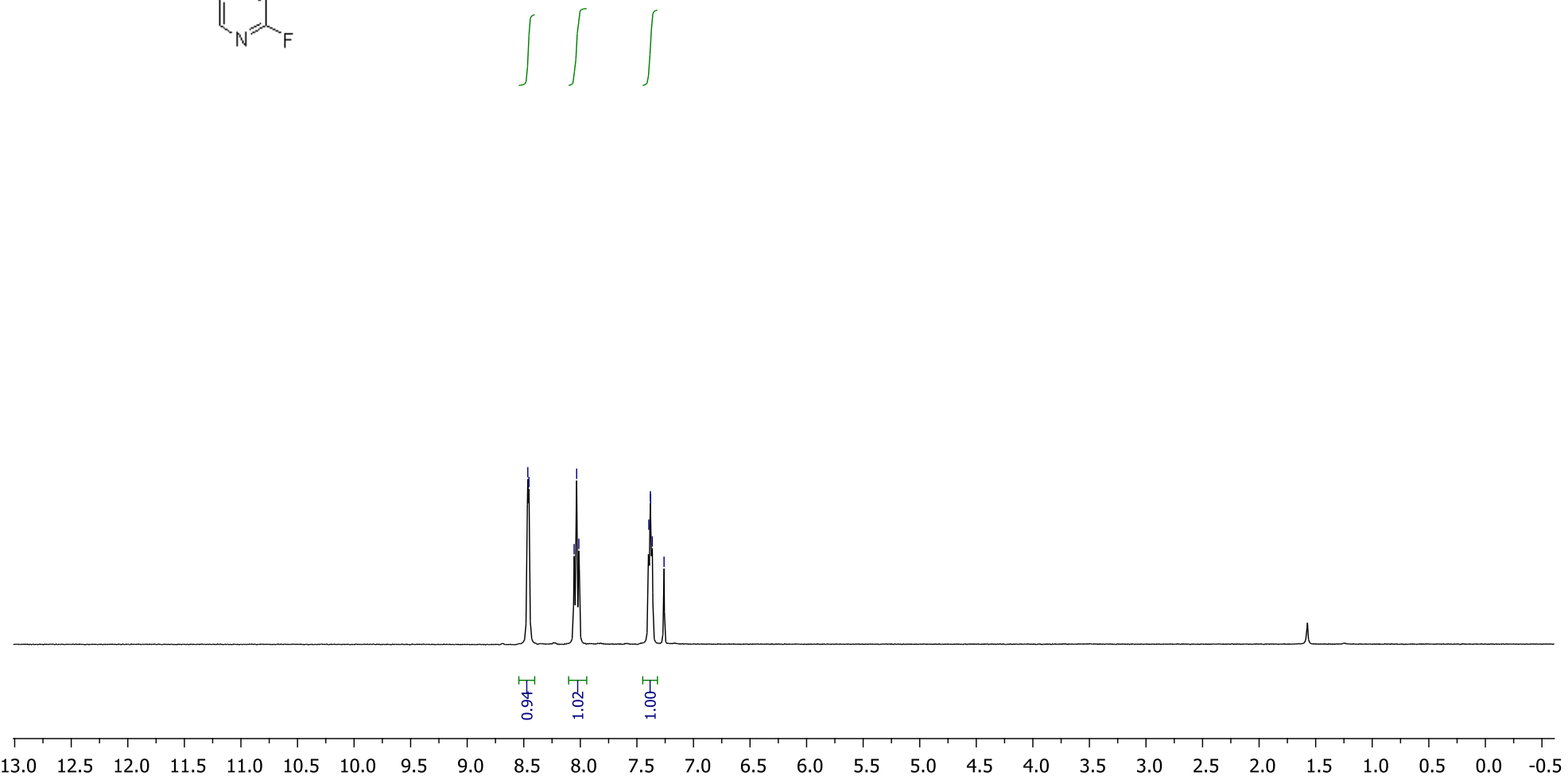
${ }^{13} \mathrm{C}\left\{{ }^{1} \mathrm{H}\right\}$ NMR $\left(126 \mathrm{MHz}, \mathrm{CDCl}_{3}\right)$

R1732494_C1:

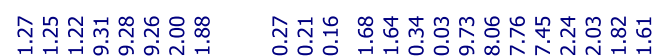

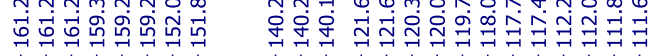

$\stackrel{?}{\stackrel{2}{1}}$

$\overbrace{n}^{C_{2} F_{5}}$

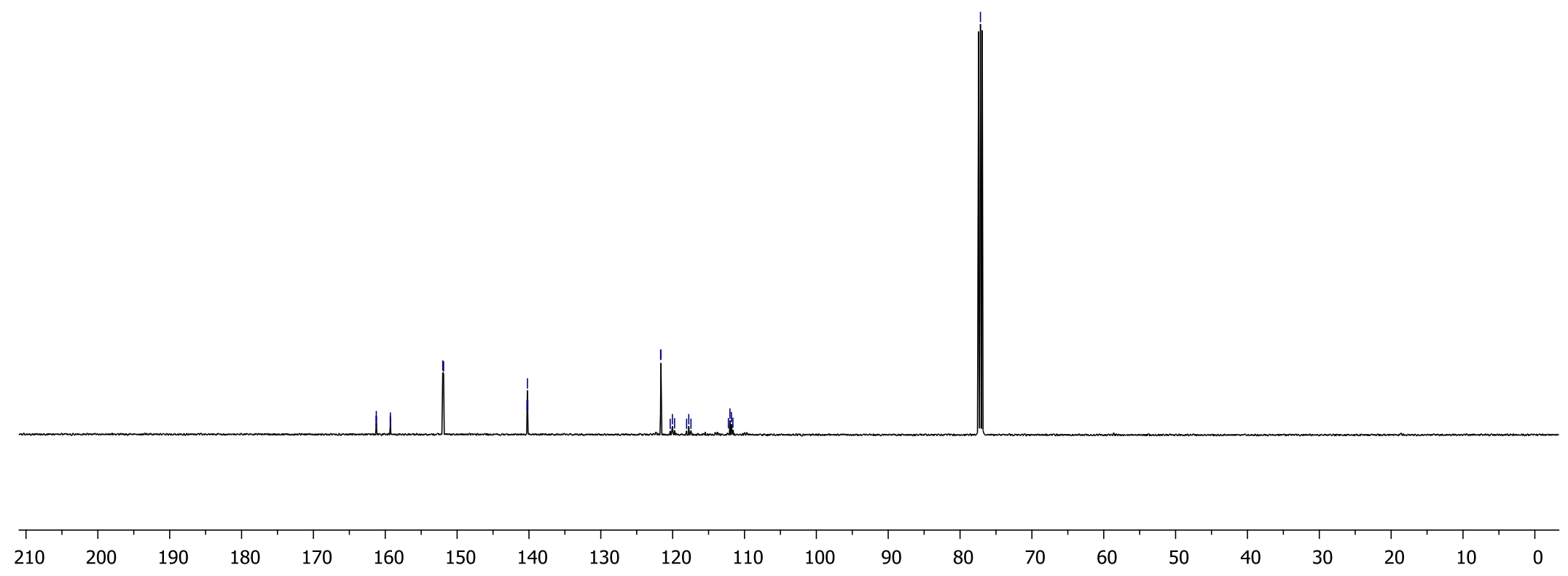


${ }^{19} \mathrm{~F}\left\{{ }^{1} \mathrm{H}\right\} \mathrm{NMR}\left(376 \mathrm{MHz}, \mathrm{CDCl}_{3}\right)$

R1732494_F19

$19 \mathrm{~F}-\{1 \mathrm{H}\}$

|

$\overbrace{\mathrm{N}}^{\mathrm{C}_{2} \mathrm{~F}_{5}}$

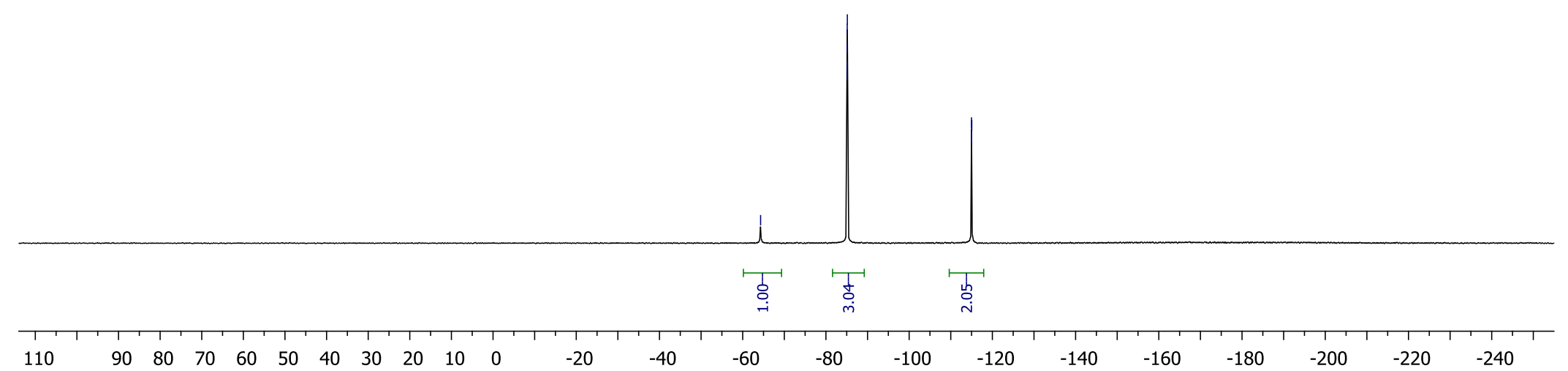


Compound 61b

${ }^{1} \mathrm{H}$ NMR $\left(400 \mathrm{MHz}, \mathrm{CDCl}_{3}\right)$

R1748686

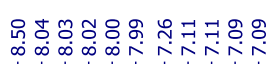

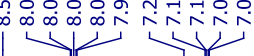

$\mathrm{C}_{2} \mathrm{~F}_{5}$ 等
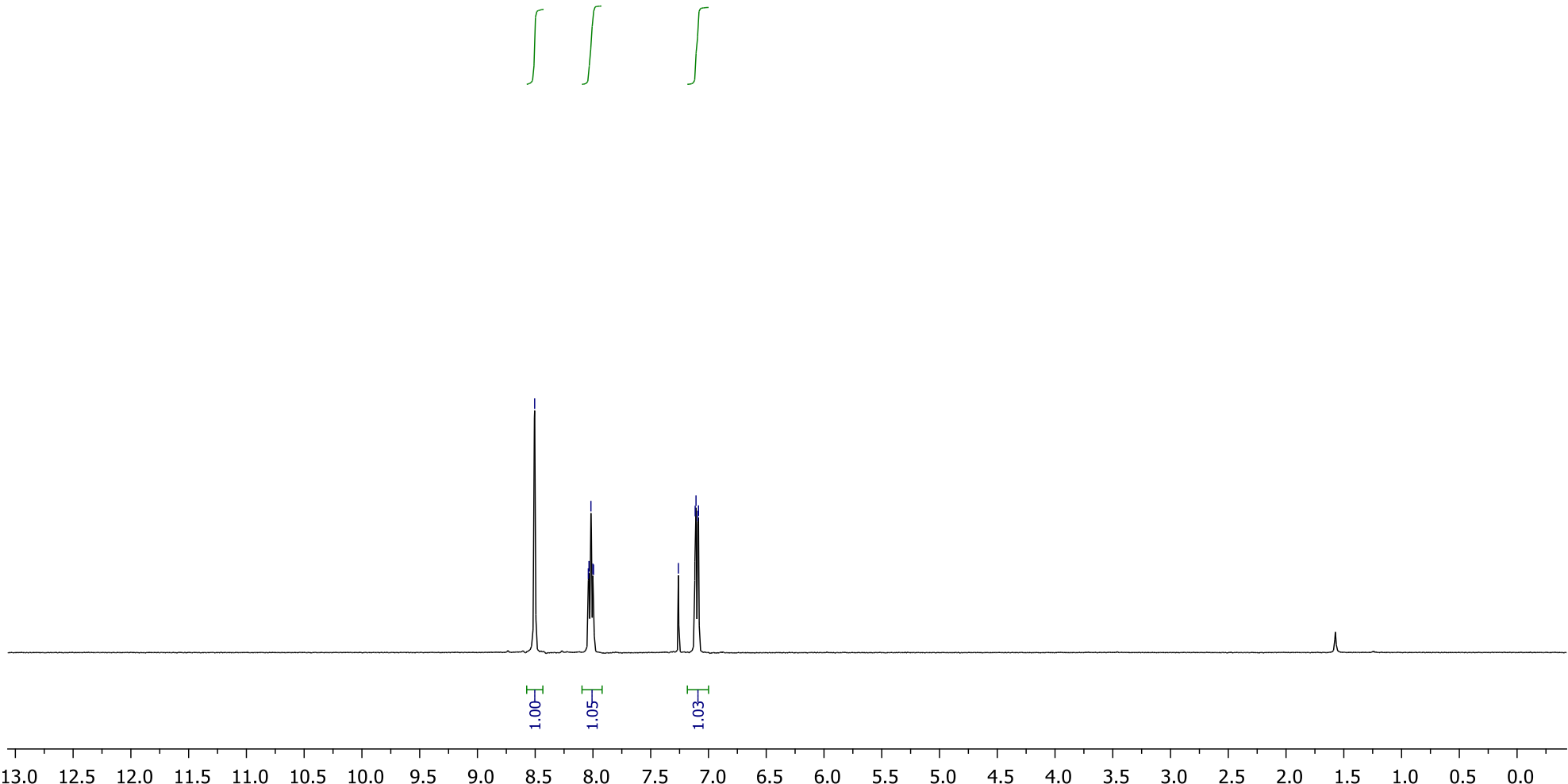
${ }^{13} \mathrm{C}\left\{{ }^{1} \mathrm{H}\right\} \mathrm{NMR}\left(151 \mathrm{MHz}, \mathrm{CDCl}_{3}\right)$

R1748686_13C

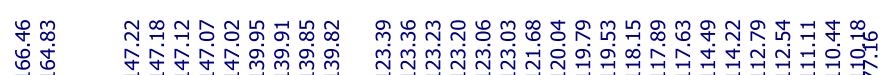

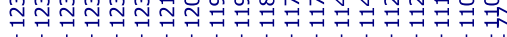<smiles>Fc1ccc(C(F)(F)F)cn1</smiles> 
${ }^{19} \mathrm{~F}\left\{{ }^{1} \mathrm{H}\right\} \mathrm{NMR}\left(376 \mathrm{MHz}, \mathrm{CDCl}_{3}\right)$

R1748686_F19 $\{H\}$<smiles>Fc1ccc(C(F)(F)F)cn1</smiles>

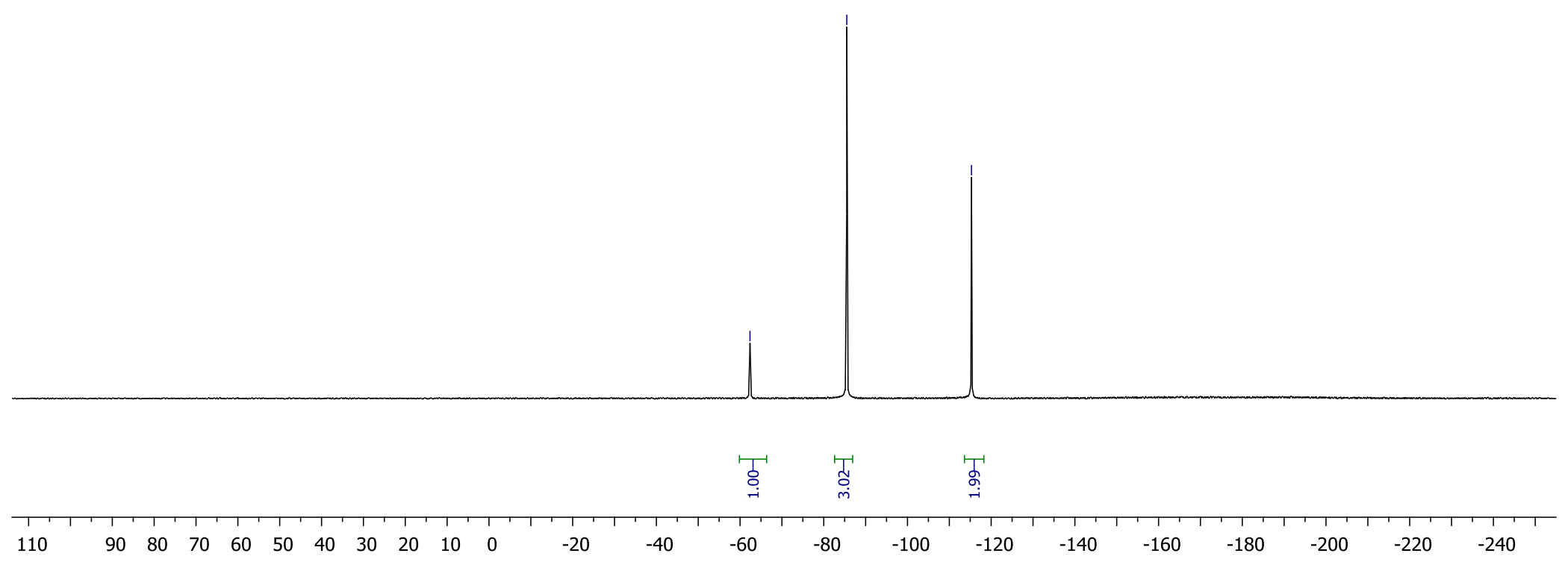




\section{Compound 63b}

${ }^{1} \mathrm{H}$ NMR (400 MHz, $\mathrm{CDCl}_{3}$ )

R2393887

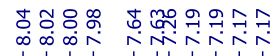

$\mathrm{C}_{2} \mathrm{~F}_{5} \overbrace{\mathrm{N}}$

$\iint$

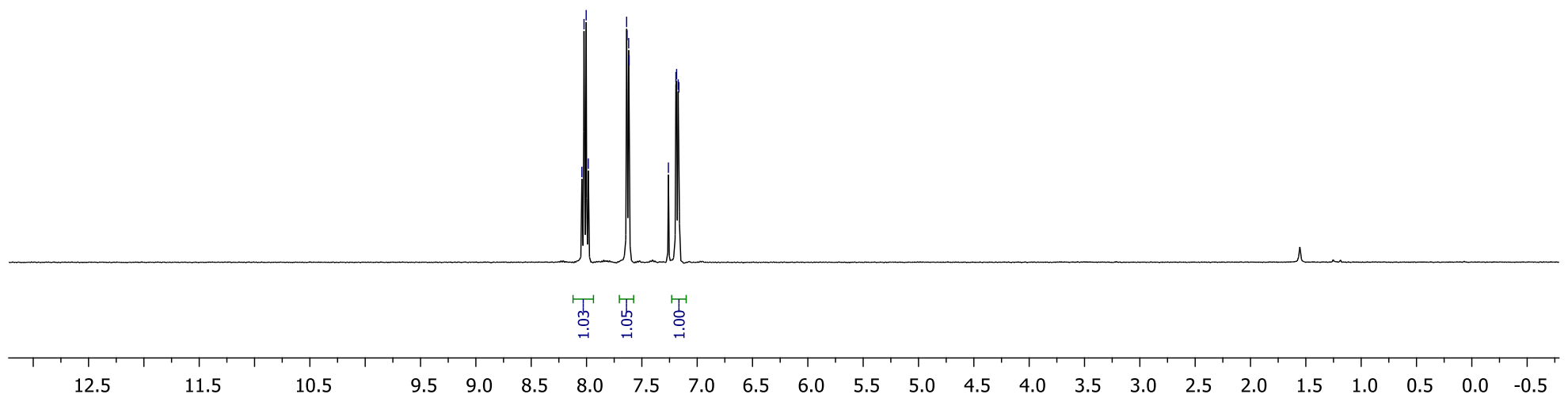


${ }^{13} \mathrm{C}\left\{{ }^{1} \mathrm{H}\right\}$ NMR (126 MHz, $\left.\mathrm{CDCl}_{3}\right)$

R2393887_13C

$13 \mathrm{C}$ (1H-decoupled)

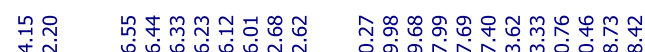

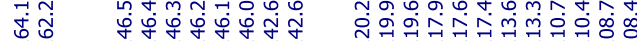

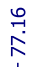
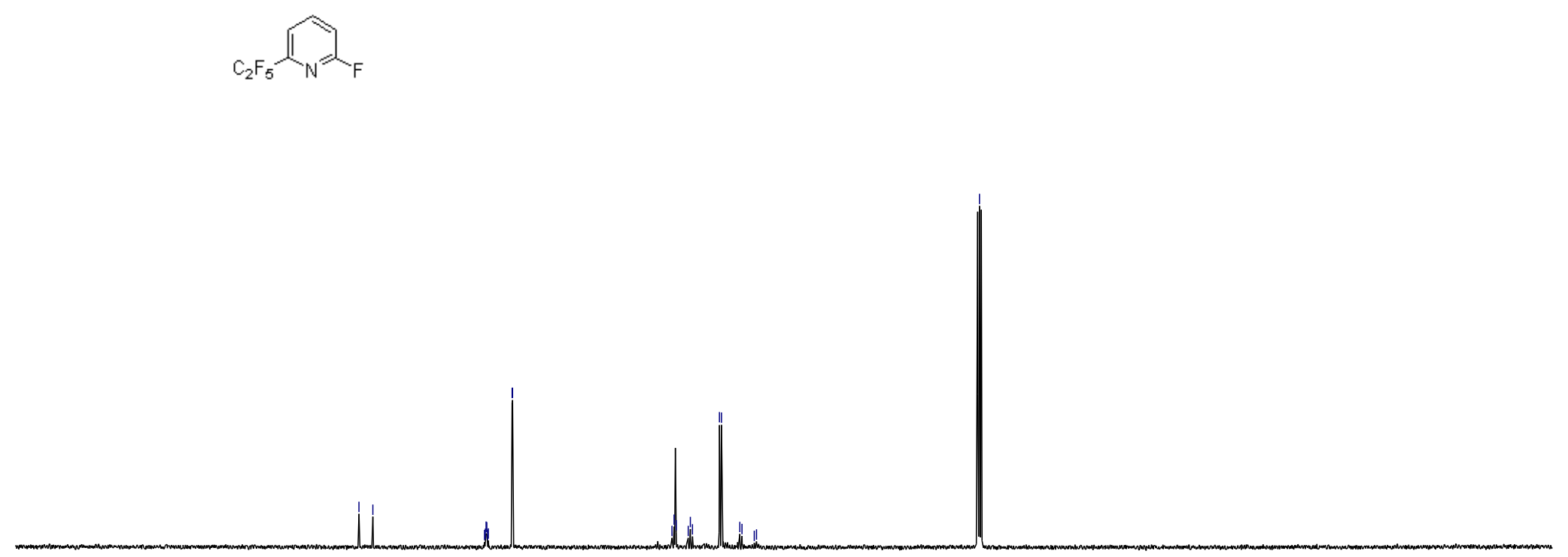
${ }^{19} \mathrm{~F}\left\{{ }^{1} \mathrm{H}\right\} \mathrm{NMR}\left(376 \mathrm{MHz}, \mathrm{CDCl}_{3}\right)$

R2393887_F19\{H\}

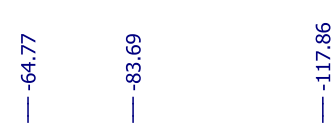

$\mathrm{C}_{2} \mathrm{~F}_{\mathrm{N}}$
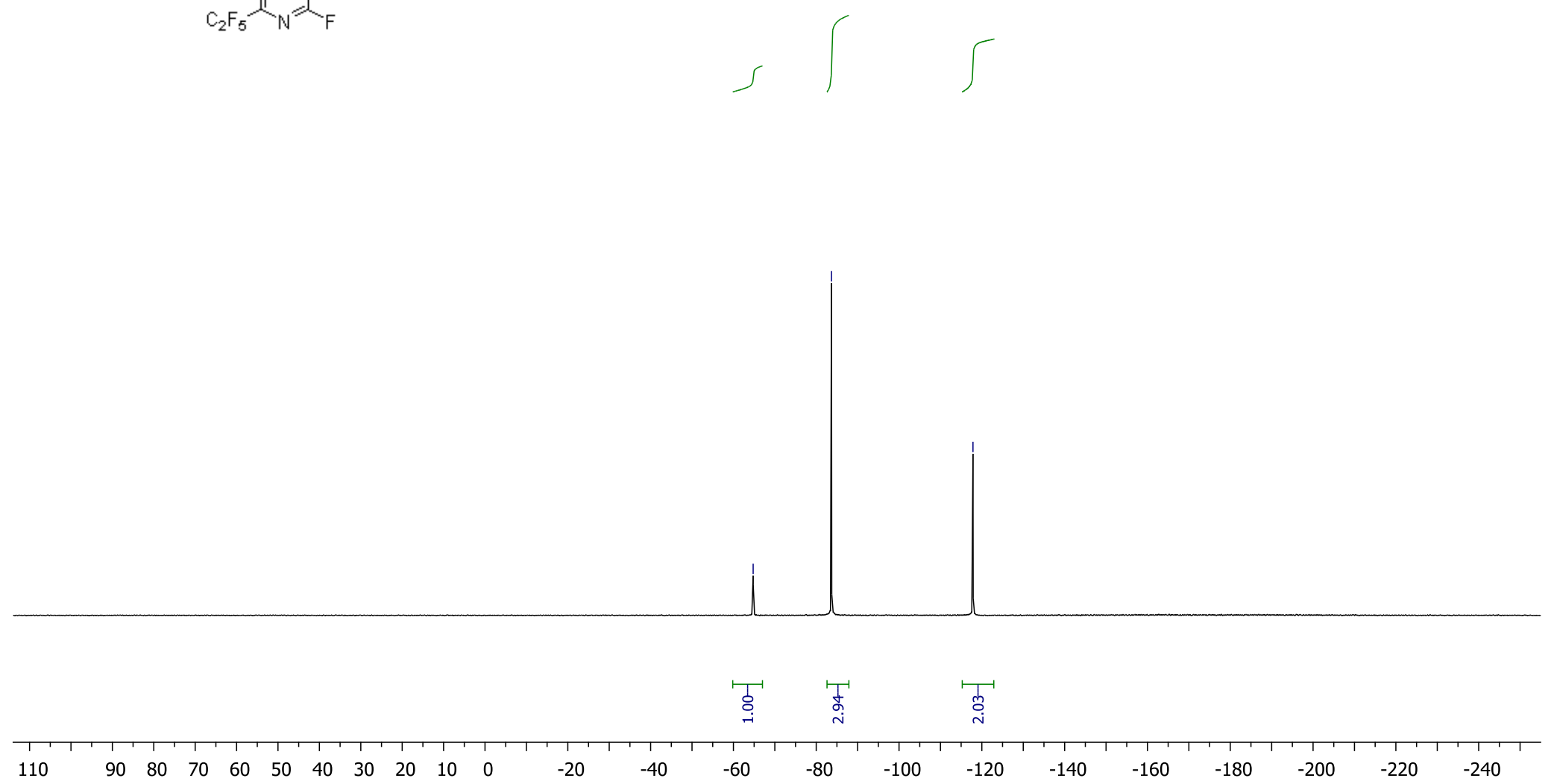
Compound 7c

${ }^{1} \mathrm{H}$ NMR $\left(400 \mathrm{MHz}, \mathrm{CDCl}_{3}\right.$ )

R1175906

$\stackrel{0}{7}$

|

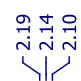
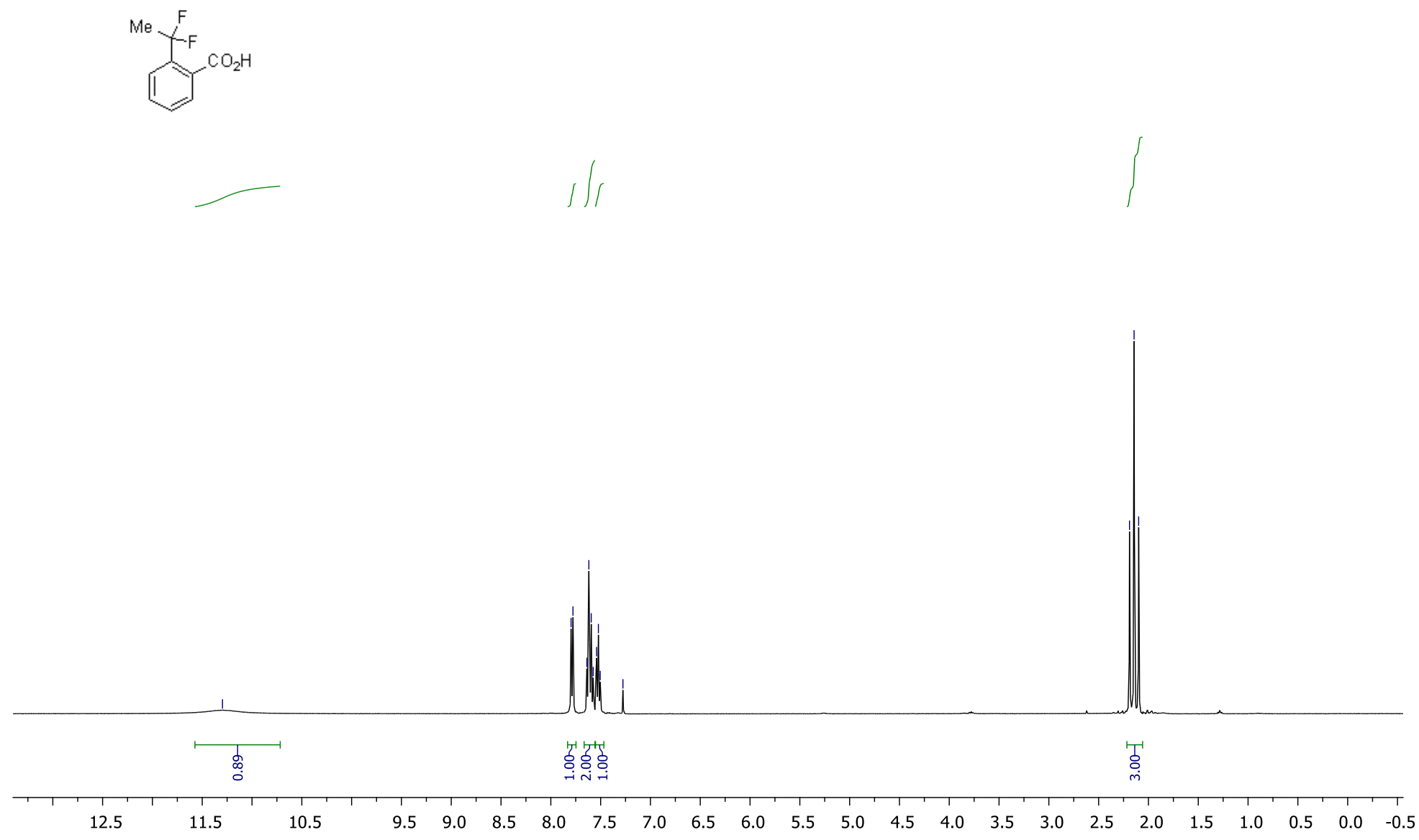

S221 
${ }^{13} \mathrm{C}\left\{{ }^{1} \mathrm{H}\right\} \mathrm{NMR}\left(151 \mathrm{MHz}, \mathrm{CDCl}_{3}\right)$

R1175906_C13

l
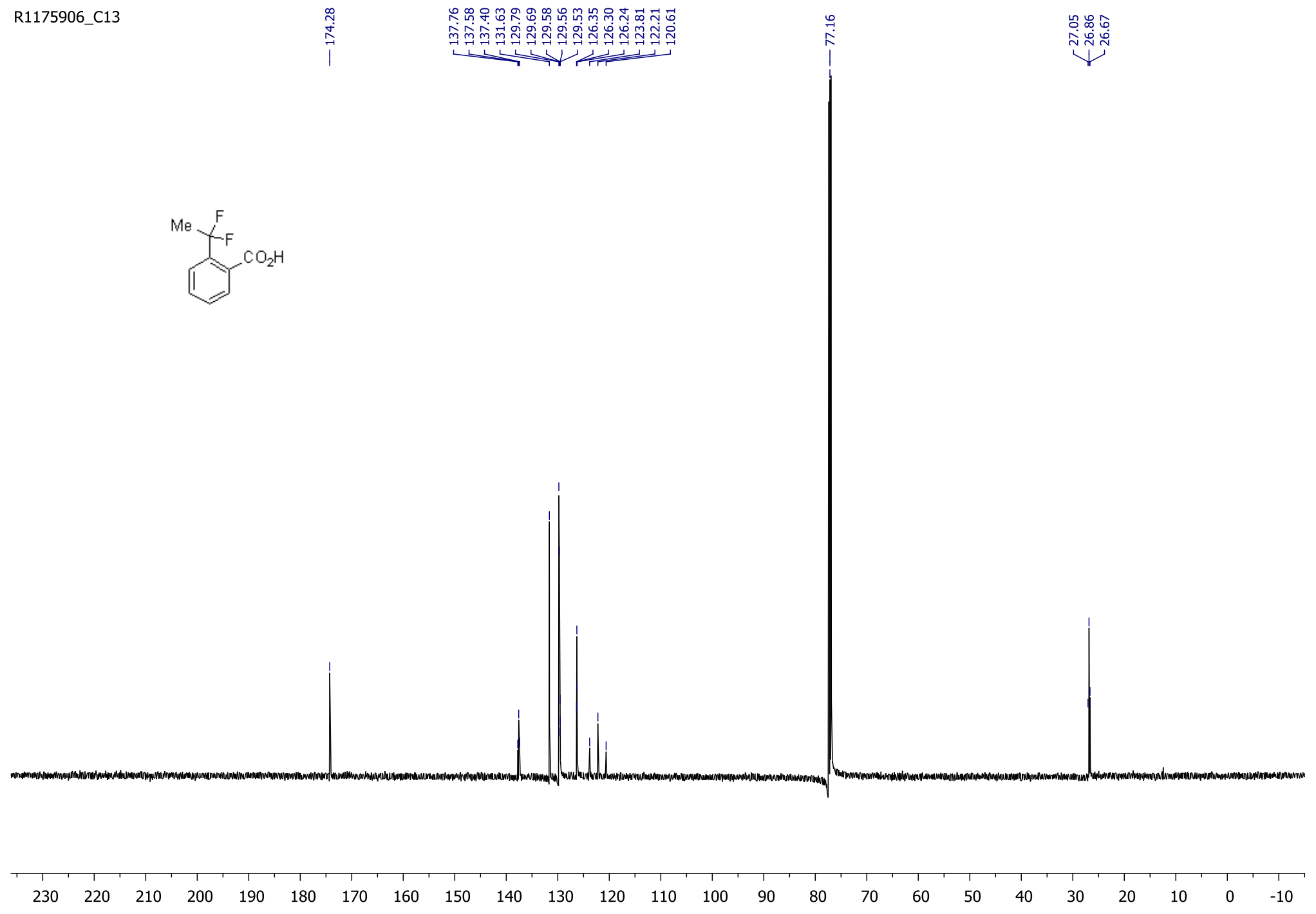

S222 
${ }^{19} \mathrm{~F}\left\{{ }^{1} \mathrm{H}\right\}$ NMR (376 MHz, $\left.\mathrm{CDCl}_{3}\right)$

R1175906_F19 $\{\mathrm{H}\}$

$19 \mathrm{~F}-\{1 \mathrm{H}\}$

$\underset{\substack{n \\ \infty}}{\substack{\infty \\ i}}$
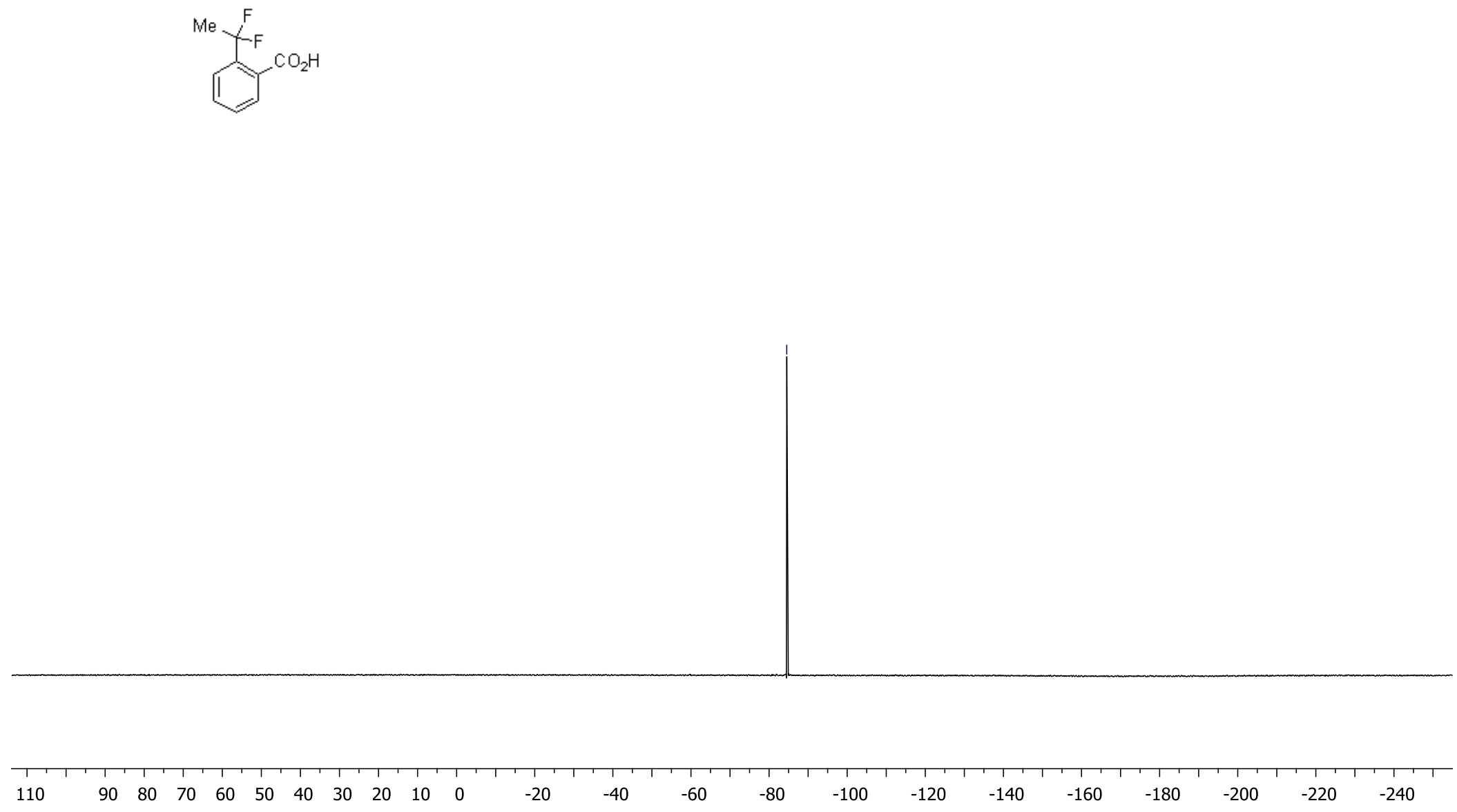

S223 


\section{Compound 11c}

${ }^{1} \mathrm{H}$ NMR (400 MHz, DMSO- $d_{6}$ )

R1133146

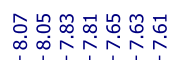

पiv

ํํํํํํำ

in

${ }^{\mathrm{F}} \mathrm{CO}_{2} \mathrm{H}$

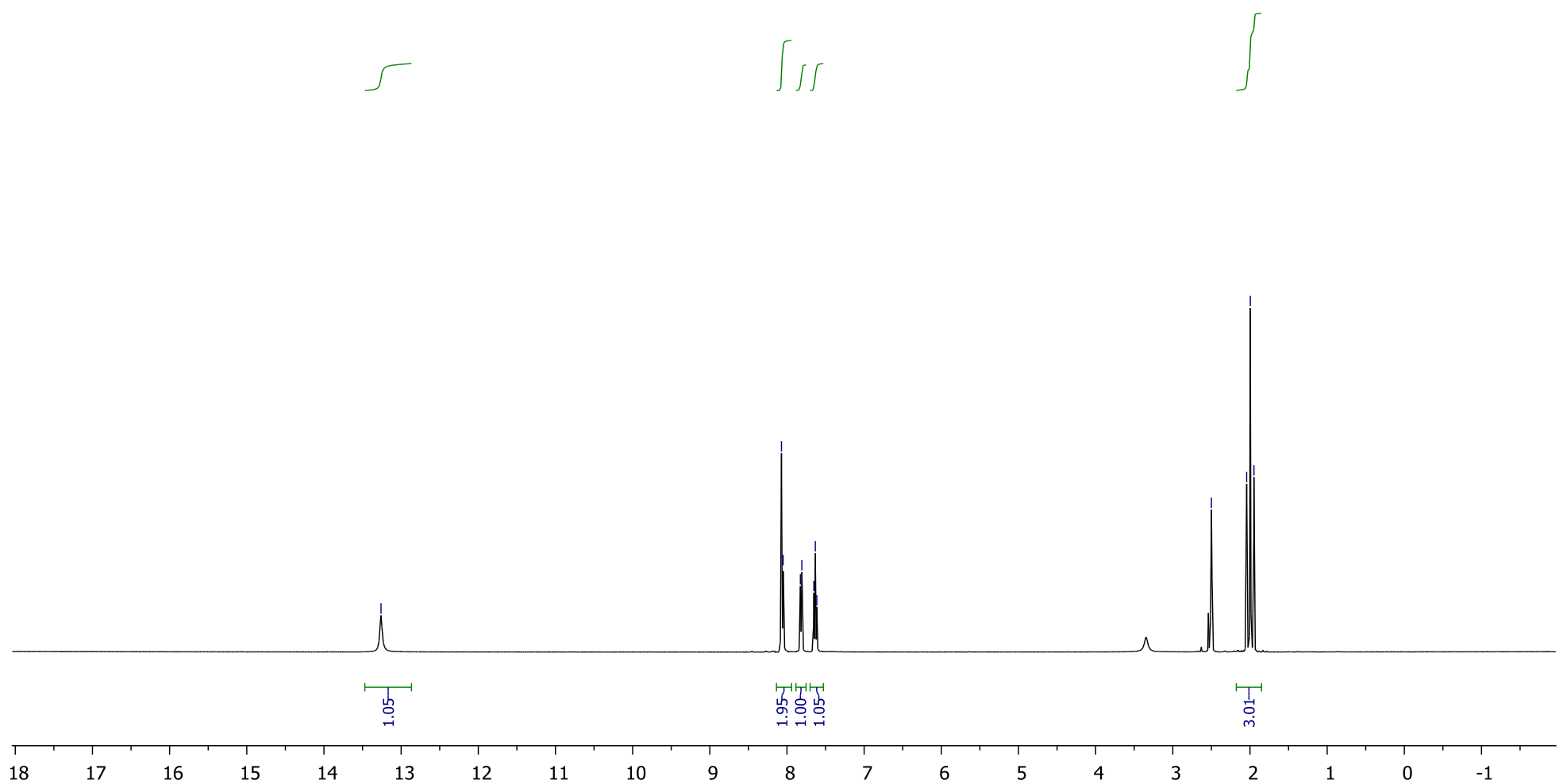


${ }^{13} \mathrm{C}\left\{{ }^{1} \mathrm{H}\right\}$ NMR (126 MHz, DMSO- $\left.d_{6}\right)$

R1133146_C1:

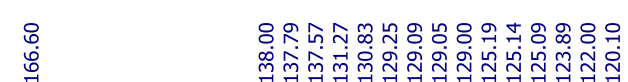

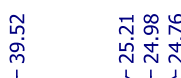

度 $\mathrm{CO}_{2} \mathrm{H}$

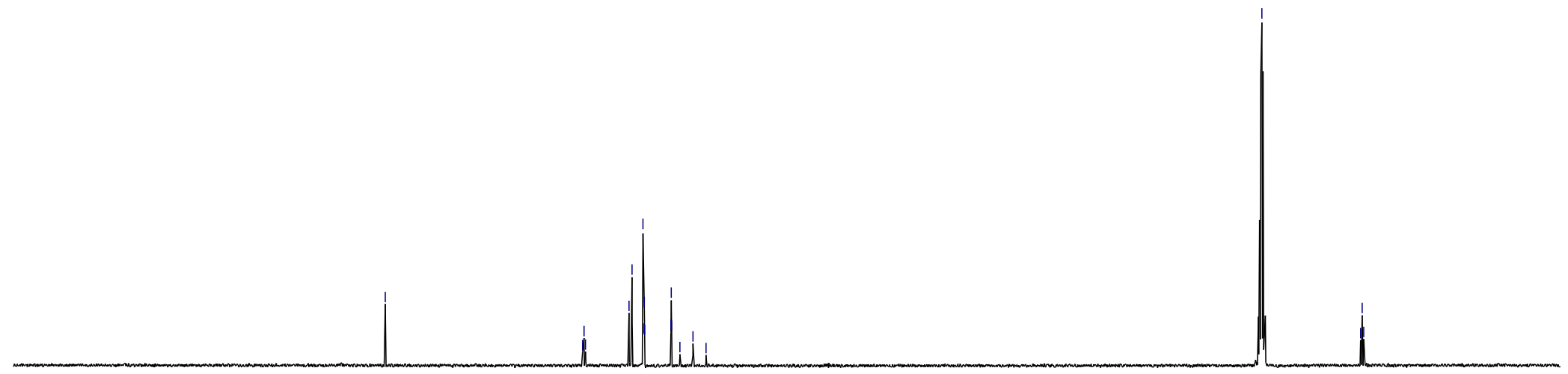

22

$210-200-190$

$180 \quad 17$
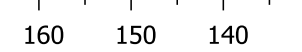

$130 \quad 120$

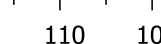

10090

$\begin{array}{llll}1 & 1 & 1 & 1\end{array}$

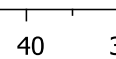

20 


\section{${ }^{19} \mathrm{~F}\left\{{ }^{1} \mathrm{H}\right\}$ NMR (376 MHz, $\left.\mathrm{CDCl}_{3}\right)$}

R1133146_F19 $\{\mathrm{H}\}$
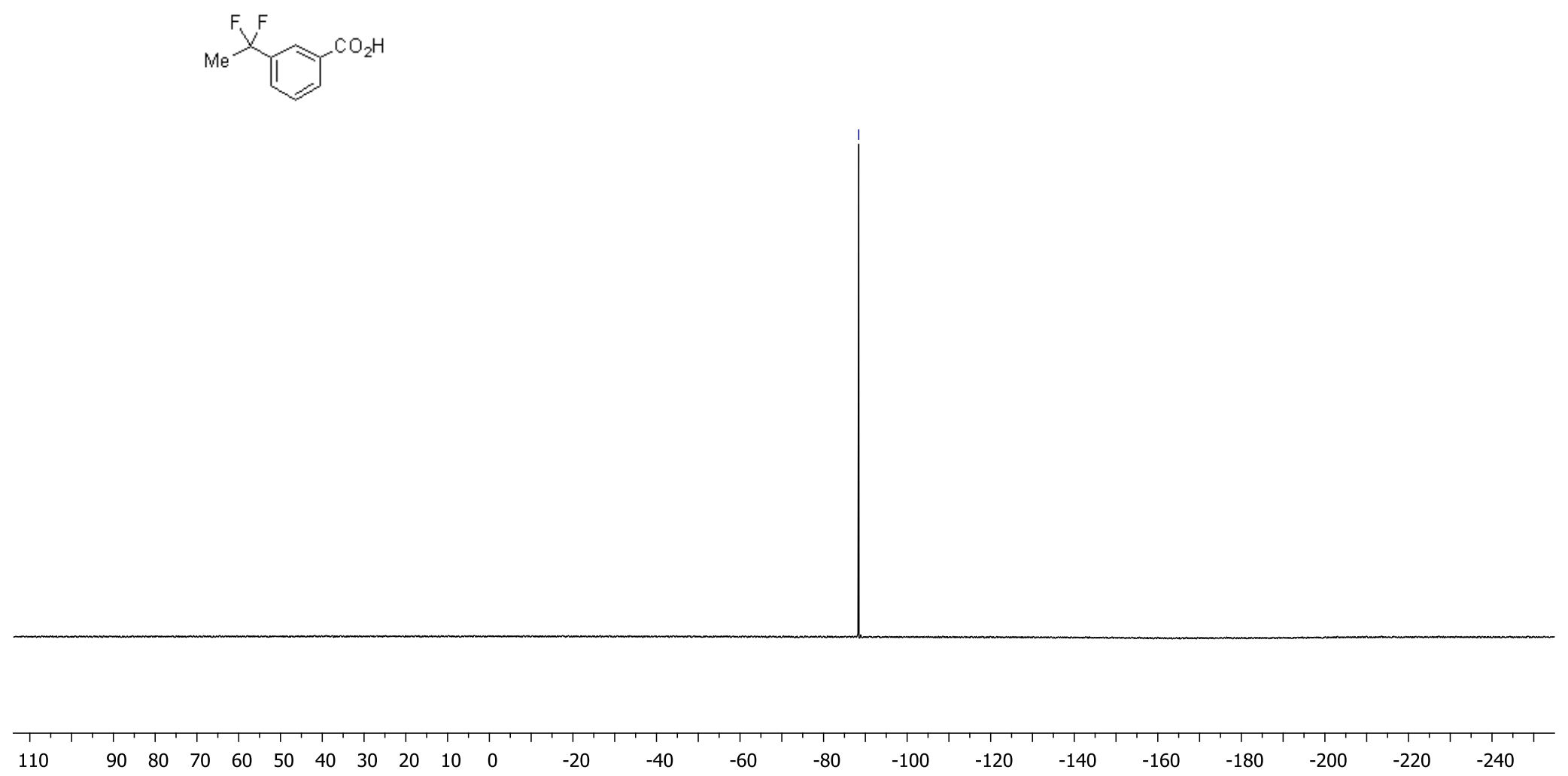


\section{Compound 15c}

${ }^{1} \mathrm{H}$ NMR (400 MHz, DMSO- $d_{6}$ )

R462224

Viv

品

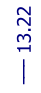

I प।

$\overbrace{F}^{\mathrm{CO}_{2} \mathrm{H}}$

$\iint$
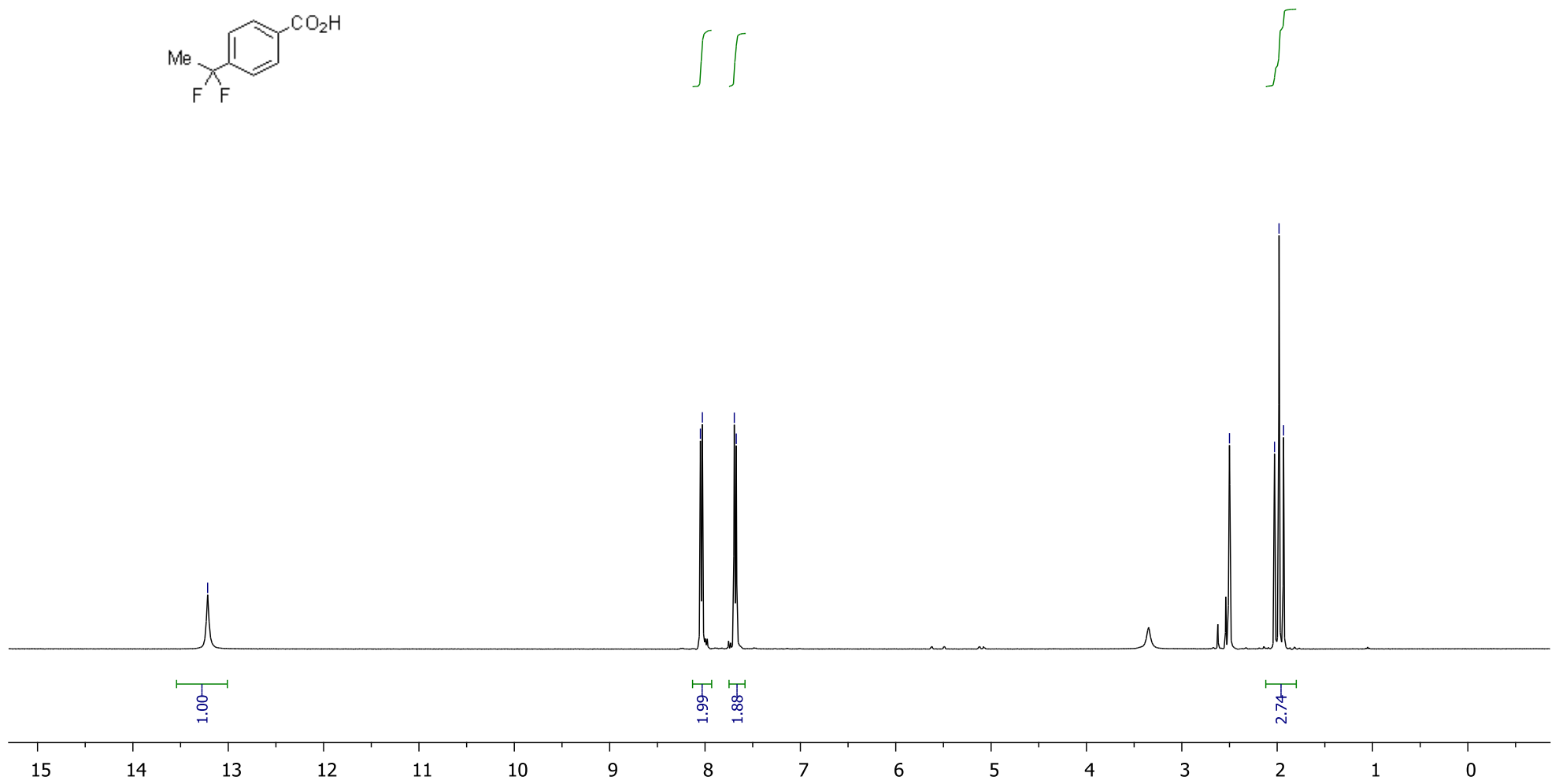


\section{${ }^{13} \mathrm{C}\left\{{ }^{1} \mathrm{H}\right\}$ NMR (101 MHz, DMSO- $\left.d_{6}\right)$}

R462224_C13

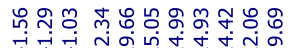

子守守

กู่

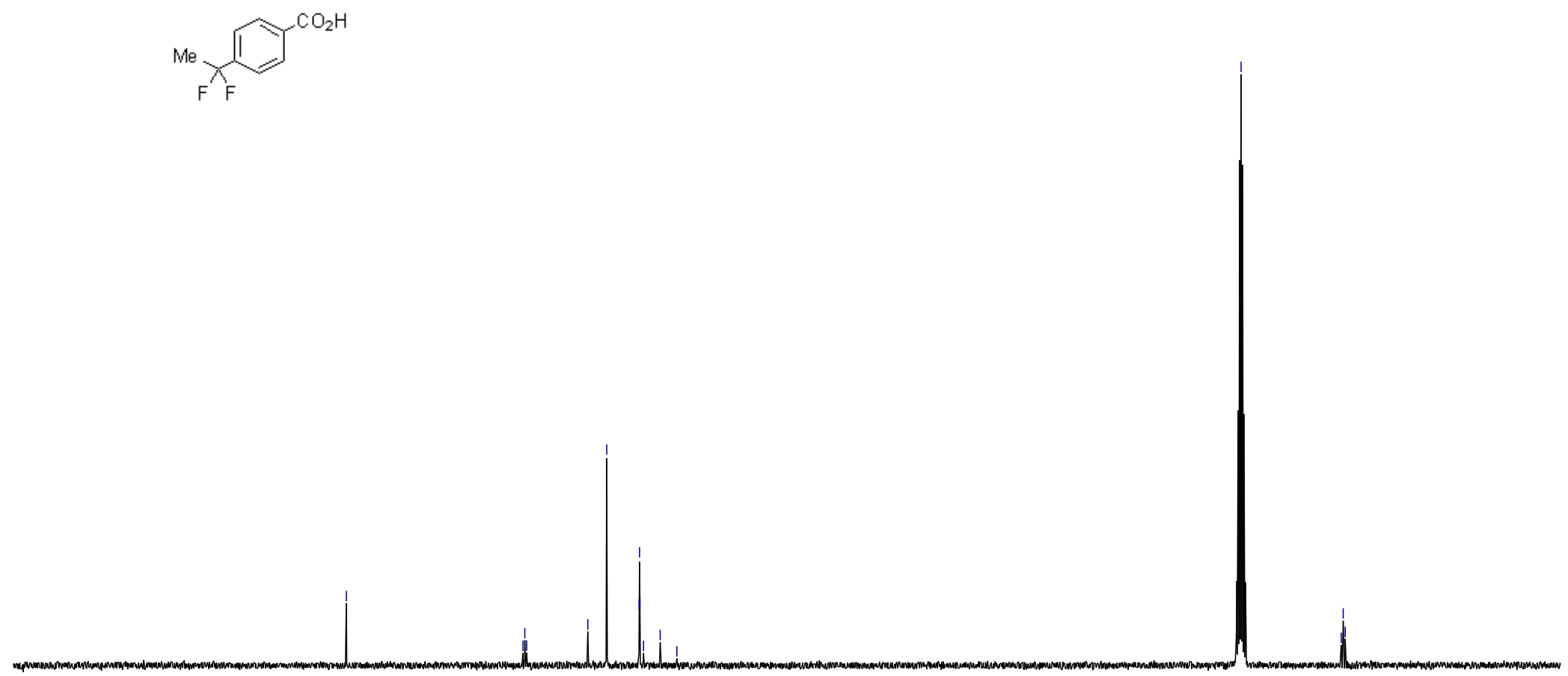

$210 \quad 200 \quad 190 \quad 180 \quad 170-160$

$140 \quad 130$

120

100

90

80

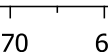

$60 \quad 50$ 


\section{${ }^{19} \mathrm{~F}\left\{{ }^{1} \mathrm{H}\right\}$ NMR (376 MHz, DMSO- $\left.d_{6}\right)$}

R462224_F19 $\{\mathrm{H}\}$

$19 \mathrm{~F}-\{1 \mathrm{H}\}$
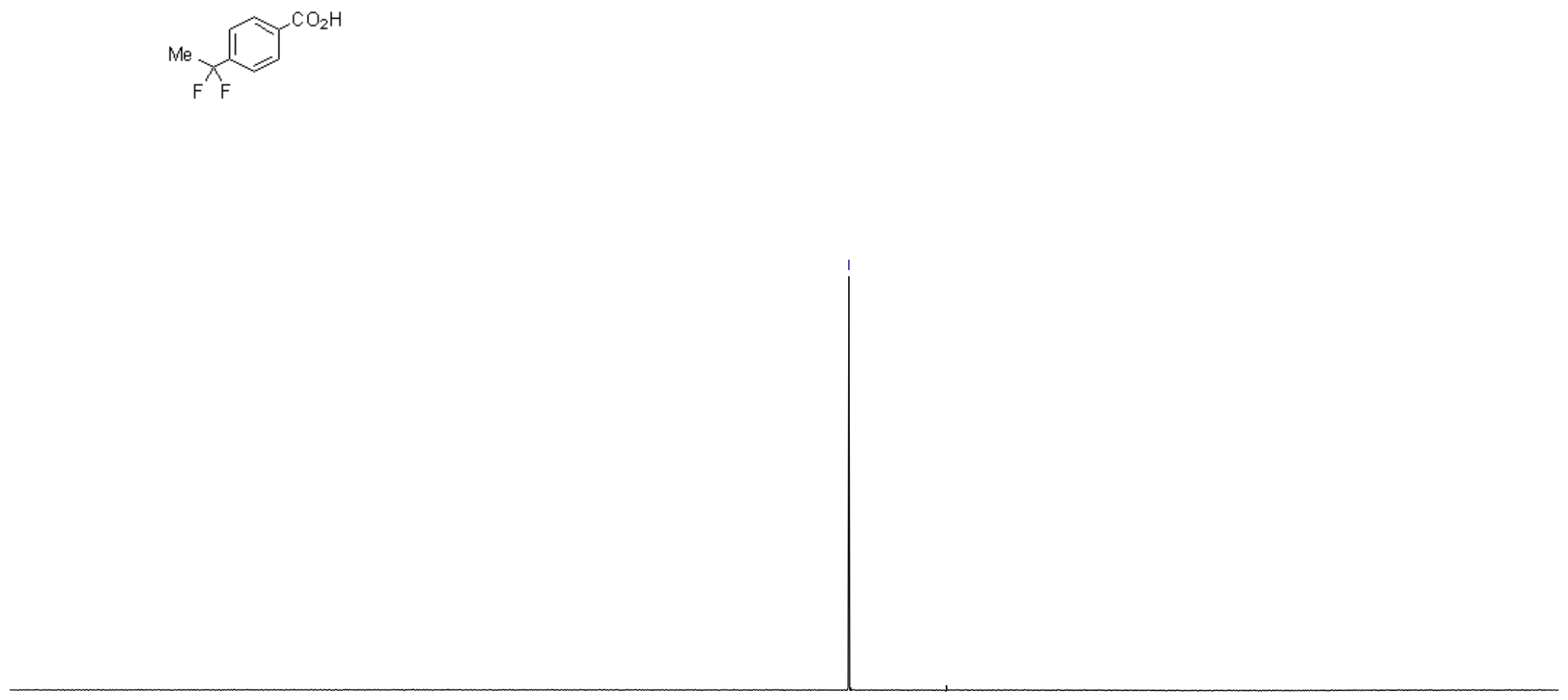

$\begin{array}{lllllllll}90 & 80 & 70 & 60 & 50 & 40 & 30 & 20 & 10\end{array}$

$-20$

$-40$

$-60$

$-80$

$-100$

$-120$

$-160-180$

$-200-220-240$ 


\section{Compound 19c}

${ }^{1} \mathrm{H}$ NMR (400 MHz, DMSO- $d_{6}$ )

R2555036

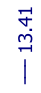

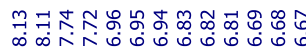

บे

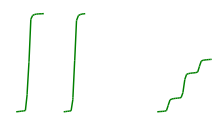

$\overbrace{F}^{C \mathrm{CO}_{2} \mathrm{H}}$

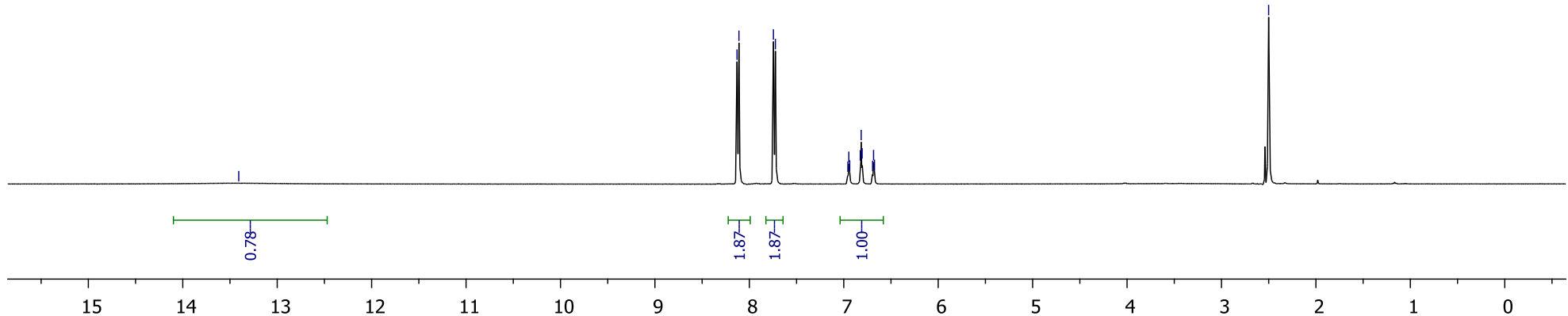


${ }^{13} \mathrm{C}\left\{{ }^{1} \mathrm{H}\right\}$ NMR (151 MHz, DMSO- $\left.d_{6}\right)$

R2555036_C13

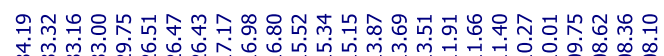

舟闻舟

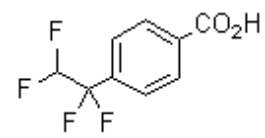

Wub 


\section{${ }^{19} \mathrm{~F}\left\{{ }^{1} \mathrm{H}\right\}$ NMR (376 MHz, DMSO- $\left.d_{6}\right)$}

R2555036_F19

$19 \mathrm{~F}-\{1 \mathrm{H}\}$

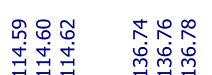

广
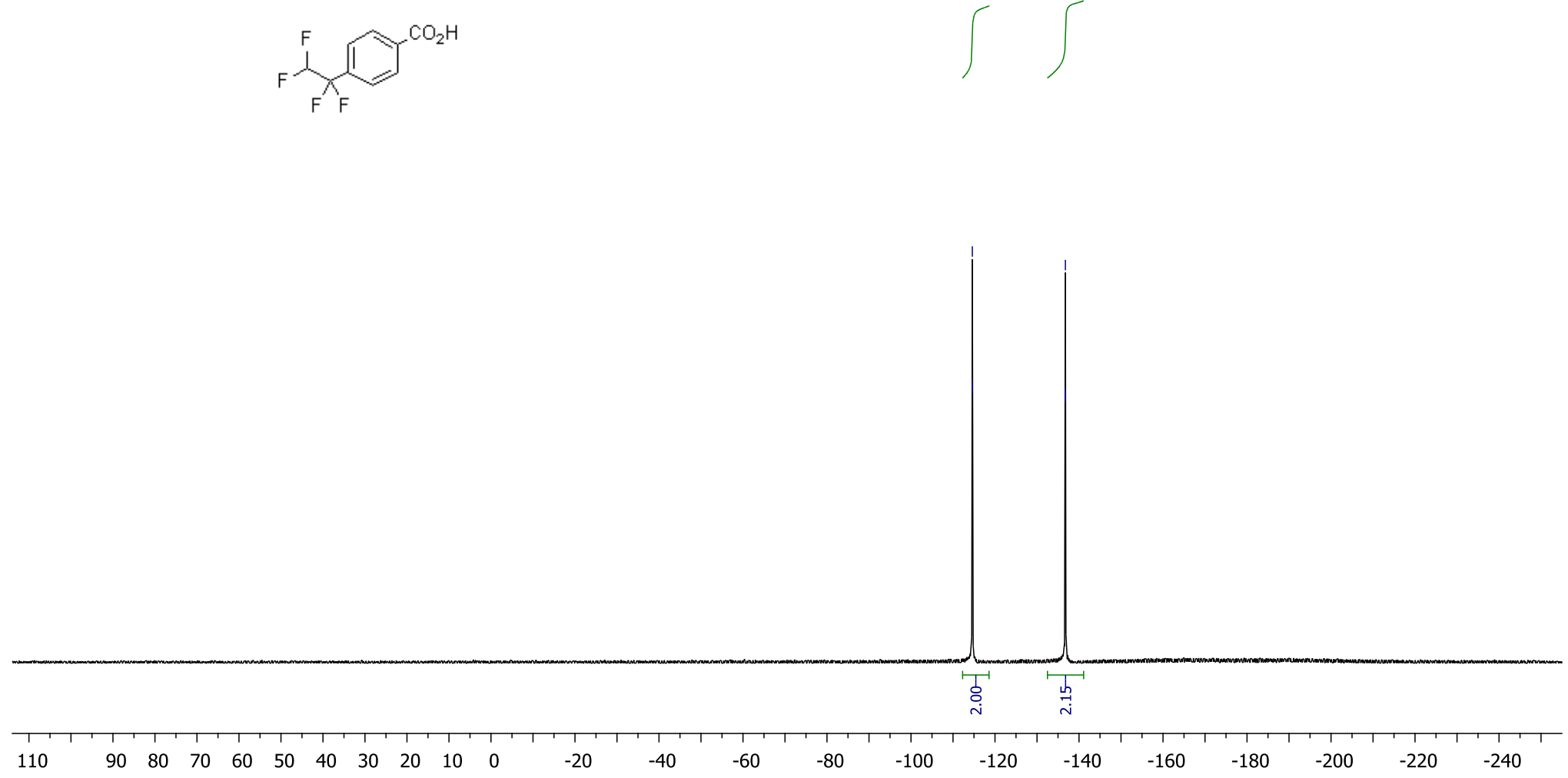


\section{Compound 31c}

${ }^{1} \mathrm{H}$ NMR (400 MHz, DMSO- $d_{6}$ )

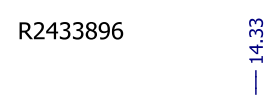

(1)

(

$\sum_{S}^{\mathrm{N}}{ }_{\mathrm{COO}}^{\mathrm{N}}$
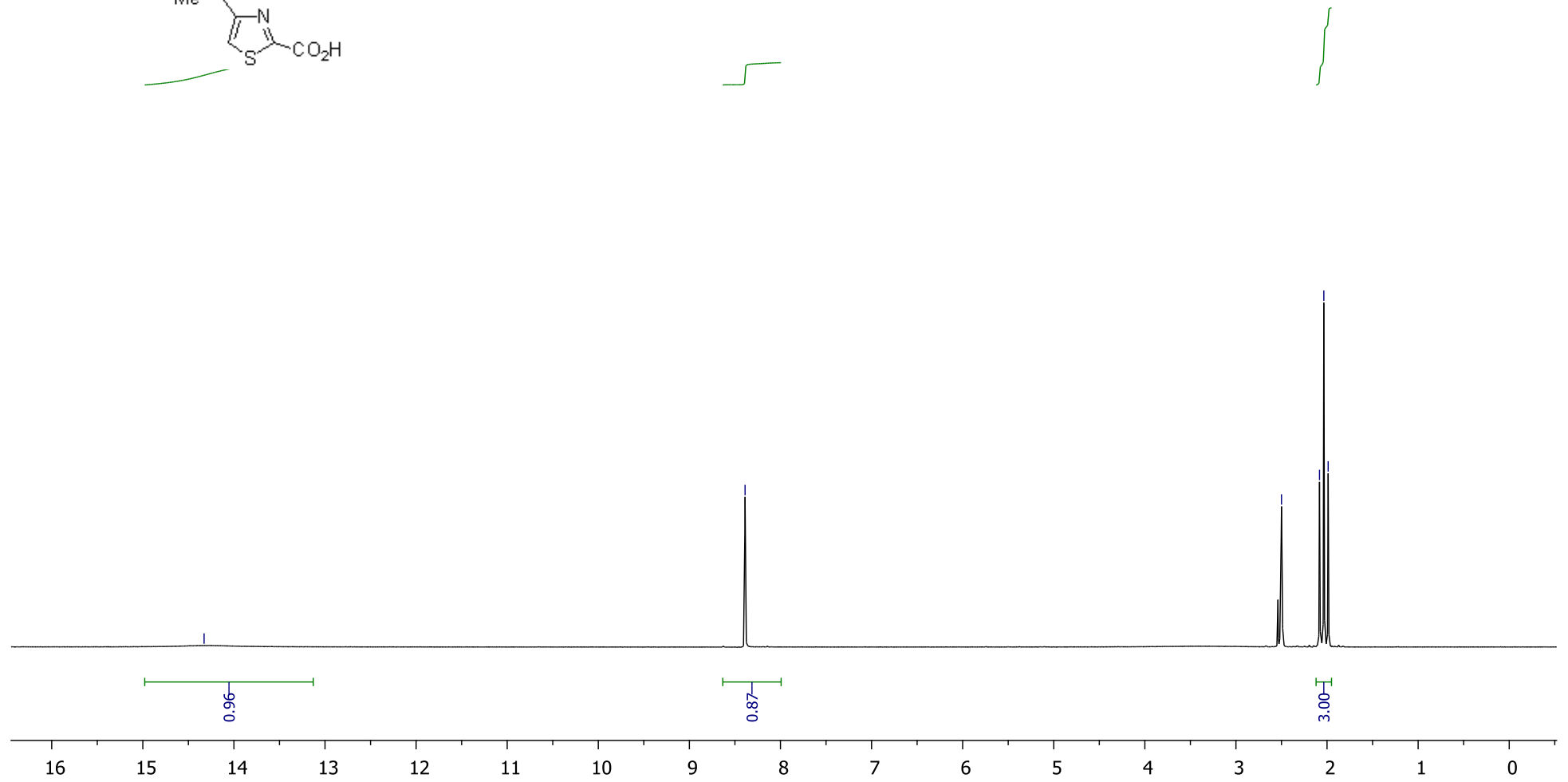
${ }^{13} \mathrm{C}\left\{{ }^{1} \mathrm{H}\right\}$ NMR $\left(126 \mathrm{MHz}, \mathrm{DMSO}-d_{6}\right)$

R2433896_C1:

$\sum_{S}^{\mathrm{N}} \mathrm{l}_{\mathrm{CO}_{2} \mathrm{H}}^{\mathrm{N}}$
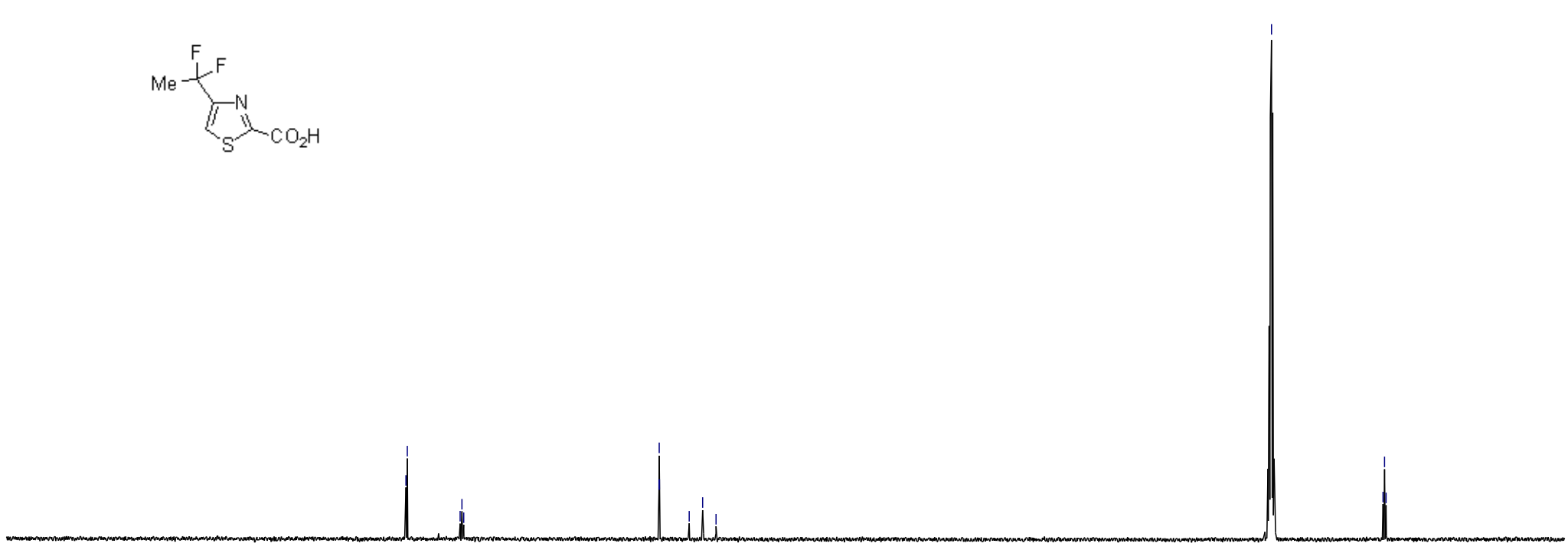

$\begin{array}{llllllllllllllllllllll}210 & 200 & 190 & 180 & 170 & 160 & 150 & 140 & 130 & 120 & 110 & 100 & 90 & 80 & 70 & 60 & 50 & 40 & 30 & 20 & 10 & 0\end{array}$ 


\section{${ }^{19} \mathrm{~F}\left\{{ }^{1} \mathrm{H}\right\}$ NMR (376 MHz, DMSO- $d_{6}$ )}

R2433896_F19 $\{\mathrm{H}\}$ $19 \mathrm{~F}-\{1 \mathrm{H}\}$
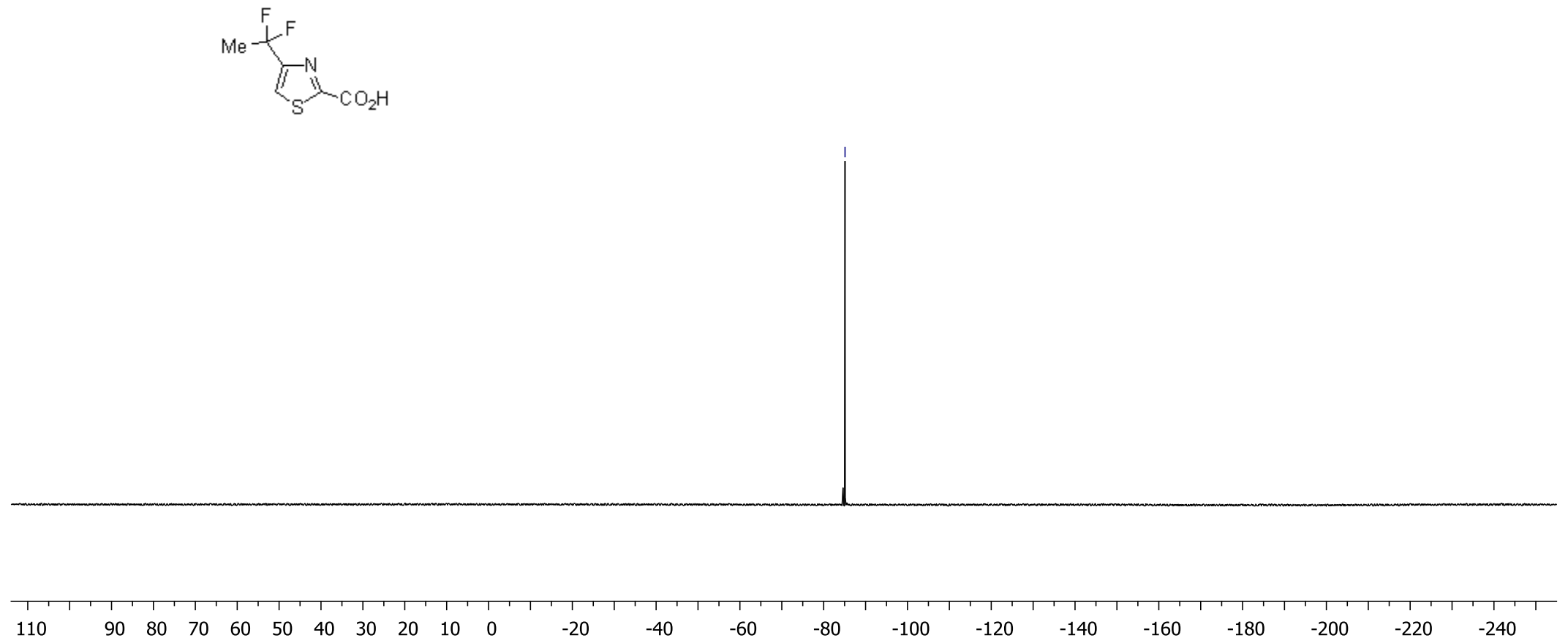


\section{Compound 42c}

${ }^{1} \mathrm{H}$ NMR (400 MHz, DMSO- $d_{6}$ )

R2464762

$\bigcup_{\substack{\infty \\ \infty}}^{\infty}$

I

Me
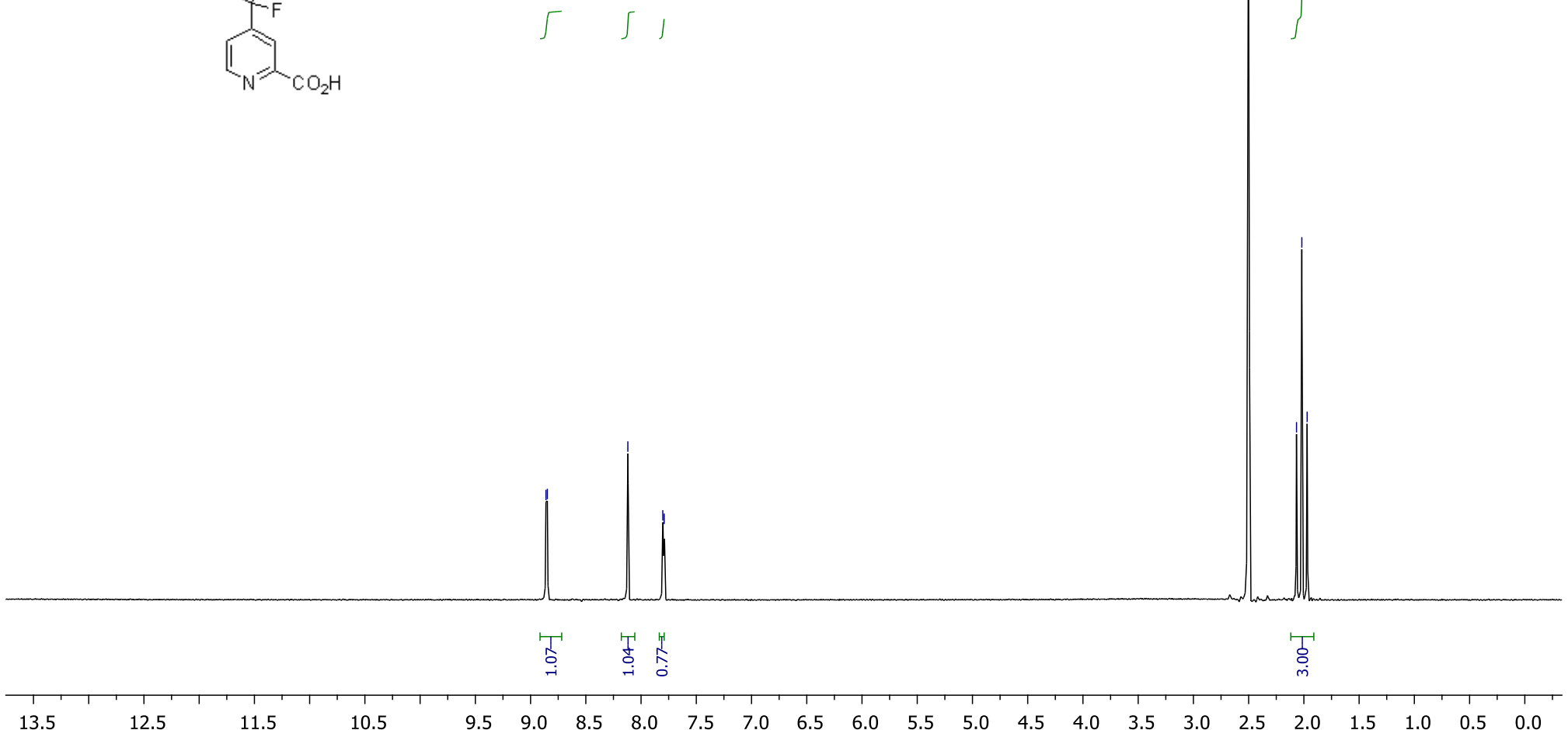
${ }^{13} \mathrm{C}\left\{{ }^{1} \mathrm{H}\right\}$ NMR (126 MHz, DMSO- $\left.d_{6}\right)$

R2464762_C1:

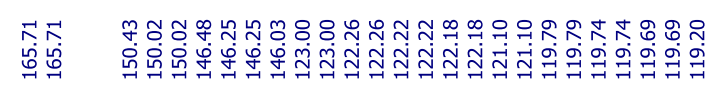

ஸุ.$$
\text { Y }
$$

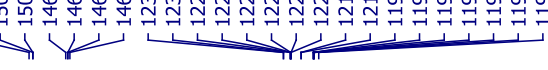
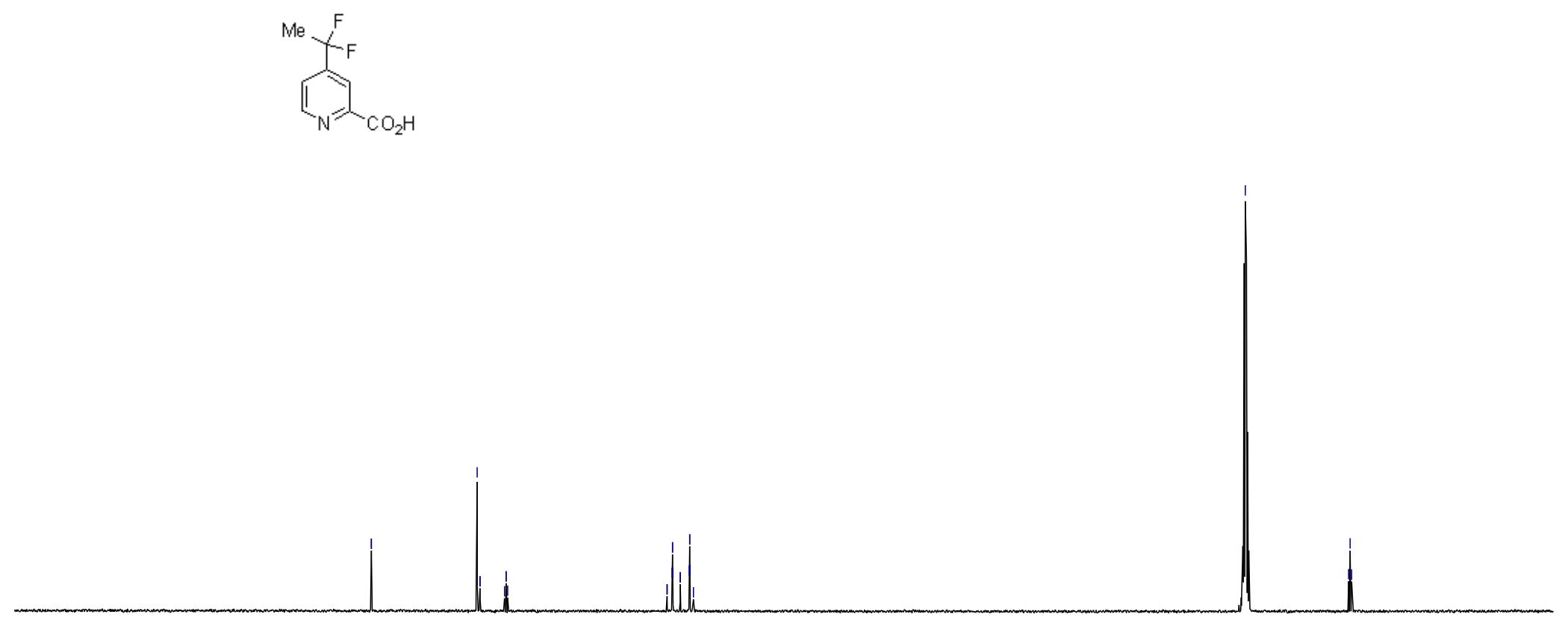

$210 \quad 200$

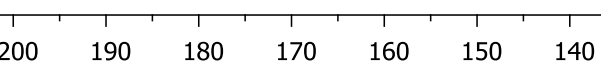

$130 \quad 12$

$\begin{array}{lll}1 & & \\ 110 & 100\end{array}$

$80 \quad 70$

60

50

$30 \quad 20$ 


\section{${ }^{19} \mathrm{~F}\left\{{ }^{1} \mathrm{H}\right\}$ NMR (376 MHz, DMSO- $\left.d_{6}\right)$}

R2464762_F19 $\{H\}$
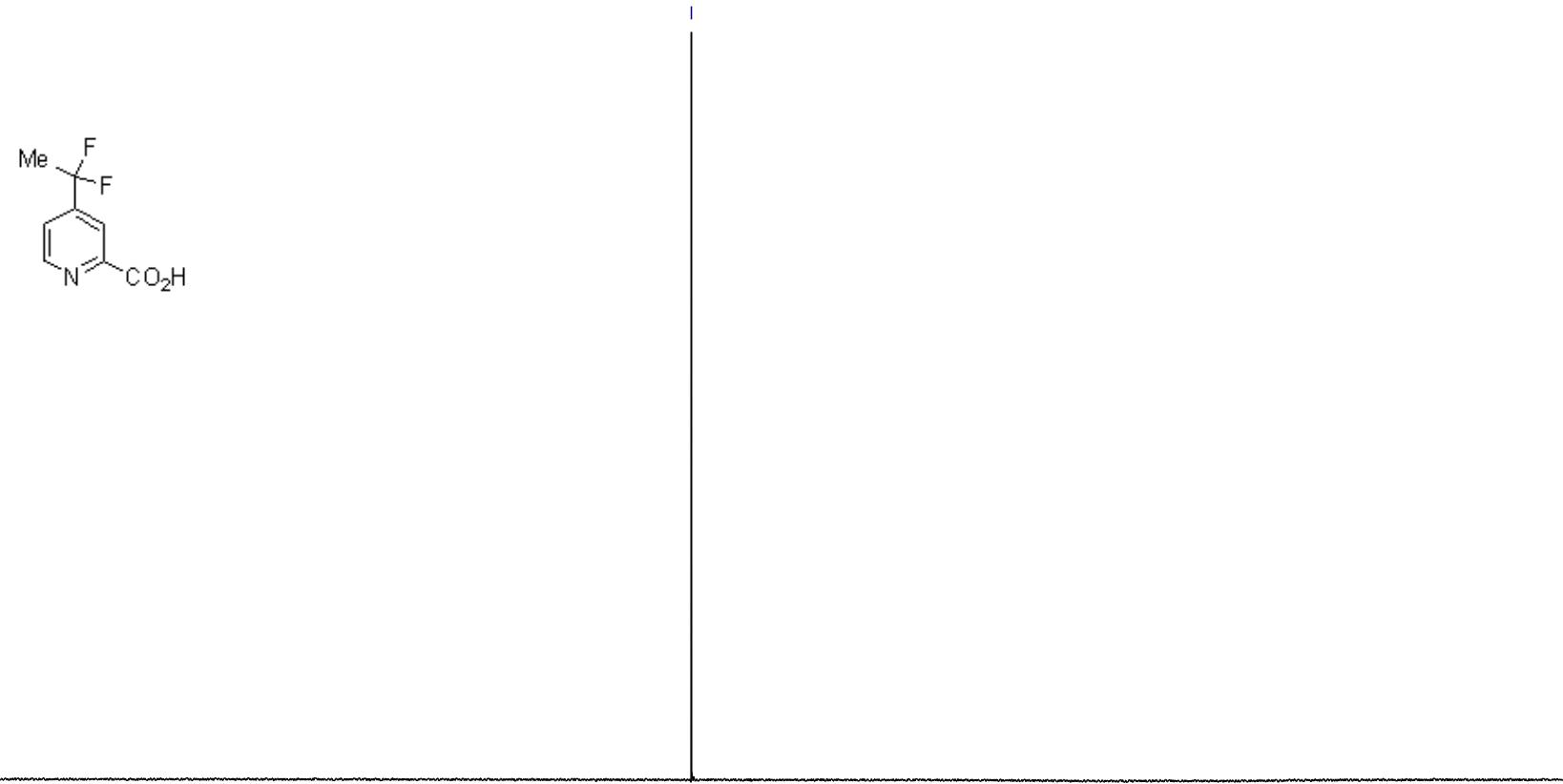

$\begin{array}{lllllllllll}110 & 90 \quad 80 \quad 70 \quad 60 \quad 50 \quad 40 \quad 30 \quad 20 & 10 & 0\end{array}$ 


\section{Compound 45c}

${ }^{1} \mathrm{H}$ NMR (400 MHz, DMSO- $d_{6}$ )

R1506386+X

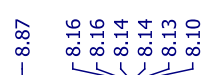

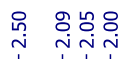

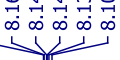

$>$

$\overbrace{\mathrm{N}}^{\mathrm{C}}$

$\int$

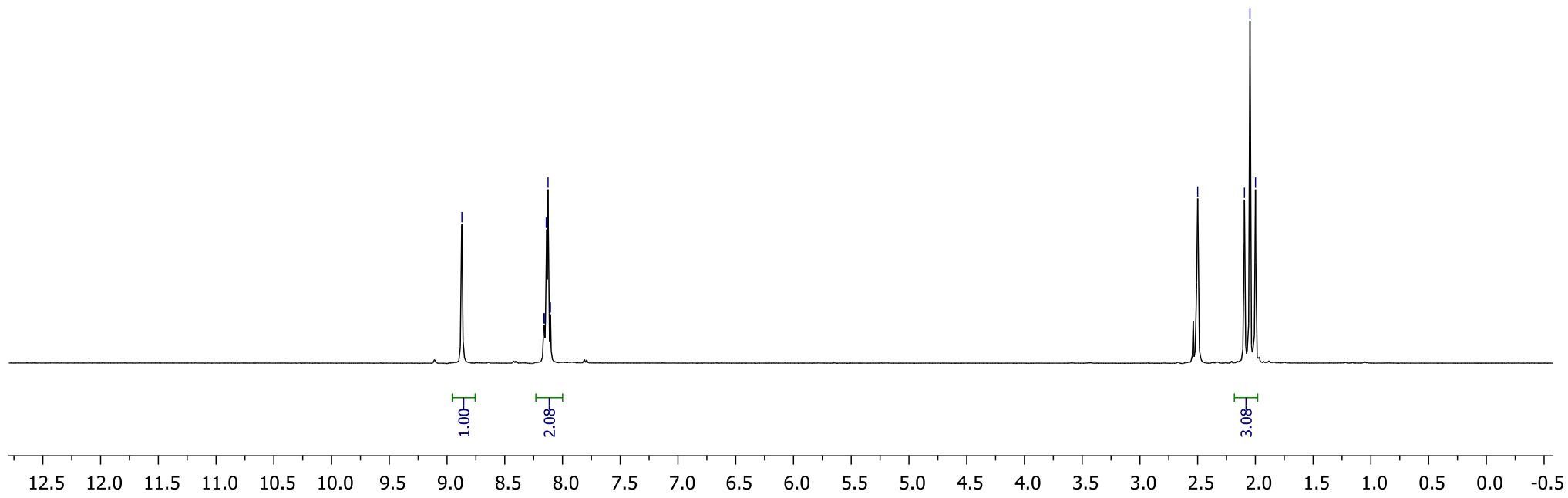


${ }^{13} \mathrm{C}\left\{{ }^{1} \mathrm{H}\right\}$ NMR $\left(126 \mathrm{MHz}, \mathrm{DMSO}-d_{6}\right)$

R1506386+X_C1: $\quad$ l
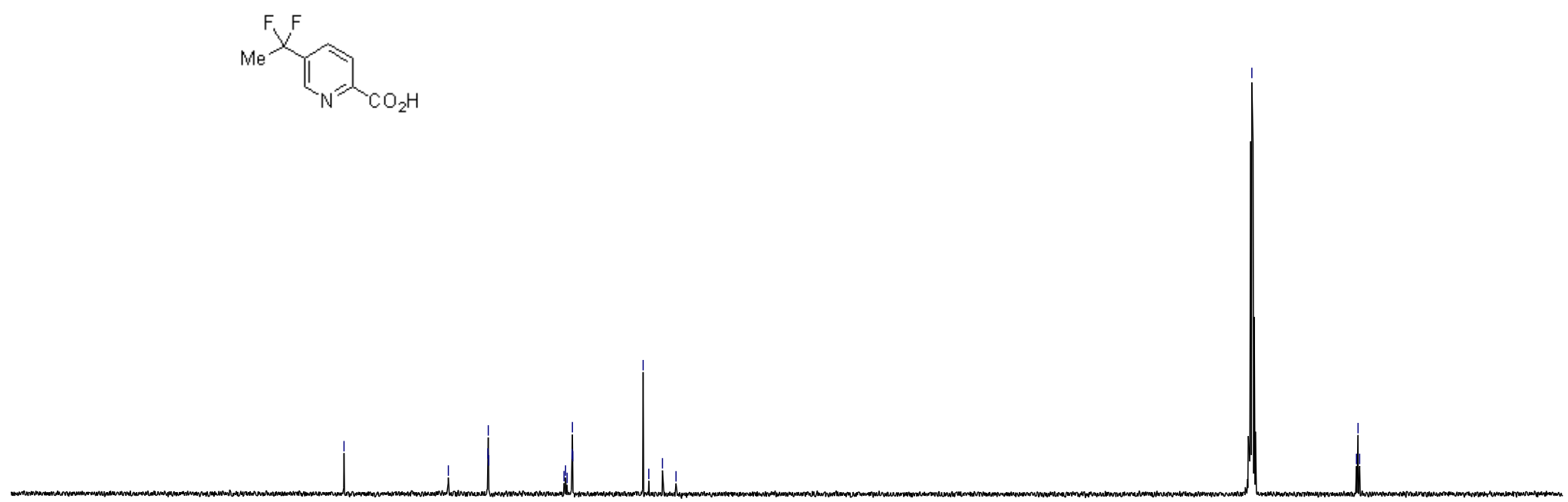

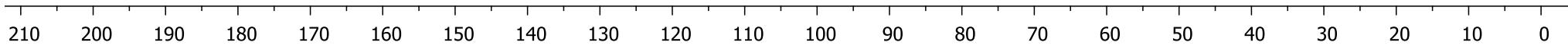




\section{${ }^{19} \mathrm{~F}\left\{{ }^{1} \mathrm{H}\right\}$ NMR (376 MHz, DMSO- $\left.d_{6}\right)$}

R1506386_F19\{H\}

$$
19 \mathrm{~F}-\{1 \mathrm{H}\}
$$
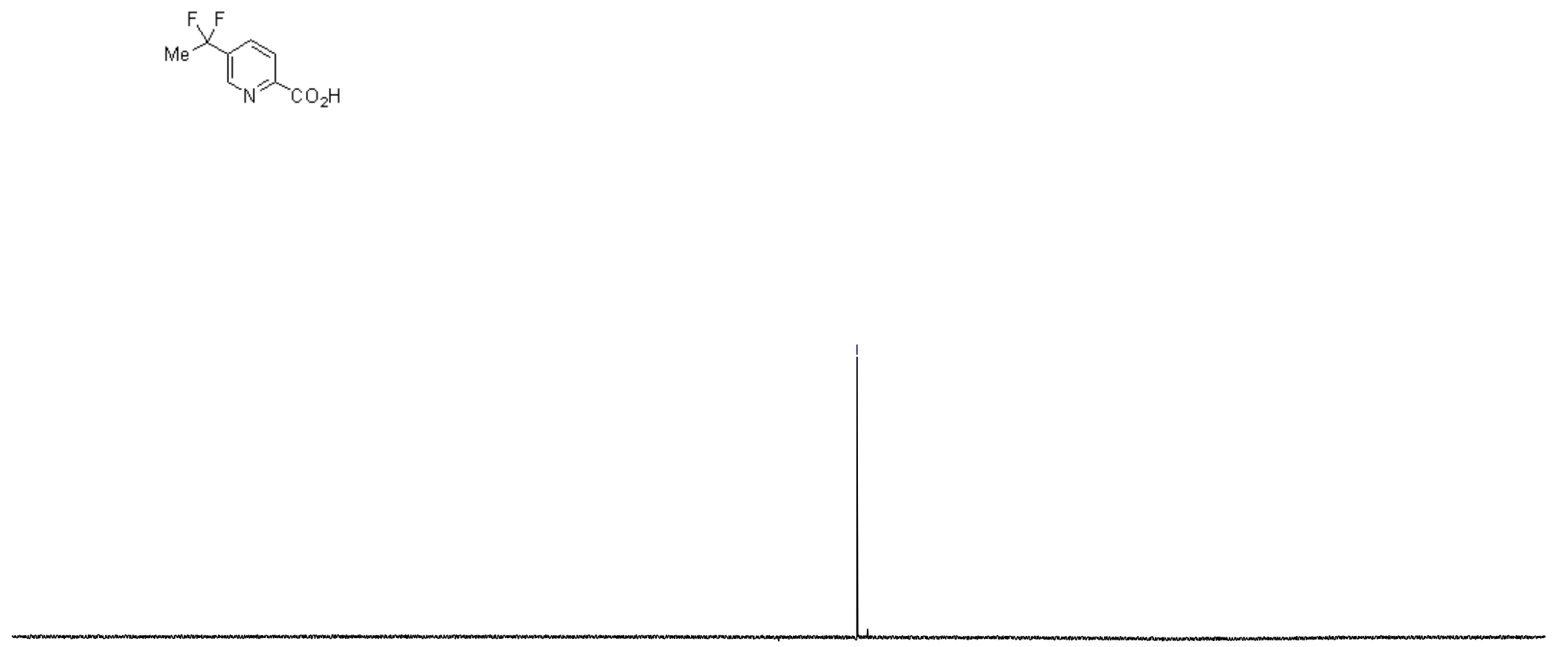

$\begin{array}{llllllllllllllllllllllllllllll} & 110 & 90 & 80 & 70 & 60 & 50 & 40 & 30 & 20 & 10 & 0 & -20 & -40 & -60 & -80 & -100 & -120 & -140 & -160 & -180 & -200 & -220 & -240 & \end{array}$ 


\section{Compound 48c}

${ }^{1} \mathrm{H}$ NMR (400 MHz, DMSO- $d_{6}$ )

R1436966

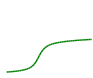

舟

$\iint \quad 5$
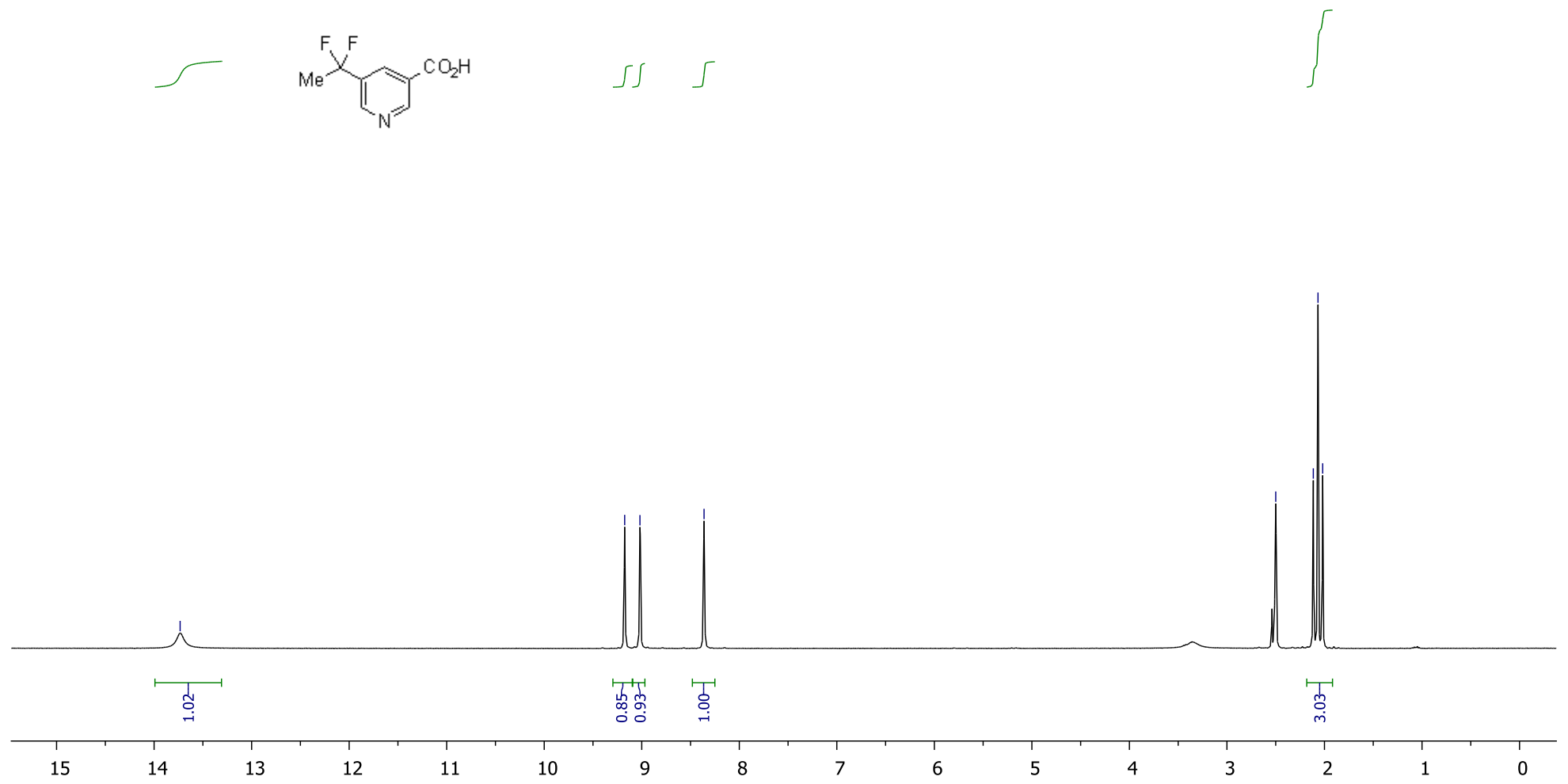
${ }^{13} \mathrm{C}\left\{{ }^{1} \mathrm{H}\right\}$ NMR (126 MHz, DMSO- $\left.d_{6}\right)$

R1436966_13C

$$
\text { | }
$$

กิ

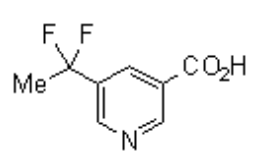

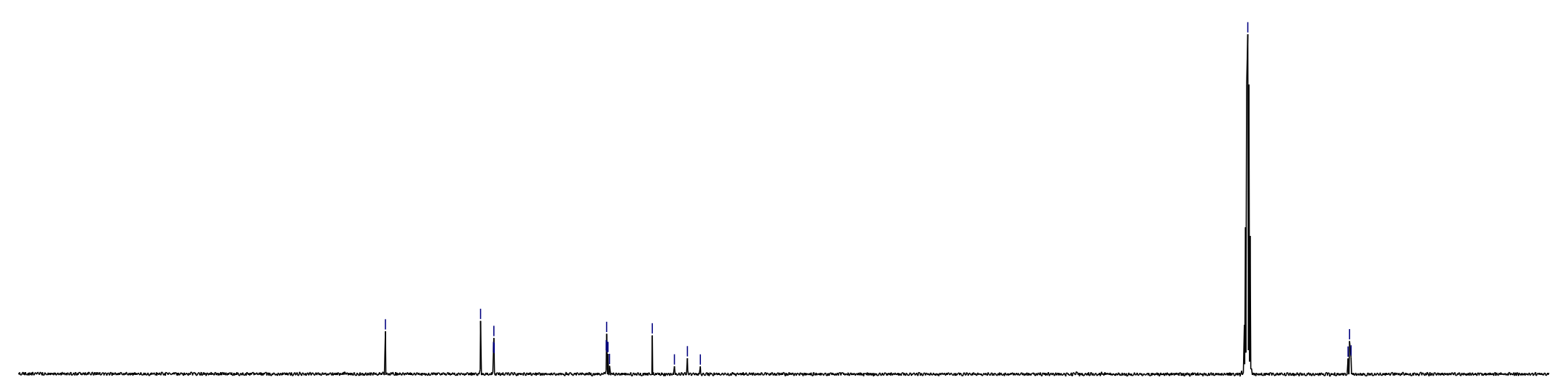

$200 \quad 190 \quad 180 \quad 170-1,1 / 2$

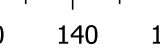

$130 \quad 120$

$110 \quad 100$

$90 \quad 80$ 


\section{${ }^{19} \mathrm{~F}\left\{{ }^{1} \mathrm{H}\right\}$ NMR (376 MHz, DMSO- $\left.d_{6}\right)$}

R1436966_F19\{H\} $19 \mathrm{~F}-\{1 \mathrm{H}\}$

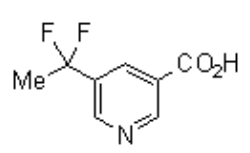

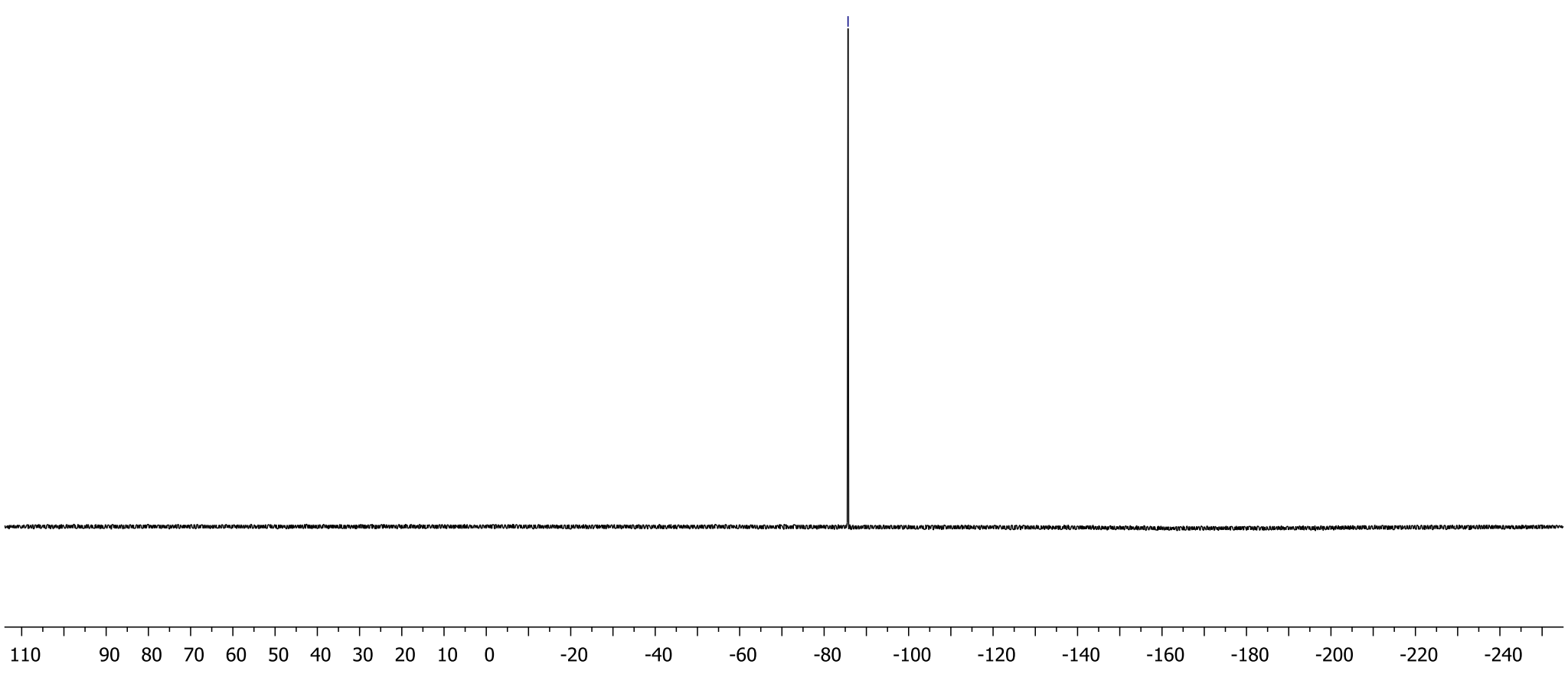




\section{Compound 50c}

${ }^{1} \mathrm{H}$ NMR (400 MHz, $\mathrm{CDCl}_{3}$ )

R1311519
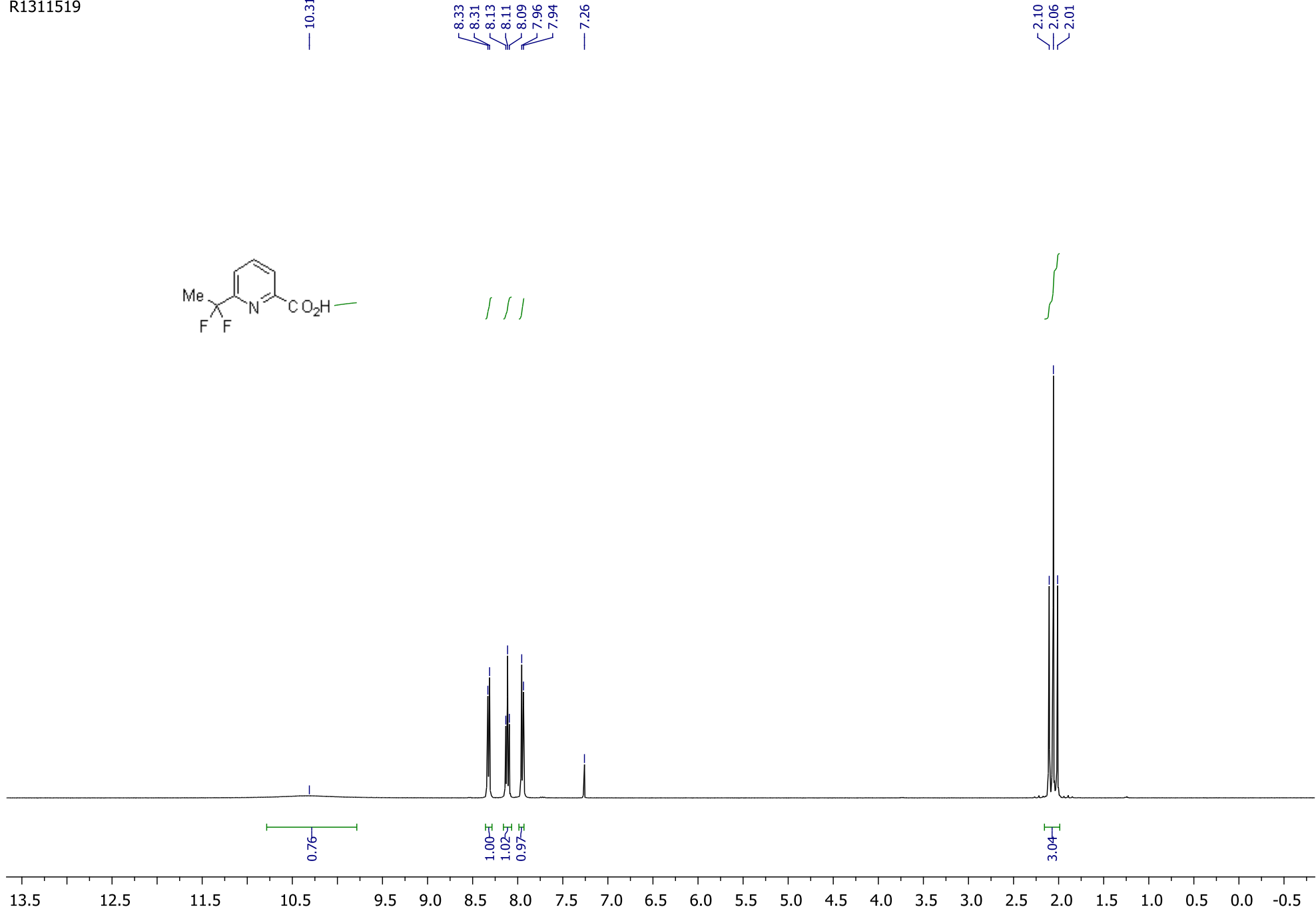


\section{${ }^{13} \mathrm{C}\left\{{ }^{1} \mathrm{H}\right\} \mathrm{NMR}\left(151 \mathrm{MHz}, \mathrm{CDCl}_{3}\right)$}

R1311519_C13

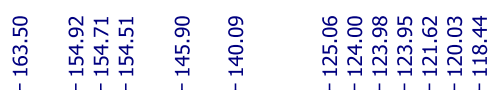

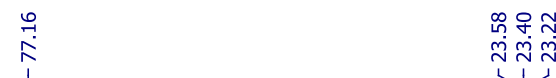

${ }_{F} \overbrace{F}{ }_{C_{2} \mathrm{H}}$

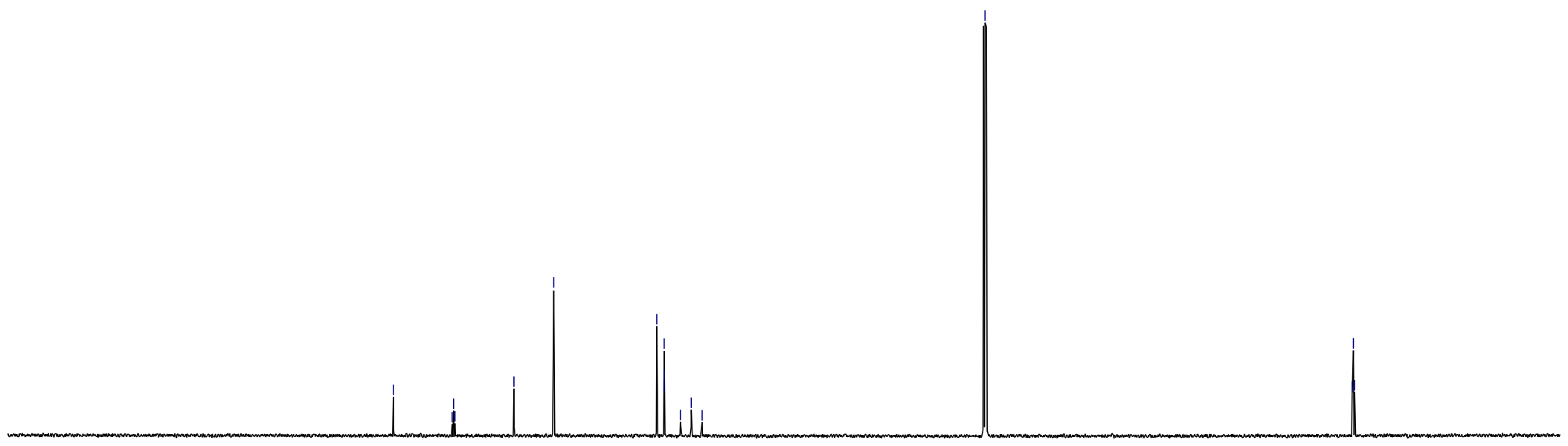

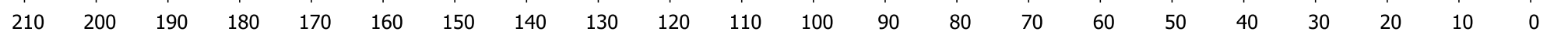


${ }^{19} \mathrm{~F}\left\{{ }^{1} \mathrm{H}\right\}$ NMR (376 MHz, DMSO- $\left.d_{6}\right)$
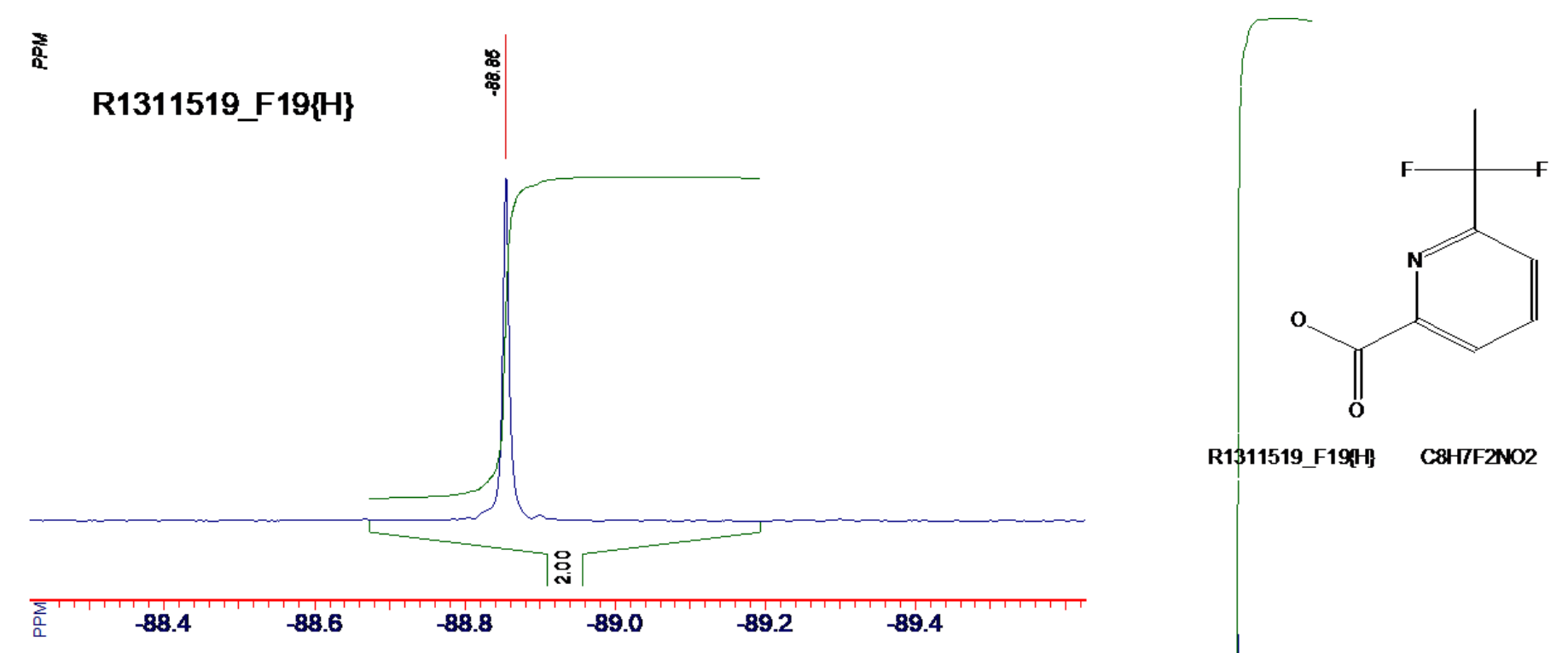

R1311519_F19H\} $\quad$ C8H7F2NO2 187.15

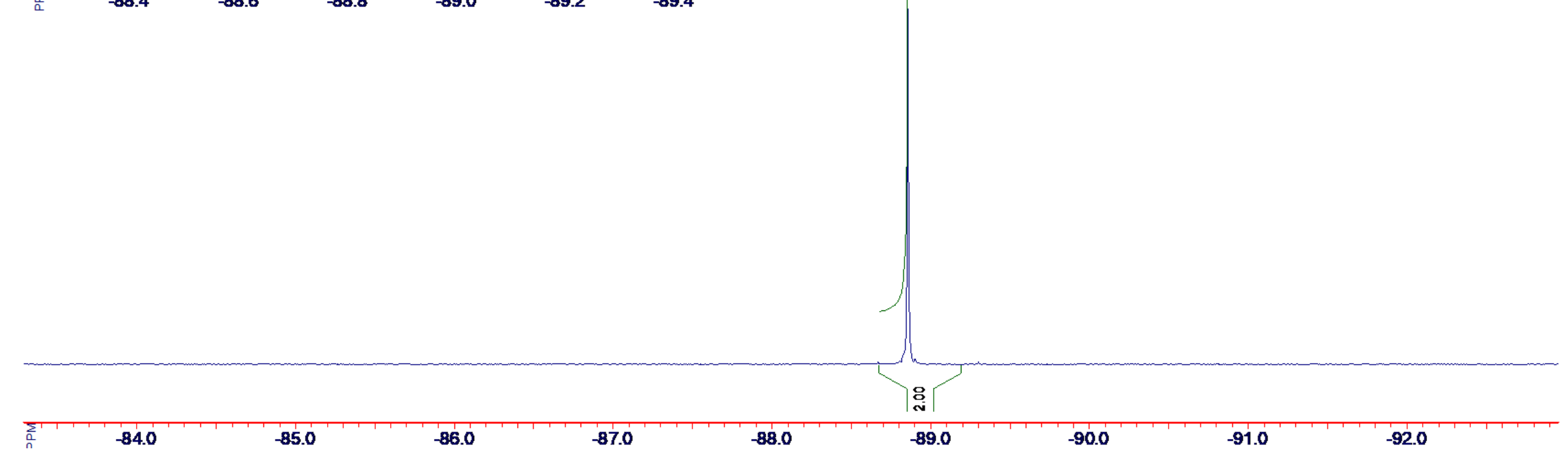




\section{Compound 62c}

${ }^{1} \mathrm{H}$ NMR (400 MHz, DMSO- $d_{6}$ )

$\stackrel{8}{\dot{+}}$

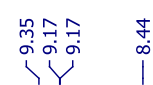
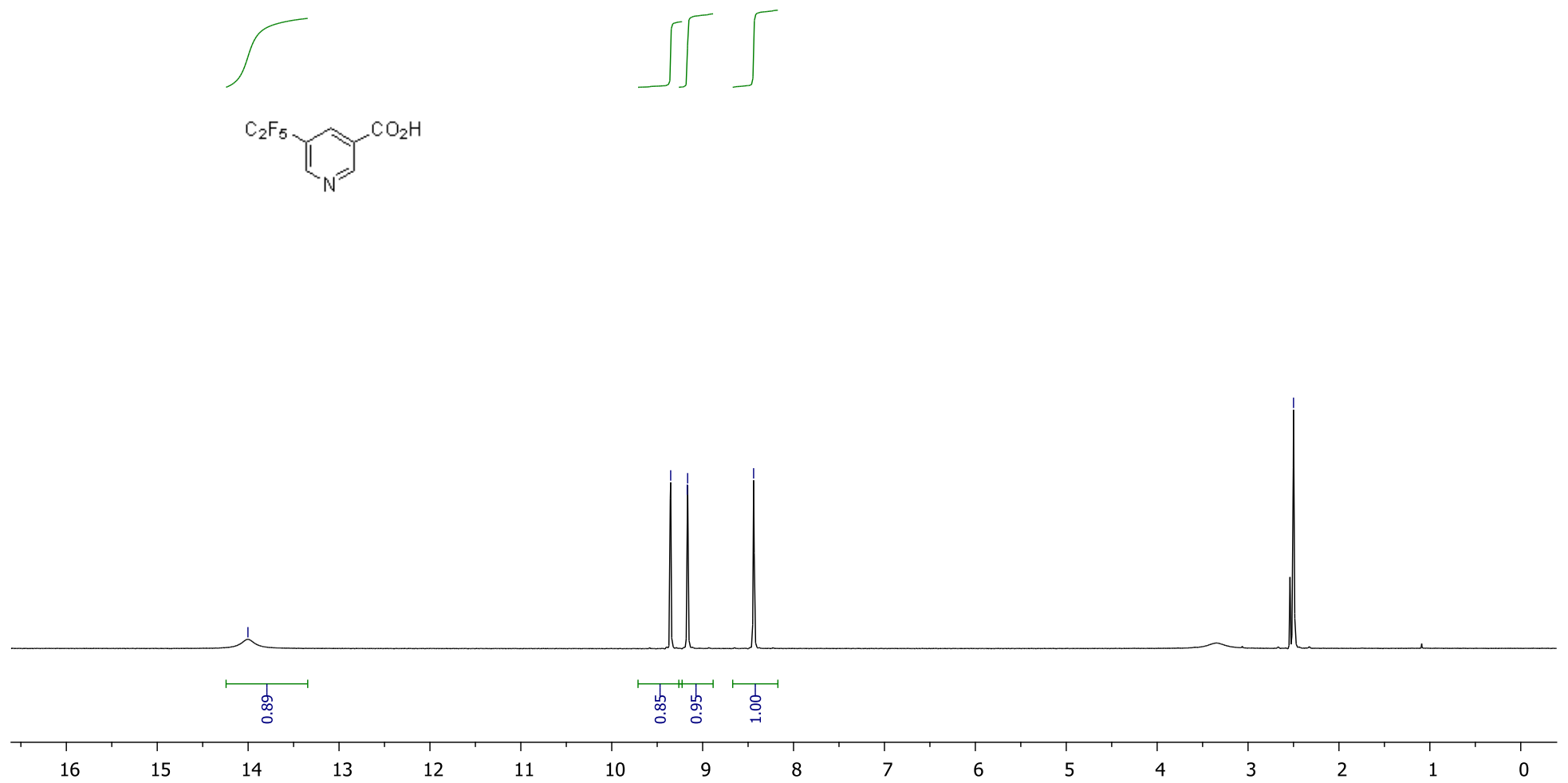
${ }^{13} \mathrm{C}\left\{{ }^{1} \mathrm{H}\right\}$ NMR (151 MHz, DMSO- $\left.d_{6}\right)$

R2428685+V_C13

t

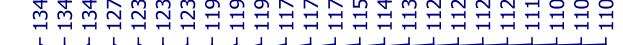
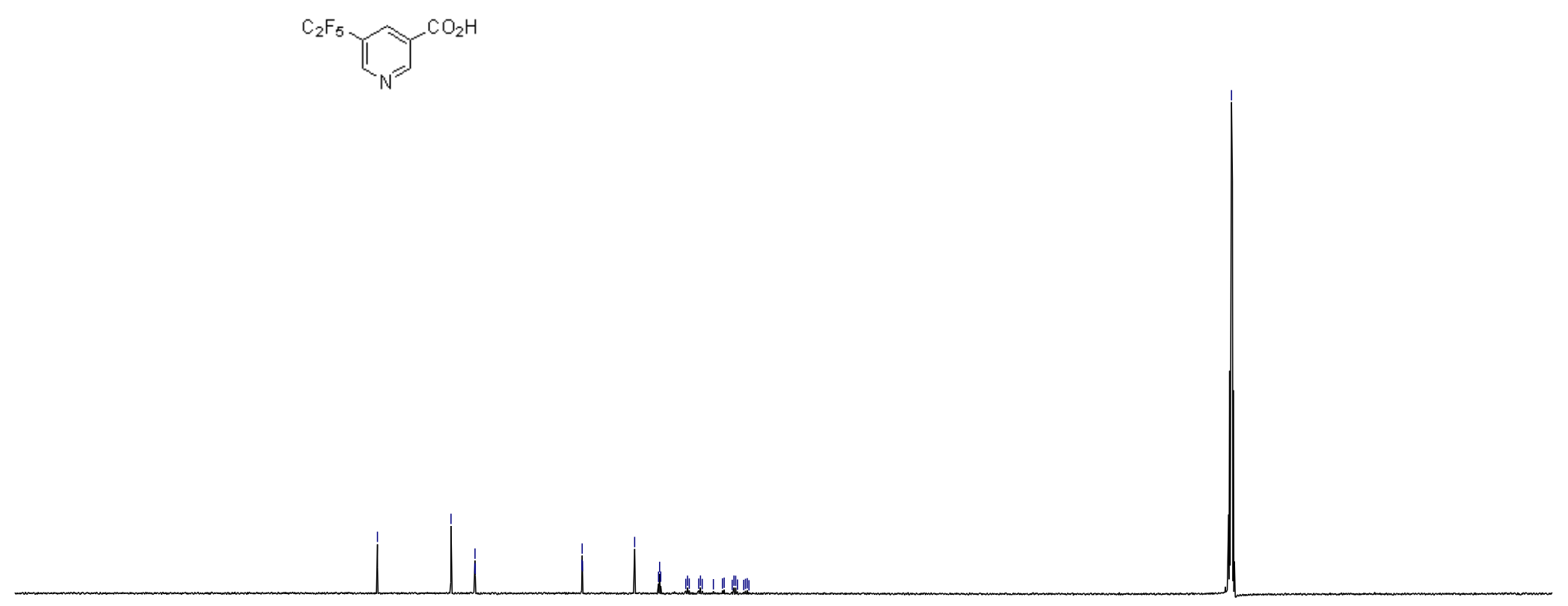


\section{${ }^{19} \mathrm{~F}\left\{{ }^{1} \mathrm{H}\right\}$ NMR (376 MHz, DMSO- $\left.d_{6}\right)$}

R2428685_F19 $\{\mathrm{H}\}$

$19 \mathrm{~F}-\{1 \mathrm{H}\}$

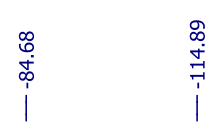

$\mathrm{C}_{2} \mathrm{~F}_{5} \mathrm{CO}_{2} \mathrm{H}$
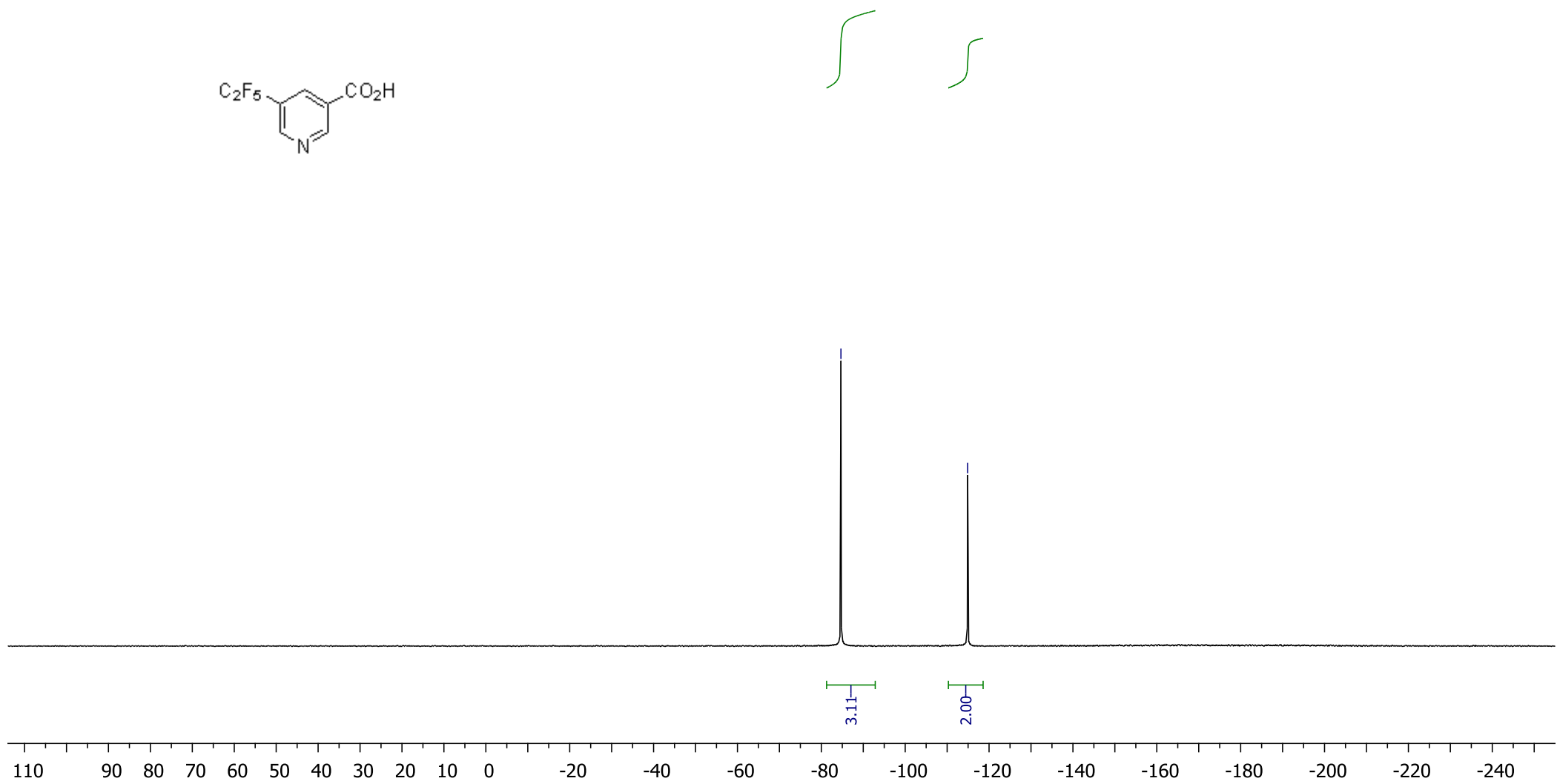


\section{Compound 64c}

${ }^{1} \mathrm{H}$ NMR (400 MHz, DMSO- $d_{6}$ )

R2425389

$\sqrt{\mathrm{C}_{2} \mathrm{~F}_{5}} \overbrace{\mathrm{N}}^{\mathrm{N}} \mathrm{CO}_{2} \mathrm{H}$

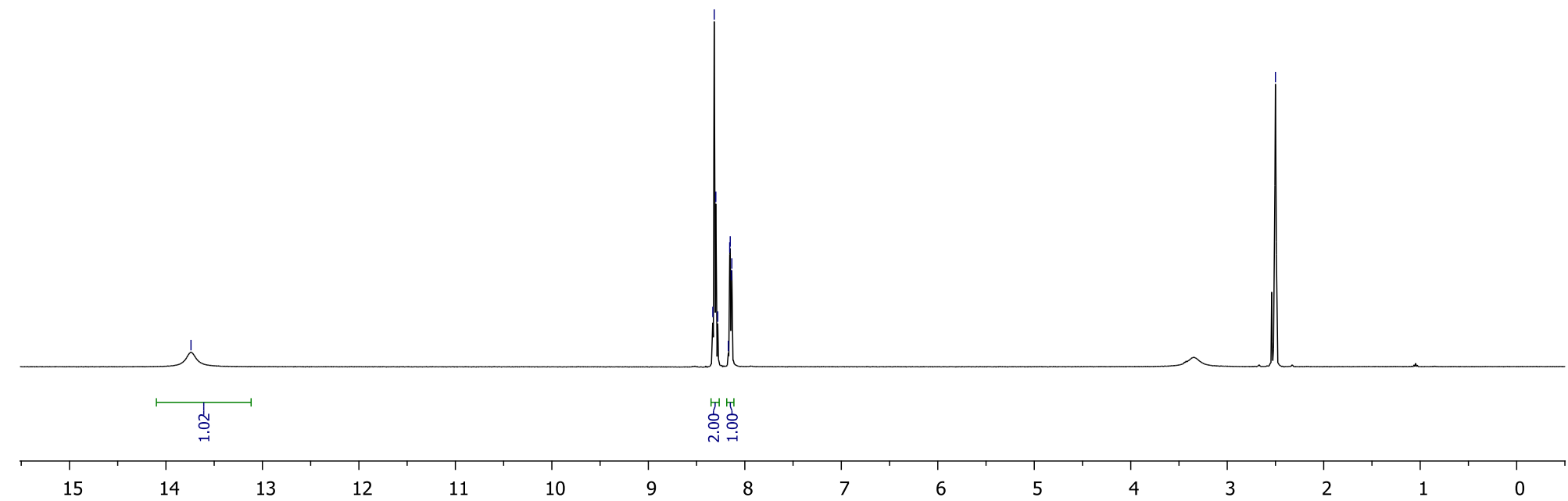


${ }^{13} \mathrm{C}\left\{{ }^{1} \mathrm{H}\right\}$ NMR (151 MHz, DMSO- $\left.d_{6}\right)$

R2425389_C13

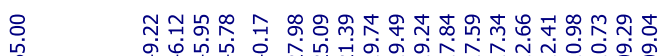

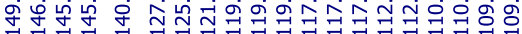

$\mathrm{C}_{2} \mathrm{~F}_{5} \underset{\mathrm{CO}_{2} \mathrm{H}}{\mathrm{N}}$

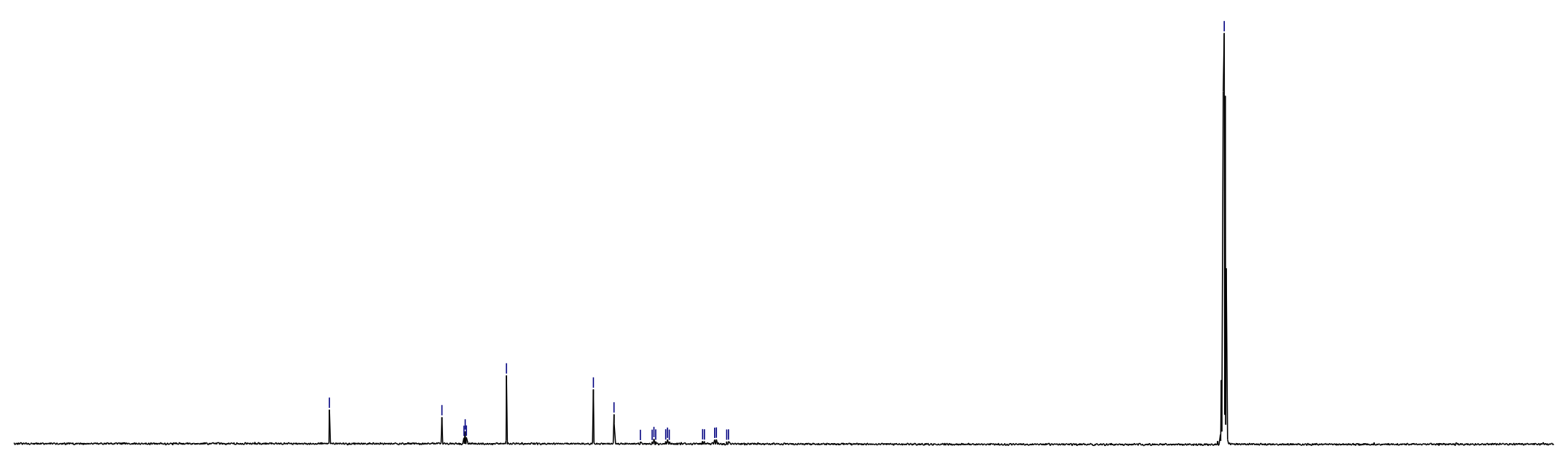




\section{${ }^{19} \mathrm{~F}\left\{{ }^{1} \mathrm{H}\right\}$ NMR (376 MHz, DMSO- $\left.d_{6}\right)$}

R2425389_F19 $\{\mathrm{H}\}$

$19 \mathrm{~F}-\{1 \mathrm{H}\}$

$\begin{array}{ll}0 & 0 \\ 0 & 7 \\ 0 & 0 \\ 0 & 7 \\ 1 & i\end{array}$

$\mathrm{C}_{2} \mathrm{~F}_{5} \mathrm{CO}_{2} \mathrm{H}$
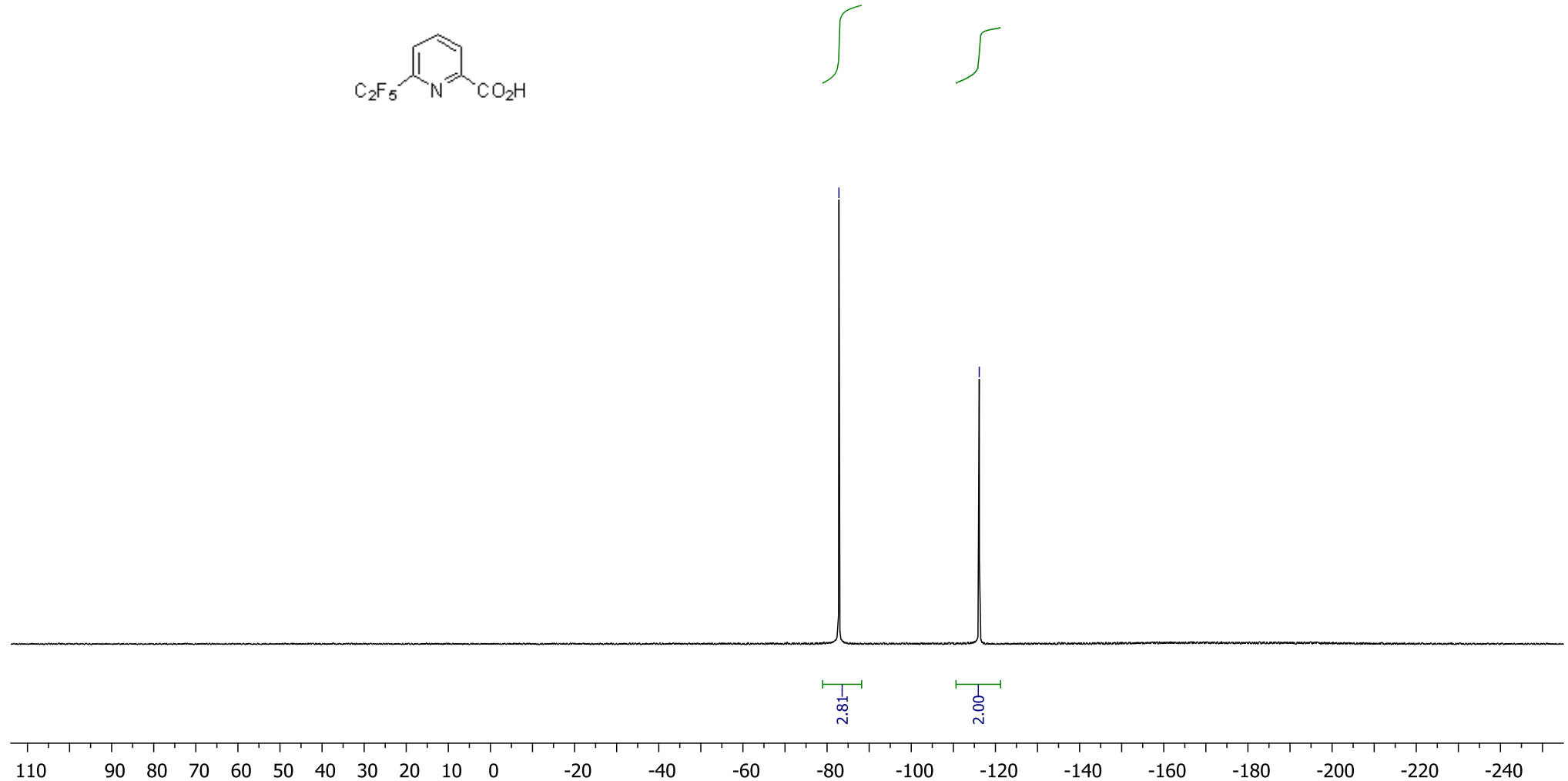


\section{Compound 65c}

${ }^{1} \mathrm{H}$ NMR (400 MHz, DMSO- $d_{6}$ )

R2433744

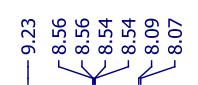
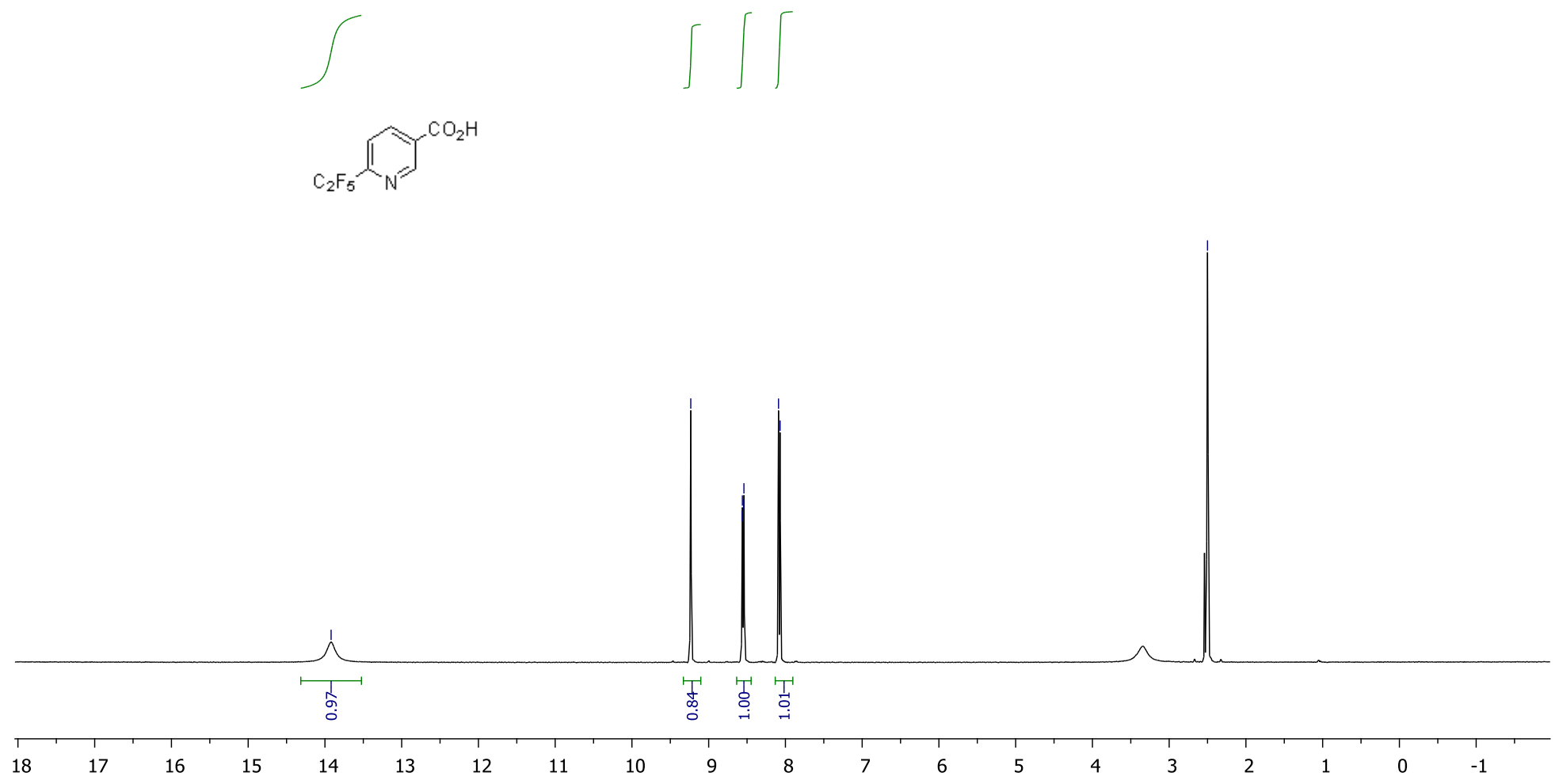


\section{${ }^{13} \mathrm{C}\left\{{ }^{1} \mathrm{H}\right\}$ NMR (151 MHz, DMSO- $\left.d_{6}\right)$}

R2433744_C13
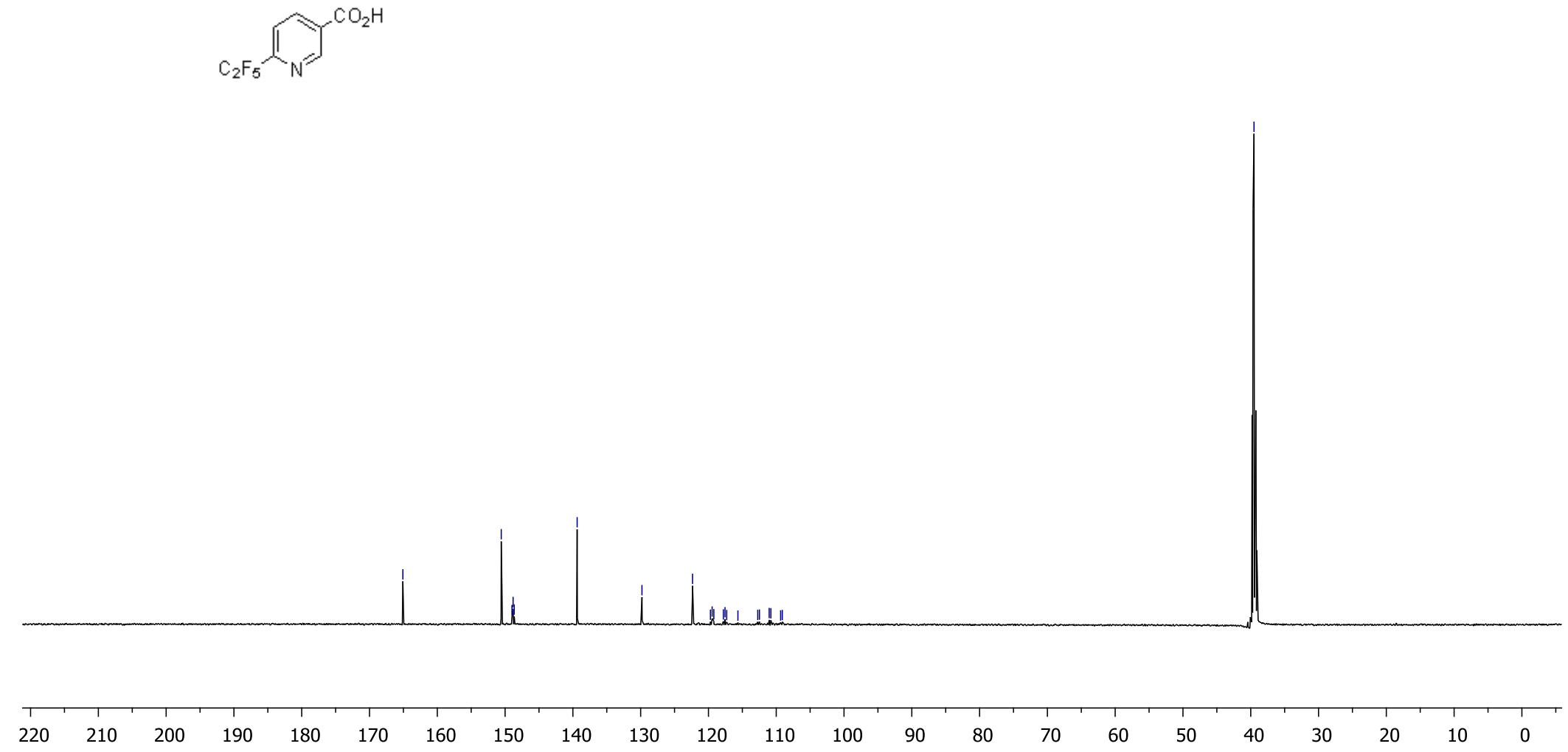


\section{${ }^{19} \mathrm{~F}\left\{{ }^{1} \mathrm{H}\right\}$ NMR (376 MHz, DMSO- $\left.d_{6}\right)$}

R2433744_F19 $\{\mathrm{H}\}$ $19 \mathrm{~F}-\{1 \mathrm{H}\}$
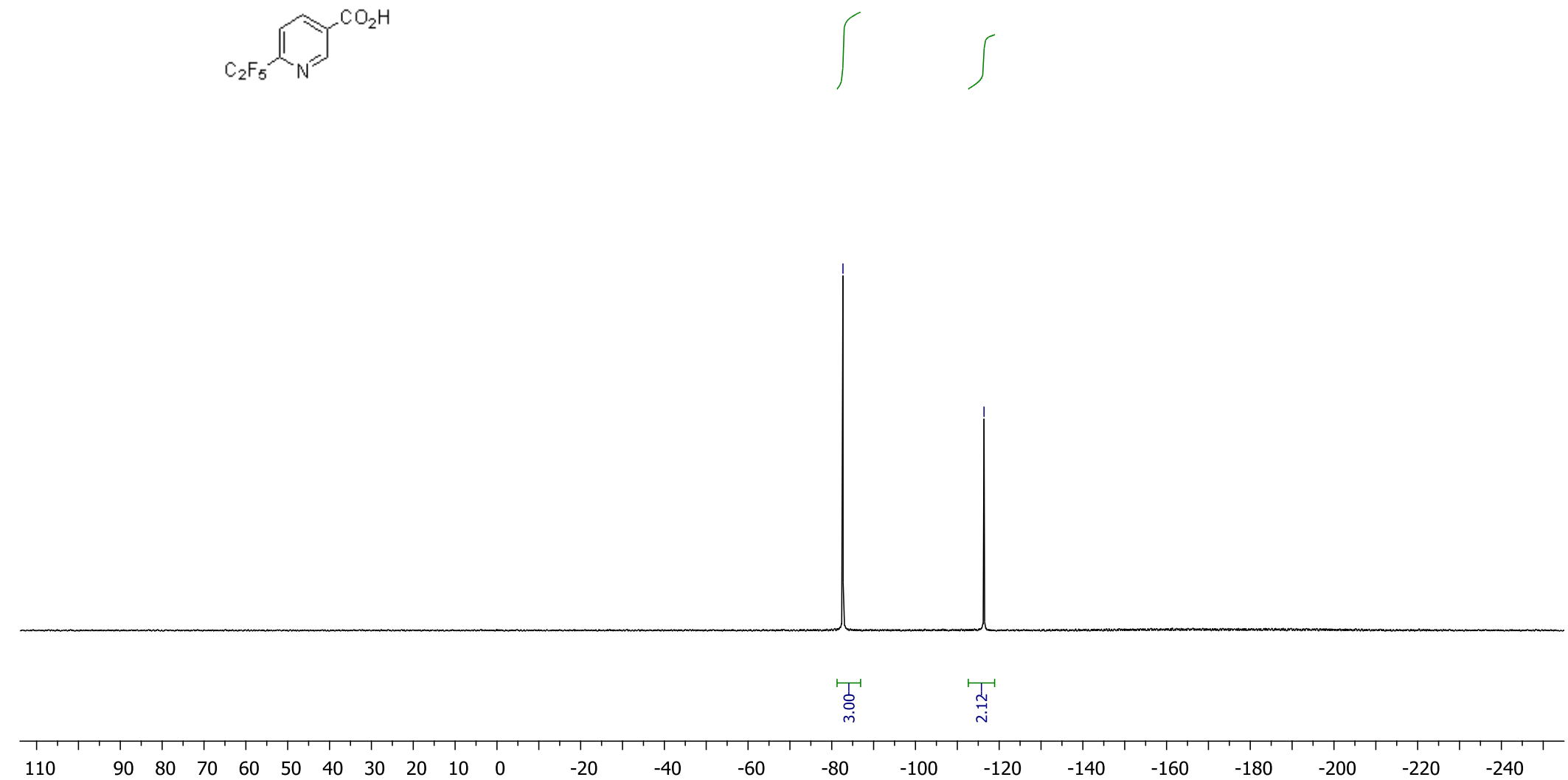


\section{Compound 9d*HCl}

${ }^{1} \mathrm{H}$ NMR (400 MHz, DMSO- $d_{6}$ )

R1505167

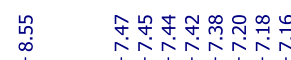

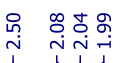

난

$\int$

$\overbrace{\mathrm{Me}}^{\mathrm{NH}_{2} \mathrm{~F}^{\mathrm{F}}}$

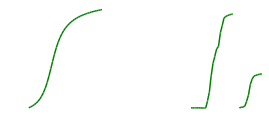

$\iint$

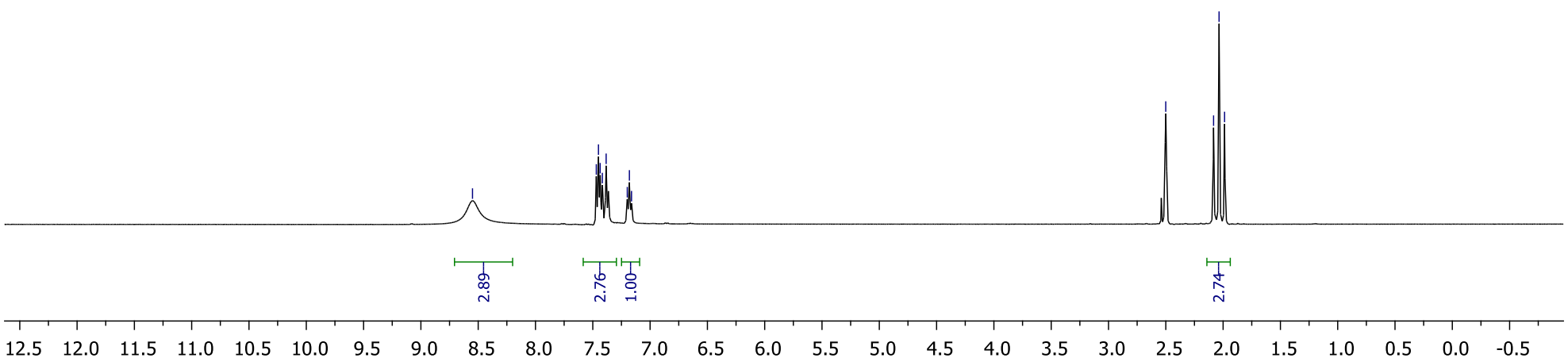




\section{${ }^{13} \mathrm{C}\left\{{ }^{1} \mathrm{H}\right\}$ NMR $\left(126 \mathrm{MHz}\right.$, DMSO- $\left.d_{6}\right)$}

R1505167_13C

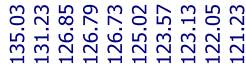

雚

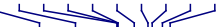

$\mathrm{NH}_{2} \mathrm{~F}_{2} \mathrm{~F}$ If

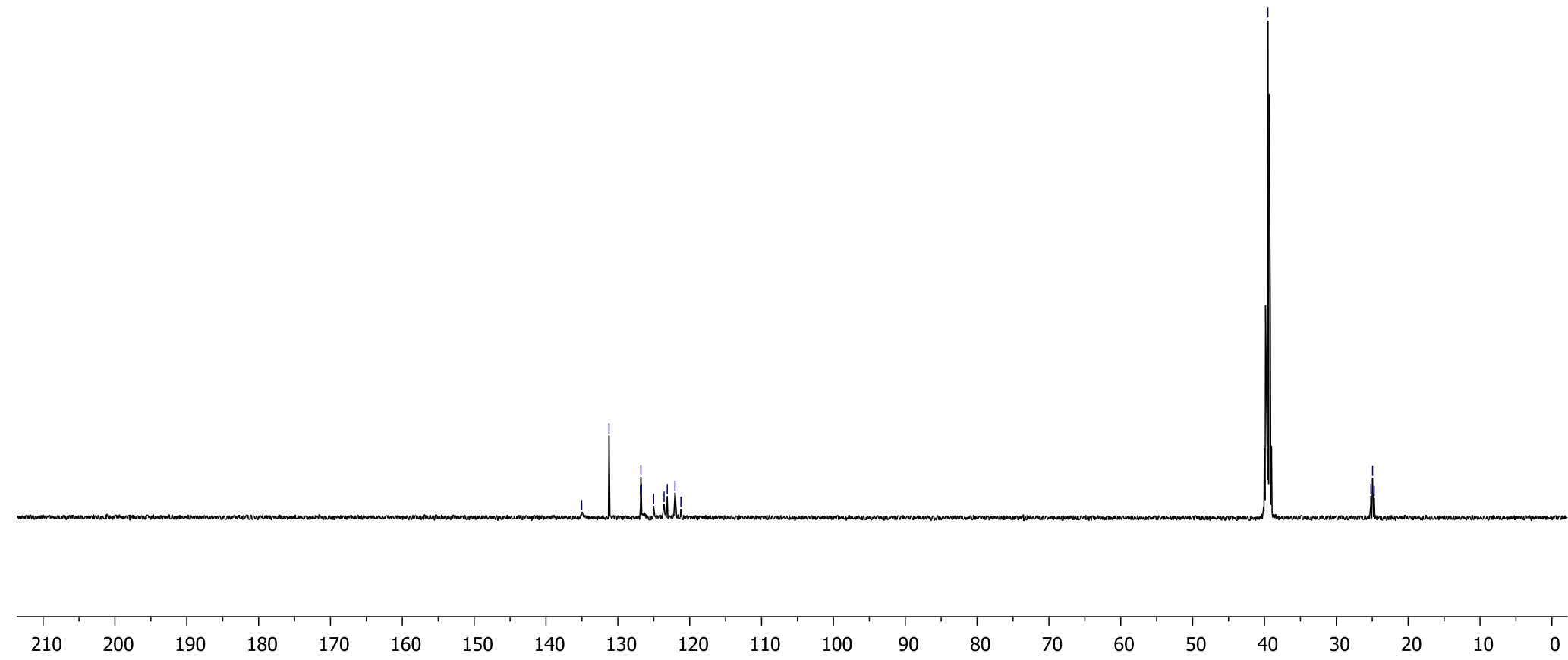




\section{${ }^{19} \mathrm{~F}\left\{{ }^{1} \mathrm{H}\right\}$ NMR $\left(376 \mathrm{MHz}\right.$, DMSO- $\left.d_{6}\right)$}

R1505167_F19\{H\}
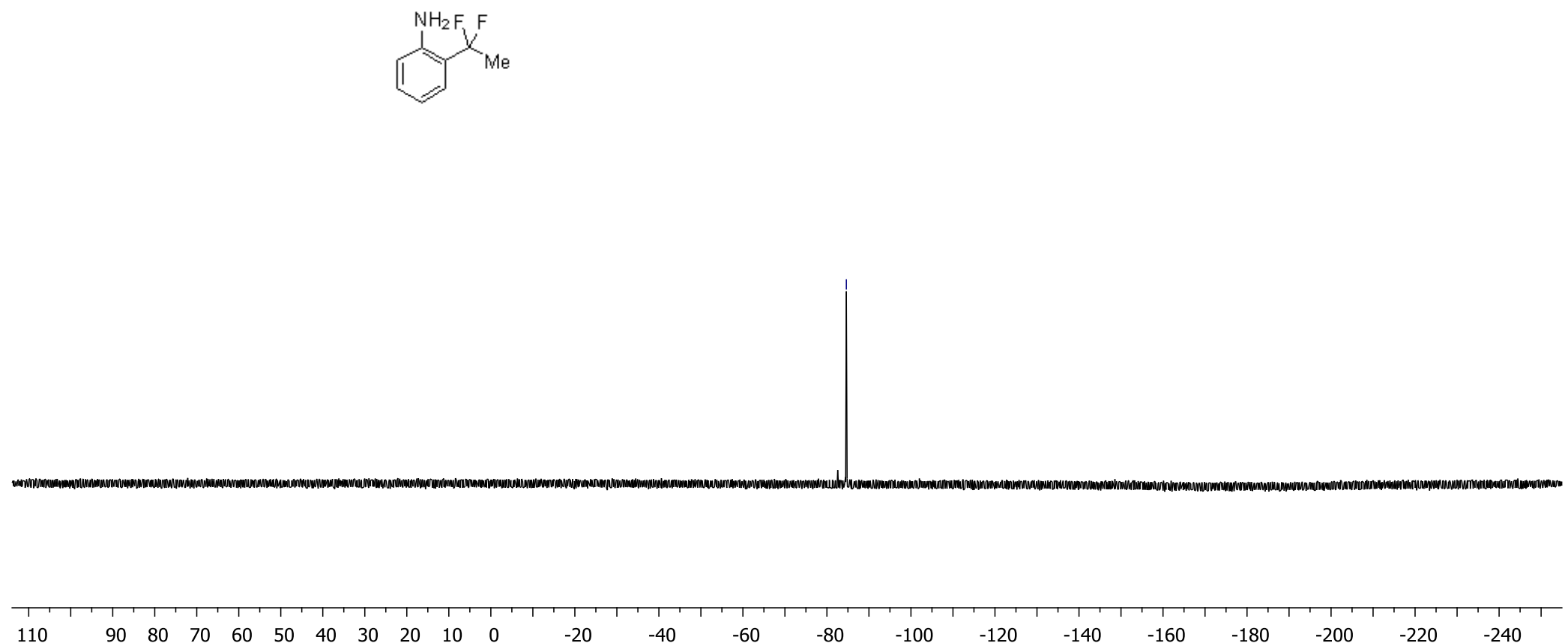


\section{Compound 10d*HCl}

${ }^{1} \mathrm{H}$ NMR (400 MHz, DMSO- $d_{6}$ )

R1474998

|

นึำ
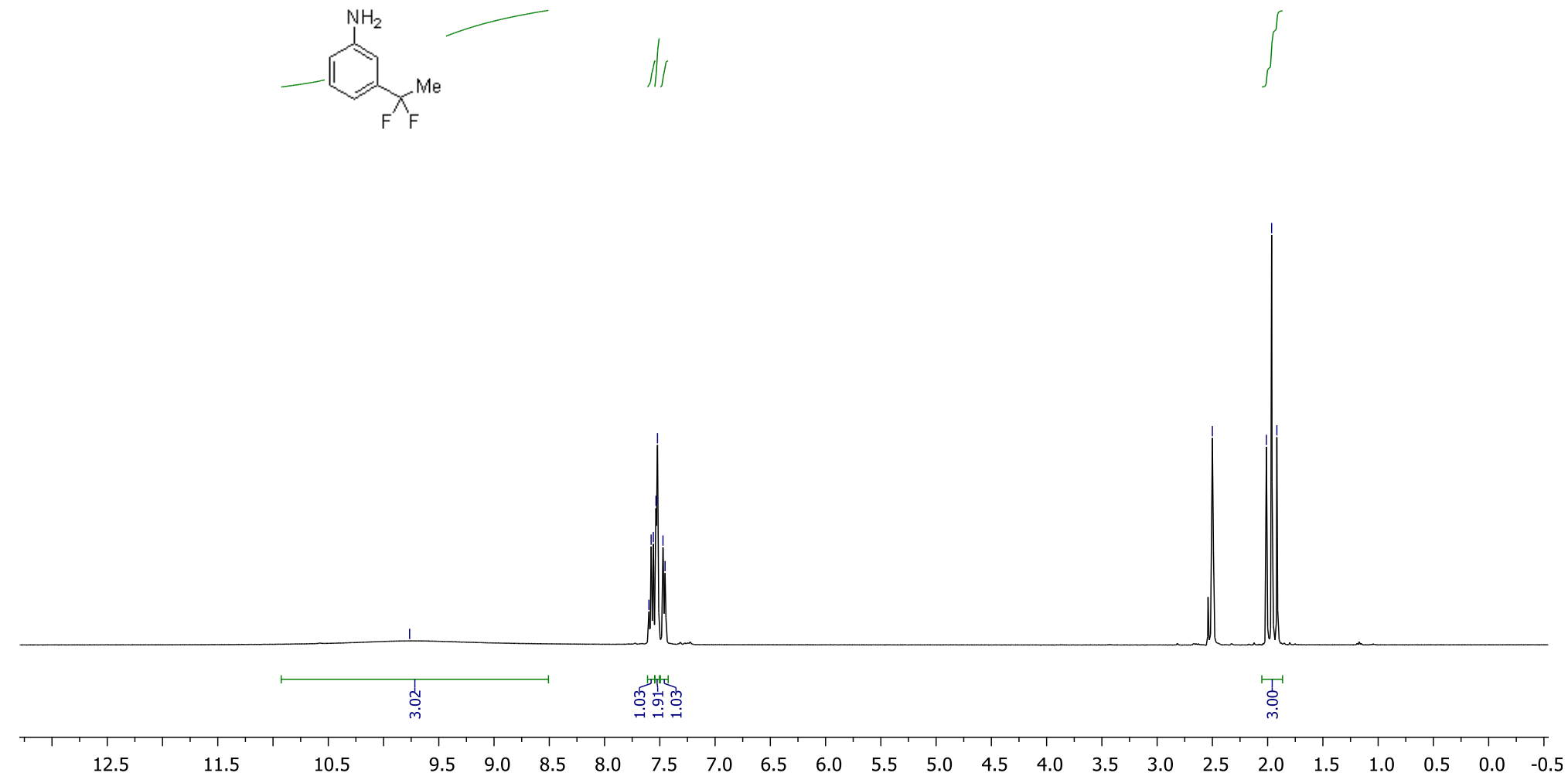
${ }^{13} \mathrm{C}\left\{{ }^{1} \mathrm{H}\right\} \mathrm{NMR}\left(126 \mathrm{MHz}, \mathrm{DMSO}-d_{6}\right)$

R1474998_C1:

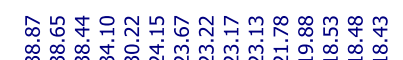

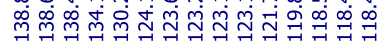

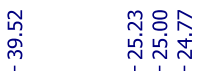

स 112

$\underbrace{M e}_{F F}$
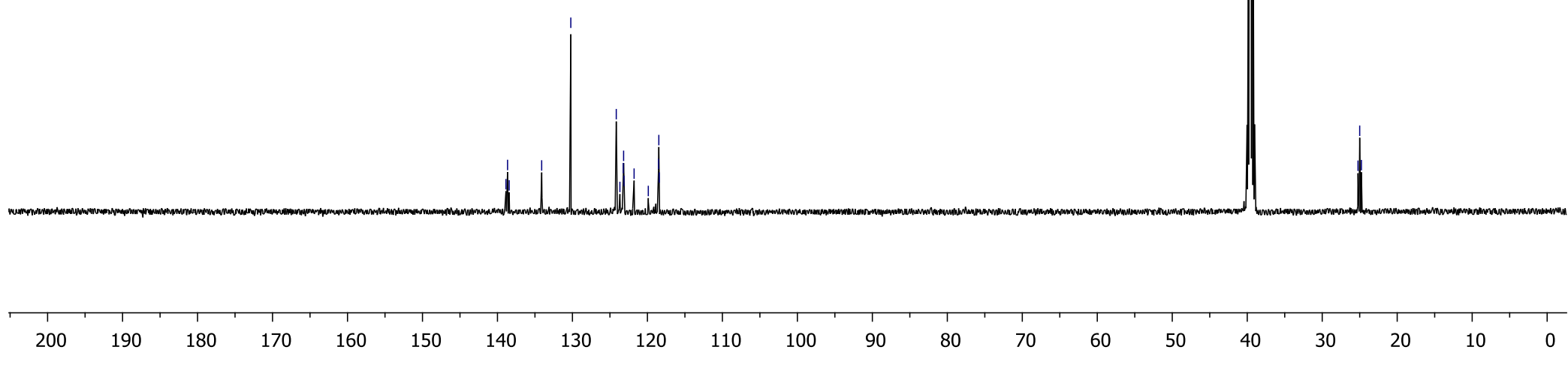


\section{${ }^{19} \mathrm{~F}\left\{{ }^{1} \mathrm{H}\right\}$ NMR $\left(376 \mathrm{MHz}\right.$, DMSO- $\left.d_{6}\right)$}

R1474998_F19 $\{\mathrm{H}\}$ $19 \mathrm{~F}-\{1 \mathrm{H}\}$
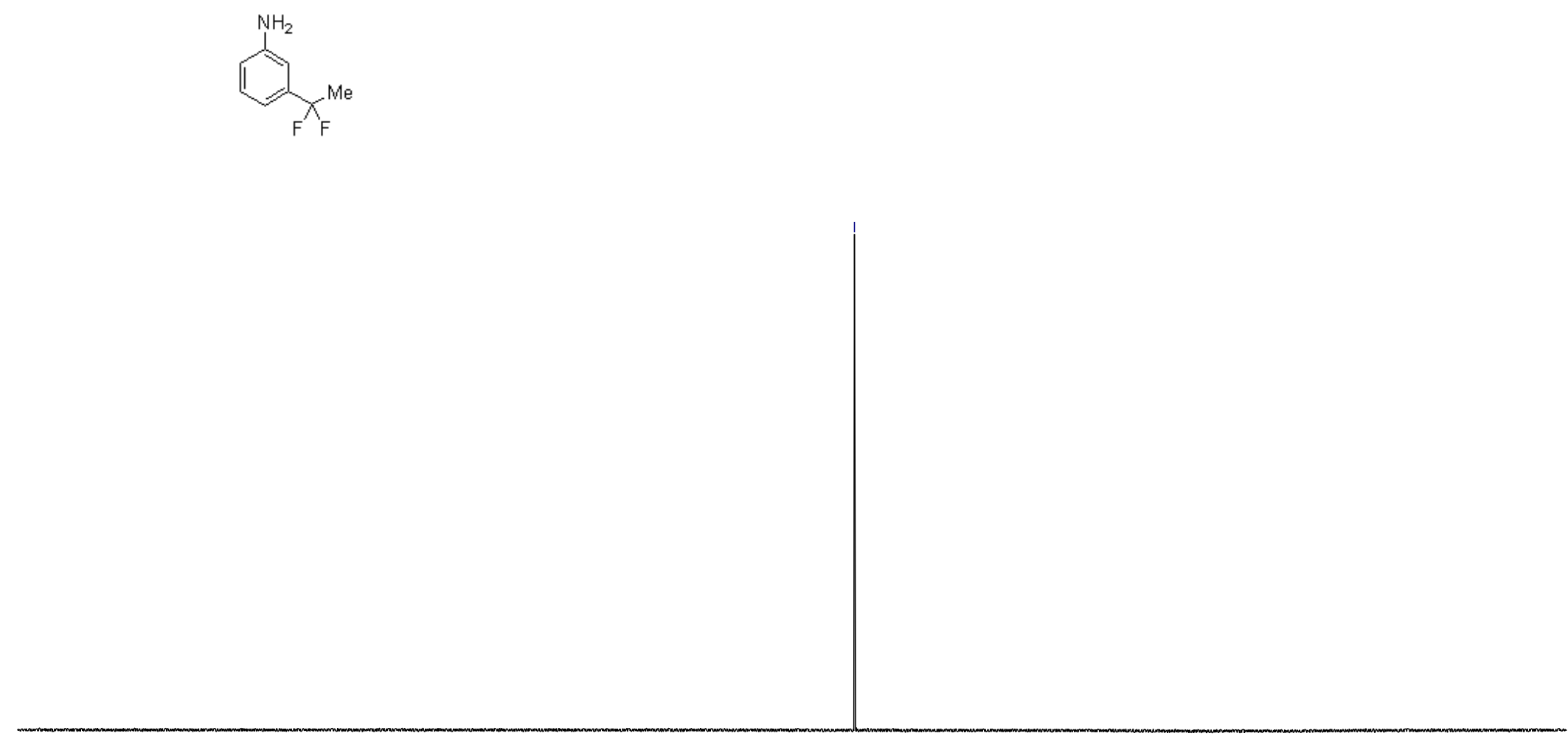


\section{Compound 17d}

${ }^{1} \mathrm{H}$ NMR (400 MHz, $\mathrm{CDCl}_{3}$ )

R1985343

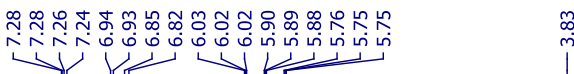
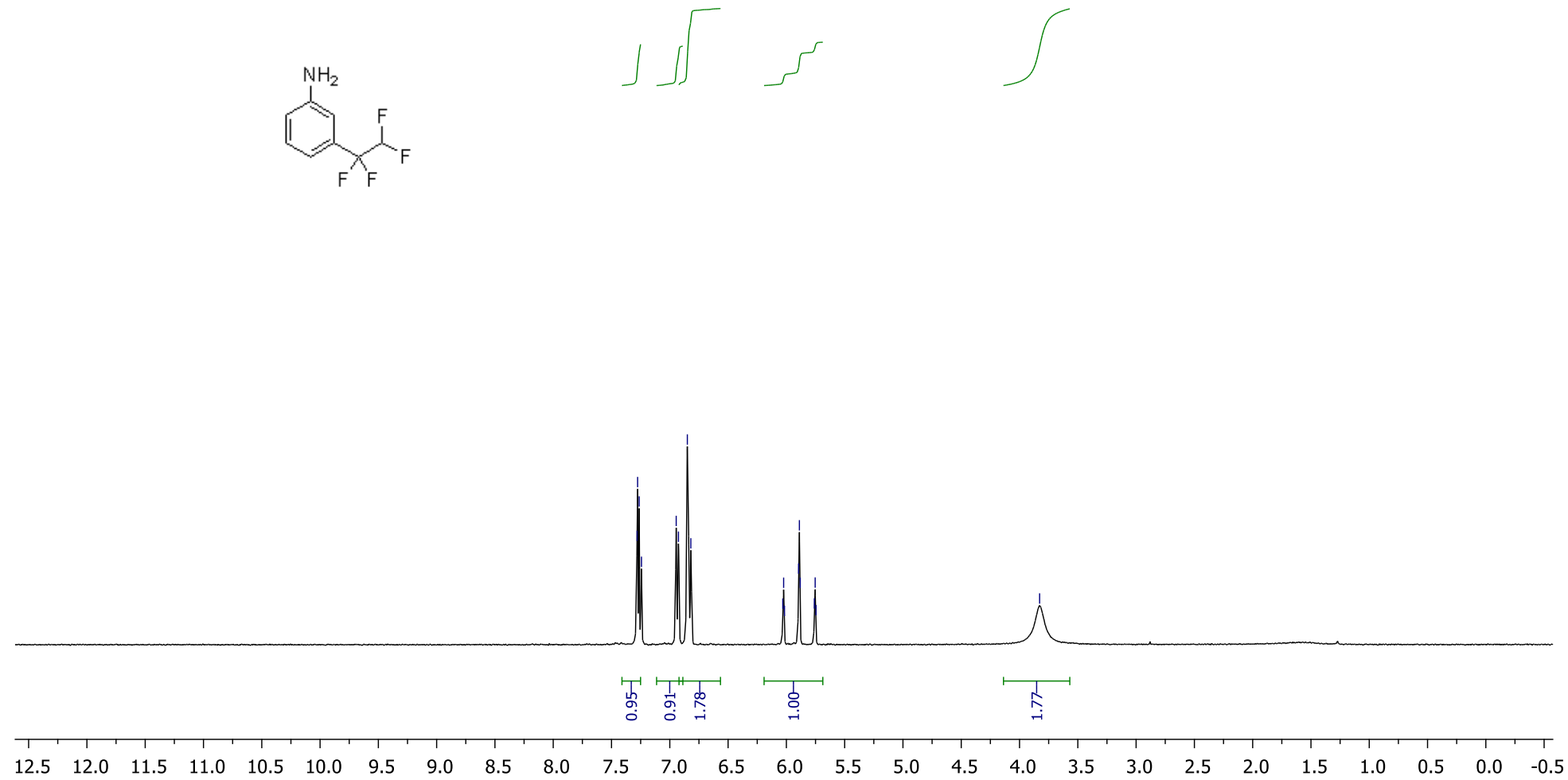
${ }^{13} \mathrm{C}\left\{{ }^{1} \mathrm{H}\right\}$ NMR (151 MHz, DMSO- $\left.d_{6}\right)$

R1985343_C13

m

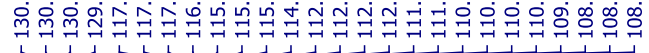

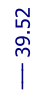
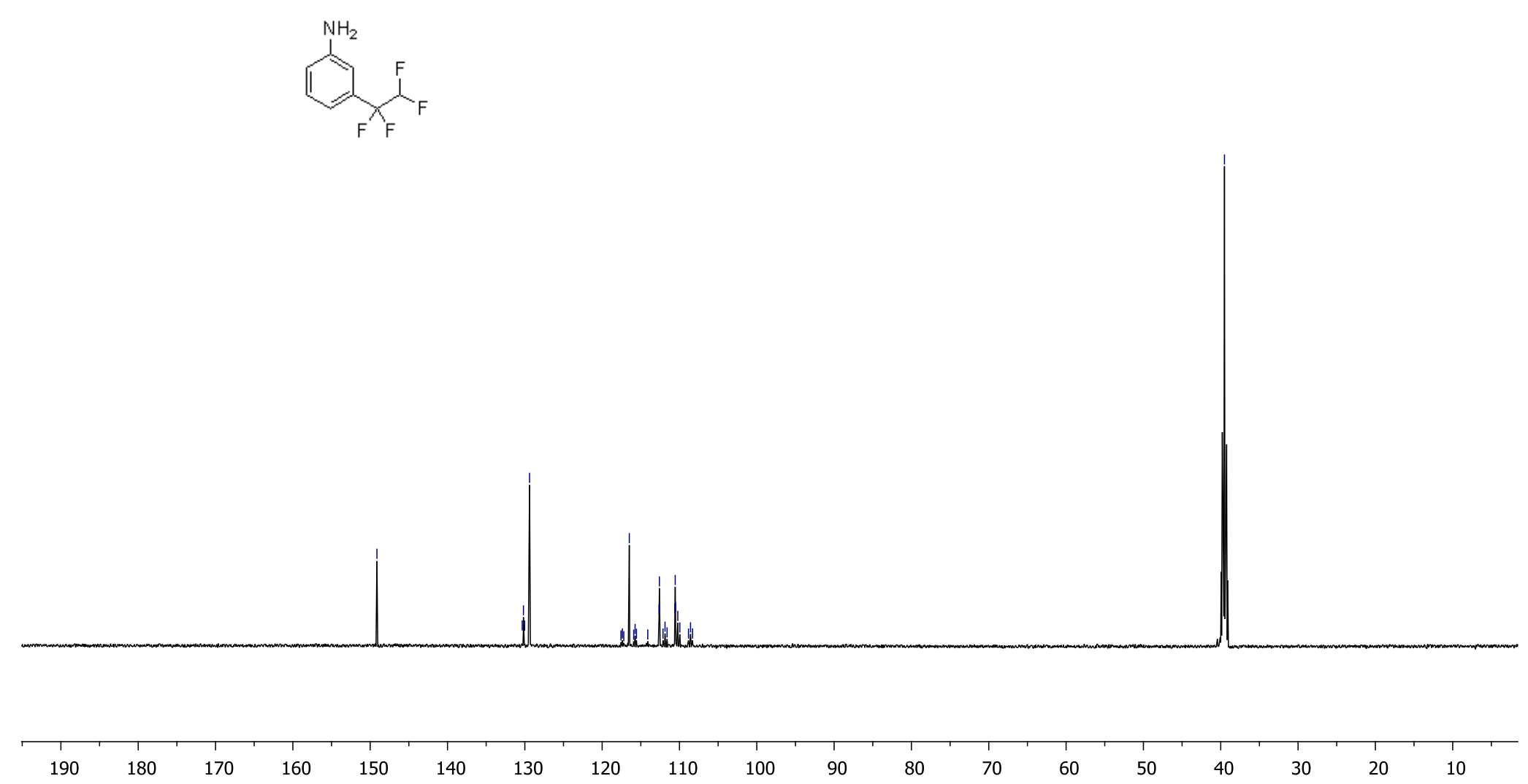
${ }^{19} \mathrm{~F}\left\{{ }^{1} \mathrm{H}\right\} \mathrm{NMR}\left(376 \mathrm{MHz}, \mathrm{CDCl}_{3}\right)$

R1985343_F19 $\{\mathrm{H}\}$

$19 F-\{1 \mathrm{H}\}$
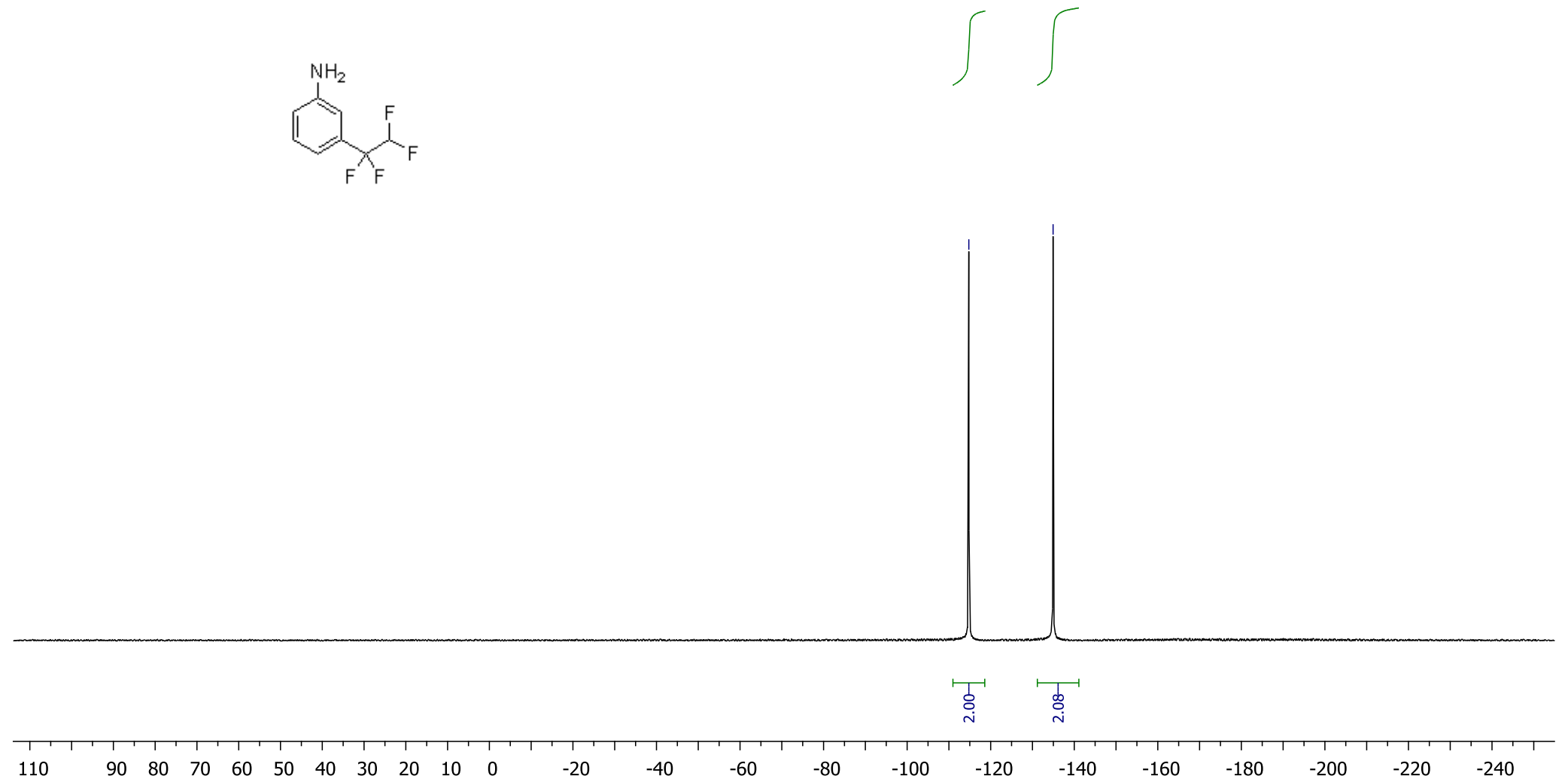


\section{Compound 40d}

${ }^{1} \mathrm{H}$ NMR (400 MHz, $\mathrm{CDCl}_{3}$ )

R1463130

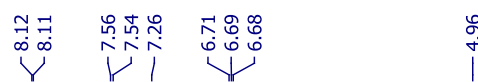

둥워

$\overbrace{\mathrm{N}}^{\mathrm{F}} \mathrm{X}_{\mathrm{Ne}}^{\mathrm{F}}$

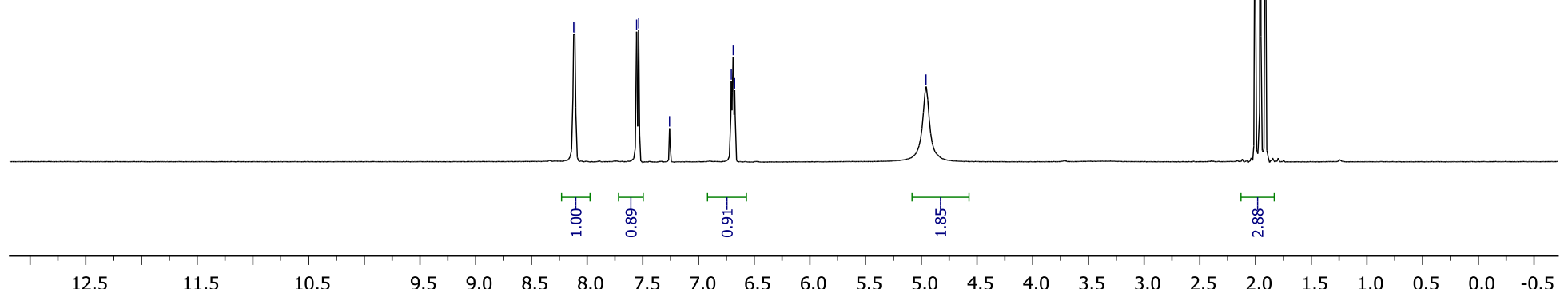


${ }^{13} \mathrm{C}\left\{{ }^{1} \mathrm{H}\right\}$ NMR (126 MHz, $\left.\mathrm{CDCl}_{3}\right)$

R1463130_136

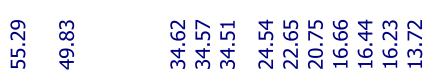

1

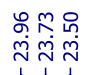

(2)
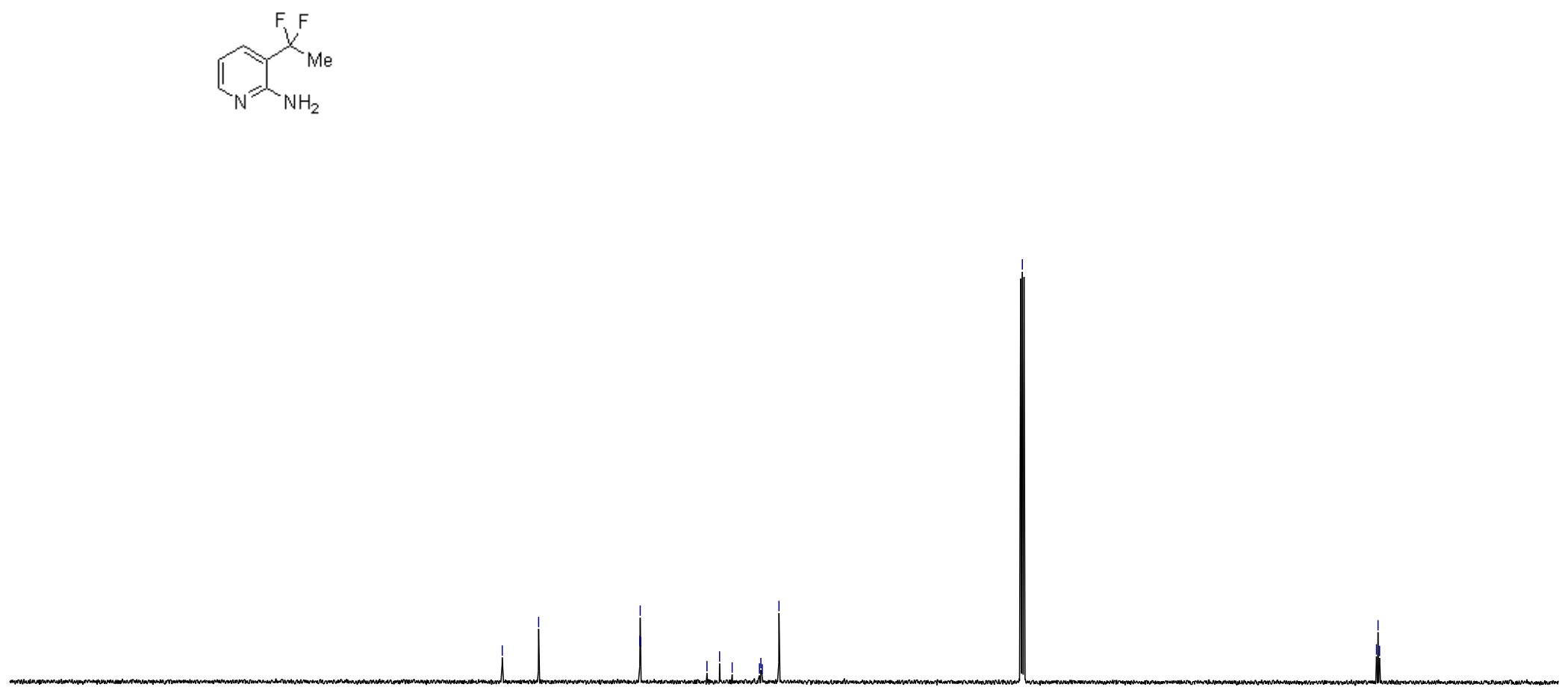

$\begin{array}{lllllllllllllllllllllll}220 & 210 & 200 & 190 & 180 & 170 & 160 & 150 & 140 & 130 & 120 & 110 & 100 & 90 & 80 & 70 & 60 & 50 & 40 & 30 & 20 & 10 & 0\end{array}$ 


\section{${ }^{19} \mathrm{~F}\left\{{ }^{1} \mathrm{H}\right\} \mathrm{NMR}\left(376 \mathrm{MHz}, \mathrm{CDCl}_{3}\right)$}

R1463130_F19

$19 \mathrm{~F}-\{1 \mathrm{H}\}$
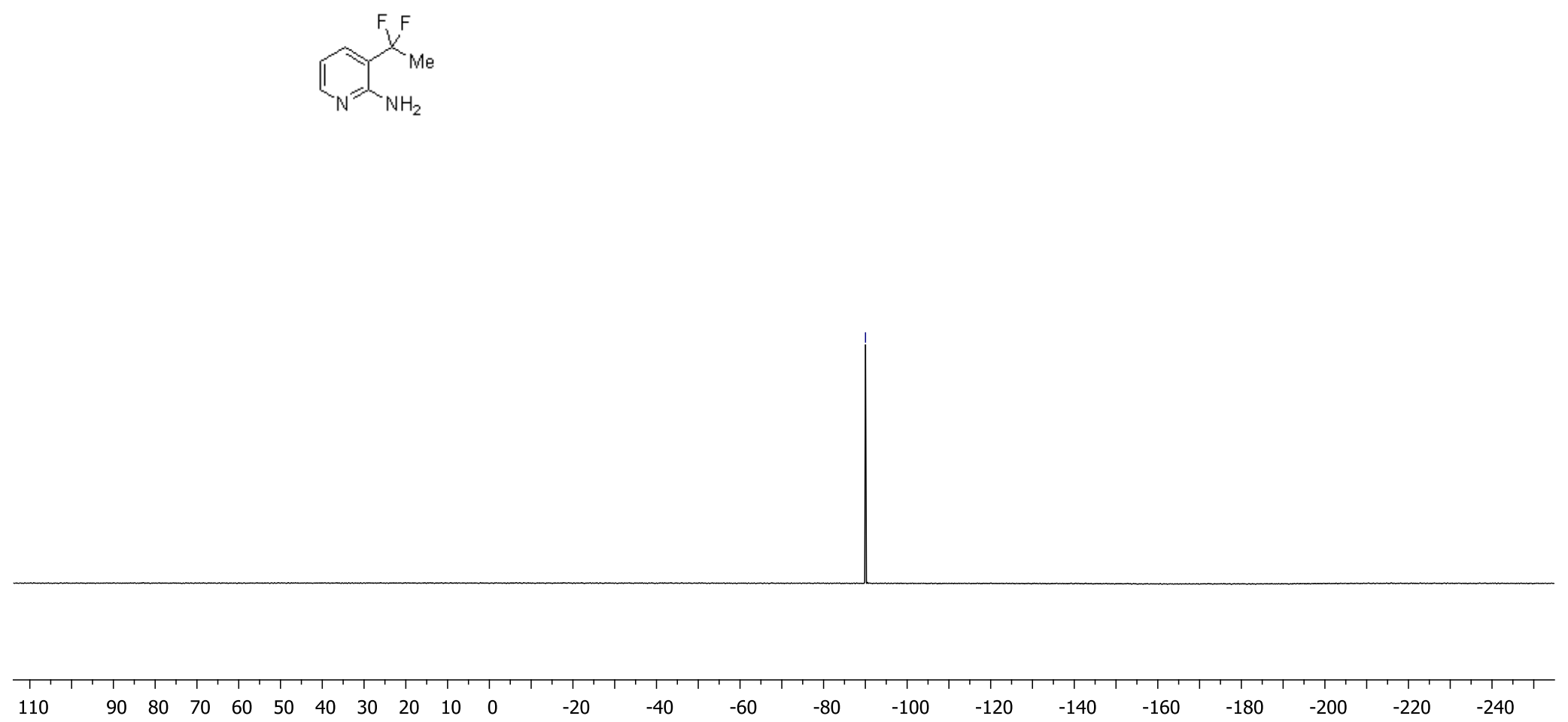


\section{Compound 43d}

${ }^{1} \mathrm{H}$ NMR (400 MHz, DMSO- $d_{6}$ )

R1878944_T

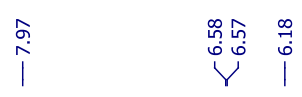

I

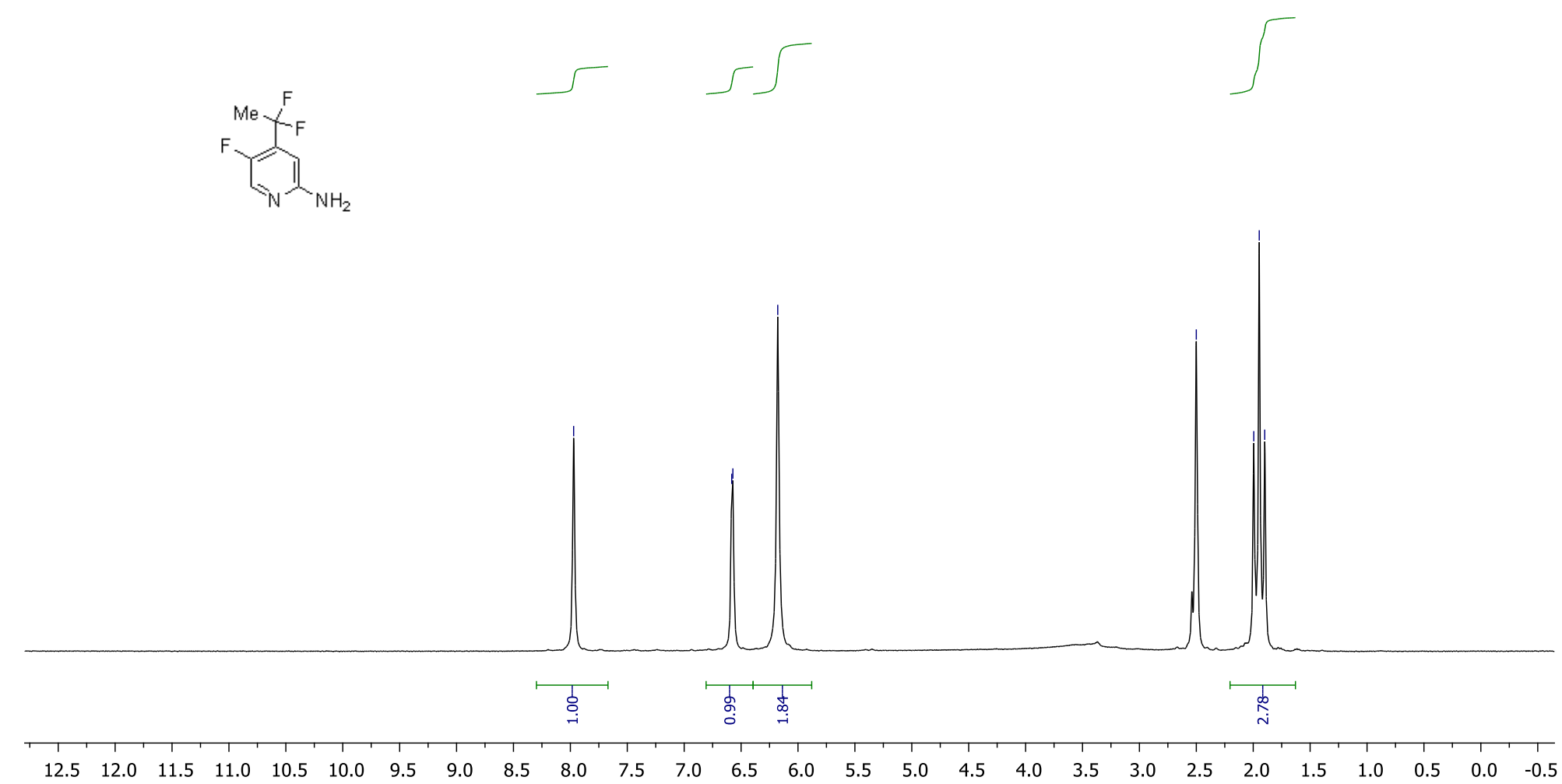


${ }^{13} \mathrm{C}\left\{{ }^{1} \mathrm{H}\right\}$ NMR (126 MHz, DMSO- $\left.d_{6}\right)$

R1878944_T_C1:

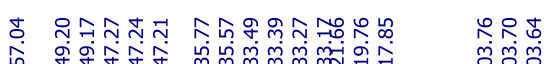

西

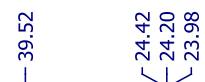
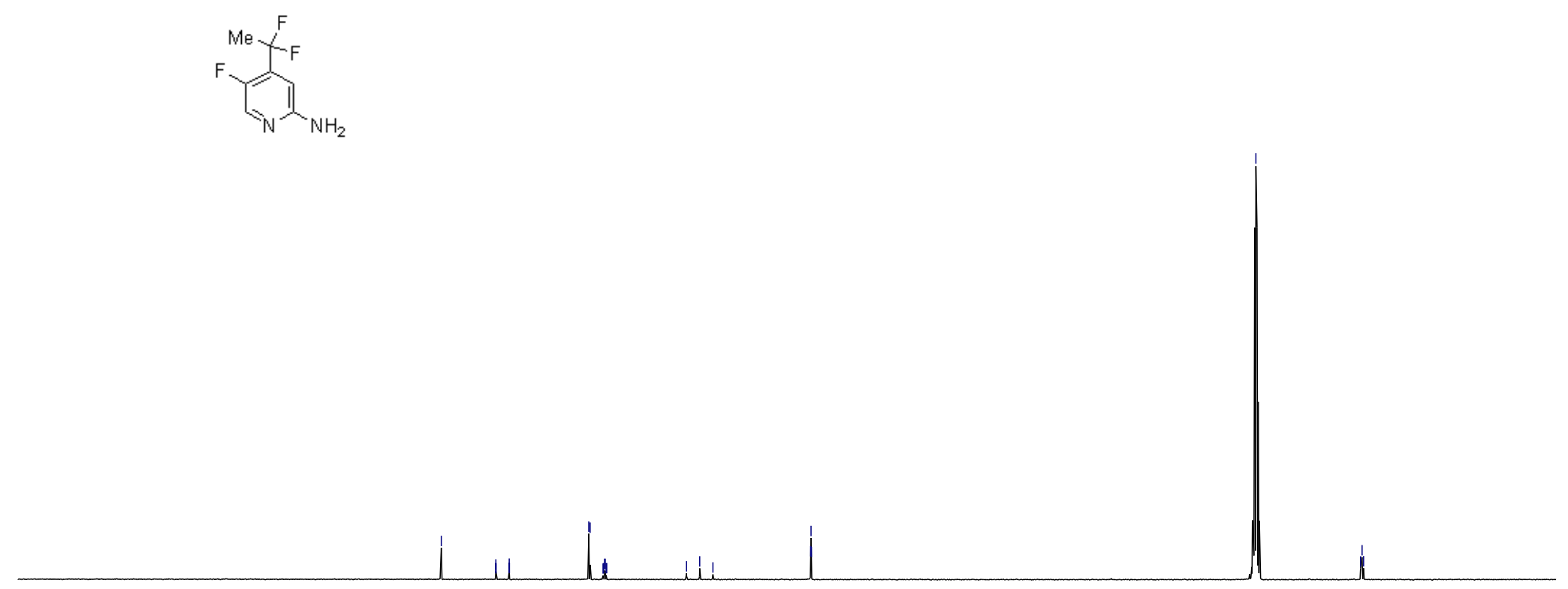

$210 \quad 200$

$190 \quad 180$

$170 \quad 160$

150

$40 \quad 130$

$\begin{array}{lll}120 & 110 & 100\end{array}$

90
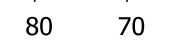
${ }^{19} \mathrm{~F}\left\{{ }^{1} \mathrm{H}\right\}$ NMR (376 MHz, DMSO- $\left.d_{6}\right)$

R1878944_F19\{H

$19 \mathrm{~F}-\{1 \mathrm{H}\}$
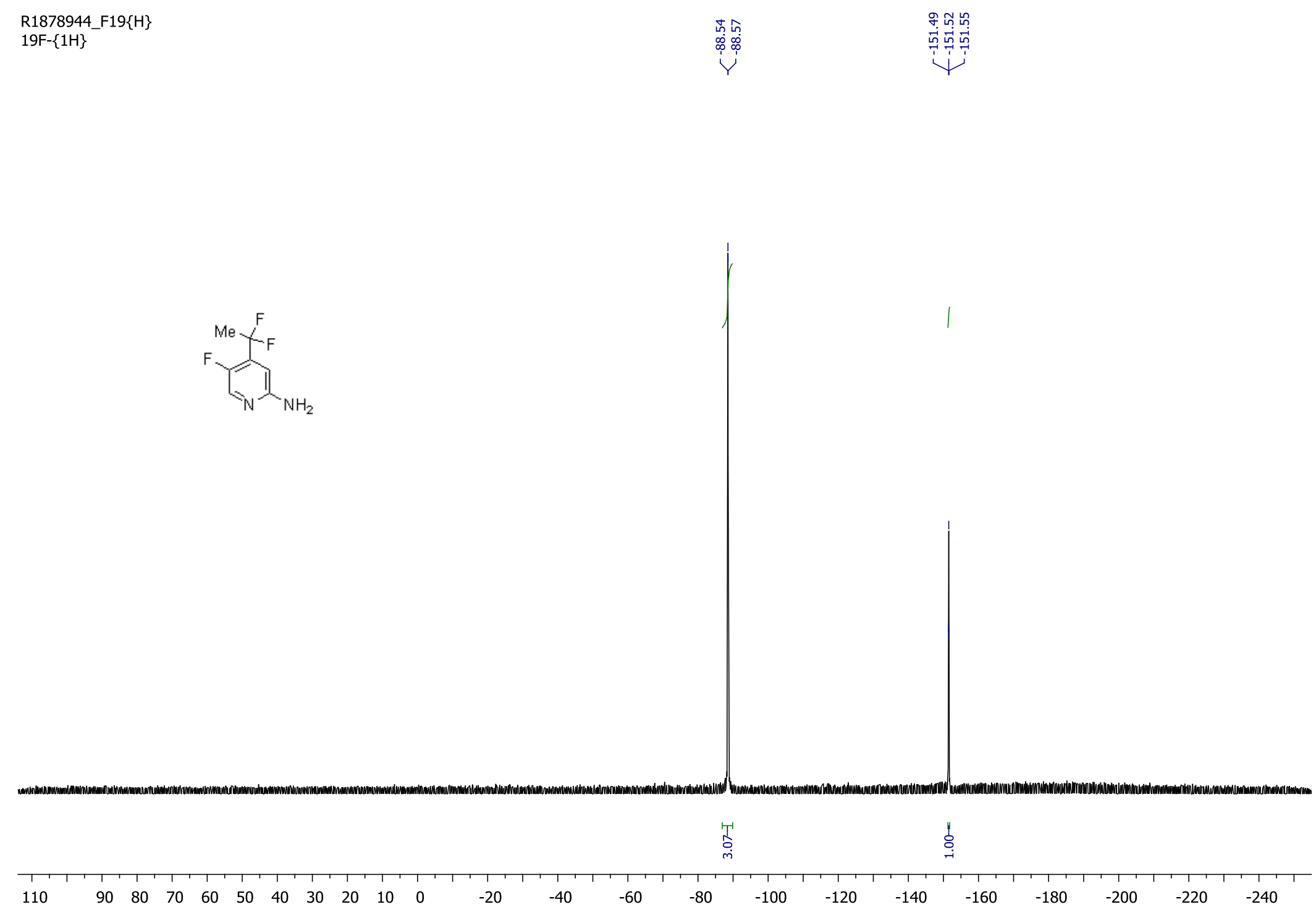


\section{Compound 58d*HCl}

${ }^{1} \mathrm{H}$ NMR (400 MHz, DMSO- $d_{6}$ )

R1732807

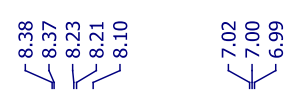

$$
\overbrace{N_{2}}^{C_{2} F_{5}}
$$
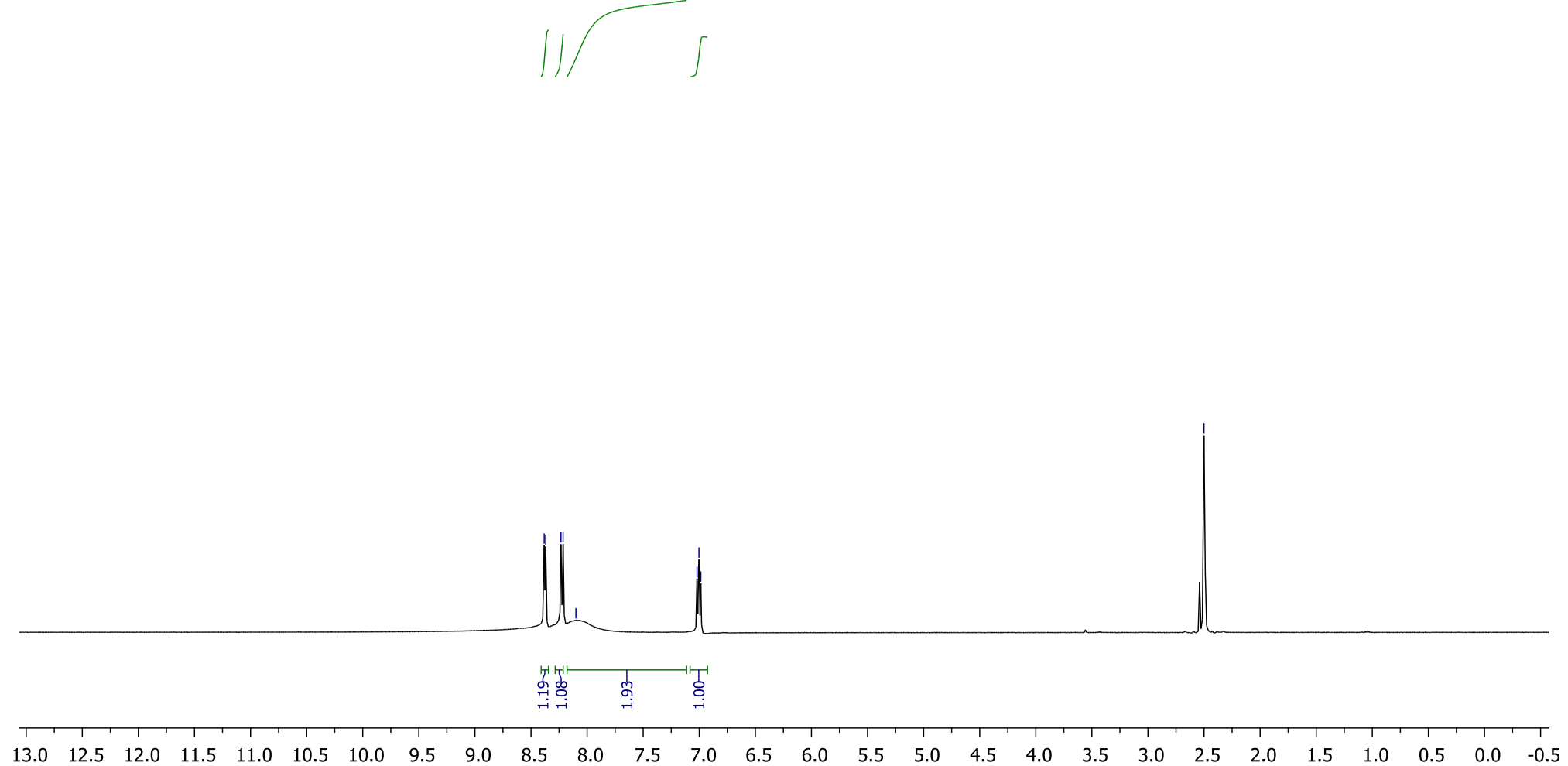
${ }^{13} \mathrm{C}\left\{{ }^{1} \mathrm{H}\right\}$ NMR (151 MHz, DMSO- $\left.d_{6}\right)$

R1732807_C13

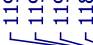

$\overbrace{N_{N}}^{C_{2} F_{5}}$

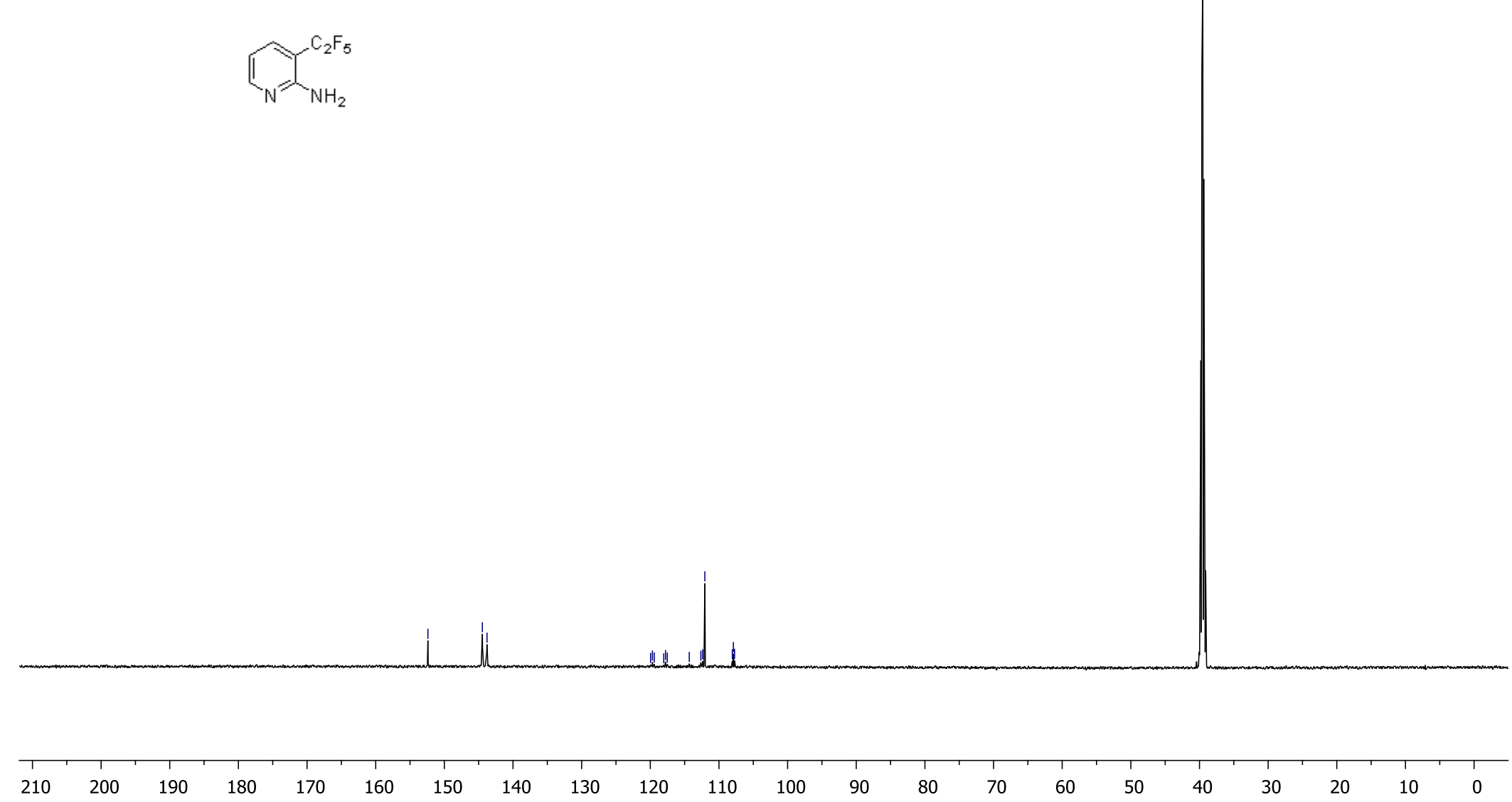




\section{${ }^{19} \mathrm{~F}\left\{{ }^{1} \mathrm{H}\right\}$ NMR (376 MHz, DMSO- $\left.d_{6}\right)$}

R1732807_F19

$19 \mathrm{~F}-\{1 \mathrm{H}\}$

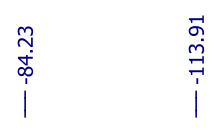

$$
\overbrace{N}^{C_{2} F_{5}}
$$
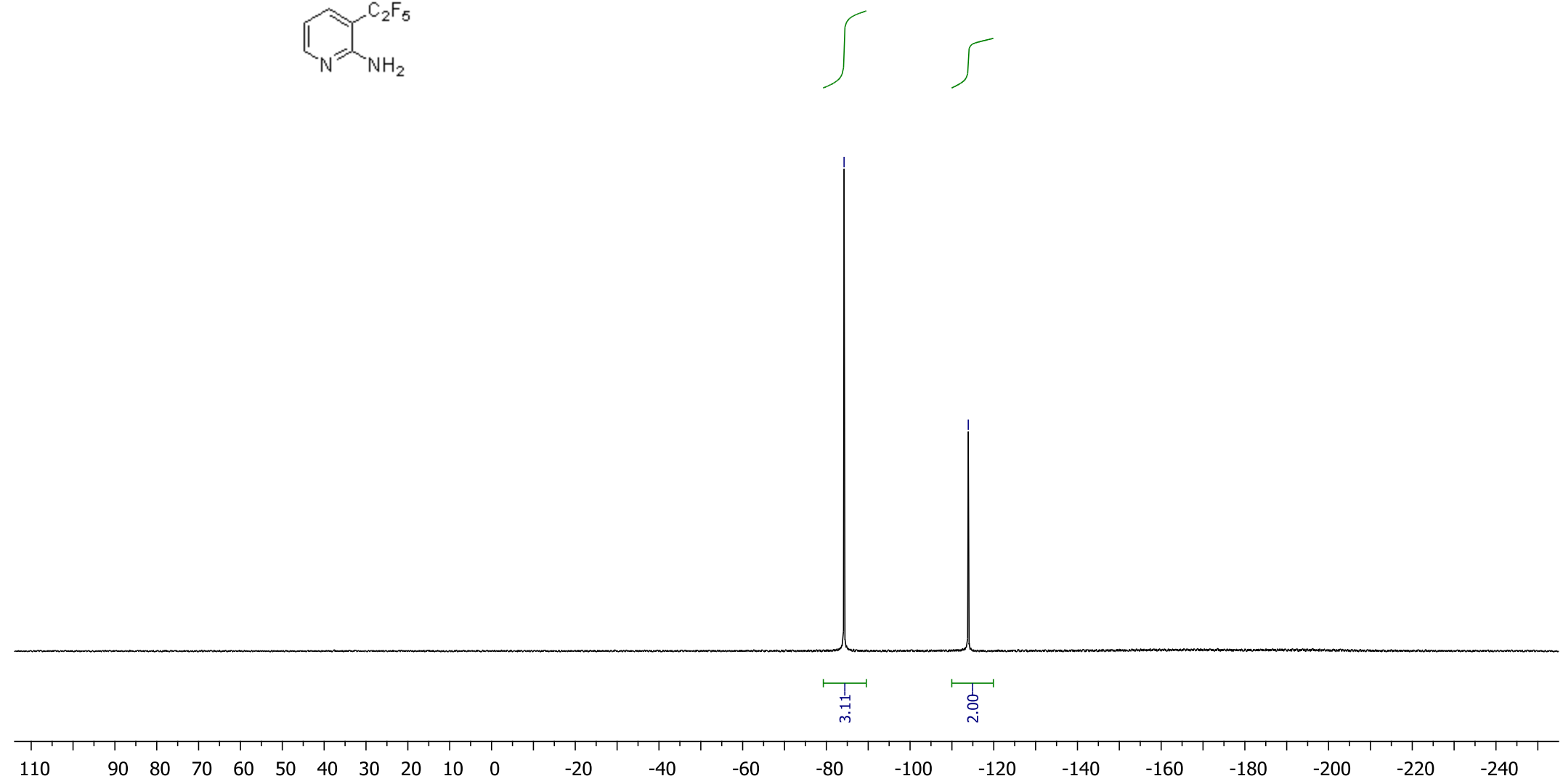


\section{Compound 59d}

${ }^{1} \mathrm{H}$ NMR (400 MHz, $\mathrm{CDCl}_{3}$ )

R1702687

|

$\overbrace{N} C_{N_{2}}^{C_{2} F_{5}}$ i.

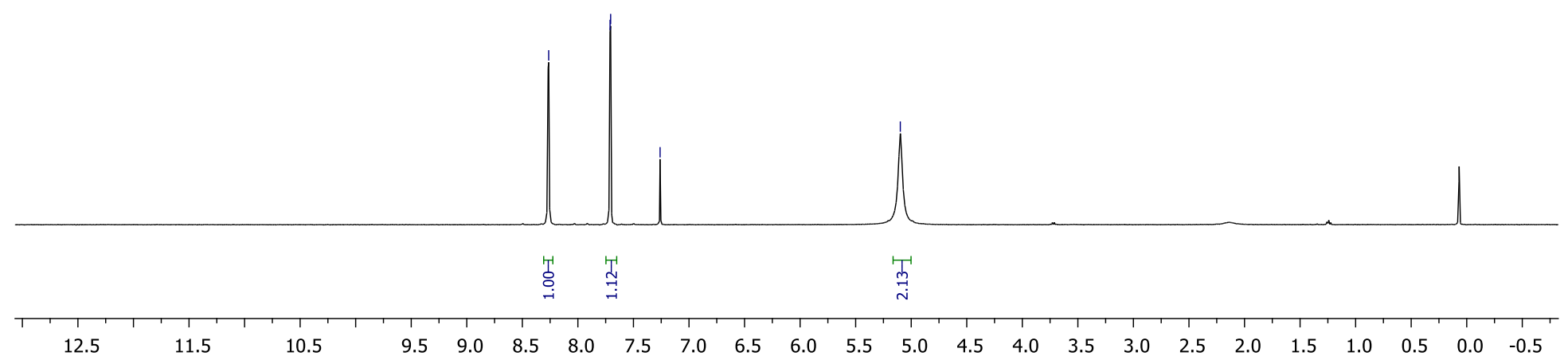


${ }^{13} \mathrm{C}\left\{{ }^{1} \mathrm{H}\right\} \mathrm{NMR}\left(126 \mathrm{MHz}, \mathrm{CDCl}_{3}\right)$

R1702687_13C

13C (1H-decoupled)

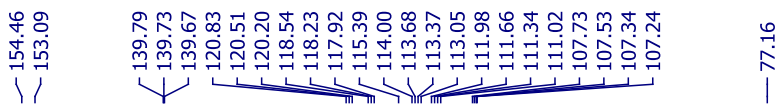
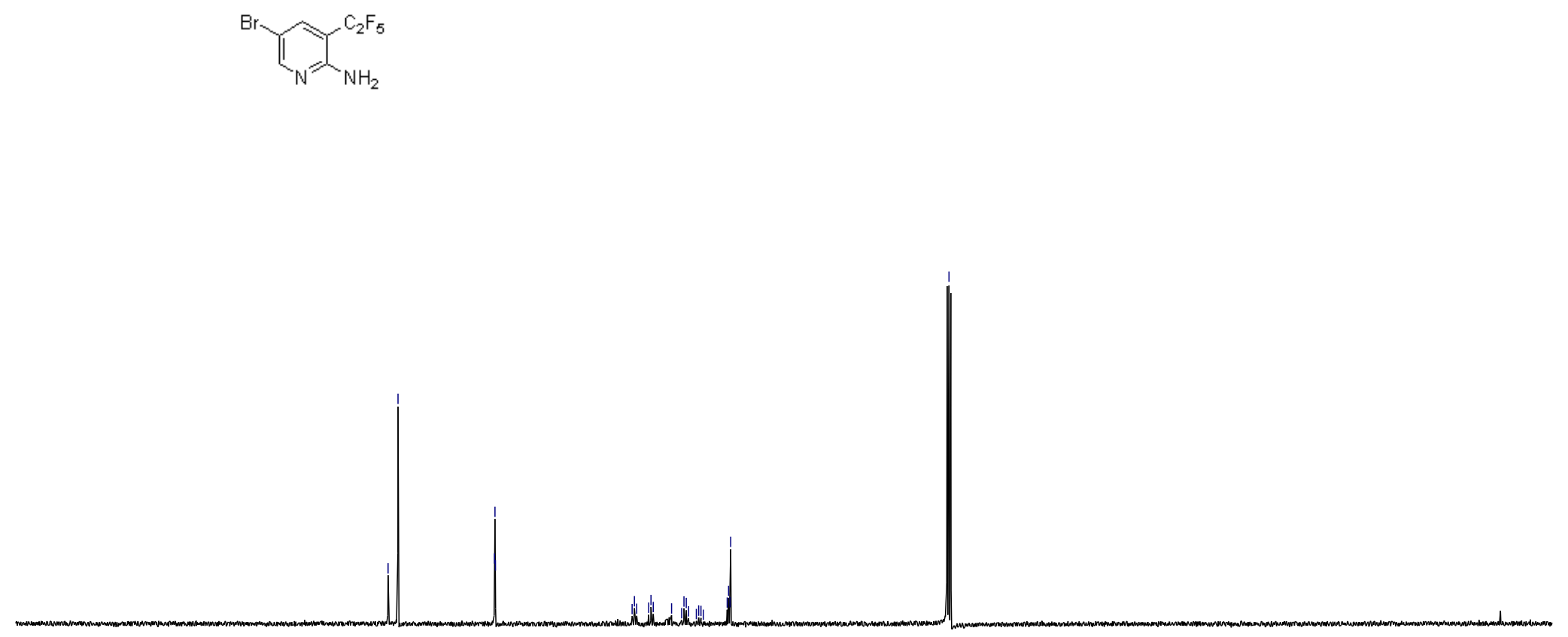

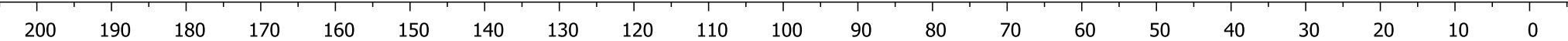


${ }^{19} \mathrm{~F}\left\{{ }^{1} \mathrm{H}\right\} \mathrm{NMR}\left(376 \mathrm{MHz}, \mathrm{CDCl}_{3}\right)$

R1702687_F19\{H\}

$\underset{\substack{\infty \\ \infty}}{\infty}$
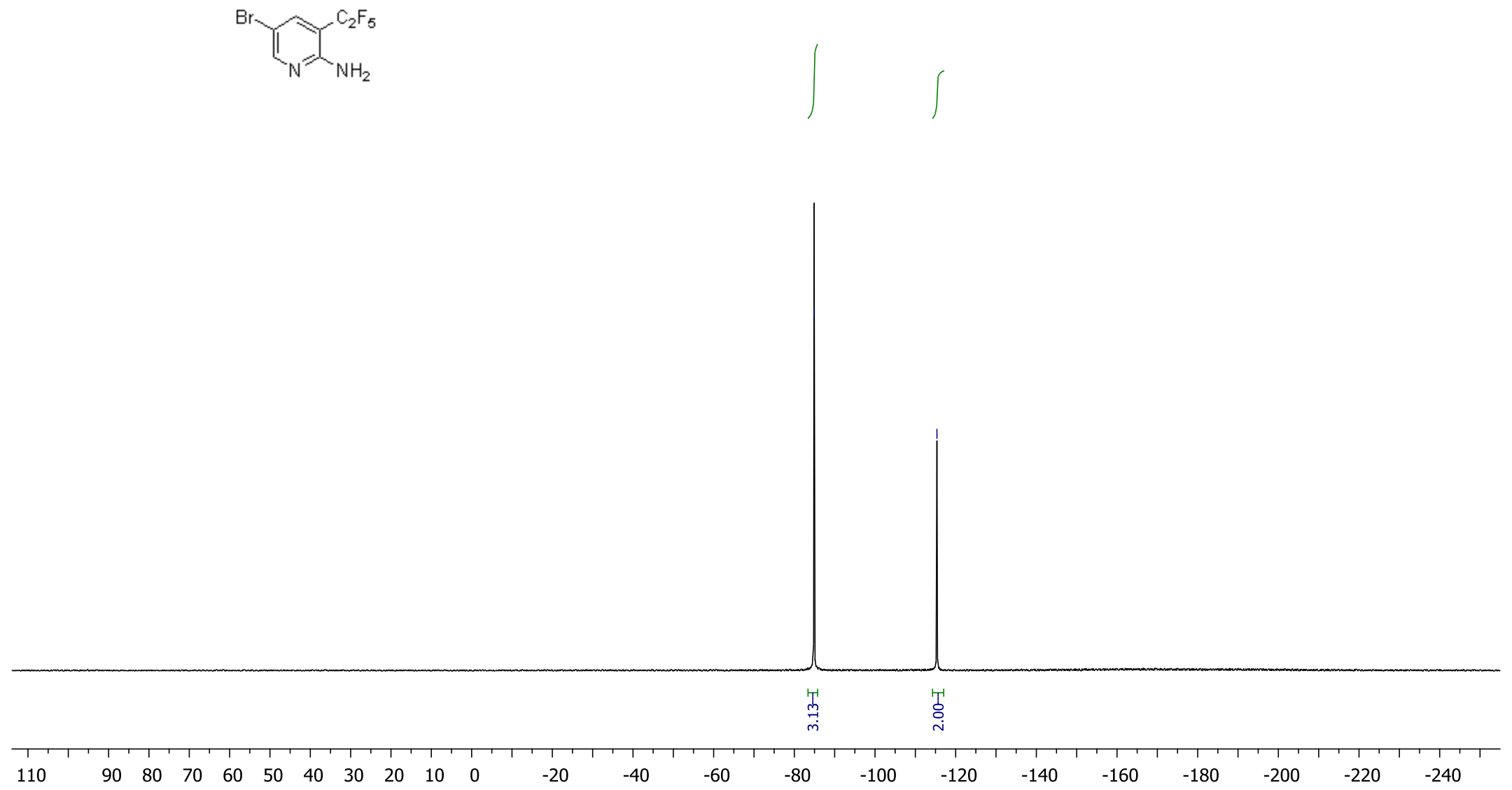


\section{Compound 63d}

${ }^{1} \mathrm{H}$ NMR (400 MHz, DMSO- $d_{6}$ )

R2399114

诲

$\mathrm{C}_{2} \mathrm{~F}_{5} \overbrace{\mathrm{N}} \overbrace{\mathrm{NH}_{2}}$
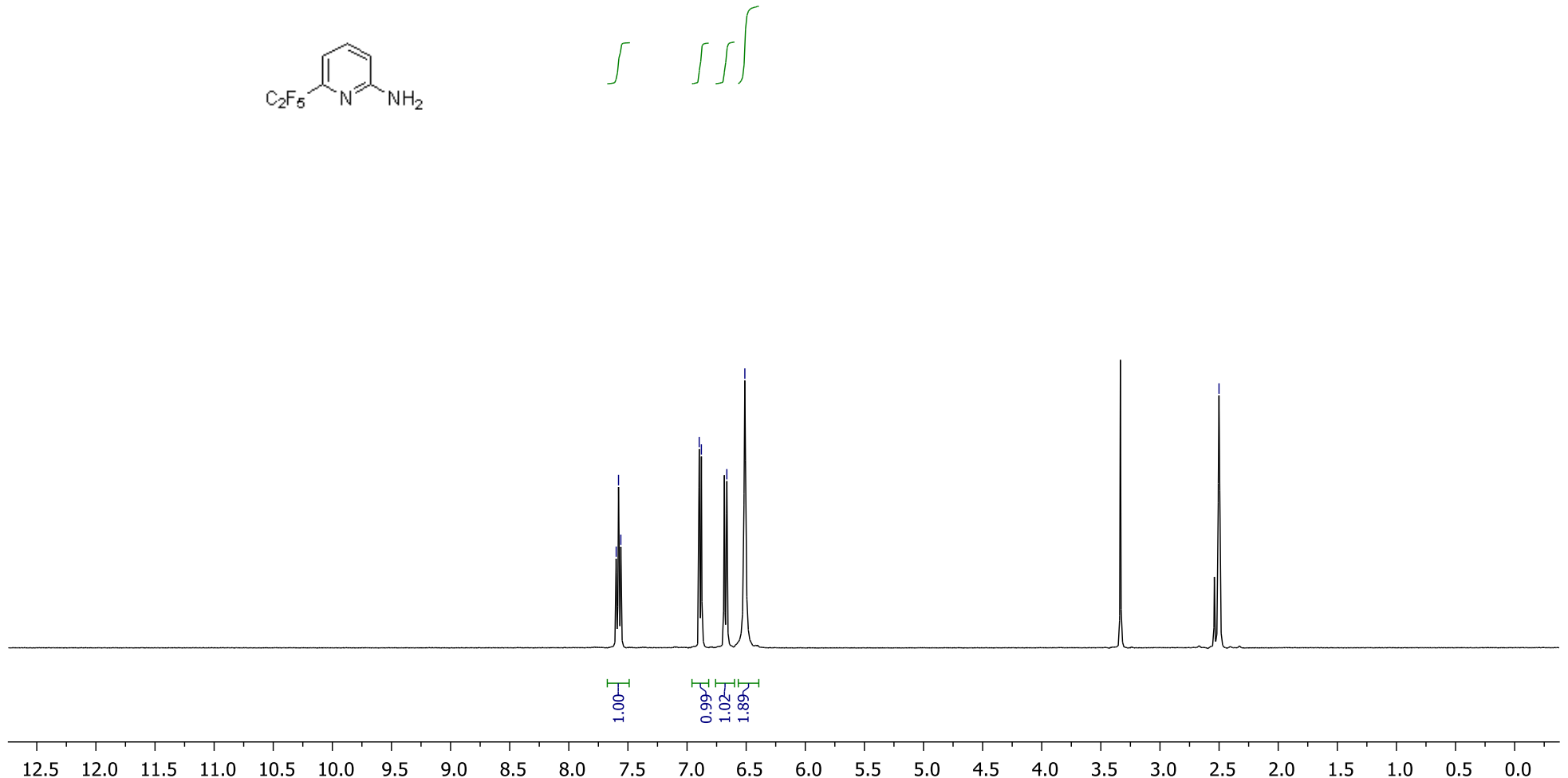
${ }^{13} \mathrm{C}\left\{{ }^{1} \mathrm{H}\right\}$ NMR (151 MHz, DMSO- $\left.d_{6}\right)$

R2399114_C13

జ

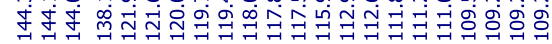

$\overbrace{2} \mathrm{~F}_{5} \overbrace{\mathrm{NH}_{2}}$

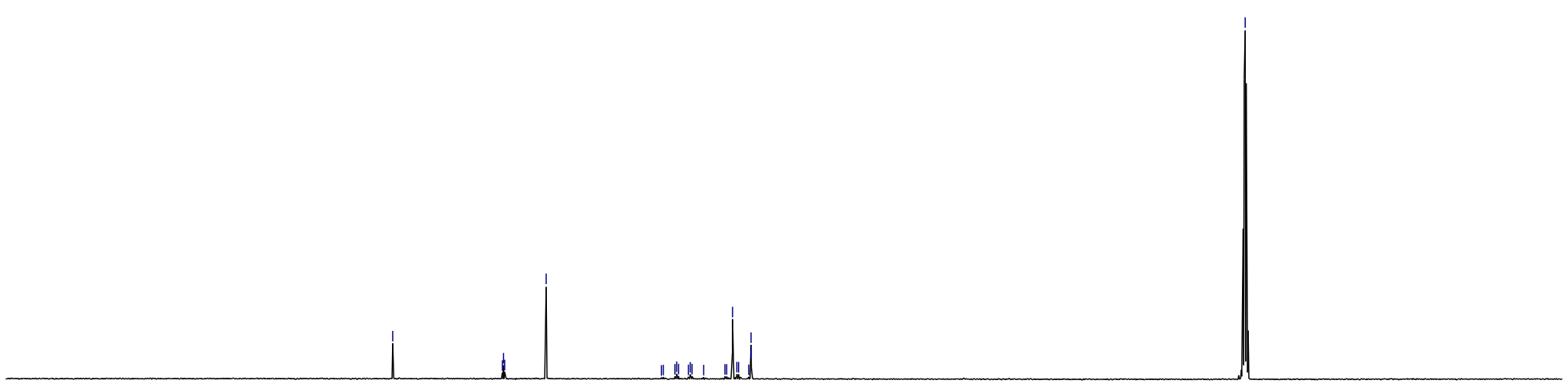

$210 \quad 200 \quad 190$

$\begin{array}{lllllllll}180 & 170 & 160 & 150 & 140 & 130 & 120 & 110 & 100\end{array}$

$90 \quad 80$

$\begin{array}{lll}70 & 60 & 50\end{array}$

$40 \quad 30$

20 


\section{${ }^{19} \mathrm{~F}\left\{{ }^{1} \mathrm{H}\right\}$ NMR (376 MHz, DMSO- $\left.d_{6}\right)$}

R2399114_F19 $\{\mathrm{H}\}$

$19 \mathrm{~F}-\{1 \mathrm{H}\}$

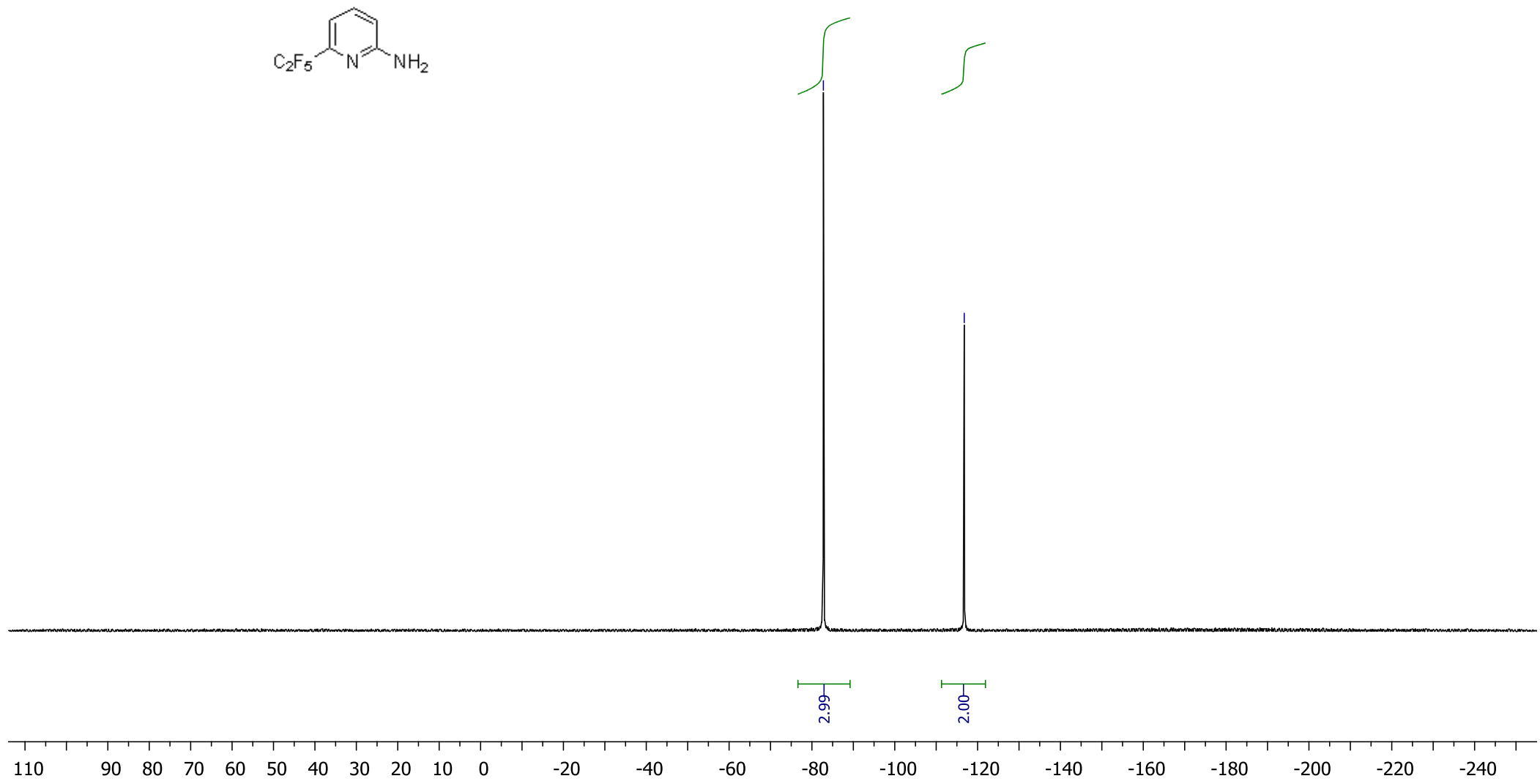




\section{Compound 15d*HCl}

${ }^{1} \mathrm{H}$ NMR (400 MHz, DMSO- $d_{6}$ )

R1571716

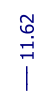

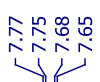

ทั่



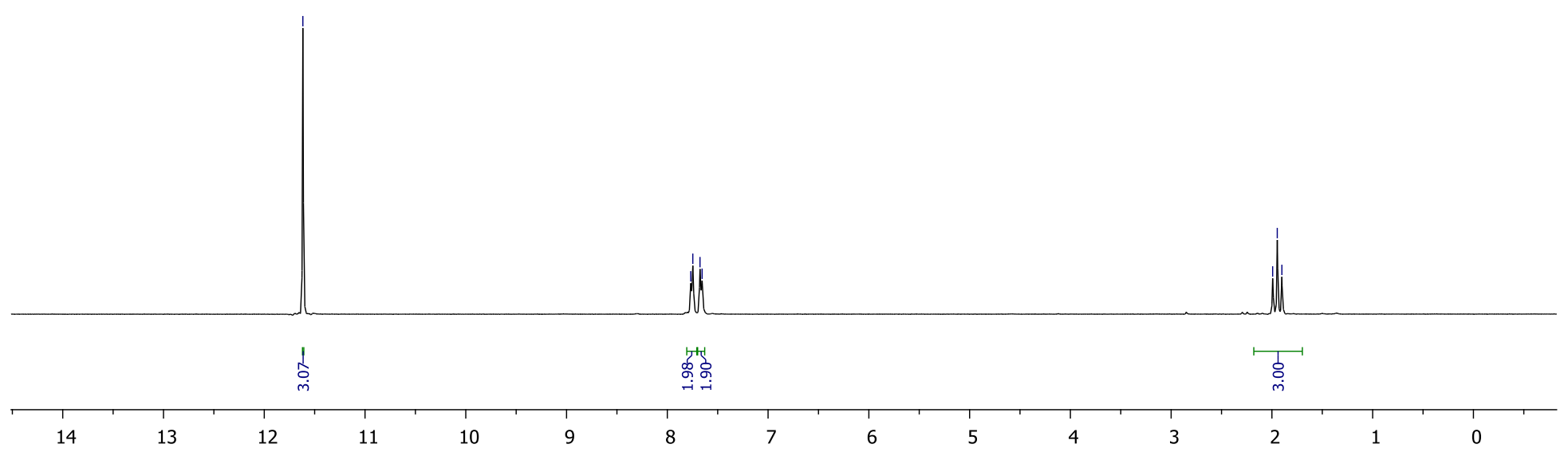


${ }^{13} \mathrm{C}\left\{{ }^{1} \mathrm{H}\right\}$ NMR (126 MHz, DMSO- $\left.d_{6}\right)$

R1571716_C1:

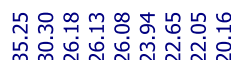

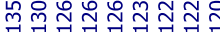

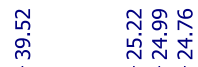

난
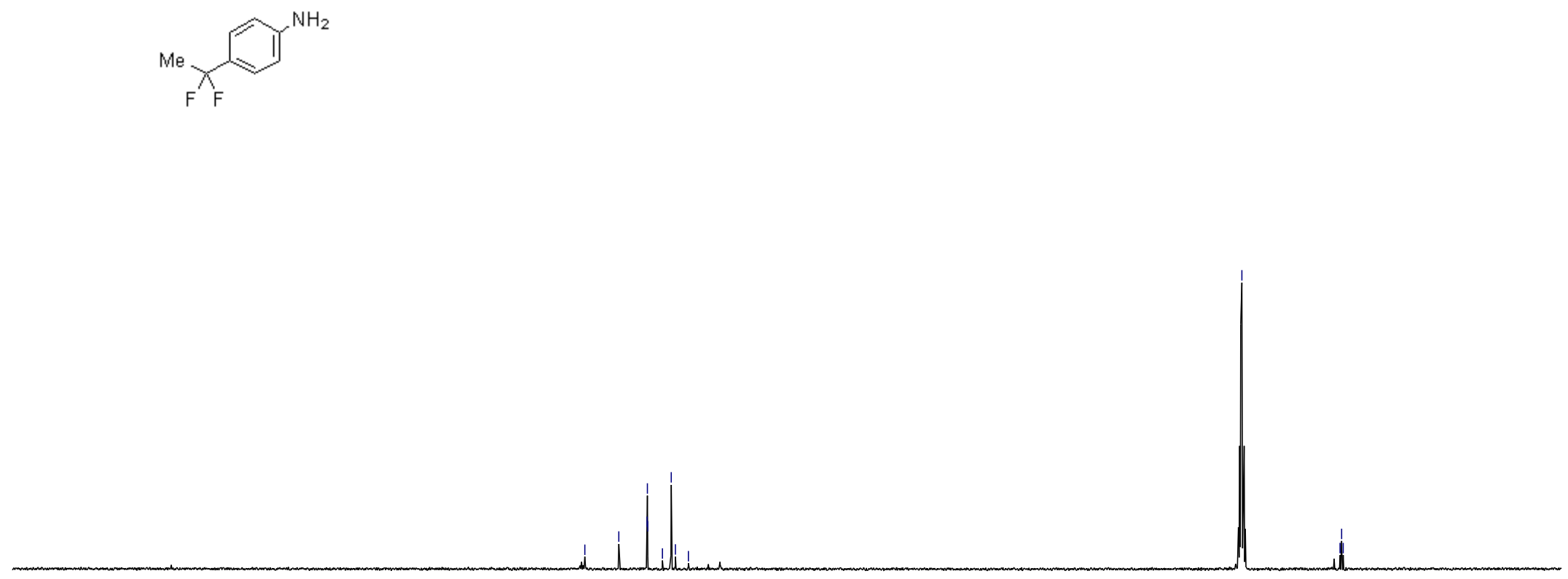

$\begin{array}{llllllllllllllllllllll}210 & 200 & 190 & 180 & 170 & 160 & 150 & 140 & 130 & 120 & 110 & 100 & 90 & 80 & 70 & 60 & 50 & 40 & 30 & 20 & 10 & 0\end{array}$ 


\section{${ }^{19} \mathrm{~F}\left\{{ }^{1} \mathrm{H}\right\}$ NMR (376 MHz, DMSO- $\left.d_{6}\right)$}

R1571716_F19

$19 \mathrm{~F}-\{1 \mathrm{H}\}$

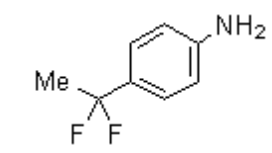

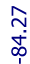

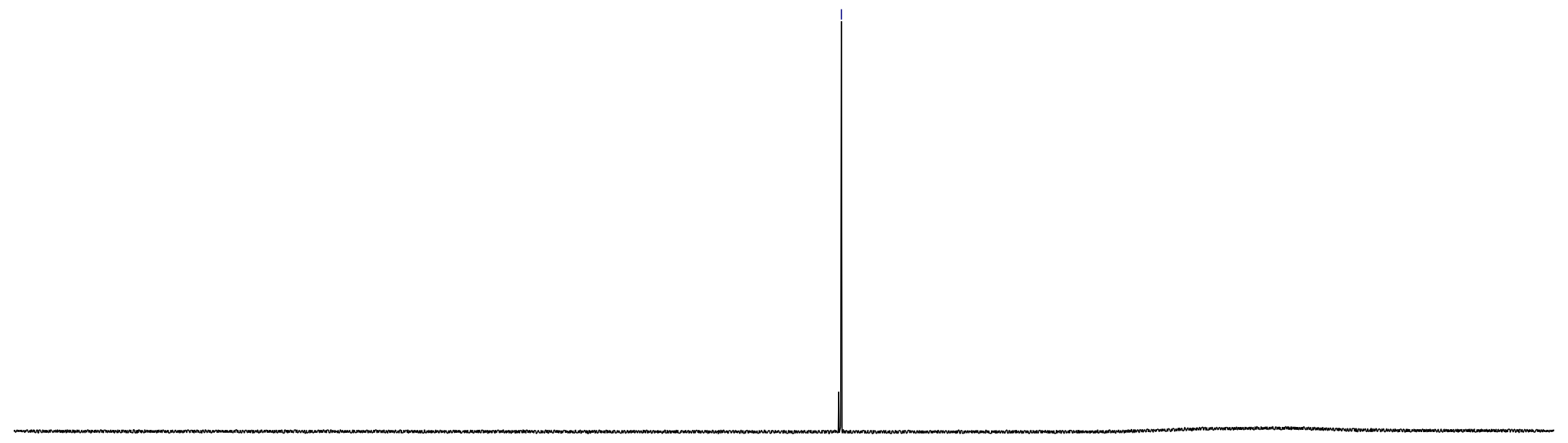




\section{Compound 50d*HCl}

${ }^{1} \mathrm{H}$ NMR (400 MHz, DMSO- $d_{6}$ )

R1343453

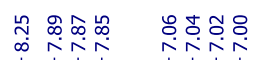

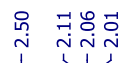

朴迕

रा
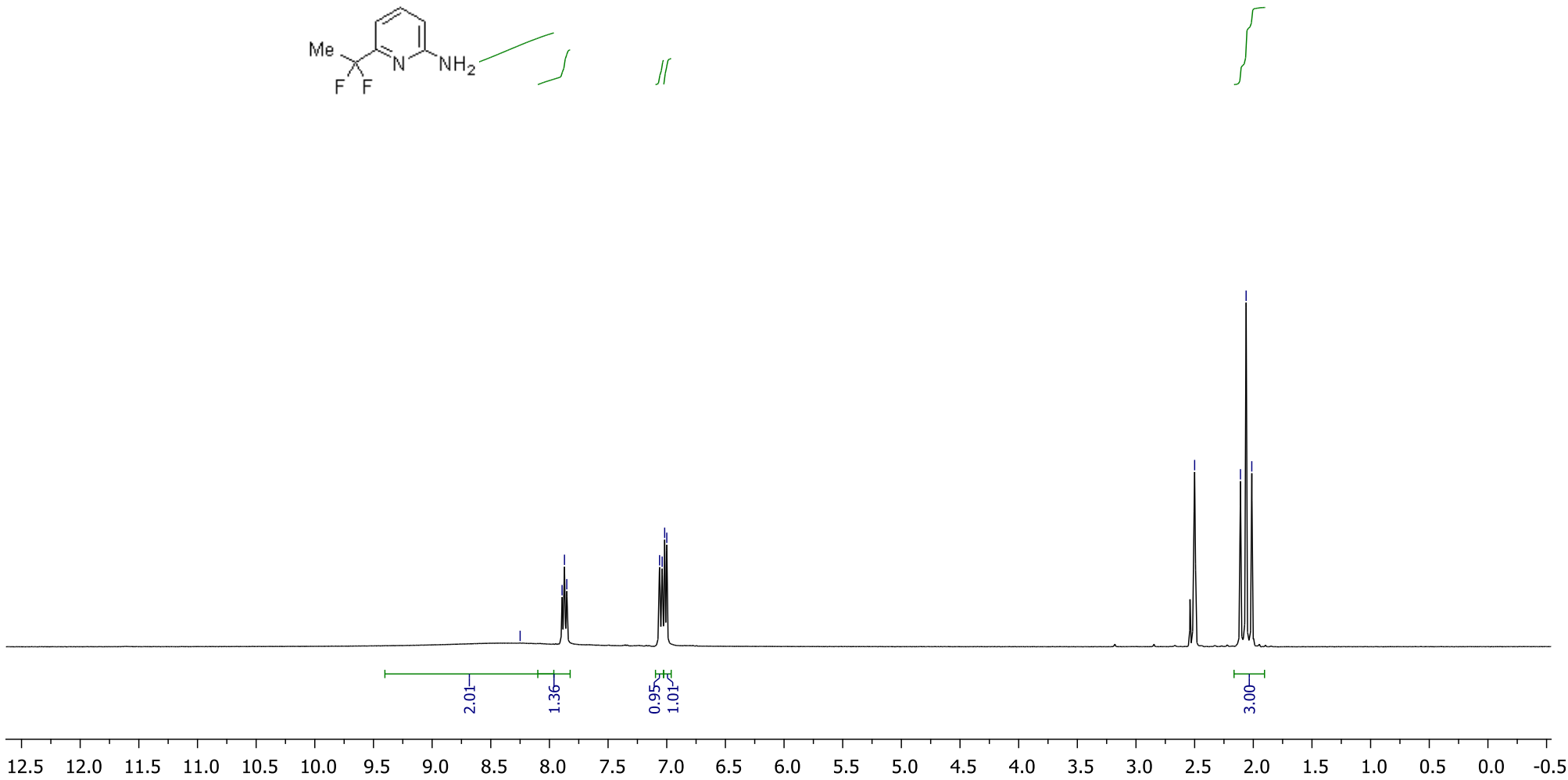
${ }^{13} \mathrm{C}\left\{{ }^{1} \mathrm{H}\right\}$ NMR (126 MHz, DMSO- $\left.d_{6}\right)$

R1343453_C1:
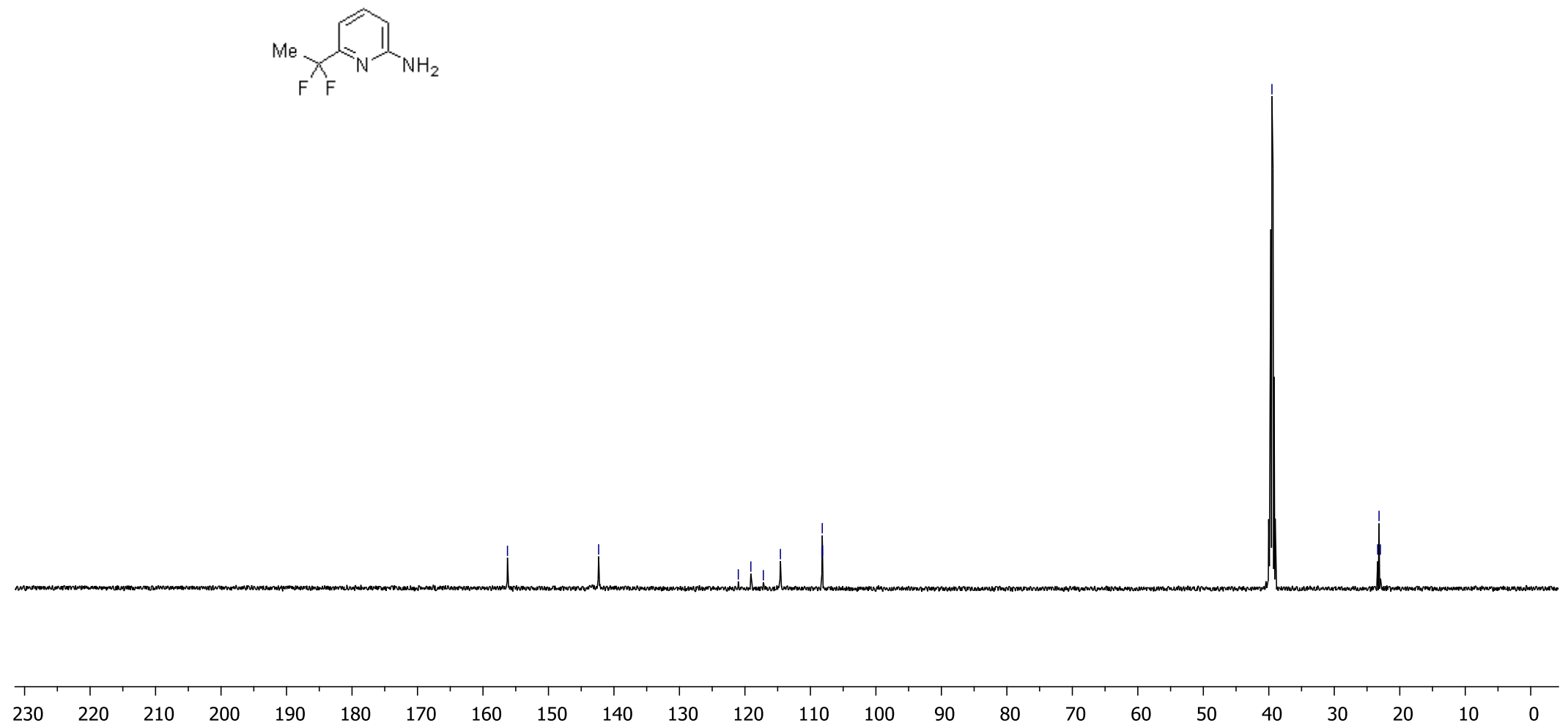
${ }^{19} \mathrm{~F}\left\{{ }^{1} \mathrm{H}\right\}$ NMR (376 MHz, $\left.\mathrm{D}_{2} \mathrm{O}\right)$

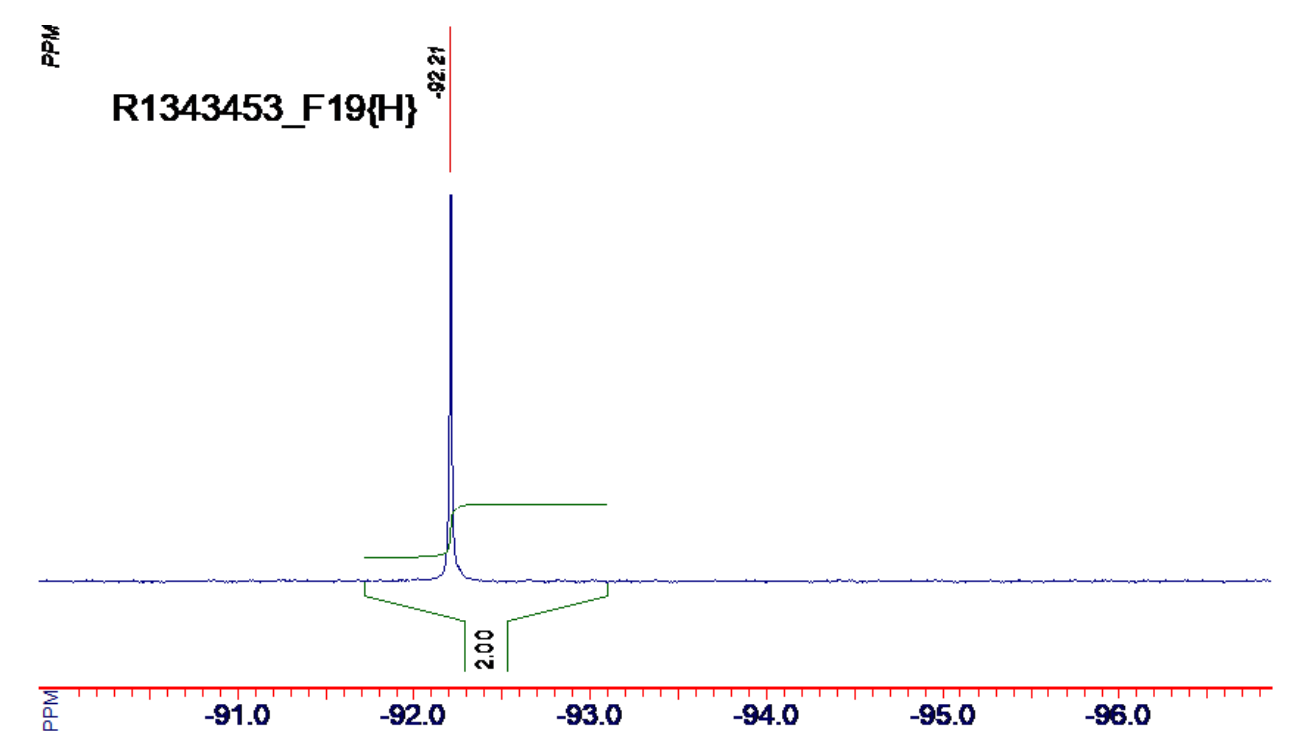

c

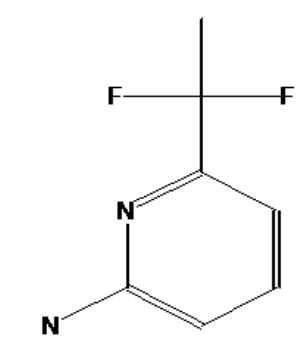

R1343453 F19H C7H9CIF2ND 194.61

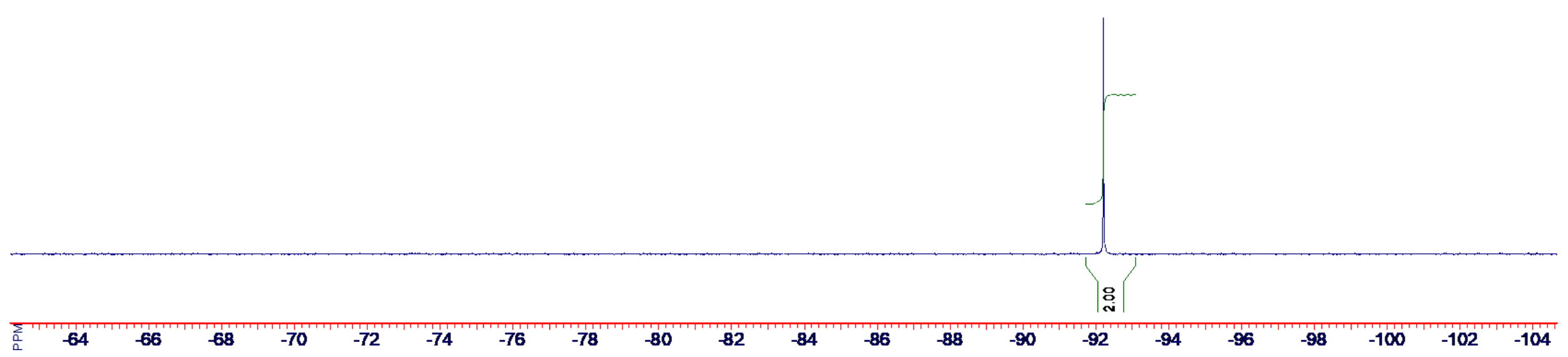




\section{Compound 48d}

${ }^{1} \mathrm{H}$ NMR (400 MHz, DMSO- $d_{6}$ )

R1436433

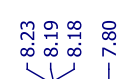

บั่

$\mathrm{CO}_{\mathrm{N}}^{\mathrm{NH}_{2}}$
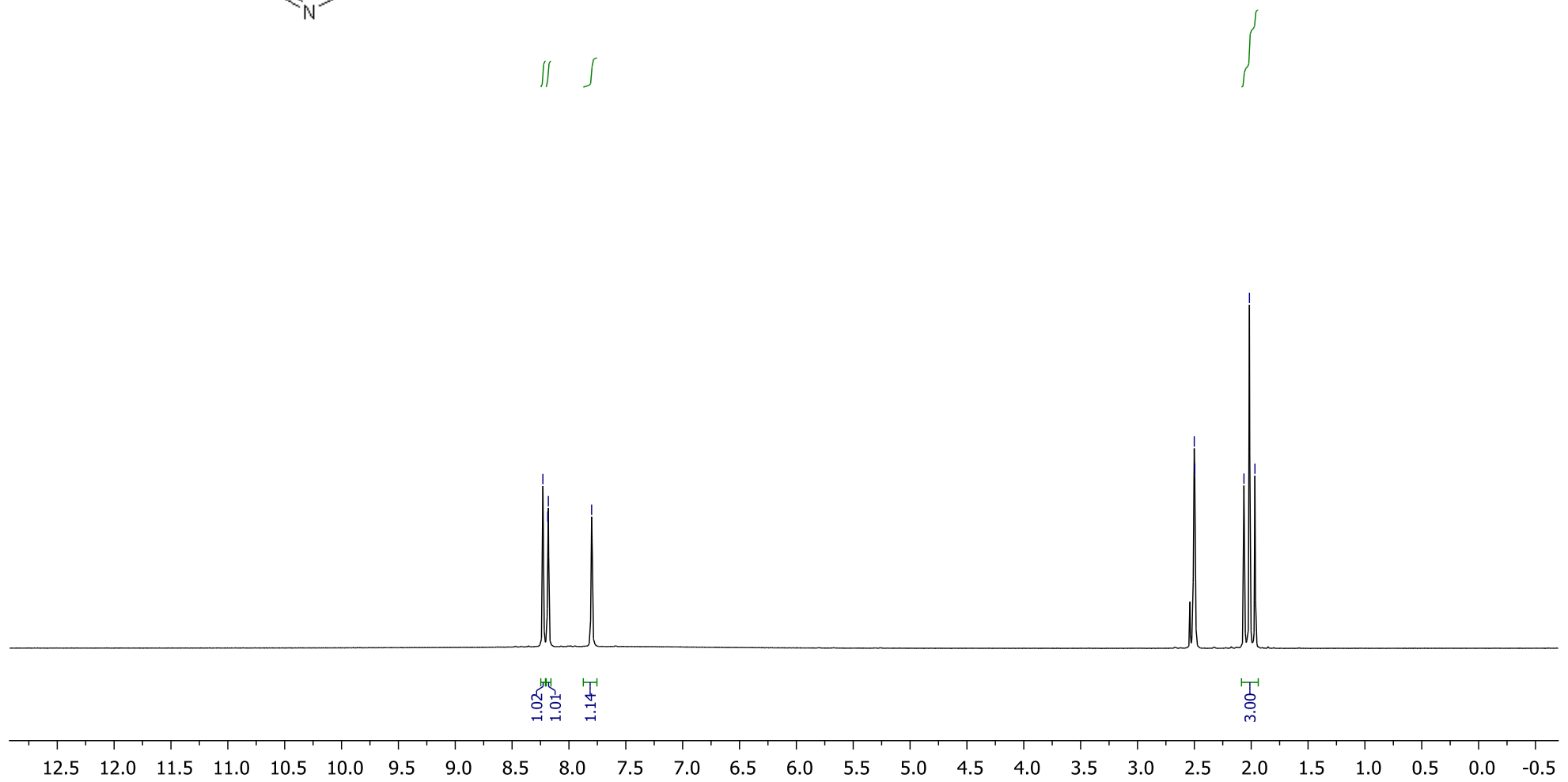
${ }^{13} \mathrm{C}\left\{{ }^{1} \mathrm{H}\right\}$ NMR $\left(126 \mathrm{MHz}, \mathrm{DMSO}-d_{6}\right)$

R1436433_C1:

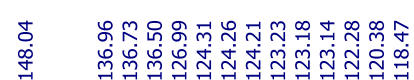

กู

$1+4$

Me

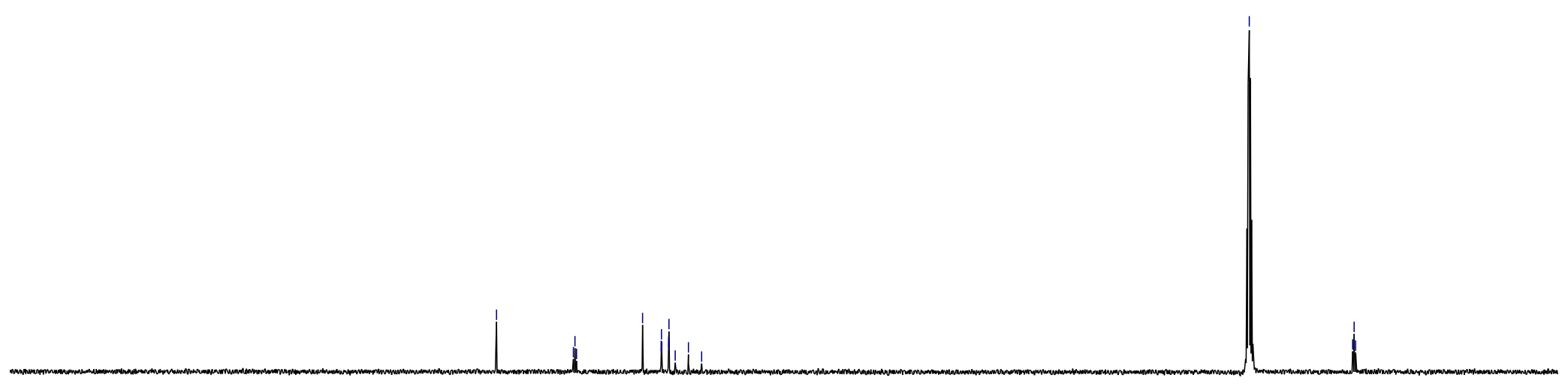

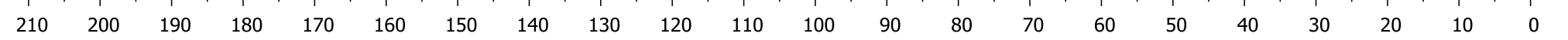




\section{${ }^{19} \mathrm{~F}\left\{{ }^{1} \mathrm{H}\right\}$ NMR (376 MHz, DMSO- $\left.d_{6}\right)$}

R1436433_F19\{H\} $19 \mathrm{~F}-\{1 \mathrm{H}\}$
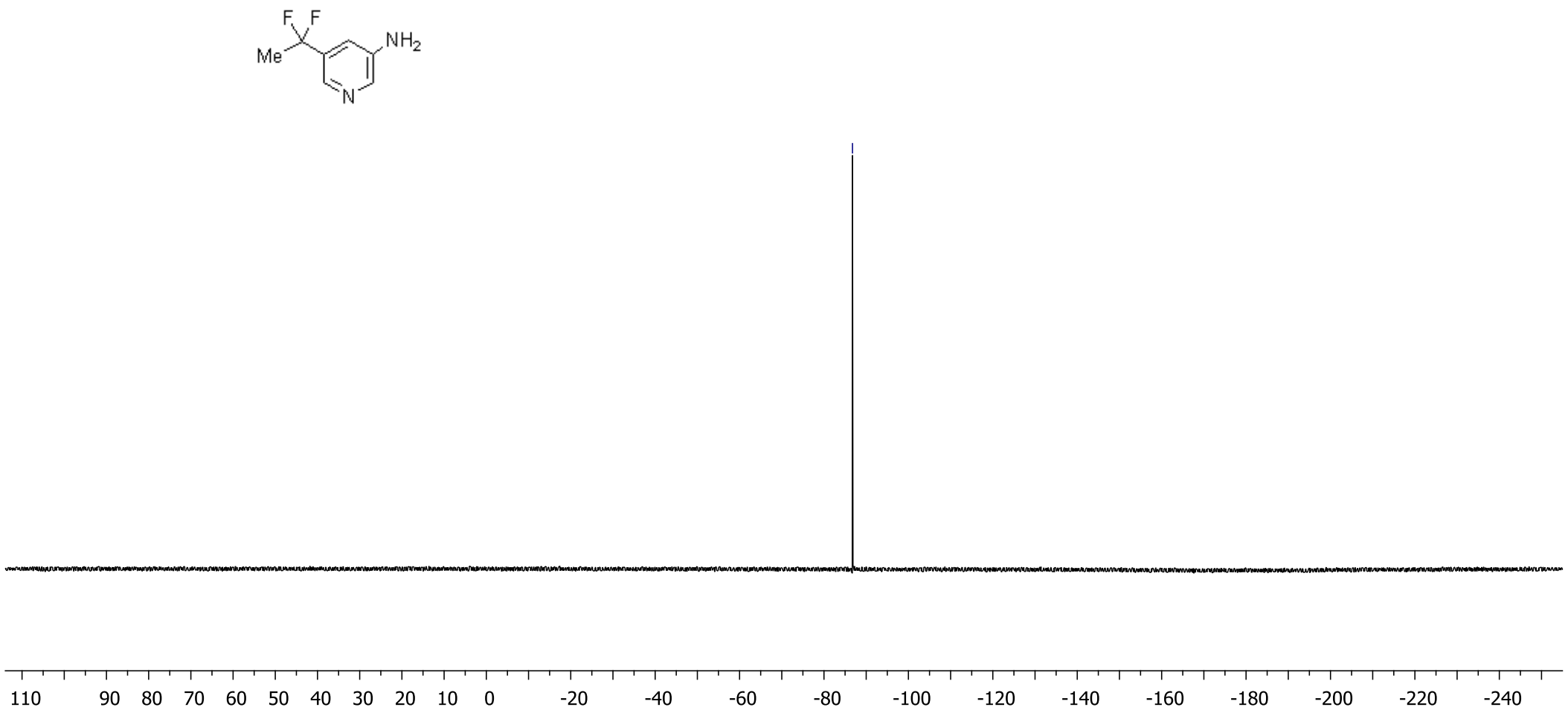


\section{Compound 62d}

${ }^{1} \mathrm{H}$ NMR (400 MHz, DMSO- $d_{6}$ )

R2498093
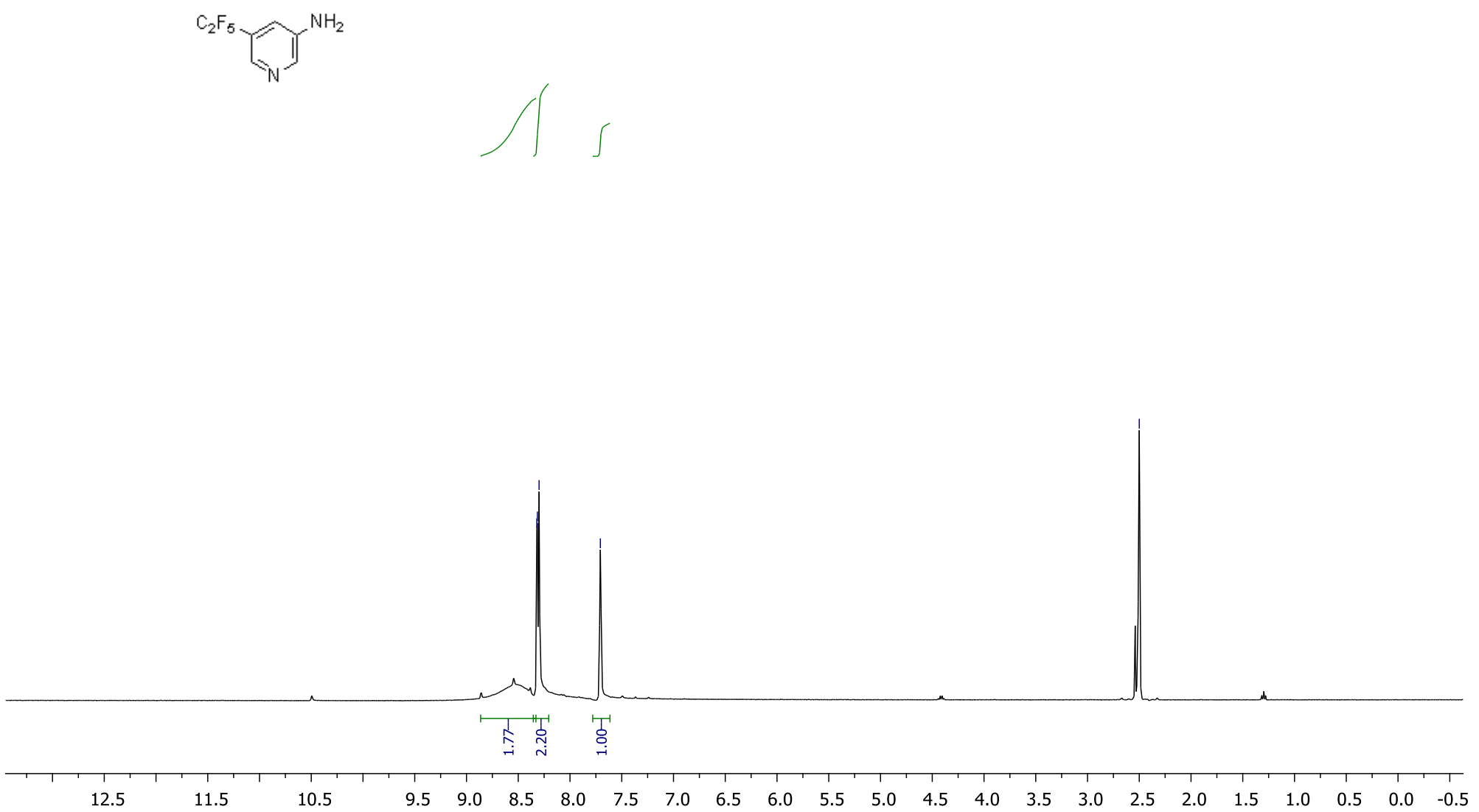
${ }^{13} \mathrm{C}\left\{{ }^{1} \mathrm{H}\right\}$ NMR (151 MHz, DMSO- $\left.d_{6}\right)$

R2498093_C13

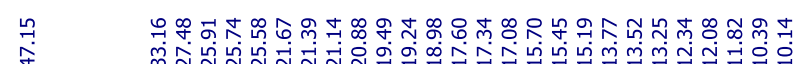

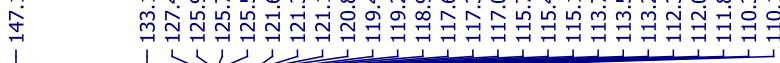

$$
\mathrm{C}_{2} \mathrm{~F}_{5}
$$

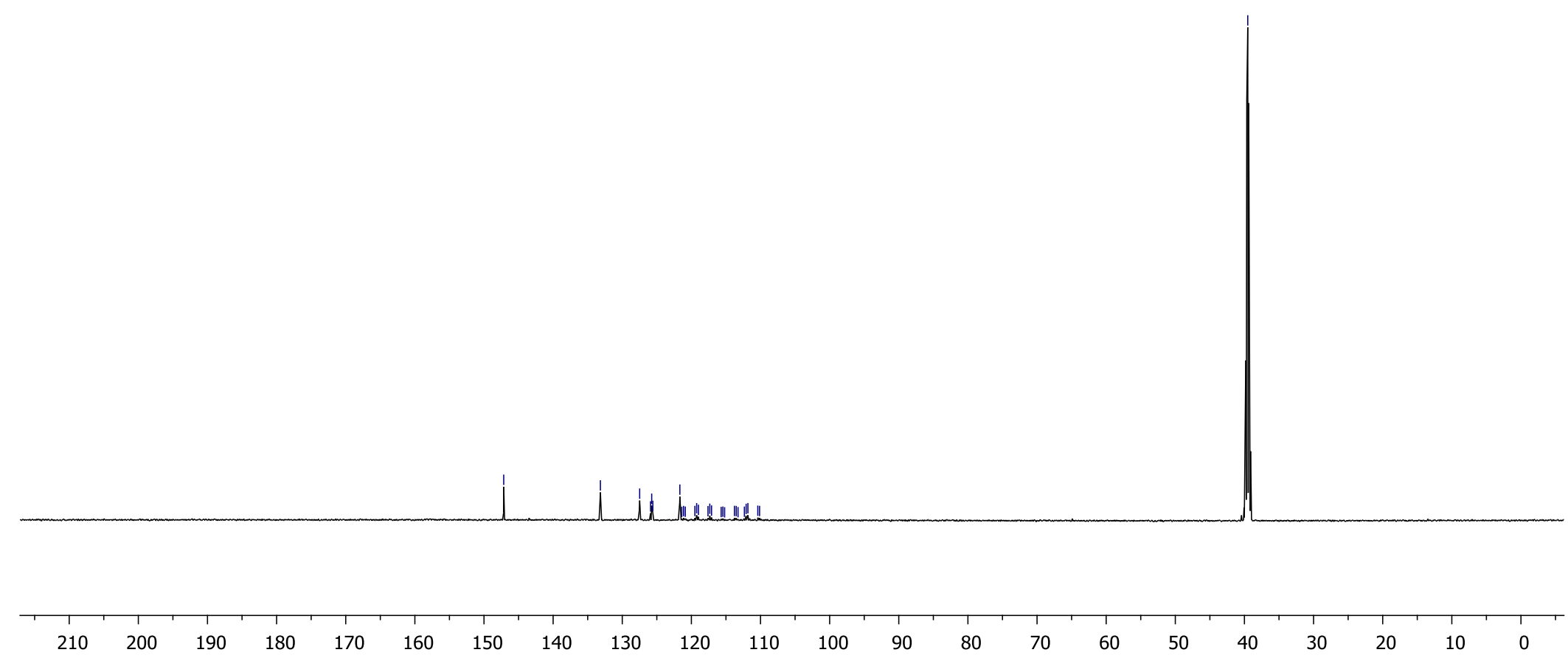




\section{${ }^{19} \mathrm{~F}\left\{{ }^{1} \mathrm{H}\right\}$ NMR (376 MHz, DMSO- $\left.d_{6}\right)$}

R2498093_F19\{H\}

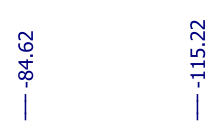

$$
\mathrm{C}_{2} \mathrm{~F}_{5}
$$
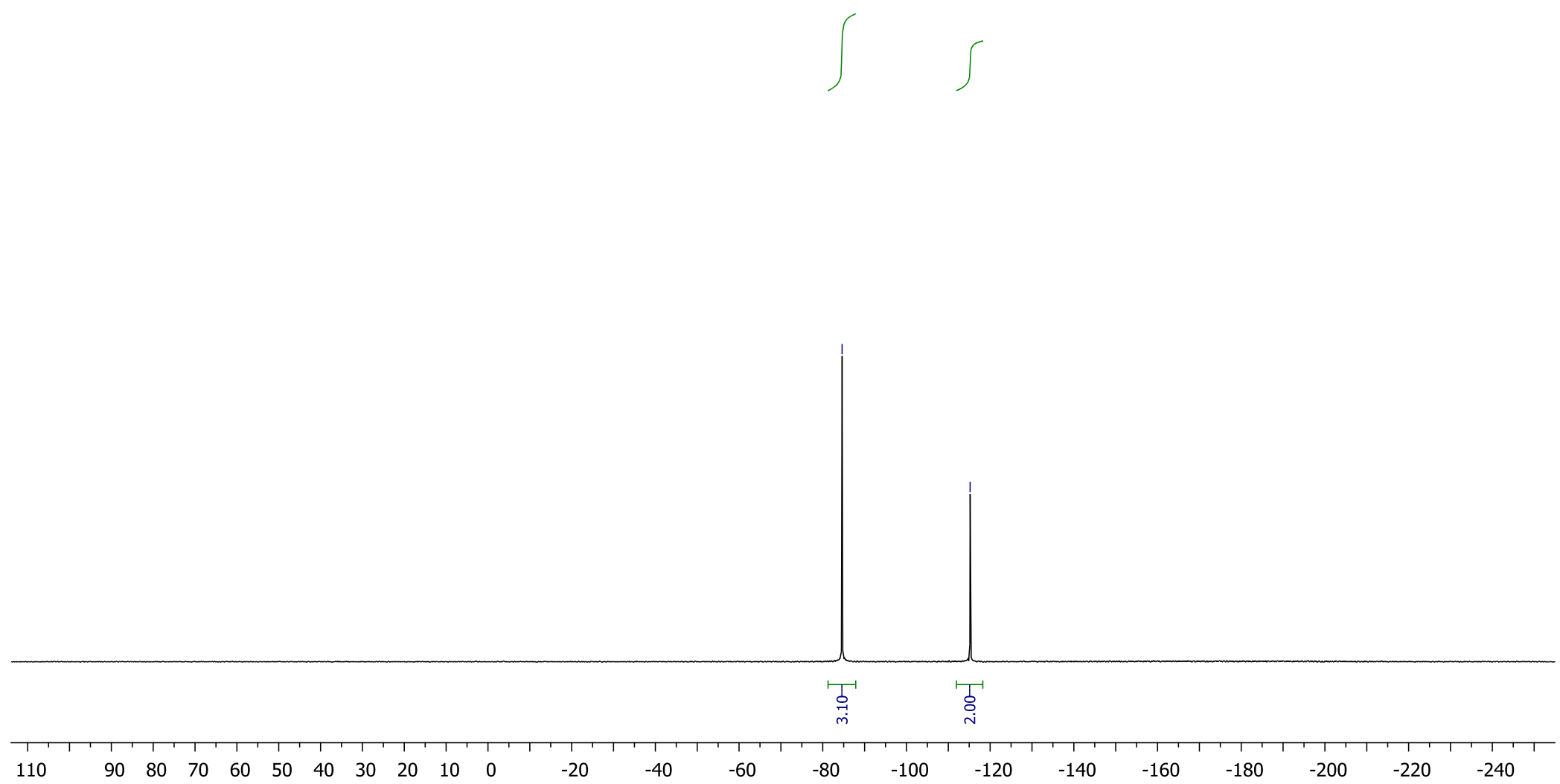


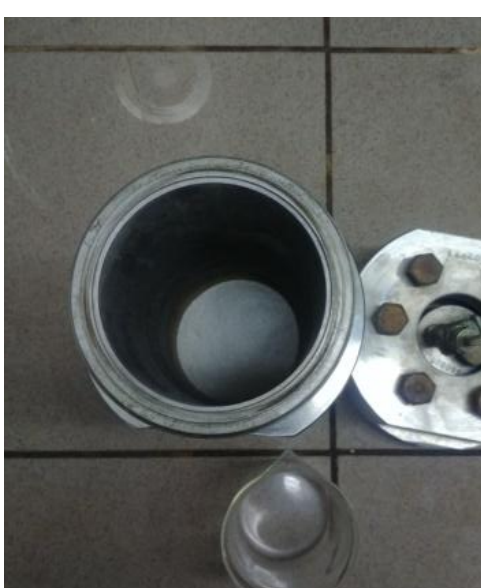

A high pressure reactor.

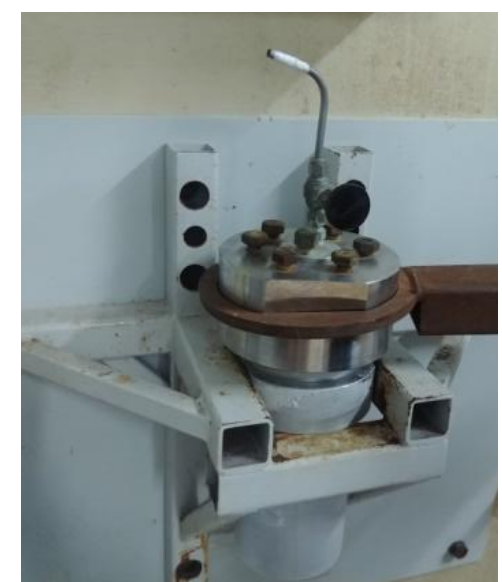

Installation of the high pressure reactor.

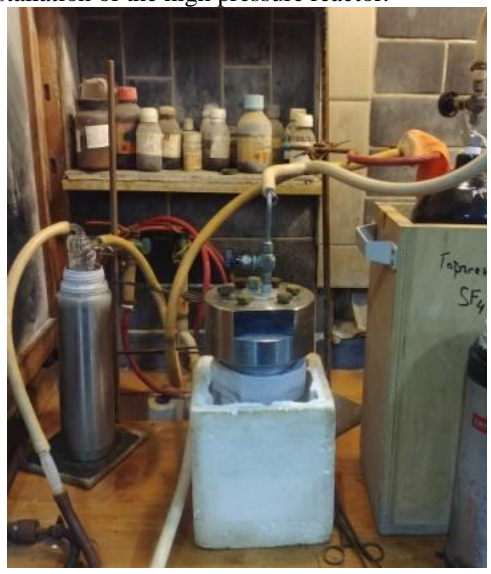

System for gas blowing.

\section{Photos}

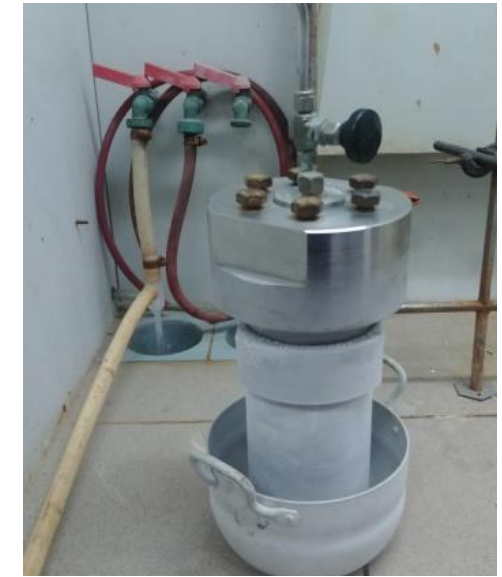

Cooling down the reaction mixture..

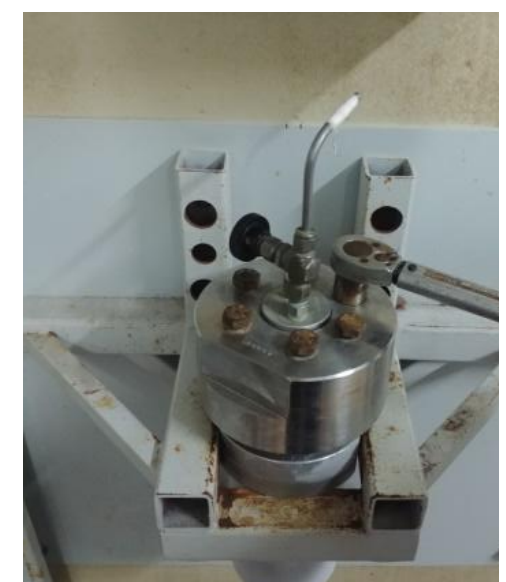

Installation of the high pressure reactor.

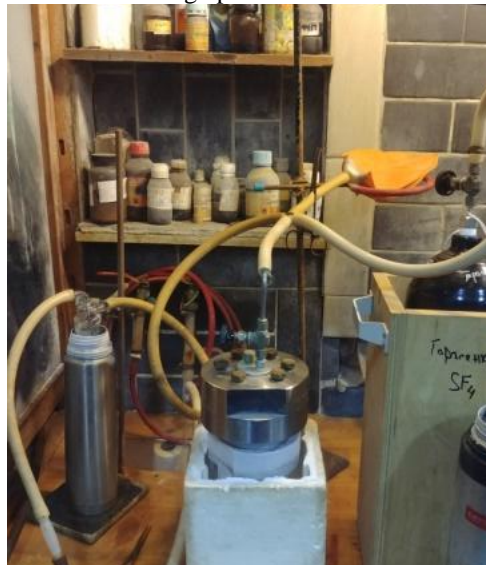

Vacuuming of the reaction system. A balloon is closed with a hemostat.

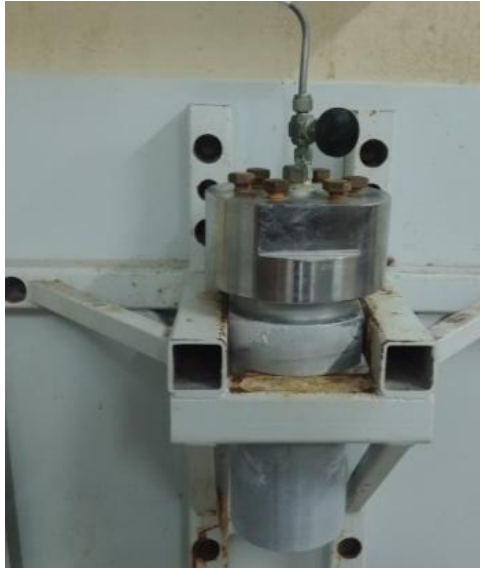

Fixing the high pressure reactor on the special

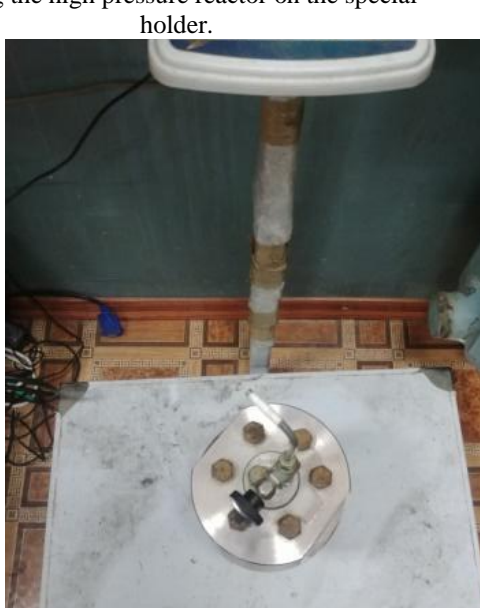

Weighing of the reaction mixture.

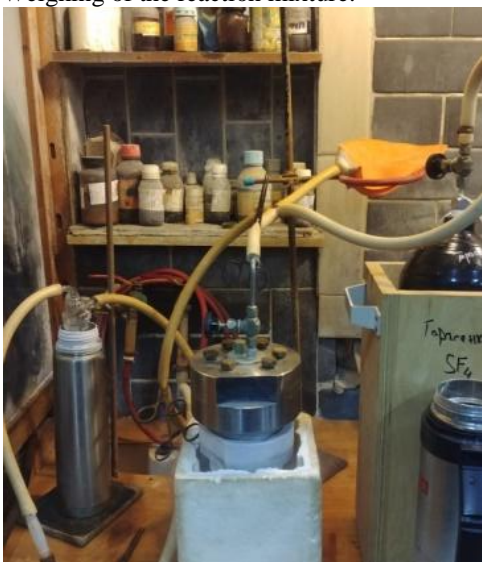

Preparation for pumping up the balloon with $\mathrm{SF}_{4}$ The high pressure reactor is closed with a hemostat. 


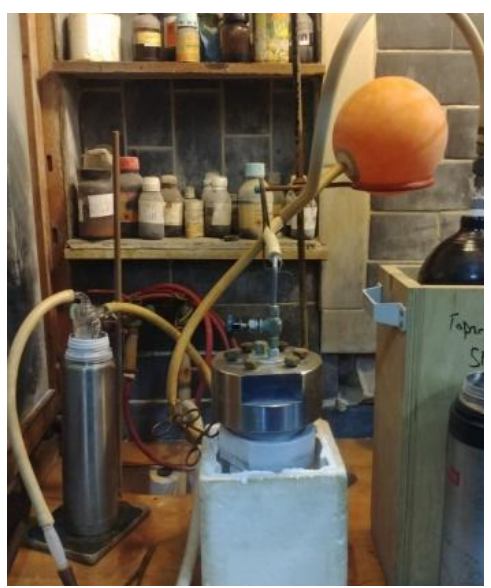

Inflating a balloon.

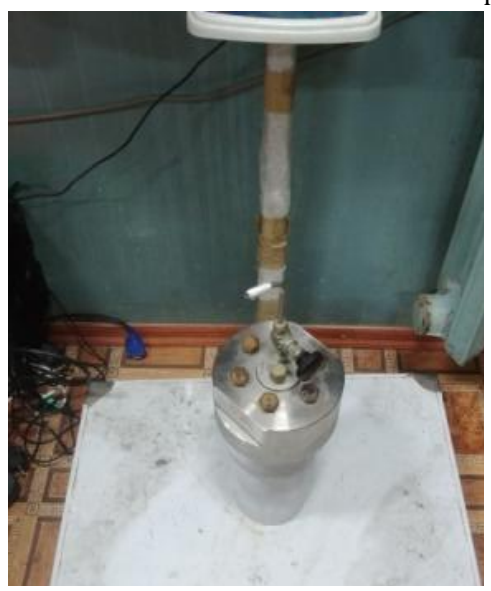

Weighing of the reaction mixture with $\mathrm{SF}_{4}$.

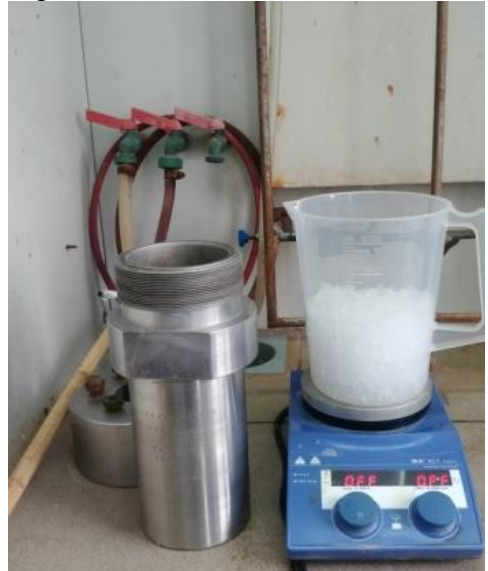

The opened high pressure reactor.

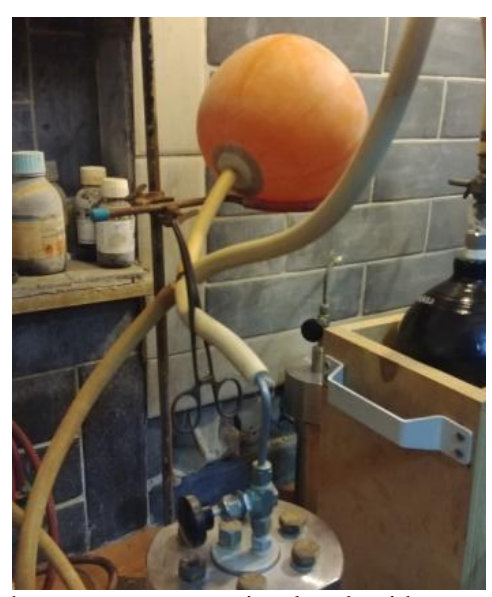

The high pressure reactor is closed with a hemostat.

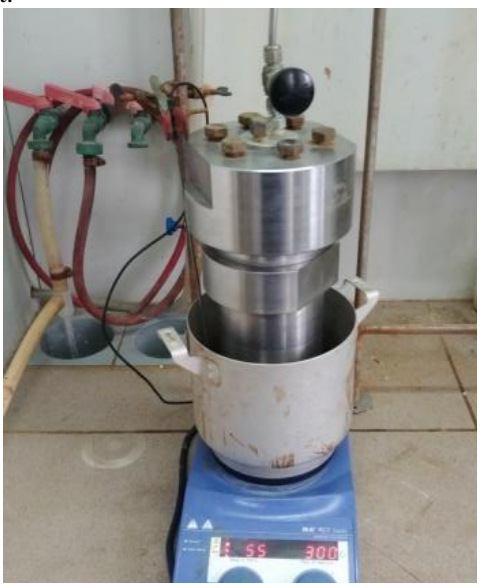

The high pressure reactor placed in oil bath.

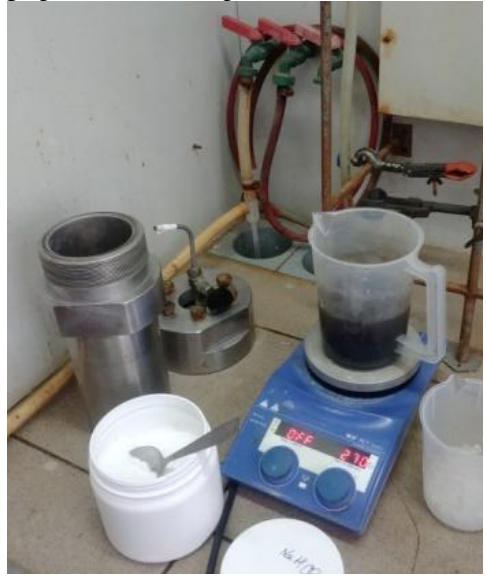

Quenching with $\mathrm{NaHCO}_{3}$

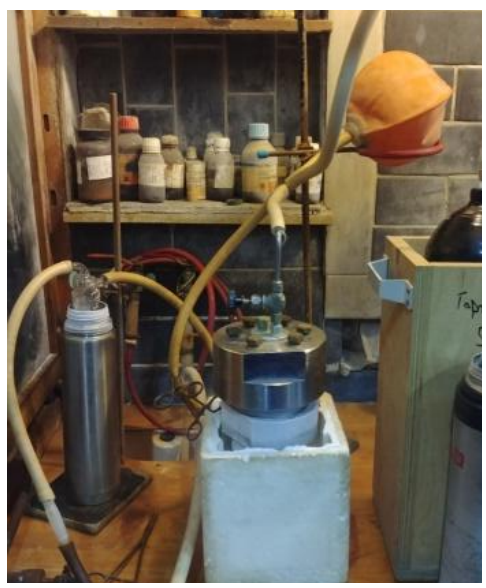

Gas injection into the reaction mixture.

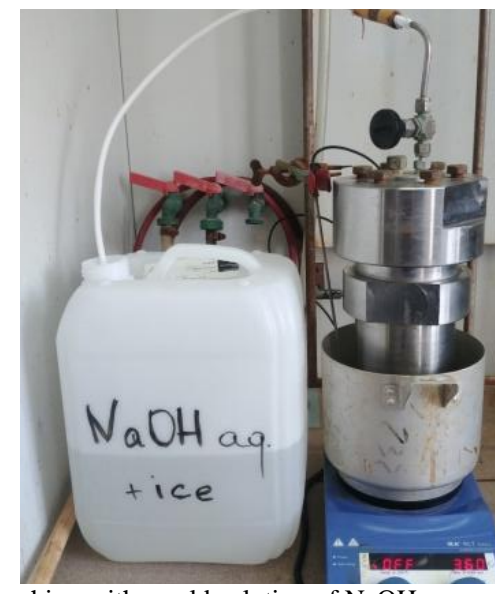

Quenching with a cold solution of $\mathrm{NaOH}$.

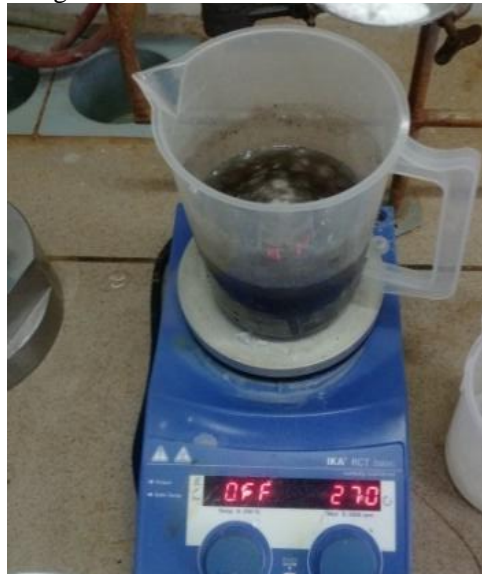

Quenching with $\mathrm{NaHCO}_{3}$. 